\title{
BREAKING DOWN THE BARRIERS
}

THE EFFECTS OFAGING, GUT MICROBIOTAAND BILE

ACIDS ON INTESTINAL HEALTH

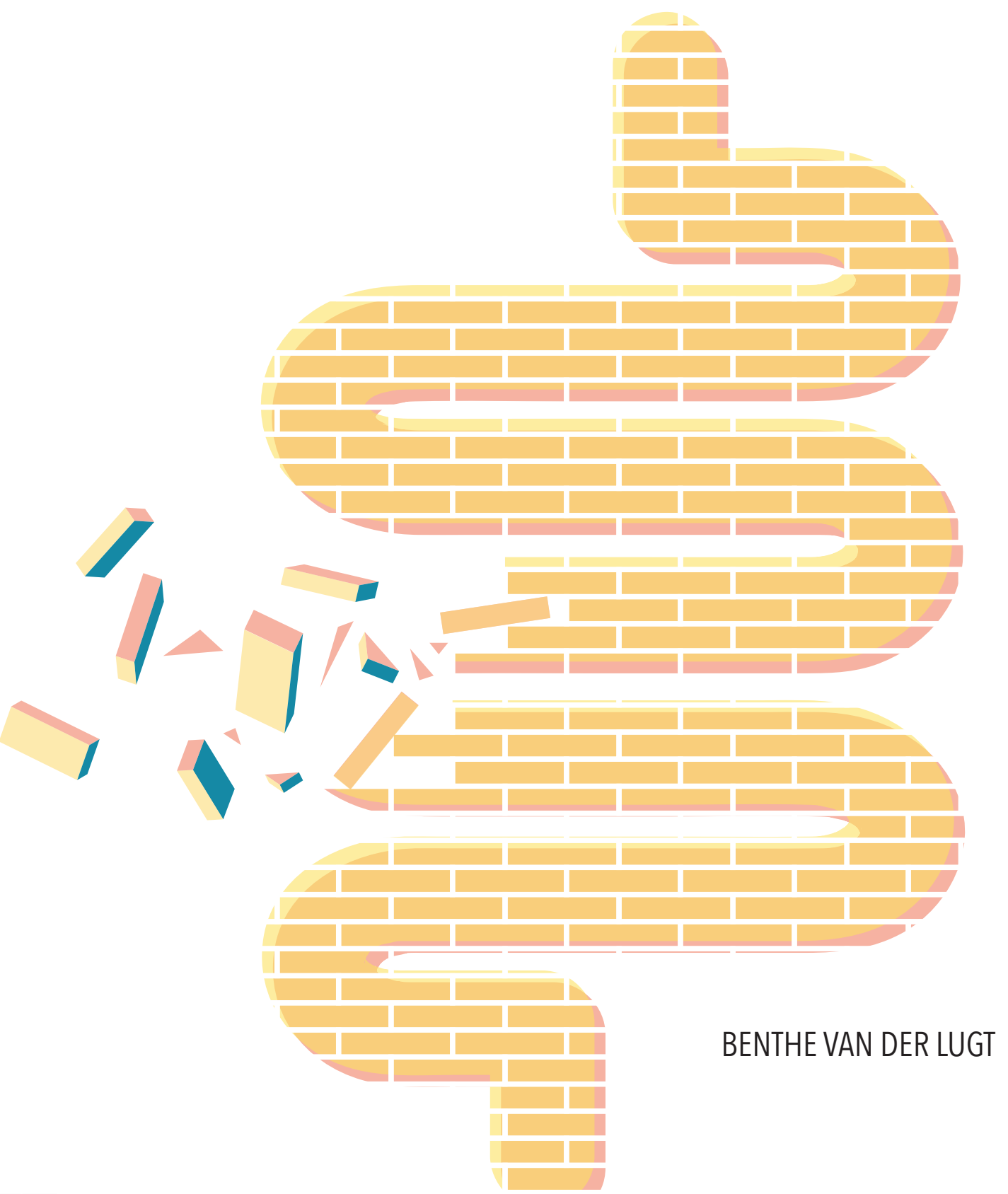




\section{PROPOSITIONS}

1. A low, rather than a high ratio of sulfated over unsulfated secondary bile acids would be beneficial for IBD patients during a flare. (this thesis)

2. To be physiologically representative, intestinal in vitro models require shear stress.

(this thesis)

3. Price-consciousness is a crucial aspect of scientific integrity.

4. The duration of a PhD track is insufficient if a fundamental part of a project requires optimization.

5. A transition from an individualistic towards a collectivist society is needed in our race against climate change.

6. The analogy between cycling and everyday life is striking, given the alternating episodes of headwind and tailwind.

Propositions belonging to the thesis, entitled

Breaking down the barriers: The effects of aging, gut microbiota and bile acids on intestinal health

Benthe van der Lugt

Wageningen, 17 September 2021 


\section{BREAKING DOWN THE BARRIERS}

The effects of aging, gut microbiota and bile acids on intestinal health

Benthe van der Lugt 


\section{Thesis committee}

\section{Promotor}

Prof. Dr A.H. Kersten

Professor of Nutrition, Metabolism and Genomics

Wageningen University \& Research

\section{Co-promotor}

Dr W.T. Steegenga

Assistant professor, Division of Human Nutrition and Health

Wageningen University \& Research

\section{Other members}

Prof. Dr J.M. Wells, Wageningen University \& Research

Prof. Dr J. Knol, Wageningen University \& Research

Prof. Dr P. de Vos, University Medical Center Groningen

$\mathrm{Dr}$ H. Bouwmeester, Wageningen University \& Research

This research was conducted under the auspices of the Graduate School VLAG (Advanced studies in Food Technology, Agrobiotechnology, Nutrition and Health Sciences). 


\section{BREAKING DOWN THE BARRIERS}

The effects of aging, gut microbiota and bile acids on intestinal health

\section{Benthe van der Lugt}

Thesis

Submitted in fulfilment of the requirements for the degree of doctor

at Wageningen University

by the authority of the Rector Magnificus

Prof. Dr A.P.J. Mol,

in the presence of the

Thesis Committee appointed by the Academic Board

to be defended in public

on Friday 17 September 2021

at 11 a.m. in the Aula. 
Benthe van der Lugt

Breaking down the barriers:

The effects of aging, gut microbiota and bile acids on intestinal health, 274 pages.

PhD thesis, Wageningen University \& Research, Wageningen, The Netherlands (2021)

With references, with summary in English

ISBN: 978-94-6395-874-5

DOI: https://doi.org/10.18174/549523 


\section{Contents}

Chapter $1 \quad$ General introduction

Chapter 2 Integrative analysis of gut microbiota composition, host colonic gene expression and intraluminal metabolites in aging C57BL/6J mice

Chapter 3 Akkermansia muciniphila ameliorates the age-related decline in colonic mucus thickness and attenuates immune activation in accelerated aging $\operatorname{Ercc}^{-/ \Delta 7}$ mice

Chapter 4 The effects of sulfated secondary bile acids on intestinal barrier function and immune response in an inflammatory in vitro human intestinal model

Chapter 5 Characterization of increased mucus production of 169 HT29-MTX-E12 cells grown under semi-wet interface with mechanical stimulation

Chapter 6 General discussion 


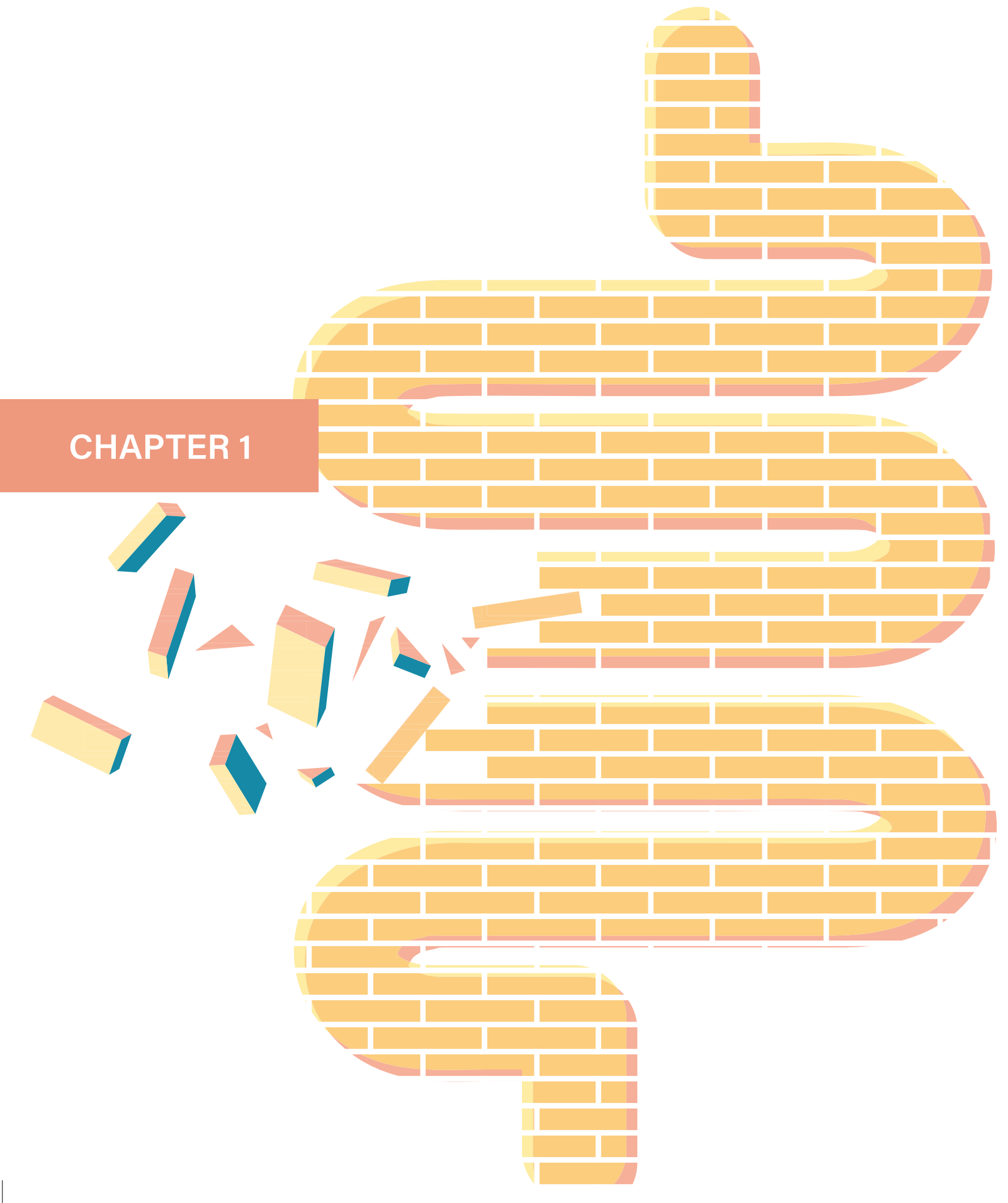


General introduction 


\section{Intestinal health}

The actuality of Hippocrates' quote "All disease begins in the gut" is still valid, given the high number of studies focusing on the relationship between the intestine and health. Although one should not interpret this quote as explicitly as it sounds, indeed, many disorders are linked to intestinal health. The intestinal tract is a large organ that harbors and interacts with trillions of micro-organisms and forms a barrier to prevent potential harmful compounds entering the bloodstream. To run all these processes smoothly, intestinal cells and their reaction to environmental stimuli should be in ingenious balance. The moment these finely regulated processes are hampered, the risk of (intestinal) diseases increases.

\section{Functions of the gastrointestinal tract}

All consumed foods and drinks pass through the gastrointestinal (Gl) tract, following the route from mouth to anus. During this journey, every part of the GI tract has its own specific role in digesting and absorbing the food. Mainly in the lower intestinal tract, i.e. the small and large intestine (colon), nutrients are taken up by intestinal absorptive cells, or enterocytes (1). In the colon, nutrients and water are absorbed and the indigestible parts that are left are ready for excretion via the feces. The structure of the epithelium in the small intestine is characterized by the presence of villi, i.e. small finger-like structures that greatly increase the surface area of the intestine and thereby maximize the absorption of nutrients (2). The epithelium of the colon does not possess villi, but has a rather flat appearance. In the small intestine and colon, crypts of Lieberkühn are located, which are epithelial invaginations where intestinal stem cells (ISCs) reside (3). ISCs are rapidly dividing cells necessary for the constant renewal of the intestinal epithelium. These ISCs give rise to either absorptive or secretory progenitors, which can differentiate into six specialized epithelial cell types (Fig. 1). Absorptive progenitors differentiate into enterocytes, or colonocytes, as they are called in the colon. Of all cell types, enterocytes are the most densely populated and are responsible for the absorption of nutrients and water. The less well known microfold (M) cells also originate from absorptive progenitors and play an important role in mucosal immunity by their ability to take up luminal antigens (2). The other four cell types originate from secretory progenitors. Goblet and enteroendocrine cells produce and secrete mucus and hormones, respectively. In contrast to other intestinal cell types, Paneth cells stay at the bottom of the crypt and are responsible for the production of antimicrobial compounds. Also the remaining cell type, the Tuft cell, is involved in the protection against intruders, since it combats parasite infection (2). 
Altogether, different types of IECs have their own specialized functions and contribute to intestinal homeostasis. It is important to realize, however, that recent studies have shown that multiple subsets exists within the same cell type. For example, a subtype of goblet cells located at the mouse colonic crypt entrances was discovered (4). These so-called sentinel goblet cells are able to protect the colonic crypts by secreting mucus in the presence of invading bacteria (4). Also, subtypes of enterocytes, enteroendocrine cells and Tuft cells are known (2), highlighting the extensiveness and complexity of IECs.

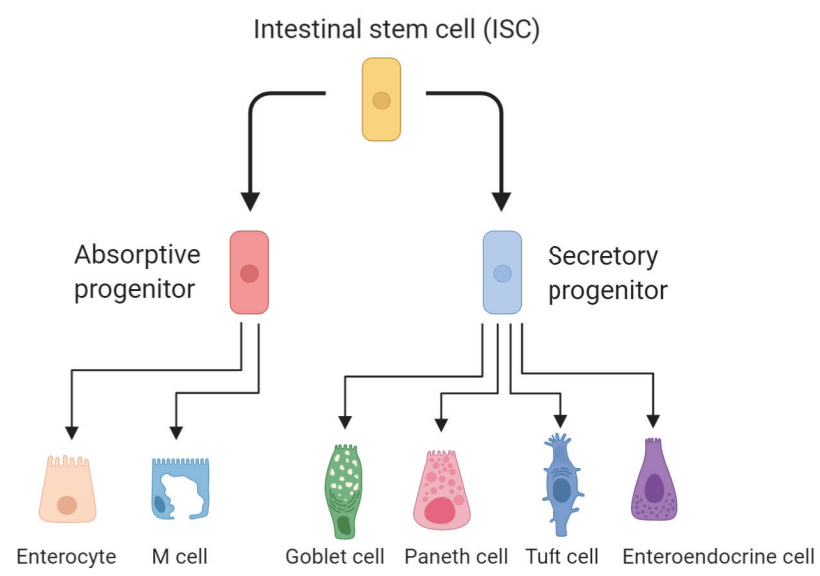

Figure $1 \mathrm{~A}$ schematic overview of the development of intestinal stem cells (ISCs) into two cell lineages: absorptive cells (enterocytes and M cells) and secretory cells (goblet, Paneth, tuft and enteroendocrine cells).

\section{Intestinal barrier function}

The intestine is continuously exposed to external factors that enter the body mainly via the ingestion of food. Exposure to these external factors might pose a risk when toxic compounds are co-ingested and exert potentially harmful effects. Moreover, the presence of an incredibly high number of bacteria in the intestine requires a well-functioning defense mechanism to avoid the invasion of bacteria. Therefore, an essential function of the intestine is to maintain a finely tuned balance between an efficient uptake of nutrients on the one hand, and the restriction of the entrance of toxic compounds and bacteria on the other hand. This highly complex task is delegated to the intestinal barrier, which consist of multiple layers that work in concert to keep intestinal homeostasis. Following the order from intestinal lumen to the mucosal tissue, these layers include the mucus layer, the intestinal 
epithelial cell layer and the immune cell 'layer.' Important similarities and differences between the intestinal barrier function in the small intestine and colon are depicted in Figure 2.

A

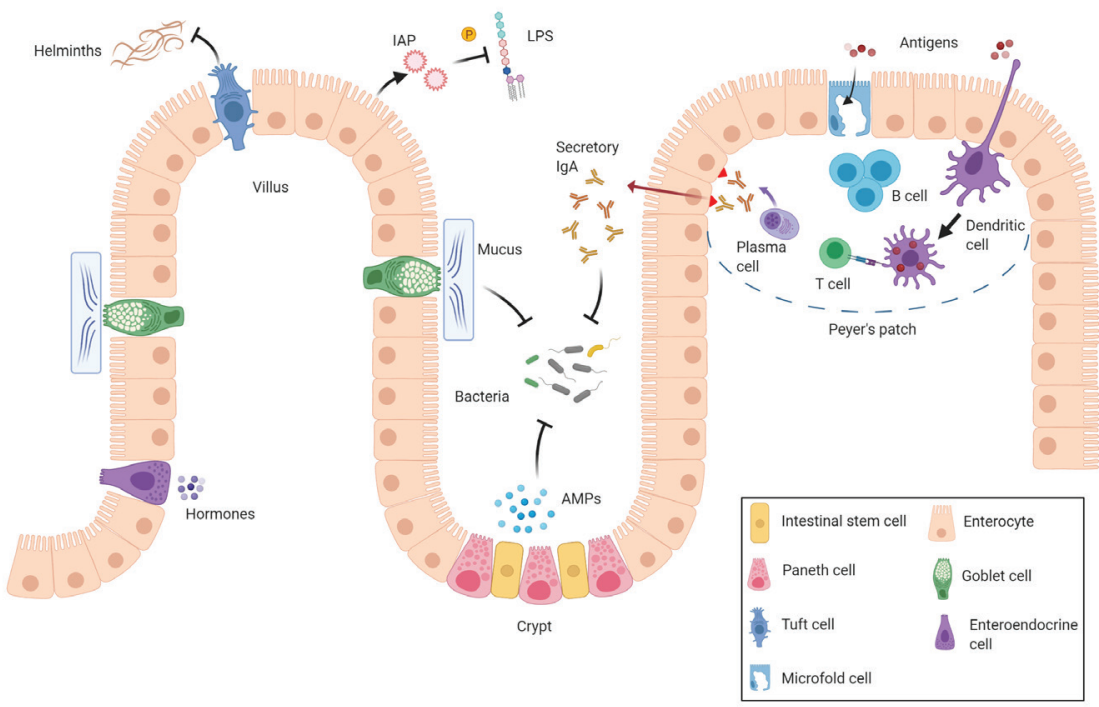

B

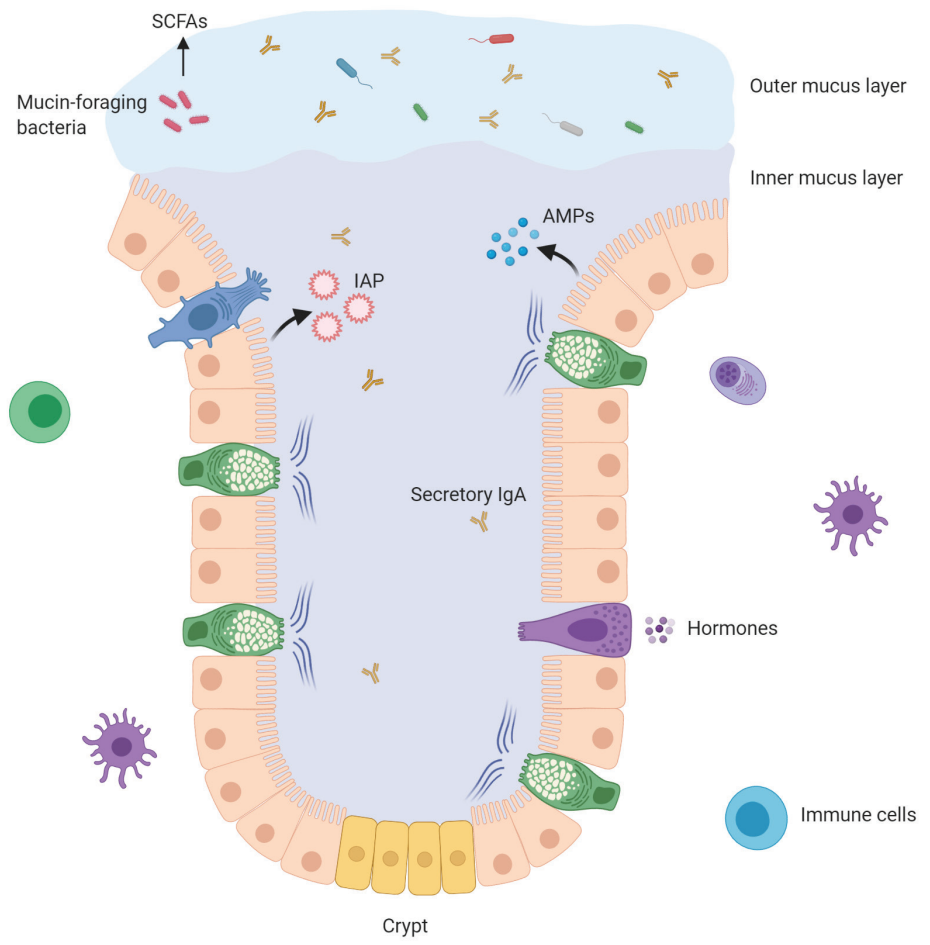


Figure 2 Schematic overview of the different components of the intestinal barrier in the small intestine (SI) and colon. (A) In the small intestine, both villi and crypts are present. Antimicrobial peptides (AMPs), Immunoglobulin A ( $\lg A)$ and intestinal alkaline phosphatase (IAP) protect against bacterial invasion. Tuft cells protect against parasites, such as helminths. The mucus layer in the SI is rather discontinuous, while in the mucus layer in the colon is thick (B) and consists of two layers: a firm inner layer that is devoid of bacteria, and a loose outer mucus layer is inhabited by bacteria. High amounts of IgA, AMPs and IAP are present in the mucus layers that protect against invasion of bacteria.

\section{Mucus layer}

The mucus layer covering the epithelial cells has a dual role: on the one hand, it acts as a protective physical barrier, and on the other hand, it serves as a nutrition source for commensal bacteria (5). These functions are accounted for by the complex structure of mucus. The mucus secreted along the intestinal tract is mainly composed of the gel-forming mucin type 2 (MUC2). MUC2 is part of a large family of mucins that consist of both secreted and transmembrane mucins. Next to MUC2, other gel-forming mucins include MUC5AC, MUC5B and MUC6. However, these mucins are mainly expressed in the stomach and are not, or very weakly, expressed in the intestine (6). In addition to secreted mucins, enterocytes produce another type of mucins: the transmembrane mucins. These membrane-bound mucins are large glycoproteins that are characterized by a long and highly glycosylated extracellular domain that is part of the intestinal barrier (7). The mucin core domain consists of repetitive tandems of proline, threonine and serine (PTS), which are heavily O-glycosylated at the hydroxyl group of serine or threonine (8) (Fig. 3). The N- and C-termini of gelforming mucins are kept together by disulfide bonds between cysteine amino acids. Transmembrane mucins are attached to the cell membrane by either sea urchin-enterokinase-agrin (SEA) domains or von Willebrand domains (VWD). The intracellular domain (cytoplasmic tail) has the potential to be phosphorylated, thereby exerting signaling functions, such as immune modulation and regulation of cell proliferation and apoptosis via different signaling pathways $(9,10)$.

The high amount of glycans makes MUC2 water soluble, reflected by the high water content of mucus (90-95\%). Together with electrolytes, lipids, immunoglobulins and many other proteins, MUC2 forms an expanded net-like structure $(11,12)$. The protective role of the mucus layer is based on the physical barrier that avoids direct contact between bacteria in the lumen and the IEC layer. Importantly, the organization of the mucus layer along the intestinal tract is different. In the small intestine, the mucus layer is loose and not attached to the underlying epithelial cells (13) (Fig. 2A). In the colon, a double mucus layer exists: an impenetrable inner layer which is firmly attached to the intestinal epithelial cells and is devoid of bacteria, and a loose and penetrable outer layer 
which is colonized by commensal bacteria $(14,15)$ (Fig. 2B). Apparently, the thickness of the mucus layer is highly dependent on the number of bacteria present, as the mucus layer is thicker in the bacteria-dense colon compared to the small intestine, where far fewer bacteria are present (16). The indispensability of bacteria for a properly functioning mucus layer was emphasized in studies which showed that the mucus layer of germfree rats was very thin. In addition, mucus composition, compactness and mucin content was negatively affected (17). Moreover, the composition of the gut microbiota was shown to be an important indicator of a well-functioning mucus layer (18).

\section{Gel-forming mucin Transmembrane mucin}

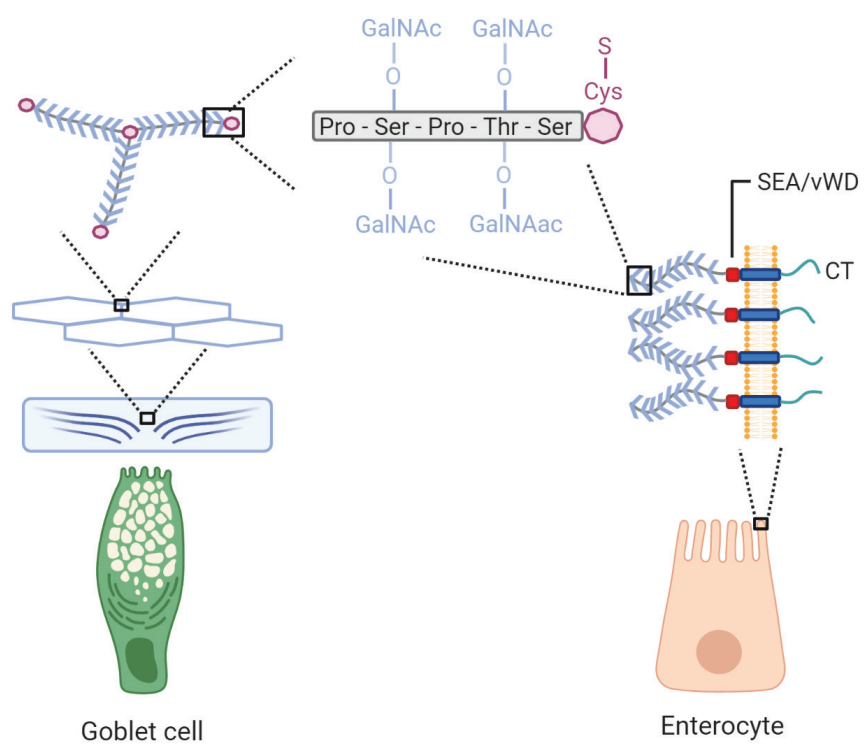

Figure 3 A schematic, simplified overview of the structure of gel-forming and transmembrane mucins. The mucin core domain consists of repetitive tandems of proline (Pro), serine (Ser) and Threonine (Thr). The latter two amino acids are attachment sites for O-glycosylation with N-acetylgalactosamine (GaINAc). Transmembrane mucins are bound to the cell membrane by sea urchin-enterokinase-agrin (SEA) domains or von Willebrand domains (VWD). The cytoplasmic tail (CT) is located inside the cytoplasm.

Next to its role as physical barrier to protect bacteria coming in contact with IECs, mucus has another beneficial function as food source for commensal bacteria (5). The so-called mucindegrading bacteria produce enzymes that allow them to metabolize mucin glycans (Fig. 2B) (6). A well-known mucin-degrading bacteria is Akkermansia muciniphila, which belongs to the phylum of Verrucomicrobia (19). Besides, many members of the phyla Bacteroidetes (Bacteroides 
thetaiotaomicron and Bacteroides fragilis) and Firmicutes (Ruminococcus torques and Ruminococcus gnavus) have been described as mucin-degrading bacteria, but also from the phyla Actinobacteria (Bifidobacterium bifidum) amongst others $(6,20)$. Mucin-degrading bacteria have several important roles in intestinal homeostasis. Under the influence of mucin-degrading bacteria, the mucus layer is continuously degraded, which stimulates the production of mucus again. This process, which is known as mucus turnover, is essential for a healthy mucus layer (19). As both the host and bacteria benefit, this microbe-mucus interaction can be seen as a mutualistic relationship. First, the degradation of mucins leads to the production of SCFAs that provides extra energy needed for the energy-demanding process of the synthesis and secretion of MUC2 (21). Second, mucin-degrading bacteria can directly inhibit the colonization of pathogens, e.g. by 1) producing antimicrobials, 2) depleting the available nutrients or 3 ) reducing oxygen levels (as most pathogens use oxygen for growth) (22). Third, the close proximity of mucin-degrading bacteria to the intestinal epithelial layer leads to a finely regulated immune response that provides protection against pathogens (22). Altogether, these examples emphasize the important role of mucus-gut microbiota interactions by maintaining a finely tuned balance between MUC2 production by the host and mucin-degradation by the gut microbiota (21).

\section{Intestinal epithelial cells}

The monolayer of IECs that is underlying the mucus layer is also a physical barrier. A crucial role of this cellular monolayer is to allow the transport of nutrients, water and ions, yet prevent the entrance of potential harmful components (23). To maintain this selective permeable barrier, IECs are strongly connected to each other by specialized proteins called tight junctions (TJs) $(23,24)$. TJs are multiprotein complexes and can be classified into families of transmembrane and cytosolic proteins. Each of these families consist of different members. The four families of transmembrane proteins include occludin, claudin, junctional adhesion molecule (JAM), and tricellulin (23). While the first three TJ proteins seal two adjacent cells, tricellulin connects the junctions of three cells (24). The TJ proteins interconnect with cytosolic proteins, such as the family of zonula occludens $(Z O 1,2,3)$ and cingulin. In turn, the ZOs and cingulin act as adaptors that form strong connections between transmembrane proteins and the perijunctional actomyosin ring, thereby anchoring the protein complex to the cytoskeleton $(24,25)$ (Fig. 4). The ingenious complex of TJ proteins and their interaction with the actomyosin ring is under strict regulation. Constant signaling between the individual components results in the opening and closure of the paracellular space upon a wide range of external stimuli 
and physiological and pathological conditions $(24,26)$. Phosphorylation of TJs plays an important role in regulation of intestinal epithelial barrier function. Key signaling proteins involved in this process include protein kinase $\mathrm{C}(\mathrm{PKC})$, mitogen-activated protein kinases (MAPK), myosin light chain kinase (MLCK), and the Rho family of small GTPases, which control the (dis)assembly and maintenance of TJs (24).

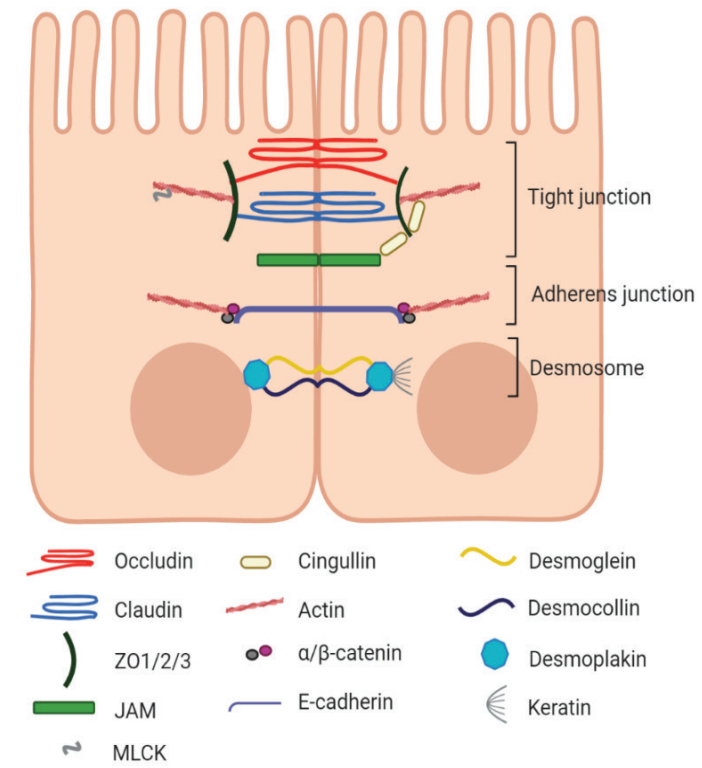

Figure 4 Schematic overview of the tight junction (TJ) proteins, adherens junctions (AJs) and desmosomes present in the intestinal cell that seal adjacent cells.

Next to the physical defense, another defense mechanism attributed to IECs is the production of intestinal alkaline phosphatase (IAP) (Fig. 2A-B). IAP is a brush border enzyme secreted at both the apical and basolateral side of the IEC. It has multiple important functions to keep intestinal homeostasis (27). In relation to the intestinal barrier, the most important function of IAP is the ability to dephosphorylate LPS. This removal of phosphate groups leads to a significant reduction in the toxicity of LPS by inhibiting downstream pro-inflammatory reactions (27). 


\section{Immune cell layer}

The third layer of defense is not an actual physical layer, but includes specialized cells of the immune system that participate in the tolerance and protection against external substances (Fig. 2A-B). The intestine is an organ with a high number of immune cells, emphasizing the important role of the intestinal immune system. The gut-associated lymphoid tissue (GALT) consists of lymphoid structures that are all dedicated to sample foreign antigens and induce an adaptive immune response (28). Peyer's patches (PPs) are the main lymphoid tissue located in the small intestine (Fig.

2A). Specialized cells in the PP include Microfold (M) cells, which are responsible for the uptake and transport of antigens to mononuclear phagocytes, such as macrophages and dendritic cells (DCs). These antigen-presenting cells present the antigens to a variety of adaptive immune cells that act in concert to detoxify the foreign substances. In the protection against pathogens and tolerance to commensal bacteria, Immunoglobulin A (IgA) plays an important role. Specific subsets of intestinal DCs induce the production of $\lg A$ by $B$ cells (29). $\lg A$ is able to interfere with gut bacteria and neutralize toxins, thereby preventing these harmful compounds from coming in contact with the IEC layer (30). In the colon, where no PPs are present, caecal patches are the equivalent of PPs. From these structures, IgA secreted by plasma cells migrates to the colon to exert its immune-related functions (31).

Although strictly not a barrier, another important antimicrobial defense mechanism is organized by Paneth cells. These cells are located in the base of the crypts in the small intestine (32). Upon bacterial stimulation via Toll like receptors (TLRs), Paneth cells produce a range of antimicrobial peptides (AMPs), such as defensins, regenerating islet-derived protein IIIA (REGIIIA) and lysozyme, amongst others $(3,33)$. The antimicrobial properties of AMPs are executed either by directly killing the bacteria by permeabilizing the membrane, or intracellularly by inhibiting essential cell processes (34). Although Paneth cells are absent in the human colon, a high amount of functional AMPs was found to be present in rectal mucus extracts, indicating that AMPs are retained in the mucus without losing their function (35). Furthermore, other sources of intestinal AMPs are also known: enterocytes, infiltrating neutrophils and the bacteria present in the intestine are three other sources of AMPs (36).

Paradoxically, to have a fully functional intestinal barrier, the host is highly dependent on bacteria present in the intestinal lumen and mucosa. The presence of bacteria results in the development and maintenance of the intestinal barrier (37). Importantly, germfree mice have an underdeveloped GALT, and also immune tissues outside the intestine (e.g. spleen and lymph nodes) are poorly developed (38)s. These immune deficits render germfree animals more prone to infections (39). 


\section{Gut microbiota}

The intestine is densely populated with a variety of microorganisms, also known as the gut microbiota. This diverse community is dominated by the presence of bacteria, but also includes fungi, viruses, archaea and protozoa (40). To date, numerous bacterial phyla have been discovered. The phyla that are mainly present in the intestine are Bacteroidetes and Firmicutes, and to a lesser extent Proteobacteria, Actinobacteria, Fusobacteria, Verrucomicrobia and Cyanobacteria (41). The number of bacteria as well as the bacterial diversity increases along the GI tract, with the highest density and diversity found in the colon (38). The human gut microbiota is of utmost importance for host health, not only with regard to intestinal health, but also beyond (42). A healthy intestine is characterized by a highly diverse microbiota composition, with the presence of beneficial commensal bacteria and the absence of infection and bacterial overgrowth (43). Many of the health effects attributed to the gut microbiota are mediated by bacterial metabolites, which are intermediates or end products of bacterial metabolism (44). In the colon, the most intensively studied bacterial metabolites are shortchain fatty acids (SCFAs) and bile acids (BAs).

\section{Short-chain fatty acids}

Diet is an important source of precursors for the production of bacterial metabolites. By far the most intensively studied diet-derived bacterial metabolites are the SCFAs butyrate, propionate, and acetate. These compounds are produced upon bacterial fermentation of indigestible carbohydrates and exert a variety of beneficial effects for the host. Amongst others, SCFAs can be used as energy source. Butyrate is mainly used as fuel for colonocytes (45), while acetate and propionate can be used in the liver as precursors for gluconeogenesis and lipogenesis, respectively (46). Furthermore, SCFAs are known to strengthen the intestinal barrier by upregulating the expression of TJ genes, increasing AMP production, and decreasing inflammation (47). SCFAs also play a role in the regulation of satiety and intestinal transit via activation of G-protein coupled receptors (GPCRs), thereby releasing PYY and GLP-1 (48). These indirect effects of SCFAs imply that their beneficial health effects extend beyond the intestinal tract.

\section{Bile acids}

Bile acids (BAs) include another group of metabolites with effects on intestinal health as well as overall health. The primary BAs cholic acid (CA) and chenodeoxycholic acid (CDCA) are produced in the liver by the conversion of cholesterol through a series of complex enzymatic pathways, with 
CYP7A1 as the rate-limiting enzyme (49). After synthesis, primary BAs are conjugated with taurine or glycine and transported via the bile salt export pump (BSEP, ABCB11). Conjugation of BAs prevents passive absorption and promotes solubility in the proximal intestine (50). These changes in their physicochemical properties are important for the maintenance of high luminal concentrations of conjugated BAs and thereby for the digestion and absorption of dietary fats (50-52). Of all produced BAs, 95\% is reabsorbed in the small intestine by the apical bile salt transporter (ABST, SLC10A2). In the enterocyte, BAs are bound to the ileal bile acid binding protein (IBABP, FABP6), are excreted via the basolateral organic solute transporters alpha and beta (OSTa/ $\beta, S L C 51 A / B)$ and travel via the portal vein back to the liver, where reuptake takes place via the $\mathrm{Na}^{+}$-taurocholate polypeptide (NTCP, SLC10A1). This elegant process of BA recirculation is called the enterohepatic cycle and is highly important for metabolic homeostasis (49) (Fig. 5). The small fraction (5\%) of BAs that escapes the enterohepatic cycle enters the colon, where the gut microbiota transform the primary BAs into secondary BAs. First, conjugated BAs are deconjugated by the bacterial enzyme bile salt hydrolase (BSH). The second pathway is the dehydroxylation at position 7 by bacterial dehydroxylases, transforming CA and CDCA in deoxycholic acid (DCA) and lithocholic acid (LCA), respectively (53). Secondary BAs can either be excreted in the feces or are absorbed by IECs and return back to the liver where they enter the enterohepatic cycle again (54) (Fig. 5).

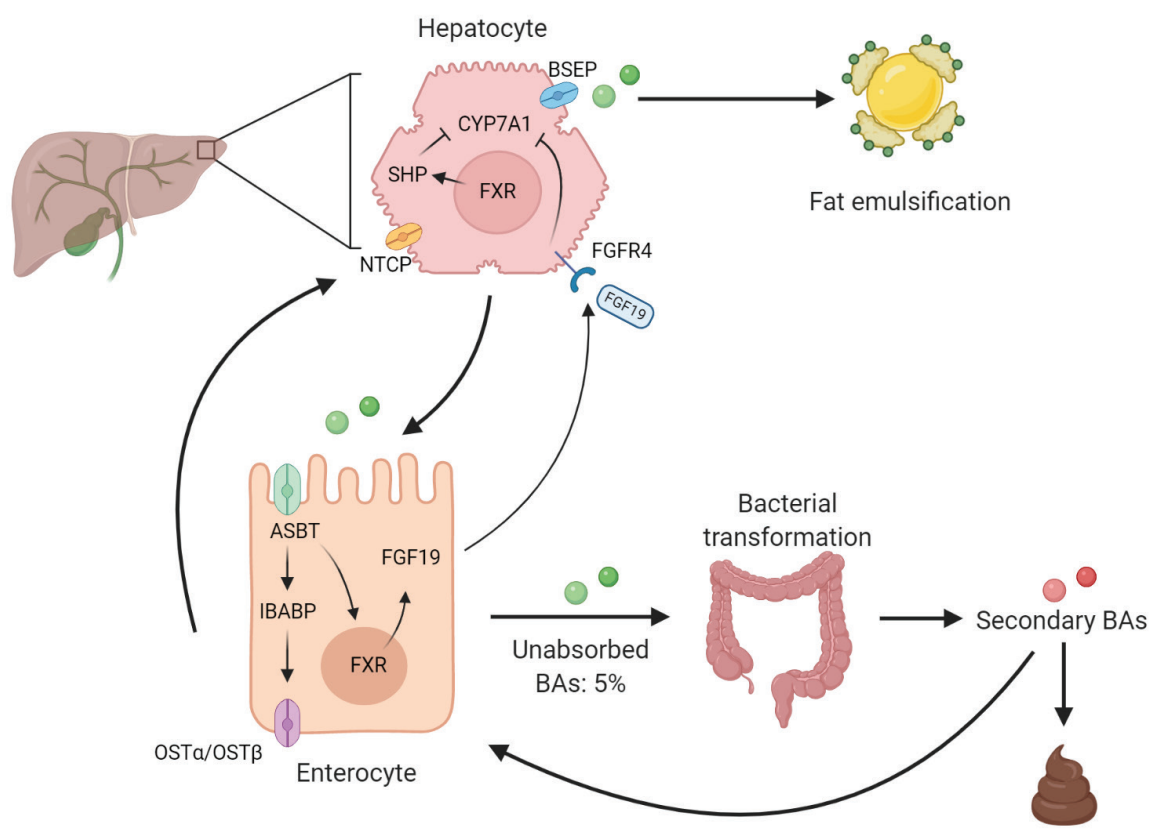


Figure 5 A schematic overview of the enterohepatic cycle. Primary bile acids (BAs) are produced in the hepatocyte. After conjugation, BAs are released in the intestine, where they aid in the emulsification of fat. BAs are then absorbed by the enterocyte and enter the liver again via the portal vein. Unabsorbed BAs are transformed by bacteria into secondary BAs, which enter the enterohepatic cycle again, or are excreted via feces. In both hepatocytes and enterocytes, FXR has an important role in the regulation of BA production.

\section{BAs as signalling molecules}

BAs have a pleotropic role in the human body. Next to their function as detergents in fat digestion and absorption, BAs also act as signalling molecules by activating the BA receptors FXR (NR1H4) and TGR5 (GPBAR1), resulting in the regulation of a wide range of biological processes (54). The nuclear receptor FXR, which is abundantly expressed in both the liver and intestine, is directly activated by BAs. The most potent FXR activator is CDCA, followed by LCA, DCA, and CA (49). FXR plays an important role in the enterohepatic cycle by regulating the expression of genes related to BA synthesis, secretion, and absorption (49) (Fig. 5). In the enterocyte, FXR activation triggers the production of fibroblast growth factor 19 (FGF19), which inhibits BA synthesis by inhibition of CYP7A1 via fibroblast growth factor receptor 4 (FGFR4). In the hepatocyte, CYP7A1 is also repressed by FXRmediated regulation of small heterodimer partner (SHP) (55) (Fig. 5). In addition to its role in the regulation of the enterohepatic cycle, FXR is also involved in mucosal protection, cell proliferation, and inflammation $(56,57)$. It was shown that FXR activation ameliorated intestinal inflammation, inhibited intestinal permeability, and protected against loss of goblet cells in mice suffering from chemically-induced colitis (58). Furthermore, FXR was also shown to be anti-tumorigenic, since FXR-deficient mice had increased colon cell proliferation rates and subsequent tumorigenesis (59).

TGR5 is a G-protein coupled receptor (GPCR) that is ubiquitously expressed throughout the GI tract, but is also expressed in innate immune cells and enteric nerves $(60,61)$. This cell surface BA receptor is most potently activated by the secondary BAs LCA and DCA, followed by CDCA and CA. However, also conjugated BAs have a high affinity for TGR5 (62). TGR5 plays an important role in several cell signalling pathways with effects on energy homeostasis, insulin secretion, cell proliferation, intestinal motility and inflammation, amongst others $(63,64)$. Although the exact mechanisms are to be unravelled, it is thought that these effect are mediated by various cyclic AMP signalling pathways (65). In addition to FXR and TGR5, BAs are also ligands for the vitamin D receptor (VDR), pregnane $X$ receptor $(P X R)$ and glucocorticoid receptor (GR), that all have important roles in physiological processes (66). 


\section{Cytotoxic effects of bile acids}

Next to their important physiological functions, BAs can also be cytotoxic, which might even have pathological consequences. The detergent properties of BAs could disturb cell membranes by solubilizing membrane lipids (67). Furthermore, high levels of hydrophobic BAs have been associated with colon cancer caused by oxidative stress and DNA damage (68). As a strategy to reduce the cytotoxicity of BAs, the liver conjugates $\mathrm{BAs}$ with a sulfonate $\left(\mathrm{SO}_{3}\right)$ group by the sulfotransferase SULT2A1 (Fig. 6) (69). In addition to hepatic BA sulfation, it is known that enterocytes also express SULT2A1, suggesting that BAs are also sulfated in the intestinal tract. Sulfation of BAs results in a hydrophilic molecule that is poorly reabsorbed by the body, and is therefore rapidly excreted via the feces and urine (69). Furthermore, sulfated BAs have less detergent capacities, which could result in less membrane-disturbing effects than their unsulfated counterparts (70). Similar to other BAs, sulfated BAs are subject to bacterial transformation. Indeed, bacterial species that have desulfating capacities include Clostridium, Pseudomonas, Peptococcus and Fusobacterium (71).

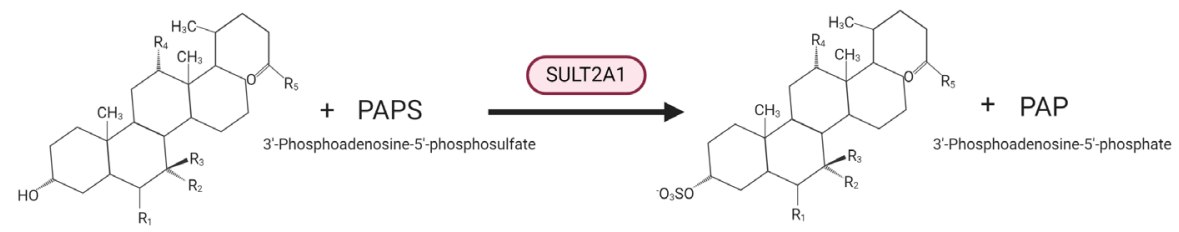

Figure 6 Schematic overview of the enzymatic formation of sulfated BAs in the human liver. During this reaction, a sulfonate group $\left(\mathrm{SO}_{3}^{-}\right)$derived from the universal sulfonate donor $3^{\prime}$-phosphoadenosine 5'-phosphosulfate (PAPS) is transferred to a hydroxyl group. The 3-OH position of BAs is the main target for sulfation in humans. The enzyme catalysing this reaction is sulfotransferase 2A1 (SULT2A1) (69).

\section{Impaired intestinal barrier function}

Following the reasoning that intestinal barrier function plays a crucial role in health, it is not surprising that impaired barrier function is associated with pathogenesis of a wide range of (inflammatory) diseases. Diet and lifestyle, e.g. nutrients, alcohol, smoking, stress and medication use could all have an impact on intestinal barrier function (72). The intestinal barrier can be directly disrupted through the damage of epithelial cells or by disrupted formation and distribution of TJ proteins (73). As a major consequence, breakdown or dysregulation of the intestinal barrier leads to increased translocation of bacterial products (e.g. LPS) and dietary antigens to enter the lamina propria (74) (Fig. 7). These stimuli trigger immune cells in the lamina propria, i.e. dendritic cells and macrophages, 
to produce pro-inflammatory cytokines. A vicious pro-inflammatory cycle might be induced, as the innate immune cells direct the activation of $\mathrm{T}$ cells, which on their turn produce signals that increase further translocation of dietary antigens and bacterial products (75). In addition to local inflammation, an increase in systemic inflammation could also occur when LPS and locally produced proinflammatory cytokines enter the bloodstream (75). Examples of diseases caused by local chronic and uncontrolled immune activation as a result of impaired barrier function include inflammatory bowel disease (IBD), coeliac disease and colorectal cancer (73). Importantly, the consequences of impaired barrier function reach far beyond the gut, as evidenced by the contribution to metabolic diseases (obesity and diabetes) (76) as wells as neurodegenerative diseases (Parkinson's disease, Alzheimer disease, multiple sclerosis and amyotrophic lateral sclerosis) (77).
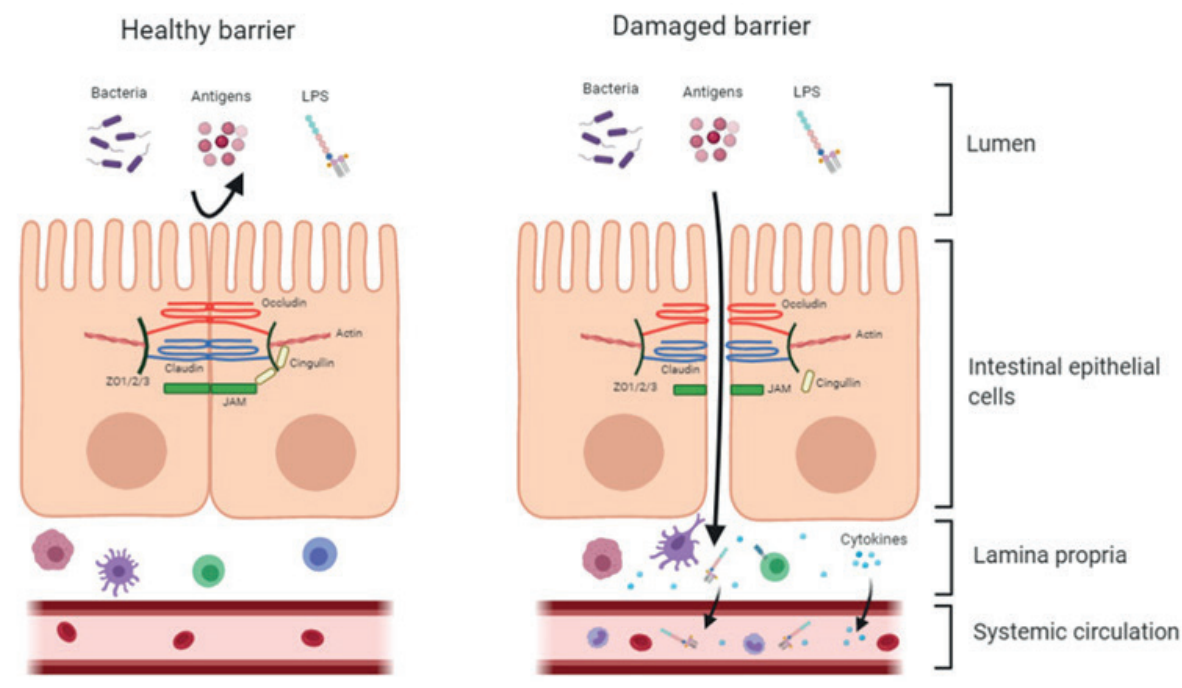

Figure $7 \mathrm{~A}$ healthy intestinal barrier is characterized by tightly sealed intestinal epithelial cells. When the intestinal barrier is damaged, disruption of TJs cause opening between the cells, allowing foreign compounds (e.g. bacteria, antigens, LPS) crossing the membrane and activate immune cells in the lamina propria. Immune cell activation leads to increased cytokine production, leading to a pro-inflammatory environment and infiltration of adaptive immune cells. When the immune response becomes uncontrolled, local inflammation extends toward the systemic circulation, which leads to systemic inflammation.

\section{Inflammatory bowel disease}

Inflammatory bowel disease (IBD) comprises the chronic and relapsing inflammatory disorders Crohn's disease and ulcerative colitis, that mainly affect the gastrointestinal tract. Symptoms of this 
disease include severe abdominal pain, diarrhea and weight loss, leading to a significant impairment of the quality of life (78). The etiology of IBD remains largely unknown, although it is clear that it is a multifactorial disorder in which genetic susceptibility, environmental stimuli, gut microbiota and exaggerated immune responses play a role (79). It is generally accepted that intestinal homeostasis is disrupted, which is characterized by an imbalance between intestinal barrier components, immune response, and the gut microbiota (80). In IBD patients, a disrupted intestinal barrier is commonly observed, revealed by increased intestinal permeability, reduced mucus layer thickness and Paneth cell dysfunction leading to decreased AMP production (81). These events contribute to the entry of bacterial products, thereby triggering the immune system and causing inflammatory responses (82). The disease course of IBD is typically characterized by alternating periods of active disease (flare) and remission. A disease flare often occurs random and the exact trigger is unpredictable (83).

\section{Bacterial metabolites in relation to IBD}

Growing evidence indicates that the gut microbiota is involved in IBD pathogenesis. Intestinal dysbiosis, i.e. an imbalance in gut microbiota composition and function, is associated with IBD (84, 85). Many studies on IBD report a reduced bacterial diversity, a decreased abundance of healthpromoting bacteria, together with an increase in potential pathogenic bacteria (86-89). Given the important function of the gut microbiota to produce bacterial metabolites, an alteration in metabolite profile is concomitant with IBD-related dysbiosis. Importantly, a shift in specific types of metabolites could contribute to the pathogenesis of IBD (44). For example, IBD is associated with a loss of butyrate-producing bacteria (90). Consistently, metabolomic studies point toward a decreased abundance of the SCFA butyrate in IBD patients (91-93).

\section{Bile acids and IBD}

Another group of bacterial metabolites known to be involved in IBD pathogenesis are BAs. As BAs are enzymatically modified by specialized bacterial species, IBD-related dysbiosis could result in BA dysmetabolism via decreased bacterial enzymatic activity (Fig. 8) (88). Indeed, a different fecal BA composition was observed in IBD patients compared to healthy controls, characterized by an increase in primary, conjugated and sulfated BAs, and a decrease in secondary BAs (88, 94-97). Mainly the decrease in secondary BAs could be of importance in relation to IBD progression, given their role as activators of the BA receptors FXR and TGR5. Reduced levels of DCA and LCA may lead to a decreased activation of FXR and TGR5, thereby decreasing their anti-inflammatory effects 
$(56,97)$. Furthermore, secondary BAs are known for their antimicrobial properties by interfering with bacterial membrane lipids (98). FXR activation was shown to promote the production of AMPs in mice (58). An IBD-related decrease in luminal secondary BAs could therefore lead to a reduced protection against bacterial overgrowth, a phenomenon which is commonly observed in IBD (99, 100).

Healthy situation

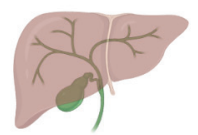

Bile acids

Healthy gut

microbiota
IBD patient
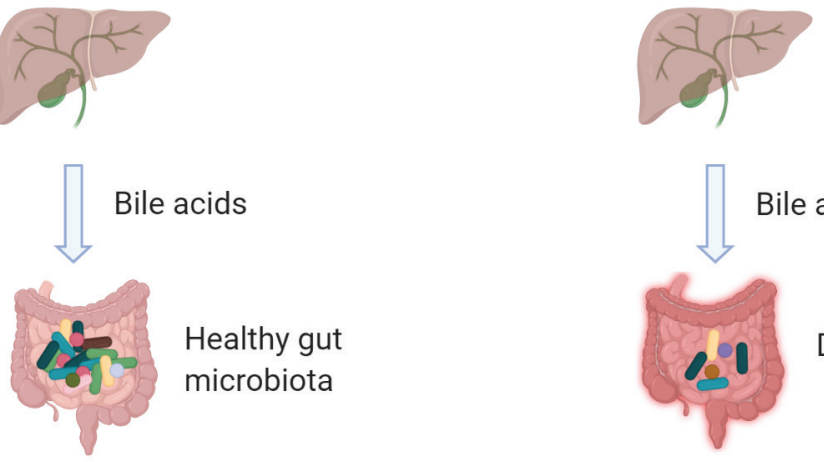

Normal enzymatic transformation
Bile acids

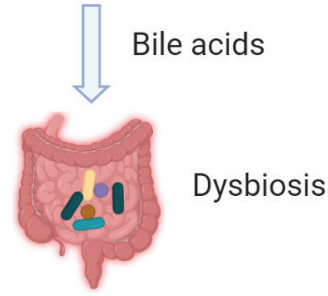

Lower enzymatic transformation

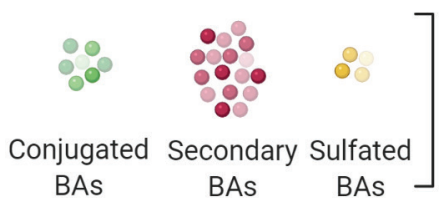

\section{Colonic
BA pool}
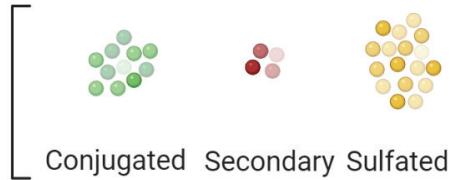

Conjugated Secondary Sulfated

BAs BAs BAs

Figure 8 Schematic overview of the differences in bile acid (BA) pool in healthy persons and IBD patients. In the healthy situation, BAs that escape the enterohepatic cycle are transformed by bacterial enzymes in the colon, leading to a normal BA pool that is dominated by secondary BAs. In IBD patients, dysbiosis leads to a reduced bacterial enzymatic activity, that results in a changed BA pool which is low in secondary BAs and high in conjugated and sulfated BAs.

\section{Aging and intestinal health}

Interestingly, IBD pathogenesis shows multiple similarities with the aging process. For example, the aging process is also associated with intestinal dysbiosis, an impaired immune response and chronic inflammation (101). Moreover, both IBD and the aging process are important risk factors 
for chronic diseases that affect the whole body. Hence, given the high similarities in underlying mechanisms, this could pose possibilities for sharing treating interventions. The aging process is associated with a functional decline of all organs and tissues in the body, including the intestinal tract (102). A commonly observed functional change of intestinal physiology include a prolonged transit time and increased prevalence of constipation (103). Moreover, the aging process is characterized by a decreased intestinal barrier function, an increase in low-grade inflammation (inflammaging) and a decline in immune response (immunosenescence) $(104,105)$ (Fig. 9).

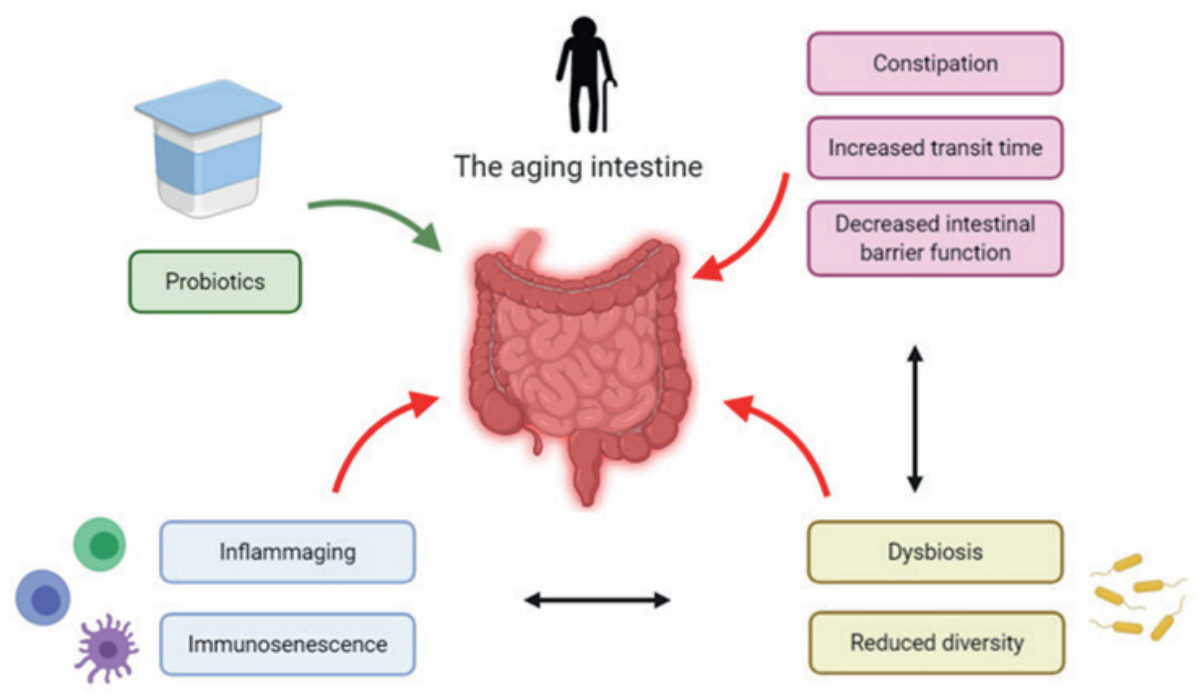

Figure 9 A schematic overview of the factors that impact intestinal health during aging. Red arrows implicate a detrimental effect on intestinal health, while green arrows could imply a positive effect on intestinal health in the aged population.

These factors are all known to alter gut microbiota composition (106). Many studies have been performed that investigated the differences in gut microbiota composition between young and old participants. Although often contradictory results have been observed, general age-related changes in gut microbiota composition include a decrease in alpha-diversity, a reduced abundance of beneficial bacteria (e.g. Bifidobacteria and Lactobacilli) $(107,108)$ and increased abundance of potential pathogens (e.g. members of the Enterobacteriaceae family) (109). Importantly, large interindividual variation was reported in elderly (110), which could pose difficulties in interpreting the 
results. Regardless, it is clear that the gut microbiota play a role in age-related diseases. Therefore, manipulating the gut microbiota to reach a more beneficially composition could be a way to combat age-related diseases. Supplementation with prebiotics or probiotics could be such an approach. However, the outcomes on infection duration and inflammatory markers are rather inconsistent (111). Importantly, the effects of pre- and probiotics on health outcomes are largely dependent on a variety of factors, such as supplementation duration, way of administration, and the targeted health outcome. This emphasizes the need of highly controlled and targeted studies.

\section{In vivo and in vitro models to investigate intestinal health}

The increased scientific interest to investigate intestinal health requires the use of representative, reliable, and reproducible study designs. For these studies, in vivo models using humans and animals are widely used. In the context of gut microbiota composition, human studies can be useful, but are often limited to the collection of fecal samples. However, the gut microbiota composition in feces is not completely representative for the luminal gut microbiota composition (112). To overcome this problem, newly developed techniques to sample luminal content in the intestine could be useful. In this regard, animal studies are valuable given the possibility to isolate not only organs and tissues, but also luminal content during sacrifice. Another advantage of animal studies is the possibility to perform genetic modifications, such as knocking out or down specific genes. This enables researchers to not only to investigate the molecular mechanisms of a gene of interest, but also to develop an animal model with a specific phenotype. For example, the use of the Ercc1 ${ }^{-/ \Delta 7}$ mouse model, which has an accelerated aging process due to a partial aberration of a DNA repair mechanism, is useful in aging research (113).

Important disadvantages of human and animal studies are the often expensive and lengthy study designs, but also ethical limitations, such as the exposure to potential harmful compounds and the isolation of tissues and organs. To overcome these limitations, in vitro models could be used as an alternative. A wide range of in vitro models exists and each has its own advantages and disadvantages. The most simple and cheap model includes a monoculture of intestinal epithelial cells, such as Caco-2 or T84 cells. To represent the intestine, these cells could be seeded on cell culture inserts that create an apical and basolateral compartment, representing the intestinal lumen and mucosal tissue, respectively. A disadvantage of using only one cell type is the lack of representativeness to the in vivo situation, as the intestine consists of several different cell types. Therefore, the monoculture grown on cell culture inserts could also be extended to a co-culture with 
another intestinal cell type, such as the goblet-like cell type HT29-MTX (114). A major advantage of using mono- and co-cultures is the relatively easy, cheap, and quick applicability. Moreover, a second cell type can be added in the basolateral compartment, such as immune cells, which allows the inclusion of the immune system to the model.

A next level model of the intestine includes the use of ex vivo organ cultures, such as intestinal organoids. Intestinal organoids are the self-propagating spheres derived from intestinal stem cells $(115,116)$ or pluripotent stem cells $(117)$. When grown in the presence of a basement membrane (i.e. Matrigel) and appropriate growth factors, intestinal stem cells will develop into a three-dimensional structure representing the in vivo organization of the intestine (118). Most often, organoid models make use of animal tissue (e.g. mouse, rat, pig), since human material is often more difficult to obtain. This may pose some difficulties in translating the results to the human situation. Another important issue is the fact that organoids are closed structures, which makes direct exposure to compounds of interest complicated. While it is possible to use microinjection techniques, this time-consuming technique restricts the performance of high-throughput studies. To overcome this limitation, organoids could also be seeded as two-dimensional monolayers, either on regular cell culture plates or cell culture inserts. Applying the right growing conditions results in a monolayer that recapitulates the in vivo intestinal epithelium in terms of cell type composition, crypt organization and tissue renewal rates (119).

Importantly, the described in vitro models are all static, while in the physiological situation, intestinal peristalsis causes a constant motion. An example of a dynamic cell culture system includes the gut-on-a-chip model, where a constant perfusion with cell culture medium generates a fluid flow that mimics the dynamic microenvironment found in vivo (120). The more sophisticated and extensive the model becomes, the more the costs increase. Furthermore, the setup of these models require detailed expertise and may therefore take a long time before the model is operational. It is, therefore, not evident that every researcher is able to use sophisticated organoid and gut-on-a-chip models, emphasizing the continuing popularity of the more accessible mono- and co-cultures.

\section{Outline of this thesis}

In this thesis, a multiperspective approach was taken to investigate intestinal health. In daily life, the intestine is exposed to a wide range of potential factors that could impair intestinal health. As the intestine plays a key role in overall health, it is of utmost importance to keep this organ in a healthy state. A plethora of factors exist that have detrimental effects on intestinal health, which 
may ultimately contribute to a decreased quality of life and increased healthcare costs. The aim of this thesis was to investigate how intestinal health is affected by the aging process, gut microbiota, and bacterial metabolites, such as bile acids. Furthermore, the increased interest in intestinal health research requires appropriate in vitro models representing the in vivo situation as close as possible. In this thesis, relevant in vitro models to investigate intestinal health are described.

It is known that the aging process has detrimental effects on intestinal health. As aging is an inescapable process, together with the fact that the life expectancy is increasing nowadays, it is important to 1) gain insight in the physiological and molecular changes that occur during aging, 2) investigate the mechanisms that are underlying these changes, and 3) seek strategies to improve healthy aging. In Chapter 2, we investigated changes in different parameters of intestinal health during the aging process in C57BL/6J mice. Feces and colonic luminal content of mice aged 6, 12, 24 and 28 months were subjected to gut microbiota composition analysis to investigate agingrelated bacterial changes. Furthermore, differences in gut microbiota composition between fecal and colonic luminal content were elucidated. We also focused on aging-related changes in colonic gene expression and colonic luminal metabolites. Lastly, multivariate analyses were performed to integrate the gut microbiota composition and transcriptome datasets.

Given the frequently observed changes in gut microbiota composition during the aging process, approaches to manipulate the gut microbiota of the aged population could be a strategy to enhance healthy aging. A nutritional strategy could be the use of probiotics, including the supplementation of one or several health-promoting bacterial strains. In Chapter 3, the progeroid $\operatorname{ErCC}^{-/ \Delta 7}$ mouse model was used to investigate the effects of supplementation of the promising health-improving probiotic strain Akkermansia muciniphila on intestinal health at high age. Special attention was given to the mucus layer, as $A$. muciniphila is a mucin-degrading bacterium with considerable effects on the mucus layer.

When investigating the aging process, it is remarkable to notice the similarities in disease pathogenesis with chronic diseases. For example, chronic (low grade) inflammation and decreased efficacy of the immune system are conditions that are also underlying a range of chronic inflammatory diseases, such as IBD. Moreover, microbial dysbiosis is an important hallmark of both the aging process as IBD. It is hypothesized that IBD-related dysbiosis plays an important role in disease aggravation that is modulated by microbial metabolites, such as BAs. Given the important physiological and regulatory functions of bile acids, bile acid dysmetabolism could have potential consequences on health. The increased abundance of fecal sulfated BAs in IBD patients is interesting, because this group of BAs has only been marginally investigated yet. In Chapter 4, 
we aimed to investigate the effects of sulfated secondary bile acids on intestinal health. To this end, we designed an in vitro model representing the inflammatory situation as seen during IBD. After exposure to sulfated secondary bile acids, the effects on intestinal barrier function and immune response were investigated.

To be able to carry out high-quality research, appropriate study designs and models are required. There is a high interest in ex vivo and in vitro models representing the intestine. One of the important aspects with regard to intestinal health, the mucus layer, is often overlooked in existing ex vivo and in vitro models, since common cell culture methods are not appropriate to create a physiologically relevant mucus layer. Furthermore, the highly aqueous properties of mucus poses difficulties in the investigation and isolation of the mucus layer. In Chapter $\mathbf{5}$, we follow-up on a study that successfully increased mucus production in the HT29-MTX-E12 cell line by applying a semi-wet interface with mechanical stimulation (SWMS) method. We aimed to unravel the underlying (molecular) mechanisms of this increased mucus production by subjecting the cells to transcriptome analysis.

Finally, a general overview, a critical discussion of the data and evaluation of the current literature is given in Chapter 6 . 


\section{References}

1. Kiela PR, Ghishan FK. Physiology of Intestinal Absorption and Secretion. Best Pract Res Clin Gastroenterol. 2016;30(2):145-59.

2. $\quad$ Allaire JM, Crowley SM, Law HT, Chang SY, Ko HJ, Vallance BA. The Intestinal Epithelium: Central Coordinator of Mucosal Immunity. Trends Immunol. 2018;39(9):677-96.

3. Clevers HC, Bevins CL. Paneth cells: maestros of the small intestinal crypts. Annu Rev Physiol. 2013;75:289-311.

4. Birchenough GM, Nyström EE, Johansson ME, Hansson GC. A sentinel goblet cell guards the colonic crypt by triggering Nlrp6-dependent Muc2 secretion. Science. 2016;352(6293):1535-42.

5. Arike L, Holmén-Larsson J, Hansson GC. Intestinal Muc2 mucin O-glycosylation is affected by microbiota and regulated by differential expression of glycosyltranferases. Glycobiology. 2017;27(4):318-28. 6. Tailford LE, Crost EH, Kavanaugh D, Juge N. Mucin glycan foraging in the human gut microbiome. Front Genet. 2015;6:81.

7. Pelaseyed T, Bergström JH, Gustafsson JK, Ermund A, Birchenough GM, Schütte A, et al. The mucus and mucins of the goblet cells and enterocytes provide the first defense line of the gastrointestinal tract and interact with the immune system. Immunol Rev. 2014;260(1):8-20.

8. Johansson ME, Larsson JM, Hansson GC. The two mucus layers of colon are organized by the MUC2 mucin, whereas the outer layer is a legislator of host-microbial interactions. Proc Natl Acad Sci U S A. 2011;108 Suppl 1(Suppl 1):4659-65.

9. $\quad$ Pelaseyed T, Hansson GC. Membrane mucins of the intestine at a glance. J Cell Sci. 2020;133(5). 10. van Putten JPM, Strijbis K. Transmembrane Mucins: Signaling Receptors at the Intersection of Inflammation and Cancer. J Innate Immun. 2017;9(3):281-99.

11. Bansil R, Turner BS. The biology of mucus: Composition, synthesis and organization. Adv Drug Deliv Rev. 2018;124:3-15.

12. Hansson GC, Johansson ME. The inner of the two Muc2 mucin-dependent mucus layers in colon is devoid of bacteria. Gut Microbes. 2010;1(1):51-4.

13. Johansson ME, Sjövall H, Hansson GC. The gastrointestinal mucus system in health and disease. Nat Rev Gastroenterol Hepatol. 2013;10(6):352-61.

14. Atuma C, Strugala V, Allen A, Holm L. The adherent gastrointestinal mucus gel layer: thickness and physical state in vivo. Am J Physiol Gastrointest Liver Physiol. 2001;280(5):G922-9.

15. Johansson ME, Phillipson M, Petersson J, Velcich A, Holm L, Hansson GC. The inner of the two Muc2 mucin-dependent mucus layers in colon is devoid of bacteria. Proc Natl Acad Sci U S A. 2008;105(39):15064-9.

16. Simon GL, Gorbach SL. Intestinal flora in health and disease. Gastroenterology. 1984;86(1):174-

93.

17. Szentkuti L, Riedesel H, Enss ML, Gaertner K, Von Engelhardt W. Pre-epithelial mucus layer in the colon of conventional and germ-free rats. Histochem J. 1990;22(9):491-7.

18. Jakobsson HE, Rodríguez-Piñeiro AM, Schütte $A$, Ermund $A$, Boysen $P$, Bemark $M$, et al. The composition of the gut microbiota shapes the colon mucus barrier. EMBO Rep. 2015;16(2):164-77.

19. Belzer C, de Vos WM. Microbes inside--from diversity to function: the case of Akkermansia. Isme j. 2012;6(8):1449-58.

20. Ouwerkerk JP, de Vos WM, Belzer C. Glycobiome: bacteria and mucus at the epithelial interface. Best Pract Res Clin Gastroenterol. 2013;27(1):25-38.

21. Birchenough G, Schroeder BO, Bäckhed F, Hansson GC. Dietary destabilisation of the balance between the microbiota and the colonic mucus barrier. Gut Microbes. 2019;10(2):246-50.

22. Stecher B, Hardt WD. Mechanisms controlling pathogen colonization of the gut. Curr Opin Microbiol. 2011;14(1):82-91.

23. Suzuki T. Regulation of intestinal epithelial permeability by tight junctions. Cell Mol Life Sci. 2013;70(4):631-59.

24. Ulluwishewa D, Anderson RC, McNabb WC, Moughan PJ, Wells JM, Roy NC. Regulation of tight junction permeability by intestinal bacteria and dietary components. J Nutr. 2011;141(5):769-76.

25. Chelakkot C, Ghim J, Ryu SH. Mechanisms regulating intestinal barrier integrity and its pathological implications. Exp Mol Med. 2018;50(8):103.

26. Marchiando AM, Graham WV, Turner JR. Epithelial barriers in homeostasis and disease. Annu Rev Pathol. 2010;5:119-44.

27. Lallès JP. Intestinal alkaline phosphatase: novel functions and protective effects. Nutr Rev. 2014;72(2):82-94.

28. Kobayashi N, Takahashi D, Takano S, Kimura S, Hase K. The Roles of Peyer's Patches and Microfold Cells in the Gut Immune System: Relevance to Autoimmune Diseases. Front Immunol. 2019;10:2345.

29. Tezuka H, Ohteki T. Regulation of IgA Production by Intestinal Dendritic Cells and Related Cells. Front Immunol. 2019;10:1891.

30. Mestecky J, Russell MW, Elson CO. Intestinal IgA: novel views on its function in the defence of the largest mucosal surface. Gut. 1999;44(1):2-5. 
31. Masahata K, Umemoto E, Kayama H, Kotani M, Nakamura S, Kurakawa T, et al. Generation of colonic IgA-secreting cells in the caecal patch. Nat Commun. 2014;5:3704.

32. Mowat AM. Anatomical basis of tolerance and immunity to intestinal antigens. Nat Rev Immunol. 2003;3(4):331-41.

33. Ouellette AJ. Paneth cells and innate mucosal immunity. Curr Opin Gastroenterol. 2010;26(6):547-

53.

34. Kumar P, Kizhakkedathu JN, Straus SK. Antimicrobial Peptides: Diversity, Mechanism of Action and Strategies to Improve the Activity and Biocompatibility In Vivo. Biomolecules. 2018;8(1).

35. Antoni L, Nuding S, Weller D, Gersemann M, Ott G, Wehkamp J, et al. Human colonic mucus is a reservoir for antimicrobial peptides. J Crohns Colitis. 2013;7(12):e652-64.

36. Furci L, Secchi M. AMPs and Mechanisms of Antimicrobial Action. Antimicrobial Peptides in Gastrointestinal Diseases: Elsevier; 2018. p. 97-131.

37. Zheng D, Liwinski T, Elinav E. Interaction between microbiota and immunity in health and disease. Cell Res. 2020;30(6):492-506.

38. Sekirov I, Russell SL, Antunes LC, Finlay BB. Gut microbiota in health and disease. Physiol Rev. 2010;90(3):859-904.

39. Luczynski P, McVey Neufeld KA, Oriach CS, Clarke G, Dinan TG, Cryan JF, Growing up in a Bubble: Using Germ-Free Animals to Assess the Influence of the Gut Microbiota on Brain and Behavior. Int J Neuropsychopharmacol. 2016;19(8).

40. Sommer F, Bäckhed F. The gut microbiota--masters of host development and physiology. Nat Rev Microbiol. 2013;11(4):227-38.

41. Structure, function and diversity of the healthy human microbiome. Nature. 2012;486(7402):207-

14.

42. Glowacki RWP, Martens EC. In sickness and health: Effects of gut microbial metabolites on human physiology. PLoS Pathog. 2020;16(4):e1008370.

43. Bischoff SC. 'Gut health': a new objective in medicine? BMC Med. 2011;9:24.

44. Lavelle A, Sokol H. Gut microbiota-derived metabolites as key actors in inflammatory bowel disease. Nat Rev Gastroenterol Hepatol. 2020;17(4):223-37.

45. Rivière A, Selak M, Lantin D, Leroy F, De Vuyst L. Bifidobacteria and Butyrate-Producing Colon Bacteria: Importance and Strategies for Their Stimulation in the Human Gut. Front Microbiol. 2016;7:979.

46. Ríos-Covián D, Ruas-Madiedo P, Margolles A, Gueimonde M, de Los Reyes-Gavilán CG, Salazar N. Intestinal Short Chain Fatty Acids and their Link with Diet and Human Health. Front Microbiol. 2016;7:185. 47. Parada Venegas D, De la Fuente MK, Landskron G, González MJ, Quera R, Dijkstra G, et al. Short Chain Fatty Acids (SCFAs)-Mediated Gut Epithelial and Immune Regulation and Its Relevance for Inflammatory Bowel Diseases. Front Immunol. 2019;10:277.

48. Roager HM, Dragsted LO. Diet-derived microbial metabolites in health and disease. Nutrition Bulletin. 2019;44:216-27.

49. Chiang JY. Bile acid metabolism and signaling. Compr Physiol. 2013;3(3):1191-212

50. Setchell KD, Heubi JE, Shah S, Lavine JE, Suskind D, Al-Edreesi M, et al. Genetic defects in bile acid conjugation cause fat-soluble vitamin deficiency. Gastroenterology. 2013;144(5):945-55.e6; quiz e14-5. 51. Hofmann AF. The enterohepatic circulation of bile acids in mammals: form and functions. Front Biosci (Landmark Ed). 2009;14:2584-98.

52. Staels B, Fonseca VA. Bile acids and metabolic regulation: mechanisms and clinical responses to bile acid sequestration. Diabetes Care. 2009;32 Suppl 2(Suppl 2):S237-45.

53. Ridlon JM, Kang DJ, Hylemon PB. Bile salt biotransformations by human intestinal bacteria. J Lipid Res. 2006;47(2):241-59.

54. de Aguiar Vallim TQ, Tarling EJ, Edwards PA. Pleiotropic roles of bile acids in metabolism. Cell Metab. 2013;17(5):657-69.

55. Lefebvre P, Cariou B, Lien F, Kuipers F, Staels B. Role of bile acids and bile acid receptors in metabolic regulation. Physiol Rev. 2009;89(1):147-91.

56. Baars A, Oosting A, Knol J, Garssen J, van Bergenhenegouwen J. The Gut Microbiota as a Therapeutic Target in IBD and Metabolic Disease: A Role for the Bile Acid Receptors FXR and TGR5. Microorganisms. 2015;3(4):641-66.

57. Fiorucci S, Mencarelli A, Cipriani S, Renga B, Palladino G, Santucci L, et al. Activation of the farnesoid-X receptor protects against gastrointestinal injury caused by non-steroidal anti-inflammatory drugs in mice. Br J Pharmacol. 2011;164(8):1929-38.

58. Gadaleta RM, van Erpecum KJ, Oldenburg B, Willemsen EC, Renooij W, Murzilli S, et al. Farnesoid $X$ receptor activation inhibits inflammation and preserves the intestinal barrier in inflammatory bowel disease. Gut. 2011;60(4):463-72.

59. Maran RR, Thomas A, Roth M, Sheng Z, Esterly N, Pinson D, et al. Farnesoid X receptor deficiency in mice leads to increased intestinal epithelial cell proliferation and tumor development. J Pharmacol Exp Ther. 2009;328(2):469-77.

60. Duboc H, Taché Y, Hofmann AF. The bile acid TGR5 membrane receptor: from basic research to clinical application. Dig Liver Dis. 2014;46(4):302-12. 
61. Poole DP, Godfrey C, Cattaruzza F, Cottrell GS, Kirkland JG, Pelayo JC, et al. Expression and function of the bile acid receptor GpBAR1 (TGR5) in the murine enteric nervous system. Neurogastroenterol Motil. 2010;22(7):814-25, e227-8.

62. Kawamata Y, Fujii R, Hosoya M, Harada M, Yoshida H, Miwa M, et al. A G protein-coupled receptor responsive to bile acids. J Biol Chem. 2003;278(11):9435-40.

63. Guo C, Chen WD, Wang YD. TGR5, Not Only a Metabolic Regulator. Front Physiol. 2016;7:646.

64. Ticho AL, Malhotra P, Dudeja PK, Gill RK, Alrefai WA. Bile Acid Receptors and Gastrointestinal Functions. Liver Res. 2019;3(1):31-9.

65. Chiang JYL, Ferrell JM. Bile acid receptors FXR and TGR5 signaling in fatty liver diseases and therapy. Am J Physiol Gastrointest Liver Physiol. 2020;318(3):G554-g73.

66. Hegyi P, Maléth J, Walters JR, Hofmann AF, Keely SJ. Guts and Gall: Bile Acids in Regulation of Intestinal Epithelial Function in Health and Disease. Physiol Rev. 2018;98(4):1983-2023.

67. Perez MJ, Briz O. Bile-acid-induced cell injury and protection. World J Gastroenterol. 2009;15(14):1677-89.

68. Bernstein H, Bernstein C, Payne CM, Dvorakova K, Garewal H. Bile acids as carcinogens in human gastrointestinal cancers. Mutat Res. 2005;589(1):47-65.

69. Alnouti Y. Bile Acid sulfation: a pathway of bile acid elimination and detoxification. Toxicol Sci. 2009;108(2):225-46

70. Donovan JM, Yousef IM, Carey MC. Pan-sulfation of bile salts markedly increases hydrophilicity and essentially abolishes self- and hetero-association with lecithin. Biochim Biophys Acta. 1993;1182(1):3745.

71. Gérard P. Metabolism of cholesterol and bile acids by the gut microbiota. Pathogens. 2013;3(1):14-

24.

72. Vancamelbeke M, Vermeire S. The intestinal barrier: a fundamental role in health and disease. Expert Rev Gastroenterol Hepatol. 2017;11(9):821-34.

73. Groschwitz KR, Hogan SP. Intestinal barrier function: molecular regulation and disease pathogenesis. J Allergy Clin Immunol. 2009;124(1):3-20; quiz 1-2.

74. Turner JR. Intestinal mucosal barrier function in health and disease. Nat Rev Immunol. 2009;9(11):799-809.

75. Ghosh SS, Wang J, Yannie PJ, Ghosh S. Intestinal Barrier Dysfunction, LPS Translocation, and Disease Development. J Endocr Soc. 2020;4(2):bvz039.

76. Chakaroun RM, Massier L, Kovacs P. Gut Microbiome, Intestinal Permeability, and Tissue Bacteria in Metabolic Disease: Perpetrators or Bystanders? Nutrients. 2020;12(4).

77. Pellegrini C, Antonioli L, Colucci R, Blandizzi C, Fornai M. Interplay among gut microbiota, intestinal mucosal barrier and enteric neuro-immune system: a common path to neurodegenerative diseases? Acta Neuropathol. 2018;136(3):345-61.

78. Knowles SR, Graff LA, Wilding H, Hewitt C, Keefer L, Mikocka-Walus A. Quality of Life in Inflammatory Bowel Disease: A Systematic Review and Meta-analyses-Part I. Inflamm Bowel Dis. 2018;24(4):742-51.

79. Kaser A, Zeissig S, Blumberg RS. Inflammatory bowel disease. Annu Rev Immunol. 2010;28:573-

621.

80. Maloy KJ, Powrie F. Intestinal homeostasis and its breakdown in inflammatory bowel disease. Nature. 2011;474(7351):298-306.

81. Xavier RJ, Podolsky DK. Unravelling the pathogenesis of inflammatory bowel disease. Nature. 2007;448(7152):427-34

82. Jäger S, Stange EF, Wehkamp J. Inflammatory bowel disease: an impaired barrier disease. Langenbecks Arch Surg. 2013;398(1):1-12.

83. Liverani E, Scaioli E, Digby RJ, Bellanova M, Belluzzi A. How to predict clinical relapse in inflammatory bowel disease patients. World J Gastroenterol. 2016;22(3):1017-33.

84. Nishida A, Inoue R, Inatomi O, Bamba S, Naito Y, Andoh A. Gut microbiota in the pathogenesis of inflammatory bowel disease. Clin J Gastroenterol. 2018;11(1):1-10.

85. Petersen C, Round JL. Defining dysbiosis and its influence on host immunity and disease. Cell Microbiol. 2014;16(7):1024-33.

86. Nishino K, Nishida A, Inoue R, Kawada Y, Ohno M, Sakai S, et al. Analysis of endoscopic brush samples identified mucosa-associated dysbiosis in inflammatory bowel disease. J Gastroenterol. 2018;53(1):95-106.

87. Frank DN, St Amand AL, Feldman RA, Boedeker EC, Harpaz N, Pace NR. Molecular-phylogenetic characterization of microbial community imbalances in human inflammatory bowel diseases. Proc Natl Acad Sci U S A. 2007;104(34):13780-5.

88. Duboc H, Rajca S, Rainteau D, Benarous D, Maubert MA, Quervain E, et al. Connecting dysbiosis, bile-acid dysmetabolism and gut inflammation in inflammatory bowel diseases. Gut. 2013;62(4):531-9.

89. Gevers D, Kugathasan S, Denson LA, Vázquez-Baeza Y, Van Treuren W, Ren B, et al. The treatment-naive microbiome in new-onset Crohn's disease. Cell Host Microbe. 2014;15(3):382-92.

90. Takahashi K, Nishida A, Fujimoto T, Fujii M, Shioya M, Imaeda H, et al. Reduced Abundance of 
Butyrate-Producing Bacteria Species in the Fecal Microbial Community in Crohn's Disease. Digestion. 2016;93(1):59-65.

91. Marchesi JR, Holmes E, Khan F, Kochhar S, Scanlan P, Shanahan F, et al. Rapid and noninvasive metabonomic characterization of inflammatory bowel disease. J Proteome Res. 2007;6(2):546-51.

92. Huda-Faujan N, Abdulamir AS, Fatimah AB, Anas OM, Shuhaimi M, Yazid AM, et al. The impact of the level of the intestinal short chain Fatty acids in inflammatory bowel disease patients versus healthy subjects. Open Biochem J. 2010;4:53-8.

93. Takaishi H, Matsuki T, Nakazawa A, Takada T, Kado S, Asahara T, et al. Imbalance in intestinal microflora constitution could be involved in the pathogenesis of inflammatory bowel disease. Int J Med Microbiol. 2008;298(5-6):463-72.

94. Franzosa EA, Sirota-Madi A, Avila-Pacheco J, Fornelos N, Haiser HJ, Reinker S, et al. Gut microbiome structure and metabolic activity in inflammatory bowel disease. Nat Microbiol. 2019;4(2):293305.

95. Jacobs JP, Goudarzi M, Singh N, Tong M, McHardy IH, Ruegger P, et al. A Disease-Associated Microbial and Metabolomics State in Relatives of Pediatric Inflammatory Bowel Disease Patients. Cell Mol Gastroenterol Hepatol. 2016;2(6):750-66.

96. Lloyd-Price J, Arze C, Ananthakrishnan AN, Schirmer M, Avila-Pacheco J, Poon TW, et al. Multiomics of the gut microbial ecosystem in inflammatory bowel diseases. Nature. 2019;569(7758):655-62.

97. Sinha SR, Haileselassie Y, Nguyen LP, Tropini C, Wang M, Becker LS, et al. Dysbiosis-Induced Secondary Bile Acid Deficiency Promotes Intestinal Inflammation. Cell Host Microbe. 2020;27(4):659-70. e5.

98. Begley M, Gahan CG, Hill C. The interaction between bacteria and bile. FEMS Microbiol Rev. 2005;29(4):625-51.

99. Klaus J, Spaniol U, Adler G, Mason RA, Reinshagen M, von Tirpitz CC. Small intestinal bacterial overgrowth mimicking acute flare as a pitfall in patients with Crohn's Disease. BMC Gastroenterol. 2009;9:61.

100. Rana SV, Sharma S, Malik A, Kaur J, Prasad KK, Sinha SK, et al. Small intestinal bacterial overgrowth and orocecal transit time in patients of inflammatory bowel disease. Dig Dis Sci. 2013;58(9):2594-8.

101. Buford TW. (Dis)Trust your gut: the gut microbiome in age-related inflammation, health, and disease. Microbiome. 2017;5(1):80.

102. O'Toole PW, Jeffery IB. Gut microbiota and aging. Science. 2015;350(6265):1214-5.

103. Saffrey MJ. Aging of the mammalian gastrointestinal tract: a complex organ system. Age (Dordr). 2014;36(3):9603.

104. Mabbott NA, Kobayashi A, Sehgal A, Bradford BM, Pattison M, Donaldson DS. Aging and the mucosal immune system in the intestine. Biogerontology. 2015;16(2):133-45.

105. Branca JJV, Gulisano M, Nicoletti C. Intestinal epithelial barrier functions in ageing. Ageing Res Rev. 2019;54:100938.

106. Popkes M, Valenzano DR. Microbiota-host interactions shape ageing dynamics. Philos Trans $R$ Soc Lond B Biol Sci. 2020;375(1808):20190596.

107. Biagi E, Franceschi C, Rampelli S, Severgnini M, Ostan R, Turroni S, et al. Gut Microbiota and Extreme Longevity. Curr Biol. 2016;26(11):1480-5.

108. Zwielehner J, Liszt K, Handschur M, Lassl C, Lapin A, Haslberger AG. Combined PCR-DGGE fingerprinting and quantitative-PCR indicates shifts in fecal population sizes and diversity of Bacteroides, bifidobacteria and Clostridium cluster IV in institutionalized elderly. Exp Gerontol. 2009;44(6-7):440-6.

109. Jackson MA, Jeffery IB, Beaumont M, Bell JT, Clark AG, Ley RE, et al. Signatures of early frailty in the gut microbiota. Genome Med. 2016;8(1):8.

110. Claesson MJ, Cusack S, O'Sullivan O, Greene-Diniz R, de Weerd H, Flannery E, et al. Composition, variability, and temporal stability of the intestinal microbiota of the elderly. Proc Natl Acad Sci U S A. 2011;108 Suppl 1(Suppl 1):4586-91.

111. An R, Wilms E, Masclee AAM, Smidt H, Zoetendal EG, Jonkers D. Age-dependent changes in GI physiology and microbiota: time to reconsider? Gut. 2018;67(12):2213-22.

112. Zoetendal EG, von Wright A, Vilpponen-Salmela T, Ben-Amor K, Akkermans AD, de Vos WM. Mucosa-associated bacteria in the human gastrointestinal tract are uniformly distributed along the colon and differ from the community recovered from feces. Appl Environ Microbiol. 2002;68(7):3401-7.

113. Vermeij WP, Hoeijmakers JH, Pothof J. Genome Integrity in Aging: Human Syndromes, Mouse Models, and Therapeutic Options. Annu Rev Pharmacol Toxicol. 2016;56:427-45.

114. Chen XM, Elisia I, Kitts DD. Defining conditions for the co-culture of Caco-2 and HT29-MTX cells using Taguchi design. J Pharmacol Toxicol Methods. 2010;61(3):334-42.

115. Jung $P$, Sato T, Merlos-Suárez A, Barriga FM, Iglesias $M$, Rossell $D$, et al. Isolation and in vitro expansion of human colonic stem cells. Nat Med. 2011;17(10):1225-7.

116. Sato T, Stange DE, Ferrante M, Vries RG, Van Es JH, Van den Brink S, et al. Long-term expansion of epithelial organoids from human colon, adenoma, adenocarcinoma, and Barrett's epithelium. Gastroenterology. 2011;141(5):1762-72.

117. Spence JR, Mayhew CN, Rankin SA, Kuhar MF, Vallance JE, Tolle K, et al. Directed differentiation 
of human pluripotent stem cells into intestinal tissue in vitro. Nature. 2011;470(7332):105-9.

118. Wallach TE, Bayrer JR. Intestinal Organoids: New Frontiers in the Study of Intestinal Disease and Physiology. J Pediatr Gastroenterol Nutr. 2017;64(2):180-5.

119. Thorne CA, Chen IW, Sanman LE, Cobb MH, Wu LF, Altschuler SJ. Enteroid Monolayers Reveal an Autonomous WNT and BMP Circuit Controlling Intestinal Epithelial Growth and Organization. Dev Cell. 2018;44(5):624-33.e4.

120. Kim HJ, Ingber DE. Gut-on-a-Chip microenvironment induces human intestinal cells to undergo villus differentiation. Integr Biol (Camb). 2013;5(9):1130-40. 


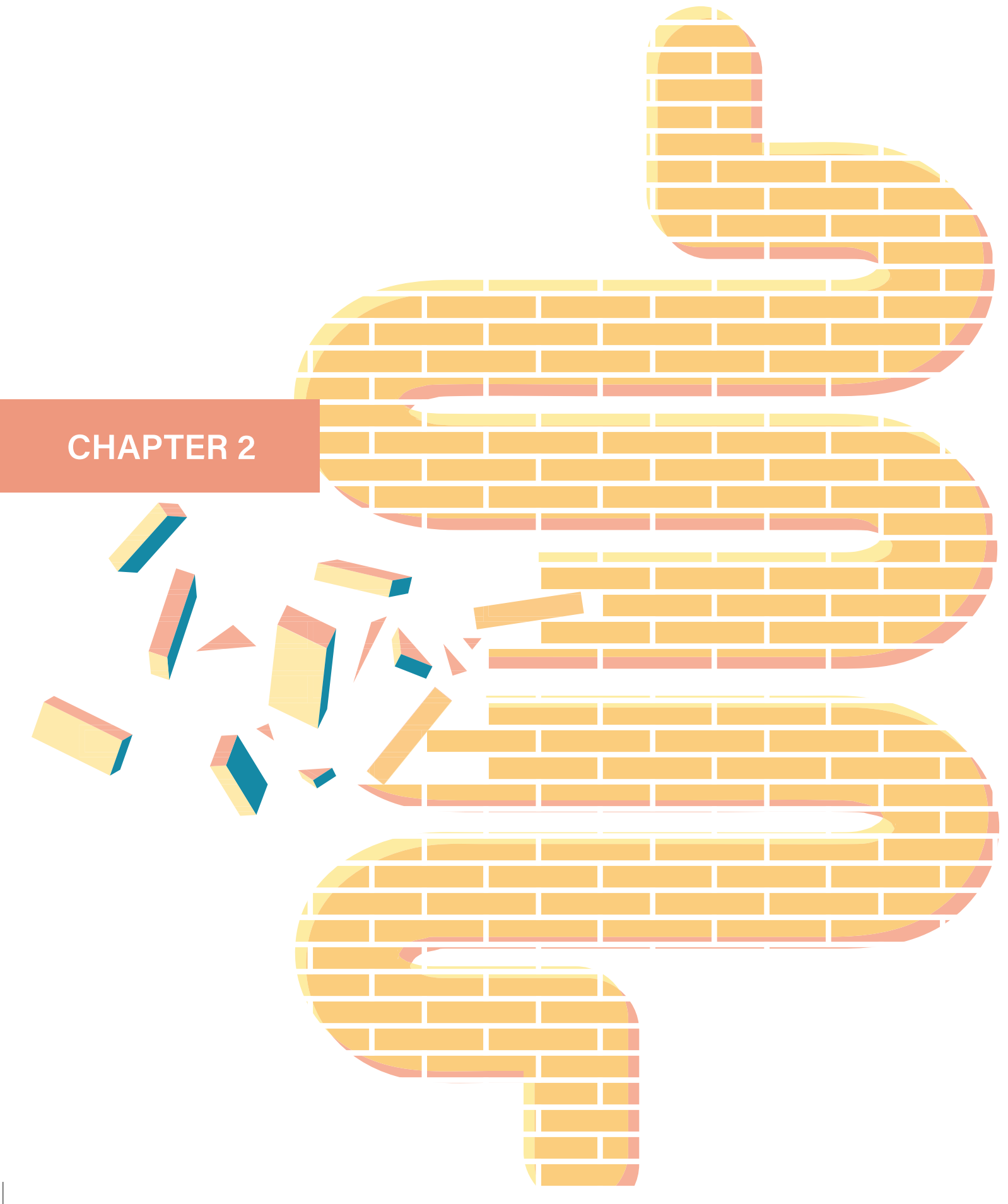




\section{Integrative analysis of gut microbiota composition, host colonic gene expression and intraluminal metabolites in aging C57BL/6J mice}

Benthe van der Lugt, Fenni Rusli, Carolien Lute, Andreas Lamprakis, Ethel Salazar, Mark V. Boekschoten, Guido J. Hooiveld, Michael Müller, Jacques Vervoort, Sander Kersten, Clara Belzer, Dieuwertje E.G. Kok, Wilma T. Steegenga 


\section{Abstract}

The aging process is associated with diminished colonic health. In this study, we applied an integrative approach to reveal potential interactions between determinants of colonic health in aging C57BL/6J mice. Analysis of gut microbiota composition revealed an enrichment of various potential pathobionts, including Desulfovibrio spp., and a decline of the health-promoting Akkermansia spp. and Lactobacillus spp. during aging. Intraluminal concentrations of various metabolites varied between ages and we found evidence for an increased gut permeability at higher age. Colonic gene expression analysis suggested that during the early phase of aging (between 6 and 12 months), expression of genes involved in epithelial-to-mesenchymal transition and (re)organization of the extracellular matrix were increased. Differential expression of these genes was strongly correlated with Bifidobacterium spp. During the later phase of aging (between 12 and 28 months), gene expression profiles pointed towards a diminished antimicrobial defense and were correlated with an uncultured Gastranaerophilales spp. This study demonstrates that aging is associated with pronounced changes in gut microbiota composition and colonic gene expression. Furthermore, the strong correlations between specific bacterial genera and host gene expression may imply that orchestrated interactions take place in the vicinity of the colonic wall and potentially mediate colonic health during aging. 


\section{Introduction}

Aging is a complex process characterized by a time-dependent loss of physical fitness accompanied by an increased risk of morbidities (1). The increase in life expectancy and the increased prevalence of age-related pathologies (1) demands further insight into the mechanisms underlying the aging process.

The colon is mainly involved in absorption of water and nutrients, but also plays an important role in general body health (2). Together with the small intestine, the colon constitutes the largest part of the whole-body immune system and is critical for an appropriate immune response towards the continuous exposure to potential inflammatory stimuli (3). One of the unique features of the colon is that it harbors a complex ecosystem of micro-organisms referred to as the gut microbiota. The gut microbiota is crucial for the development and maturation of the intestinal immune system during the early phase of life, as well as the maintenance of the intestinal epithelial barrier (4). Furthermore, the gut microbiota is involved in fermentation of non-digestible fibers and the production of shortchain fatty acids (SCFAs) (5), and as such lives in symbiosis with the host. In a healthy situation, the host is able to arrange an appropriate immune response upon microbial stimuli, which is nonresponsive towards beneficial commensal bacteria, but reacts to pathogenic bacteria and restricts bacterial overgrowth $(6,7)$. However, aging is accompanied by a decline in function of the immune system and a chronic low-grade inflammation, known as immunosenescence and inflammaging, respectively (8). These factors, together with age-related changes in diet, lifestyle as well as colon physiology, inevitably trigger changes in gut microbiota composition (9). Alterations in gut microbiota composition have been linked to intestinal inflammatory diseases, as well as the development of other pathologies, e.g. metabolic syndrome, frailty and nervous system diseases (10-12). Thus, in the context of mechanisms underlying healthy aging, gaining knowledge on novel molecular interactions between gut microbiota and the host may be the key for the prevention and treatment of age-related pathologies.

Remarkable age-related changes in gut microbiota composition were observed in large human cohort studies $(10,13-15)$. However, interpretation of the data is complex because of a wide range of interfering lifestyle factors, including diet and medication, but also other factors, such as genetic background and place of residence. Furthermore, the microbiota composition in these studies was analyzed in faecal samples, while previous studies showed distinct bacterial populations in faeces and colon lumen (16-20). This observation rises the question whether the microbiota composition in fresh faeces is representative for the microbiota composition in the colon. Besides, when exploring the effects of the microbiota composition on colonic gene expression profiles, the analysis of the 
colonic luminal content is preferred, because of its close proximity to the colonic wall. However, a major advantage of using faecal samples is the ability to investigate changes in gut microbiota composition during aging in a longitudinal manner.

To deepen our understanding regarding the effects of aging while limiting the effects of interfering factors, we generated an aging cohort of male $\mathrm{C} 57 \mathrm{BL} / 6 \mathrm{~J}$ mice. These mice received a semi-synthetic diet, were individually housed and fresh faeces was collected from the same mice at 4, 8, 12, 18, 24 and 28 months for analysis of faecal microbiota composition. After sacrifice at 6, 12, 24 and 28 months of age, scrapings of the colonic wall were isolated to investigate colonic gene expression and colonic luminal content was collected for investigation of colonic microbiota composition and metabolomics analysis.

We hypothesized that the interaction between host and the gut microbiota will change during the aging process and might contribute to a decline in colonic health at old age. To obtain potential mechanistic insights that could explain transcriptional perturbations in response to changes in colonic microbiota composition during aging, or vice versa, we used a comprehensive integrative analysis to explore interactions between the gut microbiota and host colonic gene expression.

\section{Materials and methods}

\section{Ethics statement}

The institutional and national guidelines for the care and use of animals were followed and the Local Committee for Care and Use of Laboratory Animals at Wageningen University approved the experiment (code number: drs-2010151b).

\section{Mice and study design}

In this study, 9-week old male C57BL/6J mice were housed individually and fed an ad libitum semisynthetic (AIN-93W) diet. The study design was described previously in more detail (52). In order to study the aging process, mice were randomly distributed into four groups that were sacrificed at 6 , 12,24 and 28 months ( $n=11-16$ mice per group). For 4 mice in the 24-months group and 5 mice in the 28-months group, fresh faeces were consecutively collected (directly after defecation) at the age of 4 , $8,12,18,24$ and 28 months. Bodyweight and food intake were monitored bi-weekly. During sacrifice, the colon was opened and the colonic luminal content was collected first. Then, the middle part of the colon $(\sim 1 \mathrm{~cm})$ was turned inside out, rolled and embedded in paraffin ('Swiss rolls') for histology. From the remaining colon parts, the mucosa and submucosa were scraped for RNA isolation. The 
mice included in the present study were also used as control group for diet-intervention studies in the liver and colon of which the data have been reported in previous publications $(21,52-54)$.

\section{Gut microbiota composition analysis}

For both fresh faeces (42 samples) and colonic luminal content (40 samples) the microbiota composition was determined. The detailed methods have been described previously (21). Briefly, DNA was isolated from faecal and colonic luminal content samples using the ZR Fecal DNA MicroPrep kit (ZYMO Research, Irvine, CA, USA) according to the manufacturer's instructions. Lysis of the samples was performed by bead beating using ZR BashingBead ${ }^{\text {TM }}$ Lysis Tubes (ZYMO Research, Irvine, CA, USA). Next, the V3-V4 region of the 16S rRNA gene was amplified. After purification of the amplicon, DNA quality was checked and a second PCR was performed using sample-specific barcoded primers (Nextera XT index kit, Illumina, San Diego, CA, USA). Purified PCR products were sent to BaseClear service laboratory (BaseClear BV, Leiden, The Netherlands) for sequencing on the MiSeq platform (Illumina, San Diego, CA, USA). The Casava pipeline (version 1.8.3, Illumina, San Diego, CA, USA) was used for de-multiplexing of the FASTQ files. After initial quality control, three fresh faeces samples were excluded for further analysis, as the coverage was below threshold ( $50 \%$ of the median number of reads). CLC Microbial Genomics Module version 1.2.1 (CLC Bio, Qiagen, Aarhus, Denmark) was used for further analysis of the sequencing data. FASTQ files of the fresh faeces and colonic luminal content were imported and processed simultaneously. The identified OTUs were aligned against the Silva database (version 119) at $97 \%$ similarity (55). A number of 1,034,303 reads were detected, for which 1208 OTUs were identified. 25 of these OTUs (265 reads) were unavailable. Shannon entropy was considered as a metric for alpha-diversity and the beta-diversity was determined using Bray-Curtis distances. Principal Component Analyses and Redundancy analysis were carried out using Canoco 5 (56). The input consisted of the 303 OTUs with a relative abundance of $\geq 0.1 \%$ in at least one sample. With regard to the RDA, the numbers of reads were centered and standardized using the Hellinger transformation (57). The 16S rRNA gene data of this study have been made available in the sequence read archive (SRA) at the NCBI with accession number SRP145060.

\section{Determination of metabolites in colonic luminal content}

The colonic luminal content of mice aged 6 months $(n=6), 24$ months $(n=8)$ and 28 months $(n=8)$ was prepared as described previously (21) and levels of metabolites were determined using ${ }^{1} \mathrm{H}-\mathrm{NMR}$. From the aligned spectra, integrals for SCFAs were identified. Besides, an untargeted approach 
was taken to identify metabolites that differed between age groups. After baseline correction, the concentrations of the metabolites were calculated by taking into account the dilution factor and number of hydrogen atoms. Correlations between concentrations of identified metabolites and the relative abundances of the 50 most abundant genera in colonic luminal content were determined using Spearman's correlation.

\section{Gene expression analysis}

For a number of 4 to 8 mice per age group, RNA was isolated from scrapings of the colonic wall and liver using TRIzol reagent (Invitrogen, Breda, The Netherlands). Isolated RNA was purified using RNeasy Micro columns (Qiagen, Venlo, The Netherlands) and total RNA yield (Nanodrop ND-1000, Nanodrop Products, Maarssen, The Netherlands) and RNA integrity (Agilent 2100 Bioanalyzer, Agilent Technologies, Amsterdam, The Netherlands) were measured. Only RNA was used that had a RNA integrity number (RIN) above 8.0 (Supplementary Table S4). Purified RNA (100 ng per sample) was converted to cDNA and labelled using an Ambion WT expression kit (Life Technologies, Bleiswijk, The Netherlands). Microarray hybridization and analysis was performed as previously described (52). Differences in gene expression between the age groups were analyzed using the Intensity Based Moderated T statistics (IBMT), using q-values $<0.01$ as threshold for the comparison 6 versus 12 months and $p$-values $<0.01$ for the comparison 12 versus 28 months. Microarray data has been submitted to the Gene Expression Omnibus (GEO) at the NCBI, and is accessible under number GSE113257. The Principal Component Analysis plot was generated using MultiExperiment Viewer version 4.9.0. Ingenuity Pathway Analysis (IPA) was used for identification of canonical pathways and upstream regulators (58). Gene Set Enrichment Analysis was performed to functionally interpret gene regulation (59). A false discovery rate (FDR) q-value of $<0.1$ was considered significantly enriched. Short Time-series Expression Miner (STEM) version 1.3.11 (60) was used to identify gene expression profiles during aging, using the mean Robust Multichip Average (RMA) values as input.

\section{Histology}

Swiss rolls of 6 mice per age group were cut in 5 micrometer sections and mounted on microscope slides (VWR Superfrost Plus Micro Slide). The sections were dewaxed multiple times with xylene, rehydrated in alcohol and stained with Haematoxylin/Eosin (H\&E) or Sirius red/Fast green. Pictures of the stained sections were taken using a microscope with Olympus CellSense Entry software (Olympus Europe, Hamburg, Germany). For the H\&E stained sections, the depths of the colonic crypts were measured using Olympus Cell^B software. 


\section{Multivariate analyses for gut microbiota and gene expression}

In order to integrate colonic microbiota composition and colonic gene expression, we performed multivariate analyses in R 3.3.1 using the MixOmics package (61). Partial Least Squares (PLS) regression in the canonical mode was used to determine the bi-directional relationship between the two datasets. We used data from individual mice for whom both gene expression as well as the microbiota composition from colonic luminal content were available. For the MixOmics analysis early during aging (i.e. between 6 and 12 months of age), a number of 11 mice were included ( 6 months: $n=4 ; 12$ months: $n=7$ ). A number of 14 mice were included for the analysis using the genes differentially expressed between 12 and 28 months ( $p<0.01)$ (12 months: $n=7 ; 28$ months: $n=7)$. The genera with a relative abundance of $\geq 0.1 \%$ in at least 1 sample were included, resulting in a number of 50 genera. First, the sequencing reads of the genera were transformed and zeros were imputed based on the centered log ratio (clr) using the aldex2 package with 1024 Monte Carlo permutations (62). Gene expression data were included after transformation into log2 RMA intensities. Relevance networks with the strongest positive and negative correlations $(R<-0.8$ or $R>0.8)$ were visualized using Cytoscape (63).

\section{Plasma measurements}

Plasma levels of LBP were determined using the LBP ELISA Kit Mouse (kit OKBB00573, Aviva Systems Biology, San Diego, USA), following the manufacturer's protocol. Plasma samples were diluted 100 times. Plasma levels of insulin were determined using the Mouse Adipokine kit (MADKMAG-71K, Merck Millipore, Darmstadt, Germany), according to the manufacturer's protocol.

\section{Statistical analyses}

Statistical analyses were, unless stated otherwise, carried out using GraphPad Prism 5.04 (GraphPad Software, San Diego, California, USA). The Kolmogorov-Smirnov test was used to test if data were normally distributed. In case of normal distribution, one-way analysis of variance (ANOVA) in combination with Tukey's multiple comparisons test was used to test for differences between ages. In case the data were not normally distributed, the non-parametric Kruskal-Wallis one-way analysis of variance following a Dunn's post hoc test and a Mann-Whitney test were used for independent samples. A Friedman test or Wilcoxon Signed Rank test were used in case samples were dependent. The Bonferroni or Benjamini-Hochberg approach were used to adjust for multiple testing, if appropriate. For all statistical tests, unless stated otherwise, a $p$-value of $p<0.05$ was considered as statistically significant. 


\section{Results}

\section{Physiological differences between mice during aging}

Body weight as well as food intake increased during aging (Figure S1A-B). Also liver weight and relative liver weight (\% of body weight) were significantly higher in older mice (Figure S1C-D). To determine if metabolic health altered during aging, fasting insulin levels in plasma were assessed. These levels were significantly increased at 24 and 28 months compared to 6 months $(p<0.01)$ (Figure S1E). Death rates of the mice remained low till the age of 20 months and started to increase afterwards (Figure S1F). Most mice that died prematurely suffered from multiple conditions (Data set S1).

\section{Alterations in faecal and colonic microbiota composition during aging}

Fresh faeces samples were collected longitudinally at 4, 8, 12, 18, 24 and 28 months of age in a subset of 9 mice. Sequencing of the V3-V4 region of the 16S rRNA gene revealed that the alpha-diversity, in terms of Shannon entropy, did not change during aging (Figure 1A). To explore variance in faecal microbiota composition, a Principal Component Analysis (PCA) on relative abundance of genera was performed. The PCA revealed that the first principal component, explaining $42.7 \%$ of the variation, separated the mice aged 4 and 8 months from the other age groups (Figure S2A). Dissimilarities in faecal microbiota composition between age groups were further investigated at two taxonomic levels. At the phylum level, the relative abundance of Proteobacteria was less abundant in young mice (4 months) and increased from 8 months onwards in most mice (Figure 1B). Verrucomicrobia and Actinobacteria were highly abundant at 4 months, but both disappeared in most mice between 4 and 12 months of life $(<1 \%)$. By exception, the relative abundance of Verrucomicrobia in mouse 9 at 28 months of age was high compared to the other mice at the same age (Figure 1B).

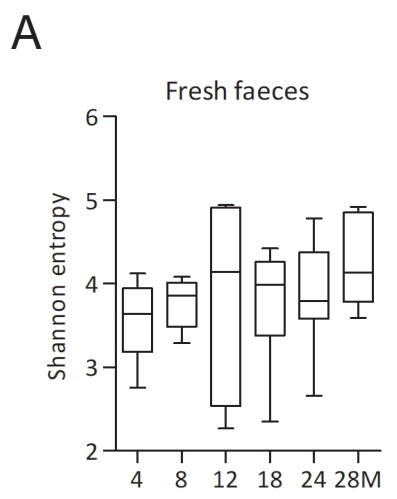




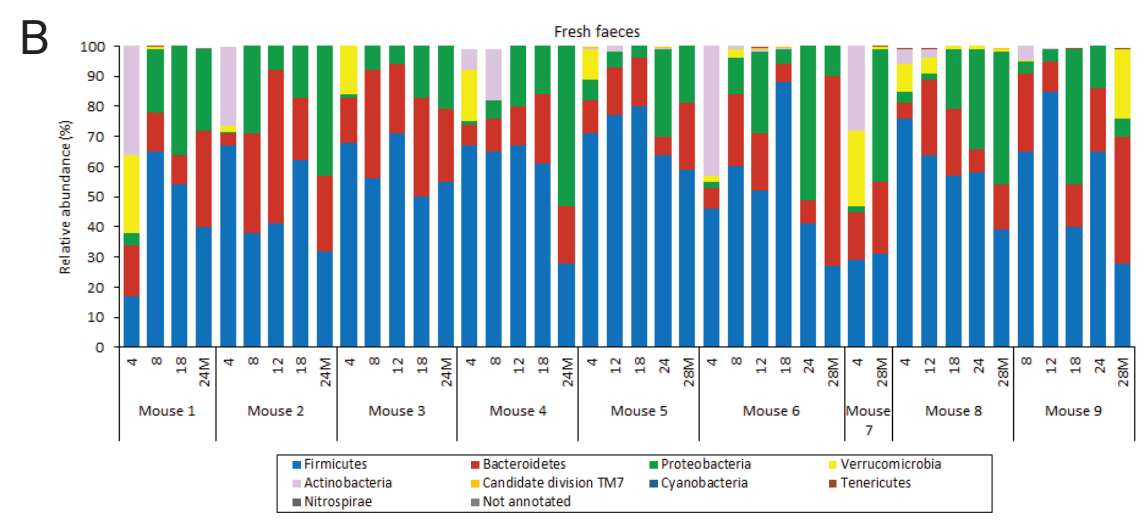

C
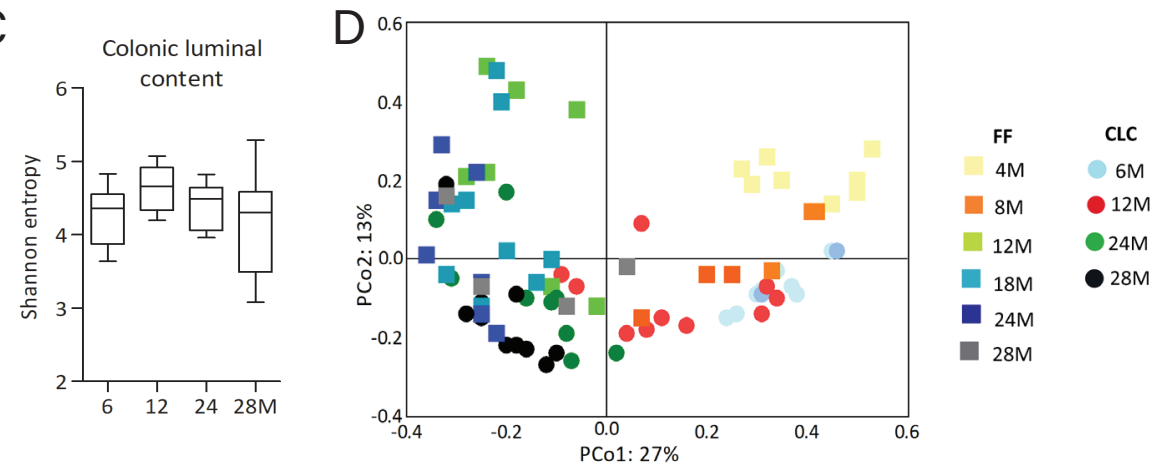

E

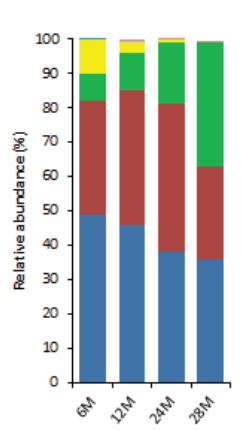

F

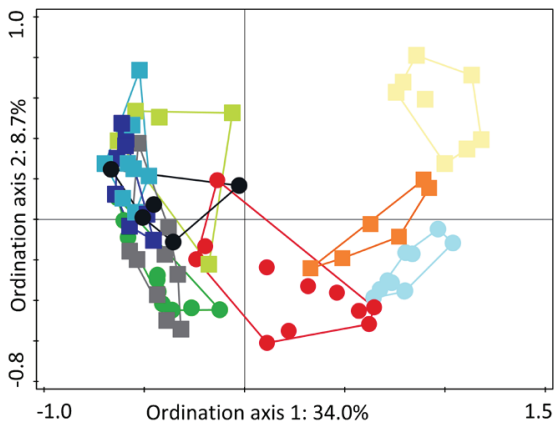

FF CLC

$4 \mathrm{M} 6 \mathrm{M}$

$8 \mathrm{M} \bigcirc 12 \mathrm{M}$

$12 \mathrm{M}$

$18 \mathrm{M}$

- $24 \mathrm{M}$

- $28 \mathrm{M}$

$24 \mathrm{M}$

- $28 \mathrm{M}$
G

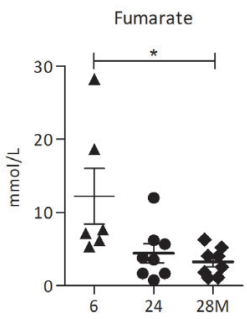

$\mathrm{H}$

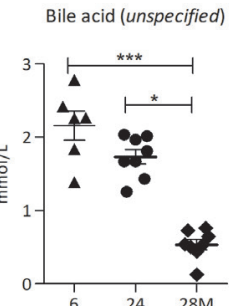

I

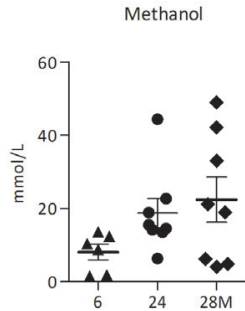

J

Genera

Desulfovibrio spp.

Ruminococcaceae Incertae Sedis spp.

Coriobacterioceae uncultured spp

Alloprevotella spp.

Parabacteroides spp.

Bilophila spp.

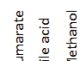


Figure 1 Alterations in gut microbiota composition and metabolites during aging. (A) The alphadiversity measured by the Shannon entropy in fresh faeces. Boxes extend from the 25th to 75th percentile, line in the middle represents median, and whiskers represent minimum and maximum values. (B) Relative abundance (\%) at phylum level in fresh faeces collected longitudinally in a subset of 9 mice. (C) The alpha-diversity measured by the Shannon entropy in colonic luminal content. (D) Principal Coordinate Analysis ( $\mathrm{PCOA}$ ) based on Bray-Curtis distances showing dissimilarities between all individual samples (FF and CLC). (E) The average relative abundance (\%) at phylum level in colonic luminal content. Legend corresponds to graph with relative abundance at phylum level in fresh faeces. (F) Redundancy Analysis (RDA) displays the part of the variation in microbiota composition explained by the age groups and source. Sample plot showing the clustering of the samples. Legend corresponds to PCoA plot. The distance between the ages and sample sources approximates the average dissimilarity of the microbiota composition. The FF analyses were based on a subset of $n=9$ mice at consecutive ages $(4,8,12,18,24$ and 28 months). For the CLC analyses, $n=10$ mice per age group $(6,12,24$ and 28 months) were taken into account. (G) Levels of fumarate, $(\mathrm{H})$ an unspecified bile acid and $(\mathrm{I})$ methanol detected by ${ }^{1} \mathrm{H}-\mathrm{NMR}$ in CLC at 6 months $(n=6), 24$ months $(n=8)$ and 28 months $(n=8)$ of age. Error bars represent standard error of the mean (S.E.M.). (J) Strongest Spearman correlation coefficients $(-0.6<r>0.6)$ for the concentrations of the identified metabolites and the relative abundances of the 50 most abundant genera in colonic luminal content.

Next to the analysis of the fresh faeces samples, the microbiota composition of the colonic luminal content of 40 mice sacrificed at the age of $6,12,24$ and 28 months of age was investigated. Similar to the results obtained from the fresh faeces samples, no significant changes in Shannon entropy were found (Figure 1C) and the PCA revealed that the youngest and oldest age groups explained most of the variation (Figure S2B). These findings were strengthened by the distancebased Principal Coordinate Analysis (PCoA), since the first principal coordinate, explaining 27\% of the variation, also separated young and old mice (Figure 1D). Comparison of the relative abundance at phylum and genus level between fresh faeces and colonic luminal content revealed mostly similar trends, although Actinobacteria phylum was hardly abundant in colonic luminal content (Figure 1E, Table 1, Figure S3). To investigate the impact of both age and source (fresh faeces and colonic luminal content) on the total variation, a Redundancy analysis (RDA) was performed. Ordination axis 1 explained $34.0 \%$ of the variation and separated the youngest age groups of both sources. Interestingly, ordination axis 2 explained $8.7 \%$ of the variation and separated the colonic luminal content and fresh faeces samples, indicating that the explanatory variable age contributed more to the variation in microbiota composition than the source variable (Figure 1F). Taken together, these results reveal pronounced changes in gut microbiota composition during aging, both in fresh faeces and more moderate in colonic luminal content. Additionally, subtle differences between the faecal and colonic microbiota composition were found. 


\section{Metabolite profiles in colonic luminal content}

To explore whether observed changes in gut microbiota composition may come with potential functional consequences, 'H-NMR was performed in colonic luminal content at 6,24 and 28 months of age. Concentrations of the SCFAs butyrate, propionate and succinate did not differ between the age groups, except for acetate that was significantly lower at 24 months compared to 6 months $(p<0.05)$ (data not shown). Intraluminal concentrations of fumarate decreased during aging, as well as an unspecified bile acid (Figure 1G-H). It was not possible to exactly identify this bile acid, since this peak in the NMR spectrum (0.17 ppm) is located in a rather unexplored region. However, it can be ruled out that this is one of the bile acids as described by Kok and colleagues (21), since these bile acids were identified in a different region of the spectrum (0.6-0.8 ppm). The concentration of methanol increased in older mice (Figure 11). A correlation analysis was performed to investigate whether the levels of these metabolites could be related to the relative abundances of the 50 most abundant genera. The strongest negative correlations were found for fumarate that was negatively correlated with the relative abundance of Desulfovibrio spp. ( $r=-0.651)$, an uncultured Ruminococcaceae spp. $(r=-0.708)$ and an uncultured Coriobacteriaceae spp. $(r=0.681)$, and positively correlated with Parasutterella spp. $(r=0.637)$ (Figure 1J).

\section{Changes in colonic gene expression in 6, 12, 24 and 28-month-old mice}

To explore the effect of aging on gene expression in the colonic wall, microarray analysis was performed on mRNA isolated from colonic scrapings from the same mice for which we also had data on the microbiota composition. A PCA carried out on the top 1,000 most variable genes revealed a clear segregation of the 6-months-old mice (Figure 2A). No clear separate clustering was observed for the mice from the other age groups, indicating that only gene expression at 6 months differed substantially from all other age groups. The highest number of significantly differentially expressed genes was found when comparing the youngest (6 months) versus the three older age groups (Figure 2B). There was a large overlap of genes $(n=610)$ that had an increased expression when comparing 6 months with 12, 24 or 28 months (Figure $\mathbf{2 C}$ ). Together, these results indicate that the expression levels of a substantial number of genes increased between 6 and 12 months, and remained increased also at higher ages. 


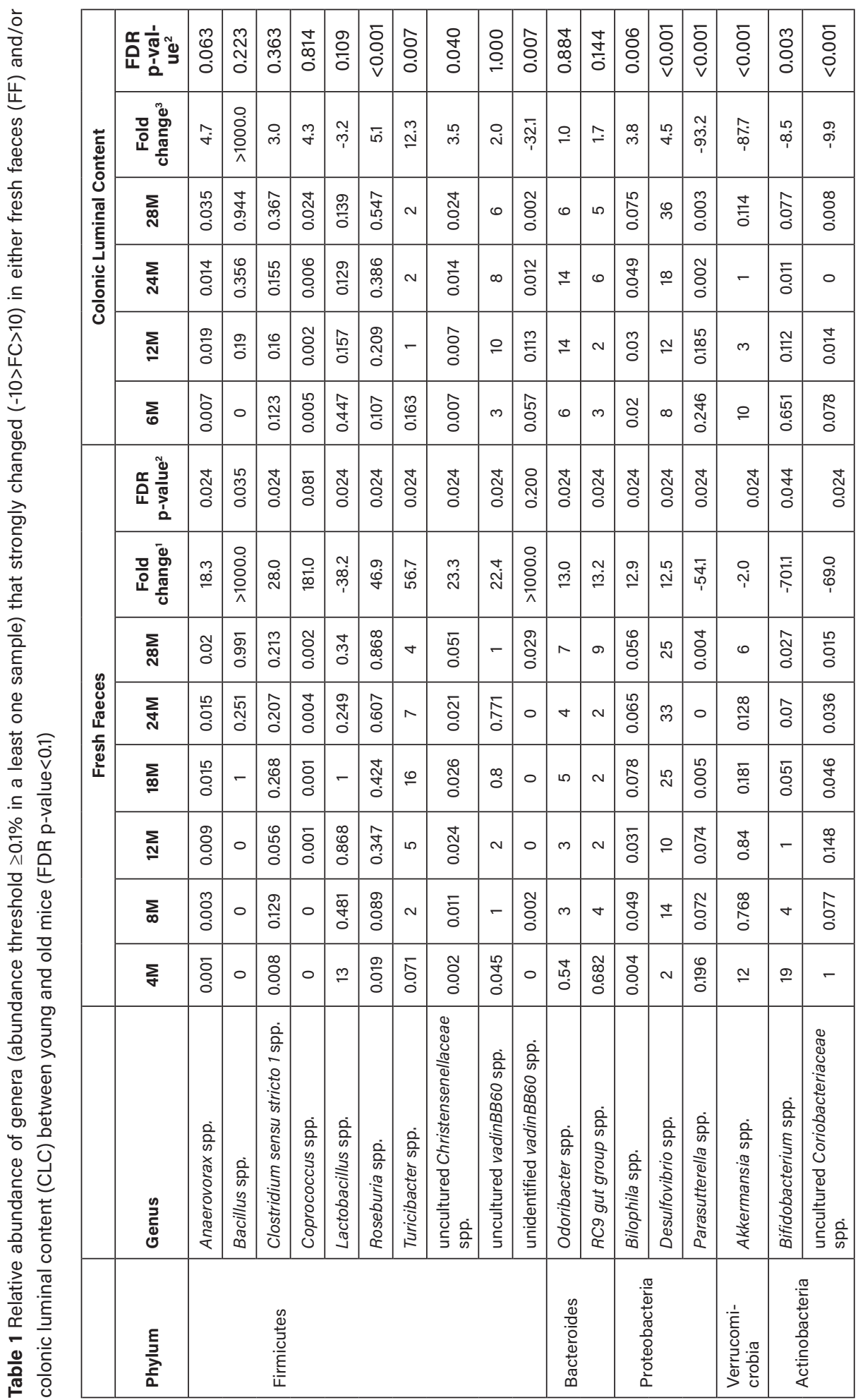




\begin{tabular}{|c|c|}
\hline $\begin{array}{l}0 \\
\text { o } \\
0\end{array}$ & $\begin{array}{l}\text { J } \\
\text { O }\end{array}$ \\
\hline$\stackrel{\circ}{m}$ & $\begin{array}{l}\stackrel{\circ}{\circ} \\
\stackrel{0}{1}\end{array}$ \\
\hline $\begin{array}{l}0 \\
\stackrel{0}{0} \\
0 \\
0\end{array}$ & $\begin{array}{l}\text { ¿ } \\
\text { O }\end{array}$ \\
\hline 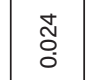 & $\begin{array}{l}\infty \\
\stackrel{0}{0} \\
0\end{array}$ \\
\hline $\begin{array}{l} \pm \\
\stackrel{0}{0} \\
0 \\
0\end{array}$ & $\begin{array}{l}8 \\
8 \\
0\end{array}$ \\
\hline $\begin{array}{l}m \\
0 \\
0 \\
0\end{array}$ & 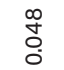 \\
\hline $\begin{array}{l}\mathbb{N} \\
\stackrel{0}{0}\end{array}$ & 䓞 \\
\hline 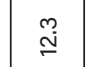 & $\stackrel{\circ}{\circ}$ \\
\hline$\hat{\ddot{\circ}}$ & 0 \\
\hline $\begin{array}{l}\stackrel{0}{0} \\
\stackrel{0}{0} \\
0\end{array}$ & $\bar{\delta}$ \\
\hline $\begin{array}{l}\hat{0} \\
\stackrel{0}{0}\end{array}$ & $\bar{\circ}$ \\
\hline$\stackrel{E}{\overline{0}}$ & 0 \\
\hline ̃o & $\circ$ \\
\hline 号 & 0 \\
\hline 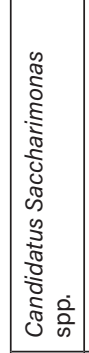 & 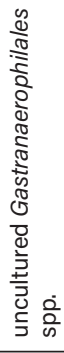 \\
\hline 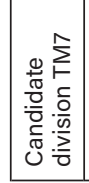 & 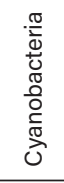 \\
\hline
\end{tabular}

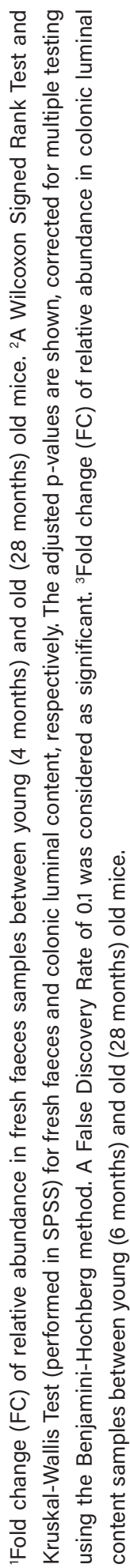




\section{Dominant cluster of genes similarly expressed between 6 and 12 months}

Visualization of the expression profiles of the 1,990 genes that were differentially expressed in the colonic wall of 6-months-old mice and the three older age groups (Data set S2) revealed a clustering of the majority of genes in four different expression profiles (Figure 2D). The profile in red contained by far the largest number of genes (817 genes) and showed the most prominent expression change between 6 and 12 months, remaining mostly stable afterwards (Figure 2D). The green profiles (602 genes in total) and the purple profile (217 genes) represent genes with decreased and increased expression levels during aging, respectively. Gene Ontology (GO) enrichment analysis of the green and purple profiles did not reveal any significant GO categories. However, analysis of the red profile revealed numerous significant GO categories that were all related to the extracellular matrix (ECM) organization and other structural development processes (Table 2). Ingenuity Pathway Analysis (IPA) and Gene Set Enrichment Analysis (GSEA) carried out on the genes differentially expressed between 6 and 12 months confirmed that similar pathways were affected (Figure 2E and Table S2A). IPA also revealed the 'Regulation of the Epithelial-to-Mesenchymal Transition Pathway' as a significantly regulated pathway in the colon between 6 and 12 months of age (Figure 2E and Table S2C). To analyze this aspect in more detail we assessed the expression regulation of genes involved in the epithelial-to-mesenchymal transition (EMT) in our microarray data set (Table S2D). Remarkably, while the expression of the epithelial markers E-cadherin (Cdh1), Occludin (OcIn) and most Claudins was minimally altered, the expression levels of the mesenchymal markers OBcadherin (Cdh11), Vimentin (Vim), Fibronectin (Fn1) and Vitronectin (Vtn) (Figure 2F and Table S2D) were strongly increased between 6 and 12 months and remained constant afterwards. In addition, the same expression pattern was found for transcription factors associated with EMT, i.e. Snail Family Transcriptional Repressor 1 and 2 (Snai1, 2), Zinc Finger E-Box Binding Homeobox 1 and $2(Z e b 1,2)$ (Figure 2F), as well as other EMT-inducers, such as Transforming growth factor beta 1 (Tgfb1) and Transcription Factor 21 (Tcf21). IPA identified the transcription factor Transforming growth factor beta 1 (Tgfb1) as the strongest activated upstream regulator associated with differential gene expression between 6 and 12 months (Table S2E). 


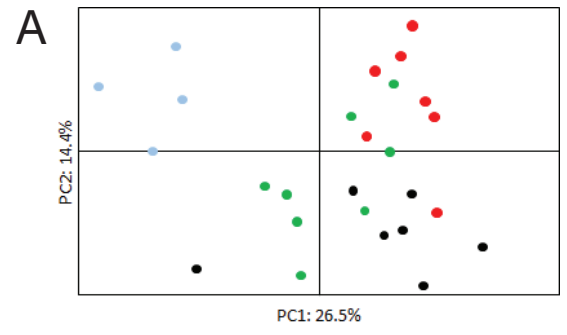

6 months $\bullet 12$ months $\bullet 24$ months $\bullet 28$ months

C

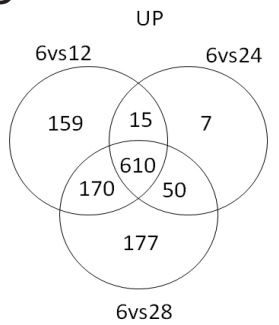

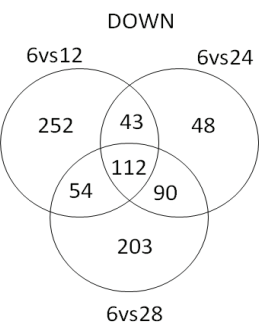

B

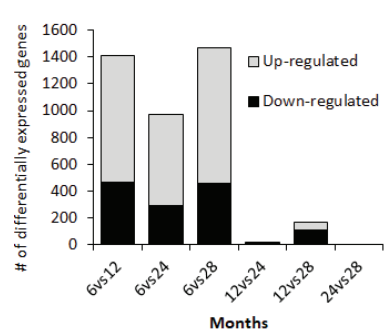

D

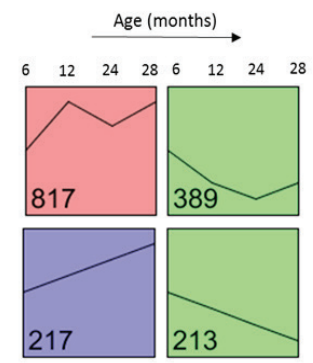

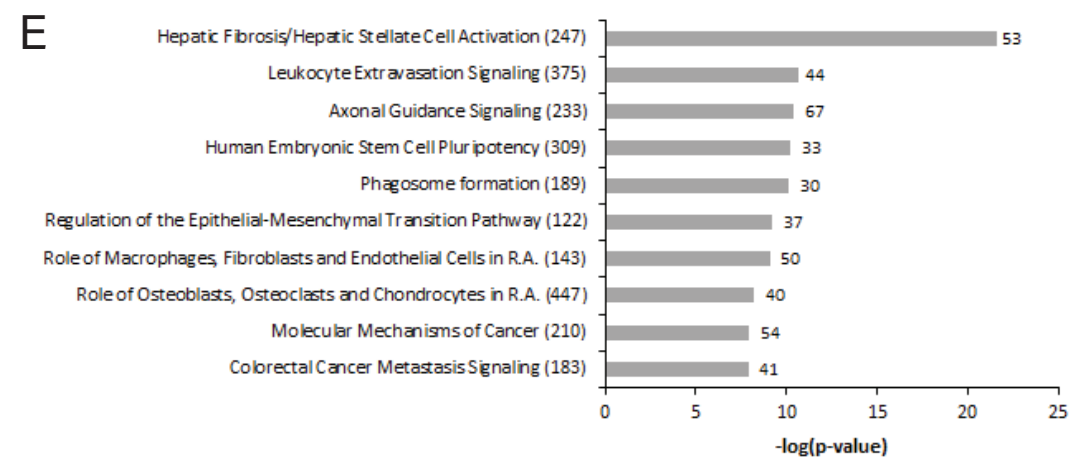

$\mathrm{F}$
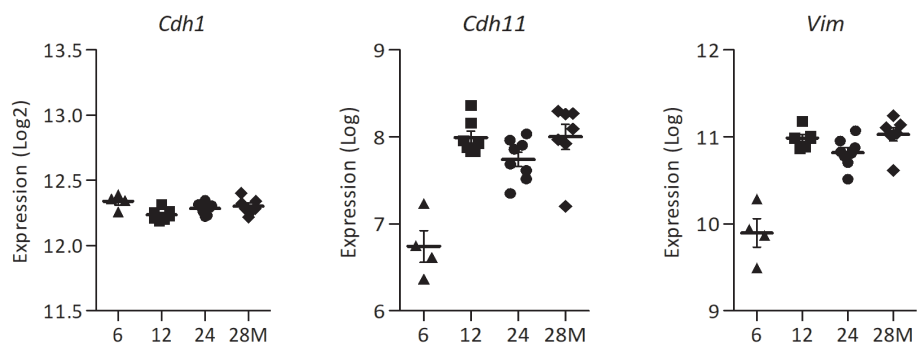

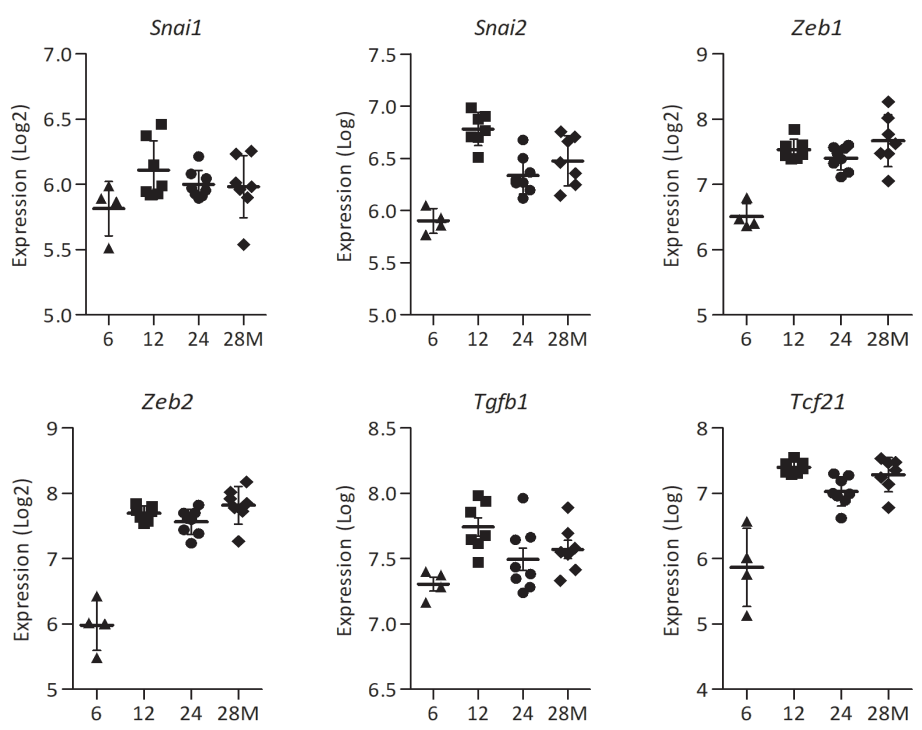

G
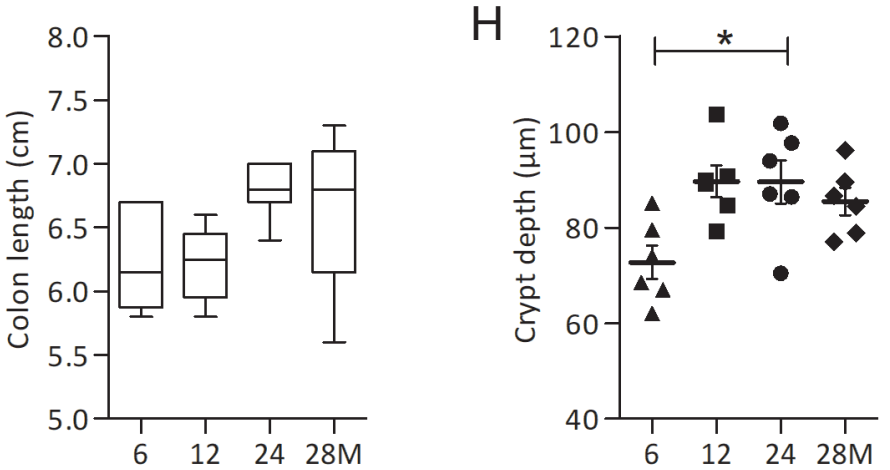

I

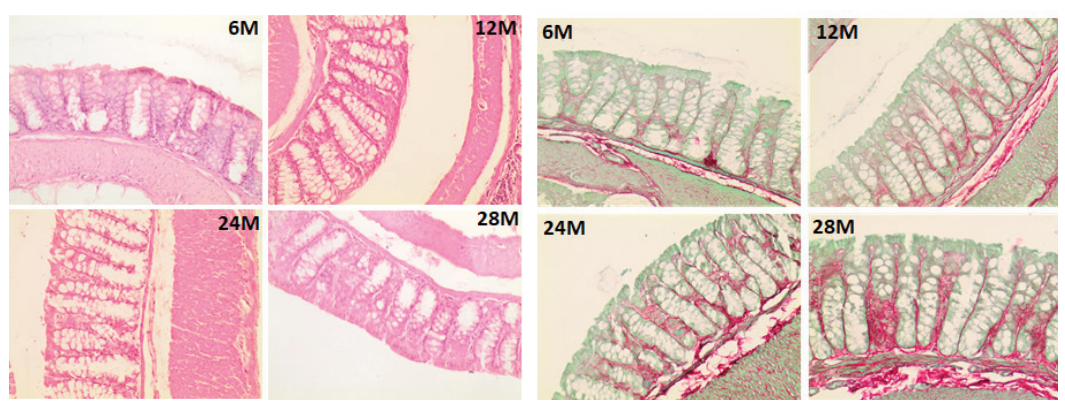


Figure 2 Changes in colonic gene expression in 6, 12, 24 and 28-month-old mice. (A) Principal Component Analysis (PCA) plot using the top 1,000 most variable genes showing the first 2 principal components. (B) The number of differentially expressed genes for all age comparisons (IBMT $q<0.01$ ). (C) Venn diagram showing the number of overlapping and unique differentially expressed genes between 6vs12, 6vs24 and $6 v s 28$ months. (D) Each significantly differentially expressed gene (6vs12, 6vs24, 6vs28 months, $q<0.01$ ) was assigned to the model profile that most closely matched their gene expression profile, executed by STEM. The model profiles with the highest number of genes assigned to (as indicated by the number in the box) are shown. (E) Top 10 most significant canonical pathways between 6 and 12 months as determined by Ingenuity Pathway Analysis (R.A. = Rheumatoid Arthritis). Numbers behind bars represent the number of differentially expressed genes (IBMT $\mathrm{q}<0.01)$ and numbers behind the pathways represent the total number of genes. (F) The gene expression (Log2) of the epithelial marker E-cadherin (Cdh1), mesenchymal markers OB-cadherin (Cdh11) and Vimentin (Vim), transcription factors Snail Family Transcriptional Repressor 1 and 2 (Snai1, 2) and Zinc Finger E-Box Binding Homeobox 1 and 2 (Zeb1, 2), Transforming growth factor beta 1 (Tgfb1) and Transcription Factor 21 (Tcf21). (G) Length of the colon ( $\mathrm{cm}$ ) at sacrifice. (H) Depth of colonic crypts $(\mu \mathrm{m})$ measured in H\&E stained colon samples. (I) Representative pictures of Haematoxylin and Eosin (H\&E) staining and Sirius Red/Fast Green staining of colon tissue at 6, 12, 24 and 28 months (200x magnification). Error bars reflect standard error of mean (S.E.M.). * $p<0.05$.

\section{Change in colon morphology during aging}

To study the potential morphological consequences of the observed changes in ECM- and EMTrelated genes we measured the colon length at sacrifice as well as colonic crypt depths in H\&Estained Swiss rolls. The obtained results revealed an increase in colon length during aging (Figure 2G). A marked deepening of the crypts between 6 and 12 months of age was observed, which minimally increased afterwards (Figure 2H). Furthermore, Sirius Red/Fast Green staining of the same Swiss rolls showed that the ECM protein collagen gradually increased with age (Figure 2I).

Table 2 Top 5 most significant Gene Ontology (GO) categories (i.e. Biological Process, Molecular Function, Cellular Component) belonging to the red, purple and combined green profiles, as determined by STEM.

\section{RED PROFILE}

\begin{tabular}{|l|l|c|c|c|c|c|c|}
\hline Category ID & Category Name & $\begin{array}{c}\text { \#Genes } \\
\text { Category }\end{array}$ & $\begin{array}{c}\text { \#Genes } \\
\text { Assigned }\end{array}$ & $\begin{array}{c}\text { \#Genes } \\
\text { Expected }\end{array}$ & $\begin{array}{c}\text { \#Genes } \\
\text { Enriched }\end{array}$ & $p$-value & $\begin{array}{c}\text { Corrected } \\
\text { p-value* }\end{array}$ \\
\hline GO:0031012 & extracellular matrix & 157 & 125 & 64.5 & 60.5 & $9.70 \mathrm{E}-25$ & $4.30 \mathrm{E}-21$ \\
\hline GO:0072359 & $\begin{array}{l}\text { circulatory system } \\
\text { development }\end{array}$ & 229 & 162 & 94 & 68 & $4.30 \mathrm{E}-22$ & $1.90 \mathrm{E}-18$ \\
\hline GO:0005578 & $\begin{array}{l}\text { proteinaceous extracel- } \\
\text { lular matrix }\end{array}$ & 129 & 103 & 53 & 50 & $1.50 \mathrm{E}-20$ & $6.80 \mathrm{E}-17$ \\
\hline GO:0072358 & $\begin{array}{l}\text { cardiovascular system } \\
\text { development }\end{array}$ & 175 & 129 & 71.8 & 57.2 & $4.80 \mathrm{E}-20$ & $2.20 \mathrm{E}-16$ \\
\hline GO:0001944 & $\begin{array}{l}\text { vasculature develop- } \\
\text { ment }\end{array}$ & 175 & 129 & 71.8 & 57.2 & $4.80 \mathrm{E}-20$ & $2.20 \mathrm{E}-16$ \\
\hline
\end{tabular}


PURPLE PROFILE

\begin{tabular}{|l|l|c|c|c|c|c|c|}
\hline Category ID & Category Name & $\begin{array}{c}\text { \#Genes } \\
\text { Category }\end{array}$ & $\begin{array}{c}\text { \#Genes } \\
\text { As- } \\
\text { signed }\end{array}$ & $\begin{array}{c}\text { \#Genes } \\
\text { Expected }\end{array}$ & $\begin{array}{c}\text { \#Genes } \\
\text { En- } \\
\text { riched }\end{array}$ & p-value & $\begin{array}{c}\text { Corrected } \\
\text { p-value* }\end{array}$ \\
\hline GO:0007416 & synapse assembly & 28 & 11 & 3.1 & 7.9 & $8.10 \mathrm{E}-05$ & 0.363 \\
\hline GO:0022607 & $\begin{array}{l}\text { cellular component } \\
\text { assembly }\end{array}$ & 307 & 52 & 33.5 & 18.5 & $3.10 \mathrm{E}-04$ & 1 \\
\hline GO:0044085 & $\begin{array}{l}\text { cellular component } \\
\text { biogenesis }\end{array}$ & 317 & 52 & 34.6 & 17.4 & $7.20 \mathrm{E}-04$ & 1 \\
\hline GO:0050808 & synapse organization & 45 & 12 & 4.9 & 7.1 & $2.30 \mathrm{E}-03$ & 1 \\
\hline GO:0031514 & motile cilium & 10 & 5 & 1.1 & 3.9 & $2.30 \mathrm{E}-03$ & 1 \\
\hline
\end{tabular}

COMBINED GREEN PROFILES

\begin{tabular}{|c|c|c|c|c|c|c|c|}
\hline Category ID & Category Name & $\begin{array}{l}\text { \#Genes } \\
\text { Category }\end{array}$ & $\begin{array}{l}\text { \#Genes } \\
\text { As- } \\
\text { signed }\end{array}$ & $\begin{array}{l}\text { \#Genes } \\
\text { Expected }\end{array}$ & $\begin{array}{l}\text { \#Genes } \\
\text { Enriched }\end{array}$ & p-value & $\begin{array}{c}\text { Corrected } \\
\text { p-value* }\end{array}$ \\
\hline GO:0005654 & nucleoplasm & 197 & 87 & 59.6 & 27.4 & $9.50 \mathrm{E}-06$ & 0.043 \\
\hline GO:0031981 & nuclear lumen & 253 & 105 & 76.5 & 28.5 & $3.10 \mathrm{E}-05$ & 0.139 \\
\hline GO:0070013 & $\begin{array}{l}\text { intracellular organelle } \\
\text { lumen }\end{array}$ & 279 & 114 & 84.4 & 29.6 & $3.10 \mathrm{E}-05$ & 0.139 \\
\hline GO:0043233 & organelle lumen & 279 & 114 & 84.4 & 29.6 & 3.10E-05 & 0.139 \\
\hline GO:0044428 & nuclear part & 286 & 114 & 86.5 & 27.5 & $1.20 \mathrm{E}-04$ & 0.517 \\
\hline
\end{tabular}

*Bonferroni correction

\section{Integrative analysis of microbiota composition and gene expression in the early phase of aging}

To obtain insight into the correlations between differential gene expression during the early phase of aging and the colonic microbiota composition, an integrative analysis was performed based on data of the individual mice. The 50 genera with a relative abundance of more than $0.1 \%$ in at least one sample and the 817 genes identified by STEM (Figure 2D, red cluster) showed several strong $(r<-0.80$ or $r>0.80)$ positive and negative correlations (top 25 strongest correlations shown in Figure 3). Bifidobacterium spp. was negatively correlated with 228 genes, including a cluster of EMT- and ECM-associated genes, e.g. Cdh11, Fn1, Zeb1, Zeb2, Vim, Vtn and Fgfr1, amongst others (Figure 3 and Data set S3). Additionally, Turicibacter spp. and an uncultured member of the Lachnospiraceae family were positively correlated with a large number of genes, including Platelet Derived Growth Factor Receptor Beta (Pdgfrb), Wnt Family Member 2B (Wnt2b) and Gremlin 2 (Grem2), amongst others. Together, these results might imply the potential role of gut microbiota in the regulation of ECM (re)organization and EMT, or vice versa. 


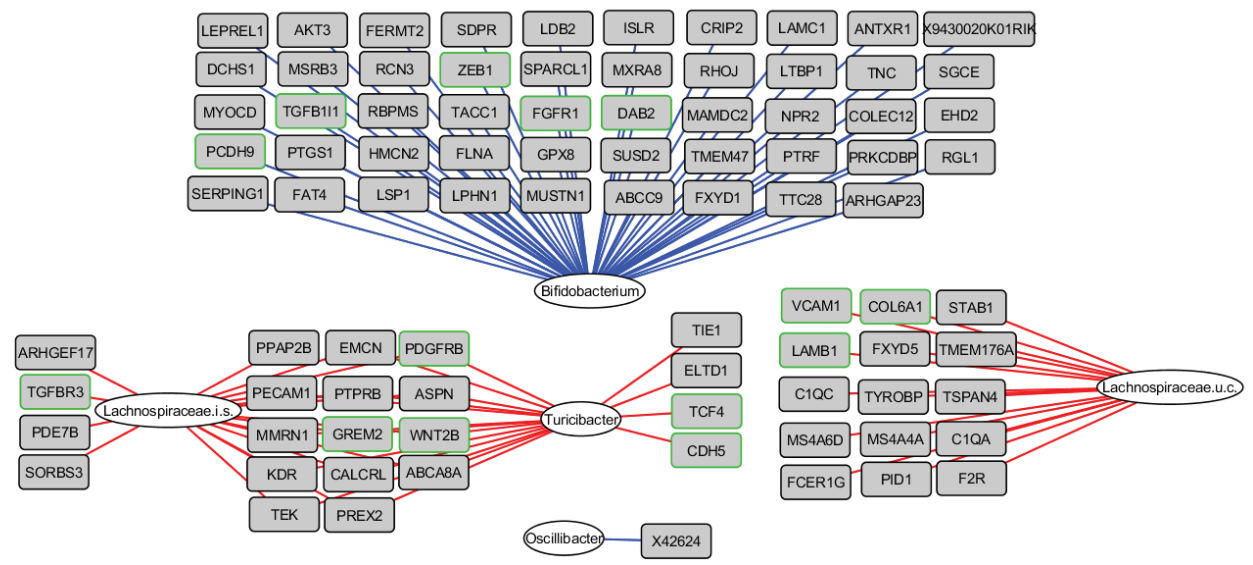

Figure 3 Integrative analysis of microbiota composition and gene expression in the early phase of aging. The 817 up-regulated genes as determined by STEM were correlated with the 50 genera with a relative abundance $\geq 0.1 \%$ in at least 1 sample, including the mice aged 6 and 12 months. Relevance network showing the genera (white circles) and genes (grey rectangles) that were correlated $(r<-0.80$ or $r>0.80)$. Red lines represent positive correlations, blue lines represent negative correlations. Regarding the negative correlations, only the top 50 strongest negative correlations are shown for visualization purposes. Genes with green frames are involved in EMT and/or ECM-related processes. i.s.=incertae sedis; u.c.: uncultured.

\section{Aging-specific changes in colonic gene expression between 12 and 28 months}

Next, we investigated alterations in gene expression during the later phase of the aging process by analyzing the changes between 12 and 28 months of age. Since the number of differentially expressed genes $(n=170, q<0.01)$ was too low to perform a functional analysis, a less stringent IBMT p-value $<0.01$ was applied to include genes displaying more subtle expression changes, resulting in 1,371 significantly differentially expressed genes (Figure 4A). The top 15 highest upand down-regulated genes presented in Table 3 show that Fatty acid binding protein 6 (Fabp6) had the strongest increased expression. The top 15 down-regulated genes were dominated by immune- and inflammatory-related genes, including 8 immunoglobulin-coding genes, Angiogenin 4 (Ang4), regenerating islet-derived 3 beta (Reg3b) and Resistin like beta (Retn/b). Another interesting immune-related gene displaying age-related reduced expression, but with a smaller fold change ranking them out of the top-15 list, was Indoleamine 2,3-dioxygenase 1 (Ido1) (Data set S4). The most significantly enriched down-regulated gene sets identified by GSEA were related to DNA replication and DNA synthesis (Table S3B). In addition, IPA identified 'Protein Ubiquitination Pathway', 'Mismatch Repair in Eukaryotes' and 'Cell Cycle Control of Chromosomal Replication' as most significantly regulated canonical pathways during the later phase of the aging process (Figure 4B) 
and X-box binding protein 1 (Xbp1) as the strongest inhibited upstream regulator (Table S3C). Taken together, these results indicate that during the later phase of aging (between 12 and 28 months), gene expression profiles pointed towards a diminished antimicrobial defense, an altered protein degradation response and aberrations in DNA repair mechanisms.

A

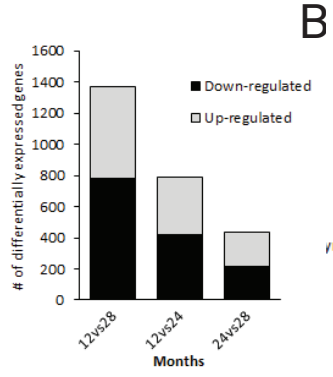

B

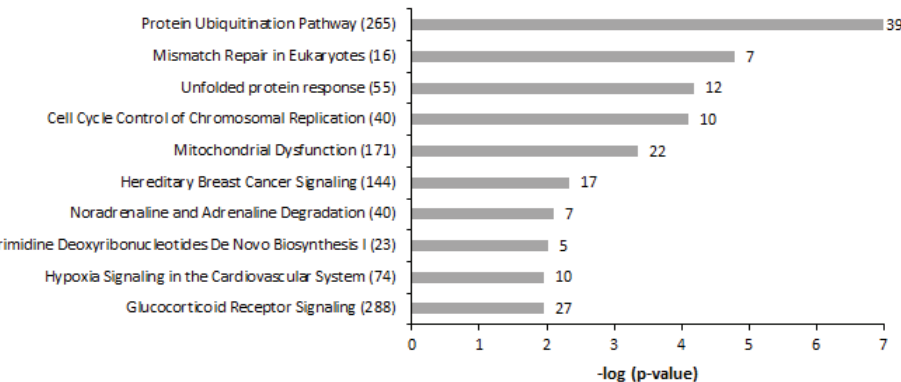

C

Guca2b

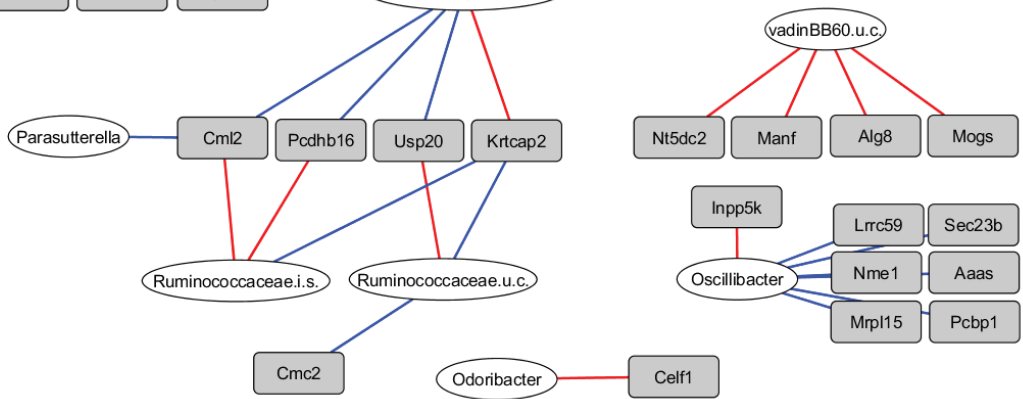

Figure 4 Aging-specific changes in colonic gene expression between 12 and 28 months and integration with colonic microbiota composition. (A) Number of differentially expressed genes between $12 \mathrm{vs} 28,12 \mathrm{vs} 24$ and $24 \mathrm{vs} 28$ months (IBMT p<0.01). (B) Top 10 most significant canonical pathways between 12 and 28 months identified by Ingenuity Pathway Analysis (IPA). Numbers behind bars represent the number of differentially expressed genes (IBMT $p<0.01$ ) and numbers behind the pathways represent the total number of genes. (C) Correlations between gut microbiota composition and gene expression during aging. The 1371 genes differentially expressed between 12 and 28 months were correlated with the 50 genera with a relative abundance $\geq 0.1 \%$ in at least 1 sample, including the mice aged 12 and 28 months. Relevance network showing the genera (white circles) and genes (grey rectangles) that were correlated $(r<-0.80$ or $r>0.80)$. Red lines represent positive correlations, blue lines represent negative correlations. For visualization purposes, only the top 25 strongest positive top 25 negative correlations are shown. i.s.=incertae sedis; u.c.: uncultured. 
Table 3 Top 15 highest up-regulated and down-regulated genes between 12 and 28 months.

\begin{tabular}{|l|l|c|c|c|}
\hline \multicolumn{2}{|l|}{ Top 15 up-regulated genes } & FC & p-value & q-value \\
\hline Fabp6 & fatty acid binding protein 6 & 3.29 & $1.59 \mathrm{E}-05$ & $2.25 \mathrm{E}-03$ \\
\hline Cm/2 & $\begin{array}{l}\text { N-acetyltransferase 8 (GCN5-related) family } \\
\text { member 2 }\end{array}$ & 2.33 & $4.42 \mathrm{E}-09$ & $1.25 \mathrm{E}-05$ \\
\hline 1700057G04Rik & RIKEN cDNA 1700057G04 gene & 2.28 & $2.54 \mathrm{E}-04$ & $1.30 \mathrm{E}-02$ \\
\hline Creb3/3 & $\begin{array}{l}\text { cAMP responsive element binding protein } \\
\text { 3-like 3 }\end{array}$ & 2.14 & $2.62 \mathrm{E}-06$ & $7.64 \mathrm{E}-04$ \\
\hline Cm/5 & $\begin{array}{l}\text { N-acetyltransferase 8 (GCN5-related) family } \\
\text { member 5 }\end{array}$ & 1.99 & $1.02 \mathrm{E}-04$ & $7.95 \mathrm{E}-03$ \\
\hline Cyp2c67 & $\begin{array}{l}\text { cytochrome P450, family 2, subfamily c, } \\
\text { polypeptide 67 }\end{array}$ & 1.89 & $3.83 \mathrm{E}-03$ & $5.02 \mathrm{E}-02$ \\
\hline Pcdhb18 & protocadherin beta 18 & 1.88 & $5.11 \mathrm{E}-11$ & $5.80 \mathrm{E}-07$ \\
\hline Snora44 & small nucleolar RNA, H/ACA box 44 & 1.88 & $1.05 \mathrm{E}-04$ & $8.03 \mathrm{E}-03$ \\
\hline Pcdhb16 & protocadherin beta 16 & 1.86 & $1.38 \mathrm{E}-08$ & $3.14 \mathrm{E}-05$ \\
\hline Abcg5 & ATP binding cassette subfamily G member 5 & 1.80 & $9.10 \mathrm{E}-06$ & $1.69 \mathrm{E}-03$ \\
\hline Gm6086 & galactose-3-O-sulfotransferase 2C & 1.79 & $5.69 \mathrm{E}-04$ & $1.92 \mathrm{E}-02$ \\
\hline Abcc2 & ATP binding cassette subfamily C member 2 & 1.77 & $3.61 \mathrm{E}-04$ & $1.53 \mathrm{E}-02$ \\
\hline Snora73b & small nucleolar RNA, H/ACA box 73B & 1.73 & $5.47 \mathrm{E}-06$ & $1.29 \mathrm{E}-03$ \\
\hline Npc1/1 & $\begin{array}{l}\text { NPC1 like intracellular cholesterol trans- } \\
\text { porter 1 }\end{array}$ & 1.70 & $2.51 \mathrm{E}-03$ & $4.11 \mathrm{E}-02$ \\
\hline Abcg8 & ATP binding cassette subfamily G member 8 & 1.70 & $1.90 \mathrm{E}-05$ & $2.49 \mathrm{E}-03$ \\
\hline
\end{tabular}

\begin{tabular}{|l|l|c|c|c|}
\hline \multicolumn{2}{|c|}{ Top 15 down-regulated genes } & FC & p-value & q-value \\
\hline Igkv8-24 & immunoglobulin kappa chain variable 8-24 & -7.63 & $9.26 \mathrm{E}-04$ & $2.48 \mathrm{E}-02$ \\
\hline Ang4 & angiogenin, ribonuclease A family, member 4 & -4.79 & $1.93 \mathrm{E}-05$ & $2.49 \mathrm{E}-03$ \\
\hline Ighg3 & immunoglobulin heavy constant gamma 3 & -3.63 & $4.46 \mathrm{E}-04$ & $1.68 \mathrm{E}-02$ \\
\hline Iglv2 & immunoglobulin lambda variable 2 & -3.50 & $1.73 \mathrm{E}-03$ & $3.53 \mathrm{E}-02$ \\
\hline Reg3b & regenerating islet-derived 3 beta & -3.30 & $9.94 \mathrm{E}-03$ & $8.25 \mathrm{E}-02$ \\
\hline Iglv1 & immunoglobulin lambda variable 1 & -3.26 & $2.01 \mathrm{E}-04$ & $1.17 \mathrm{E}-02$ \\
\hline Igkv3-12 & immunoglobulin kappa variable 3-12 & -3.21 & $9.55 \mathrm{E}-03$ & $8.04 \mathrm{E}-02$ \\
\hline Iglc2 & immunoglobulin lambda constant 2 & -2.94 & $9.64 \mathrm{E}-04$ & $2.54 \mathrm{E}-02$ \\
\hline Igkv4-57 & immunoglobulin kappa variable 4-57 & -2.61 & $8.44 \mathrm{E}-04$ & $2.32 \mathrm{E}-02$ \\
\hline Retnlb & resistin like beta & -2.59 & $9.42 \mathrm{E}-05$ & $7.64 \mathrm{E}-03$ \\
\hline Dhrs9 & $\begin{array}{l}\text { dehydrogenase/reductase (SDR family) } \\
\text { member 9 }\end{array}$ & -2.17 & $2.22 \mathrm{E}-04$ & $1.22 \mathrm{E}-02$ \\
\hline Cadps & Ca2+-dependent secretion activator & -1.98 & $6.26 \mathrm{E}-08$ & $7.37 \mathrm{E}-05$ \\
\hline Iglv3 & immunoglobulin lambda variable 3 & -1.92 & $6.12 \mathrm{E}-04$ & $1.97 \mathrm{E}-02$ \\
\hline Frmd3 & FERM domain containing 3 & -1.83 & $1.36 \mathrm{E}-07$ & $1.41 \mathrm{E}-04$ \\
\hline 2310079G19Rik & RIKEN cDNA 2310079G19 gene & -1.82 & $3.99 \mathrm{E}-04$ & $1.59 \mathrm{E}-02$ \\
\hline
\end{tabular}


Integrative analysis of microbiota composition and gene expression in the later phase of aging A second integrative analysis was carried out to investigate the correlation between gene expression and gut microbiota composition during the late phase of life. For that purpose, the data of the 50 genera with a relative abundance of more than $0.1 \%$ in at least one sample and the 1,371 genes that were differentially expressed between 12 and 28 months (IBMT $p<0.01$ ) for each individual mouse were integrated. The highest number of most pronounced correlations $(r<-0.80$ or $r>0.80)$ was found for an uncultured member of Gastranaerophilales spp. (Figure 4C). Pcdhb16, Pcdhb18 and Abcg5, all in the top 15 of strongest up-regulated genes (Table 3), as well as Ubiquitin Specific Peptidase 20 (Usp20), were negatively correlated with the relative abundance of this uncultured member. Interestingly, strong positive correlations $(\mathrm{R} \geq 0.75)$ were found for the uncultured member of Gastranaerophilales spp. with the down-regulated immune-related genes Ang4, Retnlb and Ido1 (Data set S5).

\section{Markers of gut permeability increased during aging}

To determine whether there were changes in markers of gut permeability, concentrations of lipopolysaccharide-binding protein (LBP), a marker for bacterial endotoxins, were assessed in plasma of mice aged 6, 12, 24 and 28 months. LBP concentrations were significantly higher $(p<0.05)$ in plasma from mice aged 28 months compared to 6-months-old mice (Figure 5A). Besides, the microarray data of the colonic wall revealed increased expression levels of the $L b p$ gene during aging (Figure 5B). LBP is also synthesized as an acute-phase protein in the liver and therefore we assessed expression levels of $L b p$ in liver tissue, which were increased during aging (Figure $5 C$ ). Interestingly, although we found increased plasma markers of gut permeability at higher age, there was no collective increase in expression of tight junction genes (Data set S2).

A

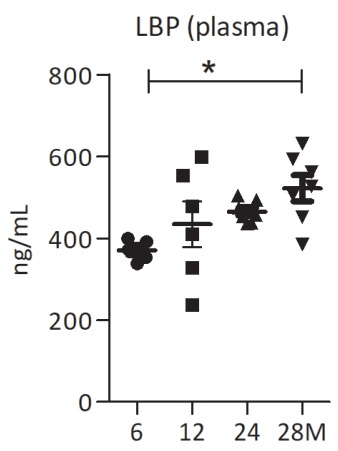

B

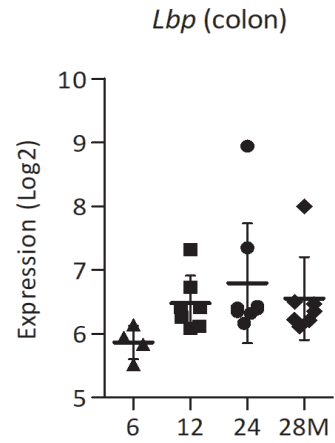

C

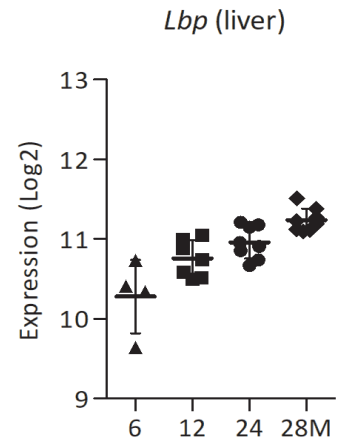

Figure 5 Markers of gut permeability during aging. (A) Concentrations of Lipopolysaccharide-binding protein (LBP) (ng/ml) in plasma. (B) Expression (Log2) of $L b p$ in the colon and (C) liver. *p $<0.05$ 


\section{Discussion}

In the present study, we report that aging is associated with pronounced changes in gut microbiota composition and colonic gene expression. In agreement with the current literature, we found an increase in potential pathobionts and a decrease in health-promoting bacteria during aging, which could potentially contribute to the development of age-related pathologies $(12,22)$. In contrast to the mild effects of aging on colonic gene expression that we found several years ago (23), we now report much stronger effects, probably because we included older mice (28 months instead of 21 months of age). Moreover, by applying an integrative approach, we show that several bacterial genera strongly correlated with colonic gene expression, providing evidence for potential host-microbe interactions.

A unique feature of this study resides in the collection of fresh faeces at consecutive time points, allowing the observation of changes in microbiota composition during the life course in the same mouse. Because environmental circumstances were strictly controlled, a broad range of external factors could be disregarded, allowing us to focus primarily on aging-related changes. However, a limitation of the $\mathrm{C} 57 \mathrm{BL} / 6 \mathrm{~J}$ mouse model in aging research is the increased vulnerability to premature hearing loss, impaired glucose tolerance and tumor formation, amongst others (24). Besides, it should be taken into account that differences exist in gut microbiota composition between mice and humans, mainly because of differences in size of the intestinal tract, metabolic rate and diet (25).

We found a strong increased relative abundance of Desulfovibrio spp. and Bilophila spp., two closely related genera that produce the potentially toxic compound hydrogen sulfide (26). It was previously shown that the relative abundance of Desulfovibrio spp. was increased in elderly humans compared to younger subjects (14). Furthermore, increased abundance of both Bilophila spp. and Desulfovibrio spp. has been associated with mucosal inflammation $(26,27)$, highlighting the presumable negative physiological consequences of an enrichment of these genera in the aged population. The increased relative abundance of these members of the gram-negative Proteobacteria could explain the significantly elevated plasma LBP levels at 28 months. LBP is an acute-phase protein that binds to LPS, resulting in an innate immune response (28). We suggest that the shift in gut microbiota composition during aging eventually caused a diminished intestinal barrier function that favored the translocation of LPS into the systemic circulation. We also found a higher relative abundance of Odoribacter spp. and Turicibacter spp. at higher age. These genera may be involved in colon tumorigenesis, since they were previously found to be enriched in tumor-bearing mice (29). Additionally, Odoribacter spp. was recently found to have a higher relative abundance in old compared to young mice (30). Turicibacter spp. was less abundant in humans with Alzheimer Disease compared to non-demented participants (31). 
We found that the relative abundance of Akkermansia spp., Bifidobacterium spp. and Lactobacillus spp. decreased at middle-age, i.e. 8 months. Each of these genera has one or more health-promoting properties, such as the production of SCFAs, enforcement of the mucus layer and stimulation of the immune system (32-34). Previous studies reported a loss of these health-associated genera in aged mice and humans $(35,36)$, but our findings implicate that in mice, these genera disappear already at middle-age. It is important to note that Akkermansia spp. was shown to be negatively correlated with adiposity (37). Accordingly, the observed decrease in relative abundance of this genus could be related to an increase in bodyweight with age. Coprococcus spp., Roseburia spp. and Christensenellaceae spp., all described as producers of the health-promoting butyrate $(38,39)$, had increased relative abundances in old mice compared to young mice in our study. This runs contrary to humans studies in which these genera have shown lower relative abundance $(14,40)$ and that elderly persons had a gut microbiota composition reflecting a lower butyrate production capacity (41). However, Christensenellaceae spp. as well as Akkermansia spp. were detected in faeces of centenarians, i.e. people over 100 years of age $(13,14)$, implicating that these genera could be linked to an extreme life span. Although the oldest mice in our study could not be considered as representative for centenarians, their age was extremely high for mice (42), possibly explaining the presence of this genus. We found relatively low concentrations of intraluminal butyrate and other SCFAs, which is possibly the consequence of the low fiber semisynthetic diets that we used.

An uncultured Coriobacteriaceae spp. followed a strong decreasing pattern during aging. The Coriobacteriaceae family is a commensal community in the gut and some members play a role in several metabolic processes, such as bile acid metabolism (43). We did not find a strong correlation between the uncultured Coriobacteriaceae spp. with the level of the unspecified bile acid, but strong negative correlations were found with both fumarate and methanol. The relative abundance of Parasutterella spp., which decreased strongly during aging in our study, was also found to be significantly reduced in patients with colorectal cancer (44), but has not been reported in relation to aging yet. We found that this genus was positively correlated with fumarate and negatively correlated with methanol. Since both the uncultured Coriobacteriaceae spp. and Parasutterella spp. showed strong decreasing abundances during aging and were correlated with various metabolites, it would be worthwhile to further investigate the role of these genera and metabolites in the aging process. A recent study discovered that the bacterial metabolite colanic acid promoted longevity in the host Caenorhabditis elegans (45), emphasizing the importance of further studies to focus on the mediating role of bacterial metabolites during aging.

In this study, we collected both faecal and colonic samples, allowing us to investigate the similarity 
in microbiota composition of both sample sources. Although age predominantly contributed to the variation in gut microbiota composition, we did observe subtle dissimilarities in relative abundances between sample sources. The most notable difference was the higher relative abundance of the Actinobacteria phylum in fresh faeces compared to colonic luminal content of young mice. A possible explanation for these dissimilarities is the fact that, in contrast to the colonic luminal content, fresh faeces passed the complete colon and may have been affected by various factors known to influence microbiota composition, such as oxygen levels and $\mathrm{pH}$-values (16). The reliability of the use of stool samples for assessment of microbiota composition in human studies is currently under debate. However, the subtle differences detected between sample sources in the present study do not directly demand for a switch in using the relatively non-invasive stool samples to colonic content isolates in aging studies.

Remarkably, both the highly abundant Akkermansia spp. and Desulfovibrio spp. displayed a strong age-related decrease or increase, respectively, but did not show strong correlations with colonic gene expression. On the other hand, Bifidobacterium spp. was negatively correlated with a considerable number of genes related to ECM and EMT processes. Strong positive correlations were also found for Turicibacter spp. and two uncultured Lachnospiraceae spp. with the same subgroup of genes. Interestingly, the expression of mesenchymal markers and related transcription factors was strongly increased between 6 and 12 months, while expression of epithelial markers was not or moderately affected. These findings suggest that a change in cellular composition of the tissue occurs, in terms of a gain of a mesenchymal phenotype, but without loss of the epithelial phenotype. Conceivably, the EMT was not of classical nature and the step of de-epithelialization was eliminated, a process called partial or intermediate EMT (46). IPA identified Tgfb1 as main upstream regulator which is a known and potent inducer of EMT during normal development, tissue repair, organ fibrosis and metastasis $(47,48)$. However, it is not plausible to assume that the observed EMT was pathological, since the mice remained in a in healthy condition far beyond the time point at which the EMT was induced, and death rates remained extremely low till the age of 20 months. More likely, the EMT contributed to normal development of the mice that was possibly induced by certain gut microbiota, since we hypothesize that the presence of Bifidobacteria spp. (or its metabolites, e.g. lactic acid) could weaken the expression of ECM- and EMT-related genes, or vice versa. It was previously shown that pathogens do have the ability to activate EMT-signaling pathways (49), and our results could indicate that also non-pathogens might be involved in the regulation of these pathways. Next, we hypothesize that the gain in mesenchymal markers resulted in an increased deposition of ECM (48, $50)$, as confirmed by the collagen staining. Ultimately, these events could have led to a change in 
colon morphology in our study, as observed by the increase in colonic crypt depth.

By exploring the changes between 12 and 28 months, we found evidence for an altered protein degradation response, together with a potential aberration of DNA repair mechanisms in the colon during the late phase of life. These processes are common events during aging and are linked to several age-related pathologies (1). Moreover, we observed a decreased intestinal immune response during aging, since several immunoglobulins, as well as the immunosuppressive Ido1 gene, were strongly down-regulated. Besides, the strong down-regulation of Ang4, Reg3b, Retnlb and Ido1 pointed toward a dysregulation of antimicrobial peptide expression at old age. Interestingly, Ang4, Retnlb and Ido1 were positively correlated with an uncultured Gastranaerophilales spp. To the best of our knowledge, little is known about Gastranaerophilales spp., however, genome sequencing revealed that this order is capable of converting glucose, mannose, starch, or glycogen into lactate, ethanol and formate (51). Notably, the semi-synthetic diet fed to the mice in our study was rich in starch, possibly explaining the presence of this bacterial strain. Usp20 was negatively correlated to the uncultured Gastranaerophilales spp., highlighting the possible role of this bacterial strain in the observed altered protein degradation response during aging.

Taken together, this study demonstrates that aging is associated with pronounced changes in gut microbiota composition and colonic gene expression, without interference of environmental factors. A presumably deleterious shift in gut microbiota composition occurred during aging, as we found an increase in potential pathobionts and a decrease in health-promoting bacteria. However, we also reported strong changes in relatively unexplored genera, which might have health effects during the aging process. Next to the pronounced differences in gut microbiota and colonic gene expression during aging, we also found strong correlations between these two aspects of colonic health. This finding implies that host-microbe interactions might play an important role during aging and therefore the newly identified molecular interactions should be investigated more extensively in future research.

\section{Acknowledgements}

We would like to thank Shohreh Keshtkar for her help during the histological analysis.

\section{Conflicts of interest}

The authors declare that they have no competing interest.

\section{Funding}

This work was financially supported by the European Union's Seventh Framework Programme 
(FP7/2007-2011) IDEAL-aging under grant agreement no 259679 and by the Netherlands Organization for Scientific Research (NWO) through the Graduate Programme on Food Structure, Digestion and Health. 


\section{References}

1. López-Otín C, Blasco MA, Partridge L, Serrano M and Kroemer G. The hallmarks of aging. Cell. 2013; 153(6):1194-1217.

2. Buford TW. (Dis)Trust your gut: the gut microbiome in age-related inflammation, health, and disease. Microbiome. 2017; 5(1):80.

3. Mowat AM and Agace WW. Regional specialization within the intestinal immune system. Nature reviews Immunology. 2014; 14(10):667.

4. Sekirov I, Russell SL, Antunes LCM and Finlay BB. Gut microbiota in health and disease. Physiological reviews. 2010; 90(3):859-904.

5. Morrison DJ and Preston T. Formation of short chain fatty acids by the gut microbiota and their impact on human metabolism. Gut Microbes. 2016; 7(3):189-200.

6. Peterson LW and Artis D. Intestinal epithelial cells: regulators of barrier function and immune homeostasis. Nature Reviews Immunology. 2014; 14(3):141-153.

7. $\quad$ Sommer F and Bäckhed F. The gut microbiota--masters of host development and physiology. Nature reviews Microbiology. 2013; 11(4):227.

8. Franceschi C, Garagnani P, Vitale G, Capri M and Salvioli S. Inflammaging and 'Garb-aging'. Trends in Endocrinology \& Metabolism. 2017; 28(3):199-212.

9. Biagi E, Candela M, Fairweather-Tait S, Franceschi C and Brigidi P. Ageing of the human metaorganism: the microbial counterpart. Age. 2012; 34(1):247-267.

10. Claesson MJ, Cusack S, O'Sullivan O, Greene-Diniz R, de Weerd H, Flannery E, Marchesi JR, Falush D, Dinan T and Fitzgerald G. Composition, variability, and temporal stability of the intestinal microbiota of the elderly. Proceedings of the National Academy of Sciences. 2011; 108(Supplement 1):45864591.

11. Konturek P, Haziri D, Brzozowski T, Hess T, Heyman S, Kwiecien S, Konturek S and Koziel J. Emerging role of fecal microbiota therapy in the treatment of gastrointestinal and extra-gastrointestinal diseases. J Physiol Pharmacol. 2015; 66(4):483-491.

12. Vaiserman AM, Koliada AK and Marotta F. Gut microbiota: A player in aging and a target for antiaging intervention. Ageing research reviews. 2017.

13. Biagi E, Franceschi C, Rampelli S, Severgnini M, Ostan R, Turroni S, Consolandi C, Quercia S, Scurti M and Monti D. Gut microbiota and extreme longevity. Current Biology. 2016; 26(11):1480-1485.

14. Biagi E, Nylund L, Candela M, Ostan R, Bucci L, Pini E, Nikkila J, Monti D, Satokari R and Franceschi C. Through ageing, and beyond: gut microbiota and inflammatory status in seniors and centenarians. PloS one. 2010; 5(5):e10667.

15. Odamaki T, Kato K, Sugahara H, Hashikura N, Takahashi S, Xiao J-z, Abe F and Osawa R. Agerelated changes in gut microbiota composition from newborn to centenarian: a cross-sectional study. BMC microbiology. 2016; 16(1):90.

16. Albenberg L, Esipova TV, Judge CP, Bittinger K, Chen J, Laughlin A, Grunberg S, Baldassano RN, Lewis JD and Li H. Correlation between intraluminal oxygen gradient and radial partitioning of intestinal microbiota. Gastroenterology. 2014; 147(5):1055-1063. e1058.

17. Eckburg PB, Bik EM, Bernstein CN, Purdom E, Dethlefsen L, Sargent M, Gill SR, Nelson KE and Relman DA. Diversity of the human intestinal microbial flora. science. 2005; 308(5728):1635-1638.

18. Lepage $P$, Seksik $P$, Sutren $M$, de la Cochetière MF, Jian R, Marteau $P$ and Doré J. Biodiversity of the mucosa-associated microbiota is stable along the distal digestive tract in healthy individuals and patients with IBD. Inflammatory bowel diseases. 2005; 11(5):473-480.

19. Mira-Pascual L, Cabrera-Rubio R, Ocon S, Costales P, Parra A, Suarez A, Moris F, Rodrigo L, Mira A and Collado M. Microbial mucosal colonic shifts associated with the development of colorectal cancer reveal the presence of different bacterial and archaeal biomarkers. Journal of gastroenterology. 2015; 50(2):167.

20. Zoetendal EG, von Wright A, Vilpponen-Salmela T, Ben-Amor K, Akkermans AD and de Vos WM. Mucosa-associated bacteria in the human gastrointestinal tract are uniformly distributed along the colon and differ from the community recovered from feces. Applied and environmental microbiology. 2002; 68(7):3401-3407.

21. Kok DEG, Rusli F, van der Lugt B, Lute C, Laghi L, Salvioli S, Picone G, Franceschi C, Smidt $H$, Vervoort J, Kampman E, Muller M and Steegenga WT. Lifelong calorie restriction affects indicators of colonic health in aging C57BI/6J mice. The Journal of nutritional biochemistry. 2018; 56:152-164.

22. Kumar M, Babaei $\mathrm{P}, \mathrm{Ji} B$ and Nielsen J. Human gut microbiota and healthy aging: Recent developments and future prospective. Nutrition and healthy aging. 2016; 4(1):3-16.

23. Steegenga WT, de Wit NJ, Boekschoten MV, ljssennagger N, Lute C, Keshtkar S, Bromhaar MM, Kampman E, de Groot LC and Muller M. Structural, functional and molecular analysis of the effects of aging in the small intestine and colon of C57BL/6J mice. BMC medical genomics. 2012; 5:38. 
24. Koks S, Dogan S, Tuna BG, Gonzalez-Navarro H, Potter P and Vandenbroucke RE. Mouse models of ageing and their relevance to disease. Mechanisms of ageing and development. 2016; 160:41-53.

25. Hugenholtz F and de Vos WM. Mouse models for human intestinal microbiota research: a critical evaluation. Cellular and molecular life sciences : CMLS. 2018; 75(1):149-160.

26. Carbonero F, Benefiel AC, Alizadeh-Ghamsari AH and Gaskins HR. Microbial pathways in colonic sulfur metabolism and links with health and disease. Frontiers in physiology. 2012; 3.

27. Earley H, Lennon G, Balfe A, Kilcoyne M, Clyne M, Joshi L, Carrington S, Martin ST, Coffey JC and Winter DC. A Preliminary study examining the binding capacity of Akkermansia muciniphila and Desulfovibrio spp., to colonic mucin in health and ulcerative colitis. PloS one. 2015; 10(10):e0135280.

28. Alexopoulou A, Agiasotelli D, Vasilieva LE and Dourakis SP. Bacterial translocation markers in liver cirrhosis. Annals of gastroenterology. 2017; 30(5):486-497.

29. Zackular JP, Baxter NT, Iverson KD, Sadler WD, Petrosino JF, Chen GY and Schloss PD. The gut microbiome modulates colon tumorigenesis. MBio. 2013; 4(6):e00692-00613.

30. Scott KA, Ida M, Peterson VL, Prenderville JA, Moloney GM, Izumo T, Murphy K, Murphy A, Ross RP, Stanton C, Dinan TG and Cryan JF. Revisiting Metchnikoff: Age-related alterations in microbiota-gutbrain axis in the mouse. Brain, behavior, and immunity. 2017; 65:20-32.

31. Vogt NM, Kerby RL, Dill-McFarland KA, Harding SJ, Merluzzi AP, Johnson SC, Carlsson CM, Asthana S, Zetterberg H, Blennow K, Bendlin BB and Rey FE. Gut microbiome alterations in Alzheimer's disease. Sci Rep. 2017; 7(1):13537.

32. Derrien M, Belzer C and de Vos WM. Akkermansia muciniphila and its role in regulating host functions. Microbial pathogenesis. 2017; 106:171-181.

33. Rivière A, Selak M, Lantin D, Leroy $F$ and De Vuyst L. Bifidobacteria and butyrate-producing colon bacteria: importance and strategies for their stimulation in the human gut. Frontiers in microbiology. 2016; 7.

34. Zhong $L$, Zhang $X$ and Covasa M. Emerging roles of lactic acid bacteria in protection against colorectal cancer. World Journal of Gastroenterology: WJG. 2014; 20(24):7878.

35. Langille MG, Meehan CJ, Koenig JE, Dhanani AS, Rose RA, Howlett SE and Beiko RG. Microbial shifts in the aging mouse gut. Microbiome. 2014; 2(1):50.

36. Woodmansey E. Intestinal bacteria and ageing. Journal of applied microbiology. 2007; 102(5):11781186.

37. Schneeberger M, Everard A, Gómez-Valadés AG, Matamoros S, Ramírez S, Delzenne NM, Gomis $\mathrm{R}$, Claret $\mathrm{M}$ and Cani PD. Akkermansia muciniphila inversely correlates with the onset of inflammation, altered adipose tissue metabolism and metabolic disorders during obesity in mice. Scientific reports. 2015; 5.

38. Louis P and Flint HJ. Diversity, metabolism and microbial ecology of butyrate-producing bacteria from the human large intestine. FEMS microbiology letters. 2009; 294(1):1-8.

39. Morotomi M, Nagai F and Watanabe Y. Description of Christensenella minuta gen. nov., sp. nov., isolated from human faeces, which forms a distinct branch in the order Clostridiales, and proposal of Christensenellaceae fam. nov. International journal of systematic and evolutionary microbiology. 2012; 62(Pt 1):144-149.

40. Conley MN, Wong CP, Duyck KM, Hord N, Ho E and Sharpton TJ. Aging and serum MCP-1 are associated with gut microbiome composition in a murine model. PeerJ. 2016; 4:e1854.

41. Hippe B, Zwielehner J, Liszt K, Lassl C, Unger F and Haslberger AG. Quantification of butyryl $\mathrm{CoA}$ :acetate $\mathrm{CoA}$-transferase genes reveals different butyrate production capacity in individuals according to diet and age. FEMS microbiology letters. 2011; 316(2):130-135.

42. Flurkey KC, J M.; and Harrison, D E. Mouse models in aging research. Faculty Research 2000 2009. 2007.

43. Clavel T, Desmarchelier C, Haller D, Gerard P, Rohn S, Lepage P and Daniel H. Intestinal microbiota in metabolic diseases: from bacterial community structure and functions to species of pathophysiological relevance. Gut Microbes. 2014; 5(4):544-551.

44. Wang T, Cai G, Qiu Y, Fei N, Zhang M, Pang X, Jia W, Cai S and Zhao L. Structural segregation of gut microbiota between colorectal cancer patients and healthy volunteers. Isme j. 2012; 6(2):320-329.

45. Han B, Sivaramakrishnan P, Lin CJ, Neve IAA, He J, Tay LWR, Sowa JN, Sizovs A, Du G, Wang J, Herman C and Wang MC. Microbial Genetic Composition Tunes Host Longevity. Cell. 2017; 169(7):12491262.e1213.

46. Lamouille S, Xu J and Derynck R. Molecular mechanisms of epithelial-mesenchymal transition. Nature reviews Molecular cell biology. 2014; 15(3):178-196.

47. Kalluri $\mathrm{R}$ and Weinberg RA. The basics of epithelial-mesenchymal transition. The Journal of clinical investigation. 2009; 119(6):1420.

48. $\mathrm{Xu} \mathrm{J,} \mathrm{Lamouille} \mathrm{S} \mathrm{and} \mathrm{Derynck} \mathrm{R.} \mathrm{TGF-a-induced} \mathrm{epithelial} \mathrm{to} \mathrm{mesenchymal} \mathrm{transition.} \mathrm{Cell}$ research. 2009; 19(2):156. 
49. Hofman P and Vouret-Craviari V. Microbes-induced EMT at the crossroad of inflammation and cancer. Gut Microbes. 2012; 3(3):176-185.

50. Hutchison N, Fligny C and Duffield JS. Resident mesenchymal cells and fibrosis. Biochimica et Biophysica Acta (BBA)-Molecular Basis of Disease. 2013; 1832(7):962-971.

51. Soo RM, Skennerton CT, Sekiguchi $Y$, Imelfort M, Paech SJ, Dennis PG, Steen JA, Parks DH, Tyson GW and Hugenholtz P. An expanded genomic representation of the phylum cyanobacteria. Genome biology and evolution. 2014; 6(5):1031-1045.

52. Rusli F, Boekschoten MV, Zubia AA, Lute C, Müller M and Steegenga WT. A weekly alternating diet between caloric restriction and medium fat protects the liver from fatty liver development in middleaged C57BL/6J mice. Molecular nutrition \& food research. 2015; 59(3):533-543.

53. Rusli F, Deelen J, Andriyani E, Boekschoten MV, Lute C, van den Akker EB, Muller M, Beekman $\mathrm{M}$ and Steegenga WT. Fibroblast growth factor 21 reflects liver fat accumulation and dysregulation of signalling pathways in the liver of C57BL/6J mice. Sci Rep. 2016; 6:30484.

54. Rusli F, Lute C, Boekschoten MV, van Dijk M, van Norren K, Menke AL, Muller M and Steegenga WT. Intermittent calorie restriction largely counteracts the adverse health effects of a moderate-fat diet in aging C57BL/6J mice. Mol Nutr Food Res. 2017; 61(5).

55. Quast C, Pruesse E, Yilmaz P, Gerken J, Schweer T, Yarza P, Peplies J and Glöckner FO. The SILVA ribosomal RNA gene database project: improved data processing and web-based tools. Nucleic acids research. 2012; 41(D1):D590-D596.

56. Braak CJF and Šmilauer P. (2012). CANOCO reference manual and user's guide: software for ordination (version 5.0): Biometris).

57. Legendre P and Gallagher ED. Ecologically meaningful transformations for ordination of species data. Oecologia. 2001; 129(2):271-280.

58. Kramer A, Green J, Pollard J, Jr. and Tugendreich S. Causal analysis approaches in Ingenuity Pathway Analysis. Bioinformatics (Oxford, England). 2014; 30(4):523-530.

59. Subramanian A, Tamayo P, Mootha VK, Mukherjee S, Ebert BL, Gillette MA, Paulovich A, Pomeroy SL, Golub TR, Lander ES and Mesirov JP. Gene set enrichment analysis: a knowledge-based approach for interpreting genome-wide expression profiles. Proceedings of the National Academy of Sciences of the United States of America. 2005; 102(43):15545-15550.

60. Ernst J and Bar-Joseph Z. STEM: a tool for the analysis of short time series gene expression data. BMC bioinformatics. 2006; 7(1):191.

61. González I, Lê Cao K-A, Davis MJ and Déjean S. Visualising associations between paired 'omics' data sets. BioData mining. 2012; 5(1):19.

62. Fernandes AD, Reid JN, Macklaim JM, McMurrough TA, Edgell DR and Gloor GB. Unifying the analysis of high-throughput sequencing datasets: characterizing RNA-seq, 16S rRNA gene sequencing and selective growth experiments by compositional data analysis. Microbiome. 2014; 2:15.

63. Shannon P, Markiel A, Ozier O, Baliga NS, Wang JT, Ramage D, Amin N, Schwikowski B and Ideker T. Cytoscape: a software environment for integrated models of biomolecular interaction networks. Genome research. 2003; 13(11):2498-2504. 
SUPPLEMENTAL MATERIAL

A

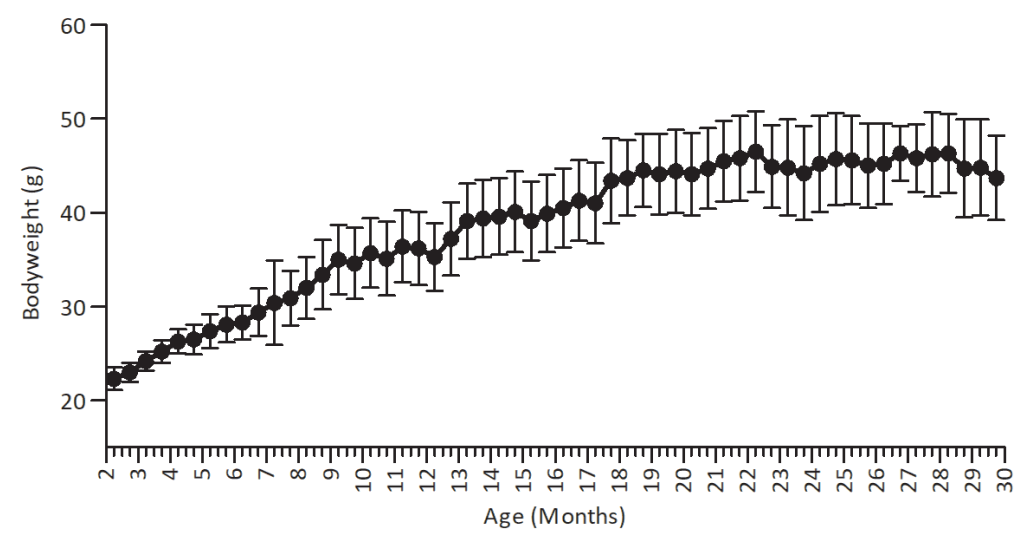

B

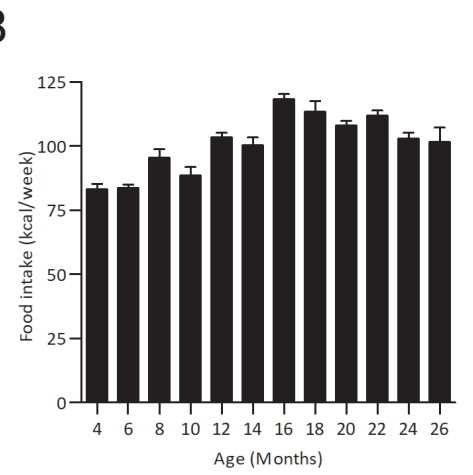

$E$

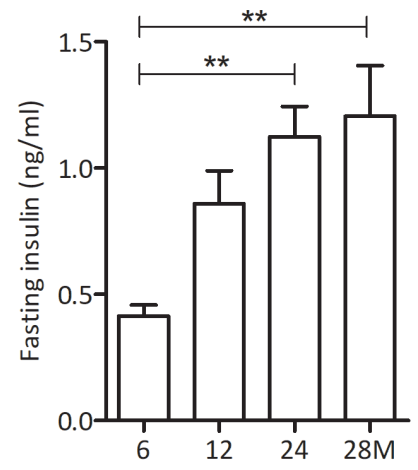

C

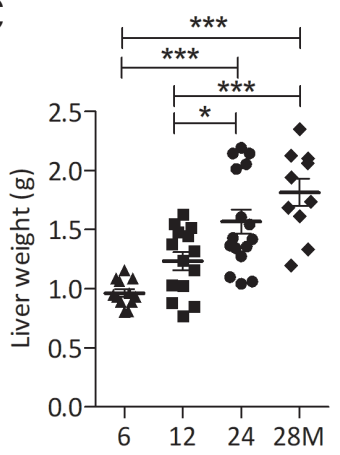

$\mathrm{F}$

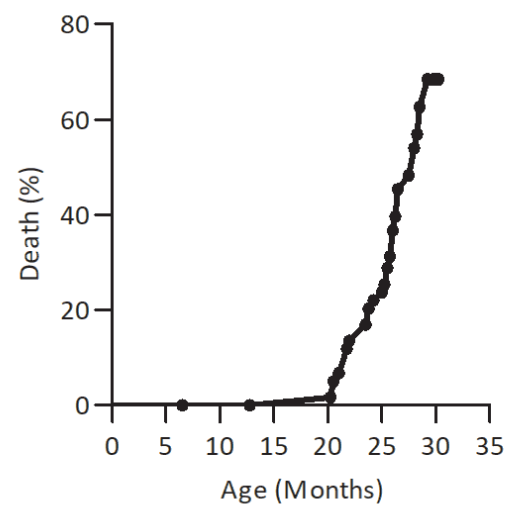

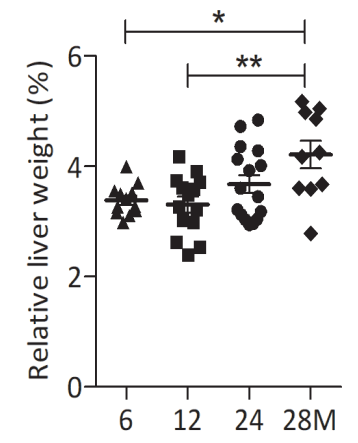


Figure S1 (A) Body weight measurements recorded every 2 weeks. (B) Mean food intake in kilocalories per week during the life span of the mice. (C) Liver weight in grams. (D) Relative liver weight as percentage of body weight. (E) Fasting insulin levels ( $\mathrm{ng} / \mathrm{ml}$ ) measured in plasma. (F) Percentage of mice that died before sacrifice.

A

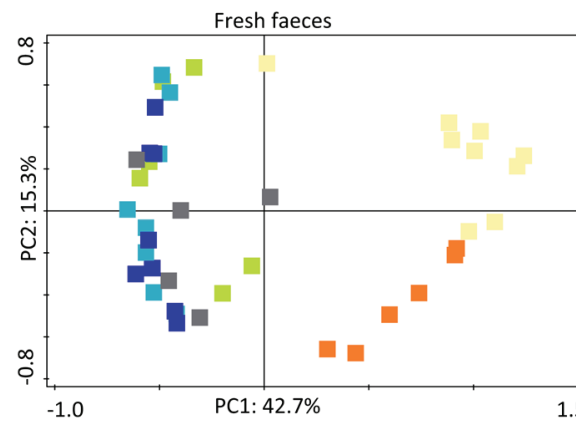

B

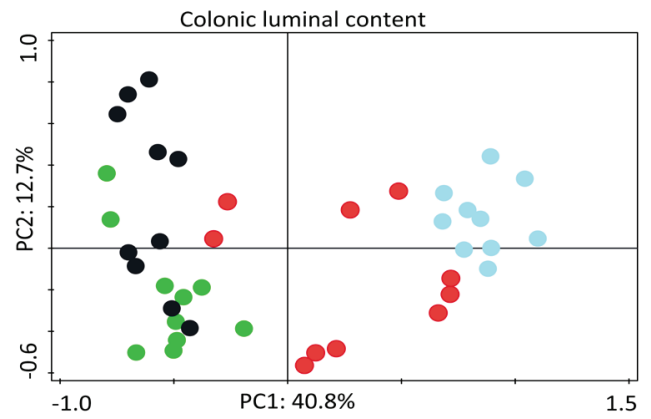

Figure S2 (A) Principal Component Analysis (PCA) displaying separation of the fresh faeces (FF) samples collected at 4, 8, 12, 18, 24 and 28 months. (B) PCA displaying separation of the colonic luminal content (CLC) samples collected at sacrifice at 6, 12, 24 and 28 months.

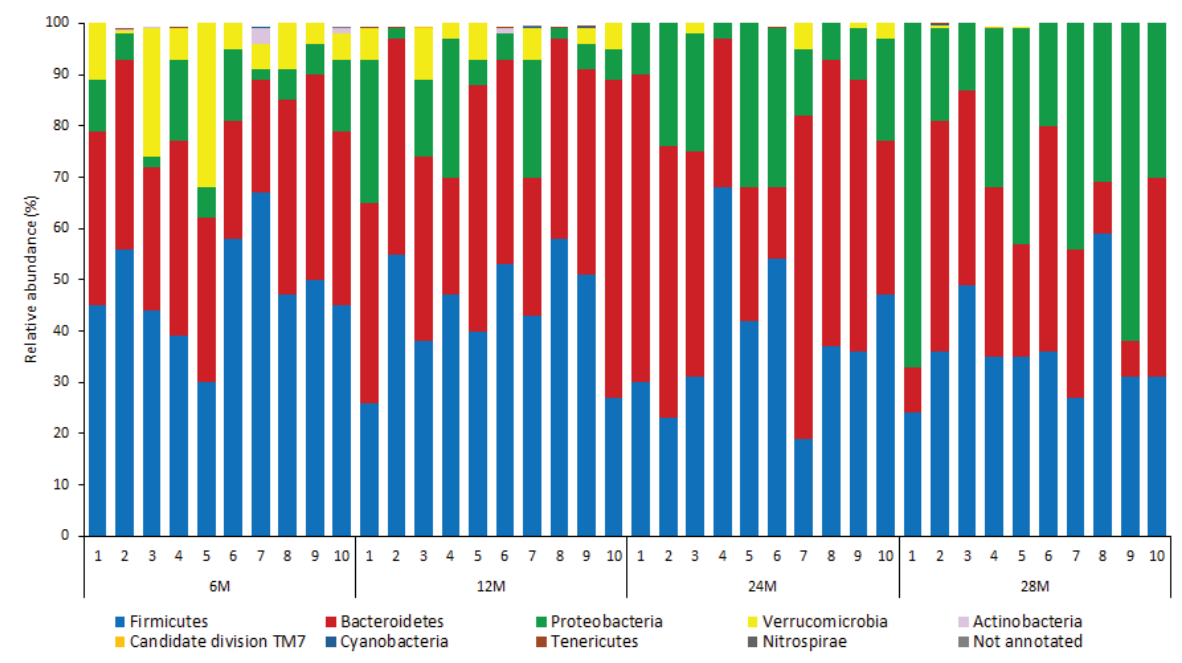

Figure S3 Relative abundance (\%) at phylum level in colonic luminal content determined in all individual mice. 


\begin{tabular}{|c|c|c|c|c|c|c|c|c|c|c|c|c|c|c|c|c|c|}
\hline 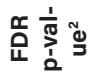 & $\begin{array}{l}0 \\
\vdots \\
0\end{array}$ & \begin{tabular}{|l|}
0 \\
\\
0 \\
0
\end{tabular} & $\begin{array}{l}0 \\
0 \\
0 \\
0\end{array}$ & $\begin{array}{l}\stackrel{n}{N} \\
0 \\
0\end{array}$ & $\begin{array}{l}0 \\
0 \\
\infty \\
0 \\
0\end{array}$ & $\begin{array}{c}\tilde{m} \\
\stackrel{p}{0} \\
0\end{array}$ & $\mid$ & $\underset{\sim}{\stackrel{0}{\circ}}$ & $\begin{array}{l}0 \\
0 \\
0\end{array}$ & 움 & $\begin{array}{l}\hat{\circ} \\
\text { o. }\end{array}$ & $\begin{array}{l}m \\
0 \\
0\end{array}$ & $\begin{array}{l}\text { ¿े } \\
\text { O̊. } \\
0\end{array}$ & $\begin{array}{l}\dddot{0} \\
0 \\
0\end{array}$ & $\begin{array}{l}\circ \\
\vdots \\
0\end{array}$ & $\frac{8}{0}$ & 웅 \\
\hline 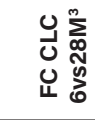 & $\hat{\sim}$ & $\stackrel{m}{m}$ & $\tilde{f}$ & $\mid \begin{array}{l}\circ \\
\stackrel{0}{\circ} \\
\end{array}$ & $\cong$ & $\stackrel{\circ}{\dot{m}}$ & $\stackrel{m}{+}$ & 0 & $\stackrel{P}{\wedge}$ & 吊 & مִ & $\bar{f}$ & 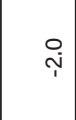 & $\hat{m}$ & 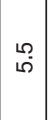 & 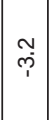 & 命 \\
\hline ن & $\circ$ & \begin{tabular}{|l|}
$m$ \\
$\frac{m}{0}$ \\
0
\end{tabular} & $\begin{array}{l}n \\
\tilde{m} \\
0 \\
0\end{array}$ & $\mid$ & م & $\begin{array}{l}\hat{0} \\
\tilde{m} \\
0\end{array}$ & 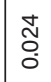 & 0 & $\begin{array}{l}\infty \\
\underset{0}{N} \\
0\end{array}$ & 克 & $\begin{array}{l}0 \\
\stackrel{N}{0} \\
0\end{array}$ & $\underset{\tilde{m}}{\tilde{O}}$ & $\begin{array}{l}\stackrel{+}{0} \\
\text { m } \\
0\end{array}$ & $m$ & $\begin{array}{l}\stackrel{0}{0} \\
\stackrel{0}{0} \\
0\end{array}$ & \begin{tabular}{|l|}
$\stackrel{9}{m}$ \\
$\stackrel{0}{0}$
\end{tabular} & $\begin{array}{l}\text { t } \\
\text { ల్ } \\
0\end{array}$ \\
\hline ن่ & 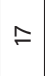 & \begin{tabular}{|l|}
0 \\
$\stackrel{0}{0}$ \\
0
\end{tabular} & $\begin{array}{l}0 \\
\vdots \\
0 \\
0\end{array}$ & \begin{tabular}{|c|}
0 \\
0 \\
0 \\
0 \\
0 \\
0
\end{tabular} & $\sim$ & $\begin{array}{l}\stackrel{\circ}{\circ} \\
0 \\
0\end{array}$ & $\begin{array}{l}0 \\
0 \\
0 \\
0\end{array}$ & 0 & $\stackrel{2}{\circ}$ & $\begin{array}{l}\bar{\sigma} \\
0\end{array}$ & $\begin{array}{l}\tilde{N} \\
0 \\
0\end{array}$ & $\begin{array}{l}0 \\
\stackrel{N}{+} \\
0\end{array}$ & $\begin{array}{l}0 \\
0 \\
0 \\
0\end{array}$ & $\sim$ & $\begin{array}{c}0 \\
\frac{\infty}{0} \\
\frac{\infty}{0}\end{array}$ & 勇 & 옹 \\
\hline نِ & $\approx$ & \begin{tabular}{|l|}
0 \\
0 \\
0 \\
0
\end{tabular} & 号 & $\frac{8}{\circ}$ & ما & $\frac{\circ}{0}$ & õ & 0 & $\begin{array}{l}\stackrel{2}{2} \\
\stackrel{0}{0}\end{array}$ & ò & $\overline{\tilde{o}}$ & $\stackrel{8}{\circ}$ & $\begin{array}{l}\widetilde{్} \\
\text { ర్ }\end{array}$ & - & $\begin{array}{l}\infty \\
0 \\
0 \\
0\end{array}$ & $\frac{10}{0}$ & $\begin{array}{l}\text { I } \\
\text { ठ } \\
0\end{array}$ \\
\hline ِّ & $\hat{m}$ & \begin{tabular}{l|} 
\\
\\
0 \\
0 \\
0
\end{tabular} & $\hat{o}$ & 0 & $m$ & $\stackrel{\stackrel{m}{0}}{0}$ & $\begin{array}{l}\text { L } \\
8 \\
0 \\
0\end{array}$ & 0 & $\begin{array}{l}\stackrel{0}{0} \\
\stackrel{m}{0} \\
\dot{0}\end{array}$ & 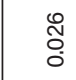 & ồ & $\frac{0}{0}$ & $\begin{array}{l}\overline{8} \\
0 \\
0\end{array}$ & $\begin{array}{c}\mathfrak{N} \\
\stackrel{0}{\infty} \\
0 \\
0\end{array}$ & $\begin{array}{l}\hat{o} \\
0 \\
0\end{array}$ & 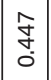 & 苞 \\
\hline 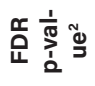 & 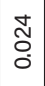 & 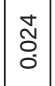 & $\begin{array}{l}0 \\
0 \\
0 \\
0\end{array}$ & \begin{tabular}{|l|}
0 \\
0 \\
0 \\
0 \\
0
\end{tabular} & $\begin{array}{l}\infty \\
\frac{0}{0}\end{array}$ & 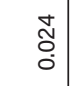 & $\begin{array}{l}\bar{\alpha} \\
0 \\
0 \\
0\end{array}$ & $\begin{array}{l}\bar{\infty} \\
\dot{o} \\
0 \\
0\end{array}$ & 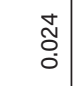 & 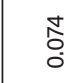 & 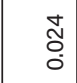 & 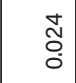 & 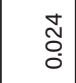 & 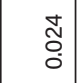 & $\frac{\infty}{0}$ & \begin{tabular}{l}
\multirow{2}{\Delta}{} \\
0 \\
0
\end{tabular} & 壱 \\
\hline 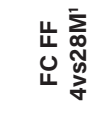 & $\stackrel{0}{\stackrel{0}{*}}$ & \begin{tabular}{|c|}
$m$ \\
$\sigma$
\end{tabular} & $\left|\begin{array}{c}m \\
\infty \\
\infty\end{array}\right|$ & 竞 & $\stackrel{\text { : }}{i}$ & 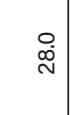 & 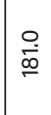 & 0 & $\bar{f}$ & $\stackrel{\leftrightarrow}{\circ}$ & 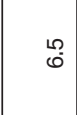 & $\stackrel{?}{\sim}$ & $\stackrel{0}{0}$ & $\stackrel{\llcorner}{\infty}$ & $\bar{f}$ & 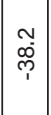 & ஸे \\
\hline 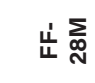 & $\approx$ & $\begin{array}{l}\bar{\delta} \\
\overline{0} \\
0 \\
\end{array}$ & 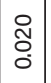 & $\begin{array}{l}\bar{\delta} \\
\bar{\delta} \\
0 \\
\end{array}$ & $m$ & 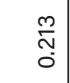 & ơ & 0 & 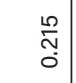 & $\begin{array}{l}\stackrel{0}{0} \\
\stackrel{0}{0} \\
0\end{array}$ & $\begin{array}{l}\overline{1} \\
0 \\
0 \\
0\end{array}$ & 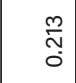 & 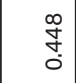 & $\sim$ & $\mid$\begin{tabular}{l|}
0 \\
$\infty$ \\
0 \\
0
\end{tabular} & 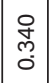 & $\stackrel{\widehat{m}}{0}$ \\
\hline 虫 导 & $\stackrel{\sim}{N}$ & \begin{tabular}{l|} 
\\
\\
0 \\
0 \\
0 \\
0
\end{tabular} & \begin{tabular}{|l|}
10 \\
0 \\
0 \\
0
\end{tabular} & $\begin{array}{l}\bar{n} \\
\stackrel{n}{0}\end{array}$ & $m$ & î̀. & $\begin{array}{l}\text { Oे } \\
0 \\
0\end{array}$ & 0 & $\begin{array}{c}\stackrel{0}{0} \\
\stackrel{\infty}{0} \\
0\end{array}$ & 음 & $\begin{array}{l}\infty \\
\tilde{o} \\
0 \\
0\end{array}$ & $\begin{array}{l}\stackrel{\circ}{\infty} \\
\stackrel{0}{0}\end{array}$ & 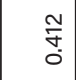 & $\sim$ & $\frac{\hat{\sigma}}{0}$ & 胥 & $\frac{\sigma}{\delta}$ \\
\hline 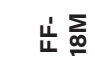 & $\stackrel{\sim}{\sim}$ & $\begin{array}{l}0 \\
0 \\
0 \\
0\end{array}$ & $\mid \begin{array}{l}10 \\
0 \\
0 \\
0\end{array}$ & - & $\sim$ & 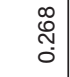 & $\begin{array}{l}\bar{\delta} \\
0 \\
0\end{array}$ & 0 & 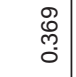 & $\begin{array}{l}\overline{0} \\
\text { Oे }\end{array}$ & $\begin{array}{l}\tilde{m} \\
0 \\
0 \\
0\end{array}$ & $\frac{2}{\stackrel{0}{0}}$ & 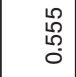 & $m$ & E & - & 壱 \\
\hline 岁 $\sum_{\mathbb{N}}$ & $g$ & $\begin{array}{l}\hat{0} \\
0 \\
0 \\
0\end{array}$ & $\begin{array}{l}0 \\
0 \\
0 \\
0\end{array}$ & 0 & r & $\begin{array}{l}0 \\
0 \\
0 \\
0 \\
0\end{array}$ & $\bar{\delta}$ & 0 & $\stackrel{\Re}{\stackrel{0}{0}}$ & $\begin{array}{l}\text { t } \\
\text { Oे }\end{array}$ & $\stackrel{m}{\vdots}$ & $\begin{array}{l}\hat{o} \\
0 \\
0 \\
0\end{array}$ & $\begin{array}{l}\infty \\
\stackrel{+}{+} \\
0\end{array}$ & $\sim$ & $\frac{\infty}{\stackrel{0}{c}}$ & \begin{tabular}{|l|}
$\infty$ \\
0 \\
$\infty$ \\
0 \\
0
\end{tabular} & 㖞 \\
\hline 岁 $\sum_{\infty}$ & $\mathscr{q}$ & $\mid \begin{array}{l}0 \\
\tilde{O} \\
0 \\
0\end{array}$ & $\begin{array}{l}m \\
0 \\
0 \\
0\end{array}$ & 0 & ص & 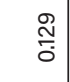 & 0 & 0 & $\begin{array}{l}1 \\
0 \\
0 \\
0 \\
0\end{array}$ & \begin{tabular}{l}
\multirow{2}{0}{} \\
0
\end{tabular} & $\overline{0}$ & $\begin{array}{l}0 \\
0 \\
0 \\
0\end{array}$ & \begin{tabular}{l}
\multirow{J}{5}{} \\
$\stackrel{0}{0}$ \\
0
\end{tabular} & - & $\mid \begin{array}{l}N \\
\hat{O} \\
0 \\
0\end{array}$ & \begin{tabular}{l|}
$\bar{\infty}$ \\
$\dot{\alpha}$ \\
0 \\
0
\end{tabular} & 范 \\
\hline 虫 $\sum_{y}$ & $\stackrel{\infty}{\sim}$ & $\begin{array}{l}0 \\
0 \\
0 \\
0 \\
0\end{array}$ & $\begin{array}{l}\overline{0} \\
0 \\
0\end{array}$ & 0 & $\simeq$ & $\begin{array}{l}\infty \\
0 \\
0 \\
0\end{array}$ & 0 & $\begin{array}{l}0 \\
0 \\
0 \\
0 \\
0\end{array}$ & $\begin{array}{l}\tilde{0} \\
0 \\
0 \\
0\end{array}$ & $\begin{array}{l}0 \\
\stackrel{0}{0} \\
0 \\
0\end{array}$ & $\begin{array}{l}\bar{\sigma} \\
\dot{0}\end{array}$ & 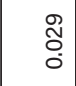 & $\begin{array}{l}\stackrel{0}{0} \\
0 \\
0 \\
0\end{array}$ & $\begin{array}{l}\infty \\
\vdots \\
0 \\
0\end{array}$ & ָ̃ & $\stackrel{m}{\sim}$ & $\mid \begin{array}{c}0 \\
\\
0\end{array}$ \\
\hline 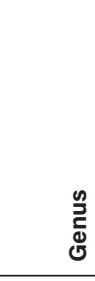 & 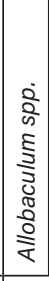 & 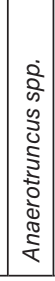 & 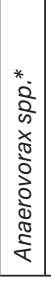 & 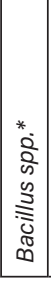 & 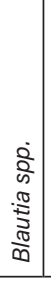 & 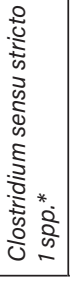 & \begin{tabular}{|c|}
$*$ \\
0 \\
0 \\
0 \\
0 \\
0 \\
0 \\
0 \\
0 \\
0 \\
0 \\
0 \\
0 \\
0
\end{tabular} & 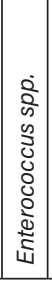 & 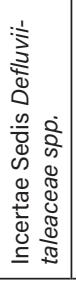 & 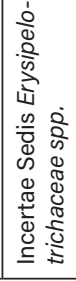 & 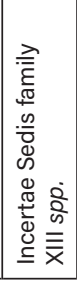 & 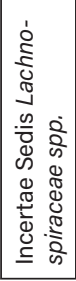 & 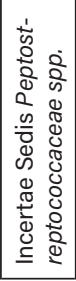 & 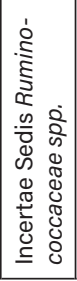 & 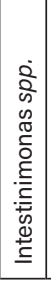 & 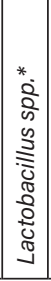 & 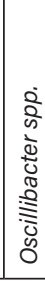 \\
\hline$\frac{\underline{E}}{\bar{\lambda}}$ & & & & & & & & & & & & & & & & & \\
\hline
\end{tabular}




\begin{tabular}{|c|c|c|c|c|c|c|c|c|c|c|c|c|c|c|c|c|c|c|}
\hline 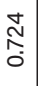 & $\stackrel{\text { ¿े }}{\circ}$ & $\begin{array}{l}m \\
0 \\
0 \\
0\end{array}$ & $\begin{array}{l}\hat{o} \\
0\end{array}$ & $\begin{array}{l}\text { ơ } \\
\text { ơ } \\
\text { O }\end{array}$ & 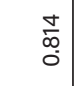 & $\begin{array}{l}0 \\
\vdots \\
0 \\
0\end{array}$ & $\begin{array}{l}0 \\
\stackrel{0}{0} \\
\end{array}$ & $\begin{array}{l}0 \\
\vdots \\
0\end{array}$ & 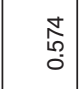 & $\begin{array}{l}0 \\
\stackrel{\infty}{0} \\
0\end{array}$ & ò & $\stackrel{\text { }}{\circ}$ & ồ̊ & $\mid$\begin{tabular}{l}
0 \\
\multirow{2}{0}{} \\
0
\end{tabular} & $\begin{array}{l}0 \\
\vdots \\
0 \\
0\end{array}$ & $\begin{array}{l}\bar{E} \\
\bar{O}\end{array}$ & $\begin{array}{l}0 \\
\infty \\
\infty \\
0 \\
0\end{array}$ & : \\
\hline$\stackrel{?}{\longrightarrow}$ & $\overline{5}$ & Б5 & $\stackrel{m}{\mathrm{I}}$ & 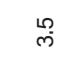 & $\stackrel{L}{N}$ & 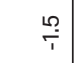 & $\stackrel{L}{N}$ & 0 & $\stackrel{\text { ?ִ }}{=}$ & $\bar{m}$ & $\stackrel{\text { I }}{\mathrm{N}}$ & $\stackrel{\circ}{\mathrm{N}}$ & 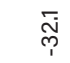 & | & 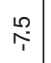 & 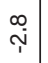 & $\stackrel{ }{-}$ & 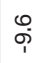 \\
\hline 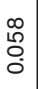 & $\begin{array}{l}\text { 告 } \\
\text { to }\end{array}$ & $\begin{array}{l}\mathscr{8} \\
\mathscr{0} \\
0 \\
0\end{array}$ & $\sim$ & 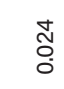 & $\stackrel{\widetilde{N}}{0}$ & $\begin{array}{l}\tilde{o} \\
0 \\
0\end{array}$ & $\sim$ & 0 & $\begin{array}{l}0 \\
0 \\
0 \\
0\end{array}$ & $\begin{array}{l}\stackrel{0}{\circ} \\
\stackrel{m}{0} \\
0\end{array}$ & เ & 0 & ơ & $\simeq$ & $\begin{array}{l}\infty \\
0 \\
0 \\
0 \\
0\end{array}$ & $\begin{array}{l}\text { స్లి } \\
0\end{array}$ & 0 & $\begin{array}{l}0 \\
0 \\
0 \\
0 \\
0\end{array}$ \\
\hline $\begin{array}{l}\mathfrak{N} \\
\stackrel{0}{0} \\
O\end{array}$ & 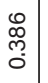 & $\begin{array}{l}\infty \\
\stackrel{0}{0} \\
0 \\
0\end{array}$ & $\sim$ & $\stackrel{\text { to }}{\circ}$ & $\begin{array}{l}\infty \\
\stackrel{0}{0} \\
\stackrel{0}{0}\end{array}$ & $\begin{array}{l}m \\
\stackrel{0}{0} \\
0 \\
0\end{array}$ & $\sim$ & $\begin{array}{l}\stackrel{0}{0} \\
\stackrel{0}{0} \\
0\end{array}$ & \begin{tabular}{l}
0 \\
\multirow{0}{0}{} \\
0
\end{tabular} & $\begin{array}{l}0 \\
\stackrel{0}{0} \\
0 \\
0\end{array}$ & $m$ & $\infty$ & ơ & $\approx$ & $\begin{array}{l}\stackrel{\infty}{0} \\
\underset{0}{0}\end{array}$ & 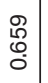 & \pm & $\frac{\tilde{0}}{0}$ \\
\hline 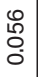 & 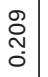 & $\begin{array}{l}\hat{\partial} \\
0 \\
0\end{array}$ & - & ò & $\begin{array}{l}\stackrel{2}{0} \\
0 \\
0\end{array}$ & $\begin{array}{l}\tilde{O} \\
\stackrel{0}{0} \\
\stackrel{0}{0}\end{array}$ & $\sim$ & $\begin{array}{l}\infty \\
\stackrel{0}{0} \\
0\end{array}$ & 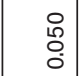 & $\begin{array}{c}\infty \\
\infty \\
\stackrel{+}{0} \\
0\end{array}$ & N & 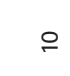 & $\frac{m}{0}$ & $\stackrel{\infty}{\simeq}$ & $\begin{array}{c}\text { fq } \\
\text { m. }\end{array}$ & 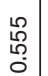 & \pm & $\frac{\tilde{\sigma}}{0}$ \\
\hline $\begin{array}{l}\stackrel{0}{o} \\
0 \\
0\end{array}$ & $\frac{\hat{0}}{0}$ & 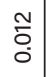 & $\begin{array}{l}0 \\
\stackrel{0}{0}\end{array}$ & ò & \begin{tabular}{l}
$\infty$ \\
\multirow{0}{0}{} \\
0
\end{tabular} & $\begin{array}{l}m \\
\stackrel{0}{0} \\
0 \\
0\end{array}$ & $\begin{array}{l}\infty \\
\infty \\
\stackrel{0}{0}\end{array}$ & 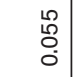 & $\begin{array}{l}\dot{J} \\
\dot{0} \\
\dot{0}\end{array}$ & $\frac{ \pm}{\square}$ & $N$ & $m$ & $\begin{array}{l}\hat{s} \\
0 \\
0\end{array}$ & $\simeq$ & 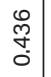 & $\begin{array}{l}\text { ㄱ. } \\
\text { ప్ }\end{array}$ & 0 & $\begin{array}{l}\stackrel{g}{m} \\
\stackrel{0}{0}\end{array}$ \\
\hline$\frac{8}{0}$ & 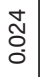 & $\begin{array}{l}0 \\
\text { ஸ் } \\
0\end{array}$ & $\begin{array}{l}\stackrel{\Delta}{\Delta} \\
0 \\
0\end{array}$ & 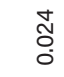 & \begin{tabular}{l}
\multirow{2}{*}{} \\
$\stackrel{0}{0}$
\end{tabular} & $\begin{array}{l}\overline{0} \\
\text { 응 }\end{array}$ & $\begin{array}{l}\stackrel{\sim}{\sim} \\
\stackrel{0}{0}\end{array}$ & $\begin{array}{l}\overline{0} \\
\text { ○. }\end{array}$ & $\frac{\infty}{\circ}$ & $\begin{array}{l}\stackrel{\sim}{0} \\
0 \\
O\end{array}$ & $\begin{array}{l}\text { J̃ } \\
\text { O. }\end{array}$ & $\begin{array}{l}\text { Ñ } \\
\text { Oे }\end{array}$ & 옹 & $\begin{array}{l}\infty \\
\frac{0}{0}\end{array}$ & $\frac{8}{0}$ & 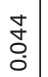 & $\begin{array}{l}\grave{\Xi} \\
0 \\
0\end{array}$ & 芯 \\
\hline$\stackrel{N}{\underset{N}{N}}$ & \begin{tabular}{l}
\multicolumn{9}{c}{} \\
$\dot{\phi}$
\end{tabular} & $\approx$ & $\begin{array}{l}\hat{0} \\
\hat{i} \\
i\end{array}$ & $\stackrel{m}{\sim}$ & 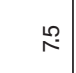 & 0 & $\begin{array}{l}0 \\
\infty\end{array}$ & 0 & $\stackrel{\grave{N}}{\mathrm{~N}}$ & $\stackrel{\sigma}{T}$ & $\stackrel{+}{+}$ & స̃ & 읏 & $\stackrel{\circ}{\stackrel{\circ}{2}}$ & $\hat{i}$ & $\cong$ & $\stackrel{\circ}{\stackrel{p}{\underline{m}}}$ & $\stackrel{ \pm}{=}$ \\
\hline $\begin{array}{l}\widetilde{D} \\
\stackrel{0}{0}\end{array}$ & $\begin{array}{l}\infty \\
\& \\
\infty \\
0\end{array}$ & $\begin{array}{l}\tilde{O} \\
\stackrel{0}{0}\end{array}$ & $\nabla$ & זٓ & $\begin{array}{l}\stackrel{9}{0} \\
0 \\
0\end{array}$ & 0 & $\sim$ & 0 & $\begin{array}{l}\hat{0} \\
\stackrel{0}{0} \\
0\end{array}$ & 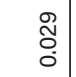 & $m$ & - & $\begin{array}{l}\text { N్ } \\
\text { Oे }\end{array}$ & $\infty$ & $\begin{array}{l}\overline{1} \\
\text { ¿ू. } \\
0\end{array}$ & $\begin{array}{l}\mathbb{m} \\
\tilde{0} \\
0 \\
0\end{array}$ & $\wedge$ & ঙ্ল \\
\hline $\begin{array}{l}\dot{0} \\
\stackrel{0}{0} \\
0\end{array}$ & $\begin{array}{l}\text { oे } \\
\text { o }\end{array}$ & $\begin{array}{l}\infty \\
0 \\
0 \\
0\end{array}$ & $\wedge$ & $\begin{array}{l}\bar{\alpha} \\
\text { Oे. }\end{array}$ & $\frac{10}{0}$ & 0 & $\sim$ & 0 & $\begin{array}{l}\stackrel{0}{ } \\
0 \\
0 \\
0\end{array}$ & $\begin{array}{l}\bar{g} \\
\ddot{\delta}\end{array}$ & $\sigma$ & 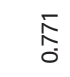 & 0 & ما & $\begin{array}{l}\mathscr{0} \\
0 \\
0 \\
0\end{array}$ & 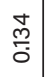 & $\nabla$ & ల్ \\
\hline$\overline{\frac{m}{o}}$ & $\begin{array}{l}\stackrel{J}{\sim} \\
\text { J }\end{array}$ & $\begin{array}{l}\tilde{O} \\
0 \\
0 \\
0\end{array}$ & $\odot$ & $\begin{array}{l}\text { 㟧 } \\
\text { O. }\end{array}$ & 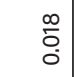 & $\begin{array}{l}0 \\
0 \\
0\end{array}$ & $\sim$ & 0 & $\begin{array}{l}: \\
: \\
0\end{array}$ & $\begin{array}{l}\stackrel{9}{0} \\
0 \\
0\end{array}$ & ما & $\begin{array}{l}\stackrel{0}{0} \\
\infty \\
0\end{array}$ & 0 & $\nabla$ & $\stackrel{\circ}{\overline{0}}$ & $\frac{\grave{m}}{\sigma}$ & 10 & $\begin{array}{l}\mathscr{O} \\
0 \\
0\end{array}$ \\
\hline $\begin{array}{l}m \\
ٍ ్ ర 0 \\
0\end{array}$ & $\begin{array}{c}\text { f } \\
\text { o } \\
\text { d }\end{array}$ & $\mid \begin{array}{l}\overline{\bar{o}} \\
\dot{0}\end{array}$ & o & 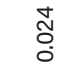 & $\begin{array}{l}\tilde{\Omega} \\
0 \\
0\end{array}$ & 0 & $\sim$ & $\begin{array}{l}0 \\
\stackrel{0}{0} \\
0\end{array}$ & $\begin{array}{l}0 \\
0 \\
0 \\
0\end{array}$ & \begin{tabular}{l}
\multirow{J}{0}{} \\
$\stackrel{0}{\circ}$
\end{tabular} & $N$ & $N$ & 0 & $\circ$ & 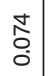 & 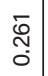 & $m$ & $\begin{array}{l}\mathscr{N}_{0} \\
0 \\
0\end{array}$ \\
\hline $\begin{array}{l}\frac{8}{0} \\
0 \\
0\end{array}$ & $\begin{array}{l}0 \\
0 \\
0 \\
0\end{array}$ & $\begin{array}{l}\hat{o} \\
0 \\
0\end{array}$ & $\sim$ & $\bar{o}$ & $\begin{array}{l}\tilde{y} \\
0 \\
0\end{array}$ & 0 & $\begin{array}{l}\infty \\
\stackrel{0}{0} \\
\stackrel{0}{0}\end{array}$ & $\begin{array}{l}m \\
0 \\
0 \\
0\end{array}$ & $\begin{array}{l}\infty \\
\tilde{y} \\
0 \\
0\end{array}$ & $\begin{array}{l}m \\
0 \\
0 \\
0\end{array}$ & $N$ & - & $\begin{array}{l}\widetilde{0} \\
\stackrel{0}{0}\end{array}$ & $\simeq$ & $\begin{array}{l}0 \\
0 \\
0 \\
0\end{array}$ & 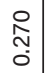 & $m$ & $\begin{array}{l}\text { Oొ } \\
0 \\
0\end{array}$ \\
\hline $\begin{array}{l}\text { ¿े } \\
0 \\
0\end{array}$ & $\begin{array}{l}\circ \\
\\
\end{array}$ & $\begin{array}{l}8 \\
0 \\
0 \\
0\end{array}$ & $\begin{array}{l}\overline{0} \\
0 \\
0\end{array}$ & $\begin{array}{l}\tilde{o} \\
\stackrel{0}{0}\end{array}$ & $\hat{o}$ & $\begin{array}{l}0 \\
0 \\
0 \\
0\end{array}$ & 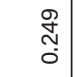 & $\begin{array}{l}\hat{o} \\
\tilde{O} \\
0\end{array}$ & $\begin{array}{l}0 \\
\tilde{O} \\
0 \\
0\end{array}$ & $\begin{array}{l}\text { Lे } \\
0 \\
0 \\
0\end{array}$ & $\begin{array}{l}\hat{o} \\
o \\
0\end{array}$ & $\begin{array}{l}\text { \& } \\
\text { Oे } \\
0\end{array}$ & 0 & is & $\begin{array}{l}\infty \\
\frac{m}{0}\end{array}$ & $\begin{array}{l}0 \\
0 \\
0 \\
0\end{array}$ & $\begin{array}{l}0 \\
\text { L } \\
0 \\
0\end{array}$ & $\begin{array}{l}\text { مू } \\
\text { N }\end{array}$ \\
\hline $\begin{array}{l}0 \\
0 \\
0 \\
0 \\
0 \\
0 \\
0 \\
0 \\
0 \\
0 \\
0 \\
0\end{array}$ & 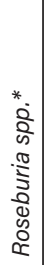 & 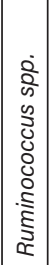 & 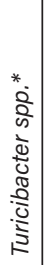 & 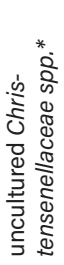 & 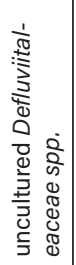 & 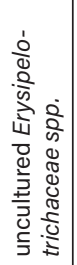 & 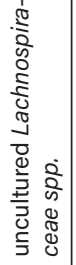 & 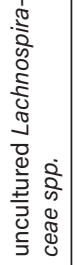 & 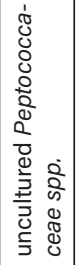 & 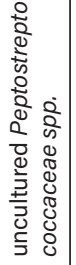 & 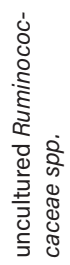 & 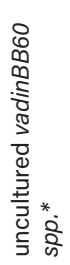 & 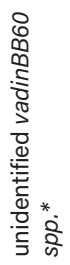 & $\begin{array}{l}\frac{1}{2} \\
0 \\
0 \\
0 \\
\frac{2}{2} \\
\frac{0}{4} \\
\end{array}$ & $\begin{array}{l}\frac{1}{2} \\
0 \\
\frac{0}{2} \\
\frac{0}{0} \\
\frac{0}{0} \\
\frac{0}{2} \\
\frac{0}{4}\end{array}$ & $\begin{array}{l}\dot{0} \\
0 \\
0 \\
0 \\
\frac{0}{0} \\
\frac{0}{0} \\
\frac{8}{0} \\
\delta \\
\infty\end{array}$ & 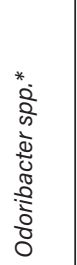 & $\begin{array}{l}\frac{2}{2} \\
0 \\
0 \\
\frac{0}{0} \\
\frac{0}{2} \\
\frac{8}{0} \\
\frac{0}{0} \\
\frac{0}{\pi} \\
0 \\
0\end{array}$ \\
\hline & & & & & & & & & & & & & & \multicolumn{4}{|c|}{ 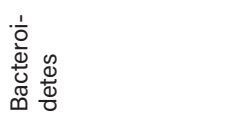 } & \\
\hline
\end{tabular}




\begin{tabular}{|c|c|c|c|c|c|c|c|c|c|c|c|c|c|}
\hline 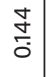 & 志 & 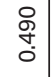 & 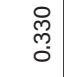 & \begin{tabular}{|c|c} 
& 8 \\
0 \\
0 \\
0 \\
0
\end{tabular} & \begin{tabular}{l|l}
0 \\
$\vdots$ \\
0
\end{tabular} & $\begin{array}{l}\circ \\
: \\
0\end{array}$ & $\begin{array}{l}\circ \\
: \\
0\end{array}$ & $\frac{d}{\delta}$ & $\begin{array}{l}8 \\
\vdots \\
0\end{array}$ & $\begin{array}{l}0 \\
0 \\
0 \\
0\end{array}$ & 웅 & $\begin{array}{l}\hat{\circ} \\
\stackrel{\circ}{0}\end{array}$ & 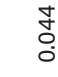 \\
\hline$\cong$ & $\stackrel{?}{\longrightarrow}$ & $\stackrel{\circ}{\therefore}$ & $\stackrel{i}{\text { i }}$ & 0 & $\stackrel{\infty}{\infty}$ & $\stackrel{L}{+}$ & 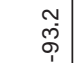 & 0 & $\begin{array}{c}\widehat{0} \\
0 \\
0\end{array}$ & \begin{tabular}{|l|}
$\mid \begin{array}{l}1 \\
0 \\
0 \\
1\end{array}$ \\
\end{tabular} & क् & $\stackrel{\circ}{\dot{m}}$ & $\begin{array}{l}\text { ㅁ } \\
\stackrel{\circ}{1}\end{array}$ \\
\hline 10 & 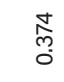 & $m$ & 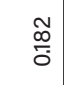 & 0 & $\begin{array}{l}0 \\
0 \\
0 \\
0\end{array}$ & $\stackrel{\infty}{\circ}$ & $\begin{array}{l}m \\
0 \\
0\end{array}$ & 0 & 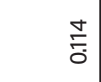 & $\hat{\hat{o}}$ & $\begin{array}{l}\infty \\
\stackrel{0}{0}\end{array}$ & 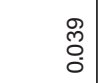 & ষ্ণ \\
\hline 0 & స్ & $m$ & $\begin{array}{l}\infty \\
\stackrel{0}{0} \\
\frac{0}{0}\end{array}$ & 0 & 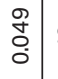 & $\stackrel{\infty}{\simeq}$ & $\begin{array}{l}\text { ò } \\
\text { o. }\end{array}$ & $\begin{array}{l}\text { 웅 } \\
\stackrel{0}{0}\end{array}$ & - & $\bar{\vdots}$ & 0 & $\begin{array}{l}\stackrel{\sim}{\Delta} \\
\stackrel{0}{0}\end{array}$ & 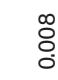 \\
\hline$\sim$ & 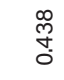 & $\nabla$ & $\frac{\circ}{\circ}$ & 0 & \begin{tabular}{l|l}
0 \\
0 \\
0 \\
0
\end{tabular} & $\simeq$ & $\stackrel{\infty}{\frac{\infty}{0}}$ & 0 & $m$ & $\stackrel{\substack{0 \\
\hdashline}}{\cong}$ & $\stackrel{\Delta}{\circ}$ & $\begin{array}{l}\stackrel{0}{0} \\
\stackrel{0}{0}\end{array}$ & : \\
\hline$m$ & $\frac{\widehat{\sigma}}{\circ}$ & $m$ & $\begin{array}{l}m \\
\text { Oे } \\
0\end{array}$ & \begin{tabular}{|}
$\bar{N}$ \\
$\underset{0}{\sim}$
\end{tabular} & 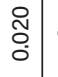 & $\infty$ & 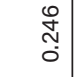 & 0 & $\circ$ & \begin{tabular}{|l|}
$\overline{5}$ \\
0 \\
0
\end{tabular} & 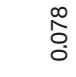 & $\stackrel{m}{0}$ & \begin{tabular}{l}
$\infty$ \\
\multirow{0}{0}{} \\
0
\end{tabular} \\
\hline $\begin{array}{l}\stackrel{\sim}{0} \\
\stackrel{O}{0}\end{array}$ & I্ & $\begin{array}{l}\stackrel{L}{\circ} \\
0 \\
0\end{array}$ & 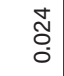 & \begin{tabular}{l}
$\stackrel{8}{0}$ \\
\multirow{2}{0}{} \\
\end{tabular} & 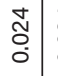 & 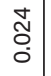 & 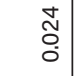 & $\begin{array}{l}\text { 옹 } \\
\text { On }\end{array}$ & 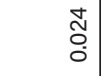 & $\begin{array}{l} \\
0 \\
0 \\
0\end{array}$ & ¿্ّ & 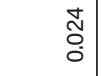 & ్ㅗㅁ \\
\hline 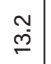 & ڤై & $\stackrel{L n}{\Gamma}$ & $\stackrel{\circ}{m}$ & 0 & 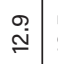 & $\begin{array}{l}\stackrel{0}{\mathrm{~N}} \\
\mathrm{~N}\end{array}$ & 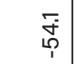 & 0 & ָ̊. & $\mid \begin{array}{l}\overline{\mathbf{z}} \\
\overline{1}\end{array}$ & $\begin{array}{l}\text { 앙 } \\
\text { in }\end{array}$ & 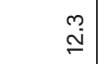 & $\circ$ \\
\hline$\sigma$ & $\begin{array}{l}\hat{A} \\
0\end{array}$ & $\wedge$ & 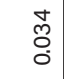 & 0 & $\begin{array}{l}\stackrel{0}{0} \\
\stackrel{0}{0}\end{array}$ & $\stackrel{\llcorner}{\sim}$ & $\begin{array}{l}\dot{0} \\
\stackrel{0}{0}\end{array}$ & 0 & 0 & $\mid \begin{array}{l}\hat{N} \\
0 \\
0\end{array}$ & $\stackrel{10}{\circ}$ & $\begin{array}{l}\hat{\phi} \\
0 \\
0\end{array}$ & $\circ$ \\
\hline$\sim$ & $\frac{d}{\delta}$ & $\nabla$ & $\begin{array}{l}\stackrel{\omega}{0} \\
0 \\
0\end{array}$ & 0 & $\begin{array}{l}0 \\
0 \\
0 \\
0\end{array}$ & $\stackrel{m}{m}$ & 0 & 0 & $\stackrel{\substack{\tilde{N} \\
\hdashline}}{0}$ & $\begin{array}{l}0 \\
0 \\
0 \\
0\end{array}$ & $\begin{array}{l}\text { Oे } \\
0 \\
0\end{array}$ & $\begin{array}{l}\mathscr{L} \\
0 \\
0 \\
0\end{array}$ & $\bar{o}$ \\
\hline$\sim$ & $\stackrel{\infty}{\infty}$ & $m$ & $\begin{array}{l}\infty \\
\vdots \\
0 \\
0\end{array}$ & 0 & $\begin{array}{l}\infty \\
\vdots \\
0 \\
0\end{array}$ & $\stackrel{\text { ని }}{ }$ & $\begin{array}{l}\text { L̊ } \\
\stackrel{0}{0} \\
0\end{array}$ & $\circ$ & $\frac{\bar{\infty}}{\bar{\sigma}}$ & $\left|\begin{array}{|l}\overline{5} \\
0 \\
0\end{array}\right|$ & \begin{tabular}{l}
0 \\
\multirow{0}{0}{} \\
0
\end{tabular} & $\begin{array}{l}\hat{0} \\
0 \\
0\end{array}$ & $\bar{\circ}$ \\
\hline$N$ & $\frac{\overline{5}}{0}$ & 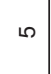 & $\stackrel{m}{0}$ & 0 & $\begin{array}{l}\bar{m} \\
\dot{0}\end{array}$ & ㅇ & 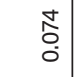 & 0 & 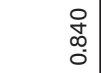 & - & 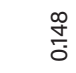 & $\bar{O}$ & 0 \\
\hline$\nabla$ & $\underset{\mathbb{N}}{\mathbb{N}}$ & $m$ & $\stackrel{\bar{m}}{\circ}$ & 0 & $\begin{array}{l}\text { ô } \\
0 \\
0\end{array}$ & 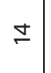 & $\begin{array}{l}\mathbb{N} \\
\hat{0} \\
0\end{array}$ & 0 & $\begin{array}{l}\infty \\
\stackrel{2}{0} \\
0\end{array}$ & $\nabla$ & $\begin{array}{l}\hat{0} \\
\text { Oे }\end{array}$ & $\begin{array}{l}0 \\
\stackrel{0}{0} \\
0\end{array}$ & 。 \\
\hline $\begin{array}{l}0 \\
0 \\
0 \\
0 \\
0\end{array}$ & 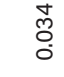 & $\sim$ & $\begin{array}{l}\text { 음 } \\
\text { 응 }\end{array}$ & 0 & \begin{tabular}{l|l}
\multirow{2}{*}{} \\
0 \\
0
\end{tabular} & $\sim$ & $\frac{\circ}{\circ}$ & o & $\simeq$ & $\mathscr{I}$ & - & $\begin{array}{l}2 \\
0 \\
0 \\
0\end{array}$ & 。 \\
\hline $\begin{array}{l}0 \\
0 \\
0 \\
0 \\
0 \\
0 \\
5 \\
5 \\
5 \\
0 \\
0 \\
0 \\
0\end{array}$ & 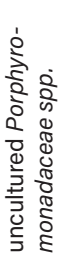 & 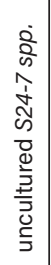 & 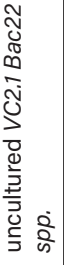 & 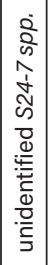 & 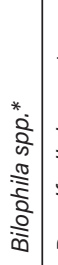 & 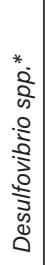 & 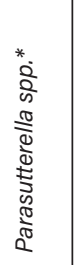 & $\begin{array}{l}0 \\
0 \\
0 \\
0 \\
0 \\
0 \\
0 \\
0 \\
0 \\
0 \\
8 \\
0 \\
\frac{1}{4}\end{array}$ & 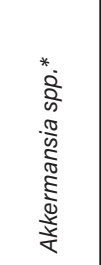 & 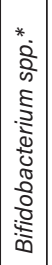 & 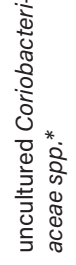 & 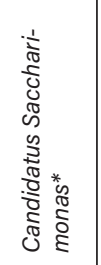 & 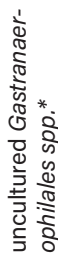 \\
\hline & & & & & \multicolumn{3}{|c|}{ 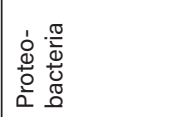 } & 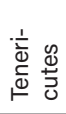 & 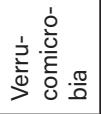 & \multicolumn{2}{|c|}{ 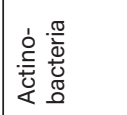 } & 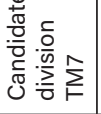 & 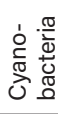 \\
\hline
\end{tabular}

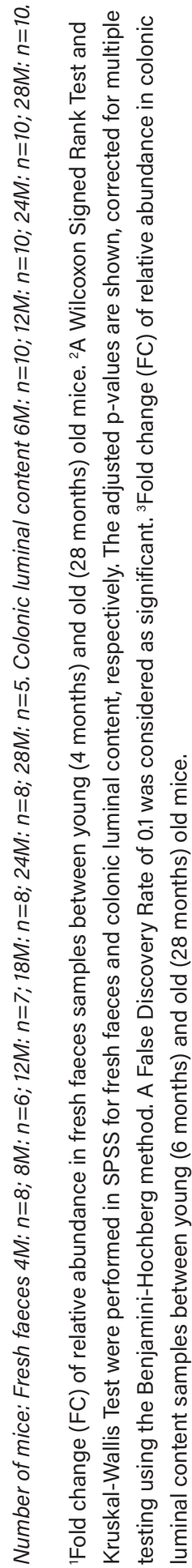


Table S2 (A) Table including the top 10 of significantly enriched up-regulated and (B) down-regulated gene sets between 6 and 12 months, as determined by GSEA. (C) Genes included in the canonical pathways 'Hepatic Fibrosis/Hepatic Stellate Cell Activation' and 'Regulation of the Epithelial-Mesenchymal Transition Pathway' identified by IPA. (D) Expression of genes described to be up- or down-regulated during EMT, selection based on literature (see references in supplemental data). (E) IPA identified the top 5 upstream regulators with the highest activation score between 6 and 12 months.

A

\begin{tabular}{|l|c|c|c|}
\hline NAME & SIZE & NES & $\begin{array}{c}\text { FDR } \\
\text { q-value }\end{array}$ \\
\hline EXTRACELLULAR MATRIX ORGANIZATION & 251 & 3.21 & $<0.001$ \\
\hline COLLAGEN BIOSYNTHESIS AND MODIFYING ENZYMES & 62 & 2.81 & $<0.001$ \\
\hline ECM PROTEOGLYCANS & 53 & 2.78 & $<0.001$ \\
\hline ELASTIC FIBRE FORMATION & 38 & 2.77 & $<0.001$ \\
\hline KEGG ECM RECEPTOR INTERACTION & 87 & 2.73 & $<0.001$ \\
\hline COLLAGEN FORMATION & 82 & 2.68 & $<0.001$ \\
\hline INTEGRIN CELL SURFACE INTERACTIONS & 64 & 2.66 & $<0.001$ \\
\hline PLATELET DEGRANULATION & 76 & 2.57 & $<0.001$ \\
\hline KEGG FOCAL ADHESION & 205 & 2.57 & $<0.001$ \\
\hline MOLECULES ASSOCIATED WITH ELASTIC FIBRES & 29 & 2.55 & $<0.001$ \\
\hline
\end{tabular}

B

\begin{tabular}{|l|c|c|c|}
\hline NAME & SIZE & NES & $\begin{array}{c}\text { FDR } \\
\text { q-value }\end{array}$ \\
\hline OLFACTORY SIGNALING PATHWAY & 308 & -1.88 & 0.242 \\
\hline KEGG MATURITY ONSET DIABETES OF THE YOUNG & 26 & -1.85 & 0.170 \\
\hline TRAF6 MEDIATED IRF7 ACTIVATION & 29 & -1.81 & 0.196 \\
\hline RIG I MDA5 MEDIATED INDUCTION OF IFN ALPHA BETA PATHWAYS & 67 & -1.78 & 0.207 \\
\hline $\begin{array}{l}\text { TRANSCRIPTIONAL ACTIVITY OF SMAD2 SMAD3 SMAD4 HETEROTRIM- } \\
\text { ER }\end{array}$ & 37 & -1.72 & 0.324 \\
\hline KEGG TYPE II DIABETES MELLITUS & 50 & -1.71 & 0.289 \\
\hline DOWNREGULATION OF SMAD2 3 SMAD4 TRANSCRIPTIONAL ACTIVITY & 19 & -1.70 & 0.287 \\
\hline SMAD2 SMAD3 SMAD4 HETEROTRIMER REGULATES TRANSCRIPTION & 25 & -1.70 & 0.252 \\
\hline WP431 NUCLEAR RECEPTORS IN LIPID METABOLISM AND TOXICITY & 30 & -1.69 & 0.258 \\
\hline RORA ACTIVATES CIRCADIAN GENE EXPRESSION & 24 & -1.68 & 0.256 \\
\hline
\end{tabular}

NES: Normalized Enrichment Score 
C

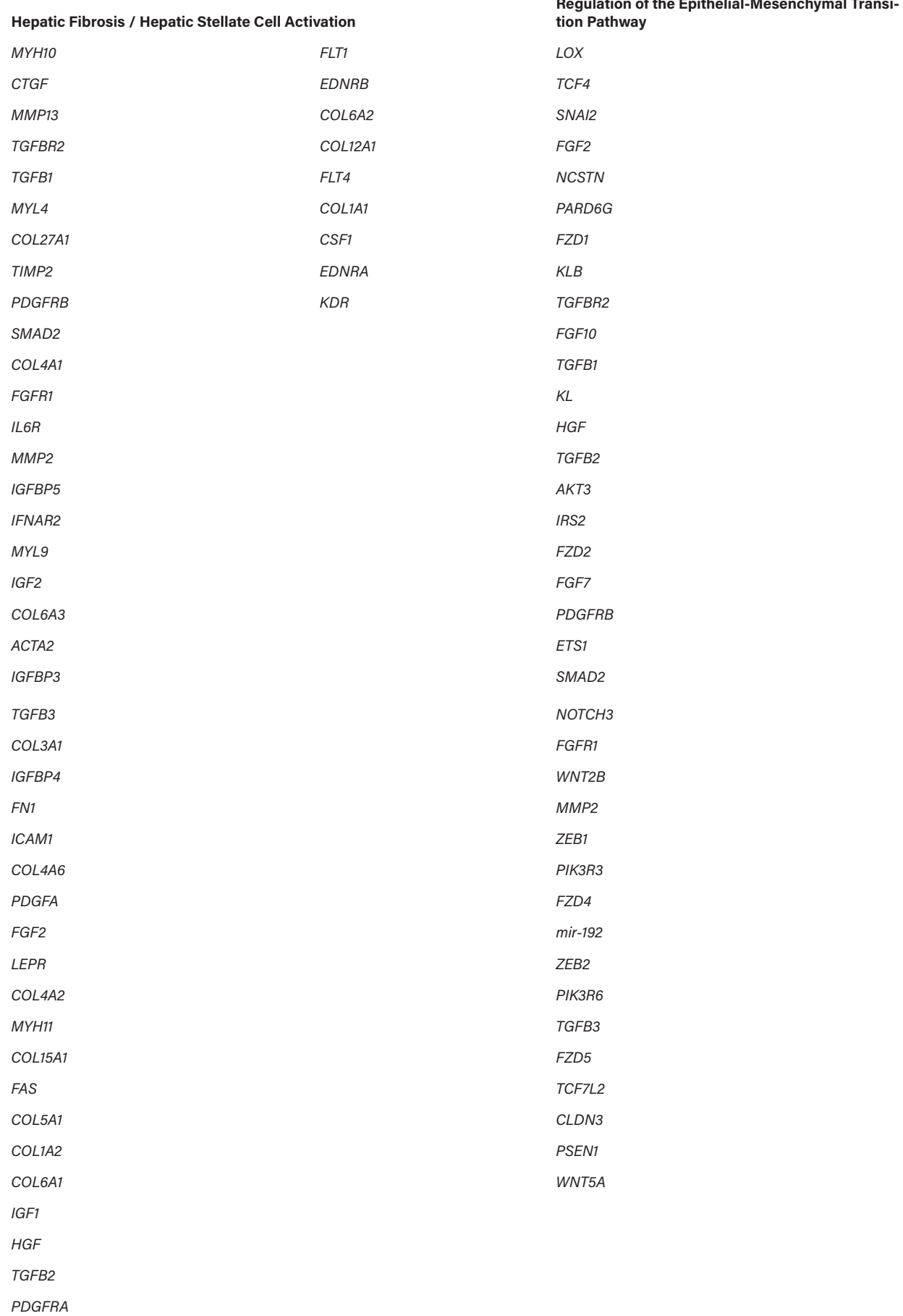


72 | Chapter 2

COL18A1

COL5A2

VCAM1

D

\begin{tabular}{|l|c|r|}
\hline \multicolumn{3}{|c|}{ TRANSCRIPTION FACTORS } \\
\hline Gene name & Fold Change & \multicolumn{1}{c|}{ IBMT q-value } \\
\hline$\uparrow$ Ets2 & 1.03 & $3.57 \mathrm{E}-01$ \\
\hline$\uparrow$ Junb & 1.04 & $5.16 \mathrm{E}-01$ \\
\hline$\uparrow$ Lef1 & 1.22 & $3.23 \mathrm{E}-02$ \\
\hline$\uparrow$ Mkl1 & -1.05 & $2.99 \mathrm{E}-01$ \\
\hline$\uparrow$ Mkl2 & -1.04 & $3.13 \mathrm{E}-01$ \\
\hline$\uparrow$ Prrx1 & 1.04 & $3.92 \mathrm{E}-01$ \\
\hline$\uparrow$ Snai1 & 1.23 & $7.72 \mathrm{E}-02$ \\
\hline$\uparrow$ Snai2 & $\mathbf{1 . 8 4}$ & $2.09 \mathrm{E}-06$ \\
\hline$\uparrow$ Twist1 & 1.14 & $1.63 \mathrm{E}-01$ \\
\hline$\uparrow$ Twist2 & 1.18 & $9.86 \mathrm{E}-02$ \\
\hline$\uparrow$ Zeb1 & $\mathbf{2 . 0 4}$ & $1.50 \mathrm{E}-05$ \\
\hline$\uparrow$ Zeb2 & $\mathbf{3 . 2 8}$ & $2.07 \mathrm{E}-09$ \\
\hline$\uparrow$ Tcf3 & -1.02 & $5.27 \mathrm{E}-01$ \\
\hline$\uparrow$ Tcf21 & $\mathbf{2 . 8 9}$ & $2.37 \mathrm{E}-07$ \\
\hline$\uparrow$ Foxc2 & 1.05 & $4.55 \mathrm{E}-01$ \\
\hline
\end{tabular}

\begin{tabular}{|l|l|}
\hline$\uparrow$ & Up-regulated during EMT \\
\hline$\downarrow$ & Down-regulated during EMT \\
\hline & $\mathrm{q}<0.01$ \\
\hline & $\mathrm{q}<0.05$ \\
\hline
\end{tabular}

\begin{tabular}{|l|c|r|}
\hline \multicolumn{3}{|c|}{$\begin{array}{c}\text { REGULATION AT RNA LEVEL \& HISTONE } \\
\text { MODIFICATIONS }\end{array}$} \\
\hline Gene name & Fold Change & \multicolumn{1}{c|}{ IBMT q-value } \\
\hline$\uparrow$ Dot11 & -1.01 & $5.69 \mathrm{E}-01$ \\
\hline$\downarrow$ Esrp1 & -1.07 & $8.70 \mathrm{E}-02$ \\
\hline$\downarrow$ Esrp2 & -1.01 & $5.86 \mathrm{E}-01$ \\
\hline$\uparrow$ Kdm1a & -1.09 & $2.21 \mathrm{E}-02$ \\
\hline$\uparrow$ Kdm3a & -1.24 & $2.87 \mathrm{E}-03$ \\
\hline$\uparrow$ Mbn11 & 1.07 & $1.01 \mathrm{E}-01$ \\
\hline$\downarrow$ Mir124a-2 & -1.13 & $1.90 \mathrm{E}-01$ \\
\hline$\downarrow$ Mir124a-3 & -1.06 & $3.14 \mathrm{E}-01$ \\
\hline$\uparrow$ Mir155 & -1.11 & $9.81 \mathrm{E}-02$ \\
\hline$\downarrow$ Mir194-1 & -1.2 & $6.06 \mathrm{E}-02$ \\
\hline$\downarrow$ Mir1a-1 & -1.02 & $4.79 \mathrm{E}-01$ \\
\hline
\end{tabular}




\begin{tabular}{|c|c|c|}
\hline$\downarrow$ Mir1a-2 & -1.04 & $3.11 \mathrm{E}-01$ \\
\hline$\downarrow$ Mir200a & -1.37 & $2.22 \mathrm{E}-02$ \\
\hline$\downarrow$ Mir200b & -1.53 & 4.12E-02 \\
\hline$\downarrow$ Mir200c & -1.14 & $2.45 \mathrm{E}-01$ \\
\hline$\downarrow$ Mir205 & -1.14 & $2.34 \mathrm{E}-01$ \\
\hline$\uparrow$ Mir24-2 & -1.11 & $2.21 \mathrm{E}-01$ \\
\hline$\downarrow$ Mir29b-1 & -1.11 & $6.62 \mathrm{E}-02$ \\
\hline$\downarrow$ Mir30a & -1.35 & $2.94 \mathrm{E}-02$ \\
\hline$\downarrow$ Mir34a & -1.11 & $3.20 \mathrm{E}-01$ \\
\hline$\downarrow$ Mir34b & -1.05 & $4.54 \mathrm{E}-01$ \\
\hline$\downarrow$ Mir34c & 1.03 & 4.76E-01 \\
\hline$\downarrow$ Mir429 & -1.54 & $1.26 \mathrm{E}-02$ \\
\hline$\downarrow$ Mir491 & 1.12 & $2.73 \mathrm{E}-01$ \\
\hline$\uparrow$ Mir9-1 & -1.18 & $9.84 \mathrm{E}-02$ \\
\hline$\uparrow$ Mir9-2 & -1.21 & $3.25 \mathrm{E}-01$ \\
\hline$\uparrow$ Mir9-3 & -1.01 & $5.95 \mathrm{E}-01$ \\
\hline$\uparrow R b f o x 2$ & -1.01 & 5.92E-01 \\
\hline$\downarrow$ Rbm47 & -1.09 & $2.55 \mathrm{E}-02$ \\
\hline$\uparrow$ Srsf1 & 1.1 & $4.49 \mathrm{E}-02$ \\
\hline
\end{tabular}

\begin{tabular}{|l|l|}
\hline$\uparrow$ & Up-regulated during EMT \\
\hline$\downarrow$ & Down-regulated during EMT \\
\hline & $\mathrm{q}<0.01$ \\
\hline & $\mathrm{q}<0.05$ \\
\hline
\end{tabular}

\begin{tabular}{|l|c|r|}
\hline \multicolumn{3}{|c|}{ GROWTH FACTORS \& OTHER SIGNALS } \\
\hline Gene name & Fold Change & IBMT q-value \\
\hline$\uparrow$ Ctgf & $\mathbf{2 . 0 7}$ & $3.22 \mathrm{E}-04$ \\
\hline$\uparrow$ Dab2 & $\mathbf{2 . 3 3}$ & $4.26 \mathrm{E}-07$ \\
\hline$\uparrow$ Egflam & 1.29 & $6.56 \mathrm{E}-03$ \\
\hline$\uparrow$ Emr1 & $\mathbf{2 . 3 2}$ & $5.87 \mathrm{E}-08$ \\
\hline$\uparrow$ Emr4 & 1.1 & $1.97 \mathrm{E}-01$ \\
\hline$\uparrow$ Fgf1 & -1.1 & $2.41 \mathrm{E}-01$ \\
\hline$\uparrow \mathrm{Hgf}$ & $\mathbf{2 . 1 6}$ & $1.42 \mathrm{E}-03$ \\
\hline$\uparrow$ lgf1 & $\mathbf{2 . 6}$ & $1.83 \mathrm{E}-07$ \\
\hline$\uparrow$ Igfbp3 & $\mathbf{3 . 6 8}$ & $1.83 \mathrm{E}-08$ \\
\hline$\uparrow l l 6$ & -1.03 & $4.20 \mathrm{E}-01$ \\
\hline$\uparrow$ Notch1 & -1 & $6.15 \mathrm{E}-01$ \\
\hline$\uparrow$ Notch2 & 1.12 & $2.41 \mathrm{E}-01$ \\
\hline$\uparrow$ Notch3 & 1.45 & $1.03 \mathrm{E}-03$ \\
\hline
\end{tabular}




\begin{tabular}{|l|c|r|}
\hline$\uparrow$ Notch4 & 1.11 & $1.87 \mathrm{E}-01$ \\
\hline$\uparrow$ Pdgfa & -1.2 & $5.33 \mathrm{E}-03$ \\
\hline$\uparrow$ Pdgfb & 1.24 & $1.97 \mathrm{E}-02$ \\
\hline$\uparrow$ Pdgfra & $\mathbf{2 . 9 2}$ & $3.73 \mathrm{E}-08$ \\
\hline$\uparrow$ Pdgfrb & $\mathbf{1 . 7 8}$ & $1.31 \mathrm{E}-07$ \\
\hline$\uparrow$ Shh & 1.01 & $6.01 \mathrm{E}-01$ \\
\hline$\uparrow$ Tgfb1 & 1.35 & $1.02 \mathrm{E}-02$ \\
\hline$\uparrow$ Tgfb2 & $\mathbf{1 . 8 1}$ & $1.26 \mathrm{E}-04$ \\
\hline$\uparrow$ Tgfb3 & $\mathbf{1 . 5 3}$ & $2.36 \mathrm{E}-03$ \\
\hline$\uparrow$ Tgfbr1 & 1.11 & $6.15 \mathrm{E}-02$ \\
\hline$\uparrow$ Tgfbr2 & 1.23 & $3.80 \mathrm{E}-04$ \\
\hline$\uparrow$ Tgfbr3 & $\mathbf{3 . 2 1}$ & $8.78 \mathrm{E}-09$ \\
\hline$\uparrow$ Vegfa & -1.06 & $3.11 \mathrm{E}-01$ \\
\hline$\uparrow W n 2$ & 1.04 & $4.83 \mathrm{E}-01$ \\
\hline$\uparrow W n t 2 b$ & $\mathbf{2 . 8 9}$ & $6.29 \mathrm{E}-09$ \\
\hline$\uparrow$ Wnt5a & 1.44 & $1.24 \mathrm{E}-02$ \\
\hline
\end{tabular}

\begin{tabular}{|l|l|}
\hline$\uparrow$ & Up-regulated during EMT \\
\hline$\downarrow$ & Down-regulated during EMT \\
\hline & $\mathrm{q}<0.01$ \\
\hline & $\mathrm{q}<0.05$ \\
\hline
\end{tabular}

\begin{tabular}{|c|c|c|c|c|c|}
\hline \multicolumn{6}{|c|}{ MARKER GENES } \\
\hline Gene name & Fold Change & IBMT q-value & Gene name & Fold Change & IBMT q-value \\
\hline$\uparrow \mathrm{Pik3ca}$ & -1.08 & $7.84 \mathrm{E}-02$ & $\uparrow B m p 4$ & 3.21 & 5.15E-09 \\
\hline$\downarrow$ Pkp2 & -1.03 & 4.23E-01 & $\uparrow$ Cav1 & 2.92 & 8.39E-06 \\
\hline$\downarrow$ Pkp3 & -1.05 & 3.33E-01 & $\uparrow \mathrm{Cd} 274$ & -1.34 & $2.63 \mathrm{E}-03$ \\
\hline$\downarrow$ Pkp4 & 1.04 & 3.76E-01 & $\uparrow D d r 2$ & 2.61 & $3.74 \mathrm{E}-06$ \\
\hline$\uparrow$ Pou5f1 & -1.03 & $5.22 \mathrm{E}-01$ & $\uparrow E d n 1$ & -1.91 & $1.16 \mathrm{E}-03$ \\
\hline$\uparrow \operatorname{Rac1}$ & -1 & $6.00 \mathrm{E}-01$ & $\uparrow E d n r a$ & 2.75 & $7.72 E-08$ \\
\hline$\uparrow R h o$ & 1.07 & $3.40 \mathrm{E}-01$ & $\uparrow$ Lcn2 & 2.44 & $6.04 \mathrm{E}-03$ \\
\hline$\uparrow$ Rhoa & 1.01 & $5.84 \mathrm{E}-01$ & 个Postn & 4.16 & $3.13 \mathrm{E}-10$ \\
\hline$\uparrow B m i 1$ & 1.06 & 3.85E-01 & $\uparrow \mathrm{Smad} 2$ & -1.21 & $1.03 E-03$ \\
\hline$\uparrow \mathrm{Cdc} 42$ & 1.03 & 3.27E-01 & $\uparrow$ Smad3 & -1.14 & $2.03 E-02$ \\
\hline$\downarrow$ Cdh1 & -1.07 & $3.49 \mathrm{E}-02$ & $\uparrow$ Smad4 & -1.07 & 3.47E-01 \\
\hline$\uparrow \mathrm{Cdh} 2$ & 1.24 & $1.08 \mathrm{E}-01$ & $\uparrow$ Smad5 & -1.02 & $4.88 \mathrm{E}-01$ \\
\hline$\downarrow$ Cldn1 & 1.55 & $1.28 \mathrm{E}-02$ & $\uparrow$ Sox2 & -1.03 & $5.01 \mathrm{E}-01$ \\
\hline$\downarrow$ Cldn25 & 1.1 & 1.37E-01 & $\uparrow$ Sparc & 3 & $9.76 \mathrm{E}-08$ \\
\hline$\uparrow A x l$ & 3.07 & $1.03 E-08$ & $\uparrow$ Vcan & 2.33 & $4.65 \mathrm{E}-06$ \\
\hline$\downarrow$ Dsg1b & -1.11 & $2.96 \mathrm{E}-01$ & $\uparrow$ Vim & 2.13 & $5.87 \mathrm{E}-08$ \\
\hline
\end{tabular}




\begin{tabular}{|l|c|c|l|c|r|}
\hline$\downarrow$ Dsg1c & -1.07 & $4.27 \mathrm{E}-01$ & $\uparrow$ Vtn & $\mathbf{1 . 6 5}$ & $5.27 \mathrm{E}-06$ \\
\hline$\downarrow$ Pkp1 & 1.02 & $5.46 \mathrm{E}-01$ & $\downarrow$ Bmp2 & $\mathbf{- 1 . 4 9}$ & $1.02 \mathrm{E}-03$ \\
\hline$\uparrow$ Pard6g & $\mathbf{1 . 5 5}$ & $5.58 \mathrm{E}-04$ & $\downarrow$ Lrp6 & $\mathbf{- 1 . 1 4}$ & $1.17 \mathrm{E}-03$ \\
\hline$\uparrow$ Col1a1 & $\mathbf{1 . 5 2}$ & $2.47 \mathrm{E}-04$ & $\downarrow$ Tjp1 & -1.15 & $9.09 \mathrm{E}-03$ \\
\hline$\uparrow$ Col3a1 & $\mathbf{1 . 8 7}$ & $7.43 \mathrm{E}-05$ & $\downarrow \mathrm{Tjp} 2$ & -1.1 & $3.01 \mathrm{E}-02$ \\
\hline$\downarrow$ Crb3 & -1.1 & $7.71 \mathrm{E}-02$ & $\downarrow$ Tjp3 & -1.1 & $2.61 \mathrm{E}-02$ \\
\hline$\uparrow$ Ctnnb1 & -1 & $6.14 \mathrm{E}-01$ & $\downarrow$ Tspan13 & 1.15 & $5.21 \mathrm{E}-02$ \\
\hline$\uparrow$ Pard3 & -1.07 & $1.85 \mathrm{E}-01$ & $\uparrow T$ Timp1 & $\mathbf{1 . 5 6}$ & $1.51 \mathrm{E}-02$ \\
\hline$\uparrow$ Pard6a & -1.12 & $1.68 \mathrm{E}-01$ & $\uparrow$ Cux1 & -1.1 & $1.20 \mathrm{E}-02$ \\
\hline$\uparrow$ Pard6b & -1.1 & $1.55 \mathrm{E}-01$ & $\uparrow$ Cd44 & 1.33 & $1.02 \mathrm{E}-02$ \\
\hline$\downarrow$ Dsg2 & -1.18 & $1.89 \mathrm{E}-03$ & & &
\end{tabular}

\begin{tabular}{|l|r|r|}
\hline$\downarrow$ Dsg2 & -1.18 & $1.89 \mathrm{E}-03$ \\
\hline$\downarrow$ Dsg3 & -1.07 & $2.38 \mathrm{E}-01$ \\
\hline$\downarrow$ Dsg4 & -1.03 & $4.97 \mathrm{E}-01$ \\
\hline$\downarrow$ Dsp & -1.09 & $1.65 \mathrm{E}-02$ \\
\hline
\end{tabular}

\begin{tabular}{|l|r|r|}
\hline$\downarrow$ Epcam & -1.09 & $1.65 \mathrm{E}-02$ \\
\hline$\downarrow$ Flnb & -1.05 & $8.31 \mathrm{E}-02$ \\
\hline
\end{tabular}


$E$

\begin{tabular}{llccc}
\hline Upstream Regulator & Molecule Type & $\begin{array}{c}\text { Predicted Activation } \\
\text { State }\end{array}$ & Activation z-score & p-value of overlap \\
\hline TGFB1 & growth factor & Activated & 6.63 & $2.34 \mathrm{E}-53$ \\
Lipopolysaccharide & chemical drug & Activated & 4.24 & $2.35 \mathrm{E}-38$ \\
Beta-estradiol & $\begin{array}{l}\text { chemical - endogenous } \\
\text { mammalian }\end{array}$ & Activated & 2.88 & $1.07 \mathrm{E}-25$ \\
TP53 & transcription regulator & Activated & 2.11 & $2.83 \mathrm{E}-25$ \\
WNT3A & cytokine & Activated & 2.07 & $7.85 \mathrm{E}-24$ \\
\hline
\end{tabular}

Table S3 (A) Table including the top 10 of significantly enriched up-regulated and (B) down-regulated gene sets between 12 and 28 months, as determined by GSEA. (C) Top 5 upstream regulators with the highest activation score between 12 and 28 months identified by IPA.

A

\begin{tabular}{|l|c|c|c|}
\hline NAME & SIZE & NES & FDR q-value \\
\hline KEGG PHOSPHATIDYLINOSITOL SIGNALING SYSTEM & 81 & 2.28 & 0.004 \\
\hline WP1259 RETINOL METABOLISM & 39 & 2.21 & 0.005 \\
\hline SYNTHESIS OF PIPS AT THE PLASMA MEMBRANE & 33 & 2.20 & 0.004 \\
\hline KEGG FATTY ACID DEGRADATION & 46 & 2.14 & 0.007 \\
\hline KEGG INOSITOL PHOSPHATE METABOLISM & 61 & 2.13 & 0.006 \\
\hline PI METABOLISM & 50 & 2.09 & 0.009 \\
\hline WP401 MITOCHONDRIAL LC FATTY ACID BETA OXIDATION & 16 & 2.07 & 0.010 \\
\hline BIOC GHPATHWAY & 23 & 1.99 & 0.031 \\
\hline PHASE 1 FUNCTIONALIZATION OF COMPOUNDS & 76 & 1.98 & 0.031 \\
\hline KEGG OTHER GLYCAN DEGRADATION & 18 & 1.95 & 0.038 \\
\hline
\end{tabular}

B

\begin{tabular}{|l|c|c|c|}
\hline NAME & SIZE & NES & FDR q-value \\
\hline DNA REPLICATION & 99 & -2.77 & $<0.001$ \\
\hline KEGG DNA REPLICATION & 35 & -2.75 & $<0.001$ \\
\hline MITOTIC M/M G1 PHASES & 280 & -2.75 & $<0.001$ \\
\hline SYNTHESIS OF DNA & 92 & -2.73 & $<0.001$ \\
\hline SEPARATION OF SISTER CHROMATIDS & 154 & -2.72 & $<0.001$ \\
\hline MITOTIC METAPHASE AND ANAPHASE & 166 & -2.71 & $<0.001$ \\
\hline MITOTIC ANAPHASE & 165 & -2.7 & $<0.001$ \\
\hline DNA REPLICATION PRE-INITIATION & 77 & -2.69 & $<0.001$ \\
\hline M G1 TRANSITION & 77 & -2.69 & $<0.001$ \\
\hline
\end{tabular}




\begin{tabular}{|l|l|l|c|}
\hline DNA STRAND ELONGATION & 31 & -2.67 & $<0.001$ \\
\hline
\end{tabular}

NES: Normalized Enrichment Score

C

\begin{tabular}{llccc}
\hline $\begin{array}{l}\text { Upstream } \\
\text { Regulator }\end{array}$ & Molecule Type & $\begin{array}{c}\text { Predicted Activation } \\
\text { State }\end{array}$ & Activation z-score & $\begin{array}{c}\text { p-value of } \\
\text { overlap }\end{array}$ \\
\hline XBP1 & transcription regulator & Inhibited & -5.34 & $2.65 \mathrm{E}-11$ \\
TBX2 & transcription regulator & Inhibited & -3.31 & $4.15 \mathrm{E}-07$ \\
ERBB2 & kinase & Inhibited & -3.42 & $1.33 \mathrm{E}-06$ \\
ATF6 & transcription regulator & Inhibited & -3.13 & $1.45 \mathrm{E}-06$ \\
FBXO32 & enzyme & Inhibited & -3.02 & $8.62 \mathrm{E}-06$
\end{tabular}

Table S4 RNA integrity numbers (RIN) of the RNA isolated from colonic scrapings that were used for microarrays.

\begin{tabular}{|c|c|c|}
\hline Mouse ID & Age (months) & $\begin{array}{c}\text { RNA integrity } \\
\text { number (RIN) }\end{array}$ \\
\hline A1_03 & 6 & 10 \\
\hline A1_07 & 6 & 10 \\
\hline A1_09 & 6 & 9.9 \\
\hline A1_11 & 6 & 10 \\
\hline A2_01 & 12 & 9.9 \\
\hline A2_03 & 12 & 10 \\
\hline A2_05 & 12 & 9.9 \\
\hline A2_07 & 12 & 10 \\
\hline A2_09 & 12 & 9.9 \\
\hline A2_11 & 12 & 10 \\
\hline A2_13 & 12 & 10 \\
\hline A3_01 & 24 & 10 \\
\hline A3_03 & 24 & 10 \\
\hline A3_05 & 24 & 10 \\
\hline A3_07 & 24 & 10 \\
\hline A3_09 & 24 & 10 \\
\hline A3_11 & 24 & 10 \\
\hline A3_13 & 24 & 10 \\
\hline A3_15 & 24 & 9.4 \\
\hline A4_01 & 28 & \\
\hline
\end{tabular}




\begin{tabular}{|l|l|l|}
\hline A4_03 & 28 & 10 \\
\hline A4_05 & 28 & 9.6 \\
\hline A4_09 & 28 & 9.6 \\
\hline A4_17 & 28 & 10 \\
\hline A4_19 & 28 & 9.8 \\
\hline A4_21 & 28 & 10 \\
\hline
\end{tabular}

Data set S1: Table with causes of death of the mice that died prior to sacrifice.

Data set S2: Genes differentially expressed between 6vs12, 6vs24 and 6vs28 months $(q<0.01)$.

Data set S3: All correlations between the 50 genera with a relative abundance $\geq 0.1 \%$ in at least 1 sample and the 817 up-regulated genes assigned to the red profile as determined by STEM. Orange cells contain $r>0.8$, blue cells contain $r<-0.8$.

Data set S4: All genes differentially expressed between 12 and 28 months of age $(p<0.01)$.

Data set S5: All correlations between the of 50 genera with a relative abundance $\geq 0.1 \%$ in at least 1 sample and the 1371 genes differentially expressed between 12 and 28 months. Orange cells contain $r>0.8$, blue cells contain $r<-0.8$.

\section{Due to the extensive size of the files, data sets S1-S5 can be found online:}

Van der Lugt B, Rusli F, Lute C, Lamprakis A, Salazar E, Boekschoten MV, Hooiveld GJ, Müller M, Vervoort J, Kersten S, Belzer C, Kok DE, Steegenga WT. Integrative analysis of gut microbiota composition, host colonic gene expression and intraluminal metabolites in aging C57BL/6J mice. Aging (Albany NY). 2018; 10:930-950. https://doi.org/10.18632/aging.101439

\section{References Supplemental Material}

1. Kalluri, R. and R.A. Weinberg, The basics of epithelial-mesenchymal transition. J Clin Invest, 2009. 119(6): p. 1420-8.

2. Lamouille, S., J. Xu, and R. Derynck, Molecular mechanisms of epithelial-mesenchymal transition. Nat Rev Mol Cell Biol, 2014. 15(3): p. 178-96.

3. Puisieux, A., T. Brabletz, and J. Caramel, Oncogenic roles of EMT-inducing transcription factors. Nat Cell Biol, 2014. 16(6): p. 488-94.

4. Serrano-Gomez, S.J., M. Maziveyi, and S.K. Alahari, Regulation of epithelial-mesenchymal transition through epigenetic and post-translational modifications. Mol Cancer, 2016. 15: p. 18.

5. $\mathrm{Yu}, \mathrm{H}_{\text {., }}$ et al., The contribution of TGF-beta in Epithelial-Mesenchymal Transition (EMT): Downregulation of E-cadherin via snail. Neoplasma, 2015. 62(1): p. 1-15.

6. Zhao, M., et al., dbEMT: an epithelial-mesenchymal transition associated gene resource. Sci Rep, 2015. 5: p. 11459. 


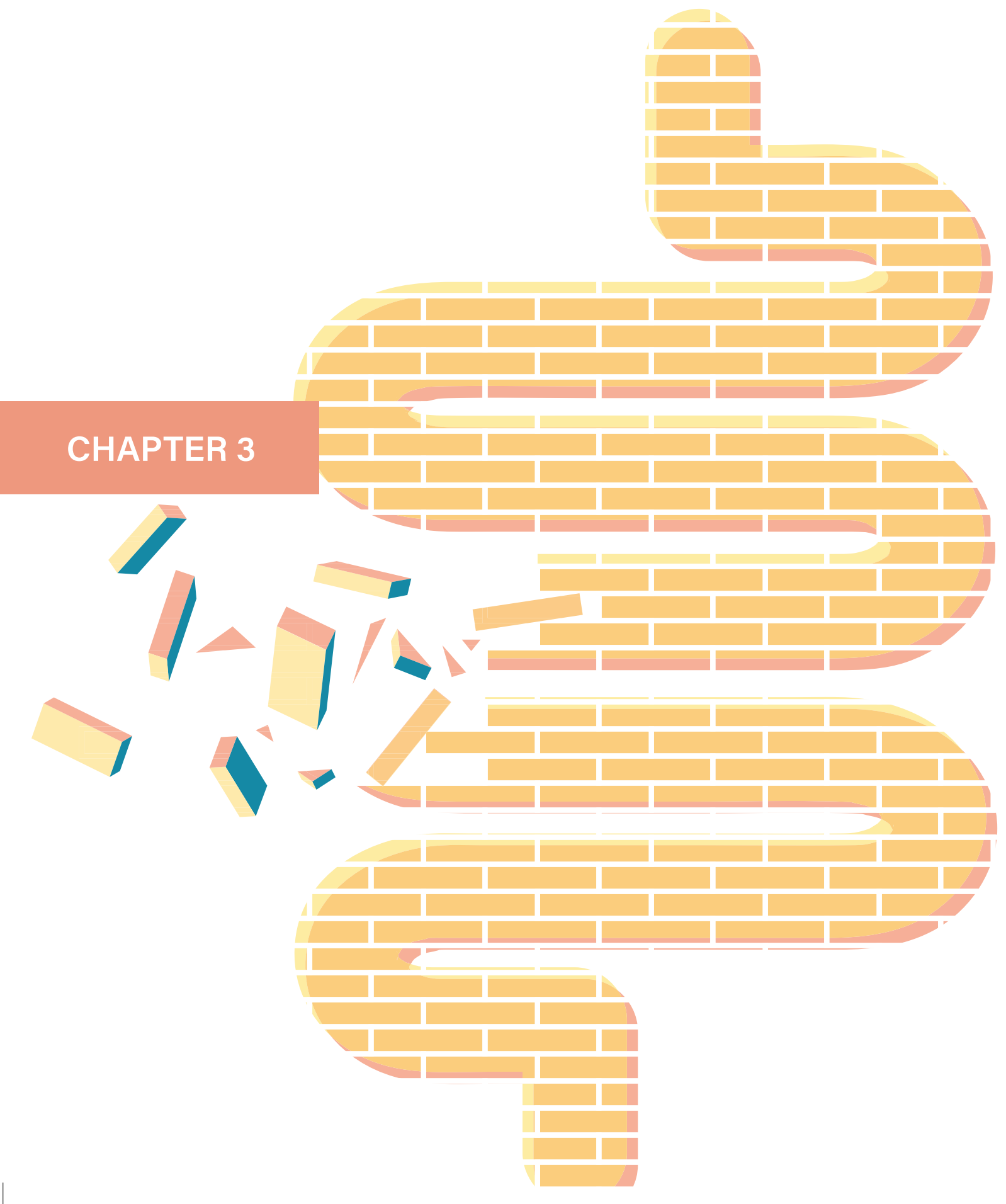




\section{Akkermansia muciniphila ameliorates the age-related decline in colonic mucus thickness and attenuates immune activation in accelerated aging $\mathrm{ErcCl}^{-/ \Delta 7}$ mice}

Benthe van der Lugt ${ }^{\dagger}$, Adriaan A. van Beek ${ }^{\dagger}$, Steven Aalvink, Ben Meijer, Bruno Sovran, Wilbert P. Vermeij, Renata M. C. Brandt, Willem M. de Vos, Huub F. J. Savelkoul, Wilma T. Steegenga and Clara Belzer

${ }^{\dagger}$ Authors contributed equally 


\section{Abstract}

\section{Background}

The use of Akkermansia muciniphila as potential therapeutic intervention is receiving increasing attention. Health benefits attributed to this bacterium include an improvement of metabolic disorders and exerting anti-inflammatory effects. The abundance of $A$. muciniphila is associated with a healthy gut in early mid- and later life. However, the effects of $A$. muciniphila on a decline in intestinal health during the aging process are not investigated yet. We supplemented accelerated aging $\operatorname{Erccr}^{-/ \Delta 7}$ mice with A. muciniphila for 10 weeks and investigated histological, transcriptional and immunological aspects of intestinal health.

\section{Results}

The thickness of the colonic mucus layer increased about 3-fold after long-term A. muciniphila supplementation and was even significantly thicker compared to mice supplemented with Lactobacillus plantarum WCFS1. Colonic gene expression profiles pointed towards a decreased expression of genes and pathways related to inflammation and immune function, and suggested a decreased presence of $B$ cells in colon. Total B cell frequencies in spleen and mesenteric lymph nodes were not altered after $A$. muciniphila supplementation. Mature and immature $B$ cell frequencies in bone marrow were increased, whereas $B$ cell precursors were unaffected. These findings implicate that $\mathrm{B}$ cell migration rather than production was affected by $A$. muciniphila supplementation. Gene expression profiles in ileum pointed toward a decrease in metabolic- and immune-related processes and antimicrobial peptide production after A. muciniphila supplementation. Besides, A. muciniphila decreased the frequency of activated $C D 80^{+} C D 273^{-}$B cells in Peyer's patches. Additionally, the increased numbers of peritoneal resident macrophages and a decrease in Ly6C $\mathrm{C}^{\text {int }}$ monocyte frequencies in spleen and mesenteric lymph nodes add evidence for the potentially antiinflammatory properties of $A$. muciniphila.

\section{Conclusions}

Altogether, we show that supplementation with A. muciniphila prevented the age-related decline in thickness of the colonic mucus layer and attenuated inflammation and immune-related processes at old age. This study implies that $A$. muciniphila supplementation can contribute to a promotion of healthy aging. 


\section{Introduction}

Coincident with the increase in the aged population that is observed nowadays, the often inevitable decline in overall health in the elderly is becoming an alarming problem. The aging process is accompanied by a chronic low-grade inflammatory state, termed 'inflamm-aging', which is a strong risk factor for many age-related pathologies (1-4). One of the organs that is affected by the aging process is the intestinal tract and the occurrence of gut-related disorders in the aged population is considerable (5).

As main inhabitant of the intestine, the gut microbiota play an essential role in the maintenance of overall health. Bacteria are able to degrade complex carbohydrates, thereby converting these substrates into metabolites that are beneficial to health, such as short-chain fatty acids (SCFAs) (6, 7). Besides, the gut microbiota interact extensively with the host immune system by the regulation of immune responses (8). During the aging process, changes in gut microbiota composition occur, such as a decreased diversity, a decrease in health-promoting bacteria and an increase in potential pathobionts. This disturbed balance in microbiota composition is thought to increase the risk of impaired intestinal barrier function and intestinal inflammation (9). In mice, transfer of microbiota from aged mice to young germfree recipient mice promoted intestinal inflammation, increased leakage of bacterial components into blood and stimulated systemic immune activation (10).

An important factor with regard to gut health is the mucus layer that covers the intestinal epithelial cell layer and serves as physical protection for bacterial penetration and harmful compounds to enter the mucosal tissue (11). Intestinal mucus is built up of mostly Mucin 2 (Muc2) proteins, which are large gel-forming proteins that are secreted by goblet cells located in the intestinal mucosa. These proteins form a net-like structure and are the building blocks of the mucus layer. The importance of the mucus layer was emphasized in studies using Muc2 knockout mice, which did not have a colonic mucus layer covering the intestinal epithelial layer $(12,13)$. These mice suffered from a decreased intestinal barrier function, an increased inflammatory status (14) and had signs of colitis (12). Next to the protective function of the mucus layer, it also serves as an energy source for bacteria. Akkermansia muciniphila is one of the bacterial species that is able to degrade mucus. This bacterium is highly abundant $(\sim 3 \%)$ in the healthy human colon (15). Upon mucus degradation, $A$. muciniphila produces several immune-stimulating compounds, such as SCFAs and pili $(16,17)$. The outer membrane pili-like protein Amuc_1100 is thought to be involved in the beneficial properties of A. muciniphila on health $(18,19)$.

Recent studies suggest that the beneficial effects of $A$. muciniphila are not limited to the intestinal tract, but extend to overall health. The abundance of $A$. muciniphila was reduced in people suffering 
from obesity, type 2 diabetes, inflammatory bowel disease, amongst others (20). Furthermore, supplementation with $A$. muciniphila in mice resulted in an improved metabolic state and reduced diet-induced obesity (ClinicalTrials.gov Identifier: NCT02637115) (21-23).

We and others previously showed that the abundance of Akkermansia spp. in colonic luminal content decreased during aging in mice $(10,24,25)$. Another study also reported an age-related loss of Akkermansia spp. in humans (26). Interestingly, the abundance of Akkermansia spp. was shown to be increased in centenarians (105-109 years old) compared to younger age groups (27). These results could indicate that a relation exists between reaching an extreme old age and the abundance of Akkermansia spp. $(24,27)$.

The numerous potential beneficial characteristics of $A$. muciniphila suggest that this bacterium could be a potent candidate for microbial supplementation. However, the effects of this bacterium on the decline in intestinal health as seen during aging are not widely investigated yet. Therefore, the aim of the present study was to investigate the effects of supplementation with $A$. muciniphila on different aspects of intestinal health. We used $\operatorname{Erccl}^{-1 \Delta 7}$ mice, an accelerated aging mouse model that has a median lifespan of $\sim 20$ weeks. Further characteristics of this mouse model were extensively described in previous studies (28-30) and indicate that the accelerated aging phenotype of Ercc1-1 ${ }^{\Delta 7}$ mice largely resembles normal aging. The $\operatorname{Ercct}^{-1 / 47}$ mice were supplemented with $A$. muciniphila for 10 weeks via oral gavage. After sacrifice, ileum and colon were subject to transcriptional analysis and the microbiota composition in these organs was investigated. Furthermore, we assessed mucus thickness in the colon and the distribution of immune cells in immune-related tissues.

\section{Materials and methods}

\section{Mice and study design}

In this study, accelerated aging Ercc1 ${ }^{-/ \Delta 7}$ mice were used. Genotyping of this mouse model was extensively described previously by others (28-30). In short, ErcC1-1/47 mice have an impaired DNA repair protein ERCC1, resulting in accumulation of a broad variety of DNA lesions and consequently accelerated aging. Mice were individually housed under SPF conditions, received an ad libitum purified diet (formula D12450B, Research Diets, Additional file 5A) and had ad libitum access to water supplied by water bottles with long nozzles. Mice were supplemented with Akkermansia muciniphila by oral gavage at a dose of $2 \times 10^{8} \mathrm{CFU} / 200 \mu \mathrm{L}$, three times a week, for a total of 10 weeks. Oral gavages were given in the morning. The control group simultaneously received oral gavages containing the same volume of PBS. A third $\mathrm{Ercct}^{-1 / \Delta 7}$ mice group was included that received the 
same dose of Lactobacillus plantarum WCFS1. These mice were only used for histological purposes. Growing procedures of the bacterial cultures was extensively described previously (29). A number of 18 mice per group (both male and female) was included and lifespan of these mice was assessed. After 10 weeks, when the mice were 16 weeks old, a number of 5-6 female mice were sacrificed. Colonic and ileal content, as well as distal ileum and proximal colon sections, were collected and snap-frozen in liquid nitrogen. A piece of ileal and colonic tissue was fixed in Carnoy's solution for histological purposes. Spleen, mesenteric lymph nodes, Peyer's patches, bone marrow and peritoneal exudate cells were isolated for immunological measurements.

\section{Histology}

After paraffin embedding, Carnoy-fixed distal ileum and proximal colon tissue were sliced in $5 \mu \mathrm{M}$ sections on poly-I-lysine coated glass slides (Thermo Scientific, Germany). Slides were dewaxed, dehydrated and stained with hematoxylin and eosin (H\&E) and PAS/Alcian blue. The thickness of the colonic mucus layer was measured using ImageJ software (NIH, MD, USA). For comparison of the mucus layer thickness, we included an extra mouse group that received Lactobacillus plantarum WCFS1.

\section{Microbiota composition analysis}

DNA was isolated from ileal and colonic content using a modified repeated bead beating method (50). Microbiota composition was assessed using 16S rRNA sequencing on the MiSeq platform (Illumina, San Diego, CA, USA). Next, the NG-Tax pipeline was used for barcode-primer filtering, demultiplexing, OTU picking and taxonomic classification (51). The generated biom-files were used for summarizing the microbiological data, i.e. alpha-diversity and beta-diversity, using the R-packages microbiome (52) and phyloseq (53).

\section{RNA isolation}

RNA was isolated from distal ileum and proximal colon tissue ( $n=5-6$ mice/group) using TRIzol reagent (Invitrogen, Breda, The Netherlands). Purification of the isolated RNA was performed using the RNeasy Mini kit (Qiagen, Venlo, The Netherlands). After measurement of the total RNA yield (Nanodrop, ND-1000, Nanodrop Products, Maarssen, The Netherlands), RNA integrity was assessed (Agilent 2100 Bioanalyzer, Agilent Technologies, Amsterdam, The Netherlands) and only RNAs were included with a RNA integrity number (RIN) above 8.0. 


\section{Microarray analysis}

Microarray analysis was performed as described previously (54). Differences in gene expression between the control and A. muciniphila supplemented mice groups were assessed using the Intensity Based Moderated T statistics (IBMT) method, with p-values $<0.05$ and fold changes $<-1.2$ or $>1.2$. Microarray data has been submitted to the NCBI Gene Expression Omnibus (GEO) (GSE126730). Gene Set Enrichment Analysis (GSEA) was used to identify significantly enriched pathways (55). Only pathways with a False Discovery Rate (FDR) $<0.2$ were taken into consideration. Ingenuity pathway analysis (IPA) was used for the identification of upstream regulators (56).

\section{CDNA synthesis and real-time quantitative PCR}

Real-time quantitative PCR ( $\mathrm{PPCR}$ ) was used to validate the expression profiles of a selection of differentially expressed genes identified in the microarray analysis. For both colon and ileum samples, complementary DNA (cDNA) was synthesized from $1000 \mathrm{ng}$ of total RNA using the RevertAid First Strand cDNA Synthesis Kit (Thermo-Fisher Scientific, Landsmeer, The Netherlands) following the manufacturer's protocol. The following thermal cycling conditions were used: $5 \mathrm{~min}$ at $25^{\circ} \mathrm{C}, 60 \mathrm{~min}$ at $37^{\circ} \mathrm{C}$ and $5 \mathrm{~min}$ at $70^{\circ} \mathrm{C}$. Primer sequences were obtained at the online PrimerBank database (Additional file 5B) (57). qPCR was performed with a CFX384 thermal cycler (Bio-Rad Laboratories, Veenendaal, the Netherlands) using the SensiMix SYBR No-ROX kit (Bioline, Alphen aan den Rijn, The Netherlands). The housekeeping gene 36B4 was used for normalization.

\section{Fluorescence-activated cell sorting (FACS) analysis}

Spleen, mesenteric lymph nodes, Peyer's patches, bone marrow and peritoneal exudate cells were all subject to FACS analysis, similar as previously reported (29). In brief, femurs, tibias, ileac crests, forelegs, and sternum were harvested and crushed with mortar and pestle. Singe-cell suspensions from each organ were prepared by passing cells through a $40-\mu \mathrm{m}$ cell strainer with a syringe. First, cells were stained for extracellular markers. Fixable live/dead eFluor506 stain (Ebioscience, San Diego, USA) was used to exclude dead cells. Cells were fixed and permeabilized with Fix/Perm buffer (Ebioscience) to stain for intracellular markers. All antibodies used for flow cytometry are enlisted in Additional file 5C. A Canto II flow cytometer was used (BD Biosciences, Erembodegem, Belgium) and data analysis was performed using FlowJo vX.07 software (Tree Star Inc, USA).

\section{Statistical analysis}

The Kolmogorov-Smirnov test was used to test if data were normally distributed and appropriate 
non-parametric statistical tests were used when data were not normally distributed. With regard to the survival analysis, the log-rank (Mantel-Cox) test was used. To test differences between the control and supplemented group, a student t-test or Mann-Whitney $U$ test was performed. With regard to the differences in mucus thickness, a Kruskal-Wallis test with Dunn's multiple comparisons test was performed, since three mouse groups were involved. Unless otherwise stated, $\mathrm{p}$-value levels of $p<0.05$ were considered as statistically significant.

\section{Results}

\section{A. muciniphila supplementation increased mucus thickness in colon of $\mathrm{Erccr}^{-/ \Delta 7}$ mice}

Since $A$. muciniphila is a mucus-colonizing bacterium and utilizes mucus as energy source, we investigated whether supplementation with $A$. muciniphila had an effect on the mucus layer in the colon of $\operatorname{Ercc1}^{-1 / 47}$ mice. Measurements of mucus thickness in PAS/Alcian Blue stained colon tissue revealed that the mucus layer was significantly thicker in the mice supplemented with $A$. muciniphila compared to the control group $(p<0.001)$ (Figure 1A-C). Besides, the results were compared with the mucus thickness of mice supplemented with L. plantarum (WCFS1), since we showed previously that supplementation with this bacterium prevented an age-related decline in mucus thickness (29). The colonic mucus layer of $L$, plantarum supplemented mice was thicker compared to the control group ( $p<0.001$ ) (Figure 1A, D), but supplementation with $A$. muciniphila resulted in a significantly thicker mucus layer than the L. plantarum supplemented mice $(p<0.001)($ Figure $1 A)$. These results show that supplementation with $A$. muciniphila contributed to the prevention of a decreased mucus layer thickness at old age.

A
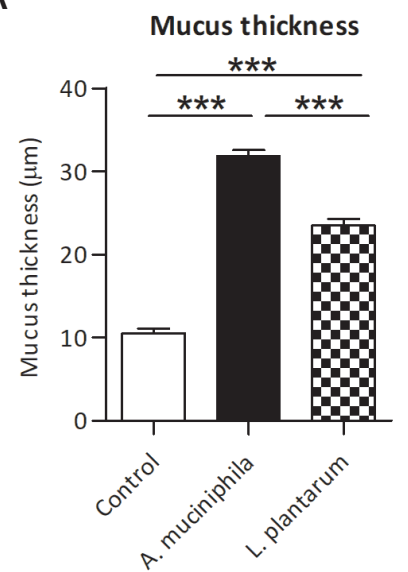

\section{B}

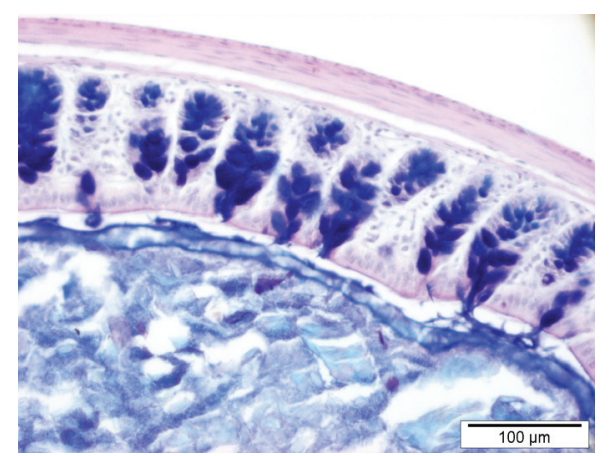


C

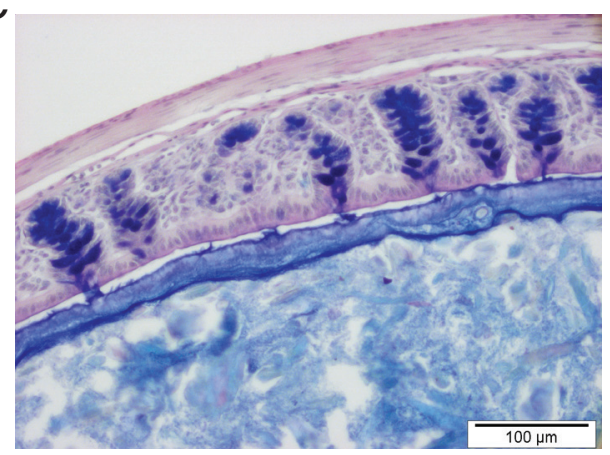

$\mathrm{D}$

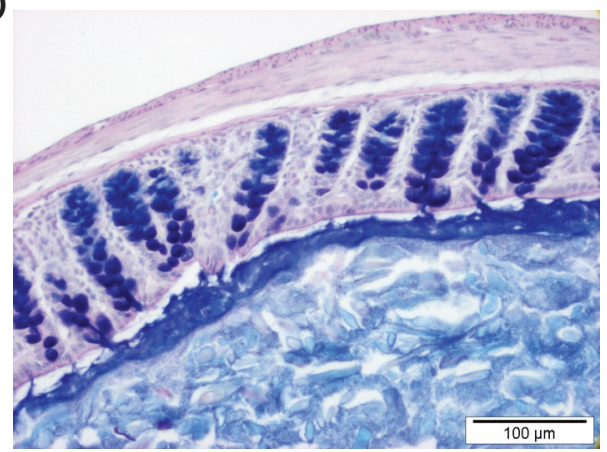

Figure 1 Mucus thickness increased in $\mathrm{Ercc1}^{-/ \Delta 7}$ mice supplemented with Akkermansia muciniphila. (A) Mucus thickness $(\mu \mathrm{m})$ measured in colon of $\operatorname{Ercc}^{-/ \Delta 7}$ mice in the control, A. muciniphila group and L. plantarum group. (B) Representative picture of PAS/Alcian Blue staining in control mouse, (C) mouse supplemented with A. muciniphila and (D) L. plantarum. Data represent the mean + SEM from three to five mice per group. ${ }^{* * *} p<0.001$. Scale bars histological images: $100 \mu \mathrm{m}$.

No differences in colonic and ileal microbiota composition after supplementation with $A$. muciniphila

In order to investigate whether supplementation with A. muciniphila caused changes in gut microbiota composition, we performed 16S rRNA gene sequencing on colonic and ileal content. Alphadiversity (Shannon entropy) of colonic and ileal content samples did not differ between the control and supplemented mice (Figure 2A). To investigate whether supplementation with A. muciniphila resulted in an increased colonization of this bacterium, the relative abundance of Akkermansia spp. in colonic content was assessed. In colonic content, the average relative abundance was slightly higher in the intervention group $(0.738 \pm 1.279 \%)$ compared to the control group $(0.252 \pm 0.503 \%)$ (Figure 2B), but this difference was not statistically significant. Akkermansia spp. was not present in ileal content, except for one mouse in the intervention group (data not shown). Variation in microbial composition between samples was represented in a principal coordinates analysis (PCoA) based on Bray-Curtis dissimilarity. In both colon and ileum samples, no clear separation was observed between the control and A. muciniphila group (Figure 2C-D). Furthermore, no statistically significant differences at genus level between the control and supplemented mice was found in both sources (data not shown). These data show that bacterial supplementation with $A$. muciniphila three times a week did not result in changes in gut microbiota composition of $\mathrm{ErcC1}^{-/ \Delta 7}$ mice. 
A

B
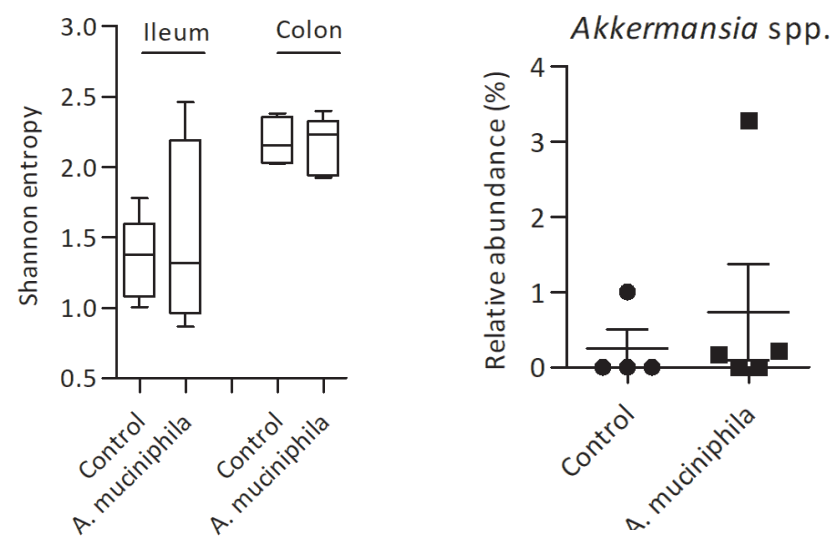

C Bray-Curtis PCoA Colon samples

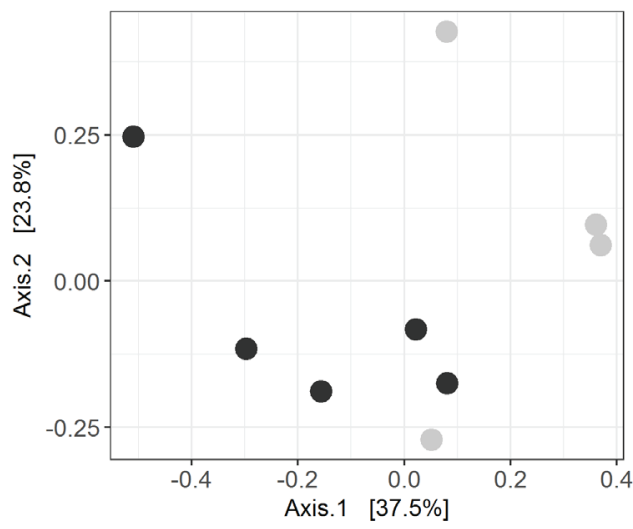

Group

Akkermansia

Control

D Bray-Curtis PCoA lleum samples

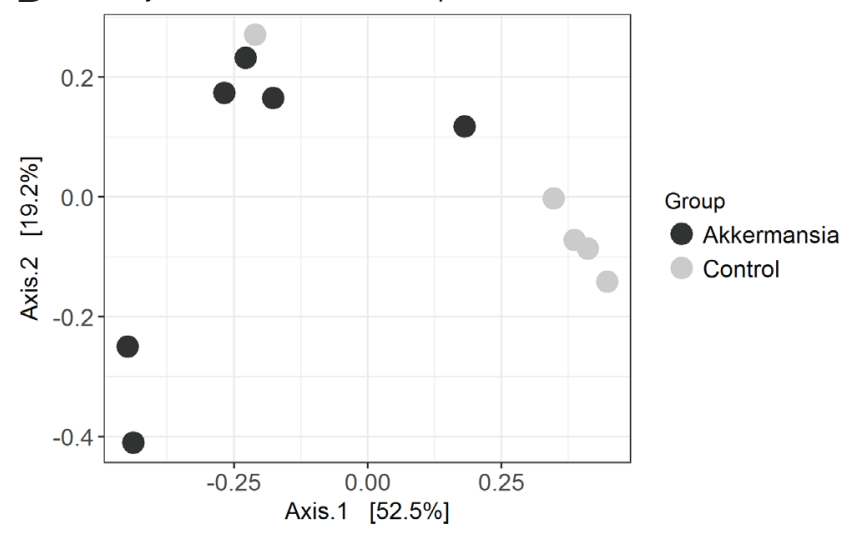


Figure 2 Microbiota composition in colon and ileum of $\mathrm{Ercc1}^{-/ \Delta 7}$ mice supplemented with Akkermansia muciniphila. (A) Alpha-diversity (Shannon entropy) measured in ileum and colon samples. Boxes extend from the 25th to 75th percentile, middle line represents median, and whiskers represent minimum and maximum values. (B) Relative abundance (\%) of Akkermansia spp. in colonic content assessed by $16 \mathrm{~S}$ rRNA sequencing. Data represent mean \pm SEM (C) Beta-diversity measured by Bray-Curtis Principal Coordinate Analysis in colon samples and (D) ileum samples. Between four and six mice per group were used for microbiota analysis.

\section{A. muciniphila supplementation minimally altered the expression of genes involved in intestinal barrier function in colon}

To explore the effects of supplementation with A. muciniphila on gene expression, transcriptome analysis was performed on mRNA isolated from colon and ileum tissue of $\operatorname{Ercct}^{-1 / \Delta 7}$ mice. In colon, a number of 427 genes was significantly differentially expressed $(p<0.05$, fold change $>1.2$ or $<-1.2$ ) between the control and A. muciniphila group, comprising 225 up-regulated and 202 downregulated genes. Since a highly significant increase in mucus thickness was observed in the colon of mice that received $A$. muciniphila, colonic expression of genes related to mucus production was investigated. No significant differential expression of mucins was observed, except Mucin like 1 (Muc/1) which was down-regulated (-1.5-fold) in the A. muciniphila group compared to the control mice (Additional file 1). Besides, to explore if an increased mucus thickness in the colon resulted in an enhanced intestinal barrier function, expression of genes related to tight junction function was investigated. The classical tight junction proteins Tjp 1-3, Jam 1-3, Claudin family and Ocln were not differentially expressed (data not shown). To investigate which pathways were regulated in colon by A. muciniphila supplementation, Gene Set Enrichment Analysis (GSEA) was performed. Significantly enriched pathways were dominated by cell cycle related processes, but no pathways related to intestinal barrier function were observed (Additional file 2).

\section{Supplementation with $A$. muciniphila decreased expression of genes and pathways related to antimicrobial activities, metabolic processes and mucus biosynthesis in ileum}

Since supplementation with $A$. muciniphila minimally altered the expression of genes related to intestinal barrier function in colon, we also investigated gene expression profiles in ileum tissue of $\operatorname{Ercct}^{-1 / 47}$ mice. A number of 795 genes was significantly differentially expressed $(p<0.05$, fold change $>1.2$ or $<-1.2$ ) between the control and A. muciniphila group in ileum, comprising 425 upregulated and 370 down-regulated genes. Interestingly, several genes encoding for antimicrobial peptides were down-regulated in the A. muciniphila supplemented mice, i.e. Reg3b and Reg3g 
(Figure 3A-B; Additional file 3). However, the expression of genes encoding for alpha-defensins and lysozymes was not affected by $A$. muciniphila supplementation. Tight junction genes were minimally differentially expressed, only Cldn2 and Cldn8 were down-regulated in ileum of mice supplemented with A. muciniphila compared to the control group (Figure 3C-D; Additional file 3). GSEA revealed that significantly enriched down-regulated pathways were dominated by metabolic processes (Additional file 2). Besides, the pathways "N-Glycan Biosynthesis" and "Biosynthesis of the N-Glycan Precursor (Dolichol Lipid-Linked Oligosaccharide, LLO) and Transfer to a Nascent Protein" were down-regulated in ileum of mice that received $A$. muciniphila supplementation compared to the control group (Additional file 2). Based on this finding, the microarray data set was searched for genes related to mucus biosynthesis. The genes Ctnna3 and St6galnac6 were downregulated in ileum in the supplemented mice versus control group (Figure 3E-F; Additional file 3).

A

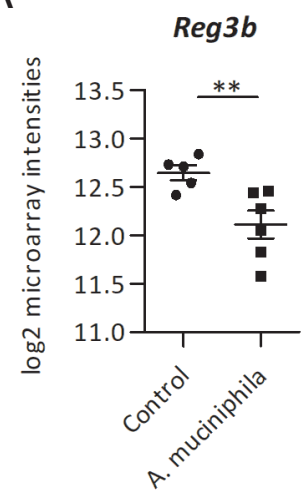

D

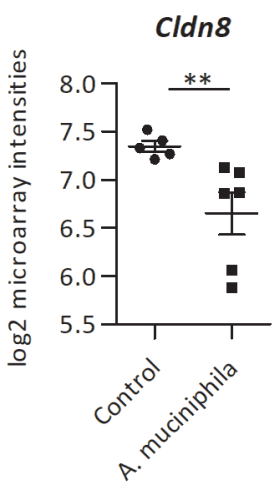

$\mathrm{B}$

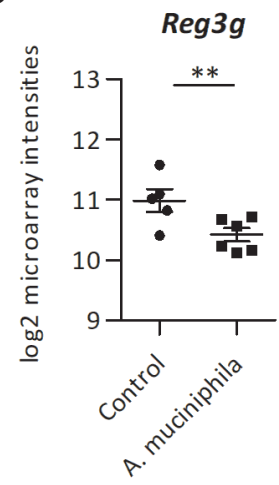

$E$

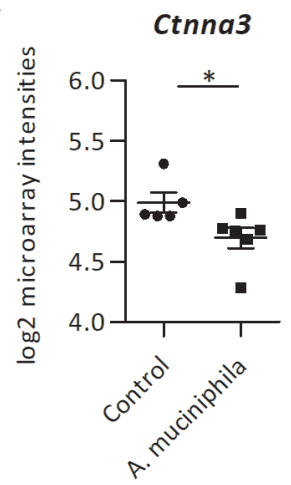

C

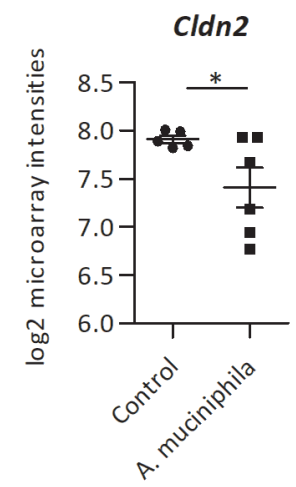

Figure 3 Microarray analysis performed on mRNA isolated from ileum tissue. (A) Log2 microarray intensities of Regenerating islet-derived 3 beta (Reg3b), (B) Regenerating islet-derived 3 gamma $(\operatorname{Reg} 3 g)$ (C) Claudin 2 (Cldn2), (D) Claudin 8 (Cldn8), (E) Catenin (cadherin associated protein), alpha 3 (Ctnna3) and (F) ST6 (alpha-N-acetyl-neuraminyl-2,3-beta-galactosyl-1,3)-N-acetylgalactosaminide alpha-2,6- 
sialyltransferase 6 (St6galnac6). Data represent mean + SEM. Control: $\mathrm{n}=5$, A. muciniphila: $\mathrm{n}=6 .{ }^{*} \mathrm{p}<0.05$; ${ }^{* *} \mathrm{p}<0.01$.

Decreased expression of genes and pathways related inflammation and immune function in colon and ileum after $A$. muciniphila supplementation

As immune function is an important factor regarding intestinal health, expression profiles of genes related to immune response were investigated. Remarkably, down-regulated genes in both colon and ileum were dominated by genes encoding for immunoglobulins (Additional file 1). In the colon, several genes encoding for chemokines, such as Cxc/13 (Figure 4A; Additional file 3) and Cc/12, as well as the cytokine $/ 15$ and the complement factors C1ra and C5ar1 were all down-regulated (Additional file 1). Also the immunoglobulin receptor Pilrb1 had a decreased expression in the colon of the A. muciniphila group (Additional file 1). Additionally, other immune-related genes were down-regulated in the colon of $A$. muciniphila supplemented mice compared to the control group, e.g. Blk, Cd4, Cd72, TIr7 and TIr12 (Figure 4B-F; Additional file 3). GSEA revealed that immunerelated pathways were down-regulated in colon, for example "Intestinal Immune Network for IgA Production", "Cytokine-Cytokine Receptor Interaction" and "Inflammatory Response Pathway", amongst others (Additional file 2). Moreover, Ingenuity pathway analysis (IPA) identified seven cytokines as upstream regulators that were predicted to be inhibited after supplementation with $A$. muciniphila, including both the pro-inflammatory Il1 and anti-inflammatory Tgf-beta (Table 1). Also other inflammation-related factors, such as Myeloid differentiation primary response 88 (Myd88), Tumor necrosis factor receptor superfamily member 1B (Tnfrsf1b) and 12 (Tnfsf12), NFKB Inhibitor Alpha (Nfkbia), T cell receptor (TCR) and Toll Like Receptor Adaptor Molecule 1 (Ticam1) were predicted inhibited upstream regulators (Table 1). In ileum, GSEA annotated the pathway "Antigen Presentation: Folding, assembly and peptide loading of class I $\mathrm{MHC}^{\prime \prime}$ as highly up-regulated (Additional file 2). The most significant upstream regulator identified by IPA in ileum tissue was Interleukin 10 Receptor Subunit Alpha (II0RA), which was predicted to be slightly inhibited after A. muciniphila supplementation (Table 2). Taken together, these results show that $A$. muciniphila supplementation decreased the expression of numerous genes and pathways related to inflammation and immune function in both colon and ileum. 
A

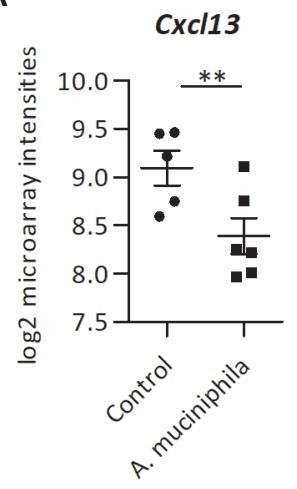

D

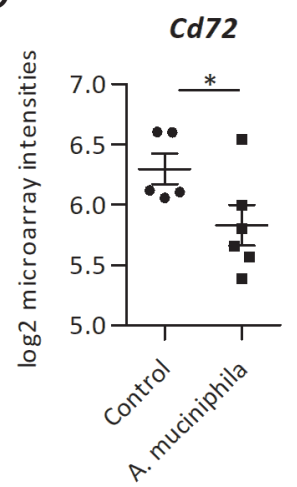

B

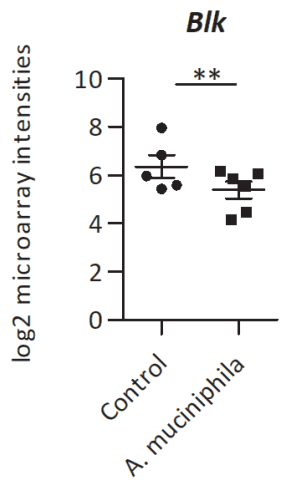

$E$

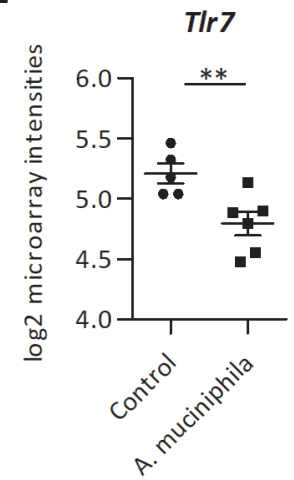

C

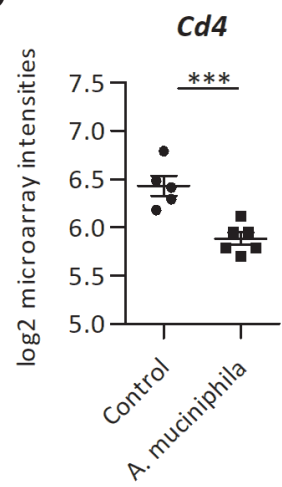

$\mathrm{F}$ TIr12

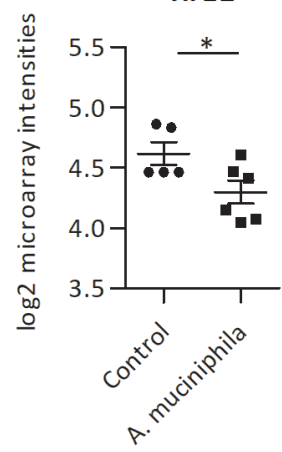

Figure 4 Microarray analysis performed on mRNA isolated from colon tissue. (A) Log2 microarray intensities of (A) C-X-C motif chemokine ligand 13 (Cxc/13), (B) B lymphoid kinase (B/k), (C) Cluster of differentiation 4 (Cd4), (D) Cluster of differentiation 72 (Cd72), (E) Toll-like receptor 7 (T/r7), (F) Toll-like receptor 12 (T/r12). Data represent mean + SEM. Control group: $\mathrm{n}=5$. A. muciniphila group: $\mathrm{n}=6 .{ }^{*} \mathrm{p}<0.05$; $* * \mathrm{p}<0.01 ; * * \mathrm{p}<0.001$

Table 1 Upstream regulators in colon identified by Ingenuity pathway analysis based on the comparison between $\operatorname{Ercc1}^{-/ \Delta 7}$ mice receiving A. muciniphila supplementation and control mice. Cut-off values include $\mathrm{p}<0.05$ and activation $z$-score $<-1.2$ or $>1.2$. Upstream regulators in bold are involved in inflammation- and immune-related processes.

\begin{tabular}{|l|c|c|}
\hline Upstream Regulator & Activation z-score & p-value of overlap \\
\hline ACOX1 & 2.24 & 0.013 \\
\hline Alpha catenin & 2.00 & 0.029 \\
\hline ID3 & 1.98 & 0.030 \\
\hline SOCS1 & 1.95 & 0.040 \\
\hline CDKN2A & 1.72 & 0.025 \\
\hline
\end{tabular}




\begin{tabular}{|c|c|c|}
\hline MYD88 & -2.41 & 0.045 \\
\hline IL1 & -2.38 & 0.049 \\
\hline TNFRSF1B & -2.22 & 0.003 \\
\hline CHUK & -2.21 & 0.017 \\
\hline GATA6 & -2.21 & 0.036 \\
\hline NFKBIA & -2.17 & 0.029 \\
\hline Akt & -2.16 & 0.020 \\
\hline cytokine & -1.99 & 0.000 \\
\hline Nfat (family) & -1.98 & 0.014 \\
\hline CCND1 & -1.98 & 0.044 \\
\hline WNT5A & -1.95 & 0.033 \\
\hline CTNNB1 & -1.95 & 0.037 \\
\hline Tgf beta & -1.94 & 0.010 \\
\hline EGR1 & -1.91 & 0.035 \\
\hline Interferon alpha & -1.78 & 0.004 \\
\hline LTBR & -1.72 & 0.001 \\
\hline STAT3 & -1.71 & 0.024 \\
\hline TCR & -1.60 & 0.029 \\
\hline TNFSF12 & -1.58 & 0.004 \\
\hline TNF & -1.54 & 0.036 \\
\hline SSB & -1.52 & 0.000 \\
\hline IL17A & -1.40 & 0.002 \\
\hline TICAM1 & -1.40 & 0.003 \\
\hline HRAS & -1.39 & 0.031 \\
\hline E2F1 & -1.36 & 0.009 \\
\hline IL6 & -1.34 & 0.005 \\
\hline PRKACA & -1.21 & 0.001 \\
\hline IL1B & -1.21 & 0.003 \\
\hline
\end{tabular}

Table 2 Upstream regulators in ileum identified by Ingenuity pathway analysis based on the comparison between mice receiving $A$. muciniphila supplementation and control mice. Cut-off values include $p<0.05$ and activation z-score $<-1.2$ or $>1.2$. Upstream regulators in bold are involved in inflammation- and immunerelated processes.

\begin{tabular}{|l|c|c|}
\hline Upstream Regulator & $\begin{array}{c}\text { Activation } \\
\text { z-score }\end{array}$ & $\begin{array}{c}\text { p-value of } \\
\text { overlap }\end{array}$ \\
\hline HMGA1 & 1.66 & 0.002 \\
\hline POR & 1.46 & 0.000 \\
\hline SYVN1 & 1.34 & 0.006 \\
\hline CFTR & 1.25 & 0.000 \\
\hline
\end{tabular}




\begin{tabular}{|l|c|l|}
\hline AR & -2.74 & 0.005 \\
\hline NR112 & -2.00 & 0.042 \\
\hline BRCA1 & -1.98 & 0.016 \\
\hline CTNNB1 & -1.72 & 0.001 \\
\hline LEP & -1.60 & 0.031 \\
\hline MAPK14 & -1.34 & 0.020 \\
\hline IL10RA & -1.31 & 0.000 \\
\hline
\end{tabular}

\section{Minor changes in local B cell distribution after A. muciniphila supplementation}

Based on the findings of the microarray analysis, we continued with the investigation of immune cell distribution in different organs of the immune system. Since several B cell related genes were down-regulated after A. muciniphila supplementation, such as immunoglobulin genes (both in ileum and colon), and Blk (only in colon), we first focused on the local distribution of B cells. In mesenteric lymph nodes (MLN) and Peyer's patches (PP), no differences in B cell frequencies between groups were observed (Figure 5A-C). However, in PP the frequency of activated CD80+CD273- B cells was significantly lower in the supplemented mice $(p=0.009)$ (Figure 5D). Furthermore, frequencies of more immature $C D 80^{-} C D 273^{-}$B cells were significantly higher in the $A$. muciniphila group $(p=0.03)$ (Figure 5E), whereas no changes were observed in $\mathrm{CD} 80^{+} \mathrm{CD} 273^{+}$memory-like and $\mathrm{CD} 80^{-} \mathrm{CD} 273^{+}$ B cells (Figure 5F-G) (31).

A

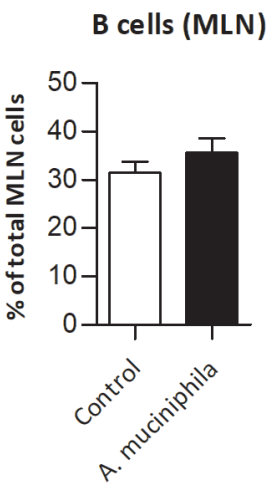

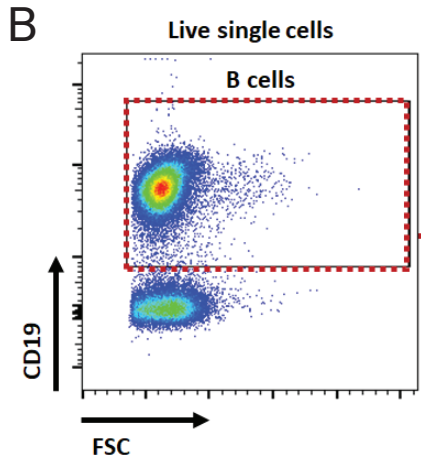

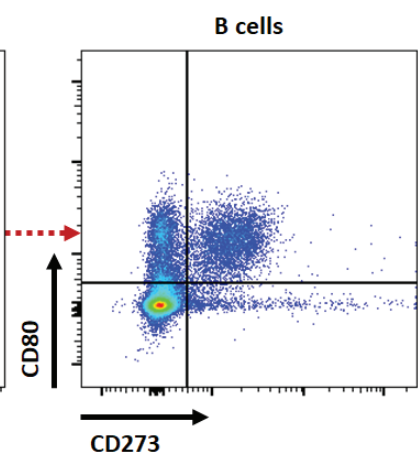


C
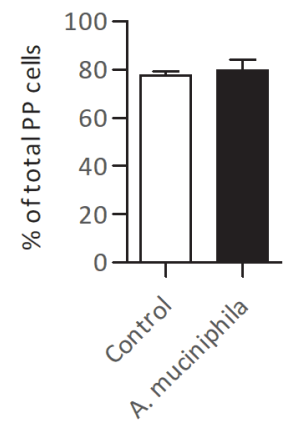

$\mathrm{F}$

$\mathrm{CD}^{+} \mathrm{CD}^{+} 23^{+}$

B cells (PP)
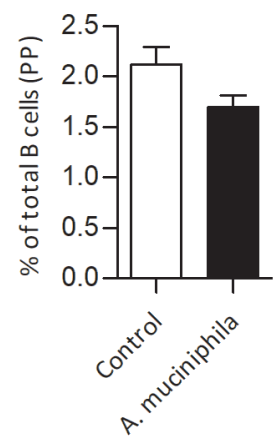

D
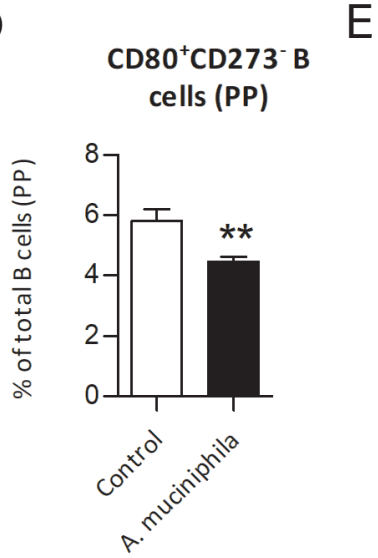

G
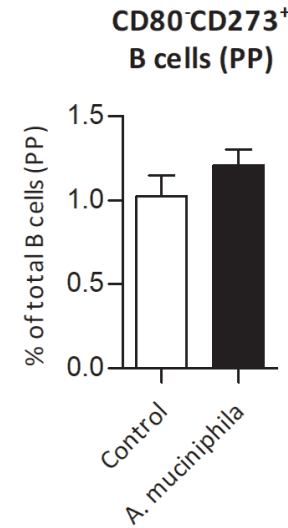

Figure 5 Distribution of B cell subsets in mesenteric lymph nodes (MLN) and Peyer's patches (PP) after supplementation with Akkermansia muciniphila. (A) Mean frequency of $B$ cells in MLN. (B) Flow cytometric analysis of $B$ cells in PP. (C) Mean frequency of $B$ cells in PP. (D) Mean frequency of CD80+CD273- B cells, (E) CD80-CD273- B cells, (F) CD80+CD273+ B cells and (G) CD80-CD273+ B cells in PP. Data represent the mean + SEM from five to six mice per group. ${ }^{*} p<0.05$; ${ }^{* *} p<0.01$.

\section{A. muciniphila supplementation increased the migration of B cells into spleen and BM}

Next, we continued with investigation of B cell subsets in spleen and bone marrow (BM). The frequency of total B cells in spleen was not different between groups (Figure 6A). Also other B cell subsets in spleen, such as immature, follicular and marginal zone B cells, were not significantly different between groups (data not shown). However, the frequency of B1 cells in spleen was significantly higher in the A. muciniphila mice $(\mathrm{p}=0.02)$ (Figure 6B-C). In bone marrow (BM), a trend of higher frequencies of total $B$ cells was observed in $A$. muciniphila supplemented mice compared 
to control mice $(p=0.07)$ and frequencies of mature and immature $B$ cells were also higher $(p=0.02$ and 0.06 , respectively) (Figure 6D-G). Frequencies of B cell precursors, i.e. pro-B cells, small resting pre-B cells and large cycling pre-B cells were not different between groups (Figure $6 \mathbf{H}-J)$. These data suggest that $A$. muciniphila supplementation did not change production of new $B$ cells in BM, but increased migration of B cells into the spleen and BM.

A

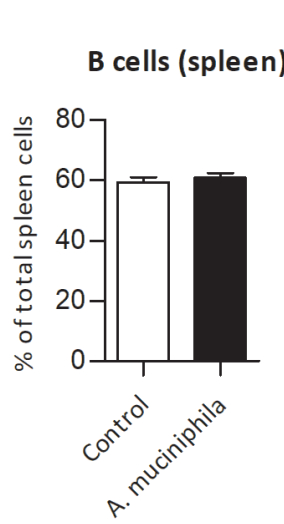

D

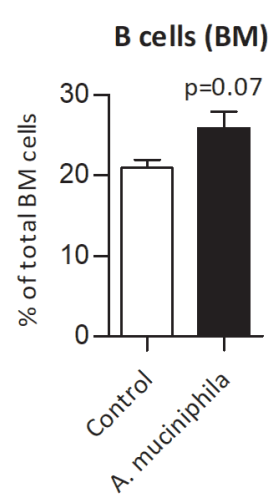

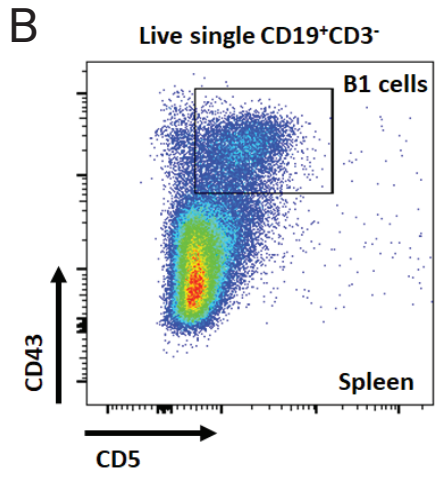

C

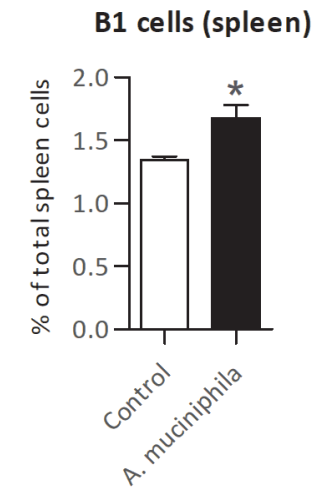

E

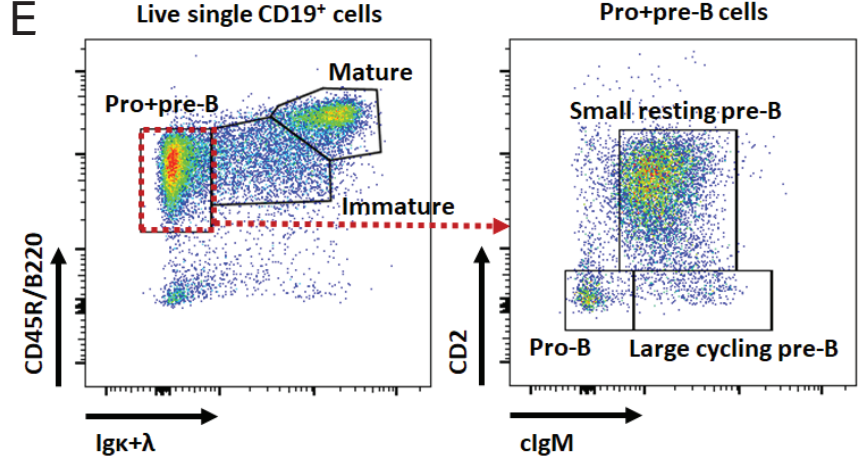


$\mathrm{F}$

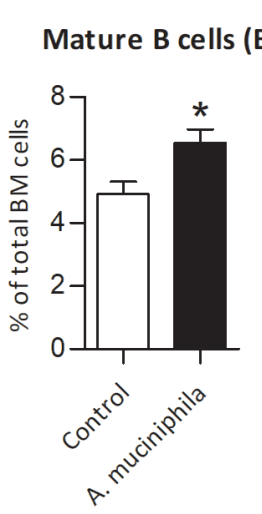

I
G Immature B cells (BM)
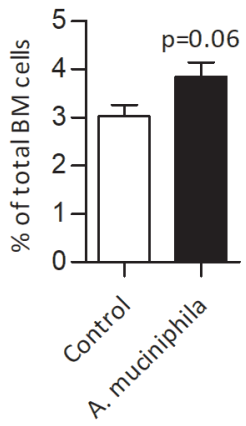

Large cycling pre-B cells (BM)

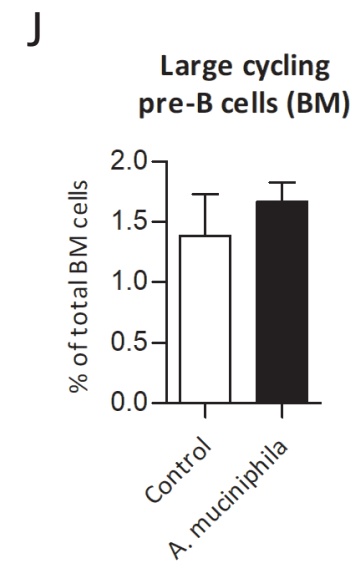

$\mathrm{H}$

Pro-B cells (BM)
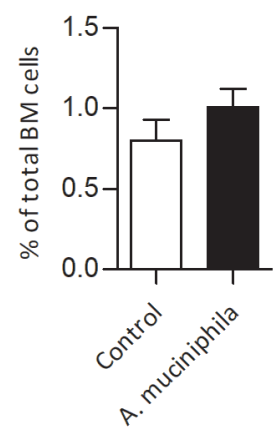

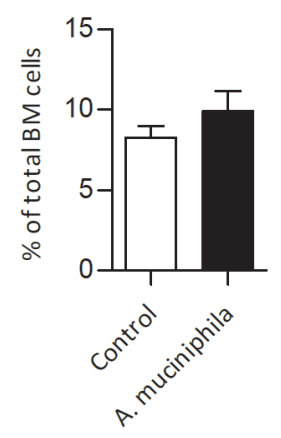

Figure 6 Distribution of $B$ cell subtypes in spleen and bone marrow (BM) after supplementation with Akkermansia muciniphila. (A) Mean frequencies of CD19+CD3- B cells in spleen. (B) Flow cytometric analysis of live single $\mathrm{CD} 19^{+} \mathrm{CD} 3^{-}$cells in spleen. (C) Mean frequencies of $\mathrm{CDF}^{+} \mathrm{CD} 43^{+} \mathrm{B} 1$ cells in spleen. (D) Mean frequencies of $B$ cells in BM. (E) Flow cytometric analysis of live single CD19+ cells, divided in pro-B cells, large cycling pre-B cells and small cycling pre-B cells in BM. (F) Mean frequencies Mature $B$ cells, (G) Immature B cells, (H) Pro-B cells, (I) Small resting pre-B cells and (J) Large cycling pre-B cells in BM. Data represent the mean + SEM from four to six mice per group. ${ }^{*} p<0.05$.

\section{T cell distribution in MLN and spleen unaltered after $A$. muciniphila supplementation}

Transcriptome analysis showed that expression of the $C d 4$ gene was decreased in the colon of mice that received A. muciniphila compared to the control mice. Moreover, IPA revealed T cell receptor (TCR) as predicted inhibited upstream regulator in colon. Therefore, we investigated whether the distribution of $\mathrm{T}$ cells was altered between groups in spleen and MLN. However, $\mathrm{CD} 4^{+}$and $\mathrm{CD} 8^{+} \mathrm{T}$ cell distributions in MLN and spleen were not different compared to the control group, neither were 
$\mathrm{CD}^{+}{ }^{+}$FoxP $3^{+}$Treg frequencies changed in both immune tissues (Figure 7A-F).

A $\mathrm{CD4}^{+} \mathrm{T}$ cells (MLN)
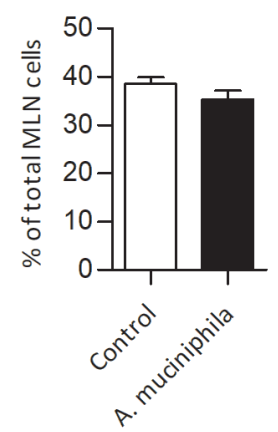

D

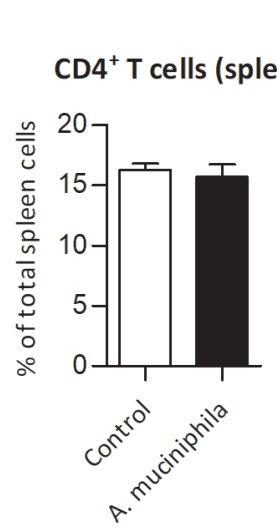

B
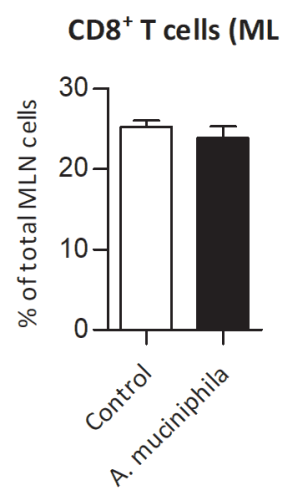

E $\mathrm{CD8}^{+} \mathrm{T}$ cells (spleen)
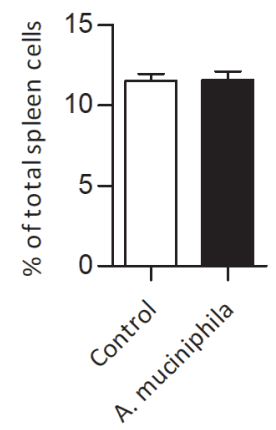

C FoxP3 $^{+}$Treg (MLN)
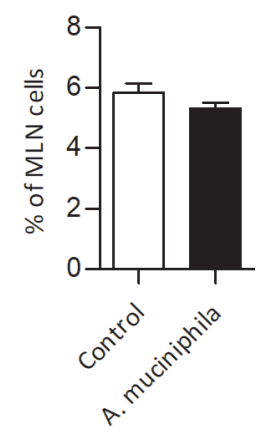

F
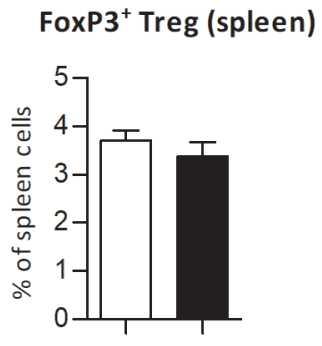

Figure 7 Distribution of T cells in mesenteric lymph nodes (MLN) and spleen after supplementation with Akkermansia muciniphila. (A) Mean frequencies of $\mathrm{CD} 4^{+} \mathrm{T}$ cells and (B) $\mathrm{CD} 8^{+} \mathrm{T}$ cells and (C) FoxP3 ${ }^{+}$ Treg cells in MLN. (D) Mean frequencies of $C D 4^{+} T$ cells and (E) CD8 ${ }^{+} T$ cells and (F) FoxP3 ${ }^{+}$Treg cells in spleen. Data represent the mean + SEM from five to six mice per group.

Decreased inflammatory cell populations in spleen and MLN after A. muciniphila supplementation

Next, since GSEA revealed an enrichment of pathways related to inflammatory response and immune function, we investigated whether inflammatory cell frequencies were altered after supplementation of $\operatorname{Ercc}^{-/ \Delta 7}$ mice. In spleen, the frequencies of total and Ly $6 C^{\text {hi }}$ monocytes were slightly lower in the $A$. muciniphila group and Ly6 $\mathrm{C}^{\text {int }}$ monocytes were significantly lower $(\mathrm{p}=0.01)$ (Figure 8A-D). Besides, the frequency of neutrophils was also slightly lower in the supplemented mice (Figure 8E). The same 
trends of these inflammatory cell populations were found in MLN, although cell frequencies were low $(<1 \%$, data not shown). These data reveal a minor decrease in inflammatory markers in spleen and MLN after A. muciniphila supplementation in $\mathrm{ErCc}^{-1 / \Delta 7}$ mice. In addition, we assessed if we could identify any signs of immune cell infiltration by investigating H\&E stained tissue. For both colon and ileum tissue, no histological signs of immune infiltration were observed in the control and $A$. muciniphila group (Figure 8F).

A

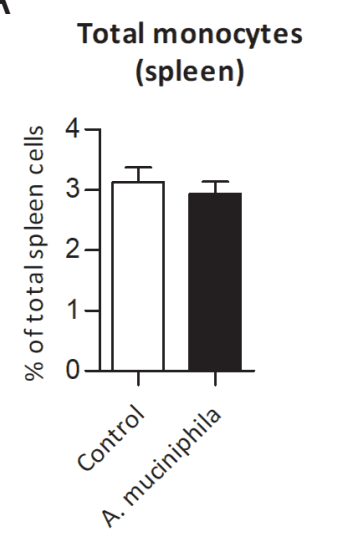

C

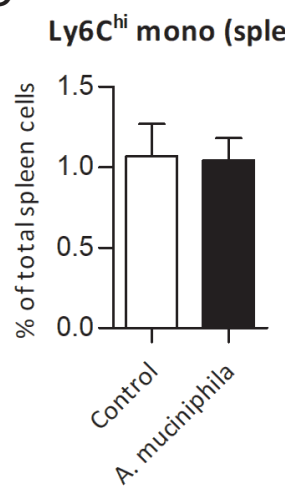

$B$

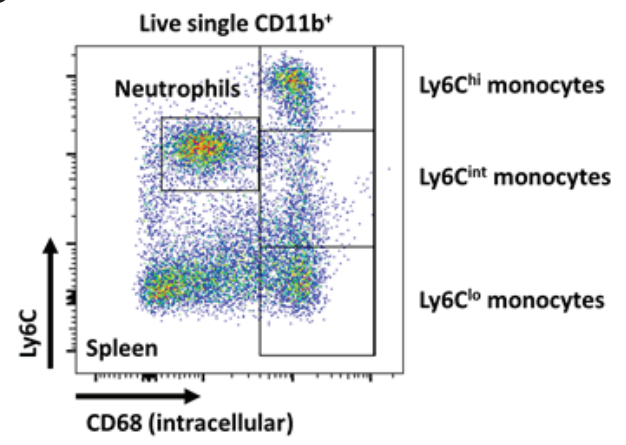

D Ly6C ${ }^{\text {int }}$ mono (spleen)

E Neutrophils (spleen)

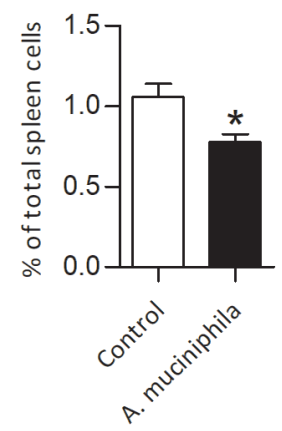

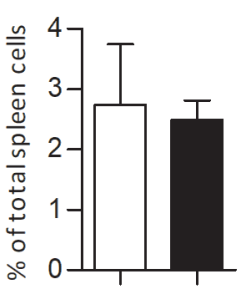

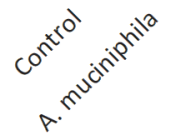


$\mathrm{F}$

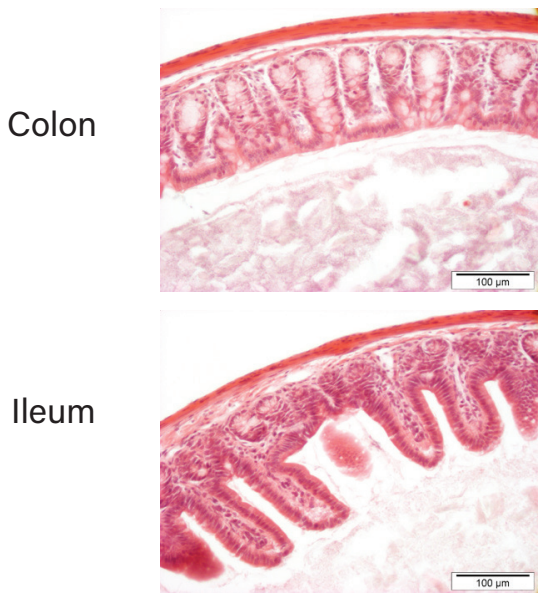

A. muciniphila
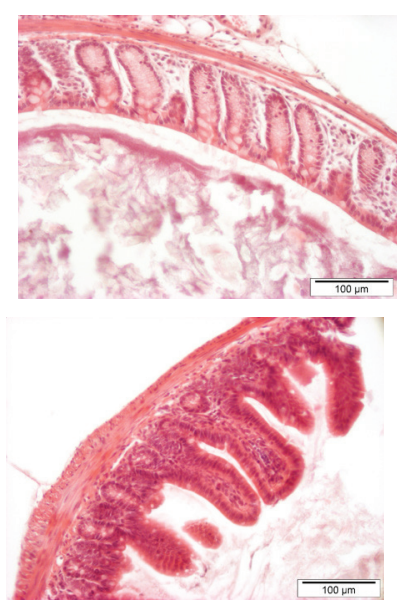

Figure 8 Distribution of inflammatory cell frequencies in spleen after supplementation with Akkermansia muciniphila. (A) Mean frequencies of total $\mathrm{CD} 11 \mathrm{~b}^{+} \mathrm{CD} 68^{+}$monocytes. (B) Flow cytometric analysis of live single $\mathrm{CD} 11 \mathrm{~b}^{+} \mathrm{Ly} 6 \mathrm{C} / \mathrm{CD} 68$ cells, divided in $\mathrm{Ly} 6 \mathrm{C}^{\mathrm{hi}}$, $\mathrm{Ly} 6 \mathrm{C}^{\text {int }}$, Ly6 $\mathrm{C}^{\mathrm{lo}}$ monocytes and CD68 ${ }^{\text {dim } L y 6 C^{\text {int/hi }}}$ neutrophils. (C) Mean frequencies of Ly6 $C^{\text {hi }}(D) L y 6 C^{\text {int }}$ monocytes and $(E)$ neutrophils. $(F)$

Representative picture of H\&E stained colon and ileum tissue of control and $A$. muciniphila supplemented mouse. Data represent the mean + SEM from five to six mice per group. ${ }^{*} \mathrm{p}<0.05$.

Increased numbers of $\mathrm{CD}_{11 \mathrm{~b}^{+}}$cells and resident macrophages in peritoneum of $\mathrm{Erccr}^{-1 / 47}$ mice supplemented with $A$. muciniphila

Since we found a higher abundance of B1 cells in spleen and this cell type is generally highly enriched in the peritoneal cavity, we also investigated the distribution of immune cells in the peritoneum. Remarkably, the total number of peritoneal cells in mice supplemented with $A$. muciniphila was nearly 3-fold higher than in the control mice $(p=0.02)$ (Figure 9A). The absolute numbers of B cells, B1 cells and T cells did not significantly differ between groups (Figure 9B-D). We found an increase in absolute numbers of $C D 11 b^{+}$cells in the peritoneum after $A$. muciniphila supplementation ( $p=0.004$ ) (Figure $9 E$ ). Investigation of $\mathrm{CD}_{11} \mathrm{~b}^{+}$cell subsets revealed that absolute numbers of neutrophils were not significantly higher after $A$. muciniphila supplementation (Figure 9F), in contrast to resident macrophages ( $\mathrm{p}=0.045)$ (Figure $9 \mathrm{G}-\mathrm{H})$. In addition, the expression of CD115 on resident macrophages was significantly higher in the $A$. muciniphila group compared to the control group $(p=0.02)$ (Figure 9I), whereas no change in the expression of CD11b and SIRPa was observed (Figure 9J-K). 
A
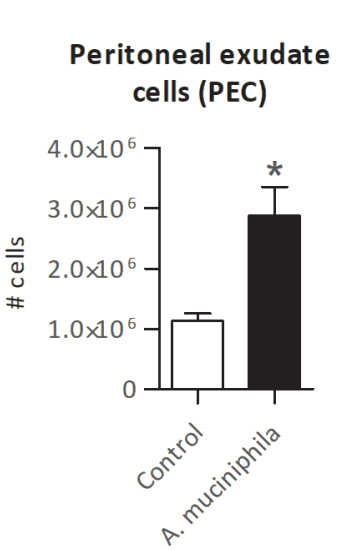

D

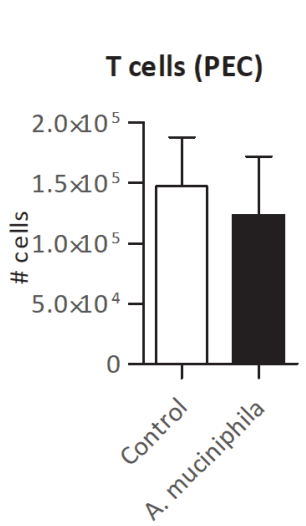

B

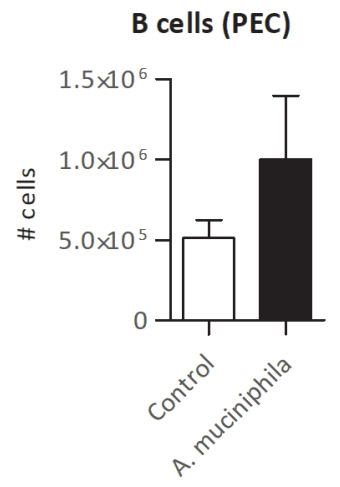

E

E
C

$$
\text { B1 cells (PEC) }
$$
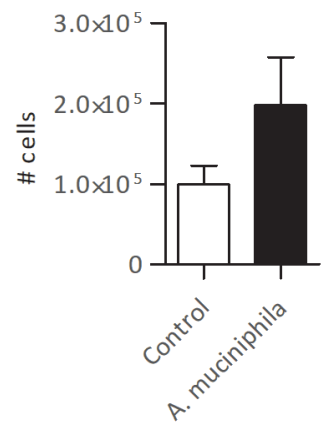

F
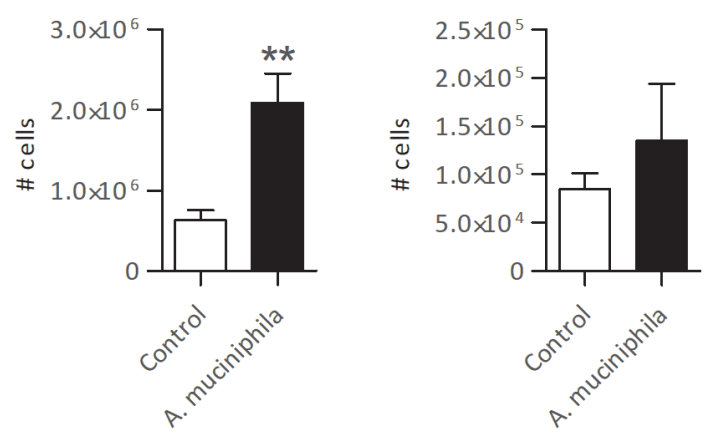

G

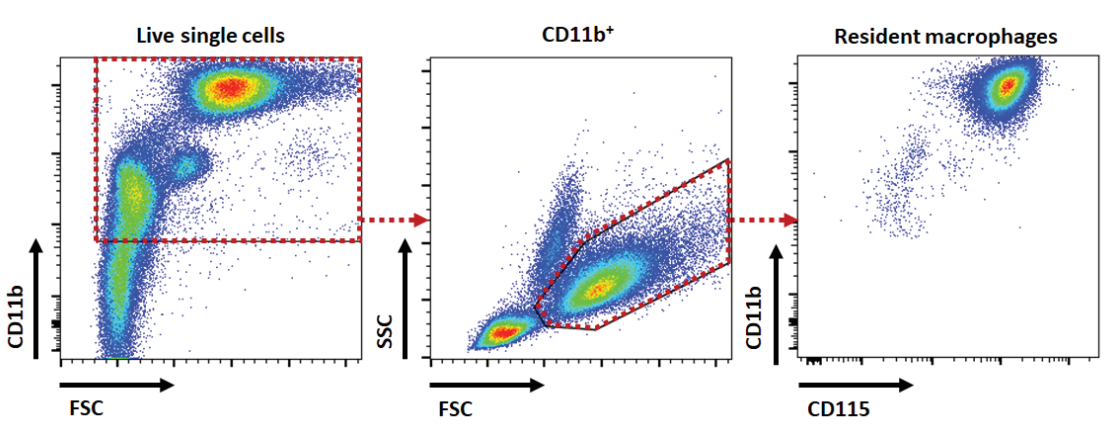


$\mathrm{H}$

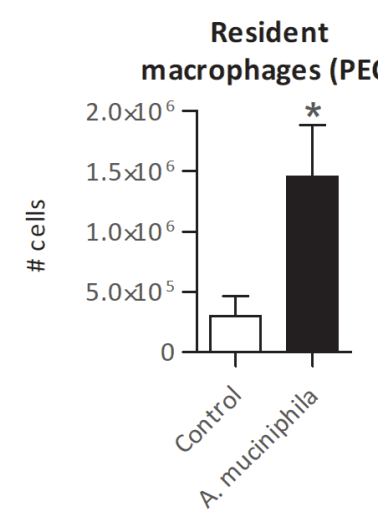

J

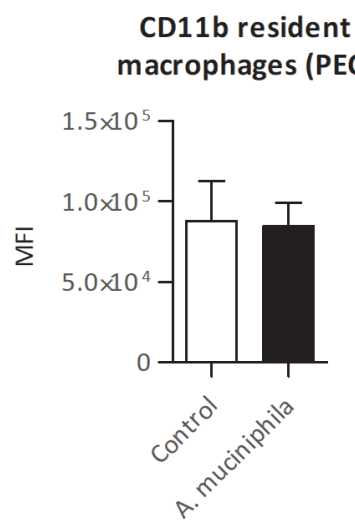

I
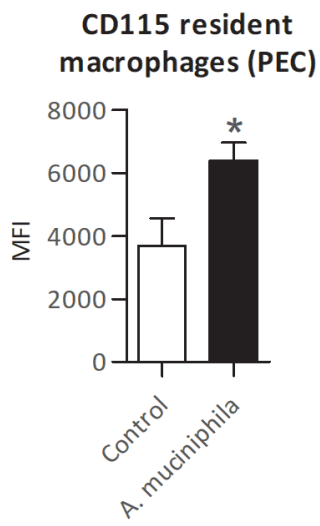

$\mathrm{K}$
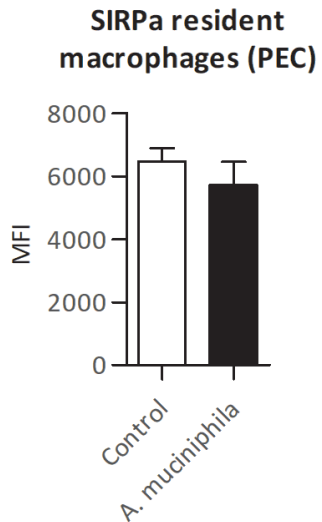

Figure 9 Distribution of immune cells in the peritoneum after supplementation with Akkermansia muciniphila. (A) Absolute number of total peritoneal exudate cells (PEC), (B) CD3-CD19+ B cells, (C) CD5+CD43+ B1 cells and (D) CD3+ T cells, (E) CD11b+ cells and (F) CD11b+Ly6G+ neutrophils. (G) Flow cytometric analysis of CD11b+ cells. $(\mathrm{H})$ Absolute numbers of CD11b+Ly6G- resident macrophages in peritoneal exudate cell suspension. (I) Median Fluorescence Intensity (MFI) of CD115 marker, (J) CD11b marker and (K) SIRPa marker expressed on resident macrophages. Data represent the mean + SEM from five to six mice per group. ${ }^{*} p<0.05 ;{ }^{* *} p<0.01$. 


\section{A. muciniphila supplementation did not alter survival, body weight and organ weights in Ercc1 ${ }^{-1}$} $\Delta 7$ mice

Supplementation with $A$. muciniphila for 10 weeks did not alter survival rates as compared to the control mice (Additional file 4). Body weight of $\operatorname{Ercc}^{-1 \Delta 7}$ mice increased in the first half of life, but decreased again from 11 weeks of age onwards (Additional file 4). No pronounced differences in body weight development were found between groups. The weight of liver, spleen and thymus measured directly after sacrifice were not significantly different between control and A. muciniphila group (data not shown).

\section{Discussion}

The bacterium Akkermansia muciniphila is suggested to be a promising microbial supplement, due to its beneficial effects on health $(20,32)$. However, its effects on the decline in intestinal health during aging are not well investigated yet. In the present study, we investigated the effects of supplementation with A. muciniphila on different aspects of intestinal health in accelerated aging $\operatorname{Ercc1}^{-1 \Delta 7}$ mice. We report that supplementation with $A$. muciniphila for 10 weeks resulted in a significantly thicker colonic mucus layer and an improvement of anti-inflammatory immune status compared to the control group.

In two recent studies, it has been shown that the colonic mucus layer decreased in aging mice, suggesting an association with bacterial penetration and immune activation (29, 33). An agingrelated decrease in mucus thickness was also observed in $\operatorname{Ercc1}^{-/ \Delta 7}$ mice (29), confirming the similarity in aging phenotype in this accelerated aging mouse model compared to a mouse model with a normal aging process. We previously showed that L. plantarum WCFS1 increased the thickness of the colonic mucus layer in $\operatorname{Ercc}^{-/ \Delta 7}$ mice (29), but we now show that $A$. muciniphila is capable to even further thicken the mucus layer. The ability of $A$. muciniphila to increase mucus thickness was also reported before (23). A. muciniphila is able to degrade mucin structures to use it as carbon and nitrogen source and is therefore called a mucus-degrader (17). Nevertheless, the observed increased mucus layer thickness after supplementation suggests that this bacterium is able to actively turn on host colonic mucus production, a suggestion that has also been made by Derrien and colleagues (34). Interestingly, we did not find differential expression of any genes encoding for mucins, apart from a down-regulation of Mucl1 in colon tissue. However, expression levels of this gene were low and it is not a typical mucin that constitutes the colonic mucus layer, such as Muc2 (35). In our previous study, supplementation with L. plantarum WCFS1 did neither result in differences in mucin 
gene expression, while significantly increased colonic mucus thickness was observed (29). Possibly, supplementation with $A$. muciniphila resulted in an increased mucus thickness by impacting mucus biosynthesis processes without affecting Muc2 expression. In order to understand the exact underlying mechanisms of the mucus turnover processes, further investigation is warranted.

Interestingly, in the ileum of $A$. muciniphila supplemented mice, GSEA revealed a downregulation of two pathways related to N-Glycan biosynthesis and besides, two genes related to mucus biosynthesis were also down-regulated. These results imply that, besides its great impact on the colonic mucus layer, A. muciniphila could also have had an effect on the ileal mucus layer. We could not verify this finding by measuring the ileal mucus layer, because of its rather discontinuous appearance due to the presence of villi.

Next to the important function of the mucus layer, tight junctions (TJs) sealing the intestinal epithelial cells also play an important role in intestinal barrier function and an age-related decreased expression of TJ genes was found in baboons (36). We and others previously showed that $A$. muciniphila improved intestinal barrier function in a Caco-2 cell model (37) as well as in mice (18, $19,23,38,39)$. In the present study, we found a down-regulation of Cldn2 and Cldn8 in the ileum of A. muciniphila supplemented mice. However, the intestinal barrier consists of a complex structure of multiple protein networks (40). Therefore, it is not possible to draw any solid conclusions on the effects of $A$. muciniphila supplementation on intestinal barrier function based on gene expression data only.

Gut microbiota composition and bacterial diversity in ileum and colon were not significantly changed after supplementation with $A$. muciniphila. This result indicates that bacterial supplementation with $A$. muciniphila does not lead to a reshaped gut microbiota composition, which was also reported before (23). Gut microbiota analysis revealed that the relative abundance of $A$. muciniphila was low in ileal and colonic content. It was previously shown that Akkermansia spp. was more present in colon than ileum in mono-colonised mice (39), but the low abundance in colon was remarkable. The dose of the bacterium, i.e. $2 \times 10^{8} \mathrm{CFU}$ for 3 times a week, was already proven effective in previous mice studies (23). Possibly, the relatively long time between the last oral gavage and sacrifice (about 24 hours) could have led to a washout of $A$. muciniphila. Though, a recent study showed that daily supplementation with 11 probiotic strains resulted in low colonization in mice which was caused by the indigenous microbiome (41). Possibly, this finding may also explain the impeded colonization of $A$. muciniphila in our study.

Bacterial supplementation with $A$. muciniphila resulted in a down-regulation of numerous immunerelated genes and pathways in colon. Notably, these included several B cell related genes, such as 
immunoglobulins, Blk and Pilrb1, amongst others. Moreover, GSEA revealed a down-regulation of the pathway "Intestinal immune network for IgA production". These results imply that $A$. muciniphila supplementation may have decreased the necessity for producing $\lg A$, i.e. exerting a mucosal protective reaction against commensal bacteria (42). In line with these results, we found a downregulation of both T/r7 and T/r12 in colon. However, we could not confirm this hypothesis, since IgA concentrations in colon were not measured during this study. We did investigate B cell frequencies in several immune tissues. Nevertheless, we could not find differences in B cell frequencies in MLNs, whereas $B$ cell frequencies were increased in spleen and BM. These findings suggest that based on transcriptome analysis, $A$. muciniphila decreased $B$ cell frequencies and $C D 4^{+} T$ cells in colon and caused a potential redistribution of B and T cells among lymphoid organs. Conversely, frequencies of total B cell subsets in BM and spleen were slightly increased after A. muciniphila supplementation, with no change in B cell precursor frequencies in BM. These findings may indicate that supplementation with $A$. muciniphila inhibited influx of $B$ cells into the colon, leading to a slightly increased mature B cell pool in spleen and BM.

Furthermore, we found a decrease in inflammatory markers in colon after A. muciniphila supplementation. Several genes encoding for chemokines, complement factors, as well as the cytokine $/ / 5$ were down-regulated after A. muciniphila supplementation. Besides, IPA identified numerous pro-inflammatory cytokines as potentially inhibited upstream regulators in colon. The anti-inflammatory properties of $A$. muciniphila are already extensively described (34). We now add evidence that $A$. muciniphila might protect against the aging-related increase in inflammation (inflamm-aging) by decreasing the colonic expression of pro-inflammatory genes and pathways. Besides, we also identified the anti-inflammatory cytokine Tgf-beta as inhibited upstream regulator. Although we only found one anti-inflammatory cytokine, this finding may point toward a general reduction of immune activation by $A$. muciniphila. Histological analysis of the colon did not reveal any clear signs of immune infiltration in both groups. In a previous study by Derrien and colleagues, an up-regulation of immune related genes with no signs of microscopically visible inflamed tissue was also observed in mice mono-colonized with A. muciniphila (39). The authors suggest that these observations were part of regulatory processes of immune tolerance toward $A$. muciniphila. However, in this study germ-free mice were used, hence any comparisons between these particular results and our results should be made with caution.

Interestingly, we found significantly lower frequencies of activated B cell subtypes and higher frequencies of more immature B cell subtypes in PP. The increased level of inactive immature B cells is in accordance with the microarray results from ileum tissue, since we also found decreased 
expression of numerous immunoglobulin-related genes. Besides, Reg3b and Reg3g were both down-regulated in ileum. We previously showed that $A$. muciniphila supplementation also decreased Reg3g expression in ileum of mice fed a high-fat diet (19). In a recent study, an increased expression of antimicrobial genes was found in the ileum of aged C57BL/6 mice, including Reg3b, Reg3g, Defb1 and Retnlb, which was suggested to be related to an increased state of epithelial distress (43). Hence, based on our findings we suggest that supplementation with A. muciniphila might contribute to prevention of the age-related state of epithelial distress in ileum.

It is well-known that T cell function declines during the aging process (44). Our previous study revealed that supplementation with L. plantarum WCFS1 and L. casei BL23 increased regulatory T cell frequencies in MLN of $\operatorname{Ercct}^{-1 \Delta 7}$ mice. However, supplementation with A. muciniphila did not lead to any changes in T cell distribution in MLN, spleen and PP, despite a down-regulation of the Cd4 gene in colon and the predicted inhibition of the upstream regulator TCR. Possibly, the increased colonic mucus layer caused by A. muciniphila supplementation resulted in an increased protective state in the colon, thereby decreasing the production of a number of $\mathrm{T}$ cell attracting chemokines and subsequently leading to a decrease of $\mathrm{CD} 4^{+} \mathrm{T}$ cell attraction and infiltration.

In the peritoneal cavity, a highly significant increase in resident macrophages was observed after A. muciniphila supplementation. Peritoneal macrophages are important in the modulation of immune responses during infections and contribute to tissue homeostasis (45). Furthermore, peritoneal resident macrophages were shown to defend against microbial invasion (46), which could explain the high presence of this cell type after a supplementation with A. muciniphila. However, this increase in peritoneal resident macrophages was not coincided with increased frequencies of neutrophils and T cells, while these cell types are expected to be highly present during inflammation. This observation implies that supplementation with $A$. muciniphila resulted in an activated state with regard to peritoneal resident macrophages, but resulted in an anti-inflammatory rather than a proinflammatory response.

\section{Conclusion}

The attention for A. muciniphila as a potential microbial supplement has increased and ample evidence exists emphasising the beneficial effects of this bacterium on low-grade inflammation and (cardio)metabolic disorders $(16,20,34,47)$. In the present study, we also observed that several metabolic processes in ileum, as well as immunological processes in both ileum and colon were affected by $A$. muciniphila, but now in an aging model. Furthermore, we convincingly showed that 
A. muciniphila has a protective effect against an age-related decline in mucus thickness, which was even stronger compared to L. plantarum WCFS1. Aging is often accompanied by low-grade inflammation and an increased risk on metabolic syndrome $(1,48)$, contributing to a decreased quality of life and a considerable rise in healthcare costs (49). Our study showed a causal relationship between $A$. muciniphila and attenuation of the aging phenotype, in terms of preventing the agerelated decline in thickness of the colonic mucus layer and improving immune status. These results therefore support the therapeutic application of $A$. muciniphila in the aging population and pave the way for further studies investigating A. muciniphila as therapeutic intervention contributing to healthy aging. Further research should focus on the practical aspects for application in humans, such as the dosage, frequency and way of administration. 


\section{List of abbreviations}

$\mathrm{BM}$, bone marrow; $\mathrm{CFU}$, colony-forming unit; $\mathrm{ErCC}^{-1 / \Delta 7}$ mice, mice with defective nucleotide excision repair gene Ercc1; FACS, fluorescence-activated cell sorting; FC, fold change; FDR, false discovery rate; GSEA, gene set enrichment analysis; H\&E, hematoxylin and eosin; IBMT, Intensity based moderated T statistics; IPA, Ingenuity pathway analysis; MLN, mesenteric lymph node; PAS, periodic acid-Schiff; PCoA, principal coordinates analysis; PP, peyer's patches; qPCR, quantitative polymerase chain reaction; SPF, specific pathogen-free; TJ, tight junction.

\section{Acknowledgments}

We would like to thank Floor Hugenholtz for her help during 16 S rRNA sequencing and Jenny Jansen for her help during microarray performance. We would also thank Jan Hoeijmakers for his excellent work on conceiving the $\operatorname{Ercc1}^{-/ \Delta 7}$ mouse model. Yvette van Loon is acknowledged for her help with breeding and genotyping the mouse cohorts.

\section{Funding}

This work was funded by the Netherlands Organization for Scientific Research (Spinoza Award and SIAM Gravity Grant 024.002.002) and through the NWO Graduate Programme on Food Structure, Digestion and Health.

\section{Ethics approval}

Experiments were performed with the Principles of Laboratory Animal Care and with Dutch legislation and approval of the Dutch Ethical Committee of Wageningen.

\section{Availability of data and material}

Microarray data are available in the NCBI Gene Expression Omnibus (GEO) with number GSE126730. The 16S rRNA gene data described in this study have been deposited in the Sequence Read Archive (SRA) at the NCBI (PRJNA525606). 


\section{References}

1. Bektas A, Schurman SH, Sen R, Ferrucci L. Aging, inflammation and the environment. Exp Gerontol. 2018;105:10-8. 1.gerontology. 2018;105:10-8.

2. Buford TW. (Dis)Trust your gut: the gut microbiome in age-related inflammation, health, and disease. Microbiome. 2017;5(1):80.

3. Sanada F, Taniyama Y, Muratsu J, Otsu R, Shimizu H, Rakugi H, et al. Source of Chronic Inflammation in Aging. Frontiers in cardiovascular medicine. 2018;5:12.

4. Franceschi C, Campisi J. Chronic inflammation (inflammaging) and its potential contribution to age-associated diseases. The journals of gerontology Series A, Biological sciences and medical sciences. 2014;69 Suppl 1:S4-9.

5. Rayner CK, Horowitz M. Physiology of the ageing gut. Current opinion in clinical nutrition and metabolic care. 2013;16(1):33-8.

6. Koh A, De Vadder F, Kovatcheva-Datchary P, Backhed F. From Dietary Fiber to Host Physiology: Short-Chain Fatty Acids as Key Bacterial Metabolites. Cell. 2016;165(6):1332-45.

7. Louis P, Hold GL, Flint HJ. The gut microbiota, bacterial metabolites and colorectal cancer. Nature reviews Microbiology. 2014;12(10):661-72.

8. Belkaid Y, Hand TW. Role of the microbiota in immunity and inflammation. Cell. 2014;157(1):121-

41.

9. Ple C, Breton J, Daniel C, Foligne B. Maintaining gut ecosystems for health: Are transitory food bugs stowaways or part of the crew? International journal of food microbiology. 2015;213:139-43.

10. Fransen F, van Beek AA, Borghuis T, Aidy SE, Hugenholtz F, van der Gaast-de Jongh C, et al. Aged Gut Microbiota Contributes to Systemical Inflammaging after Transfer to Germ-Free Mice. Frontiers in immunology. 2017;8:1385.

11. McGuckin MA, Linden SK, Sutton P, Florin TH. Mucin dynamics and enteric pathogens. Nature reviews Microbiology. 2011;9(4):265-78.

12. Van der Sluis M, De Koning BA, De Bruijn AC, Velcich A, Meijerink JP, Van Goudoever JB, et al. Muc2-deficient mice spontaneously develop colitis, indicating that MUC2 is critical for colonic protection. Gastroenterology. 2006;131(1):117-29.

13. Velcich A, Yang W, Heyer J, Fragale A, Nicholas C, Viani S, et al. Colorectal cancer in mice genetically deficient in the mucin Muc2. Science (New York, NY). 2002;295(5560):1726-9.

14. Lu P, Burger-van Paassen N, van der Sluis M, Witte-Bouma J, Kerckaert JP, van Goudoever JB, et al. Colonic gene expression patterns of mucin Muc2 knockout mice reveal various phases in colitis development. Inflammatory bowel diseases. 2011;17(10):2047-57.

15. de Vos WM. Microbe Profile: Akkermansia muciniphila: a conserved intestinal symbiont that acts as the gatekeeper of our mucosa. Microbiology (Reading, England). 2017;163(5):646-8.

16. Belzer C, de Vos WM. Microbes inside--from diversity to function: the case of Akkermansia. The ISME journal. 2012;6(8):1449-58.

17. Derrien M, Vaughan EE, Plugge CM, de Vos WM. Akkermansia muciniphila gen. nov., sp. nov., a human intestinal mucin-degrading bacterium. International journal of systematic and evolutionary microbiology. 2004;54(Pt 5):1469-76.

18. Ottman N, Reunanen J, Meijerink M, Pietila TE, Kainulainen V, Klievink J, et al. Pili-like proteins of Akkermansia muciniphila modulate host immune responses and gut barrier function. PloS one. 2017;12(3):e0173004.

19. Plovier H, Everard A, Druart C, Depommier C, Van Hul M, Geurts L, et al. A purified membrane protein from Akkermansia muciniphila or the pasteurized bacterium improves metabolism in obese and diabetic mice. Nature medicine. 2017;23(1):107-13.

20. Cani PD, de Vos WM. Next-Generation Beneficial Microbes: The Case of Akkermansia muciniphila. Frontiers in microbiology. 2017;8:1765.

21. Dao MC, Everard A, Aron-Wisnewsky J, Sokolovska N, Prifti E, Verger EO, et al. Akkermansia muciniphila and improved metabolic health during a dietary intervention in obesity: relationship with gut microbiome richness and ecology. Gut. 2016;65(3):426-36.

22. Schneeberger M, Everard A, Gomez-Valades AG, Matamoros S, Ramirez S, Delzenne NM, et al. Akkermansia muciniphila inversely correlates with the onset of inflammation, altered adipose tissue metabolism and metabolic disorders during obesity in mice. Scientific reports. 2015;5:16643.

23. Everard A, Belzer C, Geurts L, Ouwerkerk JP, Druart C, Bindels LB, et al. Cross-talk between 
Akkermansia muciniphila and intestinal epithelium controls diet-induced obesity. Proceedings of the National Academy of Sciences of the United States of America. 2013;110(22):9066-71.

24. van der Lugt B, Rusli F, Lute C, Lamprakis A, Salazar E, Boekschoten MV, et al. Integrative analysis of gut microbiota composition, host colonic gene expression and intraluminal metabolites in aging C57BL/6J mice. Aging. 2018;10(5):930-50.

25. Langille MG, Meehan CJ, Koenig JE, Dhanani AS, Rose RA, Howlett SE, et al. Microbial shifts in the aging mouse gut. Microbiome. 2014;2(1):50.

26. Biagi E, Nylund L, Candela M, Ostan R, Bucci L, Pini E, et al. Through ageing, and beyond: gut microbiota and inflammatory status in seniors and centenarians. PloS one. 2010;5(5):e10667.

27. Biagi E, Franceschi C, Rampelli S, Severgnini M, Ostan R, Turroni S, et al. Gut Microbiota and Extreme Longevity. Current biology : CB. 2016;26(11):1480-5.

28. van Beek AA, Hugenholtz F, Meijer B, Sovran B, Perdijk O, Vermeij WP, et al. Frontline Science: Tryptophan restriction arrests $B$ cell development and enhances microbial diversity in WT and prematurely aging Ercc1(-/Delta7) mice. Journal of leukocyte biology. 2017;101(4):811-21.

29. van Beek AA, Sovran B, Hugenholtz F, Meijer B, Hoogerland JA, Mihailova V, et al. Supplementation with Lactobacillus plantarum WCFS1 Prevents Decline of Mucus Barrier in Colon of Accelerated Aging Ercc1(-/Delta7) Mice. Frontiers in immunology. 2016;7:408.

30. Vermeij WP, Hoeijmakers JH, Pothof J. Genome Integrity in Aging: Human Syndromes, Mouse Models, and Therapeutic Options. Annual review of pharmacology and toxicology. 2016;56:427-45.

31. Engels $\mathrm{N}$, Wienands J. Memory control by the $\mathrm{B}$ cell antigen receptor. Immunological reviews. 2018;283(1):150-60.

32. Neef A, Sanz Y. Future for probiotic science in functional food and dietary supplement development. Current opinion in clinical nutrition and metabolic care. 2013;16(6):679-87.

33. Elderman M, Sovran B, Hugenholtz F, Graversen K, Huijskes M, Houtsma E, et al. The effect of age on the intestinal mucus thickness, microbiota composition and immunity in relation to sex in mice. PloS one. 2017;12(9):e0184274.

34. Derrien M, Belzer C, de Vos WM. Akkermansia muciniphila and its role in regulating host functions. Microbial pathogenesis. 2017;106:171-81.

35. Pelaseyed T, Bergstrom JH, Gustafsson JK, Ermund A, Birchenough GM, Schutte A, et al. The mucus and mucins of the goblet cells and enterocytes provide the first defense line of the gastrointestinal tract and interact with the immune system. Immunological reviews. 2014;260(1):8-20.

36. Tran L, Greenwood-Van Meerveld B. Age-associated remodeling of the intestinal epithelial barrier. The journals of gerontology Series A, Biological sciences and medical sciences. 2013;68(9):104556.

37. Reunanen J, Kainulainen V, Huuskonen L, Ottman N, Belzer C, Huhtinen H, et al. Akkermansia muciniphila Adheres to Enterocytes and Strengthens the Integrity of the Epithelial Cell Layer. Applied and environmental microbiology. 2015;81(11):3655-62.

38. Bodogai M, O'Connell J, Kim K, Kim Y, Moritoh K, Chen C, et al. Commensal bacteria contribute to insulin resistance in aging by activating innate B1a cells. Science translational medicine. 2018;10(467).

39. Derrien M, Van Baarlen P, Hooiveld G, Norin E, Muller M, de Vos WM. Modulation of Mucosal Immune Response, Tolerance, and Proliferation in Mice Colonized by the Mucin-Degrader Akkermansia muciniphila. Frontiers in microbiology. 2011;2:166.

40. Groschwitz KR, Hogan SP. Intestinal barrier function: molecular regulation and disease pathogenesis. The Journal of allergy and clinical immunology. 2009;124(1):3-20; quiz 1-2.

41. Zmora N, Zilberman-Schapira G, Suez J, Mor U, Dori-Bachash M, Bashiardes S, et al. Personalized Gut Mucosal Colonization Resistance to Empiric Probiotics Is Associated with Unique Host and Microbiome Features. Cell. 2018;174(6):1388-405.e21.

42. Gutzeit C, Magri G, Cerutti A. Intestinal IgA production and its role in host-microbe interaction. Immunological reviews. 2014;260(1):76-85.

43. Tremblay S, Cote NML, Grenier G, Duclos-Lasnier G, Fortier LC, Ilangumaran S, et al. Ileal antimicrobial peptide expression is dysregulated in old age. Immunity \& ageing : I \& A. 2017;14:19.

44. Haynes L, Maue AC. Effects of aging on T cell function. Current opinion in immunology. 2009;21(4):414-7.

45. Davies LC, Taylor PR. Tissue-resident macrophages: then and now. Immunology. 2015;144(4):541-

8. 
46. Davies LC, Rice CM, Palmieri EM, Taylor PR, Kuhns DB, McVicar DW. Peritoneal tissueresident macrophages are metabolically poised to engage microbes using tissue-niche fuels. Nature communications. 2017;8(1):2074.

47. Ouwerkerk JP, Aalvink S, Belzer C, De Vos WM. Preparation and preservation of viable Akkermansia muciniphila cells for therapeutic interventions. Beneficial microbes. 2017;8(2):163-9.

48. Dominguez LJ, Barbagallo M. The biology of the metabolic syndrome and aging. Current opinion in clinical nutrition and metabolic care. 2016;19(1):5-11.

49. Nichols GA, Moler EJ. Metabolic syndrome components are associated with future medical costs independent of cardiovascular hospitalization and incident diabetes. Metabolic syndrome and related disorders. 2011;9(2):127-33.

50. Salonen A, Nikkila J, Jalanka-Tuovinen J, Immonen O, Rajilic-Stojanovic M, Kekkonen RA, et al. Comparative analysis of fecal DNA extraction methods with phylogenetic microarray: effective recovery of bacterial and archaeal DNA using mechanical cell lysis. Journal of microbiological methods. 2010;81(2):12734.

51. Ramiro-Garcia J, Hermes GDA, Giatsis C, Sipkema D, Zoetendal EG, Schaap PJ, et al. NG-Tax, a highly accurate and validated pipeline for analysis of 16S rRNA amplicons from complex biomes [version 1; referees: 2 approved with reservations, 1 not approved]. F1000Research. 2016(5):1791.

52. Lathi L, Shetty S. Tools for microbiome analysis in R Version 1.1.10013. 2017.

53. McMurdie PJ, Holmes S. phyloseq: an R package for reproducible interactive analysis and graphics of microbiome census data. PloS one. 2013;8(4):e61217.

54. Sovran B, Loonen LM, Lu P, Hugenholtz F, Belzer C, Stolte EH, et al. IL-22-STAT3 pathway plays a key role in the maintenance of ileal homeostasis in mice lacking secreted mucus barrier. Inflammatory bowel diseases. 2015;21(3):531-42.

55. Subramanian A, Tamayo P, Mootha VK, Mukherjee S, Ebert BL, Gillette MA, et al. Gene set enrichment analysis: a knowledge-based approach for interpreting genome-wide expression profiles. Proceedings of the National Academy of Sciences of the United States of America. 2005;102(43):15545-50. 56. Kramer A, Green J, Pollard J, Jr., Tugendreich S. Causal analysis approaches in Ingenuity Pathway Analysis. Bioinformatics (Oxford, England). 2014;30(4):523-30.

57. Wang X, Seed B. A PCR primer bank for quantitative gene expression analysis. Nucleic acids research. 2003;31(24):e154. 


\section{Supplemental material}

Additional file 1 Gene expression profiles (Microarray analysis) of colon and ileum. Only genes that were statistically significantly different $(p<0.05)$ and had a fold change $(F C)<-1.2$ or $>1.2$ between groups are included.

\begin{tabular}{|c|c|c|c|c|c|}
\hline \multirow{3}{*}{$\begin{array}{l}\text { UP-REGULATED GENES } \\
\text { COLON } \\
\text { gene name }\end{array}$} & \multirow[b]{3}{*}{ FC } & \multirow[b]{3}{*}{$p$-value } & \multicolumn{3}{|c|}{ DOWN-REGULATED GENES } \\
\hline & & & COLON & & \\
\hline & & & gene name & $\mathrm{FC}$ & p-value \\
\hline Ighv9-4 & 6.00 & 0.011 & lgkv15-103 & -7.61 & 0.005 \\
\hline Ighv14-4 & 3.62 & 0.028 & lgkv8-21 & -6.36 & 0.007 \\
\hline Gm5169 & 1.99 & 0.003 & $\operatorname{lgkv5-43}$ & -5.86 & 0.000 \\
\hline Olfr1175-ps & 1.89 & 0.001 & $\operatorname{lghv5-4}$ & -4.79 & 0.003 \\
\hline Ssxb10 & 1.83 & 0.004 & $\operatorname{lgkv8-30}$ & -4.57 & 0.002 \\
\hline Trav13-4-dv7 & 1.77 & 0.012 & Ighv1-77 & -4.46 & 0.001 \\
\hline LOC102632658 & 1.77 & 0.020 & $\operatorname{lghg} 2 b$ & -4.01 & 0.004 \\
\hline 9430037G07Rik & 1.72 & 0.002 & Ighv1-53 & -3.89 & 0.038 \\
\hline Pramef6 & 1.65 & 0.045 & $\operatorname{lghv5-16}$ & -3.70 & 0.040 \\
\hline LOC102635127 & 1.63 & 0.005 & Ighv3-8 & -3.62 & 0.033 \\
\hline Gm2666 & 1.61 & 0.013 & lghg2c & -3.10 & 0.003 \\
\hline Mir1895 & 1.60 & 0.037 & $\operatorname{lgkv2-109}$ & -2.96 & 0.008 \\
\hline Olfr145 & 1.60 & 0.003 & $\operatorname{lgkv5-39}$ & -2.84 & 0.013 \\
\hline Gm2964 & 1.59 & 0.029 & lgkv4-86 & -2.80 & 0.032 \\
\hline LOC102635096 & 1.58 & 0.031 & lgkv1-135 & -2.80 & 0.037 \\
\hline LOC102636771 & 1.55 & 0.029 & $\lg / v 3$ & -2.65 & 0.010 \\
\hline Gm6909 & 1.54 & 0.035 & Ighv10-1 & -2.64 & 0.045 \\
\hline Fam188b2 & 1.54 & 0.004 & Ighv1-15 & -2.46 & 0.040 \\
\hline Pcdha5 & 1.53 & 0.034 & Igkv8-19 & -2.29 & 0.003 \\
\hline Gm15104 & 1.50 & 0.047 & $\operatorname{lghv1-20}$ & -2.22 & 0.001 \\
\hline Gm3453 & 1.50 & 0.036 & $\operatorname{lghv5-6}$ & -2.01 & 0.038 \\
\hline Gm13083 & 1.49 & 0.009 & lghv1-62-3 & -1.96 & 0.022 \\
\hline Gm10013 & 1.48 & 0.007 & Ighv1-85 & -1.81 & 0.041 \\
\hline Rrp7a & 1.48 & 0.002 & Zfp273 & -1.79 & 0.000 \\
\hline Rdh7 & 1.47 & 0.022 & LOC102640468 & -1.67 & 0.005 \\
\hline Mir296 & 1.47 & 0.020 & LOC102633000 & -1.63 & 0.013 \\
\hline Mir3475 & 1.45 & 0.027 & Cxc/13 & -1.63 & 0.010 \\
\hline LOC432842 & 1.45 & 0.008 & LOC102632343 & -1.62 & 0.038 \\
\hline Dppa4 & 1.44 & 0.017 & BB287469 & -1.60 & 0.011 \\
\hline LOC102639645 & 1.43 & 0.006 & $B l k$ & -1.57 & 0.004 \\
\hline Dcpp3 & 1.41 & 0.012 & Ighv1-69 & -1.55 & 0.031 \\
\hline lgkv3-9 & 1.41 & 0.027 & Pilrb1 & -1.53 & 0.005 \\
\hline LOC102637846 & 1.41 & 0.004 & Gm15527 & -1.51 & 0.019 \\
\hline Gm15448 & 1.39 & 0.032 & Igkv8-26 & -1.49 & 0.009 \\
\hline Spin2f & 1.38 & 0.047 & Vmn1r9 & -1.48 & 0.004 \\
\hline
\end{tabular}




\begin{tabular}{|c|c|c|c|c|c|}
\hline Vmn1r94 & 1.38 & 0.013 & Olfr706 & -1.47 & 0.024 \\
\hline Olfr1085 & 1.37 & 0.033 & Mucl1 & -1.47 & 0.007 \\
\hline Gm13271 & 1.37 & 0.003 & Scgb2b20 & -1.46 & 0.007 \\
\hline Gm1604A & 1.37 & 0.033 & $\mathrm{Cd} 4$ & -1.46 & 0.000 \\
\hline Serpina $3 m$ & 1.37 & 0.008 & Cc/12 & -1.44 & 0.036 \\
\hline$M n \times 1$ & 1.37 & 0.023 & LOC102631976 & -1.44 & 0.018 \\
\hline LOC102639351 & 1.36 & 0.001 & LOC102636795 & -1.44 & 0.008 \\
\hline Defb15 & 1.36 & 0.002 & LOC102631889 & -1.42 & 0.039 \\
\hline Gm4871 & 1.36 & 0.034 & Sarnp & -1.42 & 0.016 \\
\hline LOC102632332 & 1.36 & 0.048 & Olfr1487 & -1.41 & 0.023 \\
\hline Olfr691 & 1.36 & 0.025 & Cntnap5a & -1.41 & 0.045 \\
\hline Zfp787 & 1.36 & 0.002 & F830016B08Rik & -1.41 & 0.029 \\
\hline Trim43c & 1.36 & 0.037 & Olfr870 & -1.40 & 0.040 \\
\hline Orm2 & 1.36 & 0.038 & Olfr875 & -1.40 & 0.049 \\
\hline Gm15032 & 1.36 & 0.039 & Ctsr & -1.40 & 0.029 \\
\hline Gm17767 & 1.35 & 0.046 & $C d 72$ & -1.38 & 0.032 \\
\hline 2810039B14Rik & 1.35 & 0.033 & Tnip3 & -1.38 & 0.045 \\
\hline Gm10046 & 1.35 & 0.016 & LOC102635521 & -1.38 & 0.036 \\
\hline AV320801 & 1.35 & 0.023 & LOC102634822 & -1.37 & 0.004 \\
\hline Zfp772 & 1.34 & 0.004 & Socs3 & -1.37 & 0.029 \\
\hline Gm15217 & 1.34 & 0.014 & Gm4841 & -1.37 & 0.029 \\
\hline LOC102640477 & 1.34 & 0.022 & Traj37 & -1.36 & 0.030 \\
\hline Tpt1 & 1.34 & 0.028 & Pcdhgb4 & -1.36 & 0.015 \\
\hline Slc22a30 & 1.33 & 0.012 & Clec2g & -1.36 & 0.014 \\
\hline Olfr867 & 1.33 & 0.000 & LOC102634296 & -1.35 & 0.014 \\
\hline Gm4559 & 1.33 & 0.009 & Zfp867 & -1.34 & 0.028 \\
\hline Sult2a2 & 1.33 & 0.048 & Mir30d & -1.34 & 0.015 \\
\hline 5930403L14Rik & 1.33 & 0.008 & LOC102634742 & -1.34 & 0.012 \\
\hline Atp5o & 1.33 & 0.037 & 4930503B20Rik & -1.34 & 0.022 \\
\hline C1ql4 & 1.33 & 0.006 & Aoah & -1.34 & 0.041 \\
\hline Zfp442 & 1.32 & 0.043 & Abracl & -1.34 & 0.035 \\
\hline LOC102632316 & 1.32 & 0.037 & Ppih & -1.34 & 0.049 \\
\hline Olfr790 & 1.32 & 0.006 & XIr3a & -1.33 & 0.012 \\
\hline Mir3961 & 1.32 & 0.031 & 2310026/22Rik & -1.33 & 0.036 \\
\hline 2010106E10Rik & 1.32 & 0.031 & $E 2 f 2$ & -1.33 & 0.024 \\
\hline Snord64 & 1.32 & 0.016 & Tlr7 & -1.33 & 0.007 \\
\hline $2210010 C 04 R i k$ & 1.32 & 0.010 & Gm5347 & -1.33 & 0.047 \\
\hline Olfr1065 & 1.31 & 0.019 & Rp/28 & -1.33 & 0.049 \\
\hline Olfr981 & 1.31 & 0.008 & Gm19744 & -1.32 & 0.035 \\
\hline Olfr1167 & 1.31 & 0.009 & Ceacam14 & -1.32 & 0.003 \\
\hline Olfr1441 & 1.31 & 0.043 & Trav7-6 & -1.32 & 0.042 \\
\hline Vmn1r194 & 1.31 & 0.033 & Gm13272 & -1.32 & 0.032 \\
\hline Krt1 & 1.30 & 0.027 & Hells & -1.31 & 0.020 \\
\hline Olfri295 & 1.30 & 0.031 & LOC102634100 & -1.31 & 0.035 \\
\hline
\end{tabular}




\begin{tabular}{|c|c|c|c|c|c|}
\hline Nlrp9a & 1.30 & 0.006 & Krt6a & -1.31 & 0.013 \\
\hline Fgf15 & 1.30 & 0.005 & Cdkn2d & -1.31 & 0.003 \\
\hline Olfr1099 & 1.30 & 0.037 & Olfr1290 & -1.31 & 0.023 \\
\hline Cox8b & 1.30 & 0.035 & LOC102633228 & -1.31 & 0.007 \\
\hline Mir494 & 1.30 & 0.022 & Chil1 & -1.31 & 0.012 \\
\hline Cdsn & 1.30 & 0.031 & $\operatorname{lghv5-15}$ & -1.30 & 0.049 \\
\hline Gm13277 & 1.30 & 0.043 & Fgr & -1.30 & 0.008 \\
\hline Olfr1445 & 1.30 & 0.028 & Tas2r143 & -1.30 & 0.016 \\
\hline Olfr716 & 1.29 & 0.010 & Cenpk & -1.30 & 0.019 \\
\hline Olfr398 & 1.29 & 0.018 & LOC102633458 & -1.30 & 0.033 \\
\hline Olfr1361 & 1.29 & 0.019 & Hist1h2bn & -1.30 & 0.037 \\
\hline H2afy3 & 1.29 & 0.042 & Eif5a2 & -1.30 & 0.005 \\
\hline Accsl & 1.29 & 0.039 & Krtap19-9b & -1.30 & 0.016 \\
\hline Catsperg2 & 1.29 & 0.007 & Mir290a & -1.30 & 0.018 \\
\hline Olfr486 & 1.28 & 0.045 & LOC102632079 & -1.29 & 0.020 \\
\hline D830014E11Rik & 1.28 & 0.012 & S/c16a8 & -1.29 & 0.004 \\
\hline Proc & 1.28 & 0.011 & Vmn2r62 & -1.29 & 0.040 \\
\hline Olfr448 & 1.28 & 0.031 & Olfr1437 & -1.29 & 0.007 \\
\hline Slc2a2 & 1.28 & 0.017 & Hhex & -1.29 & 0.025 \\
\hline Vmn2r86 & 1.28 & 0.006 & 1830077J02Rik & -1.29 & 0.019 \\
\hline Krt8o & 1.28 & 0.038 & B3gat2 & -1.29 & 0.029 \\
\hline 2410007B07Rik & 1.28 & 0.017 & Rarb & -1.29 & 0.010 \\
\hline Ccdc177 & 1.28 & 0.017 & A330009N23Rik & -1.29 & 0.018 \\
\hline Gm6297 & 1.28 & 0.020 & Gimap8 & -1.29 & 0.024 \\
\hline C530044C16Rik & 1.28 & 0.002 & 5730522EO2Rik & -1.29 & 0.010 \\
\hline BC002059 & 1.28 & 0.040 & $\mathrm{Npl}$ & -1.29 & 0.012 \\
\hline Frmd3 & 1.28 & 0.008 & Pthlh & -1.28 & 0.007 \\
\hline Olfr628 & 1.28 & 0.011 & Clec $4 n$ & -1.28 & 0.014 \\
\hline Olfr967 & 1.28 & 0.006 & Olfr544 & -1.28 & 0.009 \\
\hline Olfr491 & 1.27 & 0.023 & Olfr1465 & -1.28 & 0.027 \\
\hline Olfr912 & 1.27 & 0.009 & $T k 1$ & -1.28 & 0.026 \\
\hline 2700038G22Rik & 1.27 & 0.038 & Gimap1 & -1.28 & 0.018 \\
\hline Gnmt & 1.27 & 0.042 & LOC102638504 & -1.28 & 0.020 \\
\hline Gemin8 & 1.27 & 0.013 & Mir350 & -1.28 & 0.010 \\
\hline Gm15638 & 1.27 & 0.022 & LOC102635305 & -1.27 & 0.011 \\
\hline LOC102638110 & 1.27 & 0.035 & Calm5 & -1.27 & 0.019 \\
\hline Snora75 & 1.27 & 0.021 & Tubg2 & -1.27 & 0.015 \\
\hline Gm765 & 1.27 & 0.002 & Trav8-1 & -1.27 & 0.030 \\
\hline Tsen2 & 1.27 & 0.028 & $\mathrm{Hmmr}$ & -1.27 & 0.002 \\
\hline Mir3081 & 1.27 & 0.049 & Gm6300 & -1.27 & 0.020 \\
\hline Tomm6os & 1.27 & 0.014 & Olfr1132 & -1.26 & 0.016 \\
\hline Gm14744 & 1.26 & 0.040 & 115 & -1.26 & 0.014 \\
\hline LOC102638623 & 1.26 & 0.045 & Chaf1a & -1.26 & 0.018 \\
\hline Tbxa2r & 1.26 & 0.024 & Cbln2 & -1.26 & 0.031 \\
\hline
\end{tabular}




\begin{tabular}{|c|c|c|c|c|c|}
\hline Ankrd60 & 1.26 & 0.007 & Olfr66 & -1.26 & 0.007 \\
\hline Gm10244 & 1.26 & 0.040 & Taf13 & -1.26 & 0.019 \\
\hline Olfr1109 & 1.26 & 0.048 & Gm2027 & -1.26 & 0.022 \\
\hline Zfp111 & 1.26 & 0.016 & Nxn/2 & -1.26 & 0.039 \\
\hline Rprml & 1.26 & 0.003 & Mir181b-1 & -1.26 & 0.047 \\
\hline Serpinb6c & 1.26 & 0.038 & Erdr1 & -1.26 & 0.010 \\
\hline Olfr328 & 1.26 & 0.027 & A530020G20Rik & -1.26 & 0.019 \\
\hline$X I r 5 c$ & 1.26 & 0.003 & Vmn1r45 & -1.25 & 0.004 \\
\hline Mir1929 & 1.26 & 0.033 & Trim52 & -1.25 & 0.014 \\
\hline Vmn2r91 & 1.26 & 0.004 & Slc5a4a & -1.25 & 0.023 \\
\hline Coprs & 1.25 & 0.019 & LOC102637926 & -1.25 & 0.019 \\
\hline 4930512B01Rik & 1.25 & 0.027 & 1700006J14Rik & -1.25 & 0.036 \\
\hline D630013N20Rik & 1.25 & 0.028 & Gm3250 & -1.25 & 0.014 \\
\hline Tha1 & 1.25 & 0.029 & Hes2 & -1.25 & 0.005 \\
\hline Zfp296 & 1.25 & 0.033 & TIr12 & -1.25 & 0.030 \\
\hline Zcwpw2 & 1.25 & 0.022 & Cstad & -1.25 & 0.045 \\
\hline Adarb1 & 1.25 & 0.024 & Gm11149 & -1.25 & 0.015 \\
\hline Olfr822 & 1.25 & 0.013 & Pcdhga7 & -1.25 & 0.030 \\
\hline LOC102634561 & 1.25 & 0.007 & Esp6Esp5 & -1.25 & 0.042 \\
\hline Cdk15 & 1.25 & 0.025 & $M s 4 a 4 d$ & -1.25 & 0.026 \\
\hline$D d n$ & 1.25 & 0.023 & Astn1 & -1.24 & 0.033 \\
\hline Tmem202 & 1.24 & 0.024 & Sox7 & -1.24 & 0.021 \\
\hline Zfp574 & 1.24 & 0.043 & Neil3 & -1.24 & 0.005 \\
\hline Spatc1l & 1.24 & 0.030 & 4930428G15Rik & -1.24 & 0.012 \\
\hline Emilin3 & 1.24 & 0.048 & Clra & -1.24 & 0.032 \\
\hline 5133400J02Rik & 1.24 & 0.004 & $B m p 8 b$ & -1.24 & 0.048 \\
\hline 1700063H04Rik & 1.24 & 0.044 & Gm9054 & -1.24 & 0.046 \\
\hline Rps11 & 1.24 & 0.023 & $F m n l 1$ & -1.24 & 0.013 \\
\hline 9330182O14Rik & 1.24 & 0.046 & Pramel1 & -1.24 & 0.008 \\
\hline Gm20742 & 1.24 & 0.010 & 5430435G22Rik & -1.24 & 0.043 \\
\hline Olfr1259 & 1.24 & 0.027 & Kcng2 & -1.24 & 0.023 \\
\hline Zc3hav1l & 1.24 & 0.009 & 9230112J17Rik & -1.24 & 0.033 \\
\hline Fitm1 & 1.24 & 0.033 & C5ar1 & -1.24 & 0.030 \\
\hline Unkl & 1.24 & 0.001 & LOC100503594 & -1.23 & 0.041 \\
\hline Olfr157 & 1.24 & 0.037 & lgfbp2 & -1.23 & 0.029 \\
\hline Olfr715 & 1.23 & 0.004 & Gpr144-ps & -1.23 & 0.038 \\
\hline Gm8221 & 1.23 & 0.031 & Gm11190 & -1.23 & 0.035 \\
\hline Gm10860 & 1.23 & 0.029 & Aida & -1.23 & 0.016 \\
\hline Foxe3 & 1.23 & 0.012 & Gpr176 & -1.23 & 0.044 \\
\hline Ube2cbp & 1.23 & 0.021 & Mir181d & -1.23 & 0.046 \\
\hline Pcgf1 & 1.23 & 0.001 & Zfp955a & -1.23 & 0.048 \\
\hline Olfr653 & 1.23 & 0.042 & LOC102640118 & -1.23 & 0.026 \\
\hline Olfr538 & 1.23 & 0.043 & Olfr1392 & -1.23 & 0.036 \\
\hline LOC102640858 & 1.23 & 0.048 & Dnah7c & -1.22 & 0.000 \\
\hline
\end{tabular}




\begin{tabular}{|c|c|c|c|c|c|}
\hline Npw & 1.23 & 0.023 & Gpm6b & -1.22 & 0.026 \\
\hline Gm6042 & 1.23 & 0.029 & Mir148b & -1.22 & 0.046 \\
\hline 2310034005Rik & 1.23 & 0.007 & Pirb & -1.22 & 0.041 \\
\hline Olfr1272 & 1.23 & 0.030 & Gatm & -1.22 & 0.028 \\
\hline $\operatorname{Sec} 14 / 3$ & 1.23 & 0.010 & Ttc26 & -1.22 & 0.010 \\
\hline Pr/3d1 & 1.23 & 0.017 & Mir344-2 & -1.22 & 0.006 \\
\hline Defb48 & 1.23 & 0.005 & Ret & -1.22 & 0.025 \\
\hline Gm3510 & 1.23 & 0.050 & Rcvrn & -1.22 & 0.038 \\
\hline Odf3 & 1.23 & 0.046 & Mir17 & -1.22 & 0.026 \\
\hline Upp2 & 1.23 & 0.020 & Klra10 & -1.22 & 0.043 \\
\hline Olfr1278 & 1.23 & 0.011 & Mir3097 & -1.22 & 0.032 \\
\hline Cisd3 & 1.23 & 0.020 & Lrrtm4 & -1.22 & 0.035 \\
\hline Olfr1000 & 1.23 & 0.017 & Sycp1-ps1 & -1.21 & 0.007 \\
\hline Pnliprp1 & 1.23 & 0.008 & Сур26с1 & -1.21 & 0.009 \\
\hline 4930465M20Rik & 1.23 & 0.018 & Aldh3b2 & -1.21 & 0.038 \\
\hline Slc6a4 & 1.23 & 0.020 & Vcam1 & -1.21 & 0.004 \\
\hline 4933404K08Rik & 1.23 & 0.014 & Esco2 & -1.21 & 0.013 \\
\hline LOC102633809 & 1.23 & 0.029 & 4930426LO9Rik & -1.21 & 0.013 \\
\hline Tymp & 1.23 & 0.043 & Neurog1 & -1.21 & 0.049 \\
\hline Olfr881 & 1.22 & 0.010 & Gm20815 & -1.21 & 0.034 \\
\hline Tmem132cos & 1.22 & 0.048 & Tnfsf14 & -1.21 & 0.011 \\
\hline Olfr1135 & 1.22 & 0.016 & Bcas3os2 & -1.21 & 0.024 \\
\hline Zfp879 & 1.22 & 0.022 & Dnajc5b & -1.21 & 0.035 \\
\hline Zfp703 & 1.22 & 0.040 & Pitx3 & -1.21 & 0.006 \\
\hline Olfr671 & 1.22 & 0.011 & Spock1 & -1.21 & 0.028 \\
\hline Gm4994 & 1.22 & 0.020 & Rnf157 & -1.21 & 0.048 \\
\hline Apol7a & 1.22 & 0.018 & Prss55 & -1.20 & 0.024 \\
\hline A730013G03Rik & 1.22 & 0.035 & AV064505 & -1.20 & 0.043 \\
\hline Ptges3 & 1.22 & 0.021 & Fam188b & -1.20 & 0.011 \\
\hline C77080 & 1.22 & 0.003 & LOC381967 & -1.20 & 0.006 \\
\hline Wdr66 & 1.22 & 0.040 & Olfr1393 & -1.20 & 0.047 \\
\hline B230398E01Rik & 1.22 & 0.041 & Htrld & -1.20 & 0.035 \\
\hline S/c5a11 & 1.22 & 0.013 & Mir130b & -1.20 & 0.048 \\
\hline Rsph3a & 1.21 & 0.048 & Pla2r1 & -1.20 & 0.004 \\
\hline Olfr272 & 1.21 & 0.019 & Krt86 & -1.20 & 0.039 \\
\hline LOC102639127 & 1.21 & 0.001 & & & \\
\hline Olfr113 & 1.21 & 0.036 & & & \\
\hline Histih4k & 1.21 & 0.047 & & & \\
\hline 8030453O22Rik & 1.21 & 0.032 & & & \\
\hline$D c c$ & 1.21 & 0.020 & & & \\
\hline Gm13034 & 1.21 & 0.017 & & & \\
\hline Sox8 & 1.21 & 0.026 & & & \\
\hline LOC102633930 & 1.21 & 0.035 & & & \\
\hline 1300017J02Rik & 1.21 & 0.009 & & & \\
\hline
\end{tabular}




$\begin{array}{lll}\text { CsI } & 1.21 & 0.031 \\ \text { H2-M1 } & 1.21 & 0.041 \\ \text { Olfr62 } & 1.21 & 0.019 \\ \text { LOC101056089 } & 1.21 & 0.006 \\ \text { Mef2b } & 1.21 & 0.041 \\ \text { 6430550D23Rik } & 1.21 & 0.006 \\ \text { Gm10389 } & 1.20 & 0.045 \\ \text { Gjd3 } & 1.20 & 0.011 \\ \text { LOC102637059 } & 1.20 & 0.040 \\ \text { Trim66 } & 1.20 & 0.003 \\ \text { LOC102635192 } & 1.20 & 0.015 \\ \text { Zbtb45 } & 1.20 & 0.029 \\ \text { Hoxd13 } & 1.20 & 0.030 \\ \text { LOC102638632 } & & 0.004\end{array}$

UP-REGULATED GENES ILEUM

\begin{tabular}{|c|c|c|}
\hline gene name & FC & p-value \\
\hline Lct & 8.11 & 0.005 \\
\hline Plb1 & 5.70 & 0.006 \\
\hline Dnase1 & 4.78 & 0.020 \\
\hline Ighv14-4 & 4.67 & 0.011 \\
\hline Fam151a & 4.34 & 0.011 \\
\hline Gm5549 & 4.08 & 0.005 \\
\hline Gata4 & 3.79 & 0.012 \\
\hline Ighv1-59 & 3.56 & 0.047 \\
\hline Asah2 & 3.46 & 0.006 \\
\hline S/c23a1 & 3.34 & 0.032 \\
\hline Gm11166 & 3.18 & 0.001 \\
\hline Kcnj13 & 3.14 & 0.008 \\
\hline Enpp7 & 3.05 & 0.004 \\
\hline S/c5a12 & 2.95 & 0.048 \\
\hline S/c5a4b & 2.83 & 0.006 \\
\hline Adh6a & 2.72 & 0.008 \\
\hline Cyp2b10 & 2.50 & 0.040 \\
\hline S/c28a1 & 2.26 & 0.033 \\
\hline Hsd17b6 & 2.26 & 0.018 \\
\hline Apol9b & 2.24 & 0.001 \\
\hline Ace & 2.16 & 0.016 \\
\hline Acot12 & 2.15 & 0.006 \\
\hline Gip & 2.15 & 0.033 \\
\hline
\end{tabular}

\section{DOWN-REGULATED GENES ILEUM}

$\begin{array}{lcc}\text { gene name } & \text { FC } & \text { p-value } \\ \text { Igkv5-43 } & -11.34 & 0.001 \\ \text { Igkv5-45 } & -7.58 & 0.004 \\ \text { Igkv4-59 } & -6.21 & 0.038 \\ \text { Slc6a14 } & -6.08 & 0.014 \\ \text { Ighv1-77 } & -5.15 & 0.007 \\ \text { Igkv8-21 } & -3.30 & 0.020 \\ \text { Igkv4-70 } & -3.22 & 0.029 \\ \text { Gm53 } & -2.92 & 0.043 \\ \text { Ighv1-58 } & -2.92 & 0.046 \\ \text { Hoxb9 } & -2.75 & 0.024 \\ \text { Mir196a-1 } & -2.63 & 0.047 \\ \text { 1810065E05Rik } & -2.59 & 0.007 \\ \text { Saa1 } & -2.57 & 0.047 \\ \text { Gm2964 } & -2.48 & 0.006 \\ \text { Igkv4-57 } & -2.48 & 0.016 \\ \text { Cyp2c55 } & -2.44 & 0.002 \\ \text { Ighv1-85 } & -2.37 & 0.042 \\ \text { S100g } & -2.21 & 0.010 \\ \text { Ces2b } & -2.20 & 0.037 \\ \text { Hoxb8 } & -2.20 & 0.003 \\ \text { Hoxb6 } & -2.18 & 0.040 \\ \text { Slc10a2 } & 0.030 \\ \text { Defb37 } & -2.09 & 0.013\end{array}$




\begin{tabular}{|c|c|c|c|c|c|}
\hline$T t / 12$ & 2.12 & 0.017 & Tat & -1.97 & 0.011 \\
\hline Sft2d1 & 2.08 & 0.019 & Tmigd1 & -1.92 & 0.021 \\
\hline S/c9a3 & 2.03 & 0.040 & B020014A21Rik & -1.89 & 0.000 \\
\hline Unc93a & 2.02 & 0.048 & S/c40a1 & -1.88 & 0.021 \\
\hline Apoa4 & 2.01 & 0.003 & Car1 & -1.88 & 0.035 \\
\hline Gm11651 & 2.00 & 0.008 & Abca12 & -1.88 & 0.009 \\
\hline Trim38 & 2.00 & 0.043 & Hoxb7 & -1.86 & 0.023 \\
\hline $\operatorname{Tm} 4 s f 5$ & 1.92 & 0.014 & Ifitm7 & -1.82 & 0.017 \\
\hline Adh4 & 1.90 & 0.015 & Gm15128 & -1.81 & 0.029 \\
\hline Acsm5 & 1.89 & 0.030 & Noxa1 & -1.79 & 0.001 \\
\hline Tymp & 1.77 & 0.011 & Sval1 & -1.79 & 0.000 \\
\hline Ms4a10 & 1.76 & 0.034 & 1700024P16Rik & -1.77 & 0.009 \\
\hline LOC102633000 & 1.76 & 0.006 & 5730507C01Rik & -1.77 & 0.016 \\
\hline S/c28a2 & 1.75 & 0.001 & Lpcat4 & -1.75 & 0.011 \\
\hline$C d a$ & 1.74 & 0.007 & 2010005H15Rik & -1.74 & 0.001 \\
\hline Rnu3a & 1.74 & 0.004 & Gmcl11 & -1.71 & 0.028 \\
\hline $\operatorname{Tm} 4 \mathrm{sf} 4$ & 1.74 & 0.021 & Gm15363 & -1.71 & 0.007 \\
\hline Rp/6 & 1.73 & 0.032 & Hoxb3 & -1.70 & 0.030 \\
\hline Pcdha1 & 1.73 & 0.029 & Lamb3 & -1.69 & 0.028 \\
\hline Mroh7 & 1.73 & 0.009 & Gm1965 & -1.67 & 0.002 \\
\hline Ano6 & 1.70 & 0.006 & $B c o 1$ & -1.67 & 0.018 \\
\hline Ptgr1 & 1.69 & 0.021 & Vmn1r91 & -1.66 & 0.025 \\
\hline Сур17a1 & 1.67 & 0.014 & 2610528A11Rik & -1.66 & 0.002 \\
\hline Rragd & 1.67 & 0.017 & $E p b 4.114 a$ & -1.66 & 0.016 \\
\hline Gm5431 & 1.64 & 0.001 & $\mathrm{Fa} 2 h$ & -1.66 & 0.035 \\
\hline Gm11517 & 1.64 & 0.014 & $\operatorname{Trgj} 3$ & -1.66 & 0.021 \\
\hline Nrii3 & 1.63 & 0.049 & Paqr5 & -1.65 & 0.018 \\
\hline LOC102634539 & 1.61 & 0.006 & Mup12 & -1.65 & 0.044 \\
\hline S/c16a10 & 1.60 & 0.020 & Gm21936 & -1.64 & 0.038 \\
\hline Gm6909 & 1.60 & 0.045 & Defb39 & -1.64 & 0.030 \\
\hline Olfr373 & 1.59 & 0.035 & Dhrs9 & -1.64 & 0.009 \\
\hline Enpp3 & 1.59 & 0.029 & Hao2 & -1.63 & 0.035 \\
\hline Gm11127 & 1.59 & 0.026 & Cldn8 & -1.62 & 0.007 \\
\hline Smpdl/3b & 1.59 & 0.014 & Plscr2 & -1.61 & 0.032 \\
\hline Pcdha11 & 1.59 & 0.004 & LOC102632682 & -1.59 & 0.028 \\
\hline Efr $3 b$ & 1.59 & 0.020 & Nptxr & -1.59 & 0.009 \\
\hline Slc7a8 & 1.58 & 0.015 & Gm3248 & -1.59 & 0.014 \\
\hline Gm6897 & 1.58 & 0.004 & Gm10505 & -1.58 & 0.007 \\
\hline Kcnj2 & 1.58 & 0.028 & $H s d 3 b 3$ & -1.57 & 0.004 \\
\hline Gpr39 & 1.57 & 0.016 & Acaa1b & -1.57 & 0.004 \\
\hline LOC102633565 & 1.56 & 0.034 & Cd177 & -1.56 & 0.019 \\
\hline /133 & 1.56 & 0.030 & Gm14327 & -1.55 & 0.003 \\
\hline Anxa13 & 1.55 & 0.013 & Hmgcs2 & -1.55 & 0.034 \\
\hline Gm14488 & 1.55 & 0.006 & Vmn1r159 & -1.54 & 0.006 \\
\hline
\end{tabular}




\begin{tabular}{|c|c|c|c|c|c|}
\hline LOC102639648 & 1.55 & 0.009 & Cpn1 & -1.54 & 0.046 \\
\hline Snora73a & 1.55 & 0.001 & Gm4907 & -1.53 & 0.018 \\
\hline Hзғза & 1.54 & 0.032 & Scd2 & -1.52 & 0.010 \\
\hline Сур4b1 & 1.54 & 0.018 & Ankrd37 & -1.51 & 0.046 \\
\hline Gm14920 & 1.52 & 0.018 & Zfp944 & -1.50 & 0.019 \\
\hline $\mathrm{Npl}$ & 1.52 & 0.002 & Nr1h4 & -1.50 & 0.012 \\
\hline$H 2-Q 2$ & 1.52 & 0.004 & Сур51 & -1.50 & 0.001 \\
\hline$H 2-Q 1$ & 1.52 & 0.021 & Mir124a-2 & -1.49 & 0.024 \\
\hline Сур 4 v3 & 1.52 & 0.021 & lgkv4-56 & -1.49 & 0.013 \\
\hline 1700110K17Rik & 1.51 & 0.017 & Slc13a1 & -1.48 & 0.031 \\
\hline Gm10229 & 1.51 & 0.047 & $A b c b 1 a$ & -1.48 & 0.010 \\
\hline S/c2a2 & 1.51 & 0.019 & Sgk2 & -1.48 & 0.037 \\
\hline Creb3/2 & 1.51 & 0.043 & $\operatorname{Reg} 3 g$ & -1.48 & 0.009 \\
\hline Mpp4 & 1.50 & 0.013 & LOC102640468 & -1.48 & 0.008 \\
\hline Arl14 & 1.50 & 0.038 & Slfn4 & -1.47 & 0.005 \\
\hline 2010109/03Rik & 1.49 & 0.005 & Car12 & -1.47 & 0.039 \\
\hline Snora44 & 1.49 & 0.005 & Vmn1r94 & -1.47 & 0.029 \\
\hline Car13 & 1.49 & 0.031 & Gm4971 & -1.46 & 0.001 \\
\hline LOC101055745 & 1.49 & 0.049 & Cutal & -1.46 & 0.024 \\
\hline Dnpep & 1.48 & 0.009 & LOC102640140 & -1.45 & 0.045 \\
\hline Bst1 & 1.48 & 0.005 & $\operatorname{Reg} 3 b$ & -1.45 & 0.004 \\
\hline Rdh7 7 & 1.47 & 0.005 & Serpina1b & -1.45 & 0.046 \\
\hline Spns2 & 1.47 & 0.010 & Acot2 & -1.45 & 0.003 \\
\hline Pisd-ps1 & 1.47 & 0.012 & LOC102637408 & -1.45 & 0.049 \\
\hline LOC102638051 & 1.46 & 0.027 & Olfr1046 & -1.44 & 0.002 \\
\hline lgkv3-9 & 1.46 & 0.033 & B3gnt6 & -1.44 & 0.004 \\
\hline Pld2 & 1.46 & 0.004 & Tspan1 & -1.44 & 0.006 \\
\hline Soat2 & 1.46 & 0.016 & Hsd3b2 & -1.44 & 0.023 \\
\hline 1810055G02Rik & 1.45 & 0.017 & Gm2464 & -1.44 & 0.039 \\
\hline Rp/35a & 1.45 & 0.026 & Fdft1 & -1.44 & 0.019 \\
\hline SIc25a45 & 1.45 & 0.023 & Sult1c2 & -1.43 & 0.008 \\
\hline Cox5b & 1.45 & 0.010 & Fgf10 & -1.43 & 0.004 \\
\hline Maob & 1.44 & 0.020 & Gm2666 & -1.43 & 0.046 \\
\hline LOC102634389 & 1.44 & 0.028 & Hsd17b13 & -1.43 & 0.014 \\
\hline S/c7a15 & 1.44 & 0.041 & St6galnac6 & -1.43 & 0.001 \\
\hline LOC102632388 & 1.44 & 0.004 & 117 & -1.43 & 0.001 \\
\hline $\mathrm{Cm} / 1$ & 1.43 & 0.036 & Ddah1 & -1.43 & 0.021 \\
\hline Gramd1b & 1.43 & 0.003 & MettI7b & -1.43 & 0.026 \\
\hline $\mathrm{Hmgb2}$ & 1.43 & 0.004 & Sema3c & -1.42 & 0.015 \\
\hline Edn3 & 1.43 & 0.002 & $\| d r 1$ & -1.42 & 0.021 \\
\hline Atp7b & 1.42 & 0.014 & Gm13034 & -1.42 & 0.015 \\
\hline Bche & 1.42 & 0.007 & Cldn2 & -1.41 & 0.029 \\
\hline Snora5c & 1.41 & 0.000 & Endod1 & -1.41 & 0.003 \\
\hline Dusp6 & 1.41 & 0.008 & $Z f p 2$ & -1.41 & 0.013 \\
\hline
\end{tabular}




\begin{tabular}{|c|c|c|c|c|c|}
\hline S/c15a1 & 1.41 & 0.010 & Renbp & -1.41 & 0.047 \\
\hline Cdr2 & 1.41 & 0.033 & Prss23 & -1.41 & 0.016 \\
\hline D130043K22Rik & 1.41 & 0.041 & $A A 467197$ & -1.40 & 0.037 \\
\hline S/c5a11 & 1.41 & 0.032 & Olfr160 & -1.40 & 0.043 \\
\hline Snord104 & 1.41 & 0.009 & Mboat1 & -1.40 & 0.036 \\
\hline Anxa4 & 1.41 & 0.009 & Gm6568 & -1.40 & 0.013 \\
\hline Trp53rk & 1.41 & 0.015 & Neu1 & -1.40 & 0.033 \\
\hline Mir6516 & 1.41 & 0.002 & Mir1946a & -1.40 & 0.013 \\
\hline Tmem86a & 1.41 & 0.022 & Ankef1 & -1.39 & 0.008 \\
\hline Pcdhgb4 & 1.41 & 0.007 & Tmem171 & -1.39 & 0.030 \\
\hline Klkb1 & 1.41 & 0.018 & Histih3h & -1.39 & 0.008 \\
\hline Snora61 & 1.40 & 0.009 & Gm2933 & -1.39 & 0.046 \\
\hline 2010107G12Rik & 1.40 & 0.003 & Ffar 4 & -1.38 & 0.004 \\
\hline S/c4a5 & 1.40 & 0.048 & Olfr1175-ps & -1.38 & 0.049 \\
\hline Usp35 & 1.40 & 0.019 & LOC102636378 & -1.38 & 0.005 \\
\hline$N r n 1$ & 1.40 & 0.011 & Gm2825 & -1.38 & 0.007 \\
\hline Rbp2 & 1.40 & 0.011 & Mir7-1 & -1.38 & 0.008 \\
\hline Rap1gapos & 1.40 & 0.003 & Trpm6 & -1.37 & 0.046 \\
\hline Pxdc1 & 1.40 & 0.007 & Nkain1 & -1.37 & 0.018 \\
\hline LOC102639005 & 1.40 & 0.002 & $K \mid k 1$ & -1.37 & 0.001 \\
\hline Tbc1d24 & 1.40 & 0.005 & Atp2b1 & -1.37 & 0.014 \\
\hline LOC102636360 & 1.39 & 0.042 & Ms4a5 & -1.36 & 0.015 \\
\hline Snora16a & 1.39 & 0.014 & Gcg & -1.36 & 0.010 \\
\hline LOC102640635 & 1.39 & 0.025 & Pcsk9 & -1.36 & 0.001 \\
\hline Oas3 & 1.39 & 0.003 & 0610040B10Rik & -1.36 & 0.022 \\
\hline Gm10471 & 1.39 & 0.026 & Bex1 & -1.36 & 0.001 \\
\hline Mir5104 & 1.39 & 0.017 & Spsb4 & -1.36 & 0.035 \\
\hline Usp17/b & 1.39 & 0.036 & Vmn1r59 & -1.36 & 0.022 \\
\hline Gprc5a & 1.39 & 0.001 & Zfp595 & -1.36 & 0.003 \\
\hline Snora34 & 1.39 & 0.026 & Cesif & -1.36 & 0.036 \\
\hline Ngp & 1.39 & 0.007 & Prkaa2 & -1.36 & 0.005 \\
\hline Eepd1 & 1.39 & 0.006 & Gnpnat1 & -1.36 & 0.002 \\
\hline Mir3081 & 1.39 & 0.016 & Baat & -1.35 & 0.014 \\
\hline Rhou & 1.38 & 0.002 & $\operatorname{lns} / 5$ & -1.35 & 0.046 \\
\hline Abca1 & 1.38 & 0.020 & Tssk3 & -1.35 & 0.033 \\
\hline Samd8 & 1.38 & 0.004 & $A b c g 2$ & -1.35 & 0.017 \\
\hline Hsf5 & 1.38 & 0.008 & Klrb1-ps1 & -1.35 & 0.020 \\
\hline Fam104a & 1.38 & 0.004 & Smok3a & -1.35 & 0.017 \\
\hline Olfr170 & 1.37 & 0.040 & LOC102640477 & -1.35 & 0.011 \\
\hline Olfr981 & 1.37 & 0.007 & Dmbt1 & -1.35 & 0.010 \\
\hline Sgp/1 & 1.37 & 0.020 & Gm15446 & -1.35 & 0.019 \\
\hline Slc14a1 & 1.37 & 0.012 & Npy4r & -1.35 & 0.008 \\
\hline Scarna6 & 1.37 & 0.010 & Slc44a4 & -1.34 & 0.020 \\
\hline Trbv12-1 & 1.37 & 0.001 & Mir1905 & -1.34 & 0.030 \\
\hline
\end{tabular}




\begin{tabular}{|c|c|c|c|c|c|}
\hline Ido1 & 1.37 & 0.001 & LOC102636235 & -1.34 & 0.045 \\
\hline Nek6 & 1.37 & 0.012 & Sqle & -1.34 & 0.044 \\
\hline Gpr155 & 1.37 & 0.032 & S/c2a13 & -1.34 & 0.007 \\
\hline$E p b 4.113$ & 1.36 & 0.010 & Acat2 & -1.34 & 0.001 \\
\hline Hen1 & 1.36 & 0.007 & Retnlb & -1.34 & 0.006 \\
\hline Zfpm1 & 1.36 & 0.047 & Trav14-1 & -1.33 & 0.027 \\
\hline Magea1 & 1.36 & 0.025 & Zfp560 & -1.33 & 0.003 \\
\hline Tdgf1 & 1.36 & 0.018 & Rnasel & -1.33 & 0.012 \\
\hline Homer2 & 1.35 & 0.014 & $F d x 11$ & -1.33 & 0.012 \\
\hline LOC102639455 & 1.35 & 0.014 & 2410012M07Rik & -1.33 & 0.014 \\
\hline Aldh4a1 & 1.35 & 0.003 & Plscr4 & -1.33 & 0.035 \\
\hline Acy1 & 1.35 & 0.024 & $J m j d 7$ & -1.33 & 0.005 \\
\hline Sec14/2 & 1.35 & 0.033 & Hmgcs1 & -1.33 & 0.015 \\
\hline Snora15 & 1.34 & 0.028 & Nipal2 & -1.33 & 0.001 \\
\hline Snora52 & 1.34 & 0.008 & Slc30a1 & -1.33 & 0.046 \\
\hline LOC102632661 & 1.34 & 0.048 & Al450353 & -1.33 & 0.010 \\
\hline Gpx2 & 1.34 & 0.003 & Grpr & -1.32 & 0.003 \\
\hline Xpnpep1 & 1.34 & 0.007 & Vsig4 & -1.32 & 0.024 \\
\hline Olfr506 & 1.34 & 0.037 & C1qtnf3 & -1.32 & 0.001 \\
\hline Ptgdr & 1.34 & 0.031 & Mir194-2 & -1.31 & 0.005 \\
\hline Snora73b & 1.34 & 0.005 & Prss 30 & -1.31 & 0.009 \\
\hline Mical1 & 1.34 & 0.029 & Ccl28 & -1.31 & 0.008 \\
\hline Gda & 1.33 & 0.006 & Olfr887 & -1.31 & 0.037 \\
\hline Bco2 & 1.33 & 0.044 & Akap17b & -1.31 & 0.009 \\
\hline Defa-ps13 & 1.33 & 0.005 & Mageb5 & -1.31 & 0.029 \\
\hline$A b c c 2$ & 1.33 & 0.031 & Sqrdl & -1.31 & 0.019 \\
\hline Krt12 & 1.32 & 0.007 & $M f s d 4$ & -1.31 & 0.007 \\
\hline Als2 & 1.32 & 0.044 & Sst & -1.30 & 0.038 \\
\hline Aldh1a3 & 1.32 & 0.003 & LOC102635261 & -1.30 & 0.020 \\
\hline Zfp820 & 1.32 & 0.007 & Itga2 & -1.30 & 0.006 \\
\hline A230103J11Rik & 1.32 & 0.007 & Slc51b & -1.30 & 0.017 \\
\hline Traj5 & 1.32 & 0.024 & Fam161a & -1.30 & 0.017 \\
\hline B4galt5 & 1.32 & 0.041 & Oxct1 & -1.30 & 0.008 \\
\hline Vwa1 & 1.32 & 0.005 & Mir5103 & -1.30 & 0.005 \\
\hline Entpd7 & 1.32 & 0.036 & LOC102632994 & -1.30 & 0.028 \\
\hline 2010003K11Rik & 1.32 & 0.019 & Glul & -1.30 & 0.016 \\
\hline Tmem86b & 1.32 & 0.043 & Ube2dn/1 & -1.30 & 0.046 \\
\hline Pcyt1a & 1.32 & 0.011 & Fbp1 & -1.30 & 0.028 \\
\hline Gm17762 & 1.32 & 0.042 & Gm17619 & -1.30 & 0.002 \\
\hline 1700019G17Rik & 1.32 & 0.010 & Gm13139 & -1.30 & 0.007 \\
\hline LOC102637966 & 1.31 & 0.018 & Msmo1 & -1.30 & 0.036 \\
\hline II27ra & 1.31 & 0.031 & Dlk2 & -1.29 & 0.028 \\
\hline Rnu73b & 1.31 & 0.020 & Сур2c54 & -1.29 & 0.024 \\
\hline Gm5965 & 1.31 & 0.044 & Vmn1r194 & -1.29 & 0.009 \\
\hline
\end{tabular}




\begin{tabular}{|c|c|c|c|c|c|}
\hline Pdcd5 & 1.31 & 0.032 & Klk1b5 & -1.29 & 0.035 \\
\hline Gm13102 & 1.31 & 0.013 & 2610044015Rik8 & -1.29 & 0.005 \\
\hline 2610018G03Rik & 1.31 & 0.014 & Plscr1 & -1.29 & 0.008 \\
\hline Trim $30 b$ & 1.31 & 0.020 & Gm6251 & -1.29 & 0.020 \\
\hline Clca6 & 1.31 & 0.001 & Pdgfc & -1.29 & 0.021 \\
\hline Creb3/3 & 1.30 & 0.011 & 2410141K09Rik & -1.29 & 0.009 \\
\hline$S g k 3$ & 1.30 & 0.020 & $A b c c 3$ & -1.29 & 0.003 \\
\hline Agmo & 1.30 & 0.039 & Olfr1099 & -1.29 & 0.045 \\
\hline Aqp1 & 1.30 & 0.014 & Efcab1 & -1.29 & 0.040 \\
\hline Adipor2 & 1.30 & 0.011 & Gm16525 & -1.29 & 0.044 \\
\hline Odf3b & 1.30 & 0.044 & Npm1 & -1.28 & 0.001 \\
\hline 1700003H04Rik & 1.30 & 0.003 & Ly6a & -1.28 & 0.008 \\
\hline Adh1 & 1.30 & 0.022 & Gm21319 & -1.28 & 0.006 \\
\hline Сурза13 & 1.30 & 0.018 & Insig1 & -1.28 & 0.010 \\
\hline $112 r b$ & 1.30 & 0.001 & $H b b-b s$ & -1.28 & 0.019 \\
\hline Mir3968 & 1.30 & 0.022 & Frrs11 & -1.28 & 0.046 \\
\hline Snora70 & 1.30 & 0.040 & B630019K06Rik & -1.28 & 0.039 \\
\hline Snora31 & 1.30 & 0.031 & Sprr2b & -1.28 & 0.039 \\
\hline LOC102639182 & 1.30 & 0.013 & Zfp599 & -1.28 & 0.003 \\
\hline Olfr1368 & 1.30 & 0.034 & Hhip/1 & -1.27 & 0.018 \\
\hline Hklos & 1.30 & 0.029 & Fgfbp1 & -1.27 & 0.004 \\
\hline Olfr338 & 1.30 & 0.013 & Vmn1r63 & -1.27 & 0.020 \\
\hline 9530003J23Rik & 1.30 & 0.031 & Plat & -1.27 & 0.044 \\
\hline Snord $49 b$ & 1.30 & 0.008 & Baalc & -1.27 & 0.040 \\
\hline Olfr299 & 1.30 & 0.002 & Rac3 & -1.27 & 0.003 \\
\hline Fbln1 & 1.30 & 0.044 & Aoah & -1.27 & 0.027 \\
\hline Snord15b & 1.29 & 0.032 & Tc2n & -1.27 & 0.010 \\
\hline Nme5 & 1.29 & 0.008 & Olfr522 & -1.27 & 0.033 \\
\hline C86187 & 1.29 & 0.004 & Gpx7 & -1.27 & 0.023 \\
\hline Al507597 & 1.29 & 0.046 & P4ha3 & -1.27 & 0.032 \\
\hline Daf2 & 1.29 & 0.013 & Klri2 & -1.27 & 0.022 \\
\hline$L y 75$ & 1.29 & 0.020 & Shc3 & -1.27 & 0.004 \\
\hline Kif27 & 1.29 & 0.005 & 9930012K11Rik & -1.27 & 0.032 \\
\hline Apol10a & 1.29 & 0.047 & Tatdn3 & -1.27 & 0.005 \\
\hline Npc111 & 1.29 & 0.006 & Gm13403 & -1.27 & 0.024 \\
\hline Zfp709 & 1.29 & 0.008 & Ampd3 & -1.27 & 0.018 \\
\hline LOC102635682 & 1.29 & 0.040 & Pyy & -1.27 & 0.010 \\
\hline LOC102636239 & 1.29 & 0.025 & Pdzklip1 & -1.26 & 0.020 \\
\hline Eno3 & 1.29 & 0.003 & Gm12814 & -1.26 & 0.036 \\
\hline Dynl/1 & 1.29 & 0.003 & $\mathrm{Ccl} / 8$ & -1.26 & 0.015 \\
\hline Zfp873 & 1.29 & 0.022 & lapp & -1.26 & 0.014 \\
\hline Ugt1a9 & 1.29 & 0.048 & Opn3 & -1.26 & 0.008 \\
\hline S/c43a2 & 1.29 & 0.031 & B4galt4 & -1.26 & 0.004 \\
\hline Fbxo47 & 1.29 & 0.023 & Clatnf9 & -1.26 & 0.015 \\
\hline
\end{tabular}




\begin{tabular}{|c|c|c|c|c|c|}
\hline Faah & 1.29 & 0.041 & Rpa3 & -1.26 & 0.017 \\
\hline Gas2/2 & 1.28 & 0.021 & Gm6642 & -1.25 & 0.018 \\
\hline Gfod1 & 1.28 & 0.005 & Ly96 & -1.25 & 0.005 \\
\hline Mir1896 & 1.28 & 0.043 & Gm10706 & -1.25 & 0.043 \\
\hline Mir3109 & 1.28 & 0.011 & Gm5595 & -1.25 & 0.039 \\
\hline Olfrio & 1.28 & 0.001 & Gm15638 & -1.25 & 0.038 \\
\hline Ly6g6d & 1.28 & 0.019 & Gm10349 & -1.25 & 0.022 \\
\hline Dpcr1 & 1.28 & 0.042 & Nbea & -1.25 & 0.027 \\
\hline Ifna15 & 1.28 & 0.022 & Gm16497 & -1.25 & 0.002 \\
\hline Vwce & 1.28 & 0.041 & Upk1a & -1.25 & 0.030 \\
\hline Map3k6 & 1.28 & 0.028 & 4931406C07Rik & -1.24 & 0.028 \\
\hline AW011738 & 1.28 & 0.007 & Cd160 & -1.24 & 0.022 \\
\hline Tmem179 & 1.28 & 0.004 & Gabarap/2 & -1.24 & 0.033 \\
\hline S/c25a15 & 1.28 & 0.006 & Pglyrp1 & -1.24 & 0.012 \\
\hline Arl5c & 1.28 & 0.035 & Zfp935 & -1.24 & 0.005 \\
\hline$M f s d 7 b$ & 1.28 & 0.024 & Nsdhl & -1.24 & 0.011 \\
\hline$W d p c p$ & 1.28 & 0.025 & Gm16287 & -1.24 & 0.030 \\
\hline Tmem151a & 1.27 & 0.027 & Mir181d & -1.24 & 0.000 \\
\hline Tmem144 & 1.27 & 0.008 & Bcas3os1 & -1.24 & 0.037 \\
\hline Gm15941 & 1.27 & 0.049 & 6330411D24Rik & -1.24 & 0.020 \\
\hline Adam39 & 1.27 & 0.012 & Gm8526 & -1.24 & 0.022 \\
\hline Frmd8os & 1.27 & 0.035 & Cyr61 & -1.24 & 0.041 \\
\hline S/c36a1 & 1.27 & 0.042 & Dhcr7 & -1.24 & 0.007 \\
\hline$A b c d 1$ & 1.27 & 0.011 & Gm4884 & -1.24 & 0.030 \\
\hline Olfr325 & 1.27 & 0.006 & 2700097009Rik & -1.24 & 0.029 \\
\hline Rsad1 & 1.27 & 0.003 & $A / 314278$ & -1.24 & 0.005 \\
\hline Ada & 1.27 & 0.040 & Edil3 & -1.24 & 0.040 \\
\hline Ptdss1 & 1.27 & 0.012 & Oit1 & -1.24 & 0.023 \\
\hline Gm13580 & 1.27 & 0.001 & Stard5 & -1.24 & 0.029 \\
\hline Cryge & 1.27 & 0.003 & Sct & -1.24 & 0.017 \\
\hline Slc39a2 & 1.27 & 0.039 & Stox 1 & -1.24 & 0.016 \\
\hline Pabpc6 & 1.27 & 0.020 & Olfr1465 & -1.24 & 0.018 \\
\hline LOC102638933 & 1.27 & 0.017 & Gm15455 & -1.24 & 0.041 \\
\hline Gm4454 & 1.27 & 0.041 & Gm3336 & -1.24 & 0.042 \\
\hline Gm10451 & 1.26 & 0.009 & Fam171a2 & -1.23 & 0.001 \\
\hline Mtmr4 & 1.26 & 0.005 & Dio2 & -1.23 & 0.047 \\
\hline Trib3 & 1.26 & 0.028 & Lss & -1.23 & 0.022 \\
\hline $\operatorname{Sec} 24 d$ & 1.26 & 0.037 & S/c5a8 & -1.23 & 0.014 \\
\hline Mir1983 & 1.26 & 0.010 & H2-M10.6 & -1.23 & 0.013 \\
\hline Dlg3 & 1.26 & 0.012 & Gent3 & -1.23 & 0.043 \\
\hline Acbd4 & 1.26 & 0.049 & A4gnt & -1.23 & 0.004 \\
\hline Lta & 1.26 & 0.026 & Chrm4 & -1.23 & 0.016 \\
\hline Gm15217 & 1.26 & 0.034 & $C d 9$ & -1.23 & 0.020 \\
\hline Olfr1418 & 1.26 & 0.041 & Fam221b & -1.23 & 0.047 \\
\hline
\end{tabular}




\begin{tabular}{|c|c|c|c|c|c|}
\hline Gata5 & 1.26 & 0.003 & 1700003F12Rik & -1.23 & 0.014 \\
\hline Ormdl3 & 1.26 & 0.008 & Slc25a4 & -1.23 & 0.044 \\
\hline 9130230L23Rik & 1.26 & 0.007 & Gm15292 & -1.23 & 0.041 \\
\hline Trim12a & 1.26 & 0.019 & Mir2136 & -1.23 & 0.005 \\
\hline Olfr1155 & 1.26 & 0.006 & 4930428N03Rik & -1.23 & 0.032 \\
\hline 1700010K23Rik & 1.26 & 0.001 & 9130024F11Rik & -1.23 & 0.048 \\
\hline$V m n 2 r 1$ & 1.26 & 0.048 & Cox20 & -1.23 & 0.003 \\
\hline Sec16b & 1.25 & 0.016 & Gsta4 & -1.23 & 0.007 \\
\hline Tas2r107 & 1.25 & 0.043 & Vcan & -1.23 & 0.007 \\
\hline Gm7030 & 1.25 & 0.016 & Myh4 & -1.23 & 0.048 \\
\hline Mett/13 & 1.25 & 0.018 & Pcdhb14 & -1.23 & 0.040 \\
\hline Olfr586 & 1.25 & 0.021 & Pnoc & -1.23 & 0.018 \\
\hline A230020J21Rik & 1.25 & 0.005 & Ldlr & -1.23 & 0.017 \\
\hline Zfp235 & 1.25 & 0.039 & Gm14295 & -1.23 & 0.047 \\
\hline Olfr539 & 1.25 & 0.013 & Olfr1360 & -1.22 & 0.031 \\
\hline Ifi27| $2 b$ & 1.25 & 0.032 & Ctnna3 & -1.22 & 0.028 \\
\hline $\operatorname{lgf2}$ & 1.25 & 0.025 & Mctp2 & -1.22 & 0.015 \\
\hline Ss $\times 9$ & 1.25 & 0.008 & Gm5947 & -1.22 & 0.011 \\
\hline Cerk & 1.25 & 0.047 & Olfr994 & -1.22 & 0.032 \\
\hline Snord16a & 1.25 & 0.033 & 4930572O13Rik & -1.22 & 0.024 \\
\hline Prss2 & 1.25 & 0.033 & Khdrbs3 & -1.22 & 0.019 \\
\hline Mme & 1.25 & 0.015 & Mid2 & -1.22 & 0.012 \\
\hline Star & 1.25 & 0.005 & Ube2v2 & -1.22 & 0.045 \\
\hline Pisd-ps2 & 1.25 & 0.024 & Cracr $2 b$ & -1.22 & 0.013 \\
\hline 4930503H13Rik & 1.25 & 0.008 & Mir759 & -1.22 & 0.006 \\
\hline Snord55 & 1.25 & 0.017 & Olfr221 & -1.22 & 0.037 \\
\hline A230057D06Rik & 1.25 & 0.005 & Rassf9 & -1.22 & 0.031 \\
\hline 2610020F03Rik & 1.25 & 0.010 & Gpx3 & -1.22 & 0.017 \\
\hline Ces2f & 1.24 & 0.030 & S/c22a29 & -1.22 & 0.031 \\
\hline 1700001J03Rik & 1.24 & 0.036 & Olfr1019 & -1.22 & 0.037 \\
\hline Ap5b1 & 1.24 & 0.014 & Hoxd8 & -1.22 & 0.034 \\
\hline D630033011Rik & 1.24 & 0.015 & Robo2 & -1.22 & 0.040 \\
\hline Gm4221 & 1.24 & 0.048 & Vmn2r56 & -1.22 & 0.018 \\
\hline Serpina11 & 1.24 & 0.004 & Nr5a1 & -1.22 & 0.020 \\
\hline Cyb5r3 & 1.24 & 0.029 & S/c39a12 & -1.22 & 0.019 \\
\hline Rag1 & 1.24 & 0.026 & Zfp429 & -1.22 & 0.045 \\
\hline Sh2d1a & 1.24 & 0.034 & D730048I06Rik & -1.22 & 0.026 \\
\hline 2610318N02Rik & 1.24 & 0.005 & Reg4 & -1.22 & 0.009 \\
\hline Obox6 & 1.24 & 0.025 & Olfr1008 & -1.22 & 0.045 \\
\hline Gm12279 & 1.24 & 0.022 & LOC100862214 & -1.21 & 0.040 \\
\hline Vmn1r86 & 1.24 & 0.011 & Gm10639 & -1.21 & 0.019 \\
\hline Slc22a4 & 1.24 & 0.048 & Zfp930 & -1.21 & 0.010 \\
\hline Kcnj15 & 1.24 & 0.017 & Pigr & -1.21 & 0.005 \\
\hline Acsf2 & 1.24 & 0.003 & Rpl14 & -1.21 & 0.032 \\
\hline
\end{tabular}




\begin{tabular}{|c|c|c|c|c|c|}
\hline 1700018C11Rik & 1.24 & 0.031 & Boc & -1.21 & 0.011 \\
\hline Serpinb9g & 1.24 & 0.034 & Rasa4 & -1.21 & 0.001 \\
\hline Pelit & 1.23 & 0.010 & Olfr68 & -1.21 & 0.035 \\
\hline Glud1 & 1.23 & 0.032 & Gpr151 & -1.21 & 0.002 \\
\hline $\operatorname{Arg} 2$ & 1.23 & 0.023 & 2810454H06Rik & -1.21 & 0.038 \\
\hline Sp6 & 1.23 & 0.033 & Aim2 & -1.21 & 0.007 \\
\hline LOC102632222 & 1.23 & 0.003 & C230072F16Rik & -1.21 & 0.005 \\
\hline Rhod & 1.23 & 0.029 & Chrna9 & -1.21 & 0.045 \\
\hline Ppp1r3fos & 1.23 & 0.031 & S/c51a & -1.21 & 0.025 \\
\hline S/c6a4 & 1.23 & 0.037 & Fam189a2 & -1.21 & 0.036 \\
\hline Gjb3 & 1.23 & 0.036 & Phgr1 & -1.21 & 0.027 \\
\hline 4933422H2ORik & 1.23 & 0.017 & Zfp9o & -1.21 & 0.021 \\
\hline Ppap2a & 1.23 & 0.023 & Krt7 & -1.21 & 0.009 \\
\hline Proc & 1.23 & 0.020 & Vav3 & -1.21 & 0.043 \\
\hline$x d h$ & 1.23 & 0.019 & $D k k 1$ & -1.21 & 0.006 \\
\hline Gm15628 & 1.23 & 0.034 & Tmem47 & -1.21 & 0.020 \\
\hline LOC101055707 & 1.23 & 0.033 & Gstm2 & -1.21 & 0.023 \\
\hline S/c3a2 & 1.23 & 0.047 & Сур8b1 & -1.21 & 0.036 \\
\hline Ncald & 1.23 & 0.017 & Tgif1 & -1.21 & 0.004 \\
\hline 1700112J16Rik & 1.23 & 0.004 & Ptprj & -1.21 & 0.018 \\
\hline$S / x 4$ & 1.23 & 0.007 & Npvf & -1.21 & 0.009 \\
\hline Pnkd & 1.22 & 0.009 & Nyx & -1.20 & 0.008 \\
\hline 1500035N22Rik & 1.22 & 0.024 & Casc4 & -1.20 & 0.031 \\
\hline Mir697 & 1.22 & 0.017 & LOC102634130 & -1.20 & 0.049 \\
\hline 4931429/11Rik & 1.22 & 0.007 & BВ031773 & -1.20 & 0.010 \\
\hline LOC102634333 & 1.22 & 0.031 & Pacsin 3 & -1.20 & 0.013 \\
\hline 10C0044D17Rik & 1.22 & 0.048 & $Z f p 759$ & -1.20 & 0.025 \\
\hline Zc2hc1c & 1.22 & 0.020 & 4931440P22Rik & -1.20 & 0.040 \\
\hline Ppid & 1.22 & 0.049 & Stim1 & -1.20 & 0.015 \\
\hline LOC102637589 & 1.22 & 0.033 & Gm12789 & -1.20 & 0.015 \\
\hline Gja6 & 1.22 & 0.016 & Hpse2 & -1.20 & 0.035 \\
\hline Rinl & 1.22 & 0.009 & Eras & -1.20 & 0.008 \\
\hline Pmaip1 & 1.22 & 0.045 & Cacnb3 & -1.20 & 0.006 \\
\hline 1700086D15Rik & 1.22 & 0.042 & Sh3bgr/2 & -1.20 & 0.002 \\
\hline Ugt1a7c & 1.22 & 0.050 & Sema6d & -1.20 & 0.049 \\
\hline Wdr20rt & 1.22 & 0.047 & Gpr37 & -1.20 & 0.050 \\
\hline Tekt4 & 1.22 & 0.009 & $L b p$ & -1.20 & 0.018 \\
\hline Zfp114 & 1.22 & 0.003 & Clca5 & -1.20 & 0.039 \\
\hline Zfp553 & 1.22 & 0.045 & Pdia5 & -1.20 & 0.004 \\
\hline$S / c 35 c 2$ & 1.22 & 0.049 & & & \\
\hline Ldoc11 & 1.22 & 0.011 & & & \\
\hline Pla2g4e & 1.22 & 0.009 & & & \\
\hline B4galt6 & 1.22 & 0.041 & & & \\
\hline Dio3 & 1.22 & 0.031 & & & \\
\hline
\end{tabular}




\begin{tabular}{|c|c|c|}
\hline 1700122E12Rik & 1.22 & 0.048 \\
\hline Naip5 & 1.22 & 0.005 \\
\hline ВС033916 & 1.22 & 0.013 \\
\hline$T k 1$ & 1.22 & 0.011 \\
\hline 5330439B14Rik & 1.22 & 0.027 \\
\hline LOC102640192 & 1.22 & 0.037 \\
\hline Arf1 & 1.22 & 0.029 \\
\hline 5033403H07Rik & 1.22 & 0.006 \\
\hline Tspan5 & 1.21 & 0.007 \\
\hline Foxo6 & 1.21 & 0.033 \\
\hline Cemip & 1.21 & 0.036 \\
\hline Olfrn1183 & 1.21 & 0.033 \\
\hline Igsf23 & 1.21 & 0.019 \\
\hline Snord $42 a$ & 1.21 & 0.034 \\
\hline Acs/5 & 1.21 & 0.044 \\
\hline Snora17 & 1.21 & 0.019 \\
\hline$P f k f b 4$ & 1.21 & 0.025 \\
\hline Plin3 & 1.21 & 0.003 \\
\hline Agpat9 & 1.21 & 0.037 \\
\hline Ssxb5 & 1.21 & 0.007 \\
\hline Tnnc2 & 1.21 & 0.010 \\
\hline Mrgprb5 & 1.21 & 0.007 \\
\hline Fut7 & 1.21 & 0.045 \\
\hline Snord15a & 1.21 & 0.020 \\
\hline Apol7a & 1.21 & 0.048 \\
\hline KIf14 & 1.21 & 0.016 \\
\hline$S c g b 2 b 23-p s$ & 1.21 & 0.038 \\
\hline Blcap & 1.21 & 0.012 \\
\hline Adcy7 & 1.21 & 0.041 \\
\hline 9330182L06Rik & 1.21 & 0.040 \\
\hline Gpt & 1.21 & 0.031 \\
\hline Snora7a & 1.21 & 0.023 \\
\hline Snord89 & 1.21 & 0.028 \\
\hline S/c34a3 & 1.21 & 0.015 \\
\hline Rnf182 & 1.21 & 0.010 \\
\hline LOC102640313 & 1.20 & 0.025 \\
\hline Nek10 & 1.20 & 0.010 \\
\hline Pi4k2b & 1.20 & 0.006 \\
\hline AA415398 & 1.20 & 0.012 \\
\hline
\end{tabular}




$\begin{array}{lll}\text { Cyp2b19 } & 1.20 & 0.006 \\ \text { Garem } & 1.20 & 0.010 \\ \text { Inpp4a } & 1.20 & 0.009 \\ \text { Otub2 } & 1.20 & 0.033 \\ \text { AA986860 } & 1.20 & 0.012 \\ \text { Btn12 } & 1.20 & 0.043 \\ \text { Fam132a } & 1.20 & 0.037 \\ \text { 4930525D18Rik } & 1.20 & 0.015 \\ \text { Kank3 } & 1.20 & 0.042 \\ \text { Fam131a } & 1.20 & 0.004 \\ \text { Snord49a } & 1.20 & 0.047\end{array}$




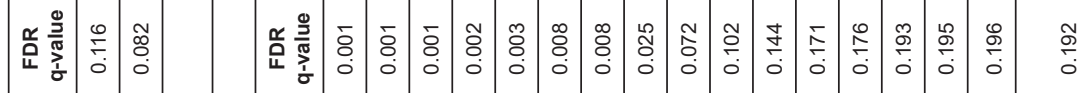

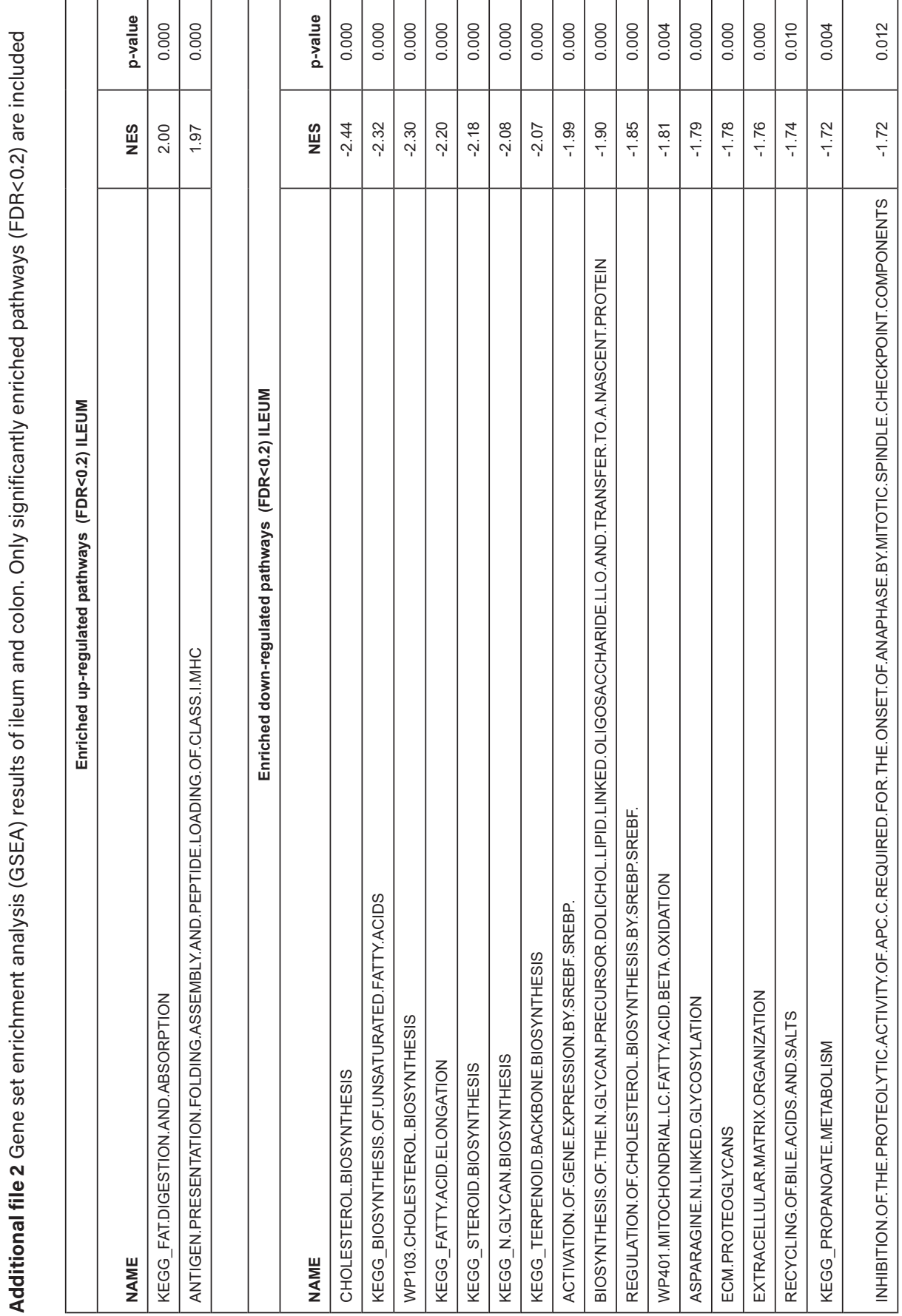


Chapter 3

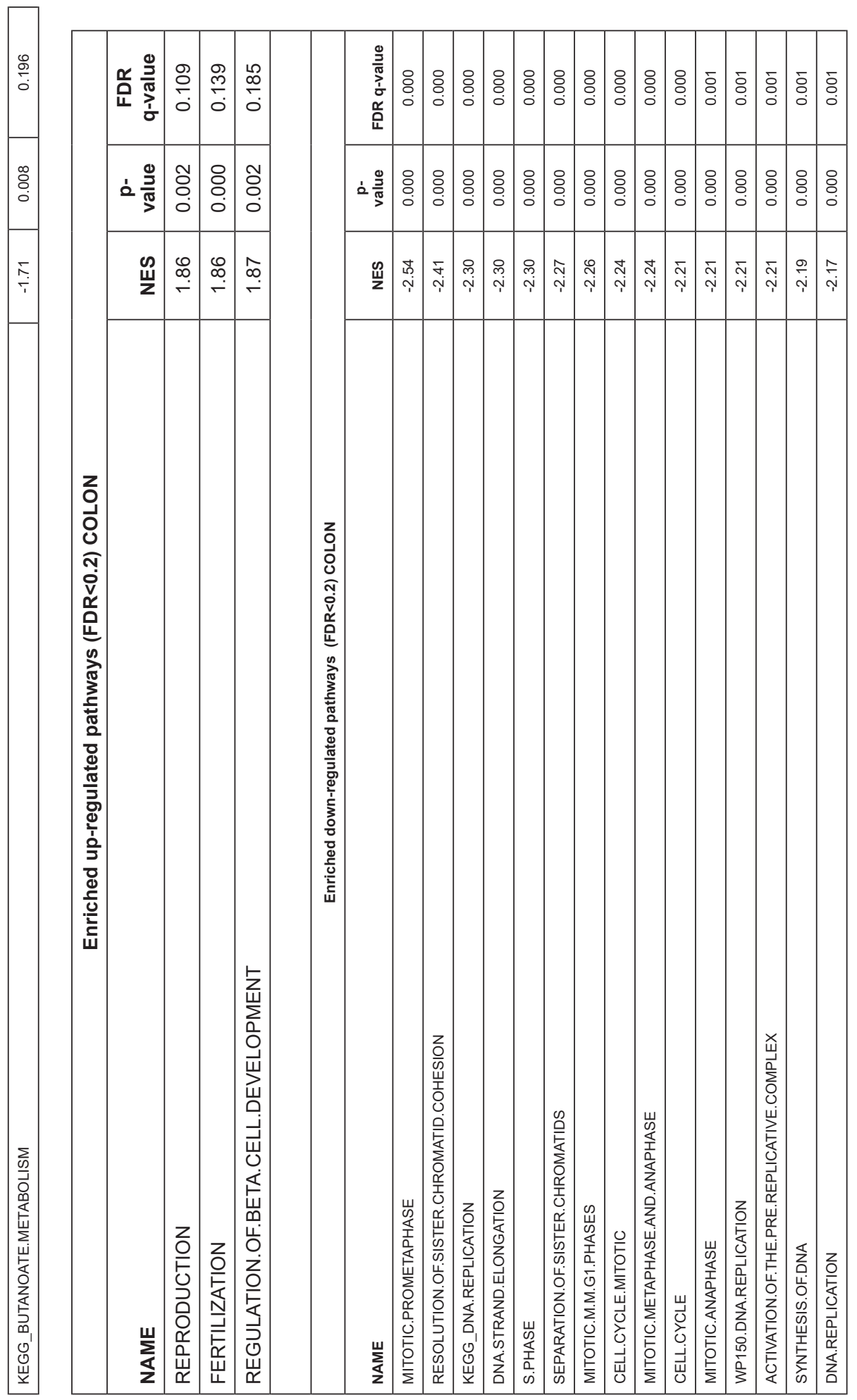




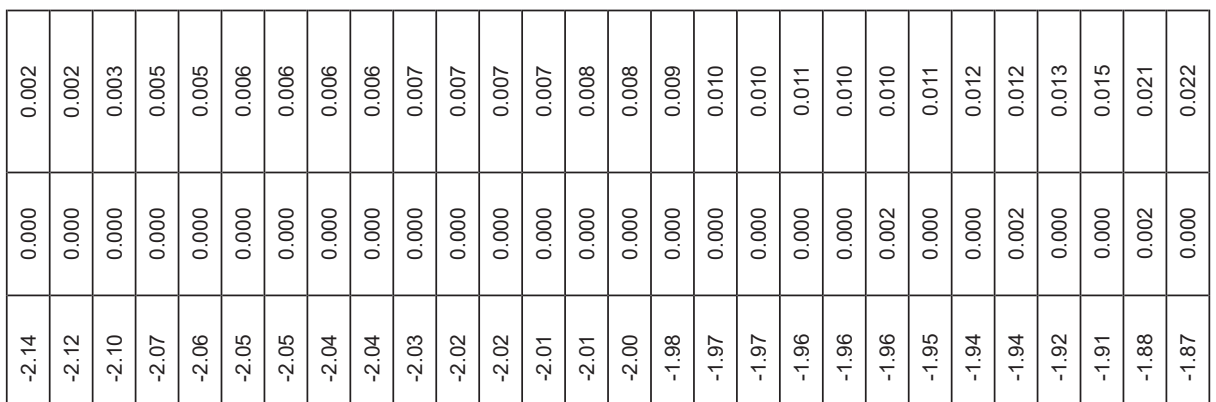




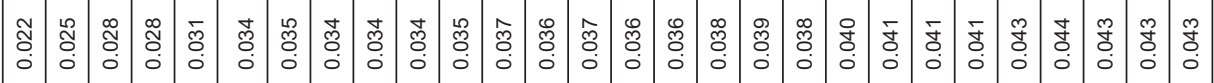
范

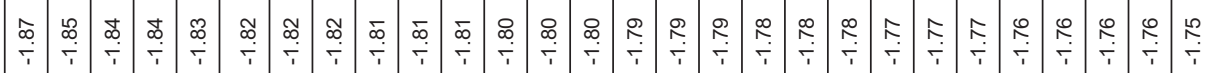




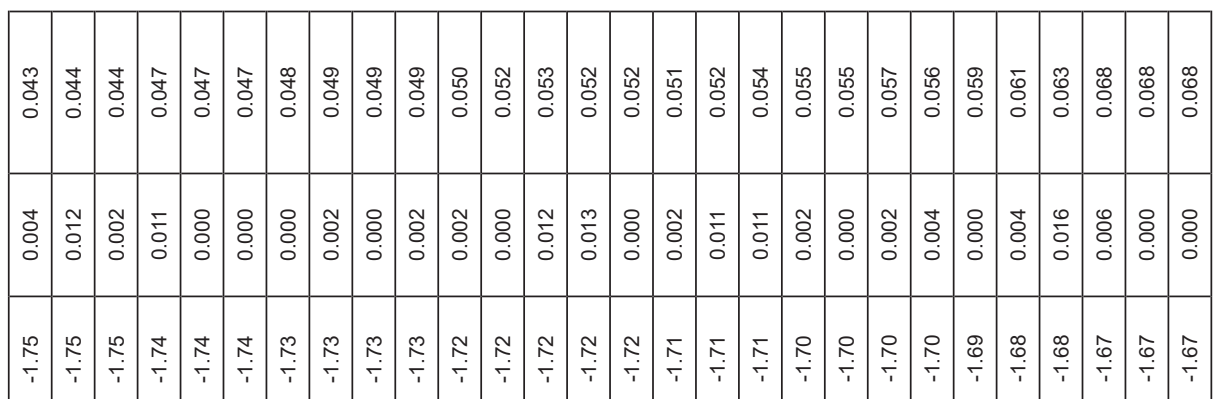




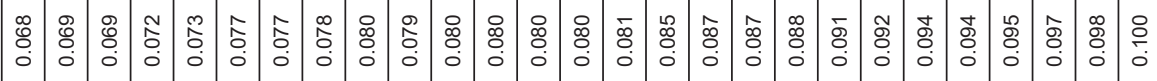

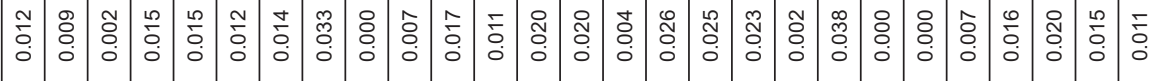

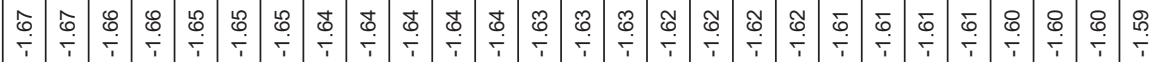

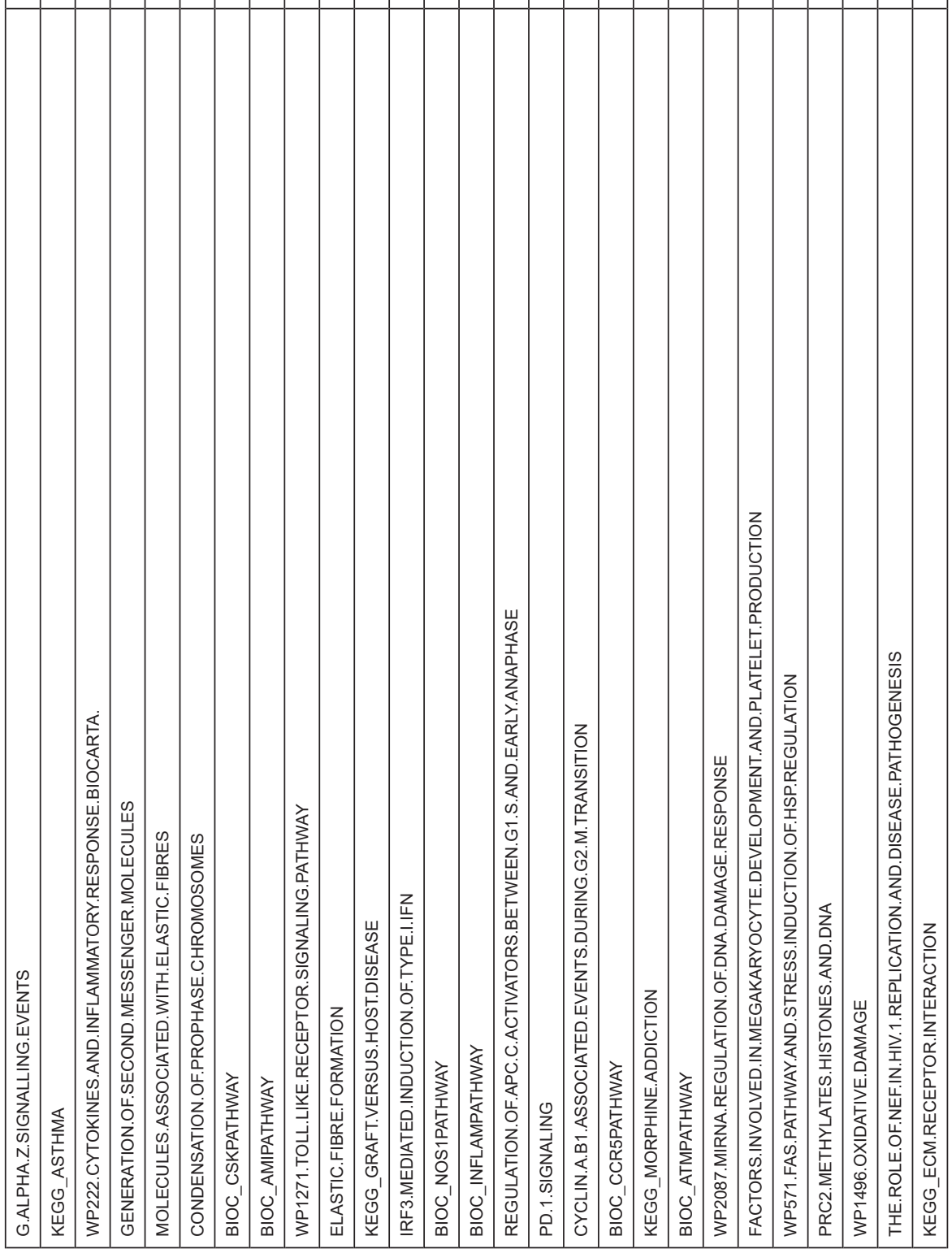




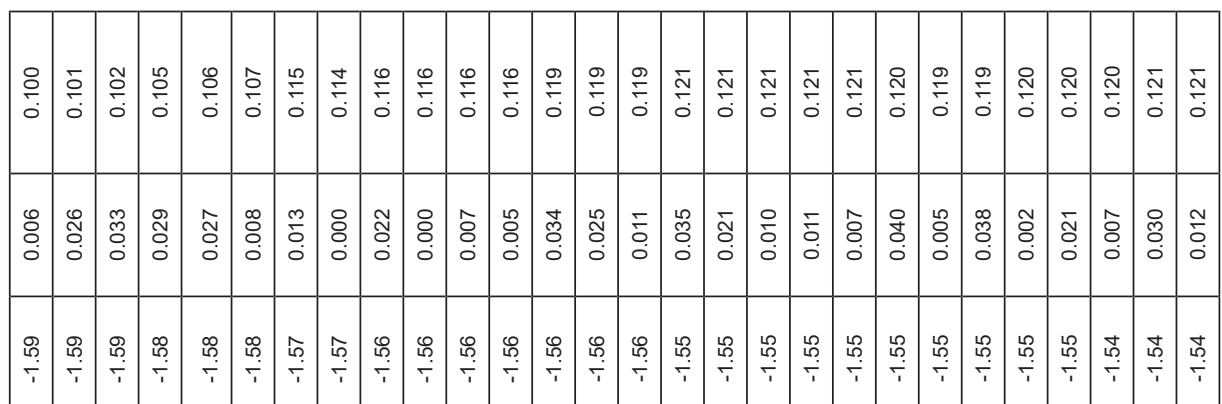




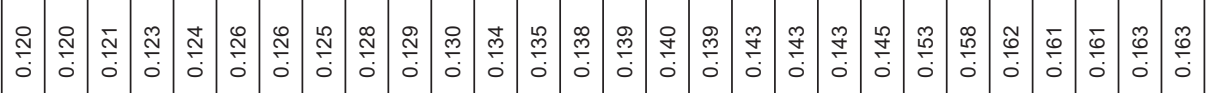

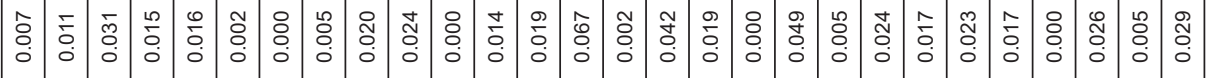

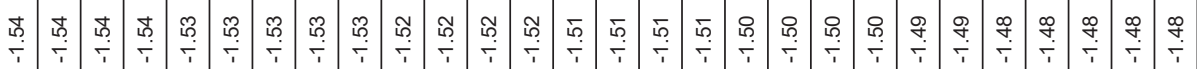

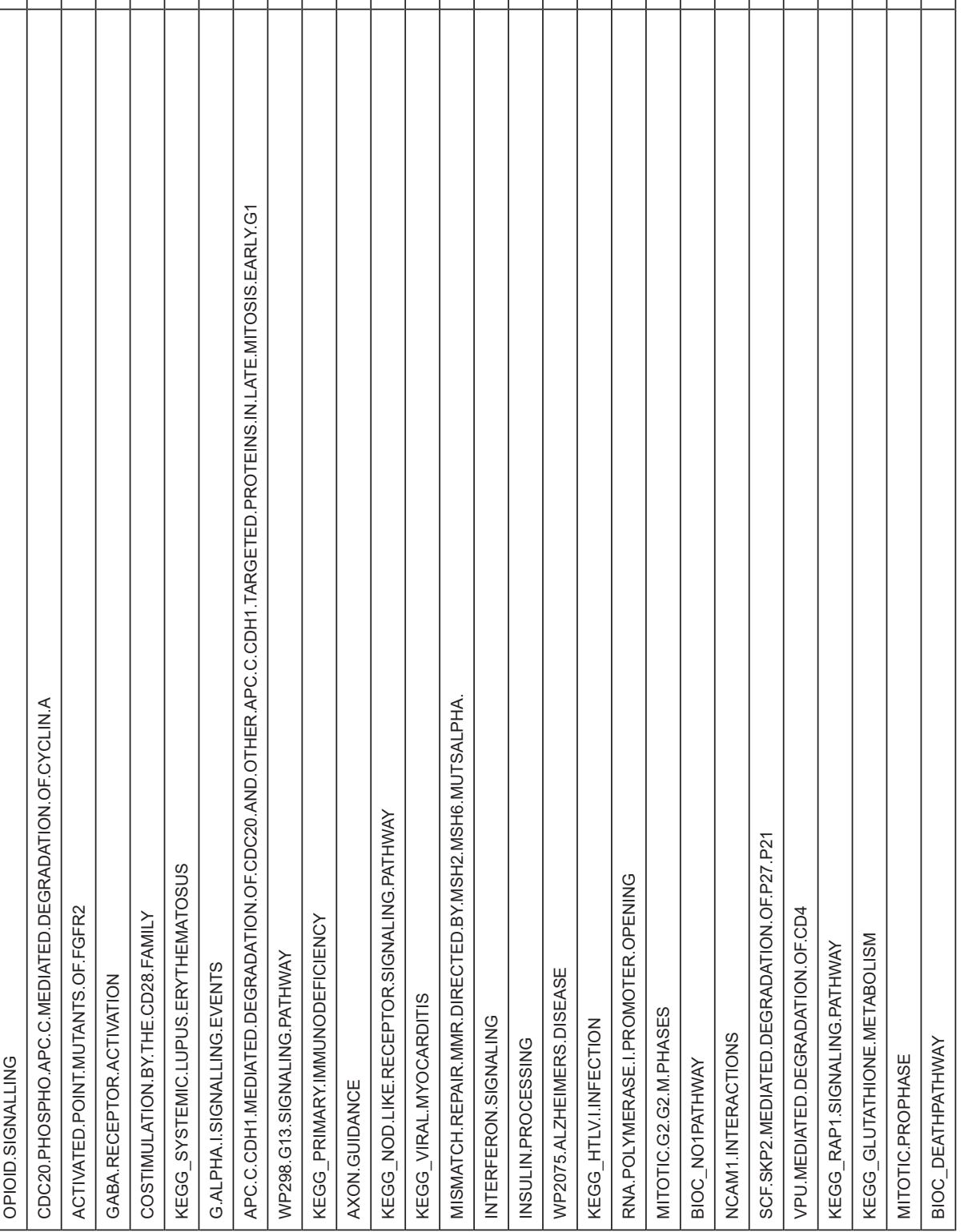




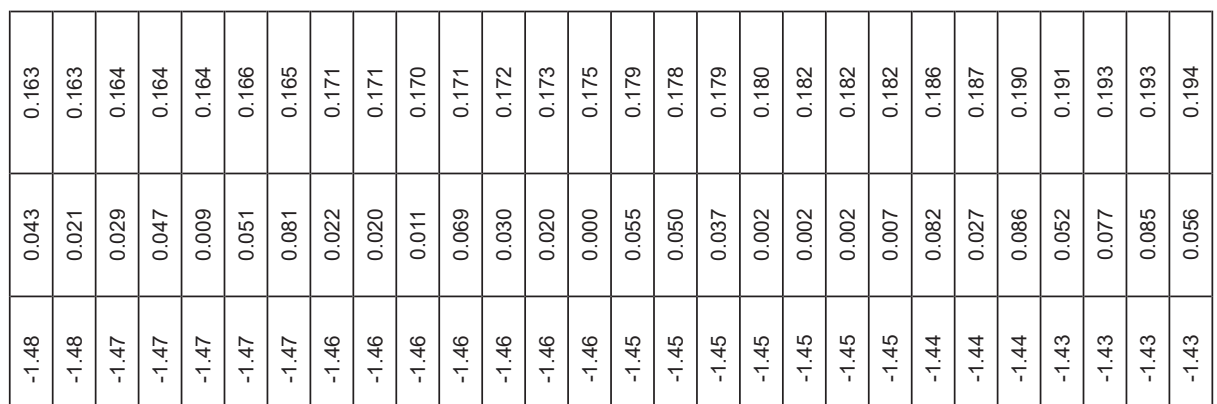

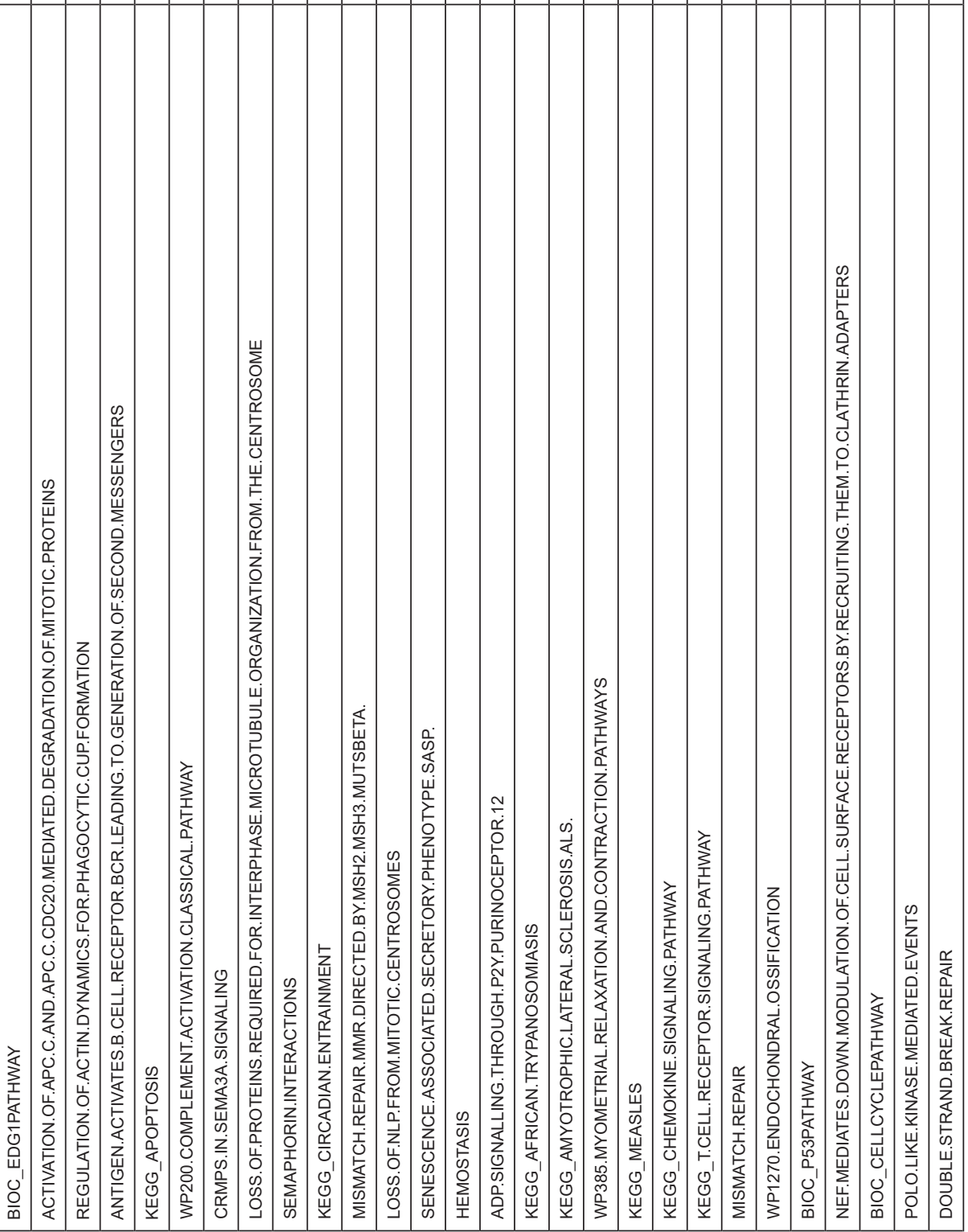




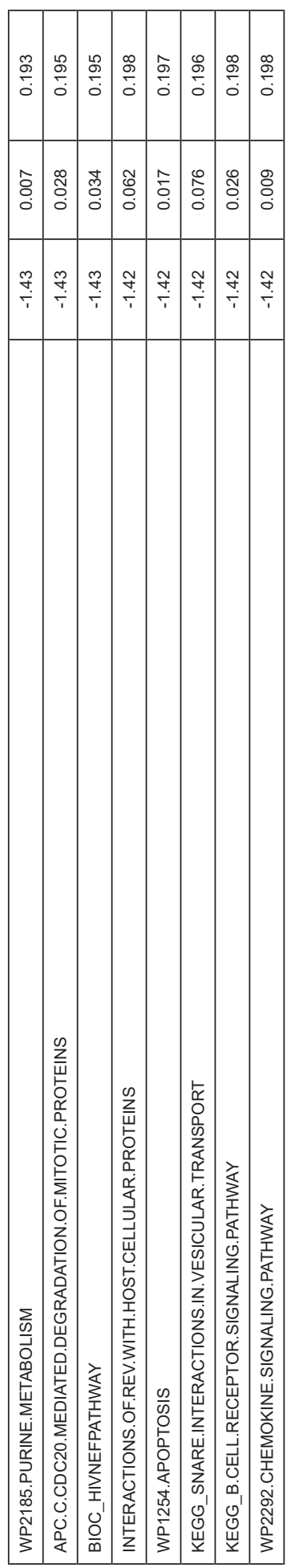


A

Reg3b

B

C

$\operatorname{Reg} 3 g$

Cldn2

Cldn8
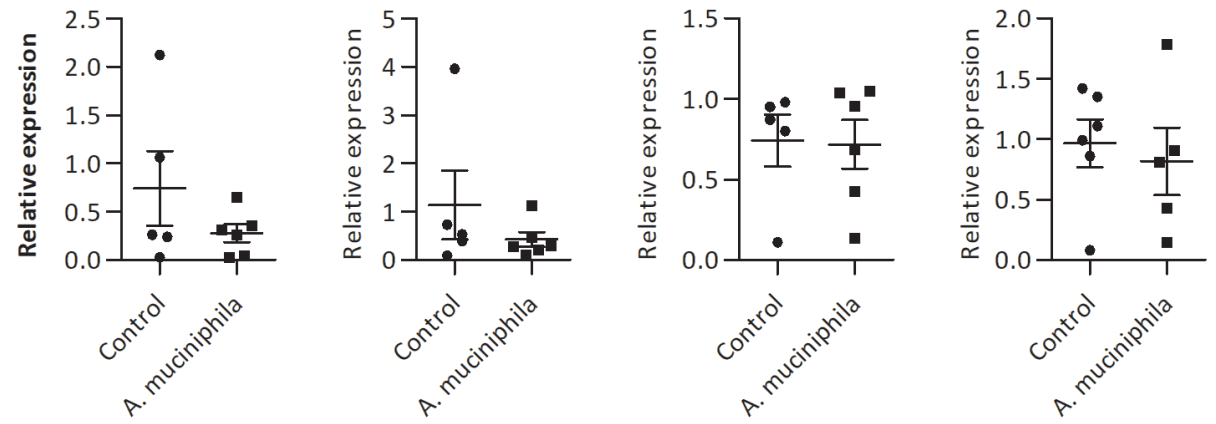

E

$\mathrm{F}$ Ctnna3 St6galnac6

G
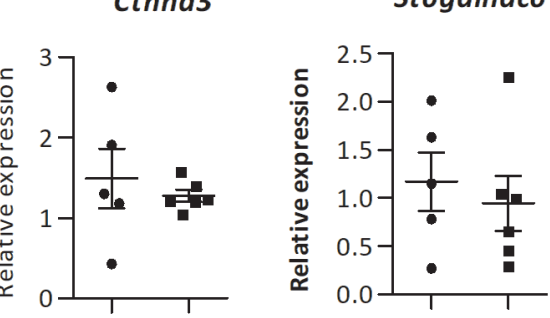

Cxcl13

BIk
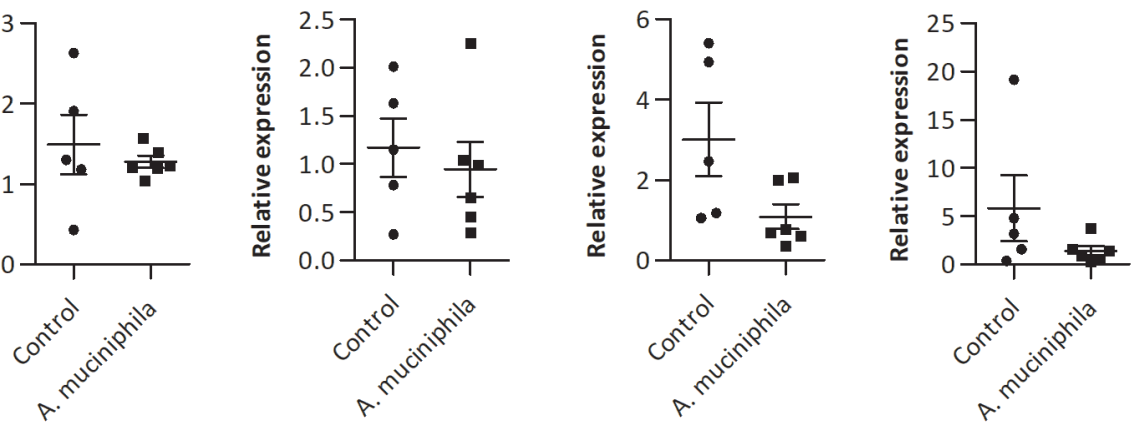

Tlr7

TIr12

Cd4

Cd72
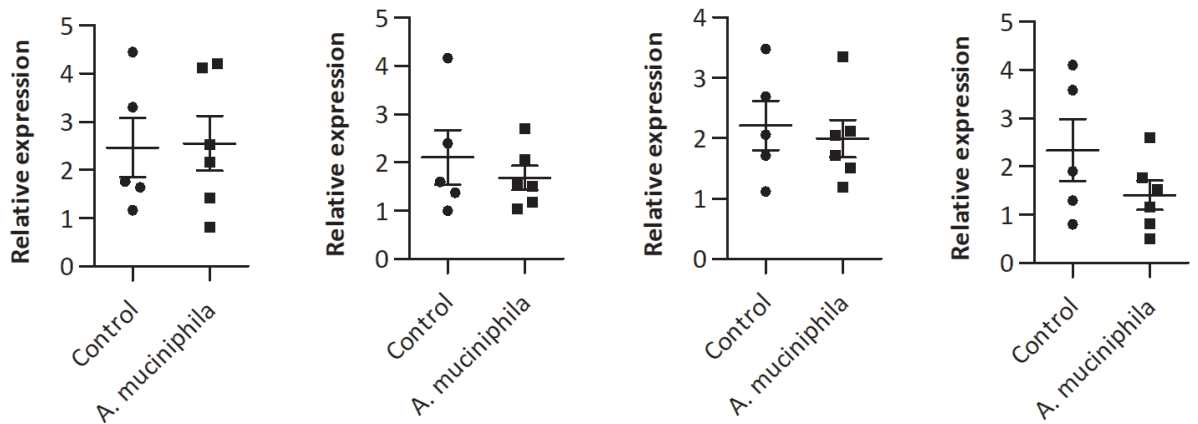

Additional file 3 Relative gene expression of (A) Regenerating islet-derived 3 beta $(\operatorname{Reg} 3 b)$, (B) Regenerating islet-derived 3 gamma (Reg3g), (C) Claudin 2 (Cldn2), (D) Claudin 8 (Cldn8), (E) Catenin (cadherin associated protein), alpha 3 (Ctnna3) and (F) ST6 (alpha-N-acetyl-neuraminyl-2,3-beta-galactosyl-1,3)$\mathrm{N}$-acetylgalactosaminide alpha-2,6-sialyltransferase 6 (St6galnac6) in ileum. (G) Relative expression of C-X-C motif chemokine ligand 13 (Cxc/13), (H) B lymphoid kinase (B/k), (I) Cluster of differentiation 4 (Cd4), (J) Cluster of differentiation 72 (Cd72), (K) Toll-like receptor 7 (T/r7), (L) Toll-like receptor 12 (T/r12) in colon. 
A

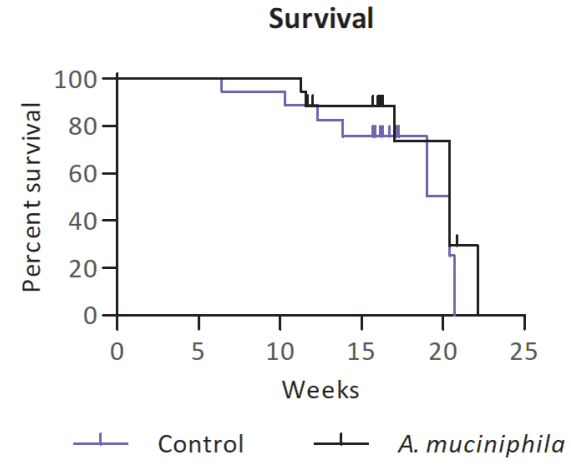

C

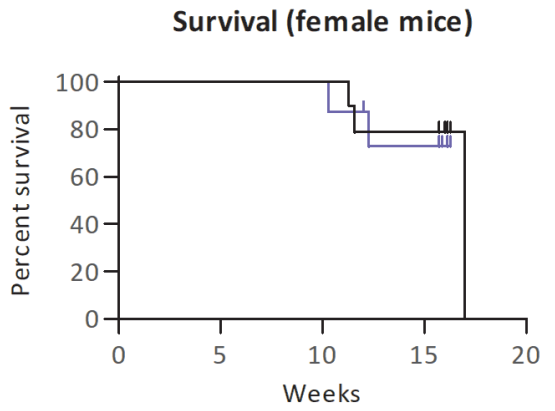

1 Control $\longrightarrow$ A. muciniphila

E

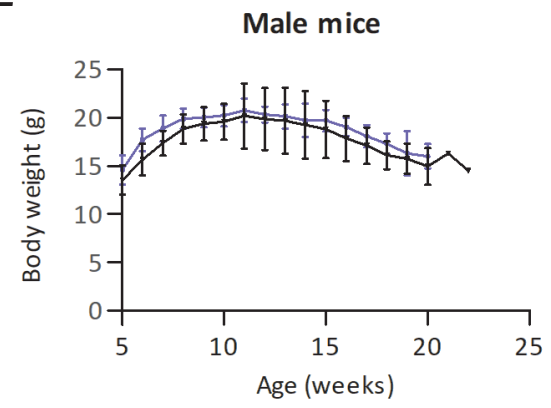

$\longrightarrow$ Control $\longrightarrow$ A. muciniphila
B

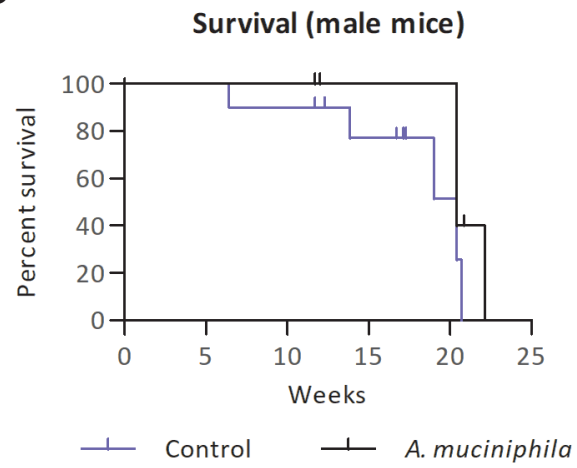

D

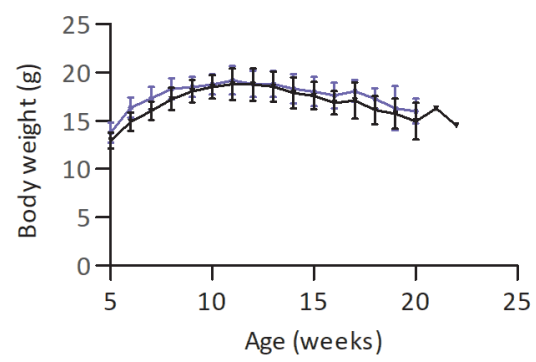

$\longrightarrow$ Control $\longrightarrow$ A. muciniphila

F

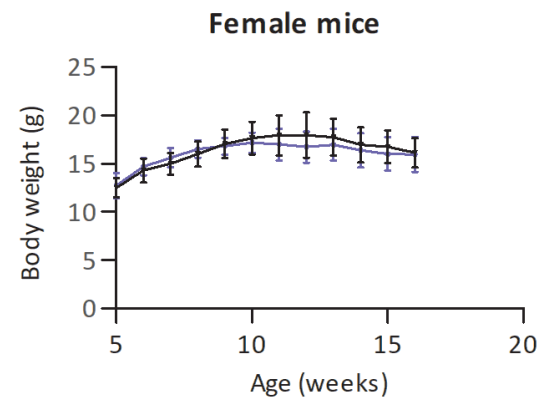

$\longrightarrow$ Control $\longrightarrow$ A. muciniphila

Additional file 4 Survival rates and body weight of Ercc1 $^{-/ \Delta 7}$ mice. (A) Percent survival of all mice. These data include 12-13 mice per group with an additional 5-6 per group censored at 16 weeks. (B) Percentage survival of only male mice ( $n=8-10 /$ group) and (C) female mice ( $n=8-10 /$ group). (D) Body weight in grams measured weekly in all mice ( $n=18$ mice per group), (E) male mice ( $n=8-10 /$ group) and $(F)$ female mice ( $n=8-10 /$ group). NB: A number of 5-6 female mice was sacrificed at 16 weeks. 
Additional file 5A Composition of the D12450B purified diet.

\begin{tabular}{|c|c|c|}
\hline \multirow[t]{2}{*}{ Based on formula \# D1245 } & \multirow[b]{2}{*}{ gm\% } & \multirow[b]{2}{*}{ kcal\% } \\
\hline & & \\
\hline Protein & 19 & 20 \\
\hline Carbohydrate & 67 & 69 \\
\hline Fat & 4 & 10 \\
\hline Other & 10 & 1 \\
\hline Total & 100 & 100 \\
\hline $\mathrm{kcal} / \mathrm{gm}$ & 3.8 & \\
\hline Ingredient & gm & kcal \\
\hline Casein, lactic & 200 & 800 \\
\hline L-Cystine & 3 & 12 \\
\hline Corn Starch & 427.2 & 1709 \\
\hline Maltodextrin & 100 & 400 \\
\hline Sucrose & 172.8 & 691 \\
\hline Cellulose, BW200 & 50 & 0 \\
\hline Soybean oil & 25 & 225 \\
\hline Palm oil & 20 & 180 \\
\hline Mineral Mix S10026* & 10 & 0 \\
\hline DiCalcium Phosphate & 13 & 0 \\
\hline Calcium Carbonate & 5.5 & 0 \\
\hline Potassium Citrate, $1 \mathrm{H} 2 \mathrm{O}$ & 16.5 & 0 \\
\hline Vitamin Mix V10001* & 10 & 40 \\
\hline Choline chloride & 2 & 0 \\
\hline Total & 1055 & 4057 \\
\hline
\end{tabular}


Additional file 5B List of all primer sequences that were used for qPCR.

\begin{tabular}{|c|c|c|}
\hline Gene Name & Forward primer $\left(5^{\prime} \rightarrow 3^{\prime}\right)$ & Reverse primer $\left(3^{\prime} \rightarrow 5^{\prime}\right)$ \\
\hline $\operatorname{Reg} 3 b$ & АСTCССTGAAGAATATACССTCC & CGCTATTGAGCACAGATACGAG \\
\hline $\operatorname{Reg} 3 g$ & AGGCCCTCAGGACATCTTGT & ATAGCCCAGTGTCGGGTCAT \\
\hline Cldn2 & CAACTGGTGGGCTACATCCTA & CCCTTGGAAAAGCCAACCG \\
\hline Cldn8 & GCAACCTACGCTCTTCAAATGG & TTCCCAGCGGTTCTCAAACAC \\
\hline Ctnna3 & AAGAATGGCCGAGTCAAGGAA & GCAGCATTTATGATCTGTGGACA \\
\hline St6galnac6 & AACAGTGCCAACGAGGTCTTC & CTTGTTGCCGAGGATAGGGAA \\
\hline Cxc/13 & GGCCACGGTATTCTGGAAGC & GGGCGTAACTTGAATCCGATCTA \\
\hline$B / k$ & GAGGCAGGTCAGTGAGAAGG & GTCCTGGTTAGGAGATGGTGG \\
\hline $\mathrm{Cd} 4$ & TCCTAGCTGTCACTCAAGGGA & TCAGAGAACTTCCAGGTGAAGA \\
\hline Cd72 & GCTCAGGGAGAAGATAAGTCAGC & GCGTCCTCGTGAGTCCTCT \\
\hline T/r7 & САССАССААТСТТАСССТТАСС & CAGATGGTTCAGCCTACGGAA \\
\hline T/r12 & TTGGAAGTTGTACCTCGGACT & GAAGTTGGGTAAGGTGCAGAC \\
\hline $36 B 4$ & ATGGGTACAAGCGCGTCCTG & GCCTTGACCTTTTCAGTAAG \\
\hline
\end{tabular}


Additional file 5C List of all antibodies used in flow cytometry.

\begin{tabular}{|c|c|c|c|}
\hline Target & Format & Clone & Company \\
\hline CD2 & PE & RM2-5 & $\mathrm{BD}$ \\
\hline CD3e & PerCP-Cy5.5 & $145-2 C 11$ & Ebioscience \\
\hline $\mathrm{CD} 4$ & $\mathrm{APC}-\mathrm{H} 7$ & GK1.5 & $\mathrm{BD}$ \\
\hline CD5 & APC & $53-7.3$ & $\mathrm{BD}$ \\
\hline CD8a & PerCP-Cy5.5 & $53-6.7$ & $\mathrm{BD}$ \\
\hline CD11b & $\begin{array}{l}\text { APC-Cy7 } \\
\text { BV421 } \\
\text { PE-Cy7 } \\
\text { PerCP-Cy5.5 }\end{array}$ & $\mathrm{M} 1 / 70$ & $\begin{array}{l}\text { BD } \\
\text { BD } \\
\text { Ebioscience } \\
\text { Ebioscience }\end{array}$ \\
\hline CD16/32 & Purified & $2.4 \mathrm{G} 2$ & $\mathrm{BD}$ \\
\hline CD19 & $\begin{array}{l}\text { APC-eFluor780 } \\
\text { PerCP-Cy5.5 }\end{array}$ & 1D3 & Ebioscience \\
\hline CD43 & PE & S11 & BioLegend \\
\hline CD45R/B220 & BV421 & RA3-6B2 & $\mathrm{BD}$ \\
\hline CD68 & FITC & FA-11 & BioLegend \\
\hline CD80 & BV421 & $16-10 \mathrm{~A} 1$ & $\mathrm{BD}$ \\
\hline CD115 & PE & AFS98 & Ebioscience \\
\hline CD172a/SIRPa & APC & P84 & Ebioscience \\
\hline CD273/PDL2 & PE & TY25 & $\mathrm{BD}$ \\
\hline FoxP3 & PE & FJK-16s & Ebioscience \\
\hline $\lg D$ & PE-Cy7 & $11-26 c$ & Ebioscience \\
\hline $\lg K$ & FITC & 187.1 & $\mathrm{BD}$ \\
\hline Igy & FITC & $\mathrm{R} 26-46$ & $\mathrm{BD}$ \\
\hline $\lg M$ & APC & II/41 & Ebioscience \\
\hline Ly6C & $\begin{array}{l}\text { AF488 } \\
\text { PerCP-Cy5.5 }\end{array}$ & $\begin{array}{l}\text { ER-MP20 } \\
\text { HK1.4 }\end{array}$ & $\begin{array}{l}\text { AbD Serotec } \\
\text { Ebioscience }\end{array}$ \\
\hline Ly6G & PE-Cy7 & $1 \mathrm{~A} 8$ & $\mathrm{BD}$ \\
\hline
\end{tabular}




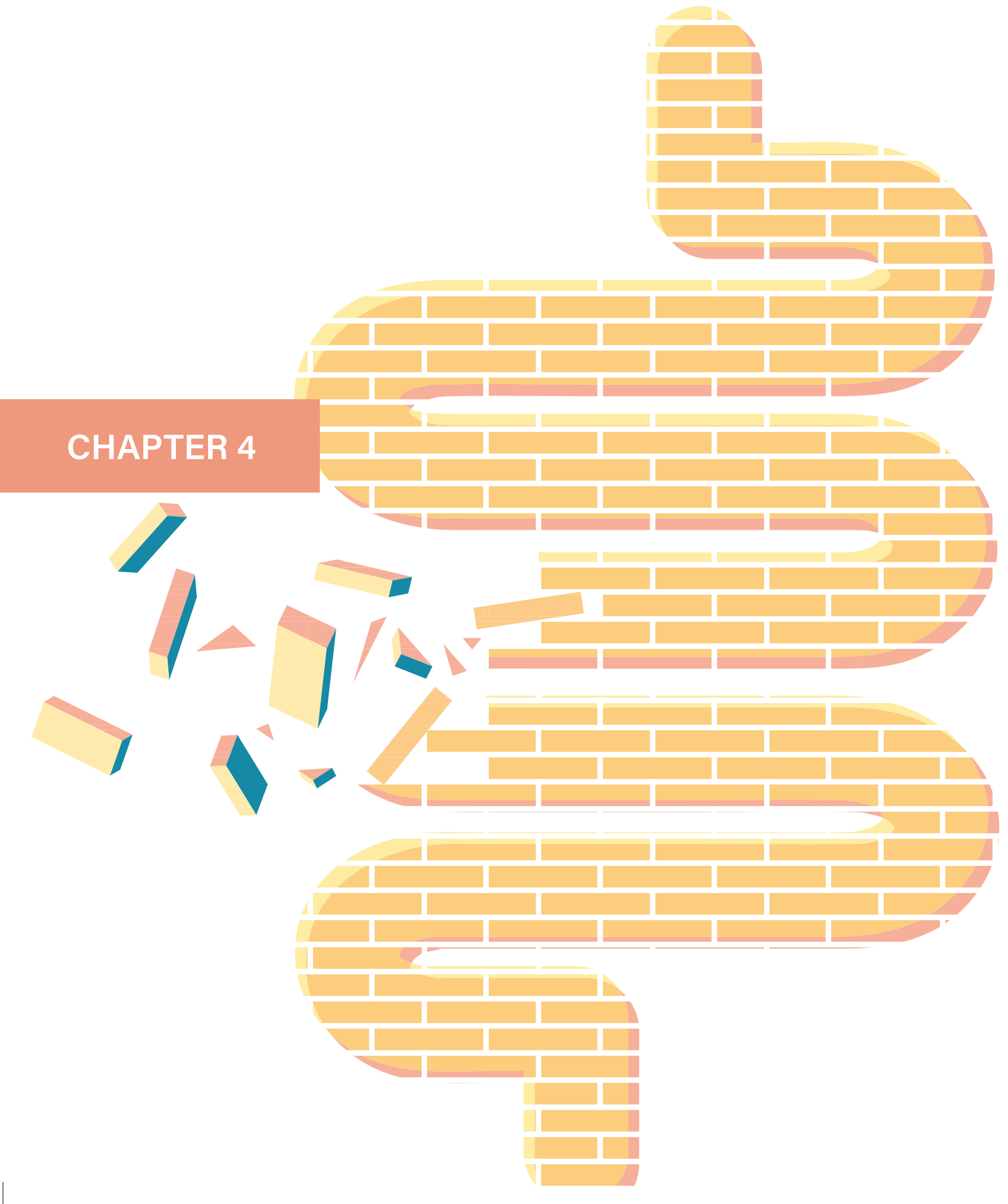




\section{The effects of sulfated secondary bile acids on intestinal barrier function and immune response in an inflammatory in vitro human intestinal model}

Benthe van der Lugt, Maartje C.P. Vos, Mechteld Grootte Bromhaar, Noortje ljssennagger, Frank Vrieling, Jocelijn Meijerink, Wilma T. Steegenga 


\section{Abstract}

Dysbiosis-related perturbations in bile acid (BA) metabolism were observed in inflammatory bowel disease (IBD) patients, which was characterized by increased levels of sulfated BAs at the expense of secondary BAs. However, the exact effects of sulfated BAs on the etiology of IBD are not investigated yet. Therefore, we aimed to investigate the effects of sulfated deoxycholic acid (DCA), sulfated lithocholic acid (LCA) and their unsulfated forms on intestinal barrier function and immune response. To this end, we first established a novel in vitro human intestinal model to mimic chronic intestinal inflammation as seen during IBD. This model consisted of a co-culture of Caco-2 and HT29-MTX-E12 cells grown on a semi-wet interface with mechanical stimulation to represent the mucus layer. A proinflammatory environment was created by combining the co-culture with LPS-activated dendritic cells (DCs) in the basolateral compartment. The presence of activated DCs caused a decrease in transepithelial electrical resistance (TEER), which was slightly restored by LCA and sulfated DCA. The expression of genes related to intestinal epithelial integrity and the mucus layer were slightly, but not significantly increased. These results imply that sulfated BAs have a minor effect on intestinal barrier function in Caco-2 and HT29-MTX-E12 cells. When exposed directly to DCs, our results point towards anti-inflammatory effects of secondary BAs, but not sulfated secondary BAs. These findings emphasize the importance of proper transformation of BAs by bacterial enzymes and the potential involvement of BA dysmetabolism in IBD progression. 


\section{Introduction}

Inflammatory bowel disease (IBD) comprises a set of disorders that causes chronic and relapsing inflammation of the gastrointestinal tract. The etiology of IBD remains largely unknown, although it is clear that it is a multifactorial disease, in which the complex interplay between genetic susceptibility, environmental stimuli and the immune system are involved $(1,2)$. Furthermore, the gut microbiota is thought to play a major role in the onset and progression of IBD, which is emphasized by studies showing that gut microbiota composition in IBD patients is dysbiotic (3-6). Dysbiosis is linked to disturbed intestinal barrier function, such as increased intestinal permeability (7) and an impaired mucus layer $(8,9)$. Impaired intestinal barrier function enables direct bacterial contact with the epithelial cell layer, thereby inducing an inflammatory response (10-13). In a healthy situation, the intestinal mucosal immune system is tolerant against commensal bacteria, a process in which intestinal dendritic cells (DCs) play a crucial role (14-16). During IBD, intestinal DCs have lost their tolerogenic function and produce elevated levels of pro-inflammatory cytokines, consequently leading to an exacerbated disease progression $(17,18)$.

Importantly, dysbiosis is also linked to an altered production of bacterial metabolites, such as secondary bile acids (BAs) $(3,19-21)$. Primary BAs are synthesized in the liver, conjugated with taurine or glycine and secreted in the small intestine, where they accomplish a major role in lipid digestion (22). BAs are actively reabsorbed in the ileum, transported back to the liver and metabolized by hepatic enzymes to be reused again, which is a process called the enterohepatic cycle (22). Approximately $5 \%$ of all BAs are not reabsorbed and enter the colon, where resident bacteria deconjugate and metabolize them into secondary BAs. These secondary BAs can be either excreted via feces or reabsorbed and transported back to the liver. However, secondary BAs might be hepatotoxic at high concentrations and are therefore first detoxified by addition of a sulfonate group ( $\mathrm{SO}^{3-}$ ) (23). As a result of IBD-related dysbiosis, the production of bacterial enzymes and thus BA metabolism can be disturbed, a process known as BA dysmetabolism (24). Indeed, the capacity of the gut microbiota to deconjugate BAs and transform primary to secondary BAs was decreased in patients with active IBD. As a consequence, increased abundance of conjugated BAs and decreased abundance of secondary BAs in feces of IBD patients during both remission and active disease was detected, as compared to healthy people $(3,25)$. Similar differences in BA composition were found in other studies investigating fecal metabolite pools in IBD patients $(19,20,26,27)$. Interestingly, dysbiosis in IBD patients was also associated with a reduced desulfation capacity, which was concomitant with $15 \%$ higher levels of fecal sulfated BAs (3). Likewise, increased levels of fecal 3-sulfodeoxycholic acid and chenodeoxycholic acid sulfate were found in Crohn's disease patients (20). The fecal abundance 
of sulfated BAs was also found to be elevated in patients with non-inflammatory intestinal disorders, such as diarrhea-predominated irritable bowel syndrome $(25,28)$.

Given the important signaling functions of secondary BAs, including their role in inflammatory pathways, a change in luminal BA composition may have consequences on the progression of IBD. However, the possible involvement of sulfated BAs is only based on associative studies and the causal effects remain elusive. Therefore, the aim of this study was to investigate the effects of sulfated BAs on intestinal barrier function and immune response. Since existing models often insufficiently approach the physiological representation of the intestinal barrier and inflammatory environment in the context of IBD, we first established a novel inflammatory in vitro human intestinal model. We included a co-culture of Caco-2 and HT29-MTX-E12 cells, which are both human colon carcinoma cell lines representing an enterotype and a mucus-producing cell line, respectively. To mimic the inflammatory state as observed during IBD, the co-culture was grown on cell culture inserts in combination with DCs in the basolateral compartment, which were activated with LPS to obtain pro-inflammatory properties. In contrast to existing models, our model had an improved mucus layer by growing the cells on a semi-wet interface with mechanical stimulation (SMWS) (29, 30). After exposure to sulfated deoxycholic acid (DCA), sulfated lithocholic acid (LCA) and their unsulfated forms for 24 hours, the effects on intestinal barrier function and immune response were investigated. New insights into the role of BA dysmetabolism in IBD may contribute to the discovery of novel therapies that may add to the treatment of IBD.

\section{Materials and methods}

\section{Cell culture}

Caco-2 cells (ATCC) and HT29-MTX-E12 cells (ECACC) were cultured in Dulbecco's Modified Eagle Medium supplemented with 10\% Fetal Bovine Serum and 1\% penicillin/streptomycin. Cells were grown until $80-90 \%$ confluence at $37^{\circ} \mathrm{C} / 5 \% \mathrm{CO}_{2}$. Passage numbers between 7 and 25 were used for Caco-2 cells and between 3 and 15 for HT29-MTX-E12 cells. Monocytes were isolated from buffy coats originated from different blood donors (Sanquin, Nijmegen, The Netherlands). First, PBMCs were isolated from the buffy coat using LeucoSep tubes (Greiner-Bio One, Alphen aan den Rijn, The Netherlands), pre-filled with Ficoll-Paque Plus (GE Healthcare via Sigma-Aldrich). PBMCs were filtered through a $70 \mu \mathrm{m}$ cell strainer (Corning) and counted using a Vi-Cell counter (Beckman Coulter, Woerden, The Netherlands). A QuadroMACS Separator (Miltenyi Biotec, Leiden, The Netherlands) 
was used to magnetically separate $\mathrm{CD} 14^{+}$monocytes, using MojoSort Human CD14 Nanobeads (BioLegend, London, UK) diluted in MACS buffer (PBS, 0.5\% BSA and 2mM EDTA) following the manufacturer's instructions. Monocytes were resuspended in RPMI, supplemented with $10 \%$ FCS, 1\% penicillin/streptomycin and 1\% GlutaMAX (Gibco). Monocytes were differentiated into dendritic cells by adding $10 \mathrm{ng} / \mathrm{mL}$ Granulocyte macrophage-colony stimulating factor (GM-CSF) (Miltenyi Biotec, Leiden, The Netherlands) and $10 \mathrm{ng} / \mathrm{mL}$ human recombinant IL-4 (PeproTech, London, UK) for 6 days at $37^{\circ} \mathrm{C} / 5 \% \mathrm{CO}_{2}$.

\section{Cell model}

A co-culture of Caco-2 cells and HT29-MTX-E12 cells was seeded in 24-well ThinCert cell culture inserts with $0.4 \mu \mathrm{m}$ pores (Greiner-Bio One, Alphen aan den Rijn, The Netherlands). Caco-2 and HT29-MTX-E12 cells were seeded in a 3:1 ratio, using a seeding density of 225,000 cells $/ \mathrm{mL}$ in a volume of $150 \mu \mathrm{L}$. A volume of $700 \mu \mathrm{L}$ DMEM was added to the basolateral compartment. Two days after seeding, media volumes were changed to $25 \mu \mathrm{L}$ and $425 \mu \mathrm{L}$ in the apical and basolateral compartment, respectively. The cell culture plates were put on a $\mathrm{CO}_{2}$ resistant shaker (Thermo Fisher Scientific, Breda, The Netherlands) at $65 \mathrm{rpm}$. Cells were differentiated for 14 days and medium was changed every other day. Immature DCs were seeded in 24-wells plates in a density of 400,000 cells per well. DCs were stimulated with $10 \mathrm{ng} / \mathrm{mL}$ LPS (L3024, Sigma-Aldrich, Darmstadt, Germany) for 24 hours. Maturation of DCs was checked on the CytoFLEX Flow Cytometer (Beckman Coulter, Woerden, The Netherlands) using CD14-ECD antibody, clone RMO52 (IM2707U, Beckman Coulter), FITC anti-human CD83, clone HB15e and PE/Cyanine7 anti-human CD209 (DC-SIGN), clone 9E9A8 antibodies (BioLegend, Amsterdam, The Netherlands). The culture inserts with Caco2 and HT29-MTX-E12 cells were transferred to the cell culture plate containing the LPS-activated DCs. The co-culture was exposed to lithocholic acid 3-sulfate disodium salt (sulfo-LCA) (SantaCruz Biotechnology, Dallas, United States), deoxycholic acid 3-O-sulfate disodium salt (sulfo-DCA) (Toronto Research Chemicals, Toronto, Canada), lithocholic acid (LCA) and deoxycholic acid (DCA) (Sigma-Aldrich, Darmstadt, Germany). LCA and sulfo-LCA were solubilized in DMEM:methanol (1:1, v/v). DCA and sulfo-DCA were solubilized in DMEM:methanol (3:1, v/v). A control without DCs and a control with LPS-activated DCs were included. Control cells were exposed to similar concentrations of methanol (0.5\%). Every condition was applied in duplicate. A total of three similar plates were seeded and exposed to BAs; plate 1 was used for permeability assays, plate 2 for RNA isolation and plate 3 three for protein isolation. Experiments where DCs were directly exposed to BAs were performed similarly, except that BAs were applied directly to the DCs. 


\section{Quantification of lactate hydrogenase release}

To investigate the effects of BA exposure on cytotoxicity of Caco-2 and HT29-MTX-E12 cells and DCs, lactate hydrogenase levels were measured in conditioned medium collected directly after 24 hours of BA exposure. To this end, a lactate dehydrogenase (LDH) cytotoxicity detection kit (Roche Applied Science; Almere, The Netherlands) was used following the manufacturer's instructions. As a control for complete cytotoxicity, cells were exposed for 15 minutes to a 1\% Triton-X100 solution.

\section{Trans- and paracellular epithelial permeability assays}

Transepithelial resistance (TEER) was measured with an EVOM2 Volt/Ohm meter using STX2 electrodes (World Precision Instruments, Sarasota, United States). To assure the electrodes were fully submerged in medium, the media volumes were adapted to $100 \mu \mathrm{L}$ apical and $700 \mu \mathrm{L}$ basolateral before the first TEER measurements were performed. The TEER values after BA exposure were expressed as percentage of the TEER value measured just before BA exposure. After 24 hours of BA exposure, culture inserts were washed twice with PBS and transferred to a new 24-wells plate. Lucifer Yellow CH dilithium salt (L0259, Sigma) was dissolved in phenol red-free medium (Gibco) to $1 \mathrm{mg} / \mathrm{mL}$ and $100 \mu \mathrm{L}$ was added to the apical compartment. In the basolateral compartment, 700 $\mu \mathrm{L}$ phenol red-free DMEM was added and afterwards the plate was incubated at $37^{\circ} \mathrm{C} / 5 \% \mathrm{CO}_{2}$ for 3 hours. Subsequently, $100 \mu \mathrm{L}$ of the basolateral compartment was collected and fluorescence was measured at 425/515 nm (excitation/emission). An empty cell culture insert served as a control for complete paracellular permeability.

\section{RNA isolation and qRT-PCR}

The cell culture inserts of plate 2 were washed twice with ice-cold PBS and subsequently, $200 \mu \mathrm{L}$ TRIzol reagent (ThermoFisher) was added per insert. The duplicates per condition were pooled to assure enough RNA yield. RNA was isolated using phenol/chloroform extraction. The RNA concentration was measured using a Nanodrop (Nanodrop ND-1000, Nanodrop Products, Maarssen, The Netherlands). A total of 1000 ng RNA was reverse transcribed using the RevertAid First Strand cDNA Synthesis kit (ThermoFisher). Real-time quantitative PCR was carried out using the SensiMix SYBR kit (Bioline, Alphen aan den Rijn, The Netherlands) in a CFX384 machine (Bio-Rad). Primer sequences are listed in Table 1. Data was normalized against the housekeeping gene GAPDH. 
Table 1 Primer sequences used for qRT-PCR

\begin{tabular}{|l|l|l|}
\hline Gene & Forward primer & Reverse primer \\
\hline GAPDH & GAAGGTGAAGGTCGGAGTC & GAAGATGGTGATGGGATTTC \\
\hline ZO1 & CGGCGAGTCCTGTGATGAG & TCTTGTATTCCTGTAGGCCAGT \\
\hline CDH1 & GAACGAGGCATCATCCCTAA & CCAGCTTCTCGAAGAACCAC \\
\hline CLDN1 & CGACCCAACCCAAGAATCTA & AGGCTGTGCCTTCCTACAGA \\
\hline MUC2 & CTTTGGGGCTTTGATCGGACT & GGAGTAGTTCAATTCCAGCAACA \\
\hline MUC5AC & ACCCGCACTATGTCACCTTC & GGACAGGACACCTTGTCGTT \\
\hline DEFB1 & CAGCACAACCCCTGTTTCAAA & GCGCACAGAGGATGACAGT \\
\hline LYZ & ATGAGAACTTCCTACCTTCTGCT & TCTGTAACAGGTGCCTTGAATTT \\
\hline CA12 & GGCCAAATGGGAGAGTGGTTA & CCAGTAGCGGCTATTGATCTGAA \\
\hline ANG & AGTGACATCCTCCAGTATGACG & GTGGCACTGTAGCGAGACT \\
\hline ASBT & CCTCCATGCCAGTACCGAG & GGACGACGGAAAATTGACTGA \\
\hline FABP6 & TGTGTTGGCTTCCTCTGTCAG & GGCAGCATCCTATAATGAGCAC \\
\hline FGF19 & GCCCGCAACTTCAAGATCG & CCTTGCCAACAGTGAACTTGT \\
\hline OSTa & CACCAGGCTTCAGGAGTAGG & CGGGACAGCAAGTTATTCTC \\
\hline OSTB & TCATTTCCCGTCAAGCCAGG & GGCGAACAAGCAATCTGCC \\
\hline SULT2A1 & TCCAGGCAAGCAGAAAAGAA & ACTGACAGCACATCTCTCTCT \\
\hline
\end{tabular}

\section{Protein isolation and Western Immunoblotting}

The cell culture inserts were washed twice with ice-cold PBS and $100 \mu \mathrm{L}$ RIPA buffer (ThermoFisher) enriched with protease- and phosphatase inhibitors (Roche Diagnostics) was added per culture insert. Duplicates were pooled to assure enough protein yield. Cell lysates were incubated on ice for 20 minutes following centrifugation for 10 minutes at 13,000 $\mathrm{g}$. Protein concentrations of the supernatants were measured using a bicinchoninic acid assay (ThermoFisher). For each sample, $14.8 \mu \mathrm{g}$ protein was loaded on a 4-15\% Mini-PROTEAN TGX Precast gel (Bio-Rad). Proteins were separated by SDS gel electrophoresis and transferred onto a polyvinylidene difluoride (Trans-Blot Turbo Midi 0.2 $\mu \mathrm{m}$ PVDF Transfer Packs, Bio-Rad) membrane using the Transblot Turbo System (BioRad). After blocking for 1 hour at room temperature, the membranes were incubated overnight at $4^{\circ} \mathrm{C}$ with anti-ZO1 (Abcam ab216880), anti-OCLN (Abcam ab216327) and anti-HSP90 (Cell Signaling Technology 4874). ZO1 and OCLN antibodies were used in 1:1000 and for HSP90 1:5000 was used. Subsequently, membranes were incubated with HRP conjugated goat anti-rabbit IgG antibody (1:5000) (GenScript A00098) for 1 hour at room temperature. All membrane incubations were in Trisbuffered saline with $0.1 \%$ Tween 20 (TBS-T) and 5\% (w/v) skimmed dry milk. Washing in between 
steps was done in TBS-T. Blots were visualized with Clarity ECL substrate (Bio-Rad) using the ChemiDoc MP system (Bio-Rad). Quantification was performed using ImageLab software (Bio-Rad).

\section{Cytokine measurements}

Medium collected from the basolateral compartments was used for the assessment of cytokines. Levels of IL-6, IL-12/IL-23 p40 and TNF- $\alpha$ were measured with human DuoSet ELISA Development kits (R\&D Systems, Abingdon, UK) following the manufacturer's instructions.

\section{Statistical analysis}

Data is presented as mean \pm standard error of mean (SEM). GraphPad Prism version 5 (San Diego, CA, USA) was used for the statistical analyses. Differences between the control and BA-exposed groups were determined with an unpaired Student's t-test, unless stated otherwise. A value of $p \leq$ 0.05 was considered as statistically significant. A total of three biological replicates were performed.

\section{Results}

Establishment of an inflammatory in vitro human intestinal model consisting of Caco-2 and HT29-MTX-E12 cells combined with LPS-activated dendritic cells

The first important step of this study was to establish an in vitro human intestinal model with an improved physiological representation of the intestinal barrier and inflammatory environment in the context of IBD. In Figure 1A, a schematic overview of the study design is given. Caco-2 and HT29MTX-E12 cells were seeded in a 3:1 ratio on cell culture inserts and SWMS conditions were applied. In parallel, primary monocytes were isolated from three human buffy coats and differentiated into DCs. Activation with $10 \mathrm{ng} / \mathrm{mL}$ LPS for 24 hours resulted in mature DCs expressing the DC surface markers CD83 and CD209 (Supplementary file 1). Activated DCs produced higher levels of IL-6 ( $p=0.0088)$ and IL-12p40 ( $p=0.1)$ compared to DCs that were not activated (Fig. 1B, C), although IL-12p40 levels of one biological replicate were relatively low (Fig. 1C). After 24 hours of LPS exposure, the cell culture inserts with the Caco-2/HT29-MTX-E12 co-culture were positioned in the cell culture plates containing activated DCs. This resulted in a model consisting of intestinal cells in the apical compartment and LPS-activated DCs in the basolateral compartment (Fig. 1D). TEER values measured at 24 and 48 hours after combination with activated DCs decreased with 12 and 45 percentage points, respectively, compared to the condition without basolateral DCs $(p<0.001$ and 
$p<0.0001$ ) (Fig. 1E). In the next BA-exposure experiments, we used a pre-incubation period of 24 hours. Altogether, we confirmed that the presence of activated DCs in the basolateral compartment caused a pro-inflammatory state, reflected by the elevated cytokine levels. This likely resulted in the observed increased intestinal permeability of the intestinal cells.

A

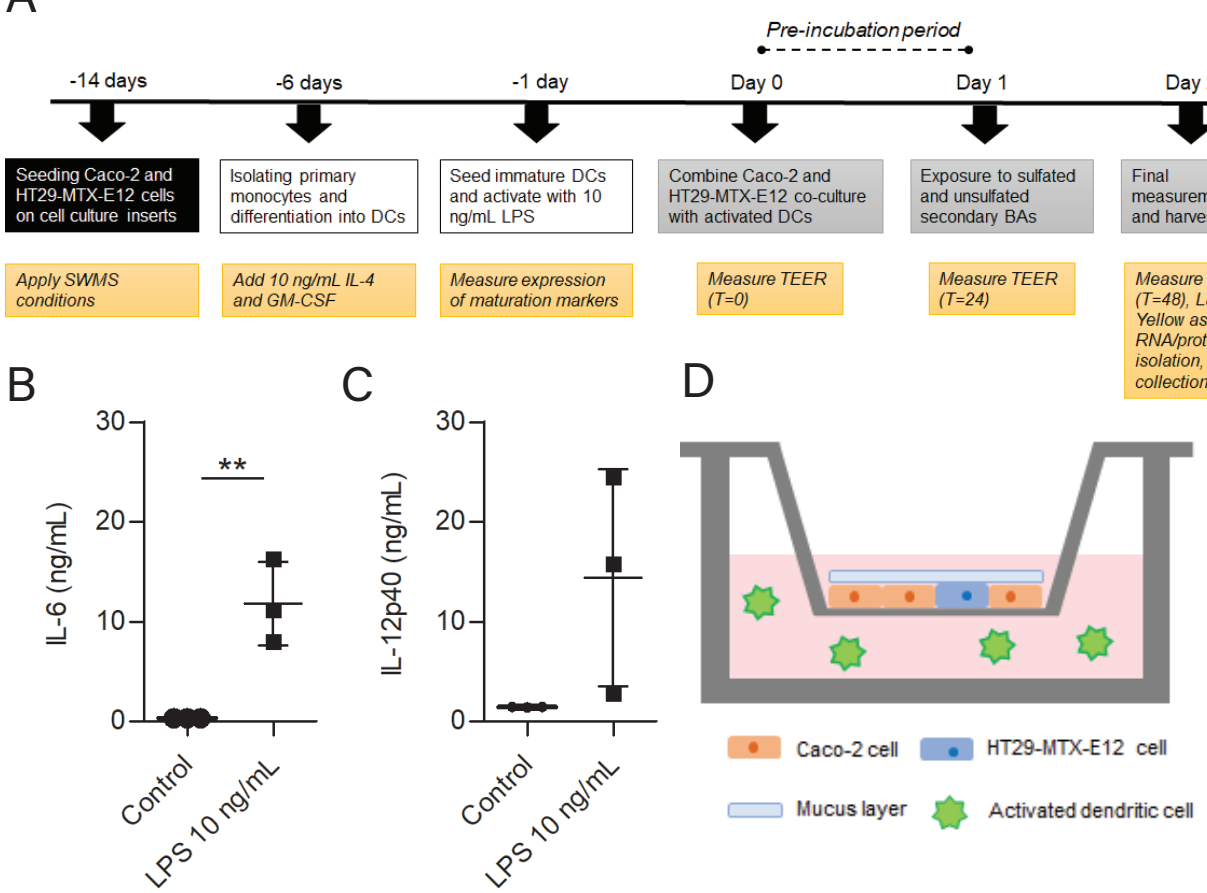

$\mathrm{E}$

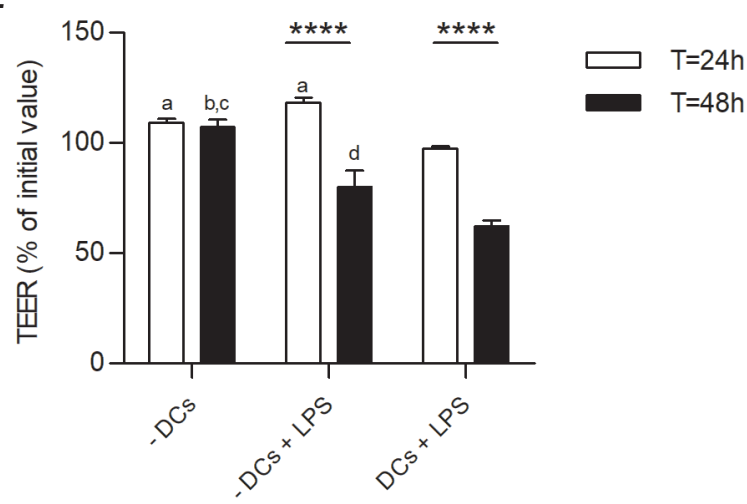

Figure 1 Establishment of a triple co-culture of Caco-2, HT29-MTX-E12 cells combined with activated dendritic cells. (A) Schematic overview of study design in chronological order. (B) Concentrations of IL-6 and (C) IL-12p40 in DC supernatant after activation with $10 \mathrm{ng} / \mathrm{mL}$ LPS for 24 hours. ${ }^{* * p}<0.01$. (D) 
Schematic overview of the Caco-2 and HT29-MTX-E12 co-culture grown in a 3:1 ratio on cell culture inserts with a the mucus layer on top and activated DCs in basolateral compartment. (E) TEER measurements of Caco-2 and HT29-MTX-E12 culture at 24 and 48 hours. TEER values are expressed as percentage of the initial value. First bar pair: control cells without DCs, second bar pair: control cells with $10 \mathrm{ng} / \mathrm{mL}$ LPS in the basolateral compartment, third bar pair: cells combined with activated DCs. Statistical differences were determined using a one-way analysis of variance (ANOVA) followed by a Bonferroni post hoc test. ap $<0.001$ at $\mathrm{T}=24 \mathrm{~h}$ compared to condition with activated DCs. bp $<0.001$ at $\mathrm{T}=48 \mathrm{~h}$ compared to condition without DCs, but with basolateral LPS. $c p<0.0001$ at $\mathrm{T}=48 \mathrm{~h}$ compared to condition with activated DCs. $\mathrm{dp}<0.05$ at $\mathrm{T}=48 \mathrm{~h}$ compared to condition with activated DCs. ${ }^{* * *} \mathrm{p}<0.0001$. Data are derived from 3 independent biological replicates.

\section{Intestinal permeability was slightly restored by LCA and sulfated DCA under inflammatory conditions}

After the pre-incubation period, the co-cultures of Caco-2 and HT29-MTX-E12 cells were exposed to sulfated DCA, sulfated LCA and their unsulfated forms in different concentrations for another 24 hours. Cytotoxicity measured by the release of LDH in the apical medium was not different between cells exposed to BAs compared to unexposed cells (data not shown). The TEER of all conditions exposed to BAs in the presence of activated DCs were significantly lower compared to the control without DCs $(p<0.0001)$ (Fig. 2A). Exposure to sulfated DCA $(200 \mu \mathrm{M})$ and both concentrations of LCA $(10 \mu \mathrm{M}$ and $50 \mu \mathrm{M})$ resulted in a slight, but significant restoration of the TEER (Fig. 2A). The same cell culture inserts were subjected to a Lucifer Yellow assay to investigate if BA treatment had an effect on paracellular permeability. The flux of Lucifer Yellow from the apical to basolateral compartment was significantly lower in cells cultured without DCs compared to the control with DCs $(p<0.05)$ (Fig. 2B). None of the BAs had a significant additional effect on paracellular permeability.

A

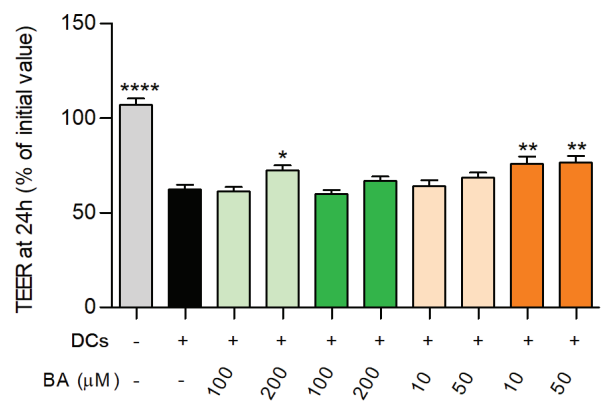

B

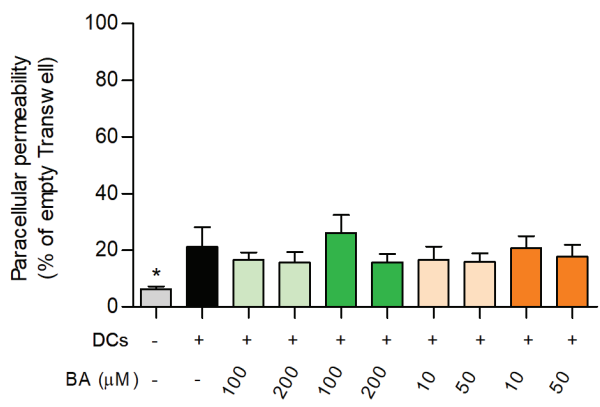


C

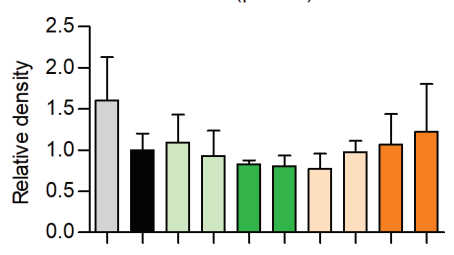

DCs

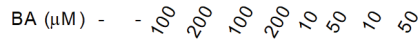

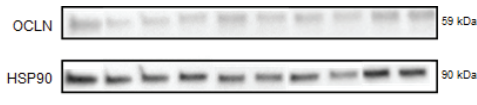

$\mathrm{D}$

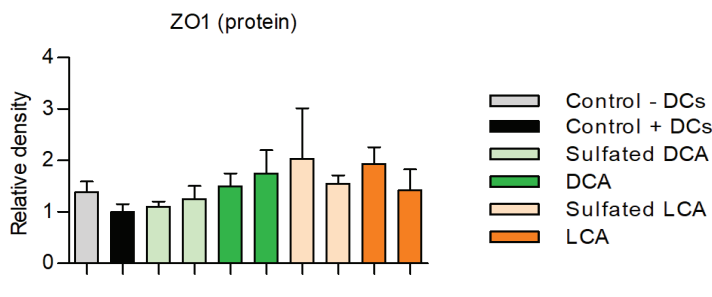

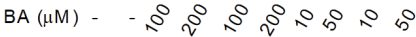

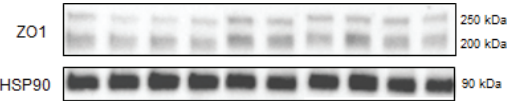

E

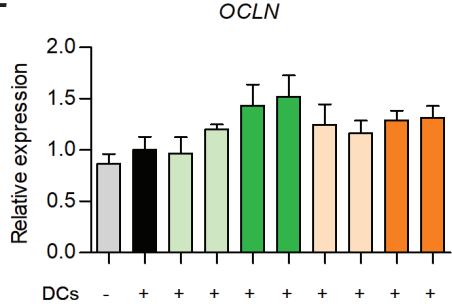

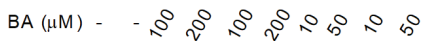

G

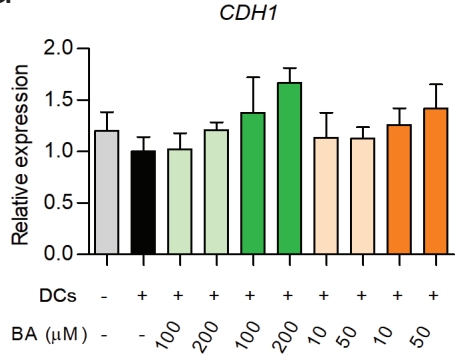

$\mathrm{F}$

F 201

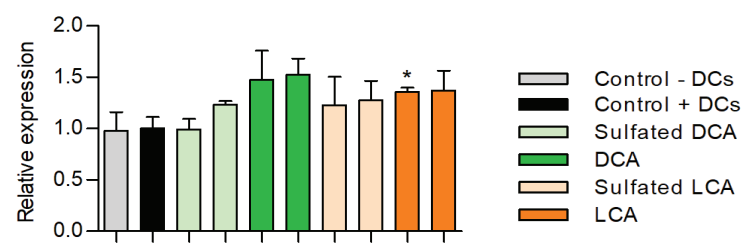

$\mathrm{DCs}-++++++++$

$\mathrm{BA}(\mu \mathrm{M})-\Omega_{0} \cong \therefore$

$\mathrm{H}$

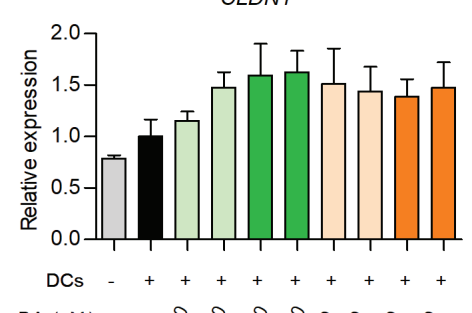

Figure 2 Intestinal permeability assays of Caco-2 and HT29-MTX-E12 cells combined with activated DCs after treatment with (sulfated) BAs for $\mathbf{2 4 h}$. (A) TEER values expressed as \% of initial values. (B) Fluorescence measured in basolateral compartment after 3h apical Lucifer Yellow incubation, expressed as \% of an empty cell culture insert (representing complete translocation). (C-D) Protein quantity of OCLN and ZO1 relative to the control. (E-H) Panel of genes related to intestinal permeability (OCLN: Occludin, ZO1: Zonula Occludens-1, CDH1: E-cadherin, CLDN1: Claudin-1). Expression of proteins and genes of interest is expressed relative to the control (Caco-2 and HT29-MTX-E12 cells exposed to activated DCs in basolateral compartment). ${ }^{*} p<0.05 ;{ }^{* *} p<0.01 ;{ }^{* * *} p<0.0001$ compared to the control condition with activated DCs. 
Expression of genes related to intestinal epithelial integrity tended to increase after BA exposure

To further investigate the effects of sulfated secondary BAs on intestinal barrier function, we measured the expression of proteins related to intestinal epithelial integrity. In line with the significant TEER reduction (Fig. 2A), lower protein levels of Occludin (OCLN) and Zonula Occludens-1 (ZO1) were measured in cells exposed to activated DCs compared to the control cells without DCs (Fig. 2CD), but these differences were not significant. Next, we investigated whether these lower protein levels were the result of decreased mRNA levels. However, OCLN and ZO1 mRNA levels were not significantly affected by the presence of activated DCs in the basolateral compartment (Fig. 2E-F). Other genes related to intestinal barrier function, E-cadherin ( $C D H 1)$ and Claudin-1 (CLDN1), were also not affected (Fig. 2G-H). Interestingly, protein levels of OCLN and ZO1 were not affected by BA exposure, whereas expression of OCLN, ZO1, CDH1 and CLDN1 followed an increasing trend after exposure to most BAs, although these differences were not significant (Fig. 2C-H). Together, these results indicate that differences in intestinal barrier function measured by TEER were partly reflected at gene and protein level.

\section{Differential expression of FXR-target genes by unsulfated, but not sulfated secondary BAs}

Next, we aimed to find out if exposure to sulfated and unsulfated secondary BAs resulted in activation of FXR. While DCA and LCA are potent activators of FXR (22), it is unknown whether the sulfated forms of these BAs also activate FXR, as these BAs are not, or poorly absorbed by enterocytes (23). To this end, we investigated if exposure to DCA, LCA and their sulfated forms resulted in differential expression of a selection of FXR-target genes: ileal bile acid binding protein (IBABP, FABP6), fibroblast growth factor 19 (FGF19), basolateral organic solute transporters alpha and beta (OST $/ \beta$, $S L C 51 A / B)$, apical bile salt transporter (ABST, SLC10A2) and sulfotransferase family $2 \mathrm{~A}$ member 1 (SULT2A1) (31-34). Interestingly, the addition of activated DCs potently reduced the expression of ABST $(p<0.05)$ and SULT2A1 $(p<0.001)$ (Fig. 3A, F). ABST was not differentially expressed by any of the BAs (Fig. 3A). In contrast, FABP6, FGF19 and OST $\beta$ were significantly upregulated in cells exposed to DCA compared to the control with activated DCs (Fig. 3B-C, E). Interestingly, exposure to $100 \mu \mathrm{M}$ DCA reduced SULT2A1 expression compared to the control cells with DCs (Fig. 3F). Altogether, these results indicate that DCA had pronounced effects on the expression of most FXRtarget genes, while LCA had only minor effects. Exposure to neither sulfated DCA nor sulfated LCA resulted in a differential expression of any FXR-target genes. Importantly, mRNA levels of $A B S T$ and SULT2A1 were significantly decreased by the presence of basolateral activated DCs. 
A

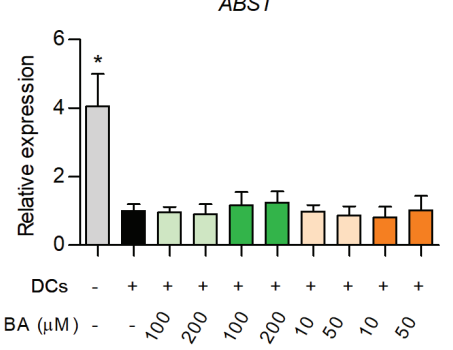

C

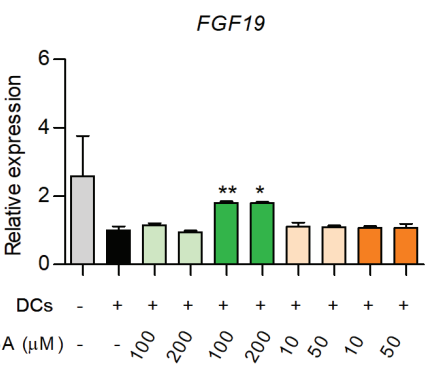

E

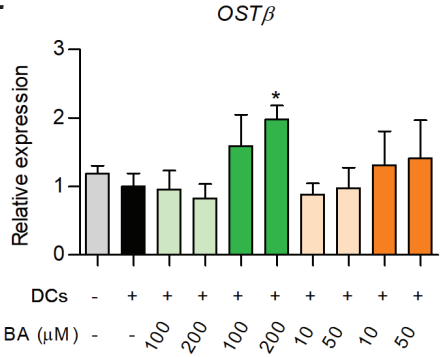

B

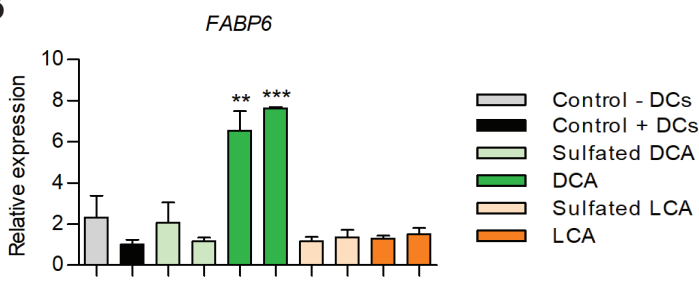

DCs

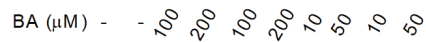

D

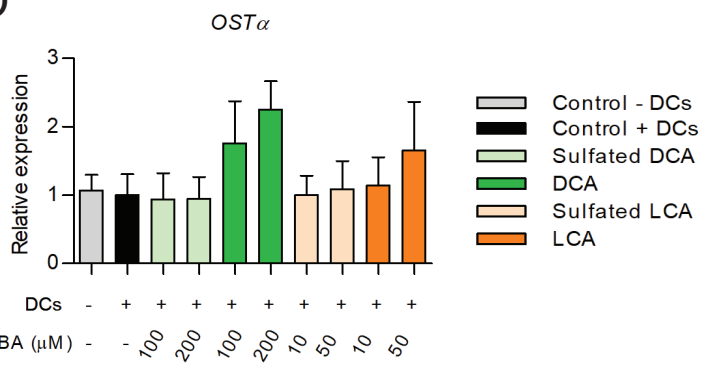

$\mathrm{F}$

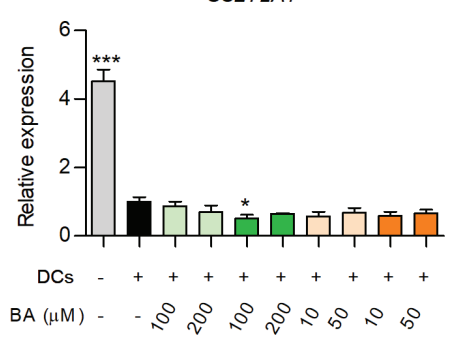

Figure 3 Expression of FXR target genes (A-F) ABST, FABP6, FGF19, OST $\alpha$, OST $\beta$ and SULT2A1. Expression of genes of interest is expressed as fold change relative to control (Caco-2 and HT29-MTX-E12 cells exposed to activated DCs in basolateral compartment). ${ }^{*} p<0.05,{ }^{* *} p<0.01,{ }^{* * *} p<0.001$ compared to the condition with activated DCs.

\section{No effects of sulfated secondary BAs on MUC2 and MUC5AC expression}

In order to determine if sulfated secondary BAs had an effect on the mucus layer, we investigated the expression of $M U C 2$, which is the most dominant gel-forming mucin present in the intestine. Moreover, we also measured expression of MUC5AC. This is another gel-forming mucin which is normally not secreted in the intestine, but is secreted in HT29-MTX-E12 cells, even after growing this cell type under SWMS conditions $(29,30)$. Interestingly, the presence of activated DCs decreased the expression of MUC2 and MUC5AC, although this effect was not statistically significant (Fig. 4A- 
B). Compared to the control with activated DCs, the expression of MUC2 seemed to increase after exposure to $100 \mu \mathrm{M}$ DCA and $10 \mu \mathrm{M} L C A$, which was borderline significant $(p=0.06$ and $p=0.08)$, respectively (Fig. 4A). Sulfated BA exposure did not have any effect on mucin mRNA expression.

\section{Subtle effect of some BAs on expression of genes encoding for antimicrobial peptides}

Antimicrobial peptides (AMPs) play an important role in intestinal innate immune defense and are known to be produced by enterocytes (35). We measured the expression of genes encoding the AMPs defensin $\beta-1$ (DEFB1) and lysozyme (LYZ), but also angiogenin (ANG) and carbonic anhydrase 12 (CA12), since the latter two AMPs are regulated by the BA receptor FXR $(36,37)$. Exposure to BAs caused slight, but non-significant changes compared to the control with activated DCs (Fig. 4C-F). Only ANG was significantly lower expressed after exposure to both $100 \mu \mathrm{M}$ sulfated DCA and DCA, as well as $50 \mu \mathrm{M}$ LCA $(p<0.05)$ (Fig. 4F).

A

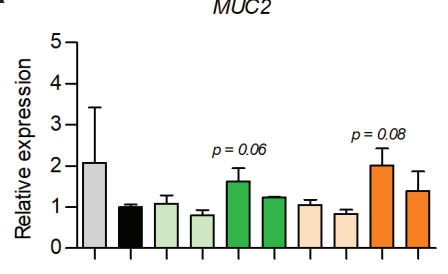

$\mathrm{DCs}-+++++++++$

BA $(\mu \mathrm{M})$ - -2000

C

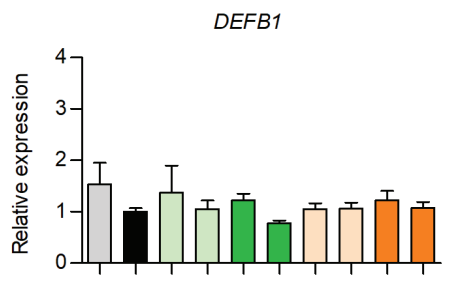

$\mathrm{DCs}-++++++++$

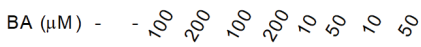

E

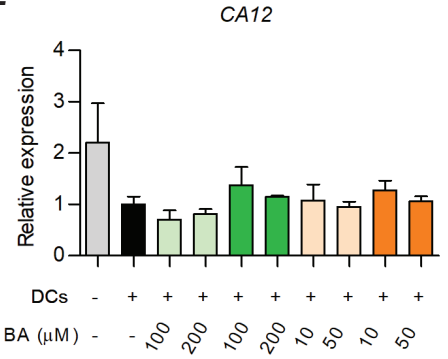

B
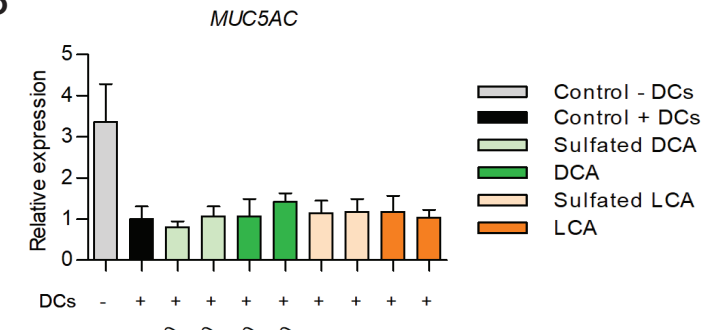

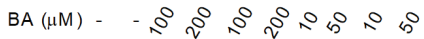

D

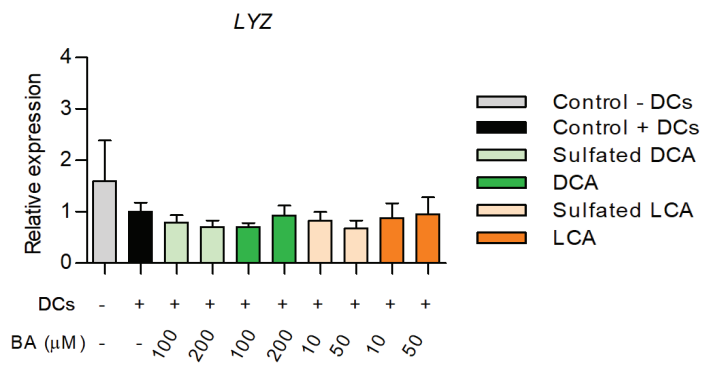

F

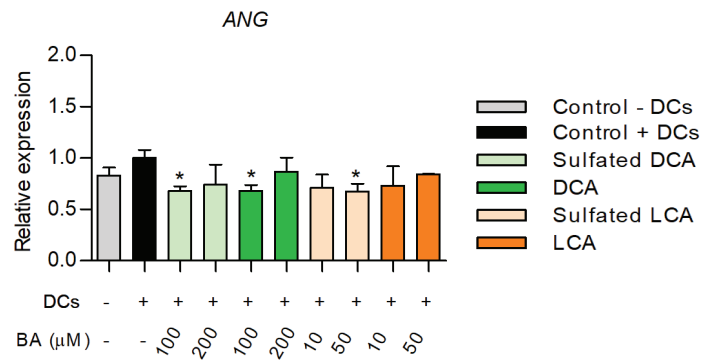


Figure 4 Expression of mucin and antimicrobial peptides. A) Expression of MUC2 and B) MUC5AC and genes encoding the antimicrobial peptides (C-F) DEFB1, LYZ, CA12 and ANG. Expression of genes of interest is expressed relative to the control (Caco-2 and HT29-MTX-E12 cells exposed to activated DCs in basolateral compartment). ${ }^{*} p<0.05$ compared to condition with activated DCs.

\section{No indirect effects of BA exposure on cytokine production by basolateral DCs}

Although the presence of activated DCs in the basolateral compartment resulted in a significant increase in permeability of the Caco-2/HT29-MTX-E12 co-culture, apical exposure to sulfated and unsulfated secondary BAs did not have a major additional effect on intestinal epithelial integrity (Fig. 2A-B). We hypothesized that BAs might have migrated from the apical to the basolateral compartment via the openings between the intestinal epithelial cells, caused by the increased intestinal permeability. In that case, BAs might have come in contact with the DCs present in the basolateral compartment. Therefore, we investigated if this potential indirect contact between BAs and DCs caused an altered immune response by DCs. To this end, TNF- $\alpha$ and IL-12p40 levels were measured in conditioned medium from basolateral DCs after apical exposure to the different BAs. No differences in either TNF- $\alpha$ or IL-12p40 levels were found (Fig. 5A-B).

A

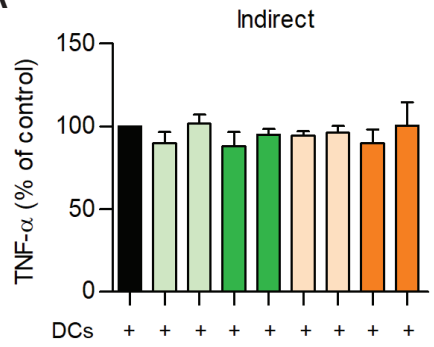

C

$\mathrm{BA}(\mu \mathrm{M})-\Omega_{0}: 0 \leqslant 6 \leqslant$

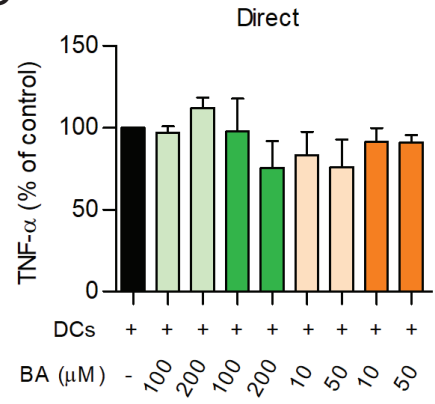

B

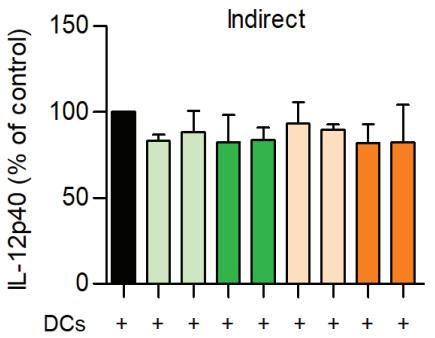

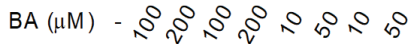

D

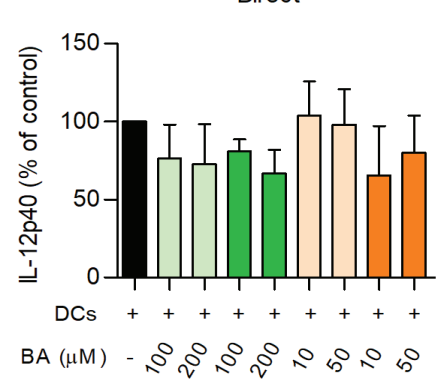

Figure 5 Cytokine levels produced by basolateral DCs after indirect BA exposure (via cell culture inserts) and direct exposure. A) TNF- $\alpha$ and B) IL-12p40 levels after indirect exposure. C) TNF- $\alpha$ and D) 
IL-12p40 levels after direct exposure. Cytokine levels are expressed as percentage of the control, i.e. DCs exposed to only medium.

Decreasing, but no significant trend in TNF- $\alpha$ and IL-12p40 production by activated DCs after direct exposure to secondary BAs

The finding that cytokine production by DCs was not affected by indirect BA exposure could either indicate that BAs were not migrated towards the basolateral compartment, or that DCs were not affected by BA exposure in terms of TNF- $\alpha$ and IL-12p40 production. To investigate if direct exposure to BAs caused an effect on immune response in DCs, we exposed activated DCs directly to sulfated and unsulfated secondary BAs under similar conditions as previous experiments with indirect exposure. DCA caused a decrease in both TNF- $\alpha$ and IL-12p40 levels compared to the control cells (Fig. 5C-D), but these differences were not significant. Lower IL-12p40 levels were found after LCA exposure, albeit variation between biological replicates was high (Fig. 5D). This was due to deviating results from one biological replicate (Supplementary file 1). No significant differences were found after exposure to sulfated BAs.

\section{Discussion}

The rising prevalence of IBD in many countries is alarming, given the concomitant increase in social and economic burden associated with this disease (38). To decrease this burden, it is of utmost importance to better understand the underlying causes of IBD, especially because the etiology of IBD is still largely unknown. Emerging evidence suggests a potential role for BA dysmetabolism in IBD, however, the exact effects of elevated levels of IBD-associated BA subtypes are not widely investigated yet. In the present study, we aimed to investigate the effects of sulfated secondary BAs on intestinal barrier function in the context of IBD. Furthermore, we also investigated if sulfated BAs had an effect on immune response in human monocyte-derived DCs.

We first aimed to establish an inflammatory in vitro human intestinal model, as existing models often insufficiently reflect the chronic inflammatory state in the context of IBD. For example, many existing models either add a cytokine cocktail to induce a pro-inflammatory state $(39,40)$ or use THP1 cells as representation of immune cells (41-46). The effectiveness of this cell line in an intestinal model is questionable. In two studies, Caco-2 cells exposed to THP-1 cells were severely damaged after 48 hours, which was reflected by the high cytotoxicity values and TEER decrease of more than $80 \%(44,46)$. Given the crucial role of intestinal DCs in IBD pathophysiology $(14,47)$, we used 
human monocyte-derived DCs in our model. After activation with LPS, these DCs produced high cytokine levels, resulting in an increased intestinal permeability without affecting cytotoxicity. To improve the physiological representativeness of our model even more, we also paid special attention to the mucus layer, since it is often underrepresented or even lacking in most existing intestinal in vitro models. Therefore, we cultured the Caco-2/HT29-MTX-E12 co-culture under SWMS conditions, which was shown to improve the quantity and quality of the mucus layer $(29,30)$.

After successful optimization, we exposed the inflammatory in vitro human intestinal immune model to sulfated and unsulfated secondary BAs for 24 hours and investigated the effects on intestinal barrier function. We found a slight TEER restoration after exposure to LCA and sulfated DCA, but not DCA and sulfated LCA. These effects on intestinal epithelial barrier integrity were partly reflected at protein level. Previous in vitro studies also showed TEER restoration by LCA in the presence of inflammatory conditions $(40,48)$. With regard to DCA, we did not find an effect on TEER, while a marked increased permeability caused by DCA was observed in several in vitro models (49$52)$ as well as in mice $(51,53,54)$. Importantly, we confirmed successful administration of DCA and LCA by measuring differential expression of FXR-target genes. Differences in incubation duration and BA concentrations might have impeded direct comparison to existing literature and results of the current study.

In line with the minor effects on intestinal epithelial barrier integrity, we did not find an effect of sulfated BAs on MUC2 and MUC5AC expression. On the contrary, DCA and LCA exposure resulted in an increased expression of MUC2, which was borderline significant. As MUC2 plays a crucial role in intestinal barrier protection (55-58), increased MUC2 mRNA expression might indicate that these BAs have a restorative effect on the mucus layer. In several human colon cancer cell lines, DCA also caused increased MUC2 expression $(59,60)$, but no effects of LCA on mucin mRNA expression have been described. Importantly, it was previously shown that prolonged exposure to pro-inflammatory cytokines strongly decreased mucin gene expression $(61,62)$. These results are in line with the decreasing trend in MUC2 and MUC5AC expression that we found after exposure to activated DCs, although this effect was not significant. Next to the effects of BAs on the mucus barrier, it is also important to consider other intestinal barrier properties, such as AMPs that are excreted in the mucus layer (63). Although DCA was previously shown to increase the expression and secretion of DEFB1/DEFB1 in vitro (64), we were not able to reproduce these results. We did find a slightly reduced expression of $A N G$ by some BAs, which might imply that these BAs have a negative effect on mucosal defense (65). However, the effects of BAs on AMPs are underexplored in current literature, indicating that more research is needed in this field. 
Secondary BAs could have anti-inflammatory effects during intestinal inflammation $(27,66,67)$. Since intestinal DCs are able to sample luminal content $(47,68)$, we hypothesized that luminal BAs could come in contact with DCs, which might result in an altered immune response. Indeed, direct exposure to secondary BAs caused a decreasing trend in cytokine production, but this effect was absent after exposure to sulfated secondary BAs. This finding might suggest that increased levels of sulfated BAs at the expense of secondary BAs could abolish the anti-inflammatory effects of secondary BAs. Similar effects were previously found in Caco-2 exposed to sulfated LCA (3), although this effect was found after exposure to relatively high concentrations of LCA and sulfated LCA (400 and $500 \mu \mathrm{M})$, which might hamper the physiological translatability of these results.

Here, we present a novel and physiological relevant in vitro human intestinal model representing a pro-inflammatory state, which can be used to study intestinal barrier function in the presence of intestinal inflammation. We used this model to investigate the effects of sulfated and unsulfated secondary BAs on intestinal barrier function and immune response in DCs. We show that these BAs had ambiguous effects on intestinal barrier integrity, as reflected by the minor effects on TEER, expression of intestinal epithelial integrity related genes, AMPs and MUC2. Our results hint towards anti-inflammatory effects of secondary BAs, but not sulfated secondary BAs on activated DCs. These findings emphasize the potential impact of BA dysmetabolism during IBD and the relevance of proper bacterial desulfation activity to assure the anti-inflammatory effects of secondary BAs.

\section{Acknowledgements}

This research was funded by the Nutricia Research Foundation with grant no. 2018-25. BvdL is supported by the NWO Graduate Programme on Food Structure, Digestion and Health (022.006.009). $\mathrm{NI}$ is supported by the MLDS career development grant (CDG16-04) and by the Wilhelmina Children's Hospital Research Fund. 


\section{References}

1. Baumgart DC, Sandborn WJ. Inflammatory bowel disease: clinical aspects and established and evolving therapies. Lancet. 2007;369(9573):1641-57.

2. Kaser A, Zeissig S, Blumberg RS. Inflammatory bowel disease. Annu Rev Immunol. 2010;28:573621.

3. Duboc H, Rajca S, Rainteau D, Benarous D, Maubert MA, Quervain E, et al. Connecting dysbiosis, bile-acid dysmetabolism and gut inflammation in inflammatory bowel diseases. Gut. 2013;62(4):531-9.

4. Frank DN, St Amand AL, Feldman RA, Boedeker EC, Harpaz N, Pace NR. Molecular-phylogenetic characterization of microbial community imbalances in human inflammatory bowel diseases. Proc Natl Acad Sci U S A. 2007;104(34):13780-5.

5. Gevers D, Kugathasan S, Denson LA, Vázquez-Baeza Y, Van Treuren W, Ren B, et al. The treatment-naive microbiome in new-onset Crohn's disease. Cell Host Microbe. 2014;15(3):382-92.

6. Nishino K, Nishida A, Inoue R, Kawada Y, Ohno M, Sakai S, et al. Analysis of endoscopic brush samples identified mucosa-associated dysbiosis in inflammatory bowel disease. J Gastroenterol. 2018;53(1):95-106.

7. Morris G, Berk M, Carvalho AF, Caso JR, Sanz Y, Maes M. The Role of Microbiota and Intestinal Permeability in the Pathophysiology of Autoimmune and Neuroimmune Processes with an Emphasis on Inflammatory Bowel Disease Type 1 Diabetes and Chronic Fatigue Syndrome. Curr Pharm Des. 2016;22(40):6058-75.

8. Heazlewood CK, Cook MC, Eri R, Price GR, Tauro SB, Taupin D, et al. Aberrant mucin assembly in mice causes endoplasmic reticulum stress and spontaneous inflammation resembling ulcerative colitis. PLoS Med. 2008;5(3):e54.

9. Johansson ME. Mucus layers in inflammatory bowel disease. Inflamm Bowel Dis. 2014;20(11):2124-

31.

10. Carvalho FA, Koren O, Goodrich JK, Johansson ME, Nalbantoglu I, Aitken JD, et al. Transient inability to manage proteobacteria promotes chronic gut inflammation in TLR5-deficient mice. Cell Host Microbe. 2012;12(2):139-52.

11. Schmitz H, Barmeyer C, Fromm M, Runkel N, Foss HD, Bentzel CJ, et al. Altered tight junction structure contributes to the impaired epithelial barrier function in ulcerative colitis. Gastroenterology. 1999;116(2):301-9.

12. Sartor RB. Microbial influences in inflammatory bowel diseases. Gastroenterology. 2008;134(2):577-94.

13. Xavier RJ, Podolsky DK. Unravelling the pathogenesis of inflammatory bowel disease. Nature. 2007;448(7152):427-34.

14. Bernardo D, Chaparro M, Gisbert JP. Human Intestinal Dendritic Cells in Inflammatory Bowel Diseases. Mol Nutr Food Res. 2018;62(7):e1700931.

15. Cerovic V, Houston SA, Scott CL, Aumeunier A, Yrlid U, Mowat AM, et al. Intestinal CD103(-) dendritic cells migrate in lymph and prime effector T cells. Mucosal Immunol. 2013;6(1):104-13.

16. Konrad A, Cong Y, Duck W, Borlaza R, Elson CO. Tight mucosal compartmentation of the murine immune response to antigens of the enteric microbiota. Gastroenterology. 2006;130(7):2050-9.

17. Bsat M, Chapuy L, Baba N, Rubio M, Panzini B, Wassef R, et al. Differential accumulation and function of proinflammatory 6-sulfo LacNAc dendritic cells in lymph node and colon of Crohn's versus ulcerative colitis patients. J Leukoc Biol. 2015;98(4):671-81.

18. Ng SC, Benjamin JL, McCarthy NE, Hedin CR, Koutsoumpas A, Plamondon S, et al. Relationship between human intestinal dendritic cells, gut microbiota, and disease activity in Crohn's disease. Inflamm Bowel Dis. 2011;17(10):2027-37.

19. Franzosa EA, Sirota-Madi A, Avila-Pacheco J, Fornelos N, Haiser HJ, Reinker S, et al. Gut microbiome structure and metabolic activity in inflammatory bowel disease. Nat Microbiol. 2019;4(2):293305.

20. Jacobs JP, Goudarzi M, Singh N, Tong M, McHardy IH, Ruegger P, et al. A Disease-Associated Microbial and Metabolomics State in Relatives of Pediatric Inflammatory Bowel Disease Patients. Cell Mol Gastroenterol Hepatol. 2016;2(6):750-66.

21. Wang S, Martins R, Sullivan MC, Friedman ES, Misic AM, El-Fahmawi A, et al. Diet-induced remission in chronic enteropathy is associated with altered microbial community structure and synthesis of secondary bile acids. Microbiome. 2019;7(1):126.

22. Chiang JY. Bile acid metabolism and signaling. Compr Physiol. 2013;3(3):1191-212.

23. Alnouti Y. Bile Acid sulfation: a pathway of bile acid elimination and detoxification. Toxicol Sci. 
2009;108(2):225-46.

24. Jones ML, Martoni CJ, Ganopolsky JG, Labbé A, Prakash S. The human microbiome and bile acid metabolism: dysbiosis, dysmetabolism, disease and intervention. Expert Opin Biol Ther. 2014;14(4):467-82. 25. Dior $M$, Delagrèverie $H$, Duboc $H$, Jouet $P$, Coffin $B$, Brot $L$, et al. Interplay between bile acid metabolism and microbiota in irritable bowel syndrome. Neurogastroenterol Motil. 2016;28(9):1330-40.

26. Lloyd-Price J, Arze C, Ananthakrishnan AN, Schirmer M, Avila-Pacheco J, Poon TW, et al. Multiomics of the gut microbial ecosystem in inflammatory bowel diseases. Nature. 2019;569(7758):655-62.

27. Sinha SR, Haileselassie Y, Nguyen LP, Tropini C, Wang M, Becker LS, et al. Dysbiosis-Induced Secondary Bile Acid Deficiency Promotes Intestinal Inflammation. Cell Host Microbe. 2020;27(4):659-70. e5.

28. Duboc H, Rainteau D, Rajca S, Humbert L, Farabos D, Maubert M, et al. Increase in fecal primary bile acids and dysbiosis in patients with diarrhea-predominant irritable bowel syndrome. Neurogastroenterol Motil. 2012;24(6):513-20, e246-7.

29. Navabi N, McGuckin MA, Lindén SK. Gastrointestinal cell lines form polarized epithelia with an adherent mucus layer when cultured in semi-wet interfaces with mechanical stimulation. PLoS One. 2013;8(7):e68761.

30. Elzinga J, Van der Lugt B, Belzer C, Steegenga WT. Characterization of increased mucus production of HT29-MTX-E12 cells grown under Semi-Wet interface with Mechanical Stimulation. 2021.

31. Gadaleta RM, van Mil SW, Oldenburg B, Siersema PD, Klomp LW, van Erpecum KJ. Bile acids and their nuclear receptor FXR: Relevance for hepatobiliary and gastrointestinal disease. Biochim Biophys Acta. 2010;1801(7):683-92.

32. Lefebvre P, Cariou B, Lien F, Kuipers F, Staels B. Role of bile acids and bile acid receptors in metabolic regulation. Physiol Rev. 2009;89(1):147-91.

33. Matsubara T, Li F, Gonzalez FJ. FXR signaling in the enterohepatic system. Mol Cell Endocrinol. 2013;368(1-2):17-29.

34. Runge-Morris M, Kocarek TA, Falany CN. Regulation of the cytosolic sulfotransferases by nuclear receptors. Drug Metab Rev. 2013;45(1):15-33.

35. Furci L, Secchi M. AMPs and Mechanisms of Antimicrobial Action. In: Cho $\mathrm{CH}$, editor. Antimicrobial Peptides in Gastrointestinal Diseases: Elsevier Ltd.; 2018. p. 97-131.

36. Inagaki T, Moschetta A, Lee YK, Peng L, Zhao G, Downes M, et al. Regulation of antibacterial defense in the small intestine by the nuclear bile acid receptor. Proc Natl Acad Sci U S A. 2006;103(10):39205.

37. Modica S, Gadaleta RM, Moschetta A. Deciphering the nuclear bile acid receptor FXR paradigm. Nucl Recept Signal. 2010;8:e005.

38. The global, regional, and national burden of inflammatory bowel disease in 195 countries and territories, 1990-2017: a systematic analysis for the Global Burden of Disease Study 2017. Lancet Gastroenterol Hepatol. 2020;5(1):17-30.

39. Dosh $\mathrm{RH}$, Jordan-Mahy N, Sammon C, Le Maitre CL. Long-term in vitro 3D hydrogel co-culture model of inflammatory bowel disease. Sci Rep. 2019;9(1):1812.

40. Sarathy J, Detloff SJ, Ao M, Khan N, French S, Sirajuddin H, et al. The Yin and Yang of bile acid action on tight junctions in a model colonic epithelium. Physiol Rep. 2017;5(10):e13294.

41. Kämpfer AAM, Urbán P, Gioria S, Kanase N, Stone V, Kinsner-Ovaskainen A. Development of an in vitro co-culture model to mimic the human intestine in healthy and diseased state. Toxicol In Vitro. 2017;45(Pt 1):31-43.

42. Makon-Sébastien N, Francis F, Eric S, Henri VP, François LJ, Laurent P, et al. Lycopene modulates THP1 and $\mathrm{CacO} 2$ cells inflammatory state through transcriptional and nontranscriptional processes. Mediators Inflamm. 2014;2014:507272.

43. Moyes SM, Morris JF, Carr KE. Macrophages increase microparticle uptake by enterocyte-like Caco-2 cell monolayers. J Anat. 2010;217(6):740-54.

44. Satsu H, Ishimoto $Y$, Nakano T, Mochizuki T, Iwanaga T, Shimizu M. Induction by activated macrophage-like THP-1 cells of apoptotic and necrotic cell death in intestinal epithelial Caco-2 monolayers via tumor necrosis factor-alpha. Exp Cell Res. 2006;312(19):3909-19.

45. Susewind J, de Souza Carvalho-Wodarz C, Repnik U, Collnot EM, Schneider-Daum N, Griffiths GW, et al. A 3D co-culture of three human cell lines to model the inflamed intestinal mucosa for safety testing of nanomaterials. Nanotoxicology. 2016;10(1):53-62.

46. Watanabe F, Satsu H, Mochizuki T, Nakano T, Shimizu M. Development of the method for evaluating protective effect of food factors on THP-1-induced damage to human intestinal Caco-2 
monolayers. Biofactors. 2004;21(1-4):145-7.

47. Stagg AJ. Intestinal Dendritic Cells in Health and Gut Inflammation. Front Immunol. 2018;9:2883.

48. Yao B, He J, Yin X, Shi Y, Wan J, Tian Z. The protective effect of lithocholic acid on the intestinal epithelial barrier is mediated by the vitamin $\mathrm{D}$ receptor via a SIRT1/Nrf2 and NF-KB dependent mechanism in Caco-2 cells. Toxicol Lett. 2019;316:109-18.

49. Chen X, Oshima T, Tomita T, Fukui H, Watari J, Matsumoto T, et al. Acidic bile salts modulate the squamous epithelial barrier function by modulating tight junction proteins. Am J Physiol Gastrointest Liver Physiol. 2011;301(2):G203-9.

50. Hughes R, Kurth MJ, McGilligan V, McGlynn H, Rowland I. Effect of colonic bacterial metabolites on Caco-2 cell paracellular permeability in vitro. Nutr Cancer. 2008;60(2):259-66.

51. Liu L, Dong W, Wang S, Zhang Y, Liu T, Xie R, et al. Deoxycholic acid disrupts the intestinal mucosal barrier and promotes intestinal tumorigenesis. Food Funct. 2018;9(11):5588-97.

52. Raimondi F, Santoro P, Barone MV, Pappacoda S, Barretta ML, Nanayakkara M, et al. Bile acids modulate tight junction structure and barrier function of Caco-2 monolayers via EGFR activation. Am J Physiol Gastrointest Liver Physiol. 2008;294(4):G906-13.

53. Murakami Y, Tanabe S, Suzuki T. High-fat Diet-induced Intestinal Hyperpermeability is Associated with Increased Bile Acids in the Large Intestine of Mice. J Food Sci. 2016;81(1):H216-22.

54. Stenman LK, Holma R, Eggert A, Korpela R. A novel mechanism for gut barrier dysfunction by dietary fat: epithelial disruption by hydrophobic bile acids. Am J Physiol Gastrointest Liver Physiol. 2013;304(3):G227-34.

55. Johansson ME, Phillipson M, Petersson J, Velcich A, Holm L, Hansson GC. The inner of the two Muc2 mucin-dependent mucus layers in colon is devoid of bacteria. Proc Natl Acad Sci U S A. 2008;105(39):15064-9.

56. Sovran B, Lu P, Loonen LM, Hugenholtz F, Belzer C, Stolte EH, et al. Identification of Commensal Species Positively Correlated with Early Stress Responses to a Compromised Mucus Barrier. Inflamm Bowel Dis. 2016;22(4):826-40.

57. Van der Sluis M, De Koning BA, De Bruijn AC, Velcich A, Meijerink JP, Van Goudoever JB, et al. Muc2-deficient mice spontaneously develop colitis, indicating that MUC2 is critical for colonic protection. Gastroenterology. 2006;131(1):117-29.

58. Velcich A, Yang W, Heyer J, Fragale A, Nicholas C, Viani S, et al. Colorectal cancer in mice genetically deficient in the mucin Muc2. Science. 2002;295(5560):1726-9.

59. Lee HY, Crawley S, Hokari R, Kwon S, Kim YS. Bile acid regulates MUC2 transcription in colon cancer cells via positive EGFR/PKC/Ras/ERK/CREB, PI3K/Akt/lkappaB/NF-kappaB and p38/MSK1/ CREB pathways and negative JNK/C-Jun/AP-1 pathway. Int J Oncol. 2010;36(4):941-53.

60. Song S, Byrd JC, Koo JS, Bresalier RS. Bile acids induce MUC2 overexpression in human colon carcinoma cells. Cancer. 2005;103(8):1606-14.

61. Enss ML, Cornberg M, Wagner S, Gebert A, Henrichs $M$, Eisenblätter R, et al. Proinflammatory cytokines trigger MUC gene expression and mucin release in the intestinal cancer cell line LS180. Inflamm Res. 2000;49(4):162-9.

62. Sharba S, Navabi N, Padra M, Persson JA, Quintana-Hayashi MP, Gustafsson JK, et al. Interleukin 4 induces rapid mucin transport, increases mucus thickness and quality and decreases colitis and Citrobacter rodentium in contact with epithelial cells. Virulence. 2019;10(1):97-117.

63. Muniz LR, Knosp C, Yeretssian G. Intestinal antimicrobial peptides during homeostasis, infection, and disease. Front Immunol. 2012;3:310.

64. Lajczak NK, Saint-Criq V, O'Dwyer AM, Perino A, Adorini L, Schoonjans K, et al. Bile acids deoxycholic acid and ursodeoxycholic acid differentially regulate human $\beta$-defensin- 1 and -2 secretion by colonic epithelial cells. Faseb j. 2017;31(9):3848-57.

65. Hooper LV, Stappenbeck TS, Hong CV, Gordon Jl. Angiogenins: a new class of microbicidal proteins involved in innate immunity. Nat Immunol. 2003;4(3):269-73.

66. Song X, Sun X, Oh SF, Wu M, Zhang Y, Zheng W, et al. Microbial bile acid metabolites modulate gut $R O R Y(+)$ regulatory T cell homeostasis. Nature. 2020;577(7790):410-5.

67. Ward JBJ, Lajczak NK, Kelly OB, O'Dwyer AM, Giddam AK, J NG, et al. Ursodeoxycholic acid and lithocholic acid exert anti-inflammatory actions in the colon. Am J Physiol Gastrointest Liver Physiol. 2017;312(6):G550-g8.

68. Schulz O, Pabst O. Antigen sampling in the small intestine. Trends Immunol. 2013;34(4):155-61.

69. Yoneno K, Hisamatsu T, Shimamura K, Kamada N, Ichikawa R, Kitazume MT, et al. TGR5 signalling inhibits the production of pro-inflammatory cytokines by in vitro differentiated inflammatory and 
intestinal macrophages in Crohn's disease. Immunology. 2013;139(1):19-29.

70. Biagioli M, Carino A, Cipriani S, Francisci D, Marchianò S, Scarpelli P, et al. The Bile Acid Receptor GPBAR1 Regulates the M1/M2 Phenotype of Intestinal Macrophages and Activation of GPBAR1 Rescues Mice from Murine Colitis. J Immunol. 2017;199(2):718-33.

71. Masyuk TV, Masyuk Al, Lorenzo Pisarello M, Howard BN, Huang BQ, Lee PY, et al. TGR5 contributes to hepatic cystogenesis in rodents with polycystic liver diseases through cyclic adenosine monophosphate/Gas signaling. Hepatology. 2017;66(4):1197-218. 


\section{Supplementary file 1}

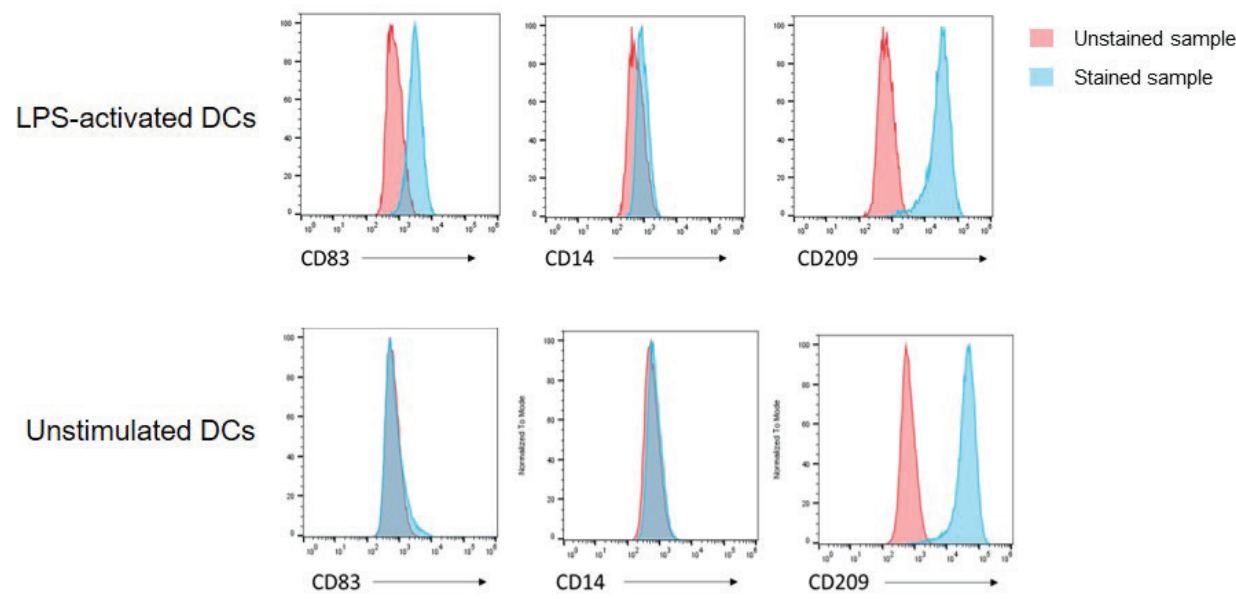

Figure 1 Expression of the cell surface markers CD83, CD14 and CD209 on DCs activated with $10 \mathrm{ng} / \mathrm{mL}$ LPS for 24 hours and unstimulated DCs. Unstained (red) and stained (blue) samples are displayed from one representative donor.

Table 1 A) TNF- $\alpha$ levels and B) IL-12p40 levels produced by basolateral DCs after direct BA exposure, expressed as percentage of control. Values of all three biological replicates (batch 1, 2, 3) are shown.

A

\begin{tabular}{|c|c|c|c|c|c|c|c|c|c|}
\hline & & \multicolumn{2}{|c|}{ Sulfo-DCA } & \multicolumn{2}{c|}{ DCA } & \multicolumn{2}{c|}{ Sulfo-LCA } & \multicolumn{2}{c|}{ LCA } \\
\hline Batch & Control & $100 \mu \mathrm{M}$ & $200 \mu \mathrm{M}$ & $100 \mu \mathrm{M}$ & $200 \mu \mathrm{M}$ & $10 \mu \mathrm{M}$ & $50 \mu \mathrm{M}$ & $10 \mu \mathrm{M}$ & $50 \mu \mathrm{M}$ \\
\hline 1 & 100 & 93.9 & 124.7 & 134.0 & 93.2 & 87.6 & 87.8 & 92.9 & 88.9 \\
\hline 2 & 100 & 105.0 & 103.4 & 64.9 & 42.7 & 56.8 & 42.8 & 76.4 & 84.5 \\
\hline 3 & 100 & 92.1 & 108.4 & 94.7 & 90.7 & 105.5 & 97.7 & 105.0 & 99.8 \\
\hline
\end{tabular}

B

\begin{tabular}{|c|c|c|c|c|c|c|c|c|c|}
\hline & & \multicolumn{2}{|c|}{ Sulfo-DCA } & \multicolumn{2}{c|}{ DCA } & \multicolumn{2}{c|}{ Sulfo-LCA } & \multicolumn{2}{c|}{ LCA } \\
\hline Batch & Control & $100 \mu \mathrm{M}$ & $200 \mu \mathrm{M}$ & $100 \mu \mathrm{M}$ & $200 \mu \mathrm{M}$ & $10 \mu \mathrm{M}$ & $50 \mu \mathrm{M}$ & $10 \mu \mathrm{M}$ & $50 \mu \mathrm{M}$ \\
\hline 1 & 100 & 80.1 & 59.9 & 66.0 & 36.5 & 59.7 & 52.3 & 39.4 & 51.8 \\
\hline 2 & 100 & 36.9 & 35.5 & 92.3 & 78.3 & 121.6 & 121.5 & 28.4 & 60.4 \\
\hline 3 & 100 & 111.9 & 122.2 & 84.1 & 85.3 & 129.9 & 120.3 & 128.4 & 127.7 \\
\hline
\end{tabular}




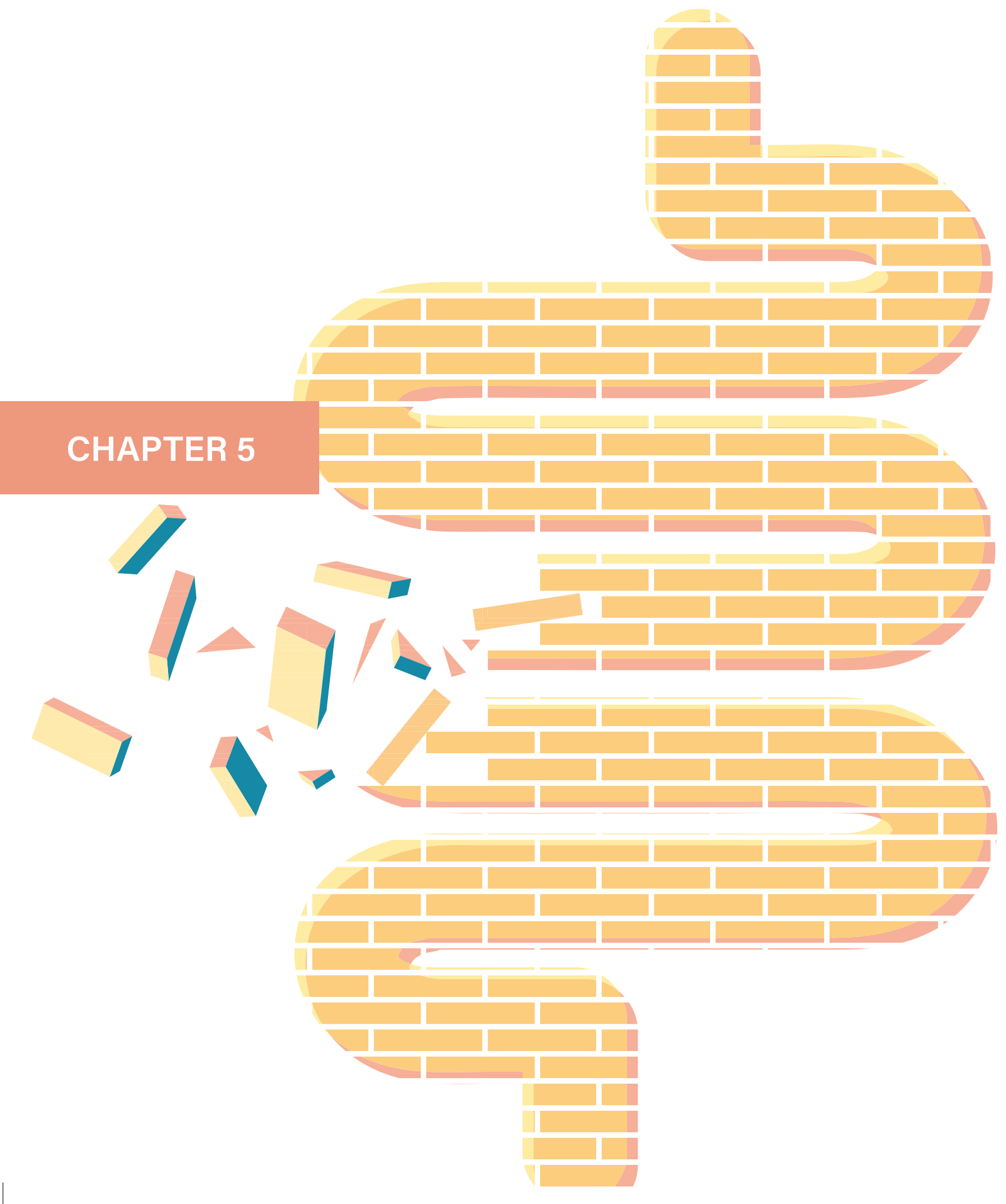




\section{Characterization of increased mucus production of HT29-MTX-E12 cells grown under Semi-Wet interface with Mechanical Stimulation}

Janneke Elzinga*, Benthe van der Lugt*, Clara Belzer\&, Wilma T. Steegenga\&

*These authors contributed equally to this work \&These authors also contributed equally to this work 


\section{Abstract}

The intestinal mucus layer plays a crucial role in human health. To study intestinal mucus function and structure in vitro, the mucus-producing intestinal cell line HT29-MTX-E12 has been commonly used. However, this cell line produces only low amounts of the intestine-specific MUC2. It has been shown previously that HT29-MTX-E12 cells cultured under Semi-Wet interface with Mechanical Stimulation (SWMS) produced higher amounts of MUC2, concomitant with a thicker mucus layer, compared to cells cultured conventionally. However, it remains unknown which underlying pathways are involved. Therefore, we aimed to further explore the cellular processes underlying the increased mucus production by HT29-MTX-E12 cells grown under SWMS conditions. Cells grown on Transwell membranes for 14 days under static and SWMS conditions (after cell seeding and attachment) were subjected to transcriptome analysis to investigate underlying molecular pathways at gene expression level. Caco-2 and LS174T cell lines were included as references. We characterized how SWMS conditions affected HT29-MTX-E12 cells in terms of epithelial barrier integrity, by measuring transepithelial electrical resistance, and cell metabolism, by monitoring $\mathrm{pH}$ and lactate production per molecule glucose of the conditioned medium. We confirmed higher MUC2 production under SWMS conditions at gene and protein level and demonstrated that this culturing method primarily stimulated cell growth. In addition, we also found evidence for a more aerobic cell metabolism under SWMS, as shown previously for similar models. In summary, we suggest different mechanisms by which mucus production is enhanced under SWMS and propose potential applications of this model in future studies. 


\section{Introduction}

The surface of the human gastro-intestinal (Gl) tract is covered by a layer of mucus, protecting the host from pathogens, harmful chemical or biological substances and physical damage (1-4). Defects in this mucus layer have been implicated in several intestinal pathologies. For instance, Muc2 knockout mice were shown to develop spontaneous colitis (5) and colorectal cancer (6), emphasizing the important protective role of mucus. Along the Gl tract, the mucus layer is thickest in the colon (7), where the number of intestinal bacteria is also highest (8). Interestingly, the mucus layer does not only protect the underlying epithelium from these high bacterial numbers, but also provides a binding site and nutrition-rich niche for residing intestinal bacteria, such as mucin-degrading bacterial species and cross-feeders which in concert produce beneficial compounds (e.g. shortchain fatty acids) for the host $(9,10)$.

The dual role of colonic mucus can be explained by the existence of two layers: The inner layer is densely packed, firmly attached to the intestinal epithelium and devoid of bacteria, whereas the outer layer is loose, constantly removed and colonized by bacteria $(7,11)$. Colonic mucus is mainly composed of the gel-forming mucin type 2 (MUC2) $(12,13)$, a heavily O-glycosylated protein secreted by intestinal goblet cells (14-16). Additionally, mucus contains salts, lipids (17) and defense-related proteins, such as antimicrobial peptides, growth factors, trefoil factors, immunoglobulins, lysozyme and other proteins (11).

Our understanding of colonic mucus structure, function and composition has been largely dependent on in vivo models, such as rodents and pigs (reviewed in Etienne-Mesmin et al. (18)) or ex vivo techniques using human mucosal biopsies (first established by Browning et al. (19)) and murine intestinal explants $(20,21)$. These models, however, show large heterogeneity between and within subjects or, in the case of animal models, have a poor translational value. Additionally, these models are often expensive, require specialized experience and pose ethical concerns. Attempts have been made to recapitulate the intestinal mucosal layer - including host cells - in vitro, varying from simple to more advanced systems. Relatively simple cell models include the use of mucusexcreting colonic cancer cell lines, such as HT29-MTX and LS174T cells $(22,23)$, but both examples have limitations. HT29-MTX cells have successfully been grown in confluent monolayers (24), but this cell type predominantly secretes MUC5AC, a mucin that is present in the stomach and airways, while producing only a limited amount of colon-specific MUC2 (22). On the other hand, LS174T cells do produce MUC2 (23), but are not capable of growing in an organized and adherent cell layer (25). More advanced models include culturing cell lines in innovative models, such as gut-on-chips (26, 27 ) or $3 \mathrm{D}$ scaffolds $(28-30)$; or the use of human colonoids $(31,32)$ and human intestinal organoids 
(33); or a combination (34), which all demonstrated increased MUC2 production in vitro compared to conventional cell culture. Although these models are supposed to resemble the in vivo colonic mucosal layer more closely in terms of mucus composition, they are highly expensive and require specialized expertise (18).

To obtain more physiologically relevant models, simpler yet effective alternative strategies have been shown to further increase mucus production in intestinal cancer cell lines, using biochemical compounds, such as prostaglandin E2 (35) and Notch y-secretase inhibitors (36), or bacteria-derived compounds (e.g. sodium butyrate (37) and LPS (38)). Physical strategies have also been applied, e.g. growing intestinal porcine epithelial cells at an air-liquid interface (ALI) $(39,40)$, stimulating cells mechanically, or a combination of both. For instance, Navabi and colleagues managed to create polarized, functional, crypt-forming intestinal cell layers with an adherent mucus layer, when growing HT29-MTX-E12 and other intestinal cell lines on Transwell membranes in semi-wet interfaces with mechanical stimulation (SWMS). SWMS conditions include decreased apical and basolateral medium volumes and continuous shaking on a rocking platform. Importantly, these cells demonstrated increased expression of MUC2, both absolute and relative to MUC5AC (25). HT29MTX-E12 cells grown under these conditions produced a thicker layer compared to static conditions. However, it remains unexplored what molecular mechanisms are involved.

In our study, we aimed to further explore the cellular processes underlying the increased mucus production by HT29-MTX-E12 cells grown under SWMS conditions. To this end, cells were subjected to transcriptome analysis after 15 days of culture to investigate underlying molecular pathways involved. As control cell lines for the transcriptomic analysis, we included Caco-2 and LS174T cells, a non-mucus producing and MUC2-producing cell line, respectively. Next, we further characterized the HT29-MTX-E12 monolayer, by measuring transepithelial electrical resistance and quantifying cell density. Additionally, as similar (semi-wet only) models have shown a more aerobic cell metabolism (41-44), we quantified $\mathrm{pH}$ and lactate production per molecule glucose of the conditioned medium. In overall, we attempted to gain more insight into the potential mechanisms underlying increased mucus production in HT29-MTX-E12 cells grown under SWMS conditions.

\section{Materials and Methods}

\section{Cell culture}

HT29-MTX-E12 cells (ECACC) were obtained from Sigma-Aldrich (Darmstadt, Germany). Caco- 
2 (ATCC HTB-37) and LS174T (ATCC CL-188) cells were purchased at LGC Standards (Wesel, Germany). All cell types were cultured in Dulbecco's Modified Eagle Medium with 4.5g/L glucose, $110 \mathrm{mg} / \mathrm{L}$ sodium pyruvate and $584 \mathrm{mg} / \mathrm{L}$ L-glutamine (Corning, NY, USA) supplemented with 10\% Fetal Bovine Serum and 1\% penicillin/streptomycin. When cells reached $80-90 \%$ confluency, they were counted and seeded. Passage numbers between 15 and 27 were used for Caco-2 cells and between 3 and 21 for HT29-MTX-E12 cells. LS174T cells were used between passage 17 and 19. Caco-2 and HT29MTX-E21 cells were seeded (day 0) at a density of 273,000 cells/mL in $275 \mu \mathrm{L}$ per well on $12 \mathrm{~mm} 0.4 \mu \mathrm{m}$-pore polyester Transwell membranes (Corning 3460). A volume of $1 \mathrm{~mL}$ medium was added to the basolateral compartment. One day after seeding (day 1), media of all Transwells was refreshed and Semi-Wet conditions with Mechanical Stimulation (SWMS) were applied $(25,30)$. To these Transwells, $75 \mu \mathrm{L}$ and $850 \mu \mathrm{L}$ of medium was added to the apical and basolateral compartments, respectively, and plates were put on a $\mathrm{CO}_{2}$-resistant shaker (Thermo Fisher Scientific, Breda, The Netherlands) at $65 \mathrm{rpm}$. Media volumes of Transwells grown under static conditions were unchanged. LS174T cells were seeded at similar seeding density on regular 12-well cell culture plates. This cell line was cultured under static conditions in a regular wells plate only, since these cells do not form a continuous monolayer of cells, but grow in a rather irregular manner (25). Medium was refreshed every Monday, Wednesday and Friday and cells were harvested 15 days after seeding ( $t=15$ days).

\section{RNA isolation}

Cells were washed with ice-cold PBS twice, trypsinized and RNA was isolated using the Maxwell囚16 LEV simplyRNA Cells Kit (Promega, cat. no. AS1270) and the Maxwell囚16 MDx Instrument (Promega), following the manufacturer's instructions.

\section{Microarray}

RNA isolate from three independent biological replicate experiments was used for microarray analysis. Total RNA yield was measured using photometry (DeNovix, USA). RNA quality was determined on an Agilent 2100 Bioanalyzer (Agilent Technologies, Amsterdam, The Netherlands). RNA was only used when the RNA integrity number (RIN) exceeded 8.0. One hundred nanogram of RNA was converted to cDNA and labelled (Ambion WT expression kit, Life Technologies, Bleiswijk, The Netherlands). Samples were hybridized to an Affymetrix Human Gene 1.1 ST array plate according to the standard Affymetrix instructions (Affymetrix, Santa Clara, CA, USA). The robust multi-array average (RMA) pre-processing algorithm in the Bioconductor library AffyPLM was used 
to obtain normalized expression estimates (45). Probe sets were defined and assigned as described by Dai et al. (46). Differences in gene expression between static and SWMS conditions per cell type were analyzed using the Intensity Based Moderated T statistics (IBMT) (47), using $p$ values $<0.05$ as threshold. The Venn diagram was created using Venny 2.1 (48). Microarray data has been submitted to the Gene Expression Omnibus (GEO) at the NCBI (GSE173729).

\section{TEER measurements}

The transepithelial electrical resistance (TEER) was measured with an EVOM2 Volt/Ohm meter using STX2 electrodes (World Precision Instruments) at day 4, 7, 9, 11 and 14. One hour prior to measuring, medium of the Transwells was refreshed and equal medium volumes were applied in all wells (275 $\mu \mathrm{L}$ apical and $1 \mathrm{~mL}$ basolateral). Before TEER measurements were performed, Transwells were put at room temperature for 5 minutes to allow temperature equilibration. After TEER measurements, the medium volumes in the wells were adapted again to volumes of the respective conditions. The background value (i.e. TEER value of an empty Transwell) was subtracted from the total TEER values.

\section{Protein isolation}

Cells were washed twice with PBS and lysed in RIPA Lysis and Extraction Buffer (ThermoFisher Scientific), supplemented with the protease and phosphatase inhibitors PhosSTOP and cOmplete (Roche Diagnostics, Almere, The Netherlands). Lysates were incubated on ice for 20 minutes following centrifugation for 10 minutes at $13,000 \times \mathrm{g}$. Supernatant was collected and protein concentrations were measured using a bicinchoninic acid assay (Thermo Fisher Scientific).

\section{Western Blot}

Protein lysates (20 $\mu \mathrm{g}$ of protein/lane) were loaded onto 4-15\% Mini-PROTEAN TGX Precast Protein Gels (Bio-Rad, Veenendaal, The Netherlands). Next, proteins were transferred onto a polyvinylidene difluoride membrane (Trans-Blot Turbo Midi $0.2 \mu \mathrm{m}$ PVDF Transfer Packs, Bio-Rad) using the Transblot Turbo System (Bio-Rad). Membranes were blocked for 1 hour and incubated overnight at $4{ }^{\circ} \mathrm{C}$ with rabbit anti-KLF4 (Sigma-Aldrich, catalogue no. SAB1300678) and rabbit anti-HSP90 (Cell Signaling Technology, cat. no. 4874). Antibodies were used in 1:500 and 1:5000 dilutions for KLF4 and HSP90, respectively. Membranes were incubated for 1 hour with goat anti-rabbit (GenScript, cat. no. A00098) diluted 1:5000. Blocking and incubation of primary and secondary antibodies were done in TBS with 0.1\% Tween 20 (TBS-T) and 5\% (w/v) skimmed milk. In between, membranes were washed in TBS-T. Signals were quantified using the ChemiDoc MP system (Bio-Rad) and Clarity ECL 
substrate (Bio-Rad).

\section{Dot Blot}

Proteins were diluted to $68.2 \mu \mathrm{g} / \mathrm{mL}$ and 6 x serially diluted 1:2 in PBS. In total 7 dilutions and one PBScontrol were blotted per condition. Of each dilution, $50 \mu \mathrm{L}$ of sample was blotted on a Pierce $0.2 \mu \mathrm{m}$ nitrocellulose membrane (Thermo Scientific) in a Dot Blot device (The Convertible, cat. series 1055. Gibco BRL) connected to a vacuum-pump. Next, membranes were blocked, incubated and imaged as described for Western Blot. Primary antibodies were diluted 1:2000 and 1:1000 for mouse antiMUC2 (Abcam, cat. no. ab11197) and-MUC5AC (Sigma-Aldrich, cat. no. WH0004586M7) respectively. Secondary goat anti-mouse antibody (Genscript, cat. no. A00160) was diluted at 1:2500. To check protein quantity on the membranes, a separate, identical membrane was blotted and incubated for 10 min. in Ponceau Red (Honeywell Fluka). Next, the blot was washed in demi-water and signals were quantified using the ChemiDoc MP system. Dot Blots with anti-MUC2 and -MUC5AC were quantified by measuring the density of the first six dots (rows) using ImageJ software. A densitybased linear trendline was calculated from the second to the sixth dot. The coefficients ( $\mathrm{per} \mu \mathrm{g} / \mathrm{mL}$ ) were corrected for Ponceau Red density. Ponceau Red signals were quantified by measuring density of the first dot (first row) only. Biological replicates per conditions were $n=2$ for HT29-MTX-E12, static, $\mathrm{n}=3$ for HT29-MTX-E12, SWMS, $\mathrm{n}=3$ for Caco-2 (static and SWMS), and $\mathrm{n}=1$ for LS174T.

\section{$\mathrm{pH}$, lactate and glucose measurements}

Every time medium of Transwells was refreshed, conditioned medium was collected from HT29MTX-E12 cells and stored at $-20{ }^{\circ} \mathrm{C}$ until further processing. Medium of apical and basolateral compartments were collected separately, but compartments were pooled per time point and condition. Samples were spun down and $200 \mu \mathrm{L}$ of supernatant was transferred to a 96-wells plate. After stabilization, absorbance was measured at 415 and $560 \mathrm{~nm}$ at $5 \% \mathrm{CO}_{2}$ in a Synergy Neo2 Hybrid Multi-Mode Reader with $\mathrm{a} \mathrm{CO}_{2}$ and $\mathrm{O}_{2}$ gas controller (1210013) (BioTek Instruments). Aliquots of growth medium (ca. $5 \mathrm{~mL}$ ) were used for preparation of a standard curve with $\mathrm{pH}$ values ranging from 2.6 to 9.9. Lactate and glucose were quantified with a Shimadzu LC2030C-Plus high-performance liquid chromatography (HPLC) system equipped with a Shodex SH1821 column kept at $45^{\circ} \mathrm{C}$ and running $0.01 \mathrm{~N}$ sulfuric acid as eluent $(1 \mathrm{~mL} / \mathrm{min})$. Compounds were detected by determining the refractive index and identified using pure lactate and glucose as external standards and crotonate as an internal standard. 


\section{Statistical analysis}

Statistical analysis of microarray data is described above. Data distribution for other (continuous) outcomes was tested with the D'Agostino-Pearson omnibus normality test using GraphPad Prism (San Diego, CA, USA). Significance of differences between two conditions was analysed using a two-tailed, unpaired Student's t-test. For non-normally distributed data, a Mann-Whitney U test was used. The data are presented as mean \pm standard deviation. A $p$-value of $\leq 0.05$ was considered significant. Unless stated otherwise, all experiments were performed in triplicate.

\section{Results}

\section{Gene expression changes in HT29-MTX-E12, Caco-2 and LS174T cells}

To investigate SWMS-specific effects leading to higher mucus production of the SWMS culturing method, HT29-MTX-E12 cells were grown for 14 days under static and SWMS conditions (after cell seeding and attachment), and microarray analysis was performed on mRNA of the cells. We included Caco-2 cells, a non-mucus producing cell line, grown on Transwell membranes under static and SWMS conditions for 14 days as a reference control. The LS174T cell line was used as a control for a mucus-producing cell line. Multilevel principal component analysis performed on the 500 most variable genes showed a strong clustering of the biological replicates per cell type with a clear separation of the three cell lines (Supplementary Fig. 1A). A number of 6,732 and 7,013 genes were differentially regulated between SWMS and static conditions in HT29-MTX-E12 and Caco-2 cells, respectively $(p<0.05)$ (Supplementary Fig. 1B-D).

\section{Increased MUC2/MUC5AC ratio in SWMS-cultured HT29-MTX-E12 cells}

We investigated the expression of genes encoding for mucins in HT29-MTX-E12 cells, since we know from previous studies that culturing this cell type under SWMS conditions led to increased MUC2 production (25). Indeed, in our study, SWMS conditions resulted in a significant 1.42-fold upregulation of MUC2 ( $p<0.05)$ (Fig. 1A). Expression of gel-forming mucins MUC5AC and MUC5B was decreased in response to SWMS (FC $=-1.56$ and -1.84 , respectively, $\left.p<1 \cdot 10^{-4}\right)$ (Fig. 1A, Supplementary File 1). Transmembrane mucins were also significantly lower expressed after SWMS conditions (Fig. 1A). MUC3A, 13, 17, 20 and 21 showed FC values between -1.52 and -2.32 (Supplementary File 1). Eight out of 19 mucins (MUC4, 6, 7, 12, 15, 16, 19 and 22) displayed very low expression levels under both static and SWMS conditions (RMA < 4) and were not significantly differentially expressed (Supplementary File 1). Taken together, these data indicate that SWMS conditions resulted in a change in expression 
of mucin encoding genes with an increased expression of $M U C 2$, while other gel-forming as well as transmembrane mucins were significantly lower or not differentially expressed. Dot Blot data supported microarray data for MUC2, as protein levels also showed a significant upregulation ( $p$ $<0.05$ ) under SWMS versus static conditions (Fig. 1B and Supplementary Fig. 2A and C). Less MUC5AC was detected under SWMS conditions, however, variation between replicates was rather high and differences were not significant $(p=0.20)$ (Fig. 1B and Supplementary Fig. 2B and C). Overall, HT29-MTX-E12 cells grown under SWMS conditions showed a significantly increased MUC2/MUC5AC-ratio (Fig. 1C, $p<0.05$ ), confirming previous findings (25). For LS174T, which we included as a control cell line reported to produce predominantly MUC2, a relatively high MUC2/ MUC5AC ratio was calculated (Supplementary Fig. 2D-E). In contrast, Caco-2 cells, which are not reported to produce mucus, showed low RMA values $(<5)$ for MUC2 and MUC5AC and protein expression of these mucins was below detection level in both conditions (Supplementary Fig. 2A-C). Furthermore, similar to HT29-MTX-E12 cells, SWMS conditions resulted in significant downregulation of $M U C 20, M U C 3 A$ and $M U C 13$ in this cell line (Supplementary File 1).

A

\section{Gel-forming mucins}
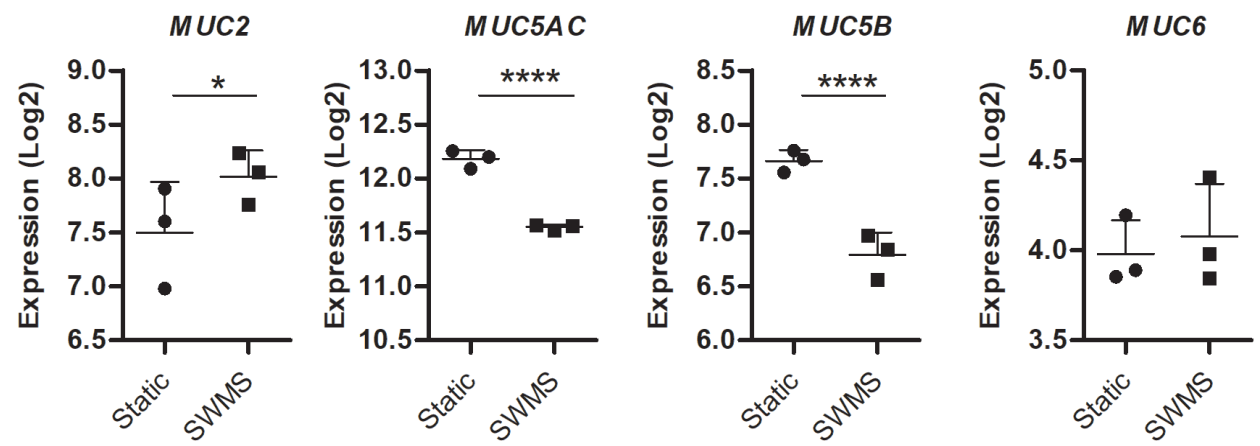

Transmembrane mucins
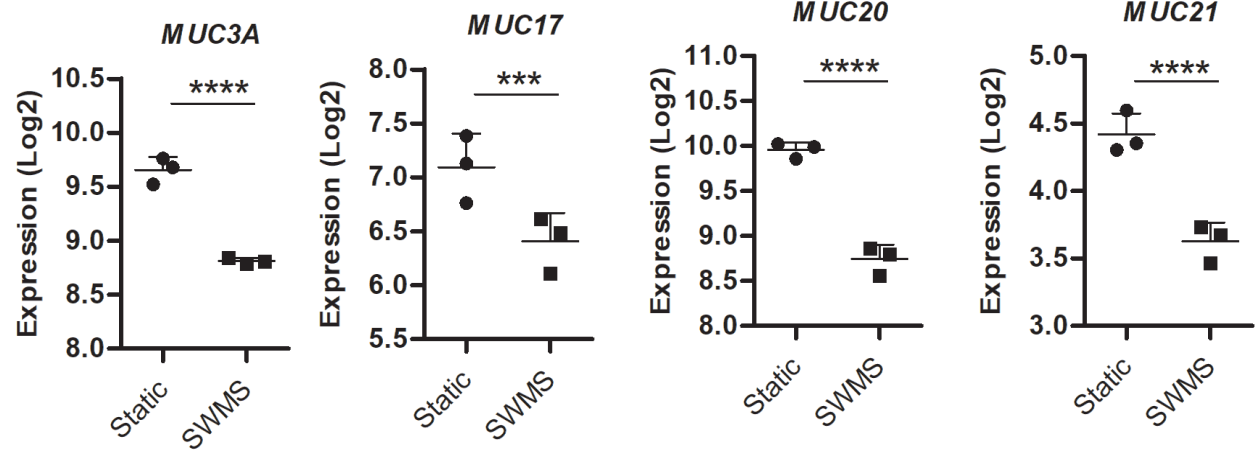
$\mathrm{B}$

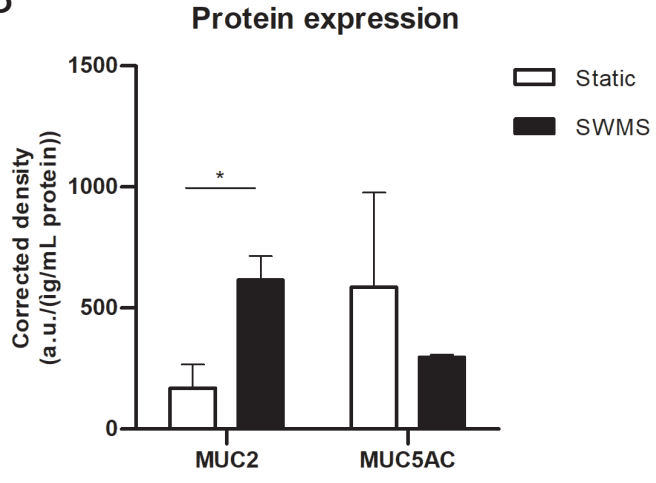

C MUC2/MUC5AC

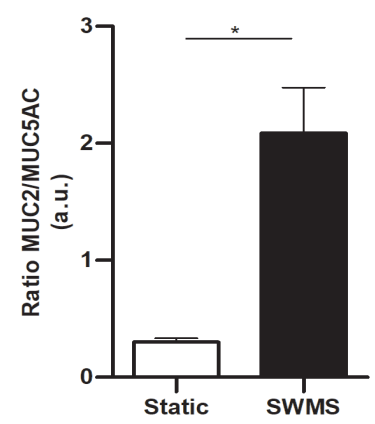

Figure 1 Mucin gene and protein expression in HT29-MTX-E12 cells grown under static and SWMS conditions. A) Microarray gene expression values (Log2) of a panel of gel-forming and transmembrane mucins B) Protein expression of MUC2 and MUC5AC, expressed as density (a.u.) per ug $/ \mathrm{mL}$ protein blotted, after correction of Ponceau Red density ( $n=2$ for static, $n=3$ for SWMS. Blot data in Supplementary Fig. 2) C) Ratio of MUC2 and MUC5AC protein expression. * $p<0.05$; ${ }^{* * *} p<0.001 ;{ }^{* * * *} p<0.0001 ; n=3$, unless stated otherwise.

Upregulation of genes and pathways related to cell cycle, cell growth and cell proliferation in cells cultured under SWMS conditions

As both HT29-MTX-E12 and Caco-2 cell lines were subjected to SWMS conditions and grown under static conditions, we compared the genes up- or downregulated in both cell lines. Of the 2,844 significantly differentially expressed genes in both cell lines, 353 genes (12.4\% of shared total significantly regulated genes) were upregulated by 1.5 -fold or higher in both cell types due to the SWMS conditions. A total of 180 genes (6.3 \%) were significantly downregulated by 1.5 -fold or higher in both cell lines and a total of 98 genes showed opposite effects when comparing the two cell lines (Supplementary Fig. 3). Interestingly, among the shared upregulated genes, a number of cell cycle related genes was included. Moreover, the top 10 most upregulated genes of HT29MTX-E12 cells under SWMS versus static conditions was dominated by genes related to cell growth, cell motility and cell proliferation, i.e. KIF14, KIF20A, DEPDC1, DLGAP5 and SPC25 (Table 1). Gene Set Enrichment Analysis (GSEA) also revealed that most significantly enriched upregulated pathways were dominated by cell cycle and DNA replication related pathways (Table 2). Furthermore, marker of proliferation Ki-67 (MKI67) was 5.03-fold upregulated by SWMS culturing conditions $\left(p<1 \cdot 10^{-10}\right)$ in HT29-MTX-E12 and 2.33-fold in Caco-2 cells $\left(p<1 \cdot 10^{-5}\right)$ (Supplementary Fig. 4A). Additionally, 
we found higher cell counts at day 15 under SWMS compared to static conditions in both cell lines (Supplementary Fig. 4B), confirming that SWMS conditions lead to an increase in cell proliferation. Altogether, these data indicate that SWMS conditions result in the activation of cell proliferation pathways compared to static conditions, independent of cell type.

Table 1 Top 10 strongest up- and downregulated genes in HT29-MTX-E12 cells grown under SWMS versus static conditions.

\begin{tabular}{|c|c|c|c|}
\hline \multicolumn{4}{|c|}{ UPREGULATED } \\
\hline Gene & Fold Change & p-value & Gene name \\
\hline RNY4P23 & 10.80 & $5.40 \mathrm{E}-06$ & RNY4 pseudogene 23 \\
\hline SUCNR1 & 8.78 & $2.55 \mathrm{E}-11$ & succinate receptor 1 \\
\hline KIF14 & 8.00 & $2.45 \mathrm{E}-12$ & kinesin family member 14 \\
\hline KIF20A & 7.99 & $1.65 \mathrm{E}-08$ & kinesin family member $20 \mathrm{~A}$ \\
\hline DEPDC1 & 7.93 & $6.11 \mathrm{E}-11$ & DEP domain containing 1 \\
\hline DLGAP5 & 7.91 & $5.15 \mathrm{E}-12$ & DLG associated protein 5 \\
\hline HMMR & 7.82 & $1.03 E-15$ & hyaluronan mediated motility receptor \\
\hline $\mathrm{H} 4 \mathrm{C} 1$ & 7.56 & $7.27 \mathrm{E}-07$ & H4 clustered histone 1 \\
\hline IL33 & 7.44 & $2.98 \mathrm{E}-13$ & interleukin 33 \\
\hline SPC25 & 7.41 & 4.47E-13 & SPC25 component of NDC80 kinetochore complex \\
\hline
\end{tabular}

\begin{tabular}{|l|c|c|l|}
\hline \multicolumn{4}{|l|}{ DOWNREGULATED } \\
\hline Gene & Fold Change & p-value & Gene name \\
\hline TFF2 & -15.75 & $2.62 \mathrm{E}-13$ & trefoil factor 2 \\
\hline SCGB2A1 & -8.91 & $1.33 \mathrm{E}-10$ & secretoglobin family 2A member 1 \\
\hline ST8SIA6 & -4.56 & $1.06 \mathrm{E}-08$ & $\begin{array}{l}\text { ST8 alpha-N-acetyl-neuraminide alpha-2,8-sialyl- } \\
\text { transferase 6 }\end{array}$ \\
\hline DUOX2 & -4.53 & $4.52 \mathrm{E}-09$ & dual oxidase 2 \\
\hline TFF1 & -4.44 & $1.37 \mathrm{E}-16$ & trefoil factor 1 \\
\hline APOL1 & -4.41 & $1.29 \mathrm{E}-06$ & apolipoprotein L1 \\
\hline ADGRF1 & -3.96 & $7.06 \mathrm{E}-08$ & adhesion G protein-coupled receptor F1 \\
\hline DUOXA2 & -3.88 & $1.67 \mathrm{E}-09$ & dual oxidase maturation factor 2 \\
\hline UPK3B & -3.80 & $4.29 \mathrm{E}-09$ & uroplakin 3B \\
\hline SPRR1B & -3.63 & $2.31 \mathrm{E}-07$ & small proline rich protein 1B \\
\hline
\end{tabular}


Table 2 Top 10 most significantly enriched upregulated pathways in HT29-MTX-E12 cells induced by SWMS conditions.

\begin{tabular}{|l|l|c|c|}
\hline Pathway entry & Enriched upregulated pathways & NES* & FDR q-value \\
\hline HSA04110 & Cell Cycle & 2.856 & 0.000 \\
\hline HSA03460 & Fanconi Anemia Pathway & 2.637 & 0.000 \\
\hline HSA03030 & DNA Replication & 2.596 & 0.000 \\
\hline HSA05322 & Systemic Lupus Erythematosus & 2.539 & 0.000 \\
\hline HSA03440 & Homologous Recombination & 2.506 & 0.000 \\
\hline HSA04114 & Oocyte Meiosis & 2.329 & 0.000 \\
\hline HSA03420 & Nucleotide Excision Repair & 2.315 & 0.000 \\
\hline HSA03430 & Mismatch Repair & 2.312 & 0.000 \\
\hline HSA00100 & Steroid Biosynthesis & 2.306 & 0.000 \\
\hline HSA04914 & Progesterone Mediated Oocyte Maturation & 2.268 & 0.000 \\
\hline
\end{tabular}

*NES: Normalised enrichment score.

Expression of target genes involved in Notch- and Atoh-key pathways did not point towards a favoured cell differentiation state by SWMS conditions

Next to the marked effects of SWMS conditions on cell proliferation in both HT29-MTX-E12 and Caco-2 cells, we took a closer look at the effects on intestinal cell differentiation. Key regulators in this process belong to the Notch/Atoh signalling pathway $(49,50)$. When it comes to cell differentiation, Notch and Atoh have opposing roles: Notch activation promotes absorptive cell differentiation, while Atoh activation favours differentiation into secretory cell types (49-51). Interestingly, in our transcriptomic dataset, both $\mathrm{NOTCH} 1$ and $\mathrm{ATOH} 1$ were significantly upregulated by SWMS conditions in HT29-MTX-E12 cells (FC $=1.61, p<0.001$ and $\mathrm{FC}=1.64, p<0.01$, respectively) and the latter also in Caco-2 cells $(F C=2.09, p<0.001)$. The stem cell marker $L G R 5$, which is known to be part of a positive feedback loop regulated by Notch (52), was significantly upregulated by SWMS conditions in HT29-MTX-E12 cells ( $\mathrm{FC}=2.33, p<1 \cdot 10^{-5}$ ), although RMA values were below 5 . In Caco-2 cells, LGR5 had higher RMA values and was even stronger upregulated $\left(\mathrm{FC}=4.37, p<1 \cdot 10^{-7}\right)$. Further downstream the Notch pathway, the target genes Hairy and enhancer of split (HES) family members HES1 and HES6 were both significantly upregulated by SWMS conditions in HT29-MTX-E12 cells (FC $=1.24, p<0.05$ and FC $=2.08, p<1 \cdot 10^{-5}$, respectively). Apart from Cyclin D1 (CCND1) $(\mathrm{FC}=-1.51, p$ $<1 \cdot 10^{-6}$ ) no other Notch-target genes were significantly differentially expressed. Regarding the Atoh pathway, the downstream target gene Neurogenin 3 (NEUROG3) was 2.07-fold and 2.41-fold higher expressed $(p<0.001)$ in HT29-MTX-E12 and Caco-2 cells, respectively. Other Atoh target genes, 
such as SPDEF and GF/1 were not differentially expressed between SWMS and static conditions in both cell types. Altogether, these transcriptomic data show that some target genes of both the Notch and Atoh pathway were differentially expressed in HT29-MTX-E12 and Caco-2 cells. However, these data do not point towards a favoured differentiation state (absorptive versus secretory cell fate).

\section{Downregulation of KLF4 at both gene and protein level under SWMS conditions in HT29-} MTX-E12 and Caco-2 cells

Another Notch-target involved in differentiation of progenitor cells into goblet cells is KLF4 (53). Given the secretory, goblet cell-like phenotype of HT29-MTX-E12 grown under SWMS conditions, the significant downregulation of KLF4 (FC $=-1.86, p<0.0001)$ is interesting. Acting as both a transcriptional repressor and activator in the gastrointestinal epithelium, this zinc-finger transcription factor plays a critical role in the decision between proliferation and cell cycle arrest/differentiation (54). We validated the downregulation of KLF4 under SWMS conditions at protein level using Western Immunoblotting. Indeed, when cultured under SWMS conditions, the expression of KLF4 protein was lower compared to the static conditions in both cell lines (Fig. 2A-B and Supplementary Fig. 5).
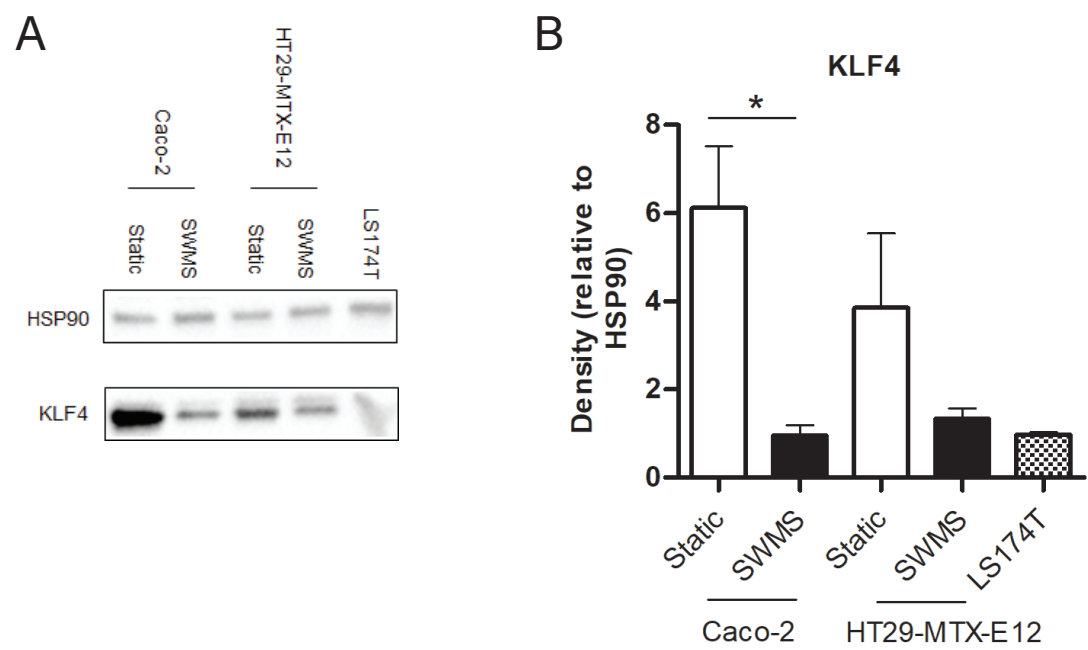

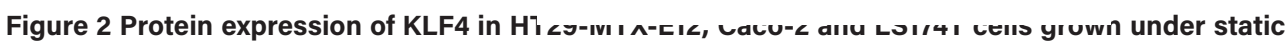
and/or SWMS conditions. A) Western Blot results of KLF4 and HSP90 (house-keeping protein) in Caco-2, HT29-MTX-E12 and LS174T-cells grown under static and SWMS conditions or static only (LS174T). Of each condition, one representative biological replicate is shown. See Supplementary Fig. 5 for all replicates. B) KLF4 protein quantity expressed as the protein band density relative to HSP90. $* p<0.05, \mathrm{n}=3$. 
We next investigated whether this downregulation of KLF4 resulted in differential expression of target genes of KLF4, as identified in literature (55). Among the most strongly regulated are genes related to cell-cycle control or essential for cell proliferation and differentiation, including cyclin B1 $\left(\mathrm{FC}=6.59, p<1 \cdot 10^{-13}\right)(56)$, cyclin E2 $(\mathrm{FC}=2.03, p<0.001)(57,58)$, ornithine decarboxylase $(\mathrm{FC}=$ $\left.1.80, p<1 \cdot 10^{-7}\right)(59)$, cyclin E1 (FC $\left.=1.62, p<1 \cdot 10^{-4}\right)(57,58)$, cyclin D1 (FC $\left.=-1.51, p<1 \cdot 10^{-6}\right)(60,61)$ and cyclin D2 (FC = 1.85, $p<0.001)(62)$. A non-exhaustive list of genes (in)directly related to KLF4 is provided in Supplementary File 1.

\section{Minor effect on intestinal epithelial barrier integrity, but difference in appearance of cells grown under SWMS conditions}

To investigate the effects of SWMS conditions on intestinal epithelial barrier integrity, transepithelial electrical resistance (TEER) was measured at multiple time points during culturing. TEER values of HT29-MTX-E12 cells increased steadily over time and no clear effects were observed between static and SWMS conditions (Fig. 3A). For Caco-2 cells, the TEER of the cells grown on SWMS conditions increased steeply during the first seven days of culturing and decreased gradually afterwards, while the TEER of cells grown on static conditions was relatively stable (Fig. 3B).

\section{A}

\section{HT29-MTX-E12}

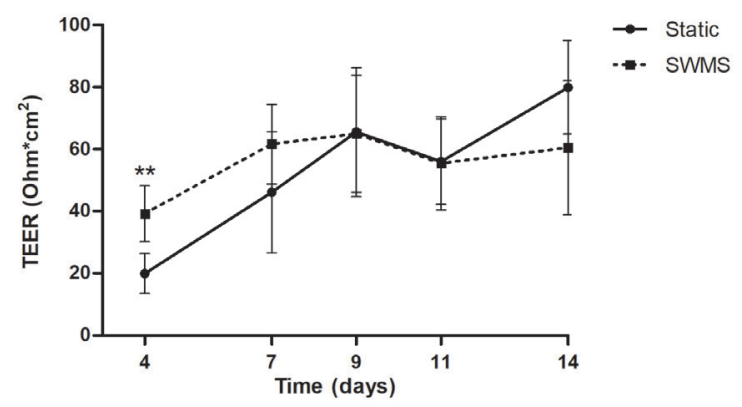

B

Caco-2

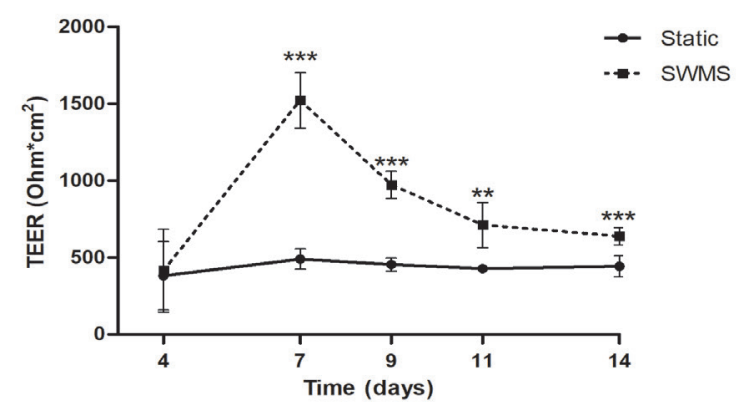


Figure 3 Transepithelial electrical resistance (TEER) of HT29-MTX-E12 and Caco-2 cells grown under static and SWMS conditions up to 14 days. TEER was measured at $t=4,7,9,11$ and 14 days and expressed in Ohm/ $\mathrm{cm}^{2}$ in A) HT29-MTX-E12 and B) Caco-2 cells. ${ }^{*} p<0.05 ; * *<0.01 ;{ }^{* * *} p<0.001 ; n=3$.

Based on available literature, we compiled a panel of genes responsible for epithelial barrier integrity (Supplementary File 1). Except for a 3.33-fold increase in expression of Catenin alpha like 1 (CTNNAL1) in HT29-MTX-E12 cells under SWMS conditions $\left(p<1 \cdot 10^{-9}\right)$, only a low number of significantly differentially expressed genes was found for both HT29-MTX-E12 and Caco-2 cells (Supplementary File 1). Together, these results indicate that SWMS conditions had negligible effects on TEER in both HT29-MTX-E12 and Caco-2 cells, which was reflected by the relatively low number of significantly differentially expressed genes related to cell integrity. Interestingly, a clear difference in cell appearance on the Transwell membranes was observed for both cell types at the time cells were harvested, as cells grown under SWMS conditions seemed to concentrate in the center of the membrane (Fig. 4A-B).

A
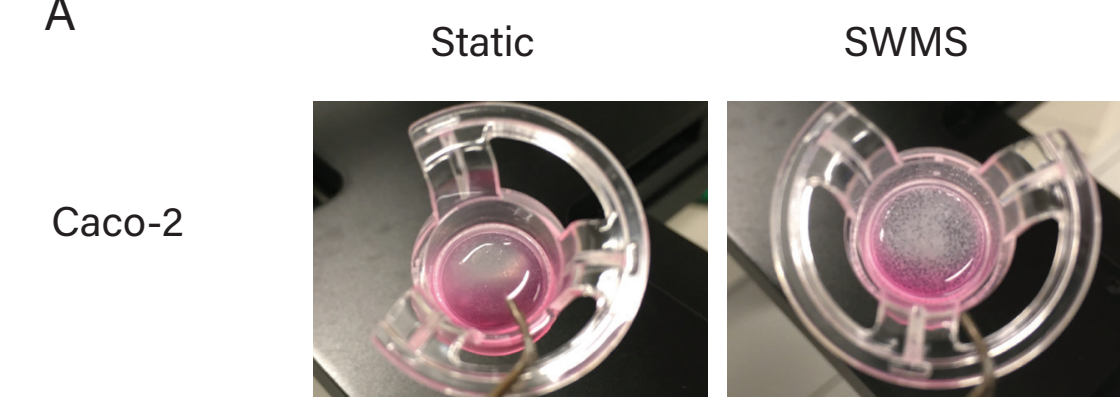

HT29-MTX-E12

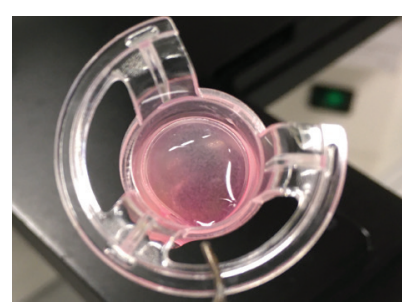


B

\section{Caco-2}

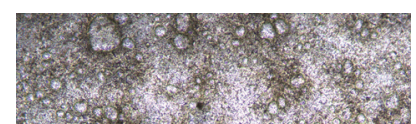

HT29MTX-E12
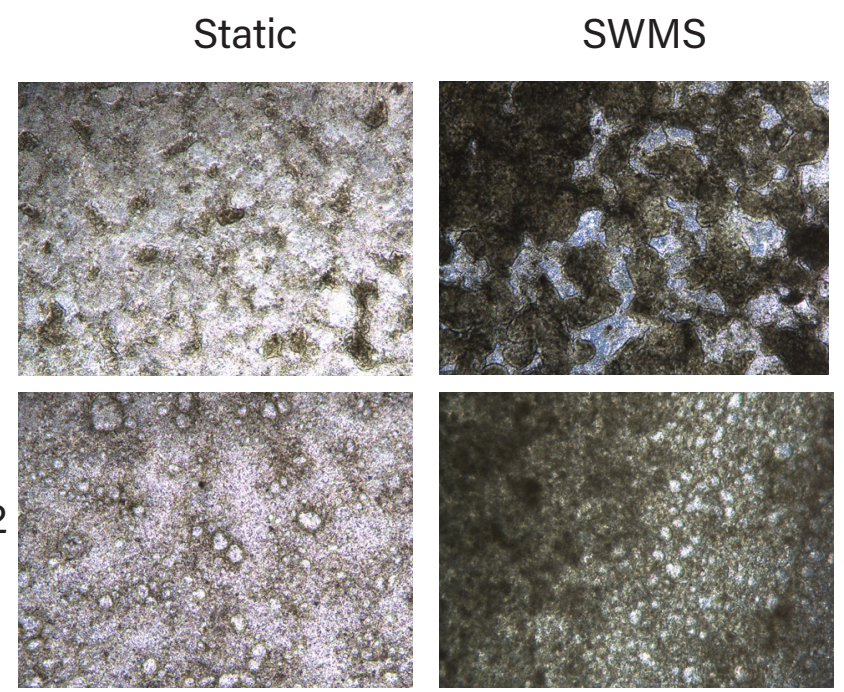

Figure 4 Images of HT29-MTX-E12 and Caco-2 cells grown under static and SWMS conditions up to 14 days. A) Pictures of HT29-MTX-E12 and Caco-2 cells grown under static and SWMS conditions at $\mathrm{t}=$ 15 days. B) Bright-field microscopy pictures of HT29-MTX-E12 and Caco-2 cells grown under static and SWMS condition, focussed on the centre of the Transwell membranes.

Downregulation of Trefoil Factor genes in HT29-MTX-E12 cells grown under SWMS conditions When focusing on the most significantly downregulated genes in HT29-MTX-E12 grown under SWMS compared to static conditions, Trefoil Factor 2 (TFF2) was most strongly downregulated (FC $=-15.75, p<1 \cdot 10^{-12}$ ) (Table 1). Although to a lesser extent, TFF1 and TFF3 were also significantly downregulated (Fig. 5). Trefoil factors are small cysteine rich peptides and form a family of mucinassociated secretory molecules involved in many physiological processes to maintain and restore gastrointestinal mucosal homeostasis (Reviewed in Aihara et al. (63)). TFF peptides are known to auto- and cross-regulate their expression via the epidermal growth factor receptor in vitro $(63,64)$. However, EGF1 was not significantly differentially expressed between culture conditions in HT29MTX-E12 cells in our dataset. On the contrary, the gene encoding Epidermal growth factor receptor (EGFR) was significantly downregulated by 1.42 -fold $(p<0.0001)$. 
TFF1

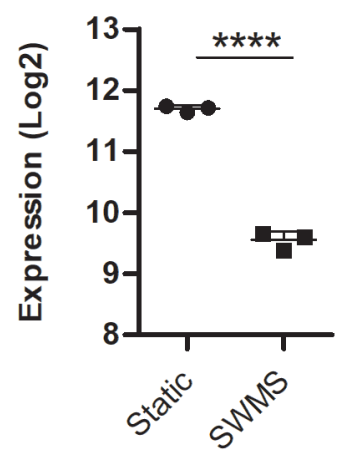

TFF2

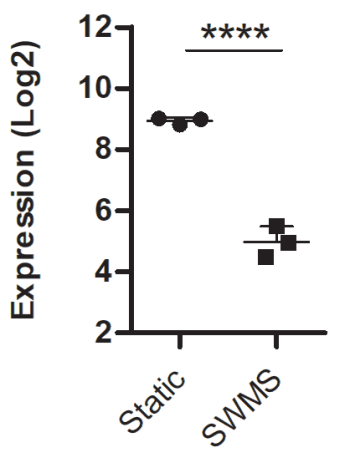

TFF3

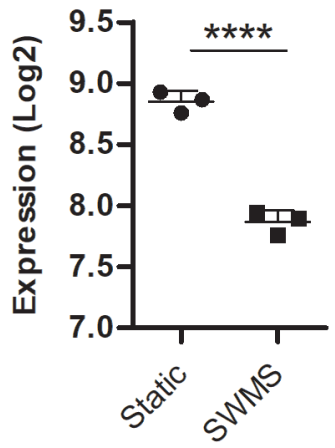

Figure 5 TFF gene expression in HT29-MTX-E12 cells grown under static and SWMS conditions. Microarray expression values (Log2) of TFF1, 2 and 3 and the corresponding fold change (FC). **** $p<$ $0.0001 ; n=3$.

Different regulation of ion transporters under SWMS conditions in both HT29-MTX-E12 and Caco-2 cells

Since ion transport has proven crucial in (intestinal) mucus production (65), we explored the effect of SWMS conditions on the expression of ion transporters and exchangers. The gene encoding Cystic Fibrosis transmembrane regulator (CFTR), a chloride and bicarbonate transporter expressed on the apical side of (intestinal) epithelial cells and demonstrated to be indispensable for normal mucus production (66), was significantly upregulated in HT29-MTX-E12 cells grown under SWMS conditions $(F C=1.68, p=<0.00001)$. Other ion transporters that were significantly regulated under SWMS conditions in HT29-MTX-E12 and Caco-2 cells include NBCe1/SLC4A4, encoding a basolateral $\mathrm{Na}^{+} /$ HCO3- importer (FC $=-1.95, p<0.01$ in HT29-MTX-E12) and NHE1/SLC9A1, encoding a basolateral $\mathrm{Na}^{+} / \mathrm{H}^{+}$exchanger $\left(\mathrm{FC}=-1.59, p<1 \cdot 10^{-5}\right)$. Additionally, the significant downregulation of $D R A /$ SLC26A3, encoding an apical $\mathrm{Cl}^{-} / \mathrm{HCO}_{3}$ - exchanger $(\mathrm{FC}=-1.25, p<0.05)$ under SWMS conditions is interesting, given its importance in intestinal salt and fluid absorption (67). This gene is, however, not highly expressed in HT29-MTX-E12 cells (RMA < 3). Whereas GSEA revealed that the pathway "Mineral absorption" was not significantly regulated in HT29-MTX-E12, interestingly, this pathway was the most enriched among all downregulated pathways in Caco-2 cells (NES = -2.58, FDR $q$-value $=0.00$, Supplementary File 2 ). These cells also showed relatively strong and significant regulation of aforementioned ion transporters $\left(\mathrm{FC}=1.78, p<1 \cdot 10^{-8}\right.$ for CFTR, FC $=-2.03, p<1 \cdot 10^{-6}$ 
for NBCe1, FC $=-1.33, p<0.01$ for NHE1 and FC $=-3.18, p<1 \cdot 10^{-8}$ for DRA). All in all, culturing under SWMS results in significant regulation of several key ion transporters in both HT29-MTX-E12 and Caco-2 cells.

Lower glucose consumption and lactate production per cell in HT29-MTX-E12 cells grown under SWMS conditions

The observed visible colour difference of cell medium in HT29-MTX-E12 cells, accompanied by significant regulation of $\mathrm{H}^{+}$and $\mathrm{HCO}^{-}$transporters, could indicate a difference in cell medium $\mathrm{pH}$ between growth conditions. The $\mathrm{pH}$ of the medium samples collected during every refreshment remained similar between the apical and basolateral compartment in both conditions. During the first 13 days, $\mathrm{pH}$ decreased to a similar extent in both conditions. Under SWMS conditions, the $\mathrm{pH}$ remained relatively stable over time after one week, whereas static conditions showed a decreased $\mathrm{pH}$ at $\mathrm{t}=14$ days in the apical compartment (difference of 0.2) (Supplementary Fig. 6A-B). At $\mathrm{t}=15$ days, one day after the last medium refreshment, trends reversed and medium of static conditions showed a significantly higher $\mathrm{pH}$ compared to SWMS conditions in both compartments (difference of 0.2) (Fig. 6A-B and Supplementary Fig. 6A, C and D).

A

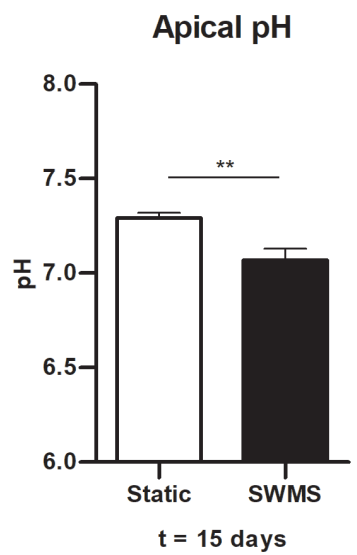

B

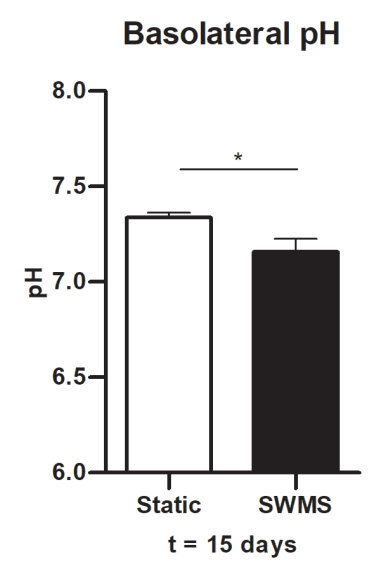

Figure $6 \mathrm{pH}$ values of medium collected at $\mathrm{t}=15$ days from HT29-MTX-E12 cells grown under static and SWMS conditions. Medium pH of $\mathbf{A}$ ) apical and $\mathbf{B}$ ) basolateral compartments of HT29-MTX-E12 cells grown under static and SWMS conditions at $\mathrm{t}=15$ days. ${ }^{*} p<0.05 ; * *<0.01, n=3$. 
A decrease in $\mathrm{pH}$ could be explained by increased lactate production and subsequent acidification of the medium. Indeed, $\mathrm{pH}$ of cell medium showed opposite trends to the amount of lactate measured in the medium, i.e. lactate concentrations increased with decreasing $\mathrm{pH}$ values and remained stable for SWMS conditions (Supplementary Fig. 7A). As medium volumes were, however, different between static and SWMS conditions, we calculated total lactate production and glucose consumption per well. Cell medium collected from cells grown under static conditions showed significantly higher lactate production at $t=7-14$ days (Supplementary Fig. 7B), accompanied with no difference in total glucose consumption per well (Supplementary Fig. 7C), indicating a lower amount of lactate produced per mole glucose under SWMS conditions. At $t=15$ days, one day after the last medium change (at which not all glucose had been consumed yet), lactate production was similar between conditions (Fig. 7A). On the contrary, cells grown under SWMS conditions had consumed significantly more glucose (Fig. 7B), again resulting in a lower lactate-per-glucose ratio under SWMS conditions. After correction for cell count at $t=15$ days, glucose consumption and lactate production per cell were still significantly lower under SWMS conditions (Fig. 7C-D, $p<0.001$ ), resulting in a significantly lower lactate-per-glucose ratio (Fig. 7E).

A

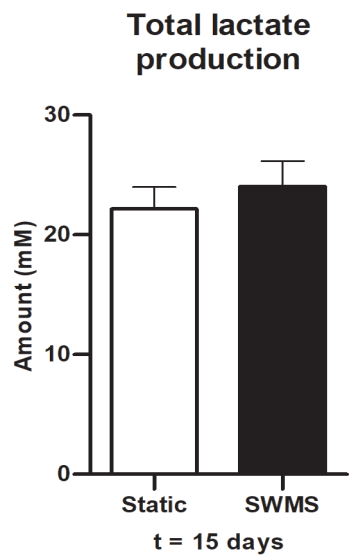

B

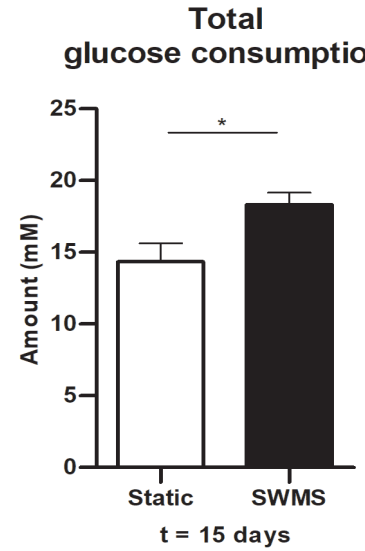

Lactate production per cell

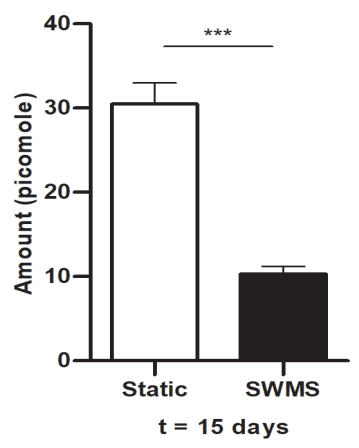


$\mathrm{D}$

Glucose consumption per cell

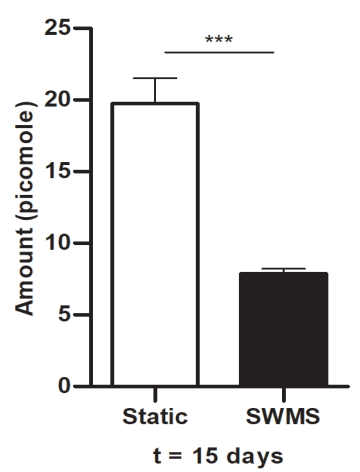

$E$

\section{Lactate/glucose}

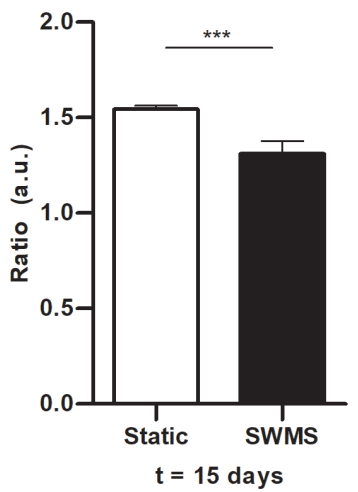

Figure 7 Lactate production and glucose consumption by HT29-MTX-E12 grown under static and SWMS conditions. A) Total lactate (micromole) produced per well in medium collected from apical and basolateral compartments of HT29-MTX-E12 cells grown under static or SWMS conditions, at $t=15$ days. B) Total glucose (micromole) consumed per well from medium collected from apical and basolateral compartments of HT29-MTX-E12 grown under static and SWMS conditions, at $t=15$ days. C) Glucose consumption (picomole) per cell by HT29-MTX-E12 grown under static and SWMS conditions at $t=15$ days. D) Lactate production (picomole) per cell by HT29-MTX-E12 grown under static and SWMS conditions at $\mathrm{t}=15$ days. E) Ratio of lactate produced glucose consumed per cell in HT29-MTX-E12 grown under static and SWMS conditions at $\mathrm{t}=15$ days. $* \mathrm{p}<0.05 ; * * \mathrm{p}<0.01 ; * * \mathrm{p}<0.001, \mathrm{n}=3$.

Lower glucose consumption under SWMS conditions coincided with a significantly lower expression of GLUT1/SLC2A1, encoding a transmembrane glucose transporter (FC $\left.=-1.46, p<1 \cdot 10^{-7}\right)$ and $H K 2$, encoding the glycolytic enzyme hexokinase $2\left(\mathrm{FC}=-2.18, p=p<1 \cdot 10^{-8}\right)$. GSEA revealed, however, no significant enrichment of the "Carbohydrate absorption pathway" or "Glycolysis pathway" in HT29MTX-E12 cells cultured under SWMS conditions. Interestingly, however, both pathways were among the top-10 enriched downregulated in Caco-2 cells (NES "Carbohydrate absorption" $=-2.35, p=$ 0.00 and NES "Glycolysis pathway" $=-2.12, p<1 \cdot 10^{-3}$, respectively). As shown previously for similar models, these data could point towards a more aerobic cell metabolism under SWMS conditions (40-44). This is further supported by a significant downregulation of the "HIF1-signalling pathway" in both HT29-MTX-E12 and Caco-2 cells, as revealed by GSEA (NES $=-1.79$, FDR q-value $=<0.05$ and NES $=-2.30$, FDR q-value $=0.01$, respectively). 


\section{Discussion}

In the present study, we aimed to further characterize the potential mechanisms underlying the increased mucus production by the SWMS culture method as described by Navabi et al. (25). To this end, we cultured HT29-MTX-E12 cells under both static and SWMS conditions and performed microarray analysis to investigate changes in gene expression by taking both a targeted and untargeted approach. First, we aimed to validate the increased mucus production under SWMS conditions. Indeed, SWMS conditions induced higher MUC2 expression in HT29-MTX-E12 cells, which was also reflected at the protein level. It seemed that the increase in MUC2 occurred at the expense of MUC5AC, since both gene and protein expression of this mucin was decreased. This resulted in a significantly increased MUC2/MUC5AC ratio, confirming previous findings (25). An overview of the most prominent changes observed at $t=15$ days is graphically summarized (Figure 8).

Effects SWMS vs. static at $\mathrm{t}=15$ days

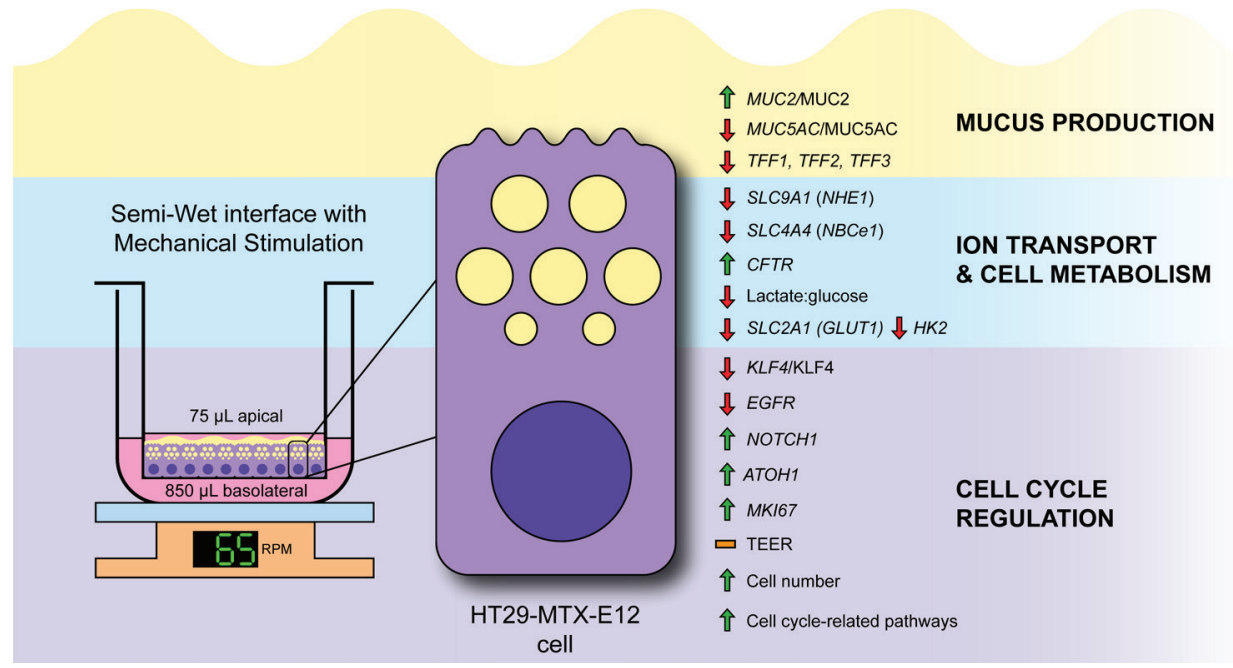

Figure 8 Graphical summary of effects observed in HT29-MTX-E12 cells grown under SWMS compared to static conditions at $\mathbf{t}=\mathbf{1 5}$ days. HT29-MTX-E12 cells were grown on Transwell under static and SWMS conditions. The most important changes between static and SWMS at $\mathrm{t}=15$ days are depicted. MUC2/MUC2 = mucin 2; MUC5AC/MUC5AC = mucin 5; TFF1/2/3 = trefoil factor 1/2/3; SLC9A1 $(N H E 1)=\mathrm{Na}^{+} / \mathrm{H}^{+}$Exchanger $1 ; \operatorname{SLC} 4 A 4(N B C e 1)=\mathrm{Na}^{+} / \mathrm{HCO}^{-}$cotransporter $1 ;$ CFTR $=$ Cystic Fibrosis transmembrane conductance regulator; lactate:glucose $=$ amount of lactate (mole) produced per mole of glucose consumed in conditioned medium; SLC2A1 (GLUT1) = Glucose Transporter Type 1; HK2 = Hexokinase 2; KLF4/KLF4 = Kruppel like factor 4; EGFR = epidermal growth factor receptor; NOTCH1 = Notch receptor 1; $A T O H 1=$ atonal $\mathrm{BHLH}$ transcription factor 1; MKI67 = marker of proliferation Ki-67; TEER $=$ transepithelial electrical resistance. 
The transcriptomic analysis of HT29-MTX-E12 cells revealed that SWMS conditions resulted in a strong upregulation of genes and pathways related to cell cycle regulation. In line with this finding, we found higher cell numbers after SWMS culturing. Although the effect size was lower, similar results were found in Caco-2 cells. Similar to our results, a previous study using ALI - which is similar to the semi-wet interface part of SWMS conditions - also showed an increase in cell number and cell layer thickness in intestinal porcine epithelial cells (39), and increased cell proliferation in intestinal organoids (68). Our experiments can, however, not answer whether the higher cell count is due to an increased height and/or a columnar shape of cells grown under SWMS, as seen in both ALI and SWMS cultures $(25,39)$ or due to stacking of the cells, although the concentration of cells in the centre of the well point to the latter. In any case, the shared upregulation of cell cycle-related pathways between colon carcinoma cell lines that are so different at the transcriptomic level (69), emphasizes the SWMS-specific effect on cell growth, independent of the cell type. As cell culturing conditions may also strongly influence cell morphology and tight junction formation (70), we explored if the SWMS method resulted in an increased resistance over the epithelial membrane by performing TEER measurements. TEER values of HT29-MTX-E12 cells were comparable to results from earlier studies $(24,71)$. No differences were found between SWMS and static conditions for HT29-MTX-E12 cells during the culturing period, which was supported by the low number of differentially regulated genes related to intestinal barrier integrity. These findings are opposing the results from Navabi et al., as they found a slight, but significant, increase in TEER values of HT29-MTX-E12 cells cultured under SWMS conditions. However, apart from the fact that measurements were performed at 21 days, Navabi et al. also used a different device (Ussing chamber) for TEER measurements. Furthermore, the higher concentration of cells in the centre of the Transwell as a result of SWMS conditions may have resulted in an underestimation of TEER values, as the chopstick electrodes do not measure the resistance over the whole membrane.

In parallel to cell proliferation, we also investigated the potential change in cell differentiation pathways as a result of the SWMS method. We focussed on the Notch/Atoh1 pathways, since these are key pathways in the decision between epithelial cell development into either absorptive or secretory cell types (51). Given the MUC2-promoting effects of SWMS conditions in HT29-MTX-E12 cells, we hypothesized that SWMS conditions inhibited Notch and promoted Atoh1, thereby favouring the differentiation of secretory goblet cells. The interplay between Notch and Atoh in the context of goblet cell differentiation was underscored by the effect of the $y$-secretase inhibitor DAPT, which further enhanced mucus production in HT29-MTX-E12 cells by indirectly inhibiting Notch, and thus promoting goblet cell differentiation (25). Although we found a number of significantly differentially 
expressed target genes of both Notch and Atoh1, the results did not point towards one particular overrepresented pathway. Based on these results, we suppose that the increased MUC2 production in HT29-MTX-E12 cells was not the result of a change towards a favoured secretory cell fate. This was further supported by a significant downregulation of the goblet cell marker KLF4 at both protein and gene level. The downregulation of KLF4/KLF4, identified as a cell cycle checkpoint protein and negative regulator of cell growth $(72,73)$, matches with the observed increased cell cycle regulation under SWMS conditions. However, based on our results, we cannot identify cause-effect relations and point at the exact trigger that led to decreased KLF4/KLF4 expression.

The three members of the TFF family (TFF1, TFF2, TFF3) were all strongly downregulated under SWMS conditions in HT29-MTX-E12 cells. TFF1 and TFF2 are predominantly expressed in the stomach and duodenum in humans (74-76), whereas TFF3 is mostly expressed in the small intestine and colon $(77,78)$. It is likely that our study confirms the previous finding that TFF1 and MUC5AC colocalize $(79,80)$, as both genes were highly expressed in HT29-MTX-E12 cells. Moreover, the strong and significant downregulation of both TFF1 and MUC5AC induced by SWMS conditions emphasizes the simultaneous regulation of these genes by the SWMS method. TFF3 is known to co-localize with $M U C 2$, however, these genes had opposite expression profiles. Interestingly, TFF expression is regulated by EGFR (64), of which the encoding gene was found downregulated in our transcriptomic dataset. Besides, EGFR activation was implicated in the production of MUC5AC in airway epithelial cells (81). However, the exact role of EGFR in the SWMS model remains elusive.

Proper mucus production depends on the activity of ion transport (65) and ALI-models with other cell types have shown changes in ion transport $(42,82-84)$. Therefore, the significant regulation of several key ion transporters in both HT29-MTX-E12 and Caco-2 cells in our microarray analysis, is interesting. It should be considered, however, that the microarray results are limited to one time point; in this case at which a lower $\mathrm{pH}$ is measured under SWMS versus static conditions. Therefore, we cannot conclude whether the change in mucus phenotype can be (partially) explained by differential regulation of ion transporters, or that this regulation is a consequence of the microclimate at the timepoint of analysis. Moreover, our method to measure $\mathrm{pH}$ did not allow us to measure potential local pH differences, as seen in vivo (85), which could further clarify the observed changes.

It has been demonstrated previously that both the semi-wet conditions/ALI and the mechanical stimulation part of SWMS separately, support a more aerobic cell culture environment in nonintestinal cell lines (41-44). More recent studies confirmed increased oxygen supply and oxidative phosphorylation, concomitant with suppressed glycolysis in intestinal epithelial cells from porcine origin $(39,40)$. Similar to the study by Klasvogt et al. $(40)$, we measured significantly lower lactate 
levels and decreased glucose consumption in HT29-MTX-E12 cells grown under SWMS conditions. Interestingly, as opposed to Klasvogt et al., we did not find a downregulation of the HIF-1a gene, but GSEA revealed significant enrichment of the HIF-1 signalling pathway among the downregulated pathways in both HT29-MTX-E12 and Caco-2 cells. This, together with the significantly decreased lactate-per glucose ratio, suggests that under SWMS conditions, cells switch to a more aerobic cell metabolism. Increased mucus production has been observed in other ALI-models (without mechanical stimulation), such as murine gastric surface mucous cells (44) and airway cells from different origins (86). MUC5AC was suggested to be transcriptionally regulated by HIF-1a in airway cells $(87,88)$, which indicates the possible involvement of HIF-1a in the downregulation of MUC5AC on both gene and protein level under SWMS conditions. In contrast, hypoxia was shown to enhance HIF-1a-binding to the MUC2 promoter, resulting in increased MUC2 mRNA and protein levels. However, this effect was transient, as MUC2 protein levels restored again after 24 hours, while hypoxic conditions were still applied (89). In any case, the increased MUC2 levels in our study cannot directly be explained by the observed downregulation of HIF-1a.

Mechanical stimulation as part of the SWMS conditions, induced by continuous shaking at $65 \mathrm{rpm}$, led to a continuous exposure to shear stress. In a model highly similar to SWMS, the shear stress value was calculated to be $1.6 \times 10^{-2} \pm 4.7 \times 10^{-3} \mathrm{dyne} / \mathrm{cm}^{2}(90)$. In other in vitro and ex vivo models, shear stress values between 1.3 and $2.0 \times 10^{-2} \mathrm{dyne} / \mathrm{cm}^{2}$ were sufficient to increase MUC2 protein or gene expression in Caco-2 cells, colon organoids or enteroids compared to static conditions (26, 91). We were able to replicate the increase in MUC2 gene and protein levels in HT29-MTX-E12, but not in Caco-2 cells, although we cannot be certain if the shear stress induced by mechanical stimulation was responsible for this observation. Importantly, cell culture conditions in aforementioned models were different than the SWMS conditions, as medium is continuously replenished, whereas under SWMS conditions, medium is refreshed every 2 or 3 days (but still constantly redistributed due to mechanical stimulation). Besides, in some of the models mentioned above, laminar flow is applied using external pumps resulting in a constant flow, which is a different approach than the circular shaking motion under SWMS conditions. Signalling pathways involved in the response of intestinal cells to mechanical forces were not significantly regulated in our study (92). Shear stress was also demonstrated to stimulate differentiation of intestinal epithelial cells $(26,91,93,94)$. Interestingly, next to its role in cell differentiation and cell cycle arrest (95), KLF4 is also known as a mechanosensitive transcription factor in vascular endothelial cells (96), osteoblasts (97) and a dermal cell line (98), in a context-dependent manner. To the best of our knowledge, there is no literature available providing evidence for a link between shear stress and KLF4 in intestinal tissues or models. 
An important limitation of our study is that our microarray was restricted to a single time point, whereas our study aimed to capture an overview of the cellular processes affected by the SWMS culture method. To increase insight in gene expression over time (e.g. with regard to cell proliferation or mucus production), future studies should include multiple time points. Another limitation is that gene expression does not always match with protein expression or activity, though protein levels of MUC2, MUC5AC and KLF4 supported the changes observed at gene levels in both HT29-MTX-E12 and Caco-2 cells. Apart from technical limitations of our study, we demonstrate that HT29-MTX-E12 cells grown under SWMS conditions, as a model developed to better represent the in vivo intestine in terms of MUC2 and cell morphology (25) still has its limitations. For instance, the downregulation of genes encoding other mucus-associated proteins, such as TFF3, suggest a less representative mucus layer in terms of whole mucus composition. This could limit the use of this model to further functionally characterize the intestinal mucus layer at a molecular level and to exploit the myriad of in vivo functions of mucus. Still, the function of the SWMS-produced mucus layer as a physical barrier was demonstrated by its interference with the genotoxic activity of colibactin, a toxin produced by certain Escherichia coli strains (30). In a similar fashion, the model could be applied to evaluate the diffusion of drugs and other particles (100). Furthermore, with MUC2 being the main component, the model could be useful to study interactions with both pathogenic and commensal bacteria, but would further require quantification of the mucin glycans present, as these have demonstrated to play a crucial role in mucin-microbe interactions (101).

Altogether, we confirm the usefulness of SWMS cell culture conditions to improve the in vivo representation of the mucus layer in vitro, with regard to the increase in intestinal mucin MUC2. Our study provides insight in potential underlying processes, which might ultimately lead to a stepby-step improvement of the representativeness of the in vitro mucus layer. For instance, our study demonstrates upregulation of cell cycle regulation, downregulation of KLF4, differential regulation of ion transporters and increased aerobic metabolism of cells cultured under SWMS versus static conditions. Further research should also focus on the qualitative aspects of the in vitro mucus layer, for example with regard to mucin glycosylation, disulphide bonds that assure firmness of the mucus, and the contribution of other proteins present in the mucus layer, such as TFFs, as observed in vivo. 


\section{Acknowledgements}

JE has been funded by a Building Blocks of Life grant from the Netherlands Organization for Scientific Research (NWO), grant no. 737.016.003. BvdL has been funded by the Nutricia Research Foundation, grant no. 2018-25 and the NWO Graduate Programme on Food Structure, Digestion and Health, grant no. 022.006.009.

\section{Conflict of interest}

Authors declare no potential conflict of interest. 


\section{References}

1. $\quad$ Allen A. Mucus-a protective secretion of complexity Trends Biochem Sci 1983;8:169-73

2. Forstner J, Forstner G. Gastrointestinal mucus In: Johnson LG, editor. Physiology of the Gastrointestinal Tract. New York: Raven Press 1994. p. 1255-83.

3. Deplancke B, Gaskins HR. Microbial modulation of innate defense: goblet cells and the intestinal mucus layer. Am J Clin Nutr. 2001;73(6):1131s-41s.

4. Allen A, Flemström G. Gastroduodenal mucus bicarbonate barrier: protection against acid and pepsin. Am J Physiol Cell Physiol. 2005;288(1):C1-19.

5. Van der Sluis M, De Koning BA, De Bruijn AC, Velcich A, Meijerink JP, Van Goudoever JB, et al. Muc2-deficient mice spontaneously develop colitis, indicating that MUC2 is critical for colonic protection. Gastroenterology. 2006;131(1):117-29.

6. Velcich A, Yang W, Heyer J, Fragale A, Nicholas C, Viani S, et al. Colorectal cancer in mice genetically deficient in the mucin Muc2. Science. 2002;295(5560):1726-9.

7. Atuma C, Strugala V, Allen A, Holm L. The adherent gastrointestinal mucus gel layer: thickness and physical state in vivo. Am J Physiol Gastrointest Liver Physiol. 2001;280(5):G922-9.

8. $\quad$ Simon GL, Gorbach SL. Intestinal flora in health and disease. Gastroenterology. 1984;86(1):17493.

9. Derrien M, van Passel MW, van de Bovenkamp JH, Schipper RG, de Vos WM, Dekker J. Mucinbacterial interactions in the human oral cavity and digestive tract. Gut Microbes. 2010;1(4):254-68.

10. Belzer C, Chia LW, Aalvink S, Chamlagain B, Piironen V, Knol J, et al. Microbial Metabolic Networks at the Mucus Layer Lead to Diet-Independent Butyrate and Vitamin B(12) Production by Intestinal Symbionts. mBio. 2017;8(5).

11. Johansson ME, Phillipson M, Petersson J, Velcich A, Holm L, Hansson GC. The inner of the two Muc2 mucin-dependent mucus layers in colon is devoid of bacteria. Proc Natl Acad Sci U S A. 2008;105(39):15064-9.

12. Jany BH, Gallup MW, Yan PS, Gum JR, Kim YS, Basbaum CB. Human bronchus and intestine express the same mucin gene. J Clin Invest. 1991;87(1):77-82.

13. Tytgat KM, Büller HA, Opdam FJ, Kim YS, Einerhand AW, Dekker J. Biosynthesis of human colonic mucin: Muc2 is the prominent secretory mucin. Gastroenterology. 1994;107(5):1352-63.

14. Strous GJ, Dekker J. Mucin-type glycoproteins. Crit Rev Biochem Mol Biol. 1992;27(1-2):57-92.

15. Chang SK, Dohrman AF, Basbaum CB, Ho SB, Tsuda T, Toribara NW, et al. Localization of mucin (MUC2 and MUC3) messenger RNA and peptide expression in human normal intestine and colon cancer. Gastroenterology. 1994;107(1):28-36.

16. Van Klinken BJ, Dekker J, Büller HA, Einerhand AW. Mucin gene structure and expression: protection vs. adhesion. Am J Physiol. 1995;269(5 Pt 1):G613-27.

17. Allen A. The structure and function of gastrointestinal mucus. In: Johnson LR, editor. Physiology of the Gastrointestinal Tract. New York: Raven Press; 1981. p. 617-39.

18. Etienne-Mesmin L, Chassaing B, Desvaux M, De Paepe K, Gresse R, Sauvaitre T, et al. Experimental models to study intestinal microbes-mucus interactions in health and disease. FEMS Microbiol Rev. 2019;43(5):457-89.

19. Browning TH, Trier JS. Organ culture of mucosal biopsies of human small intestine. J Clin Invest. 1969;48(8):1423-32.

20. Gustafsson JK, Ermund A, Ambort D, Johansson ME, Nilsson HE, Thorell K, et al. Bicarbonate and functional CFTR channel are required for proper mucin secretion and link cystic fibrosis with its mucus phenotype. J Exp Med. 2012;209(7):1263-72.

21. Yissachar N, Zhou Y, Ung L, Lai NY, Mohan JF, Ehrlicher A, et al. An Intestinal Organ Culture System Uncovers a Role for the Nervous System in Microbe-Immune Crosstalk. Cell. 2017;168(6):1135-48. e12.

22. Lesuffleur T, Porchet N, Aubert JP, Swallow D, Gum JR, Kim YS, et al. Differential expression of the human mucin genes MUC1 to MUC5 in relation to growth and differentiation of different mucussecreting HT-29 cell subpopulations. J Cell Sci. 1993;106 ( Pt 3):771-83.

23. van Klinken BJ, Oussoren E, Weenink JJ, Strous GJ, Büller HA, Dekker J, et al. The human intestinal cell lines Caco-2 and LS174T as models to study cell-type specific mucin expression. Glycoconj J. 1996;13(5):757-68.

24. Behrens I, Stenberg P, Artursson P, Kissel T. Transport of lipophilic drug molecules in a new mucus-secreting cell culture model based on HT29-MTX cells. Pharm Res. 2001;18(8):1138-45.

25. Navabi N, McGuckin MA, Lindén SK. Gastrointestinal cell lines form polarized epithelia with an adherent mucus layer when cultured in semi-wet interfaces with mechanical stimulation. PLoS One. 2013;8(7):e68761.

26. Kim HJ, Huh D, Hamilton G, Ingber DE. Human gut-on-a-chip inhabited by microbial flora that experiences intestinal peristalsis-like motions and flow. Lab Chip. 2012;12(12):2165-74.

27. Jalili-Firoozinezhad S, Gazzaniga FS, Calamari EL, Camacho DM, Fadel CW, Bein A, et al. A complex human gut microbiome cultured in an anaerobic intestine-on-a-chip. Nat Biomed Eng. 2019;3(7):520-31.

28. Costello CM, Hongpeng J, Shaffiey S, Yu J, Jain NK, Hackam D, et al. Synthetic small intestinal scaffolds for improved studies of intestinal differentiation. Biotechnol Bioeng. 2014;111(6):1222-32.

29. Chen Y, Lin Y, Davis KM, Wang Q, Rnjak-Kovacina J, Li C, et al. Robust bioengineered 3D functional human intestinal epithelium. Sci Rep. 2015;5:13708. 
30. Reuter C, Alzheimer M, Walles H, Oelschlaeger TA. An adherent mucus layer attenuates the genotoxic effect of colibactin. Cell Microbiol. 2018;20(2).

31. Jung $P$, Sato $T$, Merlos-Suárez $A$, Barriga FM, Iglesias $M$, Rossell $D$, et al. Isolation and in vitro expansion of human colonic stem cells. Nat Med. 2011;17(10):1225-7.

32. Sato T, Stange DE, Ferrante M, Vries RG, Van Es JH, Van den Brink S, et al. Long-term expansion of epithelial organoids from human colon, adenoma, adenocarcinoma, and Barrett's epithelium. Gastroenterology. 2011;141(5):1762-72.

33. Spence JR, Mayhew CN, Rankin SA, Kuhar MF, Vallance JE, Tolle K, et al. Directed differentiation of human pluripotent stem cells into intestinal tissue in vitro. Nature. 2011;470(7332):105-9.

34. Sontheimer-Phelps A, Chou DB, Tovaglieri A, Ferrante TC, Duckworth T, Fadel C, et al. Human Colon-on-a-Chip Enables Continuous In Vitro Analysis of Colon Mucus Layer Accumulation and Physiology. Cell Mol Gastroenterol Hepatol. 2020;9(3):507-26.

35. Belley A, Chadee K. Prostaglandin E(2) stimulates rat and human colonic mucin exocytosis via the EP(4) receptor. Gastroenterology. 1999;117(6):1352-62.

36. Milano J, McKay J, Dagenais C, Foster-Brown L, Pognan F, Gadient R, et al. Modulation of notch processing by gamma-secretase inhibitors causes intestinal goblet cell metaplasia and induction of genes known to specify gut secretory lineage differentiation. Toxicol Sci. 2004;82(1):341-58.

37. Hatayama H, Iwashita J, Kuwajima A, Abe T. The short chain fatty acid, butyrate, stimulates MUC2 mucin production in the human colon cancer cell line, LS174T. Biochem Biophys Res Commun. 2007;356(3):599-603.

38. Smirnova MG, Guo L, Birchall JP, Pearson JP. LPS up-regulates mucin and cytokine mRNA expression and stimulates mucin and cytokine secretion in goblet cells. Cell Immunol. 2003;221(1):42-9.

39. Nossol C, Diesing AK, Walk N, Faber-Zuschratter H, Hartig R, Post A, et al. Air-liquid interface cultures enhance the oxygen supply and trigger the structural and functional differentiation of intestinal porcine epithelial cells (IPEC). Histochem Cell Biol. 2011;136(1):103-15.

40. Klasvogt S, Zuschratter W, Schmidt A, Kröber A, Vorwerk S, Wolter R, et al. Air-liquid interface enhances oxidative phosphorylation in intestinal epithelial cell line IPEC-J2. Cell Death Discov. 2017;3:17001. 41. Dickman KG, Mandel LJ. Glycolytic and oxidative metabolism in primary renal proximal tubule cultures. Am J Physiol. 1989;257(2 Pt 1):C333-40.

42. Johnson LG, Dickman KG, Moore KL, Mandel LJ, Boucher RC. Enhanced Na+ transport in an air-liquid interface culture system. Am J Physiol. 1993;264(6 Pt 1):L560-5.

43. Kondo M, Tamaoki J, Sakai A, Kameyama S, Kanoh S, Konno K. Increased oxidative metabolism in cow tracheal epithelial cells cultured at air-liquid interface. Am J Respir Cell Mol Biol. 1997;16(1):62-8.

44. Ootani A, Toda S, Fujimoto K, Sugihara H. An air-liquid interface promotes the differentiation of gastric surface mucous cells (GSM06) in culture. Biochem Biophys Res Commun. 2000;271(3):741-6.

45. Irizarry RA, Bolstad BM, Collin F, Cope LM, Hobbs B, Speed TP. Summaries of Affymetrix GeneChip probe level data. Nucleic Acids Res. 2003;31(4):e15.

46. Dai M, Wang P, Boyd AD, Kostov G, Athey B, Jones EG, et al. Evolving gene/transcript definitions significantly alter the interpretation of GeneChip data. Nucleic Acids Res. 2005;33(20):e175.

47. Sartor MA, Tomlinson CR, Wesselkamper SC, Sivaganesan S, Leikauf GD, Medvedovic M Intensity-based hierarchical Bayes method improves testing for differentially expressed genes in microarray experiments. BMC Bioinformatics. 2006;7:538.

48. Oliveros JC. Venny. An interactive tool for comparing lists with Venn's diagrams. 2007-2015.

49. Demitrack ES, Samuelson LC. Notch regulation of gastrointestinal stem cells. J Physiol. 2016;594(17):4791-803.

50. VanDussen KL, Carulli AJ, Keeley TM, Patel SR, Puthoff BJ, Magness ST, et al. Notch signaling modulates proliferation and differentiation of intestinal crypt base columnar stem cells. Development. 2012;139(3):488-97.

51. Kazanjian A, Shroyer NF. NOTCH Signaling and ATOH1 in Colorectal Cancers. Curr Colorectal Cancer Rep. 2011;7(2):121-7.

52. Chen KY, Srinivasan T, Tung KL, Belmonte JM, Wang L, Murthy PKL, et al. A Notch positive feedback in the intestinal stem cell niche is essential for stem cell self-renewal. Mol Syst Biol. 2017;13(4):927. 53. Ghaleb AM, McConnell BB, Kaestner KH, Yang VW. Altered intestinal epithelial homeostasis in mice with intestine-specific deletion of the Krüppel-like factor 4 gene. Dev Biol. 2011;349(2):310-20.

54. Rowland BD, Peeper DS. KLF4, p21 and context-dependent opposing forces in cancer. Nat Rev Cancer. 2006;6(1):11-23.

55. Ghaleb AM, Yang VW. Krüppel-like factor 4 (KLF4): What we currently know. Gene. 2017;611:27-

37.

56. Yoon HS, Yang VW. Requirement of Krüppel-like factor 4 in preventing entry into mitosis following DNA damage. J Biol Chem. 2004;279(6):5035-41.

57. Hagos EG, Ghaleb AM, Dalton WB, Bialkowska AB, Yang VW. Mouse embryonic fibroblasts null for the Krüppel-like factor 4 gene are genetically unstable. Oncogene. 2009;28(9):1197-205.

58. Yoon HS, Ghaleb AM, Nandan MO, Hisamuddin IM, Dalton WB, Yang VW. Krüppel-like factor 4 prevents centrosome amplification following gamma-irradiation-induced DNA damage. Oncogene. 2005;24(25):4017-25.

59. Chen ZY, Shie JL, Tseng CC. Gut-enriched Kruppel-like factor represses ornithine decarboxylase gene expression and functions as checkpoint regulator in colonic cancer cells. J Biol Chem. 2002;277(48):46831-9.

60. Shie JL, Chen ZY, Fu M, Pestell RG, Tseng CC. Gut-enriched Krüppel-like factor represses cyclin 
D1 promoter activity through Sp1 motif. Nucleic Acids Res. 2000;28(15):2969-76.

61. Shie JL, Chen ZY, O'Brien MJ, Pestell RG, Lee ME, Tseng CC. Role of gut-enriched Krüppel-like factor in colonic cell growth and differentiation. Am J Physiol Gastrointest Liver Physiol. 2000;279(4):G80614.

62. Klaewsongkram J, Yang Y, Golech S, Katz J, Kaestner KH, Weng NP. Krüppel-like factor 4 regulates $B$ cell number and activation-induced B cell proliferation. J Immunol. 2007;179(7):4679-84.

63. Aihara E, Engevik KA, Montrose MH. Trefoil Factor Peptides and Gastrointestinal Function. Annu Rev Physiol. 2017;79:357-80.

64. Taupin D, Wu DC, Jeon WK, Devaney K, Wang TC, Podolsky DK. The trefoil gene family are coordinately expressed immediate-early genes: EGF receptor- and MAP kinase-dependent interregulation. J Clin Invest. 1999;103(9):R31-8.

65. Garcia MA, Yang N, Quinton PM. Normal mouse intestinal mucus release requires cystic fibrosis transmembrane regulator-dependent bicarbonate secretion. J Clin Invest. 2009;119(9):2613-22.

66. Kerem B, Rommens JM, Buchanan JA, Markiewicz D, Cox TK, Chakravarti A, et al. Identification of the cystic fibrosis gene: genetic analysis. Science. 1989;245(4922):1073-80.

67. Moseley RH, Höglund P, Wu GD, Silberg DG, Haila S, de la Chapelle A, et al. Downregulated in adenoma gene encodes a chloride transporter defective in congenital chloride diarrhea. Am J Physiol. 1999;276(1):G185-92.

68. Ootani A, Li X, Sangiorgi E, Ho QT, Ueno H, Toda S, et al. Sustained in vitro intestinal epithelial culture within a Wnt-dependent stem cell niche. Nat Med. 2009;15(6):701-6.

69. Christensen J, El-Gebali S, Natoli M, Sengstag T, Delorenzi M, Bentz S, et al. Defining new criteria for selection of cell-based intestinal models using publicly available databases. BMC Genomics. 2012;13:274.

70. Srinivasan B, Kolli AR, Esch MB, Abaci HE, Shuler ML, Hickman JJ. TEER measurement techniques for in vitro barrier model systems. J Lab Autom. 2015;20(2):107-26.

71. Hilgendorf C, Spahn-Langguth H, Regårdh CG, Lipka E, Amidon GL, Langguth P. Caco-2 versus Caco-2/HT29-MTX co-cultured cell lines: permeabilities via diffusion, inside- and outside-directed carriermediated transport. J Pharm Sci. 2000;89(1):63-75.

72. Shields JM, Christy RJ, Yang VW. Identification and characterization of a gene encoding a gutenriched Krüppel-like factor expressed during growth arrest. J Biol Chem. 1996;271(33):20009-17.

73. Chen X, Johns DC, Geiman DE, Marban E, Dang DT, Hamlin G, et al. Krüppel-like factor 4 (gutenriched Krüppel-like factor) inhibits cell proliferation by blocking G1/S progression of the cell cycle. J Biol Chem. 2001;276(32):30423-8.

74. Rio MC, Bellocq JP, Daniel JY, Tomasetto C, Lathe R, Chenard MP, et al. Breast cancer-associated pS2 protein: synthesis and secretion by normal stomach mucosa. Science. 1988;241(4866):705-8.

75. Hanby AM, Poulsom R, Singh S, Elia G, Jeffery RE, Wright NA. Spasmolytic polypeptide is a major antral peptide: distribution of the trefoil peptides human spasmolytic polypeptide and pS2 in the stomach. Gastroenterology. 1993;105(4):1110-6.

76. Jørgensen KD, Diamant B, Jørgensen KH, Thim L. Pancreatic spasmolytic polypeptide (PSP): III. Pharmacology of a new porcine pancreatic polypeptide with spasmolytic and gastric acid secretion inhibitory effects. Regul Pept. 1982;3(3-4):231-43.

77. Hauser F, Poulsom R, Chinery R, Rogers LA, Hanby AM, Wright NA, et al. hP1.B, a human $\mathrm{P}$-domain peptide homologous with rat intestinal trefoil factor, is expressed also in the ulcer-associated cell lineage and the uterus. Proc Natl Acad Sci U S A. 1993;90(15):6961-5.

78. Podolsky DK, Lynch-Devaney K, Stow JL, Oates P, Murgue B, De-Beaumont M, et al. Identification of human intestinal trefoil factor. Goblet cell-specific expression of a peptide targeted for apical secretion. J Biol Chem. 1993;268(16):12230.

79. Longman RJ, Douthwaite J, Sylvester PA, Poulsom R, Corfield AP, Thomas MG, et al. Coordinated localisation of mucins and trefoil peptides in the ulcer associated cell lineage and the gastrointestinal mucosa. Gut. 2000;47(6):792-800.

80. Ruchaud-Sparagano MH, Westley BR, May FE. The trefoil protein TFF1 is bound to MUC5AC in human gastric mucosa. Cell Mol Life Sci. 2004;61(15):1946-54.

81. Casalino-Matsuda SM, Monzón ME, Forteza RM. Epidermal growth factor receptor activation by epidermal growth factor mediates oxidant-induced goblet cell metaplasia in human airway epithelium. Am J Respir Cell Mol Biol. 2006;34(5):581-91.

82. Portier F, Kania R, Planès C, Hsu WC, Couette S, Tran Ba Huy P, et al. Enhanced sodium absorption in middle ear epithelial cells cultured at air-liquid interface. Acta Otolaryngol. 2005;125(1):16-22. 83. Robison TW, Kim KJ. Air-interface cultures of guinea pig airway epithelial cells: effects of active sodium and chloride transport inhibitors on bioelectric properties. Exp Lung Res. 1994;20(2):101-17.

84. Yamaya M, Finkbeiner WE, Chun SY, Widdicombe JH. Differentiated structure and function of cultures from human tracheal epithelium. Am J Physiol. 1992;262(6 Pt 1):L713-24.

85. Kini A, Singh AK, Riederer B, Yang I, Tan X, di Stefano G, et al. Slc26a3 deletion alters pHmicroclimate, mucin biosynthesis, microbiome composition and increases the TNFa expression in murine colon. Acta Physiol (Oxf). 2020;230(2):e13498.

86. Whitcutt MJ, Adler KB, Wu R. A biphasic chamber system for maintaining polarity of differentiation of cultured respiratory tract epithelial cells. In Vitro Cell Dev Biol. 1988;24(5):420-8.

87. Polosukhin VV, Cates JM, Lawson WE, Milstone AP, Matafonov AG, Massion PP, et al. Hypoxiainducible factor-1 signalling promotes goblet cell hyperplasia in airway epithelium. J Pathol. 2011;224(2):20311. 
88. Young HW, Williams OW, Chandra D, Bellinghausen LK, Pérez G, Suárez A, et al. Central role of Muc5ac expression in mucous metaplasia and its regulation by conserved $5^{\prime}$ elements. Am J Respir Cell Mol Biol. 2007;37(3):273-90.

89. Dilly AK, Lee YJ, Zeh HJ, Guo ZS, Bartlett DL, Choudry HA. Targeting hypoxia-mediated mucin 2 production as a therapeutic strategy for mucinous tumors. Transl Res. 2016;169:19-30.e1.

90. Alzheimer M, Svensson SL, König F, Schweinlin M, Metzger M, Walles H, et al. A threedimensional intestinal tissue model reveals factors and small regulatory RNAs important for colonization with Campylobacter jejuni. PLoS Pathog. 2020;16(2):e1008304.

91. Delon LC, Guo Z, Oszmiana A, Chien CC, Gibson R, Prestidge C, et al. A systematic investigation of the effect of the fluid shear stress on Caco-2 cells towards the optimization of epithelial organ-on-chip models. Biomaterials. 2019;225:119521.

92. Gayer CP, Basson MD. The effects of mechanical forces on intestinal physiology and pathology. Cell Signal. 2009;21(8):1237-44.

93. Sunuwar L, Yin J, Kasendra M, Karalis K, Kaper J, Fleckenstein J, et al. Mechanical Stimuli Affect Escherichia coli Heat-Stable Enterotoxin-Cyclic GMP Signaling in a Human Enteroid Intestine-Chip Model. Infect Immun. 2020;88(3).

94. Yin J, Sunuwar L, Kasendra M, Yu H, Tse CM, Talbot C, Jr., et al. Fluid Shear Stress Enhances Differentiation of Jejunal Human Enteroids in Intestine-Chip. Am J Physiol Gastrointest Liver Physiol. 2020. 95. Evans PM, Liu C. Roles of Krüpel-like factor 4 in normal homeostasis, cancer and stem cells. Acta Biochim Biophys Sin (Shanghai). 2008;40(7):554-64.

96. Niu N, Xu S, Xu Y, Little PJ, Jin ZG. Targeting Mechanosensitive Transcription Factors in Atherosclerosis. Trends Pharmacol Sci. 2019;40(4):253-66.

97. Zhang B, An L, Geng B, Ding N, Coalson E, Wan L, et al. ERK5 negatively regulates Kruppel-like factor 4 and promotes osteogenic lineage cell proliferation in response to MEK5 overexpression or fluid shear stress. Connect Tissue Res. 2021;62(2):194-205.

98. Clark PR, Jensen TJ, Kluger MS, Morelock M, Hanidu A, Qi Z, et al. MEK5 is activated by shear stress, activates ERK5 and induces KLF4 to modulate TNF responses in human dermal microvascular endothelial cells. Microcirculation. 2011;18(2):102-17.

99. $\quad$ Aihara Y, Fukuda Y, Takizawa A, Osakabe N, Aida T, Tanaka K, et al. Visualization of mechanical stress-mediated $\mathrm{Ca}(2+)$ signaling in the gut using intravital imaging. Biosci Microbiota Food Health. 2020;39(4):209-18.

100. Lock JY, Carlson TL, Carrier RL. Mucus models to evaluate the diffusion of drugs and particles. Adv Drug Deliv Rev. 2018;124:34-49.

101. Sonnenburg JL, Xu J, Leip DD, Chen $\mathrm{CH}$, Westover BP, Weatherford J, et al. Glycan foraging in vivo by an intestine-adapted bacterial symbiont. Science. 2005;307(5717):1955-9. 


\section{Supplemental material}
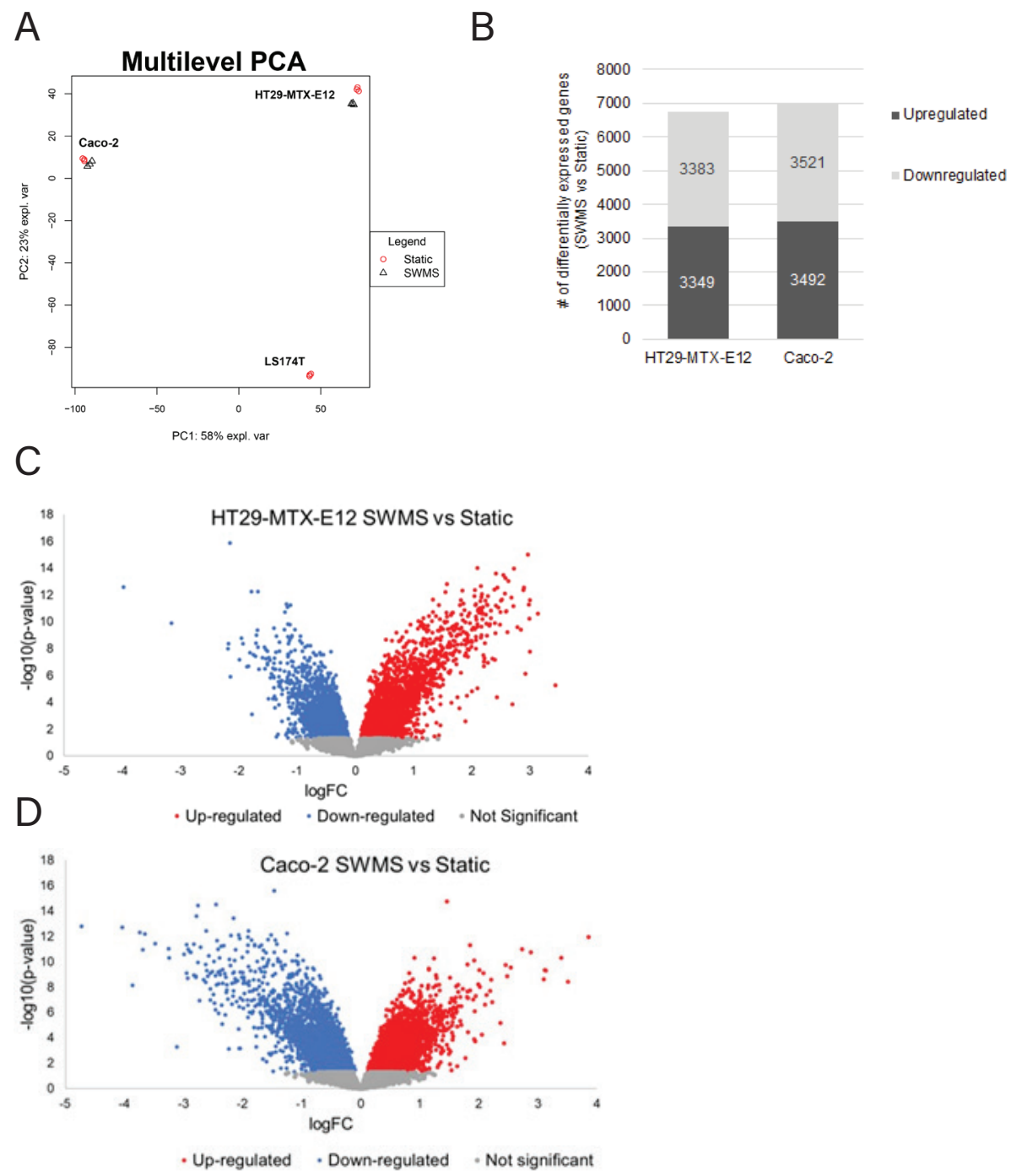

Supplementary figure 1 Overview microarray results in HT29-MTX-E12, Caco-2 and LS174T cells grown under static and/or SWMS conditions. A) Multilevel principal component analysis (PCA) of the 500 most variable genes. B) Number of differentially expressed genes between SWMS and static conditions in HT29-MTX-E12 and Caco-2 cells (IBMT $p<0.05)$. C) Volcano plot highlighting the Log Fold Change $(\log F C)$ on the $x$-axis and the corresponding $p$-values $(-\log (10))$ on the $y$-axis for the comparison SWMS versus static conditions in HT29-MTX-E12 cells and D) Caco-2 cells. $n=3$. 
A

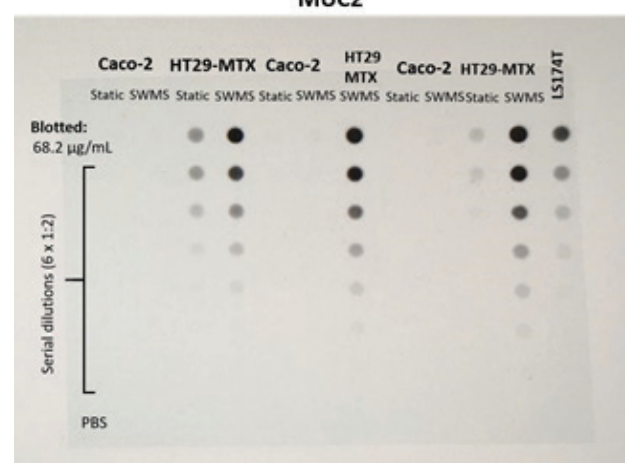

B

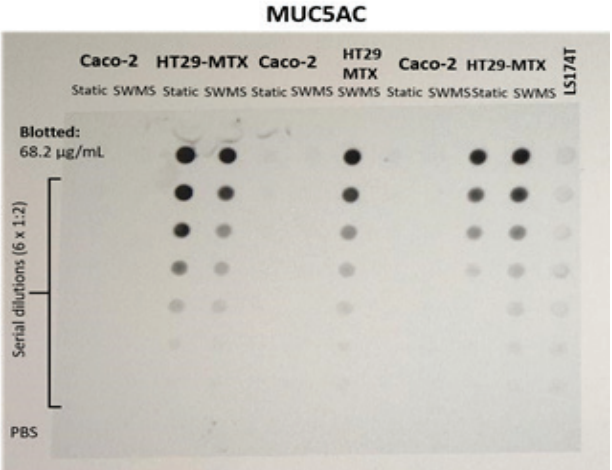

C

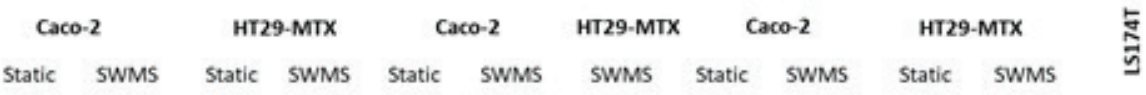

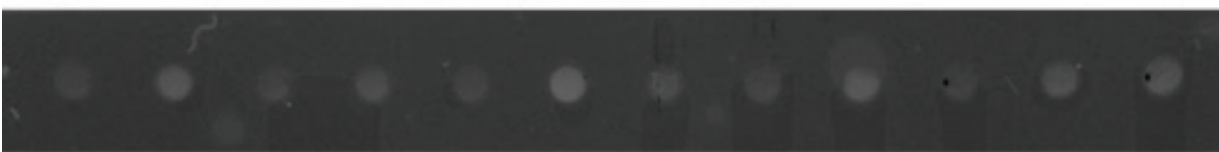

D

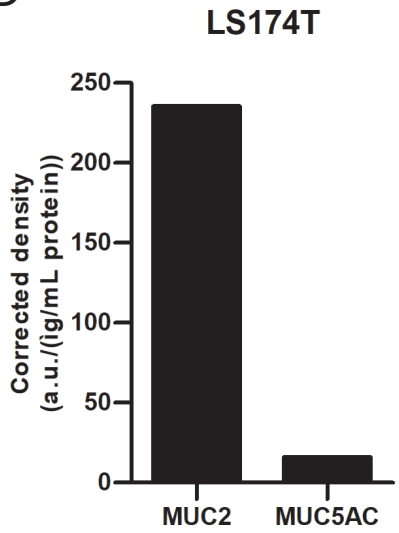

$E$

MUC2/MUC5AC

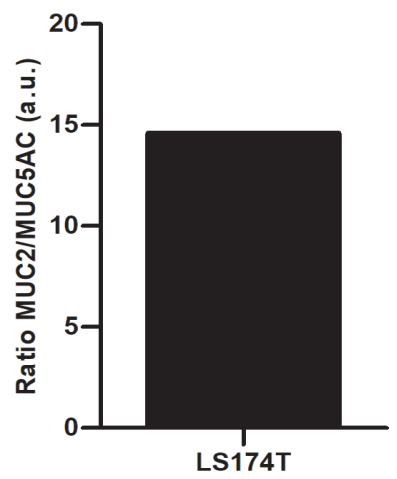


Supplementary figure 2 Overview of Dot Blot results for A) MUC2 and B) MUC5AC including Caco-2 and HT29-MTX-E12 cells cultured under static and SWMS conditions and LS174T cells. A concentration of 68.2 $\mu \mathrm{g} / \mathrm{mL}$ was blotted and six times serially diluted 1:2. In the bottom row, PBS was used as a negative control. C) Images of Ponceau Red staining (colorimetric and photographic) that were used as reference for total protein content. Protein density was based on the colorimetric image. D) Protein expression of MUC2 and MUC5AC in LS174T cells, expressed as density (a.u.) per ug $/ \mathrm{mL}$ protein blotted, after correction of Ponceau Red density. E) Ratio of MUC2 and MUC5AC protein expression in LS174T cells.

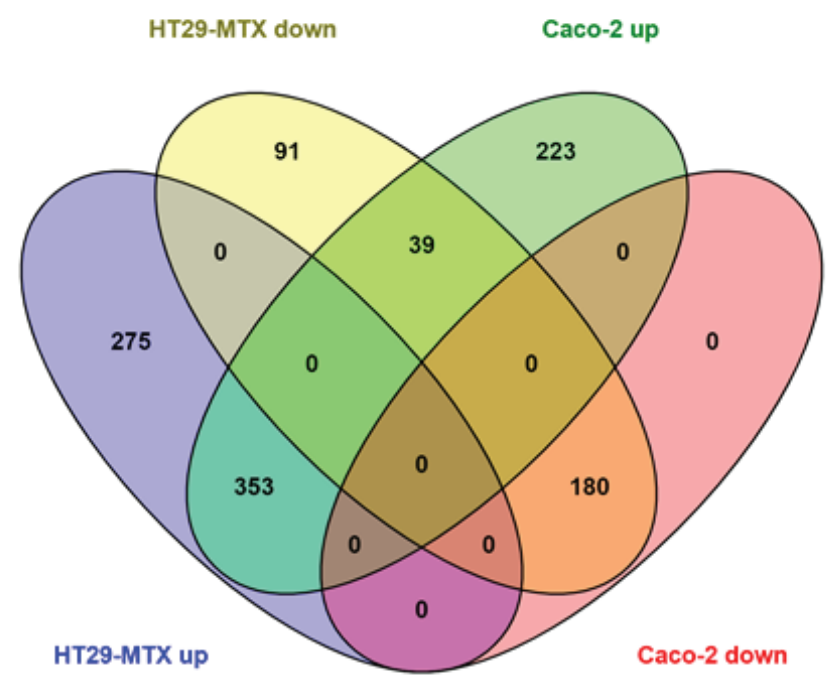

$\mathrm{Up}=\mathrm{FC} \geq 1.5$

Down $=F C \leq-1.5$ 
Top 20 upregulated genes shared between HT29-MTX and Caco-2

\begin{tabular}{|c|c|c|c|c|c|}
\hline Gene name & $\begin{array}{l}\text { FC in } \\
\text { HT29-MTX }\end{array}$ & p-value & $\begin{array}{l}\mathrm{FC} \text { in } \\
\text { Caco-2 }\end{array}$ & $\begin{array}{l}p- \\
\text { value }\end{array}$ & Gene function \\
\hline$R N Y 4 P 23$ & 10.80 & 0.00 & 2.12 & 0.05 & RNY4 pseudogene 23 \\
\hline KIF14 & 8.00 & 0.00 & 1.52 & 0.01 & kinesin family member 14 \\
\hline KIF20A & 7.99 & 0.00 & 1.76 & 0.01 & kinesin family member $20 \mathrm{~A}$ \\
\hline DLGAP5 & 7.91 & 0.00 & 2.04 & 0.00 & DLG associated protein 5 \\
\hline$H M M R$ & 7.82 & 0.00 & 1.52 & 0.00 & hyaluronan mediated motility receptor \\
\hline $\mathrm{H} 4 \mathrm{C} 1$ & 7.56 & 0.00 & 2.86 & 0.00 & H4 clustered histone 1 \\
\hline SPC25 & 7.41 & 0.00 & 2.06 & 0.00 & SPC25 component of NDC80 kinetochore complex \\
\hline $\mathrm{H} 3 \mathrm{C} 8$ & 7.20 & 0.00 & 2.17 & 0.00 & H3 clustered histone 8 \\
\hline TOP2A & 6.89 & 0.00 & 1.79 & 0.00 & DNA topoisomerase II alpha \\
\hline CCNB1 & 6.59 & 0.00 & 1.75 & 0.00 & cyclin B1 \\
\hline CENPF & 6.57 & 0.00 & 1.82 & 0.00 & centromere protein $\mathrm{F}$ \\
\hline NDC80 & 6.42 & 0.00 & 1.90 & 0.00 & NDC80 kinetochore complex component \\
\hline$H 1-5$ & 6.04 & 0.00 & 2.57 & 0.00 & H1.5 linker histone, cluster member \\
\hline$A S P M$ & 6.02 & 0.00 & 2.02 & 0.00 & abnormal spindle microtubule assembly \\
\hline PRR11 & 5.97 & 0.00 & 1.52 & 0.00 & proline rich 11 \\
\hline CENPE & 5.95 & 0.00 & 1.88 & 0.00 & centromere protein $\mathrm{E}$ \\
\hline NUSAP1 & 5.82 & 0.00 & 1.72 & 0.00 & nucleolar and spindle associated protein 1 \\
\hline FOXM1 & 5.72 & 0.00 & 1.81 & 0.00 & forkhead box M1 \\
\hline
\end{tabular}

\section{Top 20 downregulated genes shared between HT29-MTX and Caco-2}

\begin{tabular}{|c|c|c|c|c|c|}
\hline Gene name & $\begin{array}{l}\text { FC in } \\
\text { HT29-MTX }\end{array}$ & p-value & $\begin{array}{l}\text { FC in } \\
\text { Caco-2 }\end{array}$ & p-value & Gene function \\
\hline SLC6A10P & -1.51 & 0.01 & -2.29 & 0.00 & solute carrier family 6 member 10, pseudogene \\
\hline CD55 & -1.51 & 0.00 & -2.11 & 0.00 & CD55 molecule (Cromer blood group) \\
\hline$H L A-C$ & -1.51 & 0.00 & -2.07 & 0.00 & major histocompatibility complex, class I, C \\
\hline SLC46A3 & -1.51 & 0.00 & -1.97 & 0.00 & solute carrier family 46 member 3 \\
\hline NRAD1 & -1.51 & 0.00 & -1.64 & 0.00 & $\begin{array}{l}\text { non-coding RNA in the aldehyde dehydroge- } \\
\text { nase } 1 \mathrm{~A} \text { pathway }\end{array}$ \\
\hline BCAT1 & -1.52 & 0.00 & -2.60 & 0.00 & branched chain amino acid transaminase 1 \\
\hline ELF3-AS1 & -1.52 & 0.00 & -1.58 & 0.00 & ELF3 antisense RNA 1 \\
\hline$A R L 14$ & -1.52 & 0.01 & -2.08 & 0.00 & ADP ribosylation factor like GTPase 14 \\
\hline MIR4268 & -1.52 & 0.01 & -1.50 & 0.03 & microRNA 4268 \\
\hline MUC13 & -1.52 & 0.00 & -3.20 & 0.00 & mucin 13, cell surface associated \\
\hline ZNF625 & -1.52 & 0.04 & -1.66 & 0.03 & zinc finger protein 625 \\
\hline VSIR & -1.53 & 0.00 & -1.71 & 0.00 & V-set immunoregulatory receptor \\
\hline UACA & -1.53 & 0.00 & -1.53 & 0.00 & $\begin{array}{l}\text { uveal autoantigen with coiled-coil domains } \\
\text { and ankyrin repeats }\end{array}$ \\
\hline
\end{tabular}




\begin{tabular}{llllll}
\hline TSPAN18 & -1.53 & 0.00 & -3.25 & 0.00 & tetraspanin 18 \\
\hline EGFL7 & -1.53 & 0.00 & -1.57 & 0.00 & EGF like domain multiple 7 \\
\hline PHKA2-AS1 & -1.53 & 0.01 & -2.82 & 0.00 & PHKA2 antisense RNA 1 \\
\hline EPB41L1 & -1.54 & 0.00 & -1.89 & 0.00 & erythrocyte membrane protein band 4.1 like 1 \\
\hline PTPRH & -1.54 & 0.00 & -1.60 & 0.00 & protein tyrosine phosphatase receptor type H \\
\hline PDK1 & -1.55 & 0.00 & -2.79 & 0.00 & pyruvate dehydrogenase kinase 1 \\
\hline LRP1 & -1.55 & 0.00 & -1.66 & 0.00 & LDL receptor related protein 1 \\
\hline
\end{tabular}

Supplementary figure 3 Venn diagram showing the number of shared and unique differentially expressed up- and downregulated genes ( $-1.5 \geq$ Fold change $\geq 1.5$ between HT29-MTX-E12 and Caco- 2 cells cultured under static and SWMS conditions. The top 20 up- and downregulated genes shared between HT29-MTX-E12 and Caco-2 cells is given in the tables.

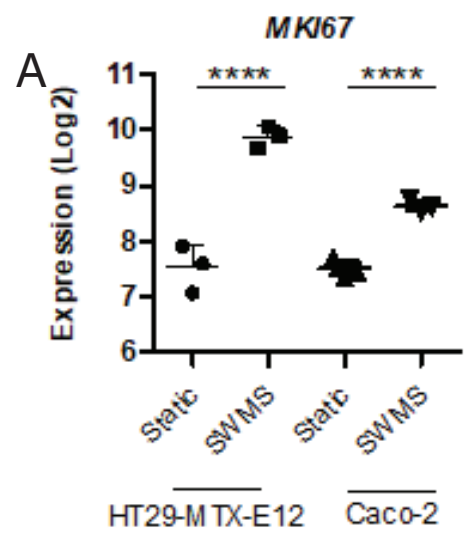

B

HT29-MTX-E12

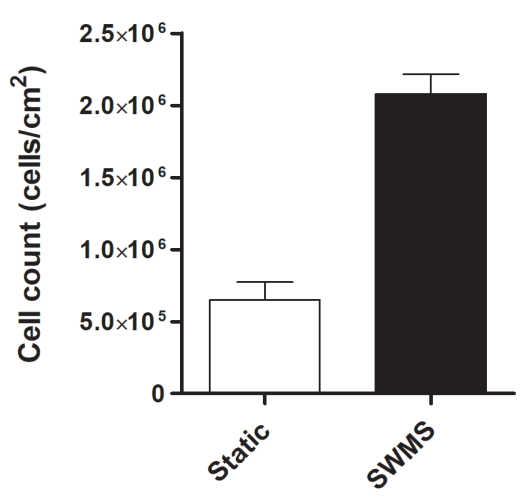

Caco-2

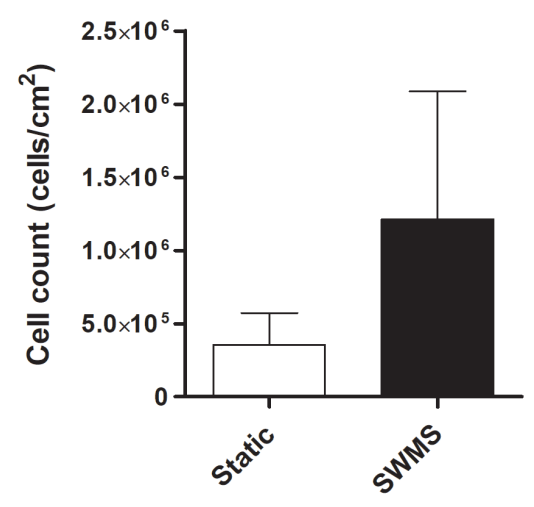


Supplementary figure 4 A) Microarray gene expression values (Log2) of MKI67 in HT29-MTX-E12 and Caco- 2 cells cultured under static and SWMS conditions. B) Cell count after $t=15$ days, expressed as cells per $\mathrm{cm}^{2}$, of HT29-MTX-E12 and Caco-2 cells cultured under static and SWMS conditions. $* * * * \mathrm{p}<0.0001$.

\section{Blot 1}

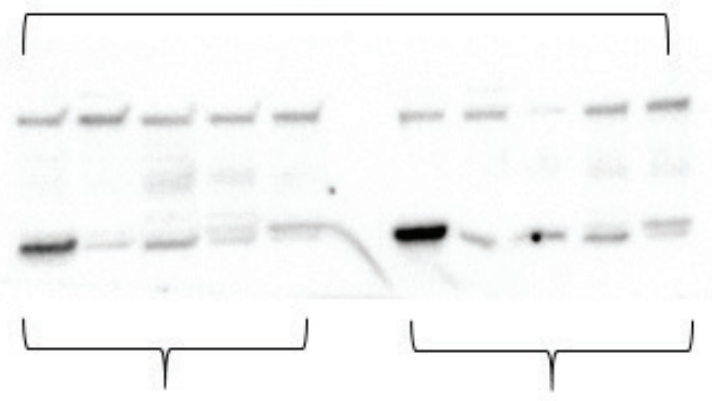

Batch 1

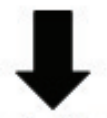

Batch 1
Batch 2

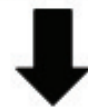

Batch 2
Blot 2

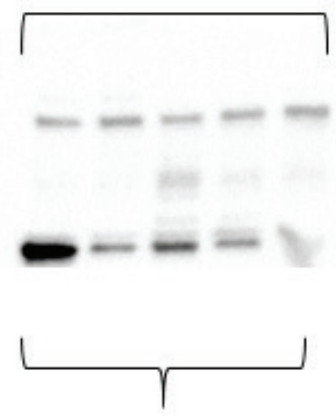

Batch 3

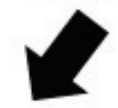

Batch 3
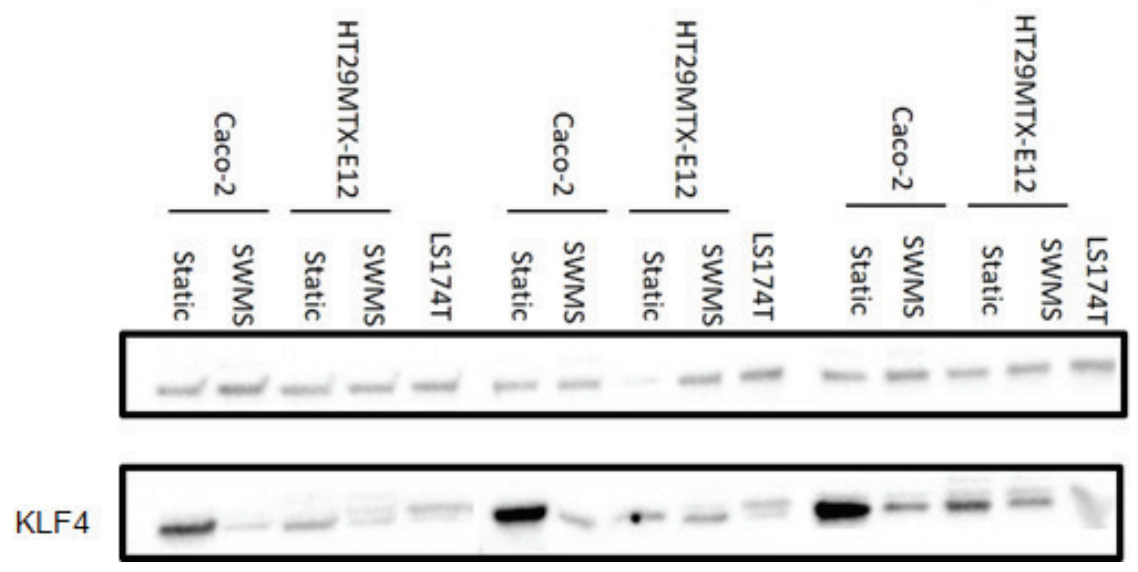

Supplementary figure 5 Western Immunoblotting results of KLF4, including all three biological replicates (batch 1, 2 and 3) in Caco-2, HT29-MTX-E12 and LS174T-cells grown under static and SWMS conditions or static only (LS174T). HSP90 was used as house-keeping protein. 
A

\section{Ratio $415 / 560 \mathrm{~nm}$}

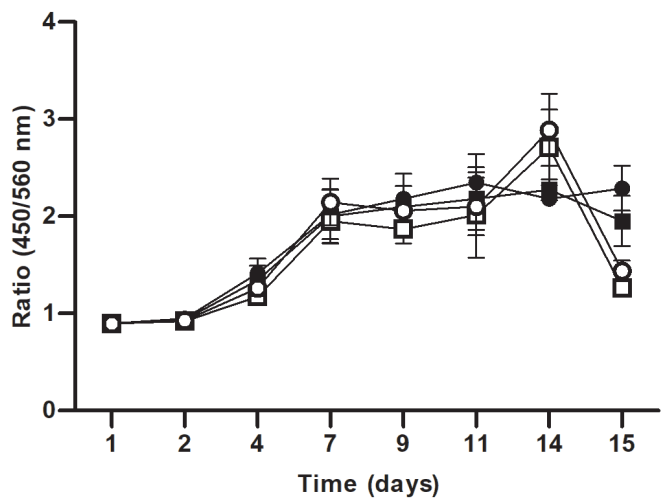

$$
\begin{aligned}
& \text { - SWMS, basolateral } \\
& - \text { - SWMS, apical } \\
& \text {-0- Static, basolateral } \\
& \text {-o- Static, apical }
\end{aligned}
$$

B

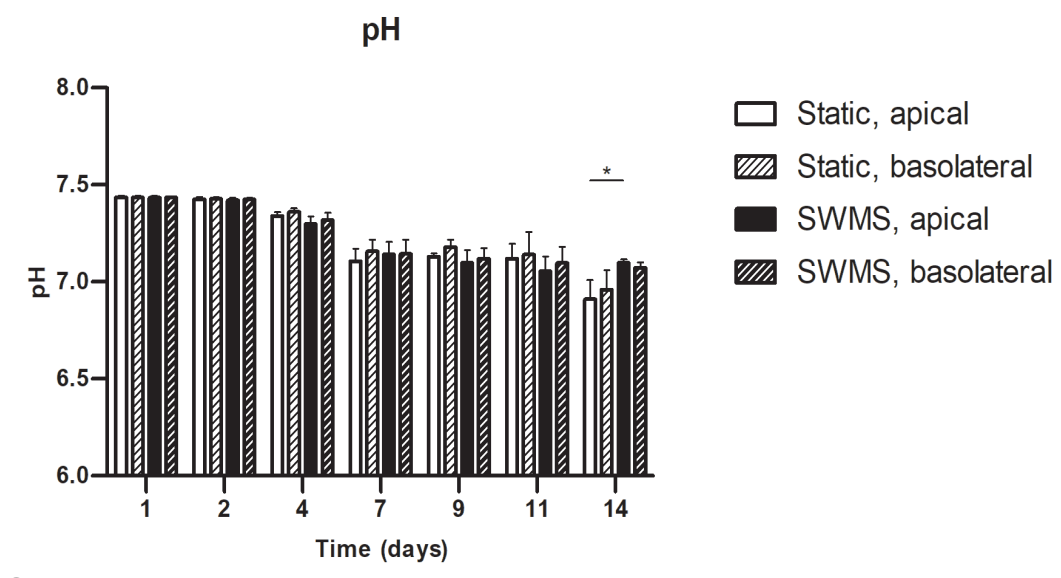

C

Phenol Red standard curve

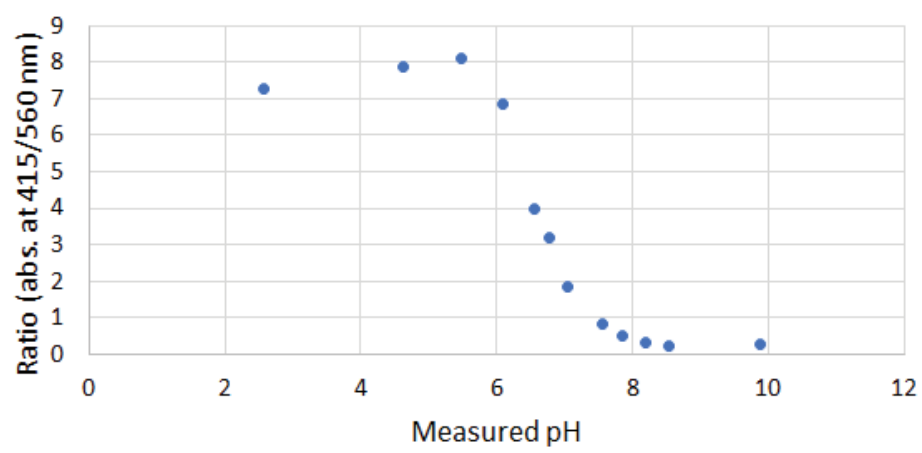


$\mathrm{D}$

\section{Trendline Linear Part}

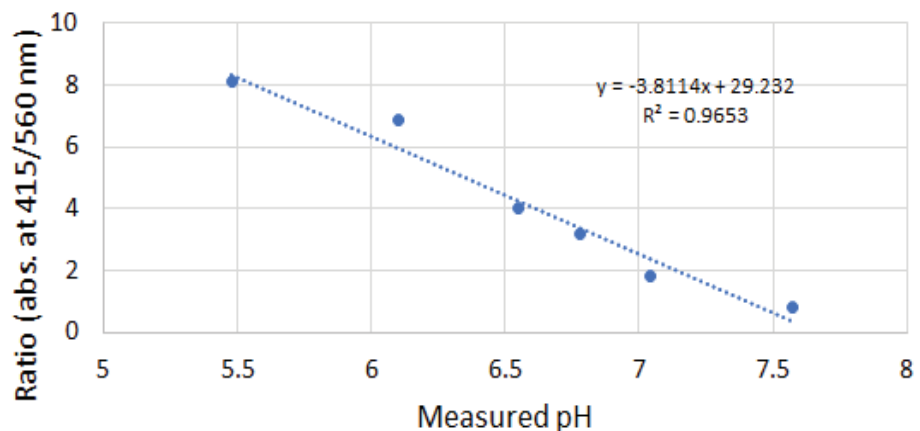

Supplementary figure 6 A) Ratio of Absorbance of cell culture medium ( 415 and $560 \mathrm{~nm}$ ) of HT29-MTX-E12 and Caco-2 cells under static and SWMS conditions measured at 5\% CO2. B) Medium pH of apical and basolateral compartments of HT29-MTX-E12 cells grown under static and SWMS conditions at $t=1-14$ days. ${ }^{*} \mathrm{p}<0.05$; ${ }^{* *} \mathrm{p}<0.01, \mathrm{n}=3 \mathrm{C}$ ) Standard curve of $\mathrm{pH}$ values and absorbance values of cell culture medium measured at $415 / 560 \mathrm{~nm}$ at $5 \% \mathrm{CO} 2$. D) The linear part the standard curve, including trendline.

A

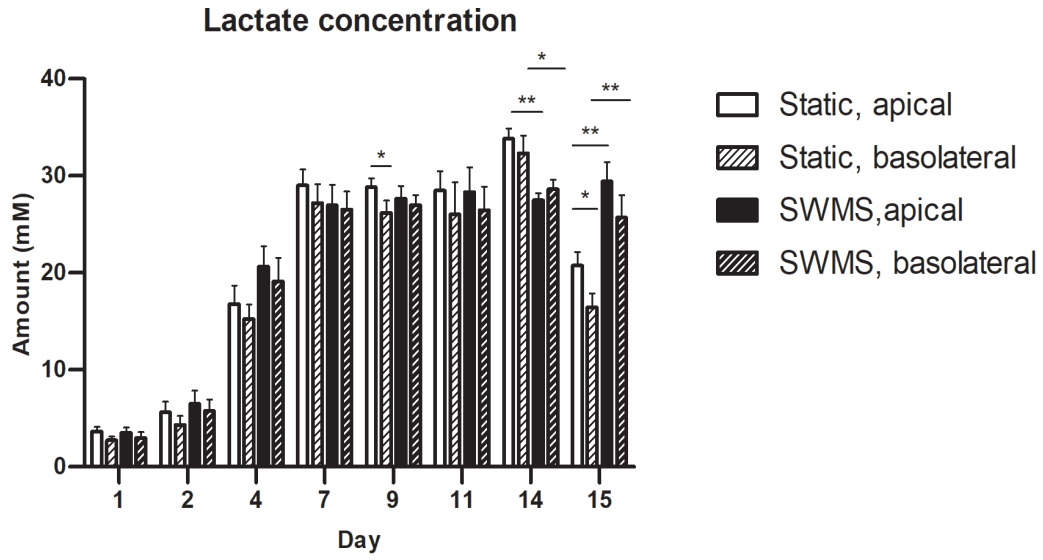


B Total lactate

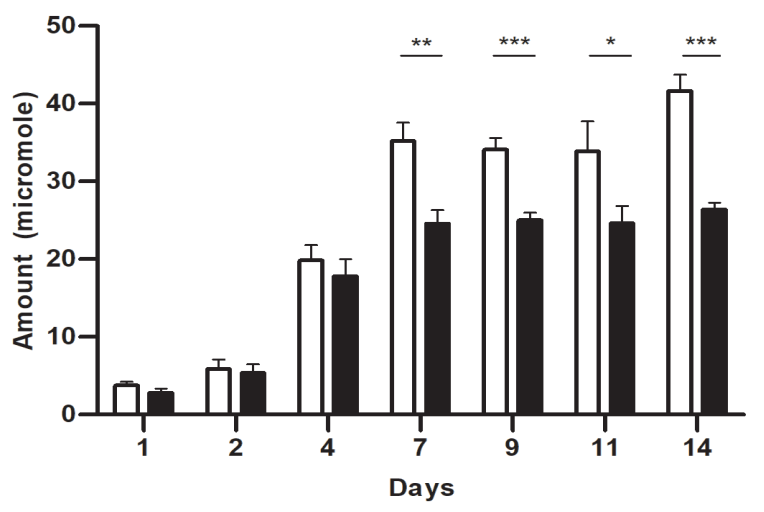

$\square$ Static

SWMS

C

\section{Glucose consumption}

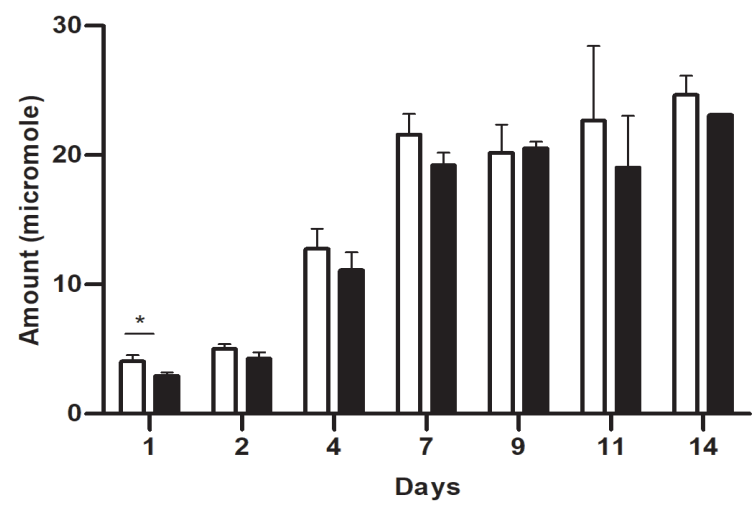

$\square$ Static

SWMS

Supplementary figure 7 A) Lactate concentration (mM) in cell culture medium of HT29-MTX-E12 cells collected during every medium refreshing moment. B) Total lactate (micromole) produced per well in medium collected from apical and basolateral compartments of HT29-MTX-E12 grown under static or SWMS conditions, at $\mathrm{t}=1-14$ days. C) Total glucose (micromole) consumed per well from medium collected from apical and basolateral compartments of HT29-MTX-E12 grown under static and SWMS conditions, at $\mathrm{t}=1-14$ days. 
Supplementary file 1 Panels of genes, including mucins, cell integrity-related genes, KLF4-related genes and genes related to ion transport in HT29-MTX-E21 and Caco-2 cells. The fold changes and $\mathrm{p}$-values between SWMS and static conditions are given.

\section{Mucins}

\begin{tabular}{|l|c|c|c|c|}
\hline & \multicolumn{2}{|c|}{ HT29MTXE12 } & \multicolumn{2}{c|}{ Caco-2 } \\
\hline SYMBOL & FC & p-value & FC & p-value \\
\hline MUC2 & 1.42 & $1.97 \mathrm{E}-02$ & 1.05 & $7.57 \mathrm{E}-01$ \\
\hline MUC12 & 1.29 & $1.07 \mathrm{E}-01$ & -1.58 & $1.43 \mathrm{E}-02$ \\
\hline MUC6 & 1.08 & $5.29 \mathrm{E}-01$ & 1.01 & $9.59 \mathrm{E}-01$ \\
\hline MUC15 & 1.05 & $7.13 \mathrm{E}-01$ & -1.09 & $5.81 \mathrm{E}-01$ \\
\hline MUC16 & 1.04 & $7.56 \mathrm{E}-01$ & -1.05 & $7.27 \mathrm{E}-01$ \\
\hline MUCL1 & 1.03 & $8.70 \mathrm{E}-01$ & -1.21 & $2.65 \mathrm{E}-01$ \\
\hline MUC7 & -1.01 & $9.38 \mathrm{E}-01$ & 1.18 & $3.42 \mathrm{E}-01$ \\
\hline MUC12-AS1 & -1.06 & $6.90 \mathrm{E}-01$ & -1.39 & $7.11 \mathrm{E}-02$ \\
\hline MUC19 & -1.08 & $4.80 \mathrm{E}-01$ & 1.02 & $8.91 \mathrm{E}-01$ \\
\hline MUC22 & -1.13 & $2.79 \mathrm{E}-01$ & 1.09 & $4.92 \mathrm{E}-01$ \\
\hline MUC1 & -1.21 & $1.21 \mathrm{E}-01$ & -1.01 & $9.19 \mathrm{E}-01$ \\
\hline MUC4 & -1.25 & $1.19 \mathrm{E}-01$ & -1.02 & $8.95 \mathrm{E}-01$ \\
\hline MUC13 & -1.52 & $1.82 \mathrm{E}-06$ & -3.20 & $5.09 \mathrm{E}-12$ \\
\hline MUC5AC & -1.56 & $6.61 \mathrm{E}-05$ & 1.21 & $5.75 \mathrm{E}-02$ \\
\hline MUC17 & -1.62 & $1.94 \mathrm{E}-03$ & -1.59 & $6.09 \mathrm{E}-03$ \\
\hline MUC5B-AS1 & -1.74 & $5.95 \mathrm{E}-04$ & 1.21 & $2.07 \mathrm{E}-01$ \\
\hline MUC21 & -1.75 & $2.04 \mathrm{E}-04$ & -1.09 & $5.46 \mathrm{E}-01$ \\
\hline MUC3A & -1.79 & $6.66 \mathrm{E}-05$ & -2.34 & $5.14 \mathrm{E}-06$ \\
\hline MUC5B & -1.84 & $1.75 \mathrm{E}-05$ & -1.03 & $8.03 \mathrm{E}-01$ \\
\hline MUCL3 & -2.24 & $1.41 \mathrm{E}-06$ & -1.32 & $3.92 \mathrm{E}-02$ \\
\hline MUC20 & -2.32 & $3.78 \mathrm{E}-10$ & -1.57 & $1.11 \mathrm{E}-05$ \\
\hline
\end{tabular}

\section{Epithelial barrier integrity}

\begin{tabular}{|l|c|c|c|c|l|}
\hline & \multicolumn{2}{|c|}{ HT29MTXE12 } & \multicolumn{2}{c|}{ Caco-2 } & \\
\hline SYMBOL & FC & p-value & FC & p-value & Gene name \\
\hline CTNNAL1 & 3.33 & $3.09 E-10$ & 1.21 & $8.26 \mathrm{E}-02$ & catenin alpha like 1 \\
\hline HEG1 & 2.67 & $3.08 \mathrm{E}-07$ & -1.61 & $2.76 \mathrm{E}-03$ & heart development protein with EGF like domains 1 \\
\hline ECT2 & 2.26 & $1.86 \mathrm{E}-10$ & 1.48 & $2.68 \mathrm{E}-05$ & epithelial cell transforming 2 \\
\hline SNA11 & 1.47 & $8.37 \mathrm{E}-03$ & 1.52 & $1.10 \mathrm{E}-02$ & snail family transcriptional repressor 1 \\
\hline CLDN6 & 1.45 & $3.90 \mathrm{E}-02$ & -1.09 & $6.40 \mathrm{E}-01$ & claudin 6 \\
\hline CDH7 & 1.42 & $7.75 \mathrm{E}-03$ & -1.02 & $8.64 \mathrm{E}-01$ & cadherin 7 \\
\hline CDH24 & 1.40 & $3.31 \mathrm{E}-03$ & 1.13 & $2.75 \mathrm{E}-01$ & cadherin 24 \\
\hline TBCD & 1.36 & $2.28 \mathrm{E}-05$ & 1.14 & $4.90 \mathrm{E}-02$ & tubulin folding cofactor D \\
\hline FLCN & 1.30 & $7.34 \mathrm{E}-03$ & -1.25 & $3.44 \mathrm{E}-02$ & folliculin \\
\hline PARD3 & 1.29 & $5.57 \mathrm{E}-04$ & 1.13 & $8.19 \mathrm{E}-02$ & par-3 family cell polarity regulator \\
\hline RAMP2 & 1.29 & $9.25 \mathrm{E}-02$ & 2.34 & $6.32 \mathrm{E}-05$ & receptor activity modifying protein 2 \\
\hline
\end{tabular}




\begin{tabular}{|c|c|c|c|c|c|}
\hline PKP2 & 1.24 & 4.97E-04 & -1.01 & 7.99E-01 & plakophilin 2 \\
\hline TGFB1 & 1.24 & $2.64 \mathrm{E}-02$ & -2.01 & $1.98 \mathrm{E}-06$ & transforming growth factor beta 1 \\
\hline CLDN20 & 1.22 & $2.17 \mathrm{E}-01$ & 1.50 & $3.16 \mathrm{E}-02$ & claudin 20 \\
\hline STRN & 1.20 & $7.38 \mathrm{E}-03$ & -1.07 & 3.12E-01 & striatin \\
\hline CLDN22 & 1.17 & $2.00 \mathrm{E}-01$ & -1.10 & $5.01 E-01$ & claudin 22 \\
\hline MPP7 & 1.17 & $1.59 \mathrm{E}-01$ & -1.57 & $1.84 \mathrm{E}-03$ & membrane palmitoylated protein 7 \\
\hline AMOT & 1.16 & $1.70 \mathrm{E}-01$ & 1.07 & $5.92 \mathrm{E}-01$ & angiomotin \\
\hline PRKCl & 1.16 & $1.06 \mathrm{E}-02$ & -1.12 & $6.24 \mathrm{E}-02$ & protein kinase $\mathrm{C}$ iota \\
\hline PARD6G & 1.16 & 3.03E-01 & -1.02 & 9.19E-01 & par-6 family cell polarity regulator gamma \\
\hline $\mathrm{CDH} 18$ & 1.15 & $2.50 \mathrm{E}-01$ & 1.03 & $8.18 \mathrm{E}-01$ & cadherin 18 \\
\hline TGFB3 & 1.15 & $2.95 \mathrm{E}-01$ & -1.45 & $1.95 \mathrm{E}-02$ & transforming growth factor beta 3 \\
\hline ACTG1 & 1.14 & $1.35 \mathrm{E}-03$ & 1.00 & $9.02 \mathrm{E}-01$ & actin gamma 1 \\
\hline $\mathrm{CDH} 13$ & 1.13 & $2.80 \mathrm{E}-01$ & -1.17 & $2.39 \mathrm{E}-01$ & cadherin 13 \\
\hline KRT5 & 1.13 & 3.74E-01 & 1.08 & $6.09 \mathrm{E}-01$ & keratin 5 \\
\hline CLDN10 & 1.13 & $2.85 \mathrm{E}-01$ & 1.01 & $9.41 \mathrm{E}-01$ & claudin 10 \\
\hline FBF1 & 1.13 & 2.87E-01 & 1.16 & $2.48 \mathrm{E}-01$ & Fas binding factor 1 \\
\hline NECTIN1 & 1.11 & 3.27E-01 & -1.38 & $1.41 \mathrm{E}-02$ & nectin cell adhesion molecule 1 \\
\hline RHOA & 1.11 & $6.46 \mathrm{E}-03$ & 1.03 & $4.97 \mathrm{E}-01$ & ras homolog family member $\mathrm{A}$ \\
\hline PARD6A & 1.11 & $3.54 \mathrm{E}-01$ & -1.20 & $1.56 \mathrm{E}-01$ & par-6 family cell polarity regulator alpha \\
\hline CLDN16 & 1.10 & 4.07E-01 & 1.48 & $8.50 \mathrm{E}-03$ & claudin 16 \\
\hline NLGN2 & 1.09 & 4.22E-01 & -1.23 & 1.03E-01 & neuroligin 2 \\
\hline KRT14 & 1.09 & $5.24 \mathrm{E}-01$ & 1.08 & $6.10 \mathrm{E}-01$ & keratin 14 \\
\hline $\mathrm{CDH} 15$ & 1.09 & $4.81 \mathrm{E}-01$ & -1.16 & $2.82 \mathrm{E}-01$ & cadherin 15 \\
\hline CCM2 & 1.08 & 4.97E-01 & 1.14 & $2.91 E-01$ & CCM2 scaffold protein \\
\hline NECTIN3 & 1.07 & $3.78 \mathrm{E}-01$ & 1.12 & $2.32 \mathrm{E}-01$ & nectin cell adhesion molecule 3 \\
\hline NEDD4L & 1.07 & 2.46E-01 & -1.06 & $3.91 \mathrm{E}-01$ & NEDD4 like E3 ubiquitin protein ligase \\
\hline CLDN8 & 1.07 & $6.03 \mathrm{E}-01$ & 1.05 & $7.26 \mathrm{E}-01$ & claudin 8 \\
\hline SRF & 1.06 & $3.20 \mathrm{E}-01$ & -1.05 & 4.58E-01 & serum response factor \\
\hline TLN2 & 1.06 & $5.58 \mathrm{E}-01$ & -1.11 & 3.29E-01 & talin 2 \\
\hline GJA4 & 1.06 & $6.83 \mathrm{E}-01$ & -1.23 & $1.96 \mathrm{E}-01$ & gap junction protein alpha 4 \\
\hline CSF1R & 1.04 & 7.41E-01 & -1.09 & 4.94E-01 & colony stimulating factor 1 receptor \\
\hline GJA1 & 1.04 & 7.93E-01 & -1.29 & $1.28 \mathrm{E}-01$ & gap junction protein alpha 1 \\
\hline SNAI2 & 1.04 & 8.09E-01 & -1.15 & 3.90E-01 & snail family transcriptional repressor 2 \\
\hline PKP4 & 1.03 & $5.08 \mathrm{E}-01$ & 1.01 & $8.22 \mathrm{E}-01$ & plakophilin 4 \\
\hline APC & 1.02 & 7.99E-01 & -1.01 & $9.63 \mathrm{E}-01$ & APC regulator of WNT signaling pathway \\
\hline RAB13 & 1.02 & $8.10 \mathrm{E}-01$ & 1.09 & $3.30 \mathrm{E}-01$ & RAB13, member RAS oncogene family \\
\hline PVR & 1.02 & $7.45 \mathrm{E}-01$ & -1.52 & $1.01 E-06$ & PVR cell adhesion molecule \\
\hline PRKACA & 1.01 & $8.65 \mathrm{E}-01$ & -1.14 & $9.30 \mathrm{E}-02$ & protein kinase cAMP-activated catalytic subunit alpha \\
\hline $\mathrm{CDH} 5$ & 1.01 & $9.33 \mathrm{E}-01$ & -1.14 & 3.40E-01 & cadherin 5 \\
\hline TJAP1 & 1.01 & $9.28 \mathrm{E}-01$ & 1.22 & $1.24 \mathrm{E}-01$ & tight junction associated protein 1 \\
\hline $\mathrm{CDH} 2$ & 1.01 & 9.49E-01 & -1.06 & $6.56 \mathrm{E}-01$ & cadherin 2 \\
\hline RAB8B & 1.01 & $9.45 \mathrm{E}-01$ & -1.00 & $9.79 \mathrm{E}-01$ & RAB8B, member RAS oncogene family \\
\hline $\mathrm{CDH} 6$ & 1.00 & $9.82 \mathrm{E}-01$ & -1.86 & $1.78 \mathrm{E}-04$ & cadherin 6 \\
\hline DLG1 & -1.00 & $9.26 \mathrm{E}-01$ & 1.08 & $1.80 \mathrm{E}-01$ & discs large MAGUK scaffold protein 1 \\
\hline
\end{tabular}




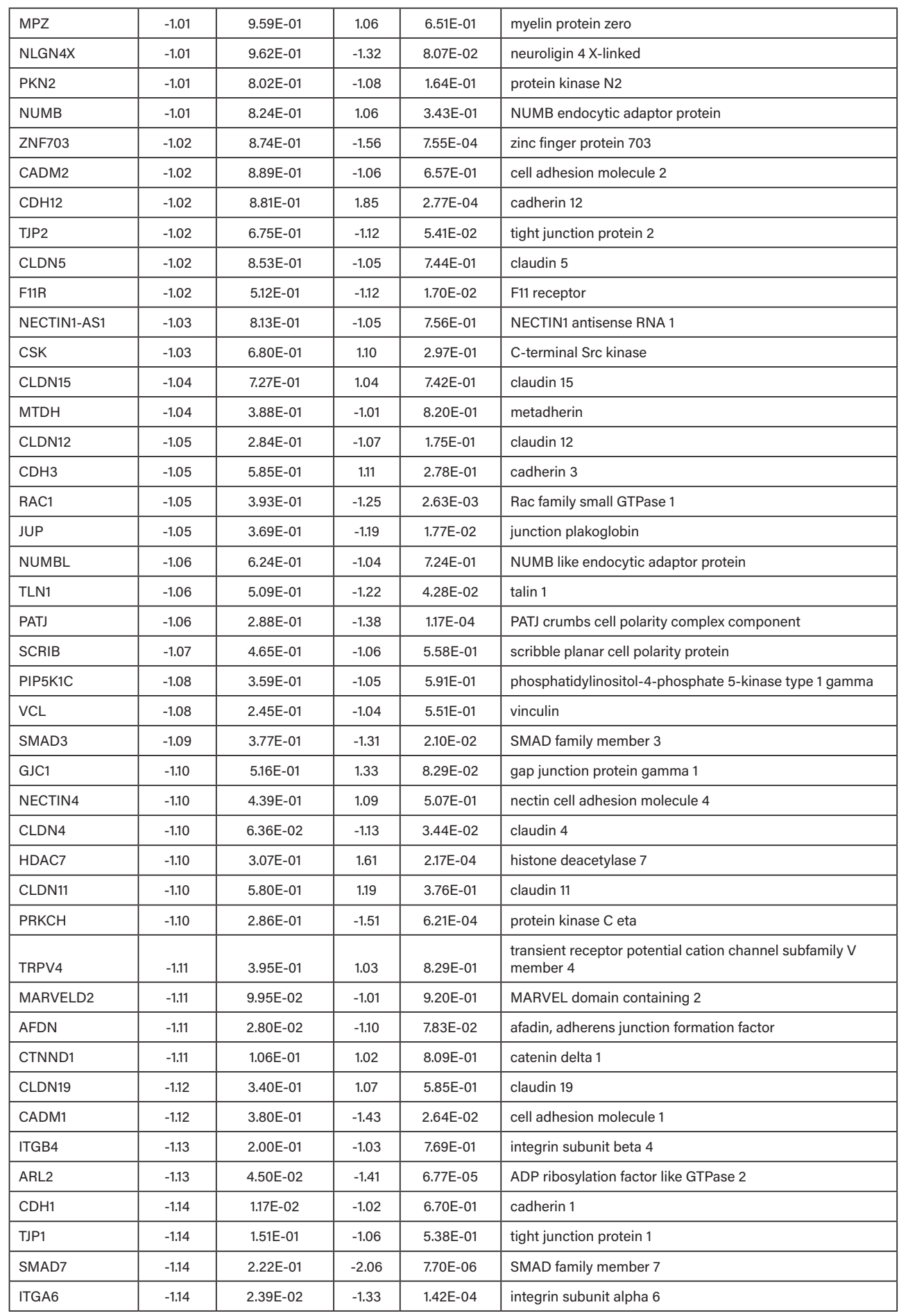




\begin{tabular}{|c|c|c|c|c|c|}
\hline CLDN3 & -1.14 & $1.49 \mathrm{E}-02$ & -1.02 & 7.02E-01 & claudin 3 \\
\hline PERP & -1.14 & $5.16 \mathrm{E}-02$ & -1.70 & $9.90 \mathrm{E}-07$ & p53 apoptosis effector related to PMP22 \\
\hline CLDN2 & -1.14 & $2.45 \mathrm{E}-01$ & -1.25 & $8.90 \mathrm{E}-02$ & claudin 2 \\
\hline NF2 & -1.14 & $3.21 \mathrm{E}-02$ & 1.18 & $2.04 \mathrm{E}-02$ & neurofibromin 2 \\
\hline CLDN14 & -1.14 & $2.61 \mathrm{E}-01$ & 1.05 & 7.20E-01 & claudin 14 \\
\hline LIM2 & -1.14 & $2.30 \mathrm{E}-01$ & 1.06 & $6.42 \mathrm{E}-01$ & lens intrinsic membrane protein 2 \\
\hline CTNNB1 & -1.15 & $5.58 \mathrm{E}-03$ & -1.04 & $4.62 \mathrm{E}-01$ & catenin beta 1 \\
\hline CDC42 & -1.15 & $9.20 \mathrm{E}-03$ & 1.09 & $1.19 \mathrm{E}-01$ & cell division cycle 42 \\
\hline LIMS2 & -1.16 & 3.23E-01 & 1.31 & $1.20 \mathrm{E}-01$ & LIM zinc finger domain containing 2 \\
\hline CD151 & -1.16 & 2.83E-03 & -1.32 & $2.86 \mathrm{E}-05$ & CD151 molecule (Raph blood group) \\
\hline MARVELD3 & -1.16 & $1.51 \mathrm{E}-02$ & -1.20 & $9.86 \mathrm{E}-03$ & MARVEL domain containing 3 \\
\hline $\mathrm{CDH} 4$ & -1.17 & $2.38 \mathrm{E}-01$ & -2.04 & 1.14E-04 & cadherin 4 \\
\hline OCLN & -1.17 & $2.69 \mathrm{E}-02$ & -1.09 & $2.32 \mathrm{E}-01$ & occludin \\
\hline NECTIN3-AS1 & -1.17 & $3.70 \mathrm{E}-01$ & 1.25 & $2.63 \mathrm{E}-01$ & NECTIN3 antisense RNA 1 \\
\hline DST & -1.17 & $5.97 \mathrm{E}-03$ & -1.64 & $1.63 \mathrm{E}-07$ & dystonin \\
\hline SHROOM2 & -1.19 & $2.13 \mathrm{E}-01$ & 1.01 & $9.50 \mathrm{E}-01$ & shroom family member 2 \\
\hline CTNNA1 & -1.20 & $3.68 \mathrm{E}-03$ & -1.11 & 1.05E-01 & catenin alpha 1 \\
\hline CADM3 & -1.20 & 1.16E-01 & 1.08 & $5.66 \mathrm{E}-01$ & cell adhesion molecule 3 \\
\hline $\mathrm{CDH} 9$ & -1.22 & $9.04 \mathrm{E}-02$ & 1.14 & $2.92 \mathrm{E}-01$ & cadherin 9 \\
\hline CRB3 & -1.22 & $7.40 \mathrm{E}-03$ & -1.59 & 9.87E-06 & crumbs cell polarity complex component 3 \\
\hline DSG1 & -1.23 & 7.83E-02 & 1.16 & $2.49 \mathrm{E}-01$ & desmoglein 1 \\
\hline TJP3 & -1.24 & $2.81 \mathrm{E}-02$ & -1.16 & 1.48E-01 & tight junction protein 3 \\
\hline CLDN9 & -1.24 & $1.50 \mathrm{E}-01$ & -1.13 & 4.77E-01 & claudin 9 \\
\hline LAMA3 & -1.25 & $9.50 \mathrm{E}-03$ & -3.36 & $1.02 \mathrm{E}-10$ & laminin subunit alpha 3 \\
\hline $\mathrm{CDH} 10$ & -1.27 & $6.56 \mathrm{E}-02$ & 1.12 & 4.25E-01 & cadherin 10 \\
\hline MPP5 & -1.28 & $9.45 \mathrm{E}-04$ & -1.64 & $1.54 \mathrm{E}-06$ & membrane palmitoylated protein 5 \\
\hline DSP & -1.28 & $1.25 \mathrm{E}-04$ & -1.56 & 4.09E-07 & desmoplakin \\
\hline NECTIN2 & -1.28 & $7.50 \mathrm{E}-04$ & 1.10 & 1.71E-01 & nectin cell adhesion molecule 2 \\
\hline CLDN7 & -1.28 & 7.83E-05 & -1.26 & 4.87E-04 & claudin 7 \\
\hline $\mathrm{CDH} 11$ & -1.28 & $7.76 \mathrm{E}-02$ & 1.06 & $6.83 \mathrm{E}-01$ & cadherin 11 \\
\hline PARD6B & -1.29 & $3.25 \mathrm{E}-04$ & -1.26 & $2.54 \mathrm{E}-03$ & par-6 family cell polarity regulator beta \\
\hline WNT11 & -1.30 & $1.97 \mathrm{E}-02$ & -2.77 & $9.18 \mathrm{E}-08$ & Wnt family member 11 \\
\hline ITGB1 & -1.31 & $1.03 \mathrm{E}-03$ & -1.33 & $1.71 \mathrm{E}-03$ & integrin subunit beta 1 \\
\hline PLEC & -1.33 & 7.60E-04 & -1.39 & $6.02 \mathrm{E}-04$ & plectin \\
\hline RHOC & -1.35 & $5.07 \mathrm{E}-04$ & -1.72 & $3.18 \mathrm{E}-06$ & ras homolog family member $\mathrm{C}$ \\
\hline CLDN17 & -1.36 & $2.29 \mathrm{E}-02$ & -1.01 & 9.17E-01 & claudin 17 \\
\hline PRKCA & -1.37 & $6.82 \mathrm{E}-05$ & 2.05 & 8.35E-09 & protein kinase $\mathrm{C}$ alpha \\
\hline $\mathrm{CDH} 8$ & -1.39 & 1.27E-02 & -1.09 & $5.41 \mathrm{E}-01$ & cadherin 8 \\
\hline CXADR & -1.41 & $1.51 \mathrm{E}-03$ & -1.55 & $6.32 \mathrm{E}-04$ & CXADR Ig-like cell adhesion molecule \\
\hline CLDN1 & -1.44 & $1.12 \mathrm{E}-05$ & 1.37 & $2.25 \mathrm{E}-04$ & claudin 1 \\
\hline MYO1C & -1.49 & $1.26 \mathrm{E}-06$ & -1.43 & $2.02 \mathrm{E}-05$ & myosin IC \\
\hline LAMC2 & -1.59 & $1.05 \mathrm{E}-05$ & -1.67 & $1.31 \mathrm{E}-05$ & laminin subunit gamma 2 \\
\hline CLDN23 & -1.62 & 1.19E-04 & -1.37 & 1.11E-02 & claudin 23 \\
\hline FRMPD2 & -1.74 & $9.47 \mathrm{E}-04$ & 1.02 & $8.80 \mathrm{E}-01$ & FERM and PDZ domain containing 2 \\
\hline
\end{tabular}




\begin{tabular}{|l|c|c|c|c|l|}
\hline LAMB3 & -1.80 & $1.87 \mathrm{E}-07$ & -1.29 & $4.28 \mathrm{E}-03$ & laminin subunit beta 3 \\
\hline CLDN18 & -1.88 & $1.31 \mathrm{E}-04$ & 1.49 & $1.39 \mathrm{E}-02$ & claudin 18 \\
\hline COL17A1 & -1.93 & $2.65 \mathrm{E}-09$ & -2.21 & $9.79 \mathrm{E}-10$ & collagen type XVII alpha 1 chain \\
\hline TGFB2 & -3.28 & $3.64 \mathrm{E}-08$ & -1.14 & $3.53 \mathrm{E}-01$ & transforming growth factor beta 2 \\
\hline
\end{tabular}

\section{KLF4-related}

\begin{tabular}{|c|c|c|c|c|c|}
\hline \multirow[b]{2}{*}{ SYMBOL } & \multicolumn{2}{|c|}{ HT29MTXE12 } & \multicolumn{2}{|c|}{ Caco-2 } & \multirow[b]{2}{*}{ Gene name } \\
\hline & FC & p-value & FC & p-value & \\
\hline CCNB1 & 6.59 & 1.07E-14 & 1.75 & $5.46 \mathrm{E}-06$ & neurogenin 3 \\
\hline NEUROG3 & 2.07 & $3.41 \mathrm{E}-04$ & 2.41 & 1.74E-04 & ornithine decarboxylase 1 \\
\hline CDK2 & 2.06 & $1.04 \mathrm{E}-08$ & 1.41 & $4.10 \mathrm{E}-04$ & atonal bHLH transcription factor 1 \\
\hline CCNE2 & 2.03 & $1.42 \mathrm{E}-04$ & 1.30 & $1.31 \mathrm{E}-01$ & notch receptor 1 \\
\hline ODC1 & 1.80 & $2.11 \mathrm{E}-08$ & 1.38 & 1.73E-04 & tumor protein p53 \\
\hline ATOH1 & 1.64 & $5.98 \mathrm{E}-03$ & 2.09 & $7.18 \mathrm{E}-04$ & TEA domain transcription factor 4 \\
\hline NOS3 & 1.63 & $1.00 \mathrm{E}-03$ & 1.00 & $9.81 \mathrm{E}-01$ & kringle containing transmembrane protein 1 \\
\hline CCNE1 & 1.63 & 4.84E-05 & 1.30 & $2.00 \mathrm{E}-02$ & protein phosphatase 2 regulatory subunit B'delta \\
\hline $\mathrm{NOTCH} 1$ & 1.61 & $1.72 \mathrm{E}-04$ & 1.11 & 3.63E-01 & MYC proto-oncogene, bHLH transcription factor \\
\hline TP53 & 1.39 & $1.71 \mathrm{E}-03$ & 1.18 & $1.22 \mathrm{E}-01$ & frizzled class receptor 1 \\
\hline TP53 & 1.39 & $1.71 \mathrm{E}-03$ & 1.18 & $1.22 \mathrm{E}-01$ & tafazzin \\
\hline TEAD4 & 1.39 & $2.05 \mathrm{E}-03$ & -1.35 & $9.47 \mathrm{E}-03$ & caudal type homeobox 2 \\
\hline KRT4 & 1.36 & 4.16E-02 & 1.07 & $6.70 \mathrm{E}-01$ & cellular communication network factor 4 \\
\hline HSP90AA1 & 1.33 & $1.64 \mathrm{E}-03$ & 1.13 & $1.68 \mathrm{E}-01$ & cyclin dependent kinase inhibitor $1 \mathrm{~A}$ \\
\hline HSP90AB4P & 1.32 & $1.92 \mathrm{E}-01$ & 1.03 & $9.01 \mathrm{E}-01$ & Kruppel like factor 6 \\
\hline KREMEN1 & 1.30 & $7.90 \mathrm{E}-03$ & -1.31 & $1.42 \mathrm{E}-02$ & SLC9A3 regulator 1 \\
\hline HDC & 1.27 & 9.07E-02 & -1.07 & $6.72 \mathrm{E}-01$ & Kruppel like factor 5 \\
\hline PPP2R5D & 1.24 & $5.96 \mathrm{E}-03$ & -1.46 & $1.50 \mathrm{E}-04$ & FRAT regulator of WNT signaling pathway 1 \\
\hline MYC & 1.24 & $1.95 \mathrm{E}-02$ & 1.45 & $1.02 \mathrm{E}-03$ & Wnt family member 16 \\
\hline TGFB1 & 1.24 & $2.64 \mathrm{E}-02$ & -2.01 & $1.98 \mathrm{E}-06$ & cytochrome P450 family 1 subfamily A member 1 \\
\hline HSP9oB1 & 1.20 & $1.53 \mathrm{E}-05$ & 1.03 & $3.58 \mathrm{E}-01$ & bone morphogenetic protein 4 \\
\hline HSP90AB1 & 1.19 & $9.32 \mathrm{E}-04$ & 1.10 & 7.10E-02 & ephrin B3 \\
\hline FZD1 & 1.16 & $1.69 \mathrm{E}-01$ & 1.02 & 8.75E-01 & Yes associated protein 1 \\
\hline CDKN1C & 1.13 & 3.69E-01 & -1.54 & $9.41 \mathrm{E}-03$ & Sp1 transcription factor \\
\hline CEBPB & 1.13 & $2.58 \mathrm{E}-01$ & -1.59 & 7.95E-04 & catenin beta 1 \\
\hline $\mathrm{TAZ}$ & 1.11 & 3.17E-01 & -1.10 & 4.17E-01 & LDL receptor related protein 5 \\
\hline ATG7 & 1.10 & $2.86 \mathrm{E}-01$ & 1.15 & $1.59 \mathrm{E}-01$ & carbonic anhydrase 1 \\
\hline IL10 & 1.10 & 4.55E-01 & -1.13 & 3.72E-01 & alkaline phosphatase, intestinal \\
\hline CLMP & 1.08 & $5.28 \mathrm{E}-01$ & -1.29 & $8.27 \mathrm{E}-02$ & ras homolog family member $U$ \\
\hline ESR1 & 1.08 & 4.91E-01 & 1.07 & $6.05 \mathrm{E}-01$ & peroxisome proliferator activated receptor gamma \\
\hline CDKN1B & 1.06 & $2.61 \mathrm{E}-01$ & -1.16 & $2.06 \mathrm{E}-02$ & ephrin B1 \\
\hline GHRL & 1.05 & 6.17E-01 & -1.09 & 4.92E-01 & cyclin D1 \\
\hline APOE & 1.05 & $6.48 \mathrm{E}-01$ & 1.11 & 4.06E-01 & GATA binding protein 4 \\
\hline SLURP1 & 1.05 & $7.46 \mathrm{E}-01$ & -1.30 & $9.97 \mathrm{E}-02$ & Kruppel like factor 4 \\
\hline CDX2 & 1.04 & $6.20 \mathrm{E}-01$ & -1.53 & 4.09E-04 & ephrin B2 \\
\hline
\end{tabular}




\begin{tabular}{|c|c|c|c|c|c|}
\hline PDGFRB & 1.04 & 7.77E-01 & 2.25 & $6.00 \mathrm{E}-05$ & platelet derived growth factor receptor beta \\
\hline $\mathrm{CCN} 4$ & 1.03 & $8.10 \mathrm{E}-01$ & -1.55 & $6.75 \mathrm{E}-03$ & cellular communication network factor 4 \\
\hline LDLR & 1.03 & $6.62 \mathrm{E}-01$ & 1.02 & 7.97E-01 & low density lipoprotein receptor \\
\hline CDKN1A & 1.02 & $8.25 \mathrm{E}-01$ & -1.58 & $5.18 \mathrm{E}-04$ & cyclin dependent kinase inhibitor $1 \mathrm{~A}$ \\
\hline KRT13 & 1.01 & $9.61 \mathrm{E}-01$ & 1.08 & $6.74 \mathrm{E}-01$ & keratin 13 \\
\hline LAMA1 & 1.01 & $9.53 \mathrm{E}-01$ & -1.48 & 7.57E-03 & laminin subunit alpha 1 \\
\hline HSPA8 & -1.01 & 7.94E-01 & 1.01 & 7.10E-01 & heat shock protein family $A(H s p 70)$ member 8 \\
\hline KLF6 & -1.01 & $9.42 \mathrm{E}-01$ & -1.61 & $3.08 \mathrm{E}-03$ & Kruppel like factor 6 \\
\hline HSP90AB2P & -1.02 & $9.49 \mathrm{E}-01$ & -1.17 & $5.72 \mathrm{E}-01$ & $\begin{array}{l}\text { heat shock protein } 90 \text { alpha family class B member } 2 \text {, } \\
\text { pseudogene }\end{array}$ \\
\hline SLC9A3R1 & -1.02 & 7.07E-01 & -1.27 & $2.45 \mathrm{E}-03$ & SLC9A3 regulator 1 \\
\hline CLDN5 & -1.02 & 8.53E-01 & -1.05 & 7.44E-01 & claudin 5 \\
\hline MAP1LC3A & -1.02 & $8.62 \mathrm{E}-01$ & -2.05 & $2.27 \mathrm{E}-04$ & microtubule associated protein 1 light chain 3 alpha \\
\hline KLF5 & -1.04 & $3.62 \mathrm{E}-01$ & -1.05 & $2.95 \mathrm{E}-01$ & Kruppel like factor 5 \\
\hline FRAT1 & -1.04 & 7.45E-01 & 1.43 & $1.88 \mathrm{E}-02$ & FRAT regulator of WNT signaling pathway 1 \\
\hline PAX6 & -1.05 & 7.11E-01 & -1.13 & 3.76E-01 & paired box 6 \\
\hline WNT16 & -1.07 & $6.46 \mathrm{E}-01$ & -1.08 & $6.31 \mathrm{E}-01$ & Wnt family member 16 \\
\hline CYP1A1 & -1.08 & $5.88 \mathrm{E}-01$ & -1.96 & 4.64E-04 & cytochrome P450 family 1 subfamily A member 1 \\
\hline BMP4 & -1.08 & $1.71 \mathrm{E}-01$ & -2.97 & $1.83 \mathrm{E}-12$ & bone morphogenetic protein 4 \\
\hline ELK3 & -1.08 & 4.69E-01 & -1.01 & $9.66 \mathrm{E}-01$ & ETS transcription factor ELK3 \\
\hline EFNB3 & -1.09 & $5.59 \mathrm{E}-01$ & 1.09 & $6.10 \mathrm{E}-01$ & ephrin B3 \\
\hline MFN2 & -1.09 & $1.70 \mathrm{E}-01$ & -1.33 & $9.33 \mathrm{E}-04$ & mitofusin 2 \\
\hline HSP90AB3P & -1.10 & $5.10 \mathrm{E}-01$ & 1.12 & $4.68 \mathrm{E}-01$ & $\begin{array}{l}\text { heat shock protein } 90 \text { alpha family class B member } 3 \text {, } \\
\text { pseudogene }\end{array}$ \\
\hline YAP1 & -1.10 & $8.21 \mathrm{E}-02$ & -1.11 & $9.56 \mathrm{E}-02$ & Yes associated protein 1 \\
\hline SP1 & -1.13 & 1.87E-02 & -1.04 & $4.50 \mathrm{E}-01$ & Sp1 transcription factor \\
\hline $\mathrm{CDH} 1$ & -1.14 & 1.17E-02 & -1.02 & $6.70 \mathrm{E}-01$ & cadherin 1 \\
\hline TJP1 & -1.14 & $1.51 \mathrm{E}-01$ & -1.06 & $5.38 \mathrm{E}-01$ & tight junction protein 1 \\
\hline CTNNB1 & -1.15 & $5.58 \mathrm{E}-03$ & -1.04 & 4.62E-01 & catenin beta 1 \\
\hline TIMP1 & -1.15 & $9.21 \mathrm{E}-02$ & -1.17 & $9.57 \mathrm{E}-02$ & TIMP metallopeptidase inhibitor 1 \\
\hline IL6 & -1.16 & $2.90 \mathrm{E}-01$ & 1.04 & 8.13E-01 & interleukin 6 \\
\hline DLK2 & -1.17 & 1.71E-01 & -1.12 & 3.64E-01 & delta like non-canonical Notch ligand 2 \\
\hline OCLN & -1.17 & $2.69 \mathrm{E}-02$ & -1.09 & $2.32 \mathrm{E}-01$ & occludin \\
\hline TAGLN & -1.17 & 3.55E-01 & -2.02 & $2.01 \mathrm{E}-03$ & transgelin \\
\hline ALPL & -1.19 & $1.28 \mathrm{E}-01$ & -1.02 & $8.70 \mathrm{E}-01$ & alkaline phosphatase, biomineralization associated \\
\hline MYOCD & -1.22 & $9.55 \mathrm{E}-02$ & 1.07 & $5.81 E-01$ & myocardin \\
\hline $\mathrm{TRH}$ & -1.23 & $1.73 \mathrm{E}-01$ & -1.08 & $6.51 \mathrm{E}-01$ & thyrotropin releasing hormone \\
\hline LRP5 & -1.24 & $2.63 \mathrm{E}-04$ & -1.14 & $2.65 \mathrm{E}-02$ & LDL receptor related protein 5 \\
\hline KRT19 & -1.25 & $2.40 \mathrm{E}-05$ & -1.75 & 3.27E-10 & keratin 19 \\
\hline DMP1 & -1.25 & $6.18 \mathrm{E}-02$ & 1.06 & $6.20 \mathrm{E}-01$ & dentin matrix acidic phosphoprotein 1 \\
\hline LAMA3 & -1.25 & $9.50 \mathrm{E}-03$ & -3.36 & $1.02 \mathrm{E}-10$ & laminin subunit alpha 3 \\
\hline CA1 & -1.30 & 4.39E-02 & -1.08 & $5.92 \mathrm{E}-01$ & carbonic anhydrase 1 \\
\hline ALPI & -1.32 & 6.67E-02 & -2.59 & $1.56 \mathrm{E}-05$ & alkaline phosphatase, intestinal \\
\hline TIMP2 & -1.33 & $7.29 \mathrm{E}-04$ & -1.56 & $2.59 \mathrm{E}-05$ & TIMP metallopeptidase inhibitor 2 \\
\hline ONECUT1 & -1.35 & $2.52 \mathrm{E}-02$ & -1.01 & $9.20 \mathrm{E}-01$ & one cut homeobox 1 \\
\hline
\end{tabular}




\begin{tabular}{|l|c|c|c|c|l|}
\hline PFKP & -1.36 & $2.67 \mathrm{E}-04$ & -4.19 & $7.97 \mathrm{E}-13$ & phosphofructokinase, platelet \\
\hline SLC39A4 & -1.36 & $1.78 \mathrm{E}-03$ & -2.20 & $2.08 \mathrm{E}-07$ & solute carrier family 39 member 4 \\
\hline RHOU & -1.36 & $3.78 \mathrm{E}-04$ & -2.33 & $5.52 \mathrm{E}-09$ & ras homolog family member U \\
\hline CYP11A1 & -1.40 & $2.78 \mathrm{E}-02$ & 1.31 & $1.08 \mathrm{E}-01$ & cytochrome P450 family 11 subfamily A member 1 \\
\hline PPARG & -1.42 & $7.58 \mathrm{E}-05$ & -1.91 & $1.73 \mathrm{E}-07$ & peroxisome proliferator activated receptor gamma \\
\hline EFNB1 & -1.43 & $1.15 \mathrm{E}-02$ & 1.17 & $2.81 \mathrm{E}-01$ & ephrin B1 \\
\hline SLC46A1 & -1.47 & $4.51 \mathrm{E}-05$ & -1.79 & $1.46 \mathrm{E}-06$ & solute carrier family 46 member 1 \\
\hline CCND1 & -1.51 & $1.04 \mathrm{E}-07$ & -1.21 & $2.06 \mathrm{E}-03$ & cyclin D1 \\
\hline GATA4 & -1.56 & $5.18 \mathrm{E}-03$ & 1.86 & $1.07 \mathrm{E}-03$ & GATA binding protein 4 \\
\hline CCND2 & -1.85 & $2.20 \mathrm{E}-04$ & 2.25 & $4.54 \mathrm{E}-05$ & cyclin D2 \\
\hline KLF4 & -1.86 & $3.92 \mathrm{E}-07$ & -2.04 & $3.24 \mathrm{E}-07$ & Kruppel like factor 4 \\
\hline EFNB2 & -2.11 & $2.07 \mathrm{E}-07$ & -2.39 & $1.31 \mathrm{E}-07$ & ephrin B2 \\
\hline GPA33 & -3.43 & $5.90 \mathrm{E}-13$ & -6.73 & $3.78 \mathrm{E}-15$ & glycoprotein A33 \\
\hline
\end{tabular}

\section{Ion transport}

\begin{tabular}{|c|c|c|c|c|c|}
\hline \multirow[b]{2}{*}{ SYMBOL } & \multicolumn{2}{|c|}{ HT29MTXE12 } & \multicolumn{2}{|c|}{ Caco-2 } & \multirow[b]{2}{*}{ Gene name } \\
\hline & FC & p-value & FC & p-value & \\
\hline ATP1A1 & 5.03 & $5.48 \mathrm{E}-11$ & 2.34 & $1.10 \mathrm{E}-06$ & ATPase $\mathrm{Na}+/ \mathrm{K}+$ transporting subunit alpha 1 \\
\hline ATP1A2 & 1.68 & $2.88 \mathrm{E}-09$ & 1.78 & 3.87E-09 & ATPase $\mathrm{Na}+/ \mathrm{K}+$ transporting subunit alpha 2 \\
\hline ATP1A3 & 1.51 & $1.04 \mathrm{E}-01$ & 1.23 & 4.59E-01 & ATPase $\mathrm{Na}+/ \mathrm{K}+$ transporting subunit alpha 3 \\
\hline ATP1A4 & 1.50 & $6.86 \mathrm{E}-06$ & -2.13 & 8.05E-09 & ATPase $\mathrm{Na}+/ \mathrm{K}+$ transporting subunit alpha 4 \\
\hline ATP1B1 & 1.46 & 1.07E-05 & 1.49 & $2.62 \mathrm{E}-05$ & ATPase $\mathrm{Na}+/ \mathrm{K}+$ transporting subunit beta 1 \\
\hline ATP1B2 & 1.45 & $3.69 \mathrm{E}-04$ & -4.98 & $5.63 \mathrm{E}-12$ & ATPase $\mathrm{Na}+/ \mathrm{K}+$ transporting subunit beta 2 \\
\hline ATP1B3 & 1.44 & $1.48 \mathrm{E}-02$ & 1.16 & $3.51 \mathrm{E}-01$ & ATPase $\mathrm{Na}+/ \mathrm{K}+$ transporting subunit beta 3 \\
\hline ATP1B4 & 1.31 & $4.40 \mathrm{E}-05$ & 1.76 & 1.37E-08 & ATPase $\mathrm{Na}+/ \mathrm{K}+$ transporting family member beta 4 \\
\hline ATP2B1 & 1.30 & $6.82 \mathrm{E}-02$ & 1.67 & $3.82 \mathrm{E}-03$ & ATPase plasma membrane $\mathrm{Ca} 2+$ transporting 1 \\
\hline ATP2B2 & 1.27 & $9.89 \mathrm{E}-05$ & -1.07 & 2.17E-01 & ATPase plasma membrane $\mathrm{Ca} 2+$ transporting 2 \\
\hline ATP2B3 & 1.23 & $1.11 \mathrm{E}-01$ & -1.26 & $1.25 \mathrm{E}-01$ & ATPase plasma membrane $\mathrm{Ca} 2+$ transporting 3 \\
\hline ATP2B4 & 1.21 & $5.16 \mathrm{E}-01$ & -1.19 & $5.92 \mathrm{E}-01$ & ATPase plasma membrane $\mathrm{Ca} 2+$ transporting 4 \\
\hline CA1 & 1.16 & $2.01 \mathrm{E}-03$ & -1.54 & $5.39 \mathrm{E}-08$ & carbonic anhydrase 1 \\
\hline CA2 & 1.16 & $2.66 \mathrm{E}-02$ & 1.29 & $2.15 \mathrm{E}-03$ & carbonic anhydrase 2 \\
\hline CFTR & 1.13 & $5.94 \mathrm{E}-01$ & -1.47 & 1.39E-01 & $\mathrm{CF}$ transmembrane conductance regulator \\
\hline CLCN2 & 1.13 & 4.67E-01 & -1.15 & $4.52 \mathrm{E}-01$ & chloride voltage-gated channel 2 \\
\hline CYBRD1 & 1.11 & 4.72E-02 & -1.03 & $6.22 \mathrm{E}-01$ & cytochrome b reductase 1 \\
\hline $\mathrm{HEPH}$ & 1.11 & $3.80 \mathrm{E}-02$ & -1.01 & $8.85 \mathrm{E}-01$ & hephaestin \\
\hline KCNE3 & 1.10 & 4.57E-01 & -1.44 & $1.66 \mathrm{E}-02$ & $\begin{array}{l}\text { potassium voltage-gated channel subfamily E regulatory subunit } \\
3\end{array}$ \\
\hline MAPK1 & 1.06 & $2.60 \mathrm{E}-01$ & 1.11 & $6.59 \mathrm{E}-02$ & mitogen-activated protein kinase 1 \\
\hline MAPK3 & 1.02 & $9.25 \mathrm{E}-01$ & 1.32 & 1.77E-01 & mitogen-activated protein kinase 3 \\
\hline MKI67 & -1.00 & $9.90 \mathrm{E}-01$ & -1.15 & $2.44 \mathrm{E}-01$ & marker of proliferation $\mathrm{Ki}-67$ \\
\hline NFKB1 & -1.01 & $9.24 \mathrm{E}-01$ & -1.62 & $6.07 E-06$ & nuclear factor kappa B subunit 1 \\
\hline PLCG1 & -1.07 & $3.50 \mathrm{E}-01$ & -1.31 & 4.87E-03 & phospholipase C gamma 1 \\
\hline PRKCA & -1.08 & 4.43E-01 & 3.14 & $2.41 \mathrm{E}-08$ & protein kinase $\mathrm{C}$ alpha \\
\hline SLC11A2 & -1.10 & 4.96E-01 & 1.21 & $2.31 \mathrm{E}-01$ & solute carrier family 11 member 2 \\
\hline SLC12A2 & -1.10 & $1.85 \mathrm{E}-01$ & -1.42 & $3.22 \mathrm{E}-04$ & solute carrier family 12 member 2 \\
\hline
\end{tabular}




\begin{tabular}{|c|c|c|c|c|c|}
\hline SLC26A3 & -1.10 & 4.03E-01 & -1.38 & $2.20 \mathrm{E}-02$ & solute carrier family 26 member 3 \\
\hline SLC26A6 & -1.13 & 3.47E-01 & -1.30 & 8.98E-02 & solute carrier family 26 member 6 \\
\hline SLC26A9 & -1.13 & $2.86 \mathrm{E}-01$ & 1.14 & 3.10E-01 & solute carrier family 26 member 9 \\
\hline SLC30A1 & -1.16 & $3.51 \mathrm{E}-01$ & -1.43 & $5.75 \mathrm{E}-02$ & solute carrier family 30 member 1 \\
\hline SLC31A1 & -1.18 & $1.41 \mathrm{E}-01$ & -2.81 & $1.33 \mathrm{E}-07$ & solute carrier family 31 member 1 \\
\hline SLC34A1 & -1.20 & $1.75 \mathrm{E}-01$ & 1.07 & $6.55 \mathrm{E}-01$ & solute carrier family 34 member 1 \\
\hline SLC34A2 & -1.20 & 7.93E-03 & -1.23 & $9.38 \mathrm{E}-03$ & solute carrier family 34 member 2 \\
\hline SLC34A3 & -1.21 & $1.04 \mathrm{E}-01$ & 1.06 & $6.76 \mathrm{E}-01$ & solute carrier family 34 member 3 \\
\hline SLC39A4 & -1.22 & 3.74E-01 & 1.28 & 3.15E-01 & solute carrier family 39 member 4 \\
\hline SLC40A1 & -1.22 & $9.88 \mathrm{E}-02$ & 1.04 & 7.70E-01 & solute carrier family 40 member 1 \\
\hline SLC46A1 & -1.22 & 2.44E-01 & 1.06 & 7.74E-01 & solute carrier family 46 member 1 \\
\hline SLC4A2 & -1.25 & $2.96 \mathrm{E}-02$ & -3.18 & $4.62 \mathrm{E}-09$ & solute carrier family 4 member 2 \\
\hline SLC4A4 & -1.27 & $1.08 \mathrm{E}-02$ & -1.18 & $1.04 \mathrm{E}-01$ & solute carrier family 4 member 4 \\
\hline SLC5A1 & -1.30 & 4.39E-02 & -1.08 & $5.92 \mathrm{E}-01$ & solute carrier family 5 member 1 \\
\hline SLC6A19 & -1.31 & $2.30 \mathrm{E}-02$ & 1.02 & $8.62 \mathrm{E}-01$ & solute carrier family 6 member 19 \\
\hline SLC8A1 & -1.33 & $1.70 \mathrm{E}-08$ & -2.76 & 2.61E-16 & solute carrier family 8 member $\mathrm{A} 1$ \\
\hline SLC8A2 & -1.36 & $1.78 \mathrm{E}-03$ & -2.20 & $2.08 \mathrm{E}-07$ & solute carrier family 8 member $\mathrm{A} 2$ \\
\hline SLC8A3 & -1.37 & $6.82 \mathrm{E}-05$ & 2.05 & 8.35E-09 & solute carrier family 8 member $\mathrm{A} 3$ \\
\hline SLC9A1 & -1.38 & $3.41 \mathrm{E}-03$ & -1.12 & 3.05E-01 & solute carrier family 9 member $\mathrm{A} 1$ \\
\hline SLC9A3 & -1.42 & $2.53 E-06$ & -2.24 & $9.02 \mathrm{E}-11$ & solute carrier family 9 member $\mathrm{A} 3$ \\
\hline STEAP1 & -1.43 & $1.08 \mathrm{E}-04$ & -3.01 & $2.31 \mathrm{E}-10$ & STEAP family member 1 \\
\hline STEAP2 & -1.43 & $2.54 \mathrm{E}-07$ & -1.63 & $1.97 \mathrm{E}-08$ & STEAP2 metalloreductase \\
\hline TF & -1.47 & 4.51E-05 & -1.79 & $1.46 \mathrm{E}-06$ & transferrin \\
\hline TNF & -1.48 & 4.37E-03 & 1.12 & 4.02E-01 & tumor necrosis factor \\
\hline TRPM6 & -1.59 & $1.88 \mathrm{E}-06$ & -1.34 & 1.10E-03 & $\begin{array}{l}\text { transient receptor potential cation channel subfamily M member } \\
6\end{array}$ \\
\hline TRPM7 & -1.95 & $2.42 \mathrm{E}-07$ & -2.03 & $5.95 \mathrm{E}-07$ & $\begin{array}{l}\text { transient receptor potential cation channel subfamily } \mathrm{M} \text { member } \\
7\end{array}$ \\
\hline TRPV 6 & -2.31 & 1.97E-11 & -1.53 & $2.21 \mathrm{E}-06$ & transient receptor potential cation channel subfamily $V$ member 6 \\
\hline
\end{tabular}


Supplementary file 2 Up- and downregulated pathways between SWMS and static conditions in HT29MTX-E12 and Caco-2 cells, obtained by Gene Set Enrichment Analysis (GSEA). NES = Normalized Enrichment Score; FDR = False Discovery Rate.

\section{HT29-MTX-E12 CELLS: UPREGULATED PATHWAYS}

\section{NAME}

HSA04110.CELL.CYCLE.KEGG

HSA03460.FANCONI.ANEMIA.PATHWAY.KEGG

HSA03030.DNA.REPLICATION.KEGG

HSA05322.SYSTEMIC.LUPUS.ERYTHEMATOSUS.KEGG

HSA03440.HOMOLOGOUS.RECOMBINATION.KEGG

HSA04114.OOCYTE.MEIOSIS.KEGG

HSA03420.NUCLEOTIDE.EXCISION.REPAIR.KEGG

HSA03430.MISMATCH.REPAIR.KEGG

HSA00100.STEROID.BIOSYNTHESIS.KEGG

HSA04914.PROGESTERONE.MEDIATED.OOCYTE.MATURATION.KEGG

HSA05034.ALCOHOLISM.KEGG

HSA03013.RNA.TRANSPORT.KEGG

HSA03010.RIBOSOME.KEGG

HSA00480.GLUTATHIONE.METABOLISM.KEGG

HSA04115.P53.SIGNALING.PATHWAY.KEGG

HSA03410.BASE.EXCISION.REPAIR.KEGG

HSA00650.BUTANOATE.METABOLISM.KEGG

HSA01524.PLATINUM.DRUG.RESISTANCE.KEGG

HSA00280.VALINE..LEUCINE.AND.ISOLEUCINE.DEGRADATION.KEGG

HSA03050.PROTEASOME.KEGG

HSA04218.CELLULAR.SENESCENCE.KEGG

HSA00071.FATTY.ACID.DEGRADATION.KEGG

HSA00900.TERPENOID.BACKBONE.BIOSYNTHESIS.KEGG

HSA01212.FATTY.ACID.METABOLISM.KEGG

HSA00020.CITRATE.CYCLE..TCA.CYCLE..KEGG

HSA00350.TYROSINE.METABOLISM.KEGG

HSA00620.PYRUVATE.METABOLISM.KEGG

HSA00640.PROPANOATE.METABOLISM.KEGG

HSA00630.GLYOXYLATE.AND.DICARBOXYLATE.METABOLISM.KEGG

HSA00380.TRYPTOPHAN.METABOLISM.KEGG

HSA00270.CYSTEINE.AND.METHIONINE.METABOLISM.KEGG

HSA05203.VIRAL.CARCINOGENESIS.KEGG

HSA01200.CARBON.METABOLISM.KEGG

HSA00061.FATTY.ACID.BIOSYNTHESIS.KEGG

HSA03022.BASAL.TRANSCRIPTION.FACTORS.KEGG

HSA00670.ONE.CARBON.POOL.BY.FOLATE.KEGG

HSA03040.SPLICEOSOME.KEGG

HSA00250.ALANINE..ASPARTATE.AND.GLUTAMATE.METABOLISM.KEGG

HSA00310.LYSINE.DEGRADATION.KEGG

\begin{tabular}{|c|c|}
\hline NES & FDR q-val \\
\hline 2.86 & 0.00 \\
\hline 2.64 & 0.00 \\
\hline 2.60 & 0.00 \\
\hline 2.54 & 0.00 \\
\hline 2.51 & 0.00 \\
\hline 2.33 & 0.00 \\
\hline 2.31 & 0.00 \\
\hline 2.31 & 0.00 \\
\hline 2.31 & 0.00 \\
\hline 2.27 & 0.00 \\
\hline 2.25 & 0.00 \\
\hline 2.20 & 0.00 \\
\hline 2.18 & 0.00 \\
\hline 2.18 & 0.00 \\
\hline 2.15 & $1.71 \mathrm{E}-05$ \\
\hline 2.08 & $6.39 \mathrm{E}-05$ \\
\hline 2.08 & $6.02 \mathrm{E}-05$ \\
\hline 2.04 & $1.48 \mathrm{E}-04$ \\
\hline 2.03 & $1.60 \mathrm{E}-04$ \\
\hline 2.03 & $1.52 \mathrm{E}-04$ \\
\hline 2.02 & $1.99 \mathrm{E}-04$ \\
\hline 2.02 & $1.98 \mathrm{E}-04$ \\
\hline 2.02 & 2.12E-04 \\
\hline 1.99 & 3.82E-04 \\
\hline 1.94 & 7.12E-04 \\
\hline 1.93 & $8.28 \mathrm{E}-04$ \\
\hline 1.91 & $1.14 \mathrm{E}-03$ \\
\hline 1.88 & $1.55 \mathrm{E}-03$ \\
\hline 1.88 & $1.55 \mathrm{E}-03$ \\
\hline 1.88 & $1.58 \mathrm{E}-03$ \\
\hline 1.88 & $1.55 \mathrm{E}-03$ \\
\hline 1.86 & $1.91 \mathrm{E}-03$ \\
\hline 1.85 & $2.18 \mathrm{E}-03$ \\
\hline 1.79 & 4.91E-03 \\
\hline 1.78 & 4.96E-03 \\
\hline 1.75 & 7.43E-03 \\
\hline 1.74 & $8.28 \mathrm{E}-03$ \\
\hline 1.73 & $8.70 \mathrm{E}-03$ \\
\hline 1.73 & 8.66E-03 \\
\hline
\end{tabular}


HSA03018.RNA.DEGRADATION.KEGG

HSA01210.2.OXOCARBOXYLIC.ACID.METABOLISM.KEGG

HSA01523.ANTIFOLATE.RESISTANCE.KEGG

HSA03008.RIBOSOME.BIOGENESIS.IN.EUKARYOTES.KEGG

HSA00983.DRUG.METABOLISM...OTHER.ENZYMES.KEGG

HSA00360.PHENYLALANINE.METABOLISM.KEGG

$1.719 .65 \mathrm{E}-03$

HSA05166.HUMAN.T.CELL.LEUKEMIA.VIRUS.1.INFECTION.KEGG

HSA00240.PYRIMIDINE.METABOLISM.KEGG

HSA00062.FATTY.ACID.ELONGATION.KEGG

HSA01040.BIOSYNTHESIS.OF.UNSATURATED.FATTY.ACIDS.KEGG

$1.71 \quad 1.02 \mathrm{E}-02$

HSA00230.PURINE.METABOLISM.KEGG

HSA03320.PPAR.SIGNALING.PATHWAY.KEGG

HSA01230.BIOSYNTHESIS.OF.AMINO.ACIDS.KEGG

HSA03015.MRNA.SURVEILLANCE.PATHWAY.KEGG

HSA00220.ARGININE.BIOSYNTHESIS.KEGG

HSA04217.NECROPTOSIS.KEGG

HSA00030.PENTOSE.PHOSPHATE.PATHWAY.KEGG

HSA00053.ASCORBATE.AND.ALDARATE.METABOLISM.KEGG

HSA00450.SELENOCOMPOUND.METABOLISM.KEGG

HSA04068.FOXO.SIGNALING.PATHWAY.KEGG

HSA04146.PEROXISOME.KEGG

HSA00330.ARGININE.AND.PROLINE.METABOLISM.KEGG

HSA05169.EPSTEIN.BARR.VIRUS.INFECTION.KEGG

HSA04714.THERMOGENESIS.KEGG

HSA05202.TRANSCRIPTIONAL.MISREGULATION.IN.CANCER.KEGG

HSA05016.HUNTINGTON.DISEASE.KEGG

HSA05206.MICRORNAS.IN.CANCER.KEGG

HSA00260.GLYCINE..SERINE.AND.THREONINE.METABOLISM.KEGG

HSA04210.APOPTOSIS.KEGG

HSA00982.DRUG.METABOLISM...CYTOCHROME.P450.KEGG

HSA05170.HUMAN.IMMUNODEFICIENCY.VIRUS.1.INFECTION.KEGG

HSA00790.FOLATE.BIOSYNTHESIS.KEGG

HSA04120.UBIQUITIN.MEDIATED.PROTEOLYSIS.KEGG

HSA02010.ABC.TRANSPORTERS.KEGG

HSA04216.FERROPTOSIS.KEGG

HSA05222.SMALL.CELL.LUNG.CANCER.KEGG

HSA00970.AMINOACYL.TRNA.BIOSYNTHESIS.KEGG

HSA05204.CHEMICAL.CARCINOGENESIS.KEGG

HSA05210.COLORECTAL.CANCER.KEGG

HSA05161.HEPATITIS.B.KEGG

HSA04152.AMPK.SIGNALING.PATHWAY.KEGG

HSA00980.METABOLISM.OF.XENOBIOTICS.BY.CYTOCHROME.P450.KEGG

HSA00561.GLYCEROLIPID.METABOLISM.KEGG

HSA05220.CHRONIC.MYELOID.LEUKEMIA.KEGG 
HSA00010.GLYCOLYSIS...GLUCONEOGENESIS.KEGG

HSA04950.MATURITY.ONSET.DIABETES.OF.THE.YOUNG.KEGG

HSA00040.PENTOSE.AND.GLUCURONATE.INTERCONVERSIONS.KEGG 
HSA04724.GLUTAMATERGIC.SYNAPSE.KEGG

$0.72 \quad 9.36 \mathrm{E}-01$

HSA04640.HEMATOPOIETIC.CELL.LINEAGE.KEGG

$0.71 \quad 9.37 \mathrm{E}-01$

HSA04730.LONG.TERM.DEPRESSION.KEGG

$0.65 \quad 9.69 \mathrm{E}-01$

\section{HT29-MTX-E12 CELLS: DOWNREGULATED PATHWAYS}

\section{NAME}

HSA04142.LYSOSOME.KEGG

HSA05416.VIRAL.MYOCARDITIS.KEGG

HSA04510.FOCAL.ADHESION.KEGG

HSA05205.PROTEOGLYCANS.IN.CANCER.KEGG

HSA00511.OTHER.GLYCAN.DEGRADATION.KEGG

HSA04928.PARATHYROID.HORMONE.SYNTHESIS..SECRETION.AND.ACTION.KEGG

HSA04070.PHOSPHATIDYLINOSITOL.SIGNALING.SYSTEM.KEGG

HSA04962.VASOPRESSIN.REGULATED.WATER.REABSORPTION.KEGG

HSA04630.JAK.STAT.SIGNALING.PATHWAY.KEGG

HSA05320.AUTOIMMUNE.THYROID.DISEASE.KEGG

HSA04144.ENDOCYTOSIS.KEGG

HSA04514.CELL.ADHESION.MOLECULES...CAMS..KEGG

HSA04066.HIF.1.SIGNALING.PATHWAY.KEGG

HSA04061.VIRAL.PROTEIN.INTERACTION.WITH.CYTOKINE.AND.CYTOKINE.RECEPTOR.KEGG

HSA04915.ESTROGEN.SIGNALING.PATHWAY.KEGG

HSA04141.PROTEIN.PROCESSING.IN.ENDOPLASMIC.RETICULUM.KEGG

HSA05145.TOXOPLASMOSIS.KEGG

HSA05321.INFLAMMATORY.BOWEL.DISEASE..IBD..KEGG

HSA05211.RENAL.CELL.CARCINOMA.KEGG

HSA01521.EGFR.TYROSINE.KINASE.INHIBITOR.RESISTANCE.KEGG

HSA05330.ALLOGRAFT.REJECTION.KEGG

HSA04130.SNARE.INTERACTIONS.IN.VESICULAR.TRANSPORT.KEGG

HSA05146.AMOEBIASIS.KEGG

HSA00562.INOSITOL.PHOSPHATE.METABOLISM.KEGG

HSA05152.TUBERCULOSIS.KEGG

HSA05140.LEISHMANIASIS.KEGG

HSA04360.AXON.GUIDANCE.KEGG

HSA04960.ALDOSTERONE.REGULATED.SODIUM.REABSORPTION.KEGG

HSA05100.BACTERIAL.INVASION.OF.EPITHELIAL.CELLS.KEGG

HSA04145.PHAGOSOME.KEGG

HSA04650.NATURAL.KILLER.CELL.MEDIATED.CYTOTOXICITY.KEGG

HSA05130.PATHOGENIC.ESCHERICHIA.COLIIINFECTION.KEGG

HSA05163.HUMAN.CYTOMEGALOVIRUS.INFECTION.KEGG

HSA05165.HUMAN.PAPILLOMAVIRUS.INFECTION.KEGG

HSA04919.THYROID.HORMONE.SIGNALING.PATHWAY.KEGG

HSA04371.APELIN.SIGNALING.PATHWAY.KEGG

HSA04530.TIGHT.JUNCTION.KEGG

FDR q-val

1.58E-04

1.50E-02

1.52E-02

2.22E-02

2.21E-02

2.22E-02

1.95E-02

1.71E-02

1.52E-02

1.43E-02

1.32E-02

1.52E-02

2.07E-02

2.03E-02

2.38E-02

2.54E-02

2.81E-02

2.91E-02

2.98E-02

3.81E-02

3.79E-02

3.72E-02

3.58E-02

3.86E-02

3.80E-02

3.97E-02

3.85E-02

5.19E-02

5.20E-02

5.12E-02

5.08E-02

5.40E-02

5.69E-02

5.78E-02

5.85E-02

5.71E-02

5.64E-02 
HSA00512.MUCIN.TYPE.O.GLYCAN.BIOSYNTHESIS.KEGG

HSA00601.GLYCOSPHINGOLIPID.BIOSYNTHESIS...LACTO.AND.NEOLACTO.SERIES.KEGG 


\begin{tabular}{|c|c|c|}
\hline HSA04923.REGULATION.OF.LIPOLYSIS.IN.ADIPOCYTES.KEGG & -1.39 & $1.09 \mathrm{E}-01$ \\
\hline HSA04966.COLLECTING.DUCT.ACID.SECRETION.KEGG & -1.39 & 1.07E-01 \\
\hline HSA00513.VARIOUS.TYPES.OF.N.GLYCAN.BIOSYNTHESIS.KEGG & -1.38 & $1.07 \mathrm{E}-01$ \\
\hline HSA00520.AMINO.SUGAR.AND.NUCLEOTIDE.SUGAR.METABOLISM.KEGG & -1.38 & $1.08 \mathrm{E}-01$ \\
\hline HSA04978.MINERAL.ABSORPTION.KEGG & -1.38 & 1.09E-01 \\
\hline HSA04910.INSULIN.SIGNALING.PATHWAY.KEGG & -1.38 & $1.08 \mathrm{E}-01$ \\
\hline HSA05412.ARRHYTHMOGENIC.RIGHT.VENTRICULAR.CARDIOMYOPATHY..ARVC...KEGG & -1.37 & $1.12 \mathrm{E}-01$ \\
\hline HSA04720.LONG.TERM.POTENTIATION.KEGG & -1.37 & 1.13E-01 \\
\hline HSA05219.BLADDER.CANCER.KEGG & -1.36 & 1.18E-01 \\
\hline HSA00140.STEROID.HORMONE.BIOSYNTHESIS.KEGG & -1.35 & $1.24 \mathrm{E}-01$ \\
\hline HSA00052.GALACTOSE.METABOLISM.KEGG & -1.35 & $1.25 \mathrm{E}-01$ \\
\hline HSA04060.CYTOKINE.CYTOKINE.RECEPTOR.INTERACTION.KEGG & -1.34 & $1.29 \mathrm{E}-01$ \\
\hline HSA00591.LINOLEIC.ACID.METABOLISM.KEGG & -1.33 & 1.39E-01 \\
\hline HSA04151.PI3K.AKT.SIGNALING.PATHWAY.KEGG & -1.33 & 1.39E-01 \\
\hline HSA04912.GNRH.SIGNALING.PATHWAY.KEGG & -1.31 & $1.50 \mathrm{E}-01$ \\
\hline HSA05230.CENTRAL.CARBON.METABOLISM.IN.CANCER.KEGG & -1.31 & $1.52 \mathrm{E}-01$ \\
\hline HSA04971.GASTRIC.ACID.SECRETION.KEGG & -1.31 & $1.54 \mathrm{E}-01$ \\
\hline HSA04370.VEGF.SIGNALING.PATHWAY.KEGG & -1.31 & $1.52 \mathrm{E}-01$ \\
\hline HSA04740.OLFACTORY.TRANSDUCTION.KEGG & -1.30 & $1.60 \mathrm{E}-01$ \\
\hline HSA00531.GLYCOSAMINOGLYCAN.DEGRADATION.KEGG & -1.30 & 1.59E-01 \\
\hline HSA04015.RAP1.SIGNALING.PATHWAY.KEGG & -1.30 & $1.58 \mathrm{E}-01$ \\
\hline HSA00564.GLYCEROPHOSPHOLIPID.METABOLISM.KEGG & -1.29 & 1.63E-01 \\
\hline HSA04612.ANTIGEN.PROCESSING.AND.PRESENTATION.KEGG & -1.29 & 1.65E-01 \\
\hline HSA05226.GASTRIC.CANCER.KEGG & -1.28 & 1.65E-01 \\
\hline HSA04917.PROLACTIN.SIGNALING.PATHWAY.KEGG & -1.28 & 1.71E-01 \\
\hline $\begin{array}{l}\text { HSA00532.GLYCOSAMINOGLYCAN.BIOSYNTHESIS...CHONDROITIN.SULFATE...DERMATAN.SULFATE. } \\
\text { KEGG }\end{array}$ & -1.27 & $1.78 \mathrm{E}-01$ \\
\hline HSA04620.TOLL.LIKE.RECEPTOR.SIGNALING.PATHWAY.KEGG & -1.27 & 1.76E-01 \\
\hline HSA04625.C.TYPE.LECTIN.RECEPTOR.SIGNALING.PATHWAY.KEGG & -1.27 & 1.77E-01 \\
\hline HSA04972.PANCREATIC.SECRETION.KEGG & -1.27 & 1.77E-01 \\
\hline HSA04927.CORTISOL.SYNTHESIS.AND.SECRETION.KEGG & -1.26 & $1.88 \mathrm{E}-01$ \\
\hline HSA00563.GLYCOSYLPHOSPHATIDYLINOSITOL..GPI..ANCHOR.BIOSYNTHESIS.KEGG & -1.25 & 1.93E-01 \\
\hline HSA00600.SPHINGOLIPID.METABOLISM.KEGG & -1.25 & 1.95E-01 \\
\hline HSA04725.CHOLINERGIC.SYNAPSE.KEGG & -1.25 & 1.93E-01 \\
\hline HSA04672.INTESTINAL.IMMUNE.NETWORK.FOR.IGA.PRODUCTION.KEGG & -1.24 & $1.98 \mathrm{E}-01$ \\
\hline HSA04211.LONGEVITY.REGULATING.PATHWAY.KEGG & -1.24 & $2.00 \mathrm{E}-01$ \\
\hline HSA05131.SHIGELLOSIS.KEGG & -1.24 & 2.02E-01 \\
\hline HSA04918.THYROID.HORMONE.SYNTHESIS.KEGG & -1.24 & 2.00E-01 \\
\hline HSA05120.EPITHELIAL.CELL.SIGNALING.IN.HELICOBACTER.PYLORI.INFECTION.KEGG & -1.23 & $1.99 \mathrm{E}-01$ \\
\hline HSA04911.INSULIN.SECRETION.KEGG & -1.23 & 1.97E-01 \\
\hline HSA04925.ALDOSTERONE.SYNTHESIS.AND.SECRETION.KEGG & -1.23 & $2.02 \mathrm{E}-01$ \\
\hline HSA05168.HERPES.SIMPLEX.VIRUS.1.INFECTION.KEGG & -1.23 & 2.03E-01 \\
\hline HSA04935.GROWTH.HORMONE.SYNTHESIS..SECRETION.AND.ACTION.KEGG & -1.22 & 2.07E-01 \\
\hline HSA05143.AFRICAN.TRYPANOSOMIASIS.KEGG & -1.22 & 2.12E-01 \\
\hline
\end{tabular}


HSA05142.CHAGAS.DISEASE..AMERICAN.TRYPANOSOMIASIS..KEGG

HSA05310.ASTHMA.KEGG

HSA05110.VIBRIO.CHOLERAE.INFECTION.KEGG

HSA04012.ERBB.SIGNALING.PATHWAY.KEGG

HSA05216.THYROID.CANCER.KEGG

HSA04977.VITAMIN.DIGESTION.AND.ABSORPTION.KEGG

HSA04926.RELAXIN.SIGNALING.PATHWAY.KEGG

HSA04080.NEUROACTIVE.LIGAND.RECEPTOR.INTERACTION.KEGG

HSA04742.TASTE.TRANSDUCTION.KEGG

HSA04014.RAS.SIGNALING.PATHWAY.KEGG

HSA00510.N.GLYCAN.BIOSYNTHESIS.KEGG

HSA04071.SPHINGOLIPID.SIGNALING.PATHWAY.KEGG

HSA04713.CIRCADIAN.ENTRAINMENT.KEGG

HSA04022.CGMP.PKG.SIGNALING.PATHWAY.KEGG

HSA04924.RENIN.SECRETION.KEGG

HSA01522.ENDOCRINE.RESISTANCE.KEGG

HSA00604.GLYCOSPHINGOLIPID.BIOSYNTHESIS...GANGLIO.SERIES.KEGG

HSA04010.MAPK.SIGNALING.PATHWAY.KEGG

HSA04270.VASCULAR.SMOOTH.MUSCLE.CONTRACTION.KEGG

HSA04024.CAMP.SIGNALING.PATHWAY.KEGG

HSA05410.HYPERTROPHIC.CARDIOMYOPATHY..HCM..KEGG

HSA04390.HIPPO.SIGNALING.PATHWAY.KEGG

HSA04261.ADRENERGIC.SIGNALING.IN.CARDIOMYOCYTES.KEGG

HSA04150.MTOR.SIGNALING.PATHWAY.KEGG

HSA05235.PD.L1.EXPRESSION.AND.PD.1.CHECKPOINT.PATHWAY.IN.CANCER.KEGG

HSA00120.PRIMARY.BILE.ACID.BIOSYNTHESIS.KEGG

HSA04380.OSTEOCLAST.DIFFERENTIATION.KEGG

HSA05162.MEASLES.KEGG

HSA04662.B.CELL.RECEPTOR.SIGNALING.PATHWAY.KEGG

HSA04657.IL.17.SIGNALING.PATHWAY.KEGG

HSA04666.FC.GAMMA.R.MEDIATED.PHAGOCYTOSIS.KEGG

HSA00760.NICOTINATE.AND.NICOTINAMIDE.METABOLISM.KEGG

HSA05133.PERTUSSIS.KEGG

HSA04622.RIG.I.LIKE.RECEPTOR.SIGNALING.PATHWAY.KEGG

HSA04611.PLATELET.ACTIVATION.KEGG

HSA04550.SIGNALING.PATHWAYS.REGULATING.PLURIPOTENCY.OF.STEM.CELLS.KEGG

HSA04610.COMPLEMENT.AND.COAGULATION.CASCADES.KEGG

HSA00860.PORPHYRIN.AND.CHLOROPHYLL.METABOLISM.KEGG

HSA04213.LONGEVITY.REGULATING.PATHWAY...MULTIPLE.SPECIES.KEGG

HSA04930.TYPE.II.DIABETES.MELLITUS.KEGG

HSA04931.INSULIN.RESISTANCE.KEGG

HSA05134.LEGIONELLOSIS.KEGG

HSA04979.CHOLESTEROL.METABOLISM.KEGG 
HSA04310.WNT.SIGNALING.PATHWAY.KEGG

$-0.97 \quad 5.56 \mathrm{E}-01$

HSA04974.PROTEIN.DIGESTION.AND.ABSORPTION.KEGG

$-0.97 \quad 5.57 \mathrm{E}-01$

HSA00534.GLYCOSAMINOGLYCAN.BIOSYNTHESIS...HEPARAN.SULFATE...HEPARIN.KEGG

$-0.97$

HSA04392.HIPPO.SIGNALING.PATHWAY..MULTIPLE.SPECIES.KEGG

HSA04921.OXYTOCIN.SIGNALING.PATHWAY.KEGG

$-0.96$

$5.55 \mathrm{E}-01$

HSA05135.YERSINIA.INFECTION.KEGG

$-0.96$

$5.69 \mathrm{E}-01$

HSA04727.GABAERGIC.SYNAPSE.KEGG

HSA00410.BETA.ALANINE.METABOLISM.KEGG

HSA04330.NOTCH.SIGNALING.PATHWAY.KEGG

HSA00514.OTHER.TYPES.OF.O.GLYCAN.BIOSYNTHESIS.KEGG

HSA05032.MORPHINE.ADDICTION.KEGG

HSA05217.BASAL.CELL.CARCINOMA.KEGG

HSA04726.SEROTONERGIC.SYNAPSE.KEGG

HSA04136.AUTOPHAGY...OTHER.KEGG

HSA04260.CARDIAC.MUSCLE.CONTRACTION.KEGG

HSA04723.RETROGRADE.ENDOCANNABINOID.SIGNALING.KEGG

CACO-2 CELLS: UPREGULATED PATHWAYS

\section{NAME}

HSA03030.DNA.REPLICATION.KEGG

HSA05322.SYSTEMIC.LUPUS.ERYTHEMATOSUS.KEGG

HSA03440.HOMOLOGOUS.RECOMBINATION.KEGG

HSA04110.CELL.CYCLE.KEGG

HSA03460.FANCONI.ANEMIA.PATHWAY.KEGG

HSA03430.MISMATCH.REPAIR.KEGG

HSA03008.RIBOSOME.BIOGENESIS.IN.EUKARYOTES.KEGG

HSA03410.BASE.EXCISION.REPAIR.KEGG

HSA03013.RNA.TRANSPORT.KEGG

HSA05034.ALCOHOLISM.KEGG

HSA04610.COMPLEMENT.AND.COAGULATION.CASCADES.KEGG

HSA03040.SPLICEOSOME.KEGG

HSA03420.NUCLEOTIDE.EXCISION.REPAIR.KEGG

HSA04914.PROGESTERONE.MEDIATED.OOCYTE.MATURATION.KEGG

HSA03050.PROTEASOME.KEGG

HSA00061.FATTY.ACID.BIOSYNTHESIS.KEGG

HSA00900.TERPENOID.BACKBONE.BIOSYNTHESIS.KEGG

HSA03015.MRNA.SURVEILLANCE.PATHWAY.KEGG

HSA03020.RNA.POLYMERASE.KEGG 


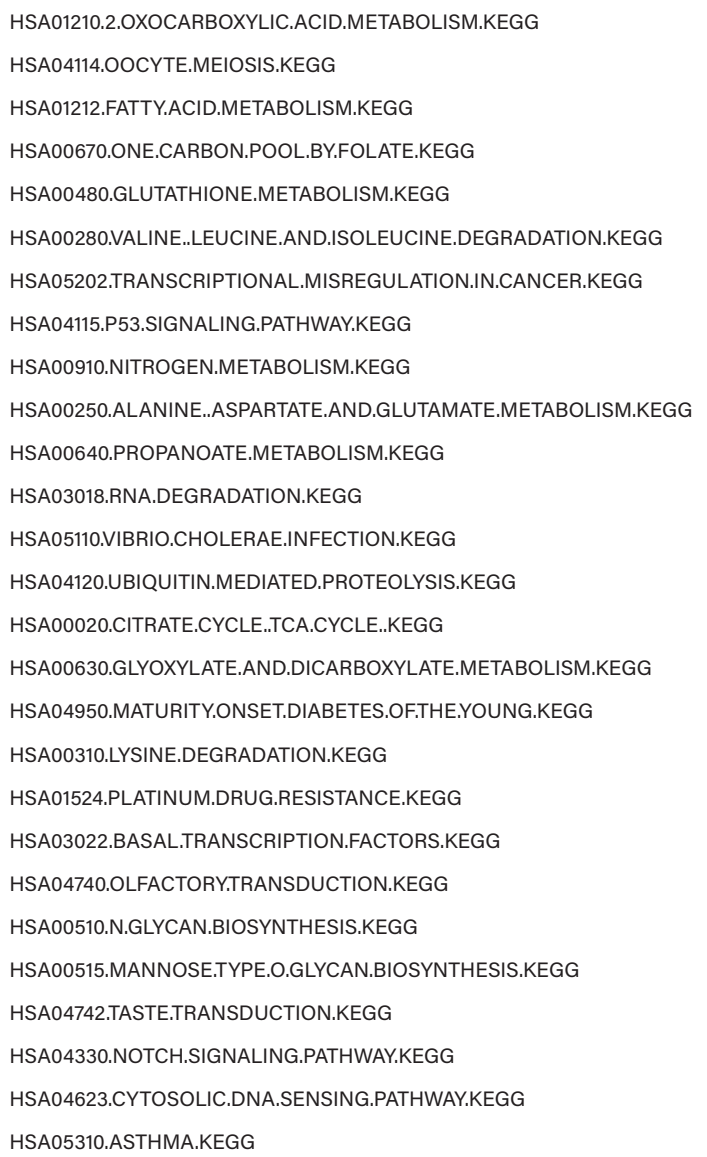

\begin{tabular}{|c|c|}
\hline 1.38 & $1.02 \mathrm{E}-01$ \\
\hline 1.38 & 9.86E-02 \\
\hline 1.37 & 1.01E-01 \\
\hline 1.35 & $1.11 \mathrm{E}-01$ \\
\hline 1.33 & 1.19E-01 \\
\hline 1.30 & $1.46 \mathrm{E}-01$ \\
\hline 1.27 & 1.69E-01 \\
\hline 1.26 & 1.70E-01 \\
\hline 1.26 & $1.65 \mathrm{E}-01$ \\
\hline 1.25 & $1.74 \mathrm{E}-01$ \\
\hline 1.21 & 2.16E-01 \\
\hline 1.20 & $2.25 \mathrm{E}-01$ \\
\hline 1.13 & 3.31E-01 \\
\hline 1.13 & 3.23E-01 \\
\hline 1.11 & 3.38E-01 \\
\hline 1.11 & 3.33E-01 \\
\hline 1.10 & 3.36E-01 \\
\hline 1.09 & $3.56 \mathrm{E}-01$ \\
\hline 1.03 & 4.66E-01 \\
\hline 0.98 & $5.89 \mathrm{E}-01$ \\
\hline 0.86 & $8.56 \mathrm{E}-01$ \\
\hline 0.86 & $8.51 \mathrm{E}-01$ \\
\hline 0.85 & $8.50 \mathrm{E}-01$ \\
\hline 0.79 & $9.32 \mathrm{E}-01$ \\
\hline 0.78 & $9.25 \mathrm{E}-01$ \\
\hline 0.77 & $9.21 \mathrm{E}-01$ \\
\hline 0.70 & $9.53 \mathrm{E}-01$ \\
\hline
\end{tabular}

CACO-2 CELLS: DOWNREGULATED PATHWAYS

NAME

HSA04978.MINERAL.ABSORPTION.KEGG

HSA04973.CARBOHYDRATE.DIGESTION.AND.ABSORPTION.KEGG

HSA00500.STARCH.AND.SUCROSE.METABOLISM.KEGG

HSA04144.ENDOCYTOSIS.KEGG

HSA00010.GLYCOLYSIS...GLUCONEOGENESIS.KEGG

HSA04974.PROTEIN.DIGESTION.AND.ABSORPTION.KEGG 


\begin{tabular}{|c|c|c|}
\hline HSA04977VITAMIN.DIGESTION.AND.ABSORPTION.KEGG & -2.00 & $5.42 \mathrm{E}-04$ \\
\hline HSA04142.LYSOSOME.KEGG & -1.99 & $6.29 \mathrm{E}-04$ \\
\hline HSA00562.INOSITOL.PHOSPHATE.METABOLISM.KEGG & -1.96 & $1.01 \mathrm{E}-03$ \\
\hline HSA05211.RENAL.CELL.CARCINOMA.KEGG & -1.94 & $1.36 \mathrm{E}-03$ \\
\hline HSA00860.PORPHYRIN.AND.CHLOROPHYLL.METABOLISM.KEGG & -1.94 & $1.53 \mathrm{E}-03$ \\
\hline HSA05230.CENTRAL.CARBON.METABOLISM.IN.CANCER.KEGG & -1.90 & 2.96E-03 \\
\hline HSA05217.BASAL.CELL.CARCINOMA.KEGG & -1.88 & $3.48 \mathrm{E}-03$ \\
\hline HSA04151.PI3K.AKT.SIGNALING.PATHWAY.KEGG & -1.88 & $3.32 \mathrm{E}-03$ \\
\hline HSA04922.GLUCAGON.SIGNALING.PATHWAY.KEGG & -1.87 & $3.58 \mathrm{E}-03$ \\
\hline HSA03320.PPAR.SIGNALING.PATHWAY.KEGG & -1.87 & $3.46 \mathrm{E}-03$ \\
\hline HSA04961.ENDOCRINE.AND.OTHER.FACTOR.REGULATED.CALCIUM.REABSORPTION.KEGG & -1.86 & 4.12E-03 \\
\hline HSA04931.INSULIN.RESISTANCE.KEGG & -1.85 & 4.07E-03 \\
\hline HSA00120.PRIMARY.BILE.ACID.BIOSYNTHESIS.KEGG & -1.85 & 4.20E-03 \\
\hline HSA00982.DRUG.METABOLISM...CYTOCHROME.P450.KEGG & -1.85 & 4.05E-03 \\
\hline HSA04216.FERROPTOSIS.KEGG & -1.84 & 4.39E-03 \\
\hline HSA00980.METABOLISM.OF.XENOBIOTICS.BY.CYTOCHROME.P450.KEGG & -1.84 & 4.49E-03 \\
\hline HSA00520.AMINO.SUGAR.AND.NUCLEOTIDE.SUGAR.METABOLISM.KEGG & -1.83 & 4.54E-03 \\
\hline HSA04140.AUTOPHAGY...ANIMAL.KEGG & -1.83 & 4.42E-03 \\
\hline HSA00830.RETINOL.METABOLISM.KEGG & -1.83 & $4.46 \mathrm{E}-03$ \\
\hline HSA05204.CHEMICAL.CARCINOGENESIS.KEGG & -1.82 & $5.25 \mathrm{E}-03$ \\
\hline HSA04512.ECM.RECEPTOR.INTERACTION.KEGG & -1.81 & $5.23 \mathrm{E}-03$ \\
\hline HSA05145.TOXOPLASMOSIS.KEGG & -1.81 & $5.39 \mathrm{E}-03$ \\
\hline HSA05205.PROTEOGLYCANS.IN.CANCER.KEGG & -1.81 & $5.49 \mathrm{E}-03$ \\
\hline HSA04152.AMPK.SIGNALING.PATHWAY.KEGG & -1.80 & $5.85 \mathrm{E}-03$ \\
\hline HSA05165.HUMAN.PAPILLOMAVIRUS.INFECTION.KEGG & -1.80 & $6.01 \mathrm{E}-03$ \\
\hline HSA00511.OTHER.GLYCAN.DEGRADATION.KEGG & -1.79 & $5.92 \mathrm{E}-03$ \\
\hline HSA01200.CARBON.METABOLISM.KEGG & -1.79 & $5.91 \mathrm{E}-03$ \\
\hline HSA05130.PATHOGENIC.ESCHERICHIA.COLIIINFECTION.KEGG & -1.78 & $6.52 \mathrm{E}-03$ \\
\hline HSA01230.BIOSYNTHESIS.OF.AMINO.ACIDS.KEGG & -1.77 & $8.23 \mathrm{E}-03$ \\
\hline HSA04810.REGULATION.OF.ACTIN.CYTOSKELETON.KEGG & -1.75 & $9.44 \mathrm{E}-03$ \\
\hline HSA04975.FAT.DIGESTION.AND.ABSORPTION.KEGG & -1.74 & $1.07 \mathrm{E}-02$ \\
\hline HSA05132.SALMONELLA.INFECTION.KEGG & -1.73 & $1.17 \mathrm{E}-02$ \\
\hline HSA04911.INSULIN.SECRETION.KEGG & -1.73 & $1.15 \mathrm{E}-02$ \\
\hline HSA04022.CGMP.PKG.SIGNALING.PATHWAY.KEGG & -1.72 & $1.28 \mathrm{E}-02$ \\
\hline HSA04130.SNARE.INTERACTIONS.IN.VESICULAR.TRANSPORT.KEGG & -1.72 & $1.26 \mathrm{E}-02$ \\
\hline HSA05222.SMALL.CELL.LUNG.CANCER.KEGG & -1.71 & $1.29 \mathrm{E}-02$ \\
\hline HSA05418.FLUID.SHEAR.STRESS.AND.ATHEROSCLEROSIS.KEGG & -1.71 & $1.34 \mathrm{E}-02$ \\
\hline HSA04520.ADHERENS.JUNCTION.KEGG & -1.70 & $1.42 \mathrm{E}-02$ \\
\hline HSA00531.GLYCOSAMINOGLYCAN.DEGRADATION.KEGG & -1.70 & $1.47 \mathrm{E}-02$ \\
\hline HSA04662.B.CELL.RECEPTOR.SIGNALING.PATHWAY.KEGG & -1.70 & 1.47E-02 \\
\hline HSA04930.TYPE.II.DIABETES.MELLITUS.KEGG & -1.70 & $1.47 \mathrm{E}-02$ \\
\hline HSA04917.PROLACTIN.SIGNALING.PATHWAY.KEGG & -1.70 & $1.44 \mathrm{E}-02$ \\
\hline HSA04668.TNF.SIGNALING.PATHWAY.KEGG & -1.69 & $1.57 \mathrm{E}-02$ \\
\hline HSA05167.KAPOSI.SARCOMA.ASSOCIATED.HERPESVIRUS.INFECTI & -1.69 & $1.55 \mathrm{E}-02$ \\
\hline
\end{tabular}


HSA00040.PENTOSE.AND.GLUCURONATE.INTERCONVERSIONS.KEGG

HSA04650.NATURAL.KILLER.CELL.MEDIATED.CYTOTOXICITY.KEGG

HSA04068.FOXO.SIGNALING.PATHWAY.KEGG

HSA05321.INFLAMMATORY.BOWEL.DISEASE.IBD..KEGG

HSA04380.OSTEOCLAST.DIFFERENTIATION.KEGG

HSA04920.ADIPOCYTOKINE.SIGNALING.PATHWAY.KEGG

HSA05416.VIRAL.MYOCARDITIS.KEGG

HSA00601.GLYCOSPHINGOLIPID.BIOSYNTHESIS...LACTO.AND.NEOLACTO.SERIES.KEGG

HSA05131.SHIGELLOSIS.KEGG

HSA04514.CELL.ADHESION.MOLECULES..CAMS..KEGG

HSA04919.THYROID.HORMONE.SIGNALING.PATHWAY.KEGG

HSA04024.CAMP.SIGNALING.PATHWAY.KEGG

HSA05163.HUMAN.CYTOMEGALOVIRUS.INFECTION.KEGG

HSA05142.CHAGAS.DISEASE..AMERICAN.TRYPANOSOMIASIS..KEGG

HSA04964.PROXIMAL.TUBULE.BICARBONATE.RECLAMATION.KEGG

HSA04630.JAK.STAT.SIGNALING.PATHWAY.KEGG

HSA00512.MUCIN.TYPE.O.GLYCAN.BIOSYNTHESIS.KEGG

HSA04261.ADRENERGIC.SIGNALING.IN.CARDIOMYOCYTES.KEGG

HSA04014.RAS.SIGNALING.PATHWAY.KEGG

HSA00513.VARIOUS.TYPES.OF.N.GLYCAN.BIOSYNTHESIS.KEGG 


\begin{tabular}{|c|c|c|}
\hline HSA05412.ARRHYTHMOGENIC.RIGHT.VENTRICULAR.CARDIOMYOPATHY..ARVC...KEGG & -1.52 & 4.45E-02 \\
\hline HSA04150.MTOR.SIGNALING.PATHWAY.KEGG & -1.51 & $4.66 \mathrm{E}-02$ \\
\hline HSA05210.COLORECTAL.CANCER.KEGG & -1.51 & 4.64E-02 \\
\hline HSA05410.HYPERTROPHIC.CARDIOMYOPATHY..HCM..KEGG & -1.51 & 4.75E-02 \\
\hline HSA05140.LEISHMANIASIS.KEGG & -1.51 & 4.81E-02 \\
\hline HSA04390.HIPPO.SIGNALING.PATHWAY.KEGG & -1.50 & 4.93E-02 \\
\hline HSA05162.MEASLES.KEGG & -1.50 & 4.90E-02 \\
\hline HSA00564.GLYCEROPHOSPHOLIPID.METABOLISM.KEGG & -1.50 & $5.01 \mathrm{E}-02$ \\
\hline HSA00592.ALPHA.LINOLENIC.ACID.METABOLISM.KEGG & -1.50 & $5.07 \mathrm{E}-02$ \\
\hline HSA04927.CORTISOL.SYNTHESIS.AND.SECRETION.KEGG & -1.49 & $5.08 \mathrm{E}-02$ \\
\hline HSA05212.PANCREATIC.CANCER.KEGG & -1.49 & $5.22 \mathrm{E}-02$ \\
\hline HSA05323.RHEUMATOID.ARTHRITIS.KEGG & -1.49 & $5.23 \mathrm{E}-02$ \\
\hline HSA04015.RAP1.SIGNALING.PATHWAY.KEGG & -1.49 & $5.21 \mathrm{E}-02$ \\
\hline HSA04625.C.TYPE.LECTIN.RECEPTOR.SIGNALING.PATHWAY.KEGG & -1.49 & $5.23 \mathrm{E}-02$ \\
\hline HSA01521.EGFR.TYROSINE.KINASE.INHIBITOR.RESISTANCE.KEGG & -1.48 & $5.32 \mathrm{E}-02$ \\
\hline HSA04614.RENIN.ANGIOTENSIN.SYSTEM.KEGG & -1.48 & $5.34 \mathrm{E}-02$ \\
\hline HSA05135.YERSINIA.INFECTION.KEGG & -1.48 & $5.37 \mathrm{E}-02$ \\
\hline HSA05231.CHOLINE.METABOLISM.IN.CANCER.KEGG & -1.48 & $5.36 \mathrm{E}-02$ \\
\hline HSA00561.GLYCEROLIPID.METABOLISM.KEGG & -1.47 & $5.55 \mathrm{E}-02$ \\
\hline HSA00591.LINOLEIC.ACID.METABOLISM.KEGG & -1.47 & $5.70 \mathrm{E}-02$ \\
\hline HSA00760.NICOTINATE.AND.NICOTINAMIDE.METABOLISM.KEGG & -1.47 & $5.70 \mathrm{E}-02$ \\
\hline HSA04072.PHOSPHOLIPASE.D.SIGNALING.PATHWAY.KEGG & -1.47 & $5.71 \mathrm{E}-02$ \\
\hline HSA00330.ARGININE.AND.PROLINE.METABOLISM.KEGG & -1.46 & $5.85 \mathrm{E}-02$ \\
\hline HSA04210.APOPTOSIS.KEGG & -1.46 & $5.85 \mathrm{E}-02$ \\
\hline HSA04658.TH1.AND.TH2.CELL.DIFFERENTIATION.KEGG & -1.46 & $5.93 E-02$ \\
\hline HSA04979.CHOLESTEROL.METABOLISM.KEGG & -1.46 & $5.96 \mathrm{E}-02$ \\
\hline HSA04728.DOPAMINERGIC.SYNAPSE.KEGG & -1.46 & $6.07 \mathrm{E}-02$ \\
\hline HSA00053.ASCORBATE.AND.ALDARATE.METABOLISM.KEGG & -1.45 & $6.48 \mathrm{E}-02$ \\
\hline HSA04060.CYTOKINE.CYTOKINE.RECEPTOR.INTERACTION.KEGG & -1.44 & $6.52 \mathrm{E}-02$ \\
\hline HSA00360.PHENYLALANINE.METABOLISM.KEGG & -1.44 & $6.48 \mathrm{E}-02$ \\
\hline HSA05219.BLADDER.CANCER.KEGG & -1.44 & $6.46 \mathrm{E}-02$ \\
\hline HSA05033.NICOTINE.ADDICTION.KEGG & -1.44 & $6.42 \mathrm{E}-02$ \\
\hline HSA00770.PANTOTHENATE.AND.COA.BIOSYNTHESIS.KEGG & -1.44 & $6.41 \mathrm{E}-02$ \\
\hline HSA04925.ALDOSTERONE.SYNTHESIS.AND.SECRETION.KEGG & -1.44 & $6.60 \mathrm{E}-02$ \\
\hline HSA05120.EPITHELIAL.CELL.SIGNALING.IN.HELICOBACTER.PYLORI.INFECTION.KEGG & -1.44 & $6.60 \mathrm{E}-02$ \\
\hline HSA05160.HEPATITIS.C.KEGG & -1.44 & $6.67 \mathrm{E}-02$ \\
\hline HSA00983.DRUG.METABOLISM...OTHER.ENZYMES.KEGG & -1.44 & $6.63 \mathrm{E}-02$ \\
\hline HSA00590.ARACHIDONIC.ACID.METABOLISM.KEGG & -1.43 & $6.64 \mathrm{E}-02$ \\
\hline HSA00565.ETHER.LIPID.METABOLISM.KEGG & -1.43 & $6.71 \mathrm{E}-02$ \\
\hline HSA04620.TOLL.LIKE.RECEPTOR.SIGNALING.PATHWAY.KEGG & -1.43 & $6.79 \mathrm{E}-02$ \\
\hline HSA04722.NEUROTROPHIN.SIGNALING.PATHWAY.KEGG & -1.43 & $6.80 \mathrm{E}-02$ \\
\hline HSA04933.AGE.RAGE.SIGNALING.PATHWAY.IN.DIABETIC.COMPLICATIONS.KEGG & -1.42 & $7.01 \mathrm{E}-02$ \\
\hline HSA04211.LONGEVITY.REGULATING.PATHWAY.KEGG & -1.42 & $6.97 \mathrm{E}-02$ \\
\hline HSA04062.CHEMOKINE.SIGNALING.PATHWAY.KEGG & -1.42 & 7.33E-02 \\
\hline
\end{tabular}


HSA04071.SPHINGOLIPID.SIGNALING.PATHWAY.KEGG

HSA04621.NOD.LIKE.RECEPTOR.SIGNALING.PATHWAY.KEGG

HSA04064.NF.KAPPA.B.SIGNALING.PATHWAY.KEGG

HSA05332.GRAFT.VERSUS.HOST.DISEASE.KEGG

HSA04360.AXON.GUIDANCE.KEGG

HSA05330.ALLOGRAFT.REJECTION.KEGG

HSA04934.CUSHING.SYNDROME.KEGG

HSA05152.TUBERCULOSIS.KEGG

HSA05215.PROSTATE.CANCER.KEGG

HSA04657.IL.17.SIGNALING.PATHWAY.KEGG

HSA04710.CIRCADIAN.RHYTHM.KEGG

HSA04940.TYPE.I.DIABETES.MELLITUS.KEGG

HSA04660.T.CELL.RECEPTOR.SIGNALING.PATHWAY.KEGG

HSA04726.SEROTONERGIC.SYNAPSE.KEGG

HSA04971.GASTRIC.ACID.SECRETION.KEGG

HSA04932.NON.ALCOHOLIC.FATTY.LIVER.DISEASE..NAFLD..KEGG

HSA04972.PANCREATIC.SECRETION.KEGG

HSA04540.GAP.JUNCTION.KEGG

HSA00730.THIAMINE.METABOLISM.KEGG

HSA04659.TH17.CELL.DIFFERENTIATION.KEGG

HSA05164.INFLUENZA.A.KEGG

HSA04670.LEUKOCYTE.TRANSENDOTHELIAL.MIGRATION.KEGG

HSA05016.HUNTINGTON.DISEASE.KEGG

HSA04935.GROWTH.HORMONE.SYNTHESIS..SECRETION.AND.ACTION.KEGG

HSA05226.GASTRIC.CANCER.KEGG

HSA04962.VASOPRESSIN.REGULATED.WATER.REABSORPTION.KEGG

HSA02010.ABC.TRANSPORTERS.KEGG

HSA04912.GNRH.SIGNALING.PATHWAY.KEGG

HSA04340.HEDGEHOG.SIGNALING.PATHWAY.KEGG

HSA04136.AUTOPHAGY...OTHER.KEGG

HSA05170.HUMAN.IMMUNODEFICIENCY.VIRUS.1.INFECTION.KEGG

HSA05218.MELANOMA.KEGG

HSA04611.PLATELET.ACTIVATION.KEGG

HSA04921.OXYTOCIN.SIGNALING.PATHWAY.KEGG

HSA05032.MORPHINE.ADDICTION.KEGG

HSA04730.LONG.TERM.DEPRESSION.KEGG

HSA00230.PURINE.METABOLISM.KEGG

HSA04218.CELLULAR.SENESCENCE.KEGG 
HSA05414.DILATED.CARDIOMYOPATHY..DCM..KEGG

$-1.26 \quad 1.68 \mathrm{E}-01$

HSA04713.CIRCADIAN.ENTRAINMENT.KEGG

$-1.25 \quad 1.76 \mathrm{E}-01$

HSA04080.NEUROACTIVE.LIGAND.RECEPTOR.INTERACTION.KEGG

$-1.25 \quad 1.79 \mathrm{E}-01$

HSA04612.ANTIGEN.PROCESSING.AND.PRESENTATION.KEGG

$-1.25 \quad 1.79 \mathrm{E}-01$

HSA04012.ERBB.SIGNALING.PATHWAY.KEGG

$-1.24 \quad 1.85 \mathrm{E}-01$

HSA04141.PROTEIN.PROCESSING.IN.ENDOPLASMIC.RETICULUM.KEGG

$-1.24 \quad 1.86 \mathrm{E}-01$

HSA00563.GLYCOSYLPHOSPHATIDYLINOSITOL..GPI..ANCHOR.BIOSYNTHESIS.KEGG

$-1.24$

HSA00410.BETA.ALANINE.METABOLISM.KEGG

$-1.23$

HSA04924.RENIN.SECRETION.KEGG

HSA04929.GNRH.SECRETION.KEGG

HSA04723.RETROGRADE.ENDOCANNABINOID.SIGNALING.KEGG

HSA04217.NECROPTOSIS.KEGG

HSA04270.VASCULAR.SMOOTH.MUSCLE.CONTRACTION.KEGG

HSA04622.RIG.I.LIKE.RECEPTOR.SIGNALING.PATHWAY.KEGG

HSA05143.AFRICAN.TRYPANOSOMIASIS.KEGG

HSA04923.REGULATION.OF.LIPOLYSIS.IN.ADIPOCYTES.KEGG

HSA00062.FATTY.ACID.ELONGATION.KEGG

HSA04970.SALIVARY.SECRETION.KEGG

HSA05320.AUTOIMMUNE.THYROID.DISEASE.KEGG

HSA05161.HEPATITIS.B.KEGG

HSA05224.BREAST.CANCER.KEGG

HSA04146.PEROXISOME.KEGG

HSA05206.MICRORNAS.IN.CANCER.KEGG

HSA04666.FC.GAMMA.R.MEDIATED.PHAGOCYTOSIS.KEGG

HSA05166.HUMAN.T.CELL.LEUKEMIA.VIRUS.1.INFECTION.KEGG

HSA04724.GLUTAMATERGIC.SYNAPSE.KEGG

HSA05030.COCAINE.ADDICTION.KEGG

HSA05133.PERTUSSIS.KEGG

HSA05221.ACUTE.MYELOID.LEUKEMIA.KEGG

HSA04213.LONGEVITY.REGULATING.PATHWAY...MULTIPLE.SPECIES.KEGG

HSA05220.CHRONIC.MYELOID.LEUKEMIA.KEGG

HSA05223.NON.SMALL.CELL.LUNG.CANCER.KEGG

HSA00071.FATTY.ACID.DEGRADATION.KEGG

HSA05225.HEPATOCELLULAR.CARCINOMA.KEGG

HSA00790.FOLATE.BIOSYNTHESIS.KEGG

HSA04061.VIRAL.PROTEIN.INTERACTION.WITH.CYTOKINE.AND.CYTOKINE.RECEPTOR.KEGG 
HSA04913.OVARIAN.STEROIDOGENESIS.KEGG

HSA00350.TYROSINE.METABOLISM.KEGG

HSA01523.ANTIFOLATE.RESISTANCE.KEGG

HSA04310.WNT.SIGNALING.PATHWAY.KEGG

HSA01040.BIOSYNTHESIS.OF.UNSATURATED.FATTY.ACIDS.KEGG

HSA00260.GLYCINE..SERINE.AND.THREONINE.METABOLISM.KEGG

HSA05017.SPINOCEREBELLAR.ATAXIA.KEGG

HSA04750.INFLAMMATORY.MEDIATOR.REGULATION.OF.TRP.CHANNELS.KEGG

HSA00532.GLYCOSAMINOGLYCAN.BIOSYNTHESIS...CHONDROITIN.SULFATE...DERMATAN.SULFATE. KEGG

HSA00190.OXIDATIVE.PHOSPHORYLATION.KEGG

HSA05031.AMPHETAMINE.ADDICTION.KEGG

HSA04744.PHOTOTRANSDUCTION.KEGG

HSA00604.GLYCOSPHINGOLIPID.BIOSYNTHESIS...GANGLIO.SERIES.KEGG

HSA00534.GLYCOSAMINOGLYCAN.BIOSYNTHESIS...HEPARAN.SULFATE...HEPARIN.KEGG

HSA05010.ALZHEIMER.DISEASE.KEGG

$-1.01 \quad 4.77 \mathrm{E}-01$

HSA04720.LONG.TERM.POTENTIATION.KEGG

HSA00220.ARGININE.BIOSYNTHESIS.KEGG

HSA04370.VEGF.SIGNALING.PATHWAY.KEGG

HSA00450.SELENOCOMPOUND.METABOLISM.KEGG

HSA00100.STEROID.BIOSYNTHESIS.KEGG

HSA01522.ENDOCRINE.RESISTANCE.KEGG

HSA04664.FC.EPSILON.RI.SIGNALING.PATHWAY.KEGG

HSA05012.PARKINSON.DISEASE.KEGG

HSA03010.RIBOSOME.KEGG

HSA04215.APOPTOSIS...MULTIPLE.SPECIES.KEGG

HSA04714.THERMOGENESIS.KEGG

HSA04392.HIPPO.SIGNALING.PATHWAY...MULTIPLE.SPECIES.KEGG 


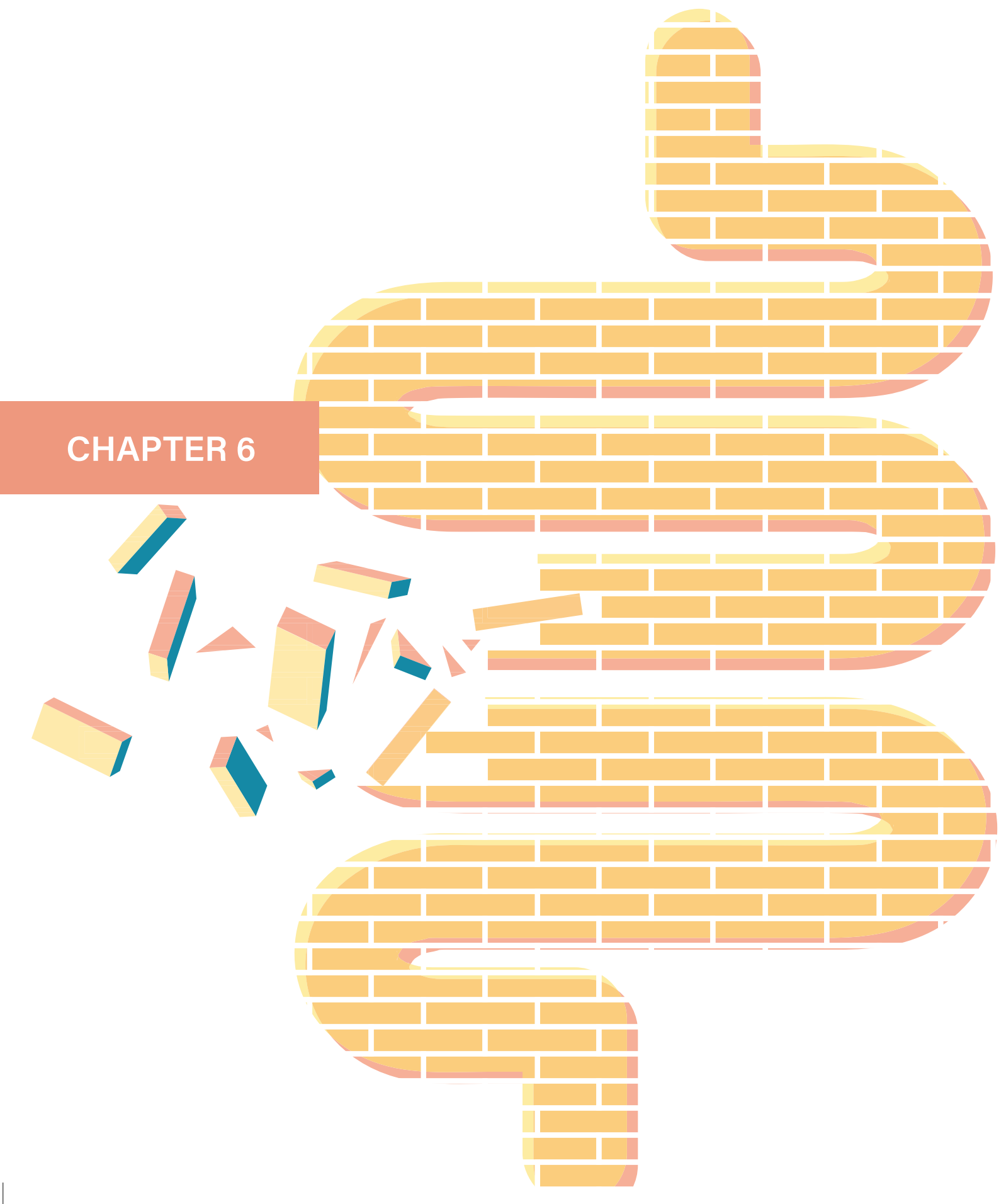


General discussion 
The urgency to obtain increased scientific knowledge in the field of gastrointestinal (GI) health is emphasized by the alarming fact that $40 \%$ of the worldwide population suffers from functional GI disorders (1). This not only results in an increase in global health care costs, but also in a decreased quality of life (1). Although gastric and esophageal disorders are all categories of GI disorders, in this thesis, we only focused on the intestine. Despite the advances in understanding intestinal health, still many underlying mechanisms remain elusive. In order to reduce the socioeconomic burden, it is of utmost important to increase our knowledge on intestinal health and the underlying mechanisms of intestinal pathophysiology. Only then, steps can be made towards reducing the prevalence of intestinal disorders on the one hand, and developing pharmacological or nutritional therapies to treat these diseases efficiently and effectively on the other hand. Therefore, the aim of this thesis was to investigate how multiple important aspects, including the aging process, gut microbiota, and bile acids, can affect intestinal health. To this end, we used physiologically relevant in vivo and in vitro models. In Chapter 2, we demonstrated that the aging process is associated with a pronounced change in gut microbiota composition and colonic gene expression, which are likely acting in concert to mediate colonic health during aging. We also observed a strong aging-related decrease in the abundance of the mucin-degrading bacterium A. muciniphila. In Chapter 3, we investigated the potential health-promoting effects of $A$. muciniphila by supplementing progeroid mice with this bacterium for 10 weeks. We found promising health-promoting effects of $A$. muciniphila with regard to the colonic mucus layer and immune-related processes. In both in vivo studies, the general dogma stating that aging-related microbial dysbiosis is related to impaired intestinal barrier function and intestinal inflammation is central. However, the molecular mechanisms underlying this negative feedback loop are not known. In this context, bacterial metabolites can act as key molecular mediators and are thus a highly relevant area of investigation. In Chapter 4, we investigated the role of sulfated BAs, which were previously shown to be elevated in IBD patients, on the progression of chronic intestinal inflammation. Although we found minor effects of sulfated secondary BAs on intestinal permeability, our evidence pointed towards that sulfated secondary BAs abolished the anti-inflammatory effects of secondary BAs when exposed directly to DCs. In in vitro research, the selection of a physiologically relevant model is a crucial aspect while designing a study. With regard to intestinal health, the mucus layer is often underrepresented in widely used models. To approach the intestinal barrier function in a more physiological way, we investigated the SWMS growing method to enhance mucus production in HT29-MTX-E12 cells in Chapter 5. This increased insight might ultimately lead to potential recommendations to further improve in vitro models to study intestinal health. 


\section{A definition of intestinal health}

Like anywhere in the body, it also applies to the intestine that homeostasis is a key aspect of health. Fundamental factors that are involved as determinants of intestinal health include a balanced microbiota composition, a well-functioning intestinal barrier, and the absence of inflammation, amongst others (2). Because of the intensive crosstalk between these factors, disturbance of one factor likely renders the others prone to disturbance as well. This vicious cycle is implied in a range of disorders and diseases of the intestinal tract, but also beyond. It is the strong involvement of the intestine in overall health that makes this a crucial area of investigation. However, before diving deeper into this research field, it is first important to discuss what intestinal health exactly involves. In 2011, a group of researchers determined five major criteria to define a healthy intestine. Some of these definitions are rather clear, such as "An effective digestion and absorption of food" and "The absence of Gl illness" (3). However, other definitions could be interpreted in a less objective way. For example, the criterium "A normal and stable microbiota" might sound straightforward, but it is unclear what the scientific definition of 'normal' and 'stable' is. In the context of a healthy microbiota composition, 'dysbiosis' is a widely used and acknowledged term. Dysbiosis is often defined as the shift in microbial composition that is associated with pathology (4). More specifically, dysbiosis is considered as a change in resident bacteria relative to the gut microbiota of healthy individuals, including a loss of beneficial bacteria, an increase in potential pathobionts, and a decreased overall diversity (5). However, results from a recent study might question the generalizability of this definition towards all diseases. This meta-analysis showed that different diseases are associated with distinct types of dysbiosis (6). For example, the gut microbiota composition of colorectal cancer patients is characterized by an increase in pathobionts, while IBD patients are characterized by a predominant depletion of beneficial bacteria (6). Additionally, an important factor that should be taken into account is the influence of age on disease-associated dysbiosis. A recent study investigated the effect of age on the gut microbiota composition in five disorders: IBD, intestinal polyps, colorectal cancer, type 2 diabetes and liver cirrhosis (7). Age-specific trends in microbiota-disease associations were found, for example, younger subjects tended to gain disease-associated gut microbiota, while elderly subjects lost gut microbiota that are usually associated with health (7). The implication of age as a confounder emphasizes the importance to control for age to improve the robustness of microbiota studies. Altogether, it can be concluded that a more detailed description of disease-specific dysbiosis should be implemented with due consideration of potential confounders. Looking more carefully into intestinal dysbiosis is important, since a specific microbial profile could serve as determinant for predicting the manifestation of a disease or disorder (8). Ultimately, this approach could serve a 
therapeutic goal by specifically targeting bacterial species, e.g. removal of harmful bacteria, and/or administration of beneficial bacteria, to eventually alleviate or cure intestinal disorders.

\section{Hallmarks of the aging intestinal tract}

Over the last decades, extensive research has resulted in a substantially increased understanding in gut microbiota composition during health and disease, but also during different stages of life. The aging process is characterized by marked shifts in gut microbiota composition, which is associated with physical fitness and frailty $(9,10)$. On the contrary, it remains relatively unexplored how this microbial shift is induced and whether this is a cause or consequence of impaired health. However, it is unlikely that the causes are constrained by only a few factors, since a plethora of lifestyle, environmental and genetic factors might be involved. A central dogma states that diet has a dominating impact on shaping the gut microbiota composition (11). However, this dogma is rejected in a recent study showing that the influence of host physiology was substantially greater than dietary factors in primates (12). It was also shown that in Drosophila, the prevention of age-related changes in intestinal physiology limited microbial dysbiosis, and resulted in extended lifespan (13). Next to the influence of physiological factors, several drugs, such as antibiotics or proton pump inhibitors, could also lead to a microbial shift $(14,15)$. These findings might lead to the large heterogeneity in aging studies. Another important factor that might add to the high variety observed in gut microbiota composition in human subjects, is that the human gut microbiota can be classified in three different enterotypes, which are quite robust among different populations (16). However, the robustness, and even the existence of enterotypes is currently under debate (17). Nevertheless, the question rises whether a random group of healthy individuals is a valid control group for gut microbiota analysis, or that control groups should match the subjects of interest more closely with regard to e.g. lifestyle, diet, gender, and, potentially, enterotype. An important disadvantage of this strategy is the increased effort, and thus time and money, that are involved in the more extensive screening process to select eligible control subjects. The highly controlled environment that is maintained during animal studies (i.e. genetic background, diet and gender) eliminate a number of aforementioned factors. Furthermore, in the context of gut microbiota analysis, the use of animals allows the sampling of colonic luminal content, instead of the dependence on fecal samples that is most often the case in human studies. It is clear that the gut microbiota composition in fecal samples is different compared to luminal content (18). Indeed, in Chapter 2, we found differences between fecal and colonic microbiota composition, although these differences were subtle. Therefore, based on our results, fecal samples could still serve as reliable source to examine gut microbiota, at least in mice. However, when sampling luminal 
content, it is important to consider the large diversity between different locations throughout the intestinal tract $(19,20)$. Newly developed techniques to sample luminal content, e.g. via catheter aspiration or other sampling devices (21), could be useful strategies to isolate luminal content from different parts of the intestine. These techniques are promising, especially with regard to sampling at different time points, which can be very useful to study time-dependent effects of gut microbiota composition. However, there are also challenges to overcome, for example to successfully sample the relatively firm colonic content, but also to preserve the intestinal sample (22).

\section{From an observational to a functional approach}

The complexity of the gut microbiota is also emphasized by the fact that a repertoire of bacterial species residing in the intestine remains to be discovered (23). Indeed, in Chapter 2, we detected a considerable number of undefined species, limiting us to fully specify bacteria at the species level. Instead, we were restricted to define some present bacteria at genus or even family or order level, which might have led to a less complete interpretation of the data, given the high variety of bacteria with different functions within taxa. In the future, this limitation will most probably become less prevalent, as the rapid development of high-throughput sequencing techniques facilitates the characterization of newly discovered species (23). Nevertheless, the question rises whether it would be more appropriate to shift our focus from the observational approach ("Who is present?") to a more functional approach ("What do they do?" and "How do they do it?"). One possible approach in this regard is to investigate the presence of bacterial metabolites, as these could have important effects on intestinal barrier function and mucosal immune response, and are therefore considered as the communicating factor between the microbe and host (24). Importantly, profiling shifts in bacterial metabolites is the first step towards the transition from observational studies to studies investigating cause-and-effect relationships of specific bacterial metabolites and their effect on (impaired) intestinal health. In Chapter 2, we determined age-related shifts in metabolites present in colonic luminal content using ${ }^{1} \mathrm{H}-\mathrm{NMR}$. It is noteworthy to mention that the metabolites present in colonic luminal content are not by definition derived from the gut microbiota, but can also be derived from food or host cell metabolism. In Chapter 2, we aimed to get clues on the origin of metabolites in colonic luminal content. Therefore, we correlated the levels of significantly altered metabolites to the relative abundances of the most present genera. These results could serve as starting point for future studies investigating the causal effects of these bacteria-derived metabolites on markers of intestinal health. However, instead of just correlation analyses, more sophisticated methods exist that can fully profile the function of the present bacterial communities by sequencing the complete 
microbial genome present in a sample. This shotgun metagenomics approach opens new and promising avenues in, amongst others, unraveling complex host-microbe interactions (25). Despite the technical breakthrough, an important limitation of this next-generation sequencing method is the high costs (25). However, the high demand for this technique already led to a rapid decline in the costs. In addition to the taxonomic approach that we performed in Chapter 2, a metagenomic approach would also have been useful for this study. Instead, we focused on multivariate methods to integrate transcriptomic and microbiome data to find clues about the interactions between bacteria and host in the vicinity of the colonic wall. For example, we found that the strong downregulation of genes encoding for AMPs at old age was positively correlated with an uncultured member of the order Gastranaerophilales. A plausible hypothesis would be that the decreased relative abundance of this bacterium could be related to an impaired intestinal barrier function by causing a downregulation of AMPs during aging. However, it is important to realize that multivariate- and metagenomic analyses do not provide information about the bacterial products that are ultimately present. In this regard, the use of metabolomic analysis is highly important.

\section{Lessons from extreme longevity studies: the case of Akkermansia muciniphila}

As important mediator of intestinal health, gut microbiota composition is proposed to be a highly relevant candidate to manipulate as a strategy to improve healthy aging. However, it is first important to obtain insight in the bacterial species that are involved in healthy aging. To this end, a highly relevant study population include subjects with extreme longevity, i.e. centenarians; people older than 100 years. These subjects are reported to reach this extreme age with lower incidence of chronic diseases (26), and lower hospitalization rates (27), as compared to younger subjects. The analysis of the gut microbiota composition in centenarians could therefore provide extremely useful information about specific bacterial species that might be related to reaching an extremely high age in a healthy way. Although it remains unknown whether the gut microbiota composition is causal or rather a consequence of the lifestyle of these subjects, it will still provide useful information that can be used for future studies. In this sense, a key example of a 'centenarian-enriched' species is $A$. muciniphila, which was found to be increased in centenarians (28-30). In mice, the abundance of this bacterium was shown to be negatively correlated with inflammation, glucose intolerance and insulin resistance $(31,32)$. In humans, negative correlations were also found between $A$. muciniphila and both overweight and obesity, as well as markers of insulin resistance and hypertension (33-35). These associative studies provide a solid base to proceed to the next step: performing proof-of-concept studies to determine a potential causal link. In Chapter 3, we showed that supplementing progeroid 
mice with A. muciniphila resulted in, amongst others, attenuation of inflammation, suggesting that supplementation could be an effective strategy to improve inflammaging. The beneficial effects of this bacterium were also proven in humans. Although this study focused specifically on metabolic effects rather than the aging process, three months of $A$. muciniphila supplementation improved metabolic and inflammatory parameters in obese and insulin-resistant human subjects (36). The same study also confirmed the safety and tolerance of supplementation in humans (36). Altogether, these data open avenues for the application of $A$. muciniphila supplementation as a strategy to improve health. Interestingly, supplementation with pasteurized A. muciniphila was also shown to be effective in mice $(37,38)$ and humans $(36)$. The use of pasteurized bacteria improves the applicability of supplementation, as the use of live bacteria may pose difficulties with regard to shelf life and viability, and thus the degree of efficacy. Moreover, pasteurized A. muciniphila was recently shown to be safe as a food ingredient (39). It is clear that $A$. muciniphila supplementation is a potent strategy to improve health, however, until now, all studies mainly focused on obese/overweight, middleaged subjects that suffer from metabolic disease. Although this study population can be generally considered as metabolically unhealthy, it is impossible to directly translate the effectiveness, safety and tolerability of $A$. muciniphila supplementation of these subjects into elderly subjects. This issue even applies more when taking into account frail elderly. Although speculative, it might be possible that the GI tract and immune system of (frail) elderly is less able to cope with a bulk of bacteria, which may result in adverse health effects, instead of the intended beneficial effects (40). In a parallel arm of the study described in Chapter 3, supplementation with the probiotic bacterium Bifidobacterium breve had adverse effects on mucus layer thickness and immune function in progeroid mice (41). With regard to $A$. muciniphila, there is evidence that the presence of this bacterium during Salmonella typhimurium infection exacerbates inflammation (42). A lower number of goblet cells was found, together with a loss of mucin sulphation, indicating that $A$. muciniphila caused disturbance of mucus homeostasis (42). The ability of $A$. muciniphila acting as a pathobiont emphasizes that probiotic supplementation of this bacterium in persons with underlying (inflammatory) diseases, e.g. frail elderly, should be considered with great awareness. Another important field of investigation is to determine the most effective mode of administration for elderly persons. For example, oral administration via a capsule may be less suitable for elderly compared to fluidic supplementation, which is more easy to swallow. Altogether, although the use of $A$. muciniphila supplementation is a promising therapeutic strategy to improve health, the effectiveness and applicability in elderly subjects should be carefully investigated in future studies. 


\section{Metabolomics and metagenomics as tools to understand healthy aging}

The relevance of discovering centenarian-specific signature species and consider these as biomarkers for healthy aging could also be debated. First, just like in any other population, high variation is observed in centenarians as well. This could lead to misinterpretation of results, ultimately leading to incorrect assignation of species to be centenarian-specific. For example, a Chinese study showed that $A$. muciniphila abundance was declined in centenarians (43), which might hamper the general consideration that this bacterium is centenarian-specific. Although this single study is unlikely to question the plethora of other studies confirming the effectiveness of $A$. muciniphila supplementation, interindividual variation could pose difficulties in interpreting results regarding other bacterial species yet to be discovered. One way to overcome this issue is to focus more on potential confounders, such as diet, living situation, and geographical location, amongst others, as these factors are known to have a profound effect on gut microbiota composition (44). Secondly, in the process of using centenarian-enriched species as a strategy to improve healthy aging, one crucial aspect is often neglected; i.e. the underlying mechanism through which the bacterium exerts the beneficial health effects. In this sense, not necessarily the presence of certain bacteria is important, but rather the function of these bacteria. To this end, bacterial metabolite analysis might provide useful information. In a large cohort study, it was shown that healthy aging was characterized by a unique gut microbiota composition, which was reflected by a distinct plasma metabolite profile (45). On the other hand, the use of metagenomic sequencing methods in elderly or centenarian subjects could provide useful information about the pathways through which the present microbiota exert their health-related effects. For example, shotgun metagenomics of fecal microbiota in an Italian cohort revealed an age-related increase in reads for genes related to xenobiotic degradation, which was even stronger in centenarians and supercentenarians (aged 104-109 years) (46). It is noteworthy to mention that this functionality was assigned to commensal bacteria that belong to the more general human core microbiome (46). The authors speculated that this observation was due to an adaptive response of the gut microbiota to the increased exposure to xenobiotics in these long-lived subjects. Exposure to xenobiotics is related to an increased risk of autoimmune disease and cancer $(47,48)$, but also to intestinal diseases $(49)$. Therefore, an increased capacity of the gut microbiota to degrade xenobiotics might therefore be considered as a health-promoting microbial shift, particularly with regard to the aging process, as accumulation of xenobiotics may occur in long-lived people. Hence, the performance of studies in this field of 'toxicomicrobiomics' is relevant particularly with regard to the aging process. 


\section{Disentangling the mechanisms underlying healthy aging through probiotics}

Given the fact that the influence of host physiology plays a major role in shaping gut microbiota composition $(12,13)$, it could be speculated that prevention of age-related changes in intestinal physiology is a useful target to improve intestinal health, instead of, for example, focus on dietary strategies. On the other hand, this approach also poses limitations. For example, techniques to determine changes in human intestinal physiology might be challenging due to technical or ethical concerns, especially in elderly. Moreover, not all bacterial species rely on nutrients derived from the food ingested by the host, but rather on alternative sources that are independent of diet. A good example includes the group of mucin-metabolizing bacteria that reside in the mucus layer and degrade mucin glycans, such as strains within the genera Bacteroides, Ruminococcus, Bifidobacterium and Akkermansia (50). This, together with the observation that the colonic mucus layer decreases with age in mice $(41,51,52)$ emphasizes the importance of investigating both the colonic mucus layer and mucin-degrading bacteria in the context of healthy aging. Unfortunately, current available data is mostly restricted to animal studies, which is most probably due to the practical issues of successfully isolating mucus in vivo. Nevertheless, an especially important candidate to manipulate the colonic mucus layer is A. muciniphila, In Chapter 3, the observed attenuated inflammation and immunerelated processes in mice supplemented with $A$. muciniphila might be the result of a reinforced mucus layer induced by this bacterium, as this is a crucial factor for a strong intestinal barrier that keeps potential health-disturbing compounds at bay. However, our study design was not suitable to establish if this role was indeed causative. Therefore, a highly relevant and fundamental issue would be the mechanism underlying the increased mucus layer thickness induced by $A$. muciniphila. The combined mucin-degrading and mucus-building capacities of this bacterium seem contradictive, although both properties are well-established (53-55). With regard to the mucus layer, another question that rises is what the effect $A$. muciniphila on mucus quality would be, i.e. the effects on firmness and stability of the mucus layer, but also the presence and accessibility of glycans, amongst others. Unraveling these mechanisms is important to be able to comprehend the health-promoting effects of $A$. muciniphila and perhaps even to (pharmacologically) target the mucus layer without the use of this mucus-promoting bacterium. This could be promising, especially with regard to susceptible elderly. Although the use of commonly used probiotics is generally safe, application in (extremely) frail elderly might pose a potential risk if side effects occur. A more direct way to target the mucus layer by using drugs that act through the same mechanism as $A$. muciniphila might be a solution to prevent these probiotic-induced side effects. 


\section{Dysbiosis as common denominator for aging and IBD}

The existence of dysbiosis during the aging process is clear. Dysbiosis is also heavily involved in the pathogenesis of IBD (56). Obviously, fundamental differences exist in pathways involved in the aging process and IBD pathology, however, a remarkable resemblance in dysbiosis is found, characterized by a loss of health-associated species (6). This could imply that therapeutic interventions aimed to restore these beneficial species, e.g. pre- and probiotic use, might be a useful treatment for both aging- and IBD-specific dysbiosis. Speculatively, the similarity in dysbiosis might also lead to a similar shift in bacterial metabolite production. However, this speculation requires more insight in specific bacterial species and the metabolites produced. In Chapter 2, we found a significant decrease in an unspecified bile acid in old mice. Although our method was not sensitive enough to profile this BA more specifically, it could be ruled out that it is one of the BAs identified in our previous study (57), i.e. cholic acid, (tauro)deoxycholic acid, or chenodeoxycholic acid. In a recent study, an increased ratio of primary to secondary BAs was found in colonic content of both male and female mice, which was concomitant with a decreased relative abundance of bacteria associated with BA deconjugation and 7-dehydroxylation (58). A decreased abundance of these bacteria, and thus decreased enzymatic activity, could have led to the observed decrease in secondary BAs. Moreover, a decreased relative abundance of Peptococcus was found in old female mice, which is a genus involved in BA desulfation (59). Although the amount of sulfated BAs was not measured, an impaired desulfation capacity may have led to an increase in sulfated BAs, which was found to be a consequence of a dysbiosis-related decrease in enzymatic activity in IBD patients (60). Interestingly, after co-housing with young mice, both the primary to secondary BA ratio and relative abundance of Peptococcus restored again to a similar extent to that of young mice (58). These results highlight the potential similarities in dysbiosis, and consequently, shifts in bacterial metabolites between the aging process and IBD. However, much more research is needed to understand the fundaments of BA-driven disease progression. In this regard, data from observational studies could serve as starting point to studies investigating the causal relationships.

\section{Secondary bile acids as drivers or preventers of intestinal inflammation: sulfation feeds the controversy}

The implication of BAs on disease progression has been subject to controversy, given the contrasting results found in several studies investigating BAs. For example, secondary BAs have been shown to cause higher intestinal permeability in Caco-2 cells $(61,62)$ and in mice (63). Furthermore, secondary BAs were also related to higher colon cancer incidence $(64,65)$. On the other hand, secondary BAs 
were shown to have anti-inflammatory properties in mice $(66,67)$ and in vitro studies $(60,68)$. These contradictive results could indicate that the effects of BAs are context-dependent, for example the concentration and type of $\mathrm{BA}$, and the tissue where the BAs are present. In the case of secondary BAs, multiple subtypes are present in the body, for example secondary BAs conjugated with taurine, glycine or sulfate-groups $(69,70)$. However, these conjugated secondary BA are less prevalent compared to their unconjugated form, which might be a plausible cause of the relatively low amount of studies performed on their potential health effects. In the context of the aforementioned dysbiosisrelated decrease in enzymatic activity in IBD patients (60), we aimed to investigate the causal role of sulfated secondary BAs on IBD progression in Chapter 4. The choice to investigate this type of BA may add to the general BA controversy, since it is generally accepted that sulfation is a process to detoxify and eliminate the normally cytotoxic secondary BAs (71). Therefore, the hypothesized involvement of sulfated BAs in IBD progression might sound counterintuitive, which is emphasized by the fact that only a low amount of studies have been published on the role of sulfated BAs in any disease. The literature that is available is often dated, as most studies are published over 40 years ago. The first matter of debate is the question whether sulfated BAs are absorbed by the intestinal cells, due to their hydrophilic character. In Chapter 4, we found that expression of FXR-target genes in intestinal cells was not altered after exposure to sulfated LCA and sulfated DCA, indicating that these BAs are most probably not taken up by the cells to activate FXR. On the other hand, sulfated BAs may possibly activate TGR5, since this is a membrane-bound BA receptor and might therefore be activated without intracellular uptake of the ligand. Unfortunately, we were not able to measure TGR5 activity. It would have been worthwhile to investigate this, for example by a luciferase reporter assay or by measuring intracellular cAMP levels, which is induced by TGR5 (72). This is particularly important, since several studies showed that TGR5 is implicated in in, amongst others, intestinal barrier integrity (73) and regeneration of injured intestinal tissue in intestinal stem cells (74). Moreover, TGR5 is also implicated in immune response, since this receptor is expressed on immune cells (68), and both natural TGR5 agonists (DCA and LCA) as well as a synthetic TGR5 agonist suppressed TNF- $\alpha$ production in macrophages via the TGR5-cAMP pathway (75). Potentially, IL-10 plays an important role, since TGR5 was shown to regulate $I L-10$ gene expression in a macrophage cell line (76). Although sulfated BAs had only minor effects on intestinal barrier function in Chapter 4, our results pointed towards an abolishment of anti-inflammatory effects exerted by secondary BAs. Future studies should focus on molecular pathways underlying these effects and the implication on IBD progression. Furthermore, a more detailed exploration of the effects on the mucus layer would also be highly relevant, given the important role of the mucus layer in intestinal health. In Chapter 
4, we only investigated the expression of MUC2 and MUC5AC, while examination of other relevant parameters regarding mucus quantity and quality will provide give more complete information. However, with regard to in-depth investigation of the mucus layer, more effort should first be made towards the establishment of relevant and reproducible techniques.

\section{Challenges of investigating the mucus layer}

Although it is clear that the mucus layer plays an extremely important role in health and disease, obtaining mechanistic insight is not straightforward. For example, mucins are extremely big proteins (MUC2 is $540 \mathrm{kDa}$ ) and are subject to complex post-transcriptional modifications $(77,78)$. Moreover, isolation of the mucus layer also faces some technical challenges. For example, mucus is transparent and has a high water content, which makes isolation and preservation of the mucus layer challenging and therefore requires specialized expertise (79). Where other aspects of the intestinal barrier are relatively easily performed, i.e. a FITC-dextran assay to measure intestinal permeability, suchlike assays to investigate the mucus layer in vivo are not widely described yet. Currently, animal models are commonly used, that allow the collection of intestinal tissue and analysis of the mucus layer upon sacrifice. In Chapter 3, we used histological techniques, including a Carnoy fixation of intestinal tissue to preserve the mucus layer, and subsequently a PAS/Alcian blue to stain mucins. The dependency on intestinal tissue to investigate mucus poses an important disadvantage, since it is not possible to measure the mucus layer in vivo at different time points. The relevance of timedependent mucus measurements is important given the fact that the mucus layer thickness is highly dynamic (80). Consequently, a single measurement could lead to incomplete or false interpretations. Alternatively, it is possible to isolate mucus derived from colonic biopsies and determine the mucus quality in an ex vivo manner, as described previously in IBD patients (79). This study indicates that it is possible to isolate mucus samples in humans, however, the feasibility remains doubtful due to the invasiveness of taking a biopsy. Furthermore, it could be questionable whether the use of ex vivo techniques to investigate mucus characteristics are completely representative for the in vivo the mucus layer, or if, for example, the composition of isolated mucus changes once outside the body. Importantly, as an increasing number of studies are focusing on the involvement of the mucus layer in health and disease, it is of utmost importance to establish methods to successfully isolate mucus in vivo, preferably in a non-invasive manner. Although speculative, a potential direction in this regard could be the use of biomarkers to assess mucus quantity and/or quality. Mucus is excreted via feces, implicating that mucus can be directly isolated from feces. Indeed, mucus isolation from feces was described in a previous study, although the authors aimed to examine colonocyte numbers 
present in mucus instead of investigating mucus properties (81). Subsequent methods to assess mucus quantity could be to measure total mucin content, for example by an Alcian Blue Mucin Assay (82). Besides, measuring other factors present in mucus that are indispensable for a strong mucus layer might also provide useful information on mucus quality. Obviously, the usefulness of these potential novel measurements should be investigated and optimized carefully before it can be applied in studies.

\section{Modeling the in vitro mucus layer: from static to dynamic conditions}

Since in vivo methods to investigate the mucus layer are to be optimized and ex vivo techniques also require specialized equipment and expertise, in vitro models still remain a useful alternative to investigate mucus properties. However, in commonly used in vitro models representing the intestine, the mucus layer is often overlooked, and if present, poorly representative. For example, frequently used colonic cell lines, including Caco-2 and T84 cells, produce only a limited amount of gel-forming mucins, which makes them irrelevant when used as a mono-culture. When grown in a co-culture with a mucus-producing cell line, e.g. HT29-MTX, the representativeness is slightly improved. However, important disadvantages of HT29-MTX cells is that, when grown statically, only a thin mucus layer is secreted, which predominantly exists of MUC5AC, which is a mucin typically present in the stomach and lungs (83). The intestinal MUC2 is also present, but to a lesser extent (84). An important factor that impedes the in vitro translatability of the in vivo situation is that current in vitro models are often static, while in the in vivo situation is highly dynamic. To overcome this, Navabi and colleagues established the semi-wet interface with mechanical stimulation (SWMS) method that resulted in a thick and adherent mucus layer, which predominantly consisted of MUC2 (84). In Chapter 5, we confirmed that the SWMS method indeed resulted in a shift in mucin composition, i.e. a higher MUC2/MUC5AC ratio. However, the exact mechanisms by which the SWMS method resulted in increased mucus production remain elusive. The aim in Chapter 5 was to improve our understanding in the underlying mechanisms, which could ultimately help to improve the representativeness of the in vitro mucus layer. We found that SWMS culture conditions resulted in increased cell growth and a shift towards a more aerobic cell metabolism, which might be the driving factors behind the increased mucus production. With regard to the implications for SWMS methods in current in vitro cell culture models, the obvious question that rises is if this method indeed results in an improved model. Based on our findings, the SWMS method can indeed result in an improved mucus layer with regard to the mucin composition. Therefore, if a model is needed that only requires the presence of a mucus layer, e.g. drug-disposition studies or the exposure of bacterial metabolites, the SWMS 
method is a relatively simple yet effective strategy. In Chapter 4, we deemed the SWMS method suitable to apply in our study design, since we aimed to establish a physiological relevant in vitro model representing the inflammatory situation as observed during IBD. As first line of defence, the mucus layer is indispensable for intestinal barrier function and is therefore crucial to include in an IBD model. By using activated DCs to create a pro-inflammatory environment, we hypothesized that both the mucus layer and intestinal epithelial cell layer were negatively affected. Indeed, we found a significantly increased intestinal permeability of the monolayer. In addition, we found a strong decreased expression of MUC2 and MUC5AC in intestinal cells exposed to activated DCs compared to unexposed control cells. However, we did not dive deeper into mucus properties, as we were unable to isolate the mucus layer from the cell culture inserts.

In mucus research, not only the mucus quantity is important, but also the mucus quality. Although the determinants of mucus quantity are sufficiently established (e.g. mucus layer thickness and mucin content), some methods to measure mucus quality are described, however, these methods are not widely used yet. An explanation for this could be similar to the aforementioned example, i.e. the specialized expertise needed to perform such methods. A relevant method with regard to mucus quality is to measure mucus penetrability using fluorescent beads that have a similar size as bacteria. This method, described by Gustafsson et al. (80), provides useful information on an important functional property of mucus, i.e. the capacity to avoid penetration of bacteria into the mucus layer. Until now, this method is only described using tissue explants grown ex vivo on a perfusion chamber (80). It would be highly relevant if suchlike methods could be applied in in vitro settings as well. In Chapter 5, we attempted to use this method on HT29-MTX-E12 cells grown on Transwell membranes. Although the preliminary outcomes seemed promising, we were unable to further optimize this protocol, due to time constraints and practical issues regarding laboratory equipment. Therefore, we considered the data not fully complete to be included in this chapter.

If the mucus layer should play a more central role in the model, e.g. in studies investigating the effects of specific compounds on mucus quality and quantity, the SWMS method might not be the best method. In this case, more relevant options include the use of ex vivo derived mucus (79), or the use of organoid or gut-on-a-chip models $(82,85)$, amongst others. Importantly, when using these more sophisticated models, researchers should not take for granted that the mucus layer is automatically representative. Instead, the mucus layer should be carefully investigated with regard to thickness, evenness, stability and composition. Furthermore, it should be taken into account that often extensive study set-ups are needed to achieve sufficient mucus yield to be able to perform quantity and quality assays. Importantly, the significance of the mucus layer in health and disease 
requires more scientific interest and therefore more research should be carried out to be able to investigate the mucus layer into more detail. For example, these models can be used to investigate the influence of dietary or microbial components on mucus properties. Eventually, these models could also be relevant to investigate the potential of (newly discovered) therapeutic interventions.

\section{The importance of approaching intestinal health from different perspectives}

The role of the intestine in a plethora of diseases and disorders emphasizes that intestinal health reaches much further than the intestinal tract alone. Increased scientific insight in underlying mechanisms is therefore crucial to keep the intestine in a healthy condition. In this thesis, we took a multiperspective approach to investigate intestinal health by focusing on the aging process, gut microbiota and bile acids. As schematically depicted in Figure 1, an intricate relationship exists between these factors.

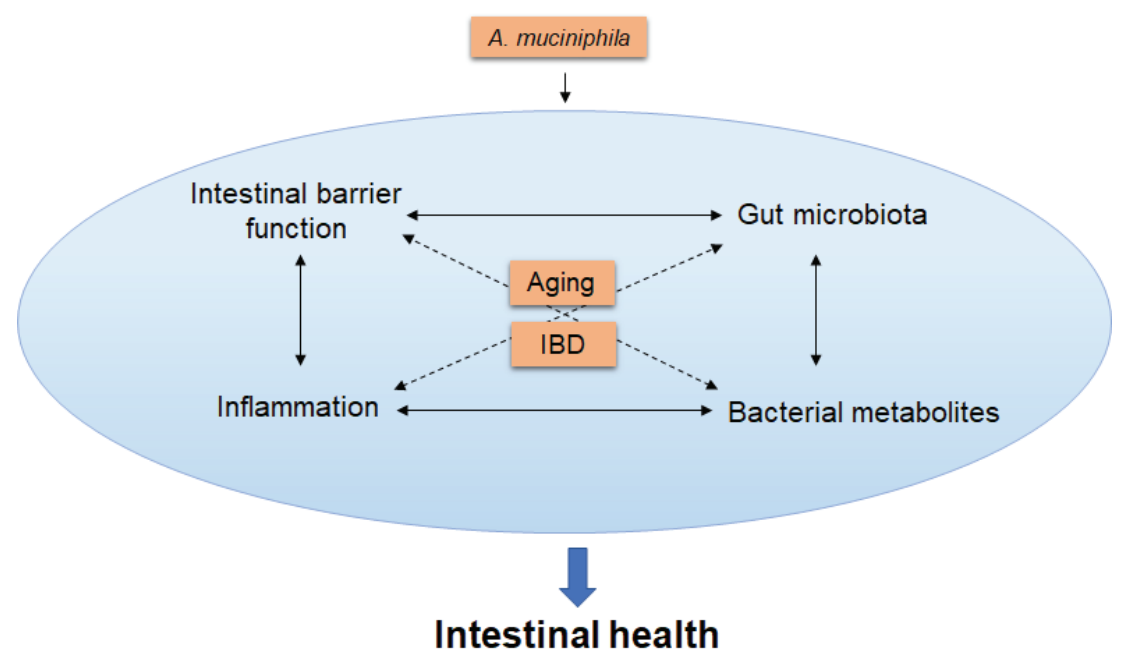

Figure $1 \mathrm{~A}$ schematic overview of the interplay between parameters of intestinal health: intestinal barrier function, gut microbiota composition, bacterial metabolites and inflammation. In this thesis, we investigated the effects of the aging process on these aspects and evaluated the use of the probiotic Akkermansia muciniphila to improve intestinal health during the aging process. We also investigated the effect of bacterial metabolites (bile acids) on intestinal barrier function in the context of inflammatory bowel disease (IBD).

Intervening in these processes, for example by bacterial supplementation (e.g. A. muciniphila) might be a promising strategy to improve intestinal health. Importantly, investigation into the role of bacterial 
metabolites (e.g. bile acids) is crucial to understand the consequences of the gut microbiota during health and disease, such as IBD. In vitro models described in this thesis might be relevant for future research that aims to investigate the role of bacterial metabolites in intestinal health. 


\section{References}

1. Sperber AD, Bangdiwala SI, Drossman DA, Ghoshal UC, Simren M, Tack J, et al. Worldwide Prevalence and Burden of Functional Gastrointestinal Disorders, Results of Rome Foundation Global Study. Gastroenterology. 2021;160(1):99-114.e3.

2. Bischoff SC, Barbara G, Buurman W, Ockhuizen T, Schulzke JD, Serino M, et al. Intestinal permeability--a new target for disease prevention and therapy. BMC Gastroenterol. 2014;14:189.

3. Bischoff SC. 'Gut health': a new objective in medicine? BMC Med. 2011;9:24.

4. Wilkins LJ, Monga M, Miller AW. Defining Dysbiosis for a Cluster of Chronic Diseases. Sci Rep. 2019;9(1):12918.

5. Petersen C, Round JL. Defining dysbiosis and its influence on host immunity and disease. Cell Microbiol. 2014;16(7):1024-33.

6. Duvallet C, Gibbons SM, Gurry T, Irizarry RA, Alm EJ. Meta-analysis of gut microbiome studies identifies disease-specific and shared responses. Nat Commun. 2017;8(1):1784.

7. Ghosh TS, Das M, Jeffery IB, O'Toole PW. Adjusting for age improves identification of gut microbiome alterations in multiple diseases. Elife. 2020;9.

8. Rajca S, Grondin V, Louis E, Vernier-Massouille G, Grimaud JC, Bouhnik Y, et al. Alterations in the intestinal microbiome (dysbiosis) as a predictor of relapse after infliximab withdrawal in Crohn's disease. Inflamm Bowel Dis. 2014;20(6):978-86.

9. Castro-Mejía JL, Khakimov B, Krych Ł, Bülow J, Bechshøft RL, Højfeldt G, et al. Physical fitness in community-dwelling older adults is linked to dietary intake, gut microbiota, and metabolomic signatures. Aging Cell. 2020;19(3):e13105.

10. Jackson MA, Jeffery IB, Beaumont M, Bell JT, Clark AG, Ley RE, et al. Signatures of early frailty in the gut microbiota. Genome Med. 2016;8(1):8.

11. David LA, Maurice CF, Carmody RN, Gootenberg DB, Button JE, Wolfe BE, et al. Diet rapidly and reproducibly alters the human gut microbiome. Nature. 2014;505(7484):559-63.

12. Amato KR, J GS, Song SJ, Nute M, Metcalf JL, Thompson LR, et al. Evolutionary trends in host physiology outweigh dietary niche in structuring primate gut microbiomes. Isme j. 2019;13(3):576-87.

13. Li H, Qi Y, Jasper H. Preventing Age-Related Decline of Gut Compartmentalization Limits Microbiota Dysbiosis and Extends Lifespan. Cell Host Microbe. 2016;19(2):240-53.

14. Bruno G, Zaccari P, Rocco G, Scalese G, Panetta C, Porowska B, et al. Proton pump inhibitors and dysbiosis: Current knowledge and aspects to be clarified. World J Gastroenterol. 2019;25(22):2706-19.

15. Zimmermann $\mathrm{P}$, Curtis N. The effect of antibiotics on the composition of the intestinal microbiota - a systematic review. J Infect. 2019;79(6):471-89.

16. Arumugam M, Raes J, Pelletier E, Le Paslier D, Yamada T, Mende DR, et al. Enterotypes of the human gut microbiome. Nature. 2011;473(7346):174-80.

17. Cheng M, Ning K. Stereotypes About Enterotype: the Old and New Ideas. Genomics Proteomics Bioinformatics. 2019;17(1):4-12.

18. Zoetendal EG, von Wright A, Vilpponen-Salmela T, Ben-Amor K, Akkermans AD, de Vos WM. Mucosa-associated bacteria in the human gastrointestinal tract are uniformly distributed along the colon and differ from the community recovered from feces. Appl Environ Microbiol. 2002;68(7):3401-7.

19. Mailhe M, Ricaboni D, Vitton V, Gonzalez JM, Bachar D, Dubourg G, et al. Repertoire of the gut microbiota from stomach to colon using culturomics and next-generation sequencing. BMC Microbiol. 2018;18(1):157.

20. He Y, Wu W, Zheng HM, Li P, McDonald D, Sheng HF, et al. Regional variation limits applications of healthy gut microbiome reference ranges and disease models. Nat Med. 2018;24(10):1532-5.

21. Tang Q, Jin G, Wang G, Liu T, Liu X, Wang B, et al. Current Sampling Methods for Gut Microbiota: A Call for More Precise Devices. Front Cell Infect Microbiol. 2020;10:151.

22. Rios-Morales M, van Trijp MPH, Rösch C, An R, Boer T, Gerding A, et al. A toolbox for the comprehensive analysis of small volume human intestinal samples that can be used with gastrointestinal sampling capsules. Sci Rep. 2021;11(1):8133.

23. Almeida A, Mitchell AL, Boland M, Forster SC, Gloor GB, Tarkowska A, et al. A new genomic blueprint of the human gut microbiota. Nature. 2019;568(7753):499-504.

24. Levy M, Thaiss CA, Elinav E. Metabolites: messengers between the microbiota and the immune system. Genes Dev. 2016;30(14):1589-97.

25. Quince C, Walker AW, Simpson JT, Loman NJ, Segata N. Shotgun metagenomics, from sampling to analysis. Nat Biotechnol. 2017;35(9):833-44. 
26. Kheirbek RE, Fokar A, Shara N, Bell-Wilson LK, Moore HJ, Olsen E, et al. Characteristics and Incidence of Chronic Illness in Community-Dwelling Predominantly Male U.S. Veteran Centenarians. J Am Geriatr Soc. 2017;65(9):2100-6.

27. Engberg H, Oksuzyan A, Jeune B, Vaupel JW, Christensen K. Centenarians--a useful model for healthy aging? A 29-year follow-up of hospitalizations among 40,000 Danes born in 1905. Aging Cell. 2009;8(3):270-6.

28. Biagi E, Franceschi C, Rampelli S, Severgnini M, Ostan R, Turroni S, et al. Gut Microbiota and Extreme Longevity. Curr Biol. 2016;26(11):1480-5.

29. Kim BS, Choi CW, Shin H, Jin SP, Bae JS, Han M, et al. Comparison of the Gut Microbiota of Centenarians in Longevity Villages of South Korea with Those of Other Age Groups. J Microbiol Biotechnol. 2019;29(3):429-40.

30. Tuikhar N, Keisam S, Labala RK, Imrat, Ramakrishnan P, Arunkumar MC, et al. Comparative analysis of the gut microbiota in centenarians and young adults shows a common signature across genotypically non-related populations. Mech Ageing Dev. 2019;179:23-35.

31. Everard A, Belzer C, Geurts L, Ouwerkerk JP, Druart C, Bindels LB, et al. Cross-talk between Akkermansia muciniphila and intestinal epithelium controls diet-induced obesity. Proc Natl Acad Sci U S A. 2013;110(22):9066-71.

32. Schneeberger M, Everard A, Gómez-Valadés AG, Matamoros S, Ramírez S, Delzenne NM, et al. Akkermansia muciniphila inversely correlates with the onset of inflammation, altered adipose tissue metabolism and metabolic disorders during obesity in mice. Sci Rep. 2015;5:16643.

33. Brahe LK, Le Chatelier E, Prifti E, Pons N, Kennedy S, Hansen T, et al. Specific gut microbiota features and metabolic markers in postmenopausal women with obesity. Nutr Diabetes. 2015;5(6):e159.

34. Dao MC, Everard A, Aron-Wisnewsky J, Sokolovska N, Prifti E, Verger EO, et al. Akkermansia muciniphila and improved metabolic health during a dietary intervention in obesity: relationship with gut microbiome richness and ecology. Gut. 2016;65(3):426-36.

35. Li J, Zhao F, Wang Y, Chen J, Tao J, Tian G, et al. Gut microbiota dysbiosis contributes to the development of hypertension. Microbiome. 2017;5(1):14.

36. Depommier C, Everard A, Druart C, Plovier H, Van Hul M, Vieira-Silva S, et al. Supplementation with Akkermansia muciniphila in overweight and obese human volunteers: a proof-of-concept exploratory study. Nat Med. 2019;25(7):1096-103.

37. Depommier C, Van Hul M, Everard A, Delzenne NM, De Vos WM, Cani PD. Pasteurized Akkermansia muciniphila increases whole-body energy expenditure and fecal energy excretion in dietinduced obese mice. Gut Microbes. 2020;11(5):1231-45.

38. Plovier H, Everard A, Druart C, Depommier C, Van Hul M, Geurts L, et al. A purified membrane protein from Akkermansia muciniphila or the pasteurized bacterium improves metabolism in obese and diabetic mice. Nat Med. 2017;23(1):107-13.

39. Druart C, Plovier H, Van Hul M, Brient A, Phipps KR, de Vos WM, et al. Toxicological safety evaluation of pasteurized Akkermansia muciniphila. J Appl Toxicol. 2021;41(2):276-90.

40. Doron S, Snydman DR. Risk and safety of probiotics. Clin Infect Dis. 2015;60 Suppl 2(Suppl 2):S129-34.

41. van Beek AA, Sovran B, Hugenholtz F, Meijer B, Hoogerland JA, Mihailova V, et al. Supplementation with Lactobacillus plantarum WCFS1 Prevents Decline of Mucus Barrier in Colon of Accelerated Aging Ercc1 $^{(-/ \Delta 7)}$ Mice. Front Immunol. 2016;7:408.

42. Ganesh BP, Klopfleisch R, Loh G, Blaut M. Commensal Akkermansia muciniphila exacerbates gut inflammation in Salmonella Typhimurium-infected gnotobiotic mice. PLoS One. 2013;8(9):e74963.

43. Luan Z, Sun G, Huang Y, Yang Y, Yang R, Li C, et al. Metagenomics Study Reveals Changes in Gut Microbiota in Centenarians: A Cohort Study of Hainan Centenarians. Front Microbiol. 2020;11:1474.

44. Wang F, Yu T, Huang G, Cai D, Liang X, Su H, et al. Gut Microbiota Community and Its Assembly Associated with Age and Diet in Chinese Centenarians. J Microbiol Biotechnol. 2015;25(8):1195-204.

45. Wilmanski T, Diener C, Rappaport N, Patwardhan S, Wiedrick J, Lapidus J, et al. Gut microbiome pattern reflects healthy ageing and predicts survival in humans. Nat Metab. 2021;3(2):274-86.

46. Rampelli S, Soverini M, D'Amico F, Barone M, Tavella T, Monti D, et al. Shotgun Metagenomics of Gut Microbiota in Humans with up to Extreme Longevity and the Increasing Role of Xenobiotic Degradation. mSystems. 2020;5(2).

47. Pollard KM, Hultman P, Kono DH. Toxicology of autoimmune diseases. Chem Res Toxicol. 2010;23(3):455-66.

48. Sajid M, Agrewala JN. Low prevalence of anti-xenobiotic antibodies among the occupationally 
exposed individuals is associated with a high risk of cancer. Cancer Med. 2019;8(1):246-60.

49. Abdelsalam NA, Ramadan AT, ElRakaiby MT, Aziz RK. Toxicomicrobiomics: The Human Microbiome vs. Pharmaceutical, Dietary, and Environmental Xenobiotics. Front Pharmacol. 2020;11:390.

50. Tailford LE, Crost EH, Kavanaugh D, Juge N. Mucin glycan foraging in the human gut microbiome. Front Genet. 2015;6:81.

51. Elderman M, Sovran B, Hugenholtz F, Graversen K, Huijskes M, Houtsma E, et al. The effect of age on the intestinal mucus thickness, microbiota composition and immunity in relation to sex in mice. PLoS One. 2017;12(9):e0184274.

52. Sovran B, Hugenholtz F, Elderman M, Van Beek AA, Graversen K, Huijskes M, et al. Ageassociated Impairment of the Mucus Barrier Function is Associated with Profound Changes in Microbiota and Immunity. Sci Rep. 2019;9(1):1437.

53. Geerlings SY, Kostopoulos I, de Vos WM, Belzer C. Akkermansia muciniphila in the Human Gastrointestinal Tract: When, Where, and How? Microorganisms. 2018;6(3).

54. van Passel MW, Kant R, Zoetendal EG, Plugge CM, Derrien M, Malfatti SA, et al. The genome of Akkermansia muciniphila, a dedicated intestinal mucin degrader, and its use in exploring intestinal metagenomes. PLoS One. 2011;6(3):e16876.

55. Zhu L, Lu X, Liu L, Voglmeir J, Zhong X, Yu Q. Akkermansia muciniphila protects intestinal mucosa from damage caused by S. pullorum by initiating proliferation of intestinal epithelium. Vet Res. 2020;51(1):34.

56. Kaur N, Chen CC, Luther J, Kao JY. Intestinal dysbiosis in inflammatory bowel disease. Gut Microbes. 2011;2(4):211-6.

57. Kok DEG, Rusli F, van der Lugt B, Lute C, Laghi L, Salvioli S, et al. Lifelong calorie restriction affects indicators of colonic health in aging C57BI/6J mice. J Nutr Biochem. 2018;56:152-64.

58. Ma J, Hong Y, Zheng N, Xie G, Lyu Y, Gu Y, et al. Gut microbiota remodeling reverses agingassociated inflammation and dysregulation of systemic bile acid homeostasis in mice sex-specifically. Gut Microbes. 2020;11(5):1450-74.

59. Gérard P. Metabolism of cholesterol and bile acids by the gut microbiota. Pathogens. 2013;3(1):14-

24.

60. Duboc H, Rajca S, Rainteau D, Benarous D, Maubert MA, Quervain E, et al. Connecting dysbiosis, bile-acid dysmetabolism and gut inflammation in inflammatory bowel diseases. Gut. 2013;62(4):531-9.

61. Hughes R, Kurth MJ, McGilligan V, McGlynn H, Rowland I. Effect of colonic bacterial metabolites on Caco-2 cell paracellular permeability in vitro. Nutr Cancer. 2008;60(2):259-66.

62. Raimondi F, Santoro P, Barone MV, Pappacoda S, Barretta ML, Nanayakkara M, et al. Bile acids modulate tight junction structure and barrier function of Caco-2 monolayers via EGFR activation. Am J Physiol Gastrointest Liver Physiol. 2008;294(4):G906-13.

63. Murakami Y, Tanabe S, Suzuki T. High-fat Diet-induced Intestinal Hyperpermeability is Associated with Increased Bile Acids in the Large Intestine of Mice. J Food Sci. 2016;81(1):H216-22.

64. Farhana L, Nangia-Makker P, Arbit E, Shango K, Sarkar S, Mahmud H, et al. Bile acid: a potential inducer of colon cancer stem cells. Stem Cell Res Ther. 2016;7(1):181.

65. Ou J, Carbonero F, Zoetendal EG, DeLany JP, Wang M, Newton K, et al. Diet, microbiota, and microbial metabolites in colon cancer risk in rural Africans and African Americans. Am J Clin Nutr. 2013;98(1):111-20.

66. Sinha SR, Haileselassie Y, Nguyen LP, Tropini C, Wang M, Becker LS, et al. Dysbiosis-Induced Secondary Bile Acid Deficiency Promotes Intestinal Inflammation. Cell Host Microbe. 2020;27(4):659-70. e5.

67. Ward JBJ, Lajczak NK, Kelly OB, O'Dwyer AM, Giddam AK, J NG, et al. Ursodeoxycholic acid and lithocholic acid exert anti-inflammatory actions in the colon. Am J Physiol Gastrointest Liver Physiol. 2017;312(6):G550-g8.

68. Ichikawa R, Takayama T, Yoneno K, Kamada N, Kitazume MT, Higuchi H, et al. Bile acids induce monocyte differentiation toward interleukin-12 hypo-producing dendritic cells via a TGR5-dependent pathway. Immunology. 2012;136(2):153-62.

69. Hofmann AF. The continuing importance of bile acids in liver and intestinal disease. Arch Intern Med. 1999;159(22):2647-58.

70. Nagana Gowda GA, Shanaiah N, Cooper A, Maluccio M, Raftery D. Bile acids conjugation in human bile is not random: new insights from (1)H-NMR spectroscopy at $800 \mathrm{MHz}$. Lipids. 2009;44(6):52735.

71. Alnouti Y. Bile Acid sulfation: a pathway of bile acid elimination and detoxification. Toxicol Sci. 
2009;108(2):225-46.

72. Pathak P, Liu H, Boehme S, Xie C, Krausz KW, Gonzalez F, et al. Farnesoid X receptor induces Takeda G-protein receptor 5 cross-talk to regulate bile acid synthesis and hepatic metabolism. J Biol Chem. 2017;292(26):11055-69.

73. Cipriani S, Mencarelli A, Chini MG, Distrutti E, Renga B, Bifulco G, et al. The bile acid receptor GPBAR-1 (TGR5) modulates integrity of intestinal barrier and immune response to experimental colitis. PLoS One. 2011;6(10):e25637.

74. Sorrentino G, Perino A, Yildiz E, El Alam G, Bou Sleiman M, Gioiello A, et al. Bile Acids Signal via TGR5 to Activate Intestinal Stem Cells and Epithelial Regeneration. Gastroenterology. 2020;159(3):956-68. e8.

75. Yoneno K, Hisamatsu T, Shimamura K, Kamada N, Ichikawa R, Kitazume MT, et al. TGR5 signalling inhibits the production of pro-inflammatory cytokines by in vitro differentiated inflammatory and intestinal macrophages in Crohn's disease. Immunology. 2013;139(1):19-29.

76. Biagioli M, Carino A, Cipriani S, Francisci D, Marchianò S, Scarpelli P, et al. The Bile Acid Receptor GPBAR1 Regulates the M1/M2 Phenotype of Intestinal Macrophages and Activation of GPBAR1 Rescues Mice from Murine Colitis. J Immunol. 2017;199(2):718-33.

77. Johansson ME, Larsson JM, Hansson GC. The two mucus layers of colon are organized by the MUC2 mucin, whereas the outer layer is a legislator of host-microbial interactions. Proc Natl Acad Sci U S A. 2011;108 Suppl 1(Suppl 1):4659-65.

78. Recktenwald CV, Hansson GC. The Reduction-insensitive Bonds of the MUC2 Mucin Are Isopeptide Bonds. J Biol Chem. 2016;291(26):13580-90.

79. van der Post S, Jabbar KS, Birchenough G, Arike L, Akhtar N, Sjovall H, et al. Structural weakening of the colonic mucus barrier is an early event in ulcerative colitis pathogenesis. Gut. 2019;68(12):2142-51.

80. Gustafsson JK, Ermund A, Johansson ME, Schütte A, Hansson GC, Sjövall H. An ex vivo method for studying mucus formation, properties, and thickness in human colonic biopsies and mouse small and large intestinal explants. Am J Physiol Gastrointest Liver Physiol. 2012;302(4):G430-8.

81. White V, Scarpini C, Barbosa-Morais NL, Ikelle E, Carter S, Laskey RA, et al. Isolation of stoolderived mucus provides a high yield of colonocytes suitable for early detection of colorectal carcinoma. Cancer Epidemiol Biomarkers Prev. 2009;18(7):2006-13.

82. Sontheimer-Phelps A, Chou DB, Tovaglieri A, Ferrante TC, Duckworth T, Fadel C, et al. Human Colon-on-a-Chip Enables Continuous In Vitro Analysis of Colon Mucus Layer Accumulation and Physiology. Cell Mol Gastroenterol Hepatol. 2020;9(3):507-26.

83. Lesuffleur T, Porchet N, Aubert JP, Swallow D, Gum JR, Kim YS, et al. Differential expression of the human mucin genes MUC1 to MUC5 in relation to growth and differentiation of different mucussecreting HT-29 cell subpopulations. J Cell Sci. 1993;106 ( Pt 3):771-83.

84. Navabi N, McGuckin MA, Lindén SK. Gastrointestinal cell lines form polarized epithelia with an adherent mucus layer when cultured in semi-wet interfaces with mechanical stimulation. PLoS One. 2013;8(7):e68761.

85. Kim HJ, Huh D, Hamilton G, Ingber DE. Human gut-on-a-chip inhabited by microbial flora that experiences intestinal peristalsis-like motions and flow. Lab Chip. 2012;12(12):2165-74. 


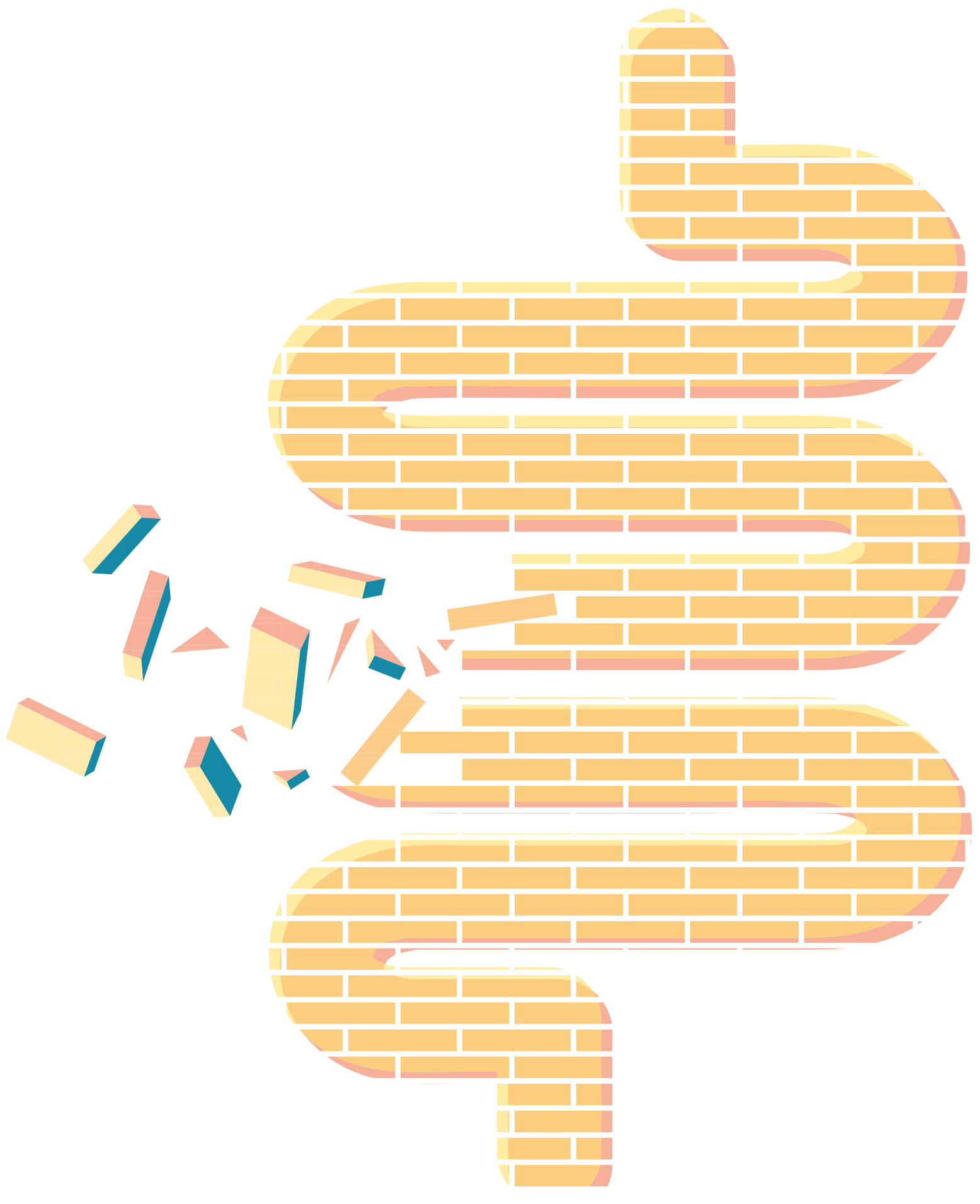


Summary 
It is widely acknowledged that the intestine plays an essential role in the maintenance of overall health. A well-regulated balance exists in the intestine, where on the one hand the uptake of nutrients is ensured, and on the other hand the entrance of bacteria and potentially harmful compounds is restricted. The intestinal barrier is responsible to carry out these highly important, but complex tasks. The intestinal barrier include the mucus layer, the intestinal epithelial cell layer and the immune cell 'layer. In a healthy condition, the trillions of micro-organisms that are present in the intestinal tract have a beneficial relationship with the host. However, in case these highly regulated processes are impaired, the risk of (intestinal) diseases increases. As the prevalence of intestinal disorders is increasing worldwide, there is a high urgency to increase the scientific knowledge in the field of intestinal health. The aim of this thesis was to investigate the effects of relevant aspects that are known to be involved in intestinal health: the aging process, gut microbiota, and bile acids. To investigate this, we used relevant in vivo and in vitro models.

The life-expectancy of humans has increased over the past centuries. However, the aging process increases the risk of disease, including intestinal disorders. To improve healthy aging, it is important to gain insight in the mechanisms underlying aging-related impaired intestinal health. In Chapter 2, we investigated the effects of the aging process on different aspects of intestinal health using C57BL/6J mice aged 6, 12, 24 and 28 months. We found pronounced changes in gut microbiota composition during aging, including an enrichment of potential pathobionts and a decline in healthpromoting bacteria, such as Akkermansia spp. Transcriptome analysis of colonic scrapings pointed towards a decreased intestinal immune response during aging. To explore interactions between the gut microbiota and host colonic gene expression, a comprehensive integrative analysis was performed. The observed correlations between specific bacterial genera and host gene expression may serve as a starting point for future studies investigating the exact host-microbe interactions that take place in the vicinity of the colonic wall.

Since a detrimental shift in gut microbiota composition is frequently observed during aging, manipulation of the gut microbiota in the aged population could be a strategy to enhance healthy aging. A highly promising candidate is Akkermansia muciniphila, since a wide range of studies showed that this bacterium has beneficial effects on low-grade inflammation and (cardio)metabolic disorders. However, the effects of $A$. muciniphila on intestinal health parameters are not widely investigated yet. In Chapter 3, we supplemented progeroid Ercc1 ${ }^{-/ 7}$ mice with $A$. muciniphila for 10 weeks and investigated histological, transcriptional and immunological aspects of intestinal health. We found that the thickness of the colonic mucus layer increased about 3-fold in supplemented mice compared to the control group that did not receive supplementation. Moreover, both transcriptomic 
and immunological analysis revealed an improved immune status. These results highlight the potential anti-inflammatory properties of $A$. muciniphila, which serve as a starting point for future studies investigating the use of this bacterium as a therapeutic intervention in the elderly population.

The involvement of the gut microbiota in inflammatory disorders of the intestine, such as IBD, is widely acknowledged. Bacterial metabolites, which are the end products of bacterial metabolism, have a great influence on intestinal health, such as immune response and intestinal barrier function. Therefore, the shift in bacterial metabolite composition as a result of IBD-related dysbiosis could play a role in IBD pathology. In a previous study, increased levels of sulfated BAs were found in IBD patients, but the exact effect of sulfated BAs on disease progression has not been investigated yet. In Chapter 4, we aimed to investigate the effects of sulfated secondary bile acids on intestinal barrier function and immune response. We first established a novel inflammatory in vitro human intestinal model which was exposed to sulfated deoxycholic acid (DCA), sulfated lithocholic acid (LCA) and their unsulfated forms. Sulfated BAs had a minor effect on intestinal barrier function. In line with previous studies, we found evidence that pointed towards anti-inflammatory effects of LCA and DCA. However, these effects were not observed for sulfated LCA and DCA. These findings might imply that impaired metabolism of BAs is involved in IBD progression.

In commonly used in vitro models used to investigate intestinal health, the mucus layer is often overlooked or underrepresented. However, the mucus layer is a highly important with regard to intestinal barrier function. To this end, it is highly important to improve the physiologically representativeness of in vitro intestinal models, for example by applying special culturing strategies. A previous study described a semi-wet interface with mechanical stimulation (SWMS) method to increase mucus production in the mucus-producing cell line HT29-MTX-E12. In Chapter 5, we aimed to investigate the underlying (molecular) mechanisms of this method. We found that SWMS culture conditions resulted in increased cell growth and a shift towards a more aerobic cell metabolism, which might be the driving factors behind the increased mucus production. This SWMS culturing method might be a simple, yet effective strategy to apply in in vitro intestinal models that require the presence of a mucus layer, e.g. e.g. studies investigating drug-disposition or the exposure of bacterial metabolites. In order to improve the physiological representativeness of the mucus layer even more, further research should focus on the qualitative aspects of the in vitro mucus layer.

In this thesis we investigated intestinal health from different perspectives. We focused on the effects of the aging process, gut microbiota and bile acids on important intestinal health parameters. We showed that the aging process has profound effects on gut microbiota composition and that supplementation with Akkermansia muciniphila might be a potential strategy to improve healthy 
aging. Besides, bacterial metabolites, such as secondary bile acids, play a crucial role in intestinal health and disease. Although the studies presented in this thesis contribute to our understanding with regard to intestinal health, still much remains to discover. An important aspect of future research on intestinal health should be to focus on the exact underlying mechanisms. To this end, the in vitro intestinal model described in this thesis could be a useful model. Ultimately, increased knowledge of intestinal health will contribute to the development of therapeutic strategies to treat, or even prevent intestinal disorders. 


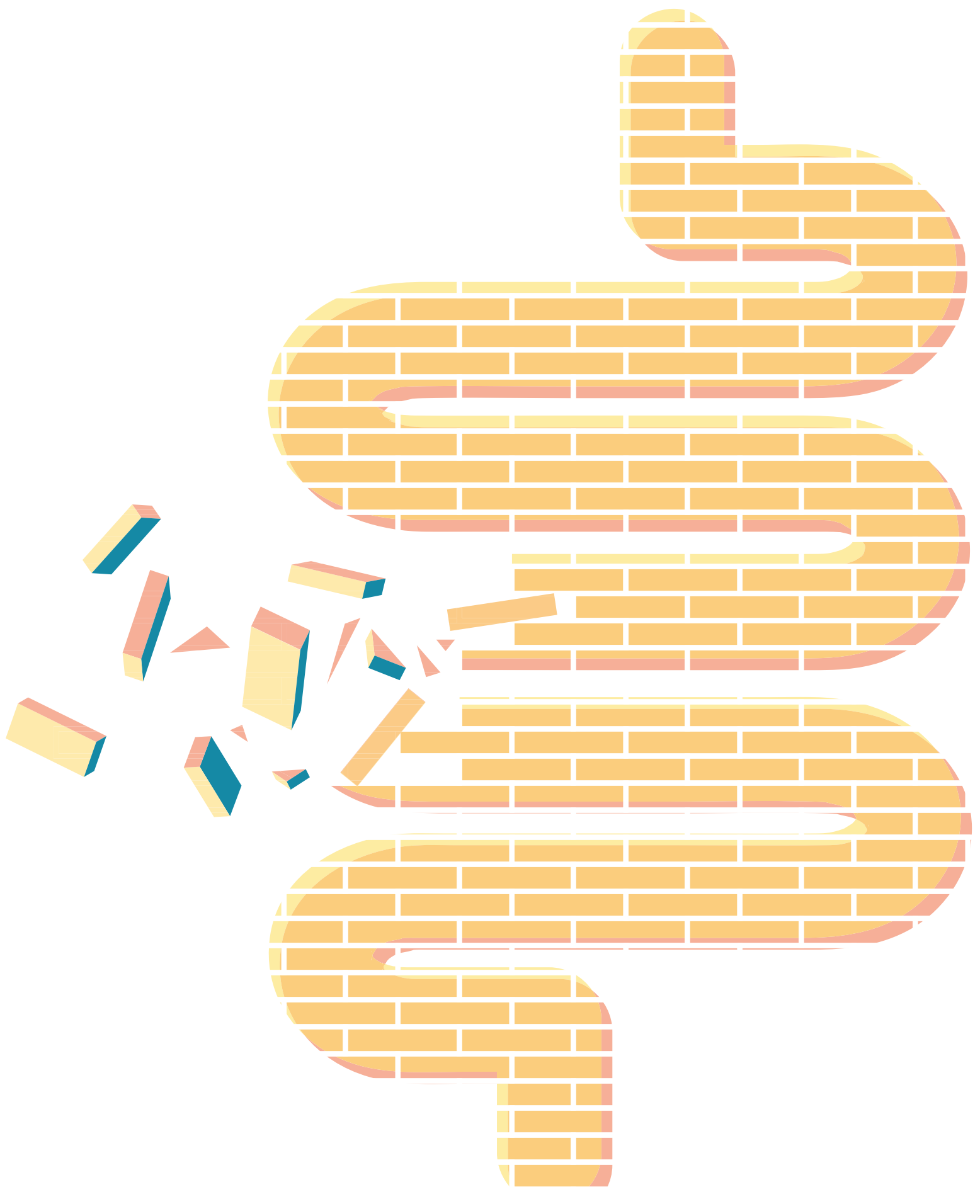




\section{Acknowledgements \\ Dankwoord}


Eindelijk is het dan zover, het allerlaatste onderdeel van dit proefschrift! En natuurlijk kan geen enkel proefschrift zonder dankwoord. Aan het einde van mijn PhD realiseer ik me nog maar eens extra wat een leuke en lieve mensen ik tot mijn collega's, vriendenkring en familie mag rekenen. Dank allemaal voor jullie bijdrage aan de leuke momenten tijdens de afgelopen jaren!

Ten eerste wil ik graag Wilma bedanken. Wij gaan al best een tijdje mee samen! Jij hebt me begeleid tijdens mijn MSc thesis, Research Master Track, stage en vervolgens natuurlijk tijdens mijn PhD. Ik heb de afgelopen jaren enorm veel van je geleerd en daar ben ik je heel dankbaar voor. Je bent altijd scherp, snel van begrip, oplossingsgericht en behulpzaam. Onze vele overleggen samen waren altijd gezellig, soms zelfs zo gezellig dat de tijd al bijna voorbij was voordat we het over ook maar iets wetenschappelijks hadden gehad. Maar altijd liep ik weer vol inspiratie en motivatie je kantoor uit. Bedankt dat ik altijd op je kon rekenen!

Sander, je hebt me regelmatig de sturing gegeven die ik op dat moment nodig had om mijn onderzoek een stap verder te brengen. Als geen ander beheers je de kunst om de juiste vragen te stellen die meer dan eens leiden tot interessante inzichten. Dank voor al je hulp de afgelopen jaren!

I would also like to thank Jerry Wells, Jan Knol, Paul de Vos and Hans Bouwmeester for their willingness to be part of my committee. Thank you for critically reviewing and judging my PhD thesis.

Merel, ik vind het zo leuk dat wij elkaars paranimfen zijn! Heel erg bedankt dat jij als paranimf aan mijn zijde wilt staan. Jij staat echt altijd voor iedereen klaar en met je docentenskills kan je alles ook nog eens heel goed uitleggen. Ik ben je enorm dankbaar voor de vele labtechnieken die je mij door de jaren heen hebt geleerd. Het is alleen wel verstandiger om ons samen niet meer te wagen aan 'moeilijke' berekeningen, want dat gaat gegarandeerd mis;-) De ontelbare koffie-haalmomentjes, wandelingen, spelletjesavonden, borrels en barbecues waren onvergetelijk. Uiteraard kan ik Ingmar dan ook niet vergeten te bedanken! Jullie kwaliteiten om feestjes te organiseren zijn echt onvoorstelbaar goed. Of het nou om een trouwfeest, verjaardag of voetbal/fiets-kijkavond gaat, het ontbrak werkelijk nooit aan iets! Het is fantastisch om ongeveer bij elkaar in de achtertuin te wonen, laten we nog heel veel gezellige momenten beleven met $z$ 'n allen, inclusief Tristan!

Janneke, wat een toeval dat wij een aantal jaren geleden bij elkaar gebracht zijn door onze gemeenschappelijke interesse: mucus. En wat blijkt, de sticky eigenschappen van mucus hebben 
ervoor gezorgd dat wij sindsdien bij elkaar zijn blijven plakken! Het bleek namelijk niet onze enige overeenkomst, en van mucusmaatjes werden we al snel framemaatjes. Tijdens de duizenden kilometers die wij samen op de fiets hebben doorgebracht, kwamen we vaak achter nóg meer gemeenschappelijke interesses. Dit resulteert nog steeds regelmatig in het feit dat ik na afloop van onze fietsritjes vaak vermoeider ben van het kletsen dan van het fietsen zelf. Ik bewonder je tomeloze energie en inzet, je rotsvaste vertrouwen dat er een Elfstedentocht aankomt en uiteraard je onophoudelijke stroom aan woordgrappen. Ik vind het super dat jij mij wilt bijstaan als paranimf, heel erg bedankt daarvoor!

Daarnaast wil ik graag al mijn (oud)-collega's bedanken! Xanthe en Mara, wij gaan al terug tot onze Masters! Samen stortten wij ons in het avontuur van de RMT en begonnen daarna (ongeveer) gelijktijdig aan onze PhDs. Het was ontzettend fijn om deze tijd samen met jullie mee te maken en wat hebben we een leuke momenten beleefd! Xanthe, ons tripje naar Barcelona (incl. onze dagelijkse patatas bravas) zal ik nooit vergeten. Net zoals onze imitaties van internetgekkies en slappe lach over Xasse en Besse ;-) Mara, nog een darm-partner in crime! Dankjewel dat ik altijd met jou kon sparren over darm-vraagstukken! Onze wandelingetjes, borrels, barbecues of 'walking dinners' waren altijd super gezellig. Hopelijk gaan we nog vaak samen herinneringen ophalen met z'n allen! Charlotte, ook wij hebben ontelbaar veel gezellige momenten beleefd tijdens borrels, etentjes, 5 mei-feestjes, nog meer borrels, enzovoorts. Ongetwijfeld gaan er nog veel meer volgen, ik kijk er nu al naar uit! En uiteraard heel erg bedankt voor je hulp met alle vragen die ik had over het in elkaar zetten van dit boekje ;-) Danny, I always enjoyed our conversations about the differences between China and the Netherlands. I really admire the way you adapted so quickly to the Dutch culture. If you want to have more tips on sightseeing in the Netherlands, you can always contact me! Anouk, Brecht, Frank (speciale dank voor het bijbrengen van de fijne kneepjes van het DC-vak), Lisa, Miranda, Monique, Montse, Tessa, Xiaolin, Philip, Antwi, Susanne en Shauna ontzettend bedankt/thank you all voor alle gezelligheid op werk, op het lab en natuurlijk tijdens de lunch, picknicks en borrels!

Karin, het was altijd gezellig om met je te kletsen op werk of in de moestuin (tijdens het meditatief onkruidwieden). De jaarlijkse tomatenplanten en moestuinoogst heb ik altijd zeer gewaardeerd! Mechteld, nog een fietsende collega! Dankjewel dat je altijd voor me klaarstond om te helpen tijdens mijn experimenten. Het was niet alleen gezellig met je op het lab, maar ook op de mountainbike of racefiets! Ik hoop dat we nog veel ritjes gaan maken in de toekomst. Mieke, enorm bedankt voor alle gezelligheid, hulp en af en toe een luisterend oor. Het was top om samen met jou de celkweek 
filmpjes op te nemen. Wij hebben nu eeuwige roem en daar mogen we heel trots op zijn! Jenny, het kweken van organoids, cellen, co-culturen, niks was ons te gek! Ik vond het heel leuk met je samen te werken, bedankt voor de gezellige momenten! Shohreh, you already supervised me during my MSc thesis, longggg time ago. I really appreciate that you were always willing to help, and of course thanks for all the cookies and candy :-) Marlies, als overbuurvrouw wil ik jou ook bedanken voor alle gezelligheid en je interesse. Wij namen altijd steevast het weekend door op de maandagochtend, dat ga ik zeker missen! Guido, bij jou kon ik altijd terecht voor prangende vragen over alles wat met data analyse te maken had. Ook wist ik je te vinden als ik weer eens een GEO nummer nodig had. Daarnaast heb ik je nuttige en leerzame feedback op mijn resultaten tijdens de labmeetings en Gut Health meetings altijd zeer gewaardeerd, dank daarvoor! Carolien, jij hebt me van begin af aan geholpen in het lab en ik kon me geen betere begeleider wensen! Ik ben je ontzettend dankbaar voor alles wat ik van je heb geleerd. Daarnaast was het ook nog eens heel gezellig om je collega te zijn! Dieuwertje, wij hebben elkaar ook al tijdens mijn Master thesis leren kennen. Ik heb veel van je geleerd in de periode dat wij parallel aan de IDEAL samples werkten. Dank voor de momenten waarop we samen over de resultaten konden sparren! Jocelijn, bedankt voor je hulp en feedback op mijn in vitro darmmodellen. Ook je grondige feedback op de bile acid paper was zeer nuttig! Rinke, ik gok dat 9 van de 10 gesprekken die wij voerden over de fiets gingen ;-) Dankjewel voor de gezelligheid en uiteraard ook voor je hulp/ideeën over de experimenten met de DCs. Lydia, ook onze gesprekken gingen vaak over uiteenlopende onderwerpen. Maar er was altijd genoeg om het met je over te hebben en het was in ieder geval altijd erg gezellig, dank hiervoor! Pieter, Mark, Klaske, Michiel, Marco, Nhien, bedankt voor jullie interesse in mijn onderzoek! Nikkie, ik vind het leuk dat je weer (deels) terug bij Humane Voeding bent teruggekomen. Dank voor je interesse en dat je me op gang hebt geholpen met de organoids! Anna, so nice that you joined our group! I really like working together with you and talk about our experiments. Thanks a lot for your help in the lab and your interest in my research! Rieneke, onze gesprekken bij de koffiemachine gingen uiteraard altijd over onze gezamenlijke passie: sport! Ik vond het heel leuk om jouw inzichten en ervaringen hierover te horen. Wie weet komen we elkaar nog een keer tegen bij een RBR of triatlon! Pol, wij hebben de afgelopen jaren ook heel wat onvergetelijke momenten op de fiets meegemaakt, zoals onze 200 km-rit, samen tegeltjes zoeken, op KOM/QOMetjes jacht, MTB met hongerklop (incl. saucijzenbroodje-to-the-rescue) en zo kan ik nog wel even doorgaan ;-) Maar altijd met serieuze en soms minder serieuze gesprekken. Heel erg bedankt voor alle gezelligheid, en natuurlijk ook dat ik af en toe even in je wiel mocht kruipen! Aafke, het was super fijn om als groentje naast zo'n ervaren aio te mogen zitten op kantoor. Dank voor al je wijze raad! Daarnaast konden we goed 
met elkaar over ons onderzoek sparren, daar heb ik veel van geleerd! Maarten, wat was het altijd relaxed om met jou het practicum in goede banen te leiden. Jij had altijd wel een goede oplossing als het in de soep dreigde te lopen. Ook op de fiets hebben we heel wat gezellige momenten beleefd, dankjewel daarvoor! Maartje, wat ben ik blij dat je ik je heb mogen begeleiden tijdens je stage! Jouw gedrevenheid, motivatie, labskills, harde werk en natuurlijk je gezelligheid heb ik altijd enorm gewaardeerd. Dank voor al je hulp tijdens mijn onderzoeken!

Clara, de afgelopen jaren hebben wij heel wat keren samengewerkt. Heel erg bedankt voor je hulp op microbiologisch gebied en natuurlijk voor de kans die je me gegeven hebt om aan de Akkermansia studie te werken!

Noortje, dankjewel dat ik altijd bij je terecht kon voor mijn vragen over galzouten en in vitro modellen! Ik vond het altijd heel leuk om bij je langs te komen in Utrecht en te sparren over m'n onderzoek. Heel erg bedankt voor je behulpzaamheid en je goede inzichten. Wie weet mogen we in de toekomst nog eens samenwerken!

Lianne, wij hebben van begin af aan onze studie samen doorlopen! Ik heb zo'n goede herinneringen aan deze tijd, van samen uitbrakken in de collegezaal, ochtendzwemsessies met een Hema-ontbijtje, samen hardlopen in het Arboretum en natuurlijk alle hardloopwedstrijden. Ik vind het super dat jij ook aan een $\mathrm{PhD}$ bent begonnen! Ook al wonen we niet meer zo dicht bij elkaar, laten we nog heel vaak afspreken en samen gouden medailles gaan winnen!

Lily, als ervaringsdeskundige kon ik altijd bij je terecht voor raad en daad op het gebied van promoveren. Eerst als collega's, daarna tijdens urenlange telefoongesprekken of wandelingen met slechte cappuccino. Dankjewel voor al deze momenten en natuurlijk ook voor je cursus 'Omgaan met een PhD voor dummies' ;-) Onze epische fietsritjes (inclusief kuitkrampen, gigantische verdwalingen en irritante vouwfietsers) zullen me altijd bijblijven, ik hoop dat er nog heel veel gaan komen (maar we zijn weer op de goede weg)!

Alle supermenckies: Frans, Gerrie, Hidde en Suzanne, heel erg bedankt voor jullie gezelligheid de afgelopen jaren. Ik vond het super dat jullie altijd zo geïnteresseerd in mijn onderzoek waren. Dank voor alle fijne momenten samen! Jasmijn, ik vond het zo leuk dat jij er voor koos om naar Wageningen te komen om ook Voeding \& Gezondheid te gaan studeren! Het was altijd gezellig om met je te kletsen over je studie en mijn PhD. Ook de etentjes samen met Bob en Thijs erbij waren erg geslaagd! 
Roel, Josta, llona en Ruben, ik kan me geen betere broer, zus, schoonzus en zwager wensen. De bezoekjes aan jullie behoren tot mijn meest favoriete bezigheden! Maar ik zal niet onder stoelen of banken steken dat Fedde, Thijmen en Boris hier een grote rol in spelen ;-) En uiteraard speciale dank voor Ruben voor de omslag van dit boekje! Papa en mama, dank voor jullie onvoorwaardelijke steun en vertrouwen in mij. Ik vond het altijd fijn om het met jullie te hebben over het wel en wee van mijn PhD. En natuurlijk dank voor de regelmatige etentjes met de hele bups, die boden altijd een welkome afleiding!

Tenslotte gaat mijn dank uit naar de allerliefste Bob. De afgelopen jaren hebben maar weer eens bewezen dat team B\&B alles aankan. Je altijd bemoedigende woorden als het wat minder ging ('kom op slappe'), je 'pragmatische' instelling en natuurlijk je bijzonder gedetailleerde Excel-planningen hebben mij in het gareel gehouden, waardoor ik de eindstreep heb kunnen halen. Het leven samen met jou is een groot feest. Laten we samen nog veel avonturen gaan beleven! 


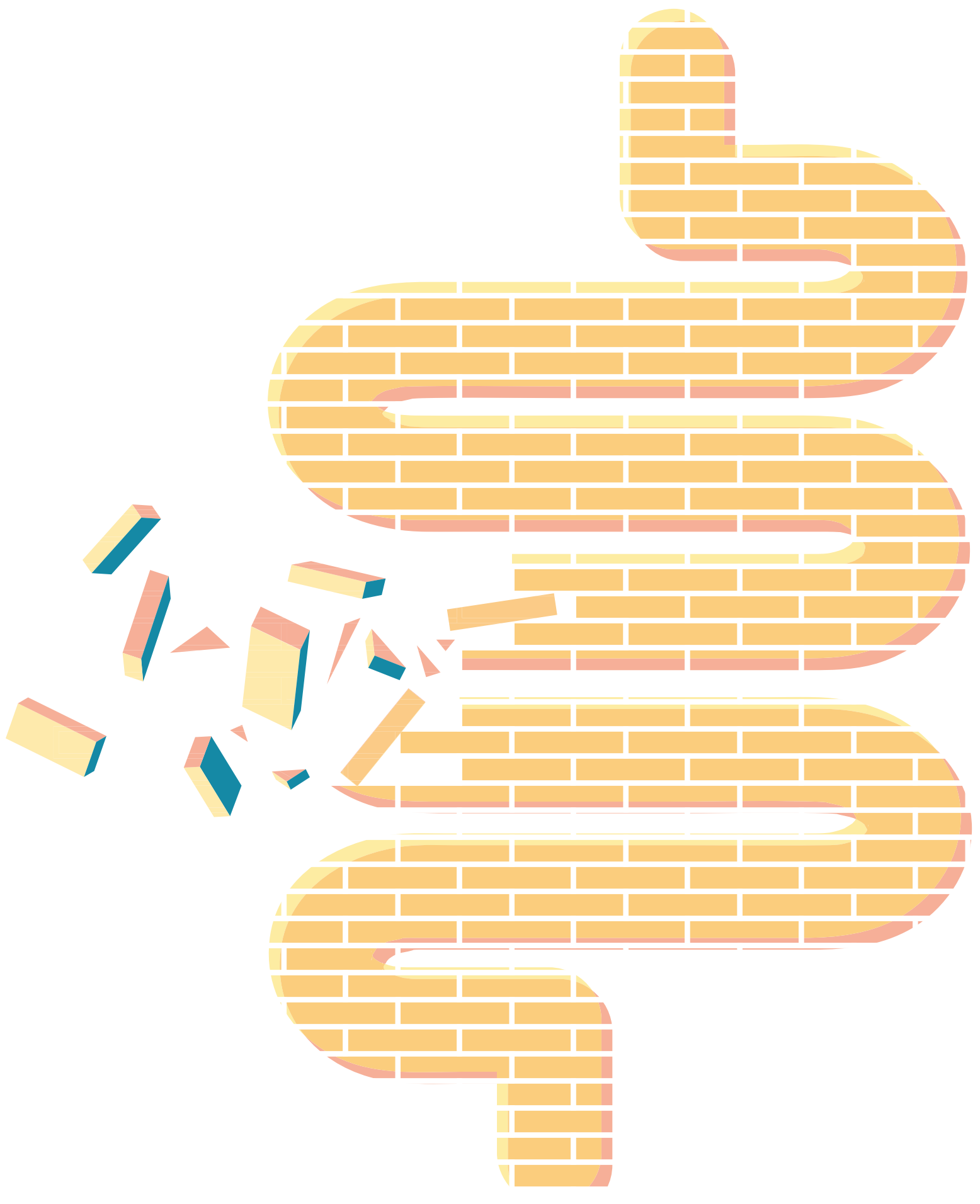


About the author 


\section{CURRICULUM VITAE}

Benthe Mathilde van der Lugt was born on June 8, 1993 in Wanroij, the Netherlands. After completing secondary school at the Merletcollege in Cuijk in 2011, she started the bachelor Nutrition and Health at Wageningen University. After successful completion in 2014, she continued with the master program Nutrition and Health with the specialization Molecular Nutrition \& Toxicology. During her master program, she enrolled in the Research Master Cluster, which is a program initiated by VLAG that prepares MSc students in the process of becoming a PhD student. During the Research Master Cluster, she participated in several courses and activities and wrote a full PhD proposal. In parallel, she completed a MSc thesis at the Nutrition, Metabolism and Genomics group at Wageningen University under supervision of $\mathrm{dr}$. Wilma Steegenga. She investigated the effects of a life-long intermittent diet, alternating between caloric restriction and a medium fat diet, on colonic health. For her internship, she moved out of Wageningen and ended up in the south of Germany. At the Technische Universität München, she investigated the role of the MFS transporter in IL-10 ${ }^{-/}$mouse monoassociated with E. faecalis, under supervision of prof. dr. Dirk Haller. In the meantime, she received the great news that her PhD proposal was selected for funding. In September 2016, after receiving her master's degree, she started her PhD project under the supervision of dr. Wilma Steegenga and prof. $d r$. Sander Kersten. The results of this $\mathrm{PhD}$ project are described in this thesis, entitled: 'Breaking down the barriers: The effects of aging, gut microbiota and bile acids on intestinal health'. 


\section{LIST OF PUBLICATIONS}

\section{This thesis}

Van der Lugt, B., Rusli, F., Lute, C., Lamprakis, A., Salazar, E., Boekschoten, M. V., Hooiveld, G. J., Müller, M., Vervoort, J., Kersten, S., Belzer, C., Kok, D. E. G., Steegenga, W. T., Integrative analysis of gut microbiota composition, host colonic gene expression and intraluminal metabolites in aging C57BL/6J mice. Aging (Albany NY). 2018 May 16;10(5):930-950. doi: 10.18632/aging.101439

Van der Lugt, B.*, Van Beek, A. A.*, Aalvink, S., Meijer, B., Sovran, B., Vermeij, W. P., Brandt, R. M. C., de Vos, W. M., Savelkoul, H. F. J., Steegenga, W. T., Belzer, C. (2019). Akkermansia muciniphila ameliorates the age-related decline in colonic mucus thickness and attenuates immune activation in accelerated aging Ercc1-/L7 mice. Immunity \& Ageing. 2019 Mar 8; 16:6. doi: 10.1186/s12979-019-0145-z. eCollection 2019

Van der Lugt, B., Vos, M.C.P.., Grootte Bromhaar, M., IJssennagger, N., Vrieling, F., Steegenga, W. T. The effects of sulfated secondary bile acids on intestinal health in the context of inflammatory bowel disease. Submitted for publication.

Elzinga, J.*, van der Lugt, B.*, Belzer, C., Steegenga, W. T. Characterization of increased mucus production of HT29-MTX-E12 cells grown under Semi-Wet interface with Mechanical Stimulation. Submitted for publication.

\section{Other}

Kok, D. E. G., Rusli, F., van der Lugt, B., Lute, C., Laghi, L., Salvioli, S., Picone, G., Franceschi, C., Smidt, H., Vervoort, J., Kampman, E., Müller, M., Steegenga, W. T. (2018). Lifelong calorie restriction affects indicators of colonic health in aging C57BI/6J mice. The Journal of Nutritional Biochemistry, Jun;56:152-164. doi:10.1016/j.jnutbio.2018.01.001. Epub 2018 Mar 20.

*these authors share first authorship 
OVERVIEW OF COMPLETED TRAINING ACTIVITIES

\section{Discipline specific activities}

Courses

\begin{tabular}{|l|l|l|l|}
\hline Name & Organizer & Location & Year \\
\hline $\begin{array}{l}\text { The Intestinal Microbiome and Diet in Human and Animal } \\
\text { Health }\end{array}$ & VLAG & Wageningen, NL & 2017 \\
\hline Epigenesis and Epigenetics & VLAG & Wageningen, NL & 2017 \\
\hline
\end{tabular}

Conferences and symposia

\begin{tabular}{|l|l|l|l|}
\hline Name & Organizer & Location & Year \\
\hline $\begin{array}{l}\text { Exploring Human Host-Microbe Interac- } \\
\text { tions in Health and Disease }\end{array}$ & Wellcome Genome Campus & $\begin{array}{l}\text { Cambridge, } \\
\text { UK }\end{array}$ & 2018 \\
\hline Gut Day & $\begin{array}{l}\text { Laboratory of Microbiology, } \\
\text { WUR }\end{array}$ & $\begin{array}{l}\text { Wageningen, } \\
\text { NL }\end{array}$ & 2018 \\
\hline $\begin{array}{l}\text { Wageningen Molecular Life Sciences } \\
\text { Seminar Series }\end{array}$ & WUR & $\begin{array}{l}\text { Wageningen, } \\
\text { NL }\end{array}$ & $\begin{array}{l}\text { 2018- } \\
2019\end{array}$ \\
\hline NWO Life Sciences & NWO & Bunnik, NL & 2019 \\
\hline Mucins in Health and Disease & Kings College London & $\begin{array}{l}\text { Cambridge, } \\
\text { UK }\end{array}$ & 2019 \\
\hline $\begin{array}{l}\text { Gut Day } \\
\text { In vitro studies of the human intestinal } \\
\text { microbiota }\end{array}$ & $\begin{array}{l}\text { Laboratory of Microbiology, } \\
\text { WUR }\end{array}$ & $\begin{array}{l}\text { Online } \\
\text { NL }\end{array}$ & 2020 \\
\hline
\end{tabular}

General courses

\begin{tabular}{|l|l|l|l|}
\hline Name & Organizer & Location & Year \\
\hline VLAG PhD week & VLAG & Baarlo, NL & 2017 \\
\hline Project and time management & WGS & Wageningen, NL & 2018 \\
\hline Brain friendly working and writing & WGS & Wageningen, NL & 2018 \\
\hline Teaching lab practicals & WGS & Wageningen, NL & 2018 \\
\hline Scientific writing & WGS & Wageningen, NL & 2018 \\
\hline Reviewing a Scientific paper & WGS & Wageningen, NL & 2018 \\
\hline Supervising Gifted MSc students & WGS & Wageningen, NL & 2019 \\
\hline Supervising MSc students & WGS & Wageningen, NL & 2019 \\
\hline Career Assessment & WGS & Wageningen, NL & 2020 \\
\hline
\end{tabular}


Optionals

\begin{tabular}{|l|l|l|l|}
\hline Name & Organizer & Location & Year \\
\hline Preparation of research proposal & VLAG & Wageningen, NL & 2016 \\
\hline $\begin{array}{l}\text { Weekly group meetings NMG/Nutrition and Biol- } \\
\text { ogy scientific meetings }\end{array}$ & WUR & Wageningen, NL & $2016-2021$ \\
\hline Bimonthly Gut Health Meeting/Journal Club & WUR & Wageningen, NL & $2017-2021$ \\
\hline Staff seminars Human Nutrition and Health & WUR & Wageningen, NL & $2016-2021$ \\
\hline PhD study tour to the UK & & UK & 2017 \\
\hline
\end{tabular}




\section{COLOPHON}

The research described in this thesis was financially supported by the NWO Graduate Programme on Food Structure, Digestion and Health (grant no. 022.006.009), the European Union's Seventh Framework Programme (FP7/2007- 2011) IDEAL-aging (grant no. 259679), the NWO Spinoza Award and SIAM Gravity Grant (grant no. 024.002.002), the Nutricia Research Foundation (grant no. 2018-25) and the NWO Building Blocks of Life grant (grant no. 737.016.003).

The financial support from Wageningen University for printing this thesis is gratefully acknowledged.

Cover design Ruben Otten

Lay-out Benthe van der Lugt

Printed by Digiforce | ProefschriftMaken

(OBenthe van der Lugt, 2021 